\title{
NUCLEAR TECHNOLOGY DIVISION ANNUAL PROGRESS REPORT FOR PERIOD ENDING JUNE 30, 1971
}

\section{Aerojet nuclear Company \\ NATIONAL REACTOR TESTING STATION Idaho Falls Idaho - 8340 I \\ DATE PUBUISHED OCTOBER 1971}

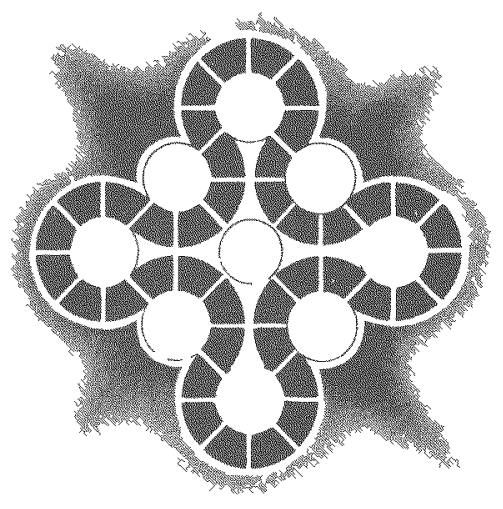




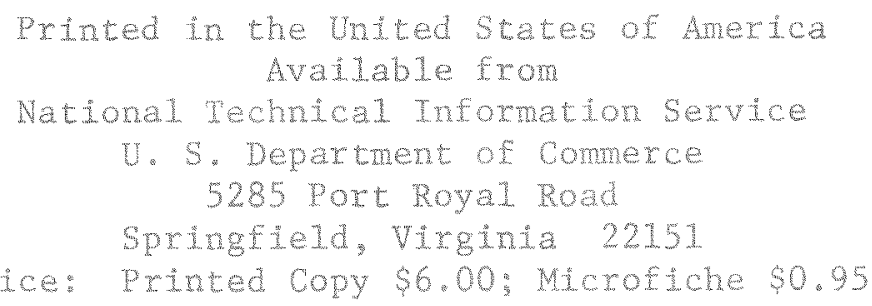

\section{LEGAL NOTICE}

This report was prepered as ais account af work sponsored by the united states Government. Neither the Unted States mon he United states Atonic Energy Commission, nor any of their employees, nor any of thetr cantractors. subcontractors, or the r employes, makes any warranty, express or implied. or assumes any legal liablliy ar reaponsibility for the accuracy, campleteness or usembess of am latormation, apparatus, product or process disw closed, of represents that its use would not intrige privately owned pights. 


\section{DISCLAIMER}

This report was prepared as an account of work sponsored by an agency of the United States Government. Neither the United States Government nor any agency Thereof, nor any of their employees, makes any warranty, express or implied, or assumes any legal liability or responsibility for the accuracy, completeness, or usefulness of any information, apparatus, product, or process disclosed, or represents that its use would not infringe privately owned rights. Reference herein to any specific commercial product, process, or service by trade name, trademark, manufacturer, or otherwise does not necessarily constitute or imply its endorsement, recommendation, or favoring by the United States Government or any agency thereof. The views and opinions of authors expressed herein do not necessarily state or reflect those of the United States Government or any agency thereof. 


\section{DISCLAIMER}

Portions of this document may be illegible in electronic image products. Images are produced from the best available original document. 
NUCLEAR TECHNOLOGY DIVISION ANNUAL PROGRESS REPORT FOR PERIOD ENDING JUNE 30, 1971

\author{
R. M. Brugger \\ MANAGEF
NUCLEAR TECHNOLOG DIVISION \\ E. E. Burdick W. C. Francis R. L. Heath J. F. Kunze \\ REACTOR TECHNOLOGY METALLURGY AND MATERIALS PHYSICS REACTOR DEVELOPMENT
BRANCH
}

\title{
AEROETT NUCLEAR COMPANY
}

Date Published - October 1971

PREPARED FOR THE U. S. ATOMIC ENERGY COMMISSION IDAHO OPERATIONS OFFICE

UNDER CONTRACT NO. AT $(10-1)-1375$ 


\begin{abstract}
This document is a report of progress on technical programs of the Nuclear Technology Division of Aerojet Nuclear Company* for FYTI ending June 30,1971 . It contains abstracts or expansions of abstracts of papers which have been published within the year. In these cases preprints or reprints of the articles are available. Results of work in progress are also reported; since this work is of a preliminary nature, the authors should be contacted before including any reference to these works in other publications.

On June 1, 1970 a reorganization within Idaho Nuclear Corporation split the Nuclear and Chemical Technology Division and formed the Nuclear Technology Division. This new Division contained some parts of the old Nuclear Technology Branch plus Metallurgy and Materials Science Branch and Reactor Technology Branch. This year's annual is being assembled at the Division level instead of at the Branch level. Previous reports related to the Nuclear Technology Branch are IN-1218, IN-1317 and IN-1407; previous reports related to the Metallurgy and Materials Science Branch are IN-1228, IN-1335, and IN-1437, and that related to the Reactor Technology Branch is ANCR-1013.
\end{abstract}

*On July I, 1971 Aerojet Nuclear Company took over the responsibilities of Idaho Nuclear Corporation. 


\section{CONTENTS}

ABSTRACT . . . . . . . . . . . . . . . . . . ii

A. NEUTRON INTERACTIONS WITH NUCLEI . . . . . . . . . . . I

Total Neutron Cross Sections of the Cm Isotopes from 0.01

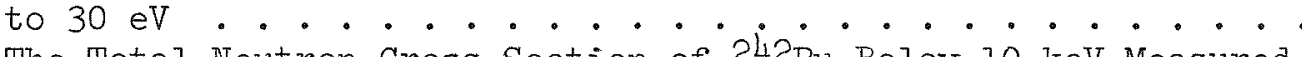

The Total Neutron Cross Section of $242 \mathrm{Pu}$ Below $10 \mathrm{keV}$ Measured

at Liquid Nitrogen Temperature . . . . . . . . . . . . . II

The Total Neutron Cross Section of $\mathrm{Cm}-244$ from 0.01 to

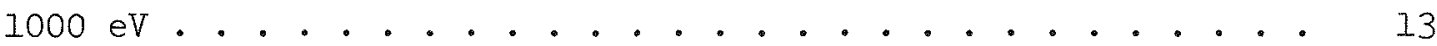

Fission Product Integral Measurements in the CFRMF . . . . . 15

Estimates of Fission Product Activities for Short

Irradiation and Decay Times . . . . . . . . . . . . . 20

Sample Preparations . . . . . . . . . . . . . 26

B. NUCLEAR PROPERTIES . . . . . . . . . . . . . . . 27

Neutron Capture $\gamma$-Ray Studies using the 2-keV Neutron Beam

Facility. . . . . . . . . . . . . . . . . . . . . . .

A Survey of Nonrotational States of Deformed Odd-A Nuclei

$(150<A<190)$. . . . . . . . . . 42

Note on the Decays of $44 \mathrm{~m}_{\mathrm{Sc}-44 \mathrm{Sc}}$ and $44 \mathrm{Ti}-44 \mathrm{Sc} . . . . . .43$

Decay Scheme Studies of $18 \mathrm{~min} 141 \mathrm{Ba}$ and $11 \mathrm{~min} 142 \mathrm{Ba}$. . . . 48

Internal Conversion Coefficients in the Decay of $160 \mathrm{~Tb} . . .249$

Gamma-Ray Transitions De-Exciting States Near $1 \mathrm{MeV}$ in $183 \mathrm{~W}$

Observed in the $(n, \gamma)$ Reaction ............. 54

The Decay of $183 \operatorname{Re}$ i . . . . . . . . . . . . . 57

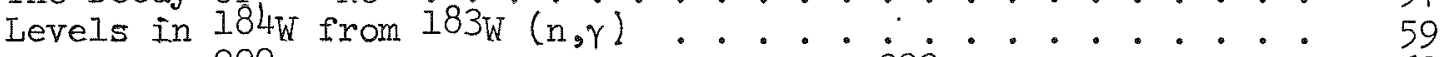

Levels in ${ }^{229} \mathrm{Th}$ Excited in the $\alpha$-Decay of $233 \mathrm{U} . . . . .268$

Levels in 23lTh Excited in the a-Decay of 235 . . . . . . 75

Limits on the Precision of Measured Relative Internal-

Conversion Coefficients - 192Ir . . . . . . . . . . 77

Gamma Rays Emitted by the Fissionable Nuclides and Associated
Isotopes. . . . . . . . . . . . . . . . . . . 86

Spallation Reaction Products from Irradiation of Gold by

C. MATERIALS . . . . . . . . . . . . . . . . 91

Short and Intermediate Range Crystal Potentials in Terms of Valence Symmetry Coordinates . . . . . . . . . . . 9 93

Calculation of Force Constants in Molecules and Crystals . . . 95

Transmission Electron Microscopy Surveillance of Fatigue and

Irradiation Damage Interaction . . . . . . . . . . . 96

Elevated Temperature Fatigue ............... 105

Calculation of Creep Stress in Power Burst Facility (PBF)

Fuel Grains . . . . . . . . . . . . . . . . . . . . . 123

Experimental Breeder Reactor (EBR-II) Mark II Fuel Examination

By Microprobe . . . . . . . . . . . . . . . . . 127

Examination of Waste Calciner Fuel Nozzle Caps by Microprobe. . 129 
Irradiation Testing of Sample Fuel Plates to Very High

Burnups . . . . . . . . . . . . . . . . . . . . .

Irradiation Testing to Verify Failure Predictions Produced by

Post-Irradiation Testing . . . . . . . . . . . . 135

Corrosion of Aluminum in Critical Facilities and Canals . . . 137

FTR Fuel Oxide Investigation ${ }^{4}$ - Bulk Transformation of

Aluminum Oxide .................. . I40

Metallurgical Support for BWR-FLECHT. . . . . . . . . . . . 142

Metallurgical Support for PWR-FLECHT. . . . . . . . . . . . I49

Metallurgical Examinations of Stainless Steel Samples from the

Waste Calcining Facility . . . . . . . . . . . . . . .

Metallographic Examination of Borated Stainless Steel Corrosion

Coupons . . . . . . . . . . . . . . . . 155

D. TEST REACTOR OPERATIONS SUPPORT . . . . . . . . . . 159

Measurement of $\mathrm{B} / \mathrm{l}$ in the MTR Phoenix Core . . . . . . 161

ETR Power Coefficient Measurements . . . . . . . . . 163

ETR Bulk Water Temperature Coefficient . . . . . . . . . 165

ATR and ETR Instrumentation Upgrade . . . ......... 168

ATR Level Channel Modification . . . . . . . . . . . 170

Effects of Installing a Large In-Pile Tube in the ATR . . . . . 173

Gamma Heat Measurements in the Large In-Pile Tube Experiment

Mock-Up at the ATRC . . . . . . . . . . . . . . 174

ATR Loop Temperature Effects . . . . . . . . . . . . 176

Prediction of Shim Positions for Criticality for ATR Startups . 178

Effects of Installing the ATR Beam Tube-Rabbit Facility . . . I79

Expanded ATR Data System . . . . . . . . . . . . . I8I

Reactivity Meter Checkout and Use . . . . . . . . . 183

Reactivity Meter Theory and Design ............. 186

Reactor Operator Certification Program . . . . . . . . . 197

Non-Destructive Measurement of Oxide Film Buildup on

Reactor Fuel Elements . . . . . . . . . . . . . . 199

Post-Irradiation Examination of Oxide Films on ETR Fuel

Elements. . . . . . . . . . . . . . . 206

Clad Thickness Measurements of ATR Fuel Elements . . . . . 213

Surveillance of ATR Reactor Materials . . . . . . . . . . 223

Preparation of Test Reactor Fuel Plates Containing Fissium. • . 231

Compressive Yield Strength Determinations on ETR Fuel Plates

Clad with 2219 Aluminum . . . . . . . . . . . . . 233

Performance Evaluation of Fuel Elements used in Startup

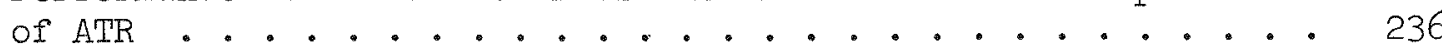

Phase I Report for the Safety and Waste Management Studies

Priority Number 22, Study 15, Health Physics Instrumentation

Reliability and Performance Study . . . . . . . . . . 239

ATR Extended Burnup Program Support............. 243

Fuel Procurement Support . . . . . . . . . . . . 244

Shelf Life of UAl . . . . . . . . . . . . . . 245

Delayed Neutron Fission Break Monitor . . . . . . . . . 247 
E. REACTOR DEVELOPMENTI . . . . . . . . . . . . $25 I$

Portable Neutron Radiographic Reactor . . . . . . . . . 253 Transfer Cask Designs for the Fuel Element Failure

Propagation Loop (FEFPL). . . . . . . . . . . . 258

Fuel Element Failure Propagation Loop Instrumentation . . . . 259

Preliminary Design for LOFT 5-I/2 Foot Core I. . . . . . . . 261

Flowing Gas Tests for the Gas Core Reactor Concept. . . . . . 267

Gamma Heating Rate Measurements in the Spherical Cavity

Reactor Critical Experiment . . . . . . . . . . . . 271

Measurements of Control Methods Concepts for the Gas Core

Reactor . . . . . . . . . . . . . . . 274

Fission Chamber Analysis of the CFRMF . . . . . . . . . 277

Comparison of Spectrum Determinations in the CFRMF. . . . . . 279

Consistency Checks on Evaluated CFRMF Spectra . . . . . . . . 281

Evaluation of the $239 \mathrm{Pu}$ Cross Sections in the Resonance Region

Below $45 \mathrm{eV}$ for the ENDF/B Version III Data File. . . . . . 283

U-235 Resonance Parameters for ENDF/B-III . . . . . . . . . 292

Revision of the Low Energy ENDF/B Files for $244 \mathrm{~cm}$. . . . . 304

Pù-242 Resonance Parameters for ENDF/B-III. . . . . . . . 306

Reactor Physics Code Modifications for EIDF/B Cross

Section Data. . . . . . . . . . . . . . . . 309

Fast Critical Benchmark Calculations using ENDF/B Version

II Data . . . . . . . . . . . . . . . . . . . . . 311

Monte Carlo Development ................ 313

Monte Carlo Calculations for a Fuel Pin Cell. . . . . . . . . 314

Methods used for Physics Analysis of the Power Burst Facility

and Computed Results for the Design Core. . . . . . . . . 315

IOFT Core I Physics Methods Verification. . . . . . . . . 316

Metallography for LOFT . . . . . . . . . . . . . 377

PIutonium Detection in Bone Sections. . . . . . . . . . . 320

Design and Performance Analysis of the PBF Experiment Loop

for Thermal Volume Swell. . . . . .......... 324

PBF Experiment Loop Control Study . . . . . . . . . . 332

Design and Reliability Analysis of LOFT PPS Logic Circuitry . . 342

F. RESEARCH AND DEVELOPMENT FOR NONREACTOR RELATED DISCIPLINES • - 357

Large Scale Irradiation of Biological Samples for

Mercury Analysis. . . . . . . . . . . . . . . 359

Aerospace Medical Activation Analysis Program . . . . . . . . 362

Elemental Determinations in Idaho Wildlife Samples and in Air

Filter Samples . . . . . . . . . . . . . 364

Telemetered Profiling Isotopic Snow Gauge . . . . . . . . . 375

PPM Measurements of Iron-In-Oil By Radioactive X-Ray

Fluorescence Analysis . . . . . . . . . . . . 377

G. EXPERIMENTAL FACILITIES . . . . . . . . . . . . . 379

Upgrade of the ETR Pneumatic Rabbit Irradiation Facility. . . 381

Comparison of Spectrum Determinations in the CFRMF. . . . . 383

Subcritical Multiplication of $252 \mathrm{Cf}$ Neutrons . . . . . . 385

Split Table Reactor Startup and Autorod Testing . . . . . 388 
Electromagnetic Mass Separator . . . . . . . . . . 392

New Metallurgy Facility . . . . . . . . . . . . . 393

The Oak Ridge Electron Linear Accelerator Radioactive Sample

Changer . . . . . . . . . . . . . . . . 394

The Need for High Flux Neutron Beams. . . . . . . . . . 399

H. TECHNIQUES AND INSTRUCTIONS . . . . . . . . . . 401

I. GAMMA-RAY AND NEUTRON SPECTRISCOPY . . . . . . . . . 403

Precise Comparison and Measurement of Gamma-Ray Energies

With a Ge(Li) Detector III: $1300-3600 \mathrm{keV}$. . . . . .

Precise Comparison and Measurement of Gamma-Ray Energies

With a Ge(Li) Detector II: 400 to $1300 \mathrm{keV}$....... 408

Determination of Relative Photopeak Efficiency and

System Linearity for Ge(Li) Gamma-Ray Spectrometers. . . 421

Calibration of the Efficiency of a Si(Li) Photon

Spectrometer in the Energy Region 5 to $125 \mathrm{keV}$. . . . . 422

Calibration of Ge(Li) Spectrometer Systems . . . . . 423

Prompt Neutron Activation Analysis with a 252Cf Source. . 424

A Technique for Assay of I-10 Bottles of Plutonium

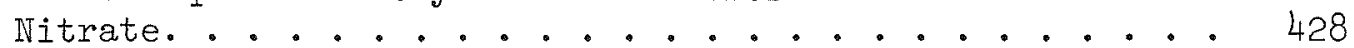

Evaluation of a Gas Seintillation Counter for Measuring

Fission Cross Sections in High Alpha Environment . . . . 429̄

Fast Neutron Measurement Methods . . . . . . . . . 432

Analysis of Dow Chemical Company, Rocky Flats Division

Plutonium Waste Barrels ... . . . . . . . . 436

Plastic Irradiation Capsules . . . . . . . . . . 445

II. EXPERIMENTAL REACTOR TECHNOLOGY . . . . . . . . . 448

Fuel Cladding Thickness Measurements Using X-Ray

Fluorescence Spectroscopy. . . . . . . . . . . 448

Iron Influence on Fast Neutron Flux Distributions. . . . 450

In-Plant Fuel Clad Thickness Measurement Facility Using

the Beta Autoradiography Technique . . . . . . . . . 453

Use of Metal Alloys for Neutron Spectrum Measurements. • . 457

Improvements in Foil Activation Data Analysis. . . . . . . 460

Fabrication and Evaluation of Samples for the Irradiation

Portion of the FBR Physics Constants Program . . . . . . 462

Non-Destructive Analysis of Fuel Pins in the ARMF. . . . 464

Preparation of Standards for Beta Autoradiography. . . . . 469

Preparation of Test Capsules for NASA. . . . . . . . 469

Forming AU-NI Alloy Bellows . . . . . . . . . . . 470

Preparation of Transducer Housings by EB Welding . . . . 472

Fabrication of Flux Monitor Foils. . . . . . . . . 475

Chopper Test Instrumentation for Use in Quality Assurance

and Maintenance. . . . . . . . . . . 478

Automatic Underwater Radioactive Wire Scanner. . . . . . 483 
III. APPLIED MATHEMATICS AND COMPUTER SOFTWARE. • . . . . . 487

New Software for Gamma-Ray Analysis. . . . . . . . . 487 Automatic Peak Location and Library Look-Up. . . . . . . 489 Spectrum Stripping Using a PDP-9. . . . . . . . . . 490 Computer Program for the Analysis of Continuous

Electron Spectra................ 492 Analysis of Conversion-Electron Spectra. . . . . . . 494 Plotting Routine for Gamma-Ray Spectra . . . . . . . 498 "Best" Level Energy Program. . . . . . . . . . . . 500 Automated Cross Section Analysis Program (ACSAP) . . . . 502 Spectrum Calculations for the CFRMF. . . . . . . . . 507 Comparison of Calculated and Measured Reactivities in the CFRMF. . . . . . . . . ........ 510 Diffusion Theory Calculations for Various Sleeve Configurations of the CFRMF. . . . . . . . . . 513 Snow Gauge Control Software. . . . . . . . . . . 519 Computer Reliability Assurance . . . . . . . . . 528 NASA X-Ray Fluorescence and Diffraction Software . . . 529 Real-Time Debugging Software for the PDP-8 . . . . . . 530 Using Function Box for a Backup Control Device. . . . . 531 Device-Level Software for a PDP-9 or -15 Serial Data Multiplexer (IT-19) and a Computek Display Terminal. . . . 533 A Small Floating Point Arithmetic Package for the PDP-8 Family of Minicomputers. . . . . . . . . . . . . 534 A Comprehensive Basic Data Acquisition and Control Monitor for the PDP-9 and PDP-15 Computers . . . . . . . 535

I. COOPERATIVE PROGRAMS WITH EDUCATIONAL INSTITUTIONS. . . . . 537 Cooperative Programs with Educational Institutions. . . . . 539

J. RELATED ACTIVITIES OF THE BRANCH PERSONNEL . . . . . . . 543 Related Activities of Division Personnel. . . . . . . . 545

K. PUBLICATIONS. . . . . . . . . . . . . . . 547

Presentation of Technical Papers. . . . . . . . . . 549 Papers Published in the Open Literature . . . . . . . . . 553 Reports Issued During Fy 1971 . . . . . . . . . . 555

\section{FIGURES}

1. Neutron transmission of the mixed $\mathrm{Cm}$ isotopes as a function of time of flight......................

2. Neutron transmission of the mixed $\mathrm{Cm}$ isotopes as a function of time of flight................... 
3. Neutron transmission of the mixed $\mathrm{Cm}$ isotopes as a function of time of flight. . . . . . . . . . . . . . . .

1. The number of resonances below energy $E$ (keV) as a function of energy for 242Pu. . . ............ 12

1. Number of nuclear levels observed in $\mathrm{Cm}-244$ as a function of neutron energy. . . . . . . . . . . . . . 14

1. Integral capture cross section relative to that of gold versus target neutron number. . . . . . . . . . . . . . 16

1. Fission product activities for the thermal irradiation of

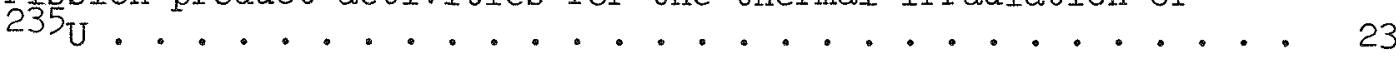

2. Fission-product decay ( 30 sec. irradiation)........ 24

3. Fission-product decay (60 sec. irradiation). . . . . . 25

1. The high-energy portion of the prompt $\gamma$-ray spectra resulting from the capture of thermal and $2-\mathrm{keV}$ neutrons in natural tungsten .................... 41

1. Decay scheme of $44 \mathrm{~m}_{\mathrm{Sc}}$ and ${ }^{44} \mathrm{Sc}$ as proposed by Okano et al. . 46

2. Decay scheme of $44 \mathrm{Ti}$ as proposed by Ristinen and Sunyar. . . 46

1. Portions of internal-conversion electron spectrum from

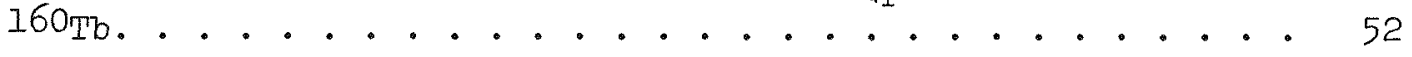

2. Gamma-ray spectrum of $160_{\mathrm{Tb}}$............... 53

1. A portion of the prompt $\gamma$-ray spectrum resulting from $2-\mathrm{keV}$ neutron capture in $182_{\mathrm{W}}$............... 56

1. A portion of the prompt $\gamma$-ray spectrum resulting from $2-\mathrm{keV}$

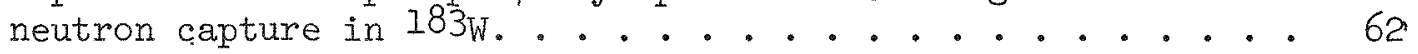

2. A plot of $I_{\gamma} E^{-5}$ versus the energy, $E_{\gamma}$, of the prompt $\gamma-$ ray transitions ${ }^{\gamma} \gamma$ observed from $2-\mathrm{keV}$ neutron capture in $183 \mathrm{~W} . .63$

3. Levels in ${ }^{184} \mathrm{~W}$ populated by the ${ }^{183} \mathrm{~W}(\mathrm{n}, \gamma)$ reaction ..... 64

1. Proposed decay scheme for ${ }^{233}$ U. . . . . . . . . . 73

2. Coriolis-mixing analysis of the energy-level structure of the negative-parity states in 229Th. . . . . . . . . . 74

1. Proposed decay scheme for ${ }^{235} U$. . . . . . . . . . 76

1. Three $K$ conversion electron lines from the decay of ${ }^{192}$ Ir measured at $0.016 \%$ resolution ............ 82 
2. I and $M$ conversion electron lines from the $205-\mathrm{keV}$ transition in 1920s. . . . . . . . . . . . . . .

3. I, $M$ and $N$ conversion electron lines from the $316-\mathrm{keV}$ transition in 192Pt. . . . . . . . . . . . . . 84

4. Lines shapes used in the analysis of the ${ }^{192}$ Ir conversion electron spectra. . . . . . . . . . . . . . . . .

1. Gamma-ray spectrum from gold sample about four days after irradiation with $600 \mathrm{MeV}$ protons. . . . . . . . . . . .

2. Gamma-ray spectrum from gold sample about sixty-five days after irradiation with $600 \mathrm{MeV}$ protons . . . . . . . . .

1. Micrographs showing difference in dislocation cell formation between unirradiated (a) and irradiated (b) 316 SS fatigue specimens... . . . . . . . . . . . . . . . 100

2. Micrographs showing effect of irradiation retarding dislocation cell formation of 304 SS (a) and (b) tested at a total strain range of $0.35 \%$ and $304 \mathrm{~L}$ (Ti) SS (c) and (d) tested at a total strain range of $0.36 \%$.............. I0I

3. Micrographs illustrating the difference in distance between extended dislocations between unirradiated (a) and irradiated (b) 304 SS fatigue specimens tested at $0.35 \%$ total strain range

4. Micrographs showing distribution of voids and/or helium bubbles in $304 \mathrm{SS}$ (a), $304 \mathrm{~L}$ (Ti) SS (b), $316 \mathrm{SS}$ (c), and

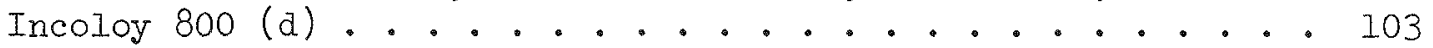

5. Micrographs showing strings of voids probably left behind from moving grain boundary (a) in $304 \mathrm{~L}$ (Ti) and bubbles occur at major grain boundaries in Incoloy 800 (b) . . . . . . . . 104

1. Engineering stress-strain behavior of irradiated and unirradiated stainless steel obtained from constant strain rate testing of hourglass shaped specimens at $700^{\circ} \mathrm{C}$. . . . . . . . 111

2. True stress-strain curves for irradiated and unirradiated stainless steels tested at $700^{\circ} \mathrm{C}$. . . . . . . . . . . 112

3. Irradiated and unirradiated fatigue behavior of Type 304 stainless steel tested at $700^{\circ} \mathrm{C}$ compared with predictions based on tensile data................. 113

4. Irradiated and unirradiated fatigue behavior of Type $304 \mathrm{~L}$ (Ti mod.) stainless steel tested at $700^{\circ} \mathrm{C}$ compared with predictions based on tensile data. 
5. Irradiated and unirradiated fatigue behavior of Incoloy 800 tested at $700^{\circ} \mathrm{C}$ compared with predictions based on tensile data . . . 115

6. Comparison of the pre- and post-irradiated fatigue behavior of several stainless steels tested at $700^{\circ} \mathrm{C}$. . . . . . . II6

7. Comparison of fatigue life of solution annealed and aged 316 stainless steel tested at $593^{\circ} \mathrm{C}$. . . . . . . . . . 117

8. Cyclic hold time testing of unirradiated stainless steel

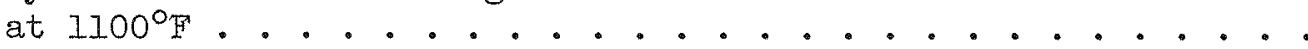

9. Comparison of the mode of fatigue crack propagation in two specimens of unirradiated 316 stainless steel . . . . . . .

10. Scanning electron microscope fractographs showing the fatigue surfaces of 316 stainless steel tested at a total strain range of $1.0 \%$ at $1100^{\circ} \mathrm{F}$. . . . . . . . . . . . . .

11. Influence of tensile hold periods on the fatigue life of preand post-irradiated 316 stainless steel tested at $700^{\circ} \mathrm{C}$. .

12. Stress relaxation at various fatigue cycles during hold time period. . . . . . . . . ... . . . . . . . . . .

1. Calculated strain rate $\dot{\varepsilon}$ in PBF fuel grains as a function of time $t$ after the initiation of $1.3 \mathrm{msec}$ power burst for three cases assuming different fuel - insulator contact coefficients and heat capacities . . . . . . . . . . . . . . . . .

2. Creep stress $\sigma$ vs strain rate $\dot{\varepsilon}$ for three different grain sizes calculated for PBF fuel using Folweiler's equation. . . . . .

1. Line scans data for uranium, iron, and chromium across the fuel-cladding interface revealing the chromium enhancement at the interface and iron diffusion into the fuel region . . . . . .

1. Carbon $\mathrm{K} \alpha \mathrm{X}$-ray intensities observed during microprobe point counting on waste calciner fuel nozzle caps . . . . . . .

1. Post-irradiation blister failure-no-failure curve for $\mathrm{UAI}_{x}$ fuels........................

1. Post-irradiation blister failure-no-failure curve for UAI fuels.......................

2. Typical corrosion patterns - normal and inverse plate orientations

1. A cross section macrograph of Tube 11 at the $8 \mathrm{ft}$ elevation from Test $\mathrm{Zr}-3 . . . . . . . . . . . . .$.

2. Comparison of control sample (a) to posttest samples with intermediate attack (b) and severe attack (c)........... 
3. Electron microscope fractography of the ring compression sample from Rod 12 at the $6 \mathrm{ft}$ elevation ........... 146

4. Electron microscope fractography of the ring compression sample from Rod 27 at the 6 ft elevation . . . . . . . . 147

5. Photograph of "Lantern spring" after Test Zr-3. . . . . . . . . 148

1. Coupon $444-27.2$ months exposure . . . . . . . . . . 156

2. Coupon $446-27.2$ months exposure in uranium nitrate - nitric acid solution . . . . . . . . . . . . . 156

3. Control \#690....................... . I57

1. Calibration curve of ETR regulating rod . . . . . . . 166

2. Reactivity change as a function of the temperature of ETR primary coolant water ............... . . I67

1. Distribution of the measured relative gamma heating values around the periphery of the $\mathbb{N E}$ large in-pile tube (numbers in the center of the flux trap) and the relative fuel element power distribution around the $\mathrm{NE}$ lobe (numbers in the fuel element positions)................ . 175

1. ATR 1oop temperature reactivity effect as a function of $100 p$ metal-to-water ratio. . . . . . . . . . . . . 177

I. Schematic representation of beam tube mock-up . . . . . . . . 180

1. Block diagram of the components of the expanded ATR Data System. . . . . . . . . . . . . . . . . . . 182

1. Reactivity as a function of time during the ATR regulating rod calibration .................. 184

2. Regulating rod worth as a function of position. . . . . . . 185

3. Block diagram of reactivity meter . . . . . . . . . . 194

4. Simplified circuit diagram. . . . . . . . . . . 195

5. Simplified circuit diagram for source and power feedback. . . . 196

1. Probe drift compensation circuit. . . . . . . . . . 201

2. Probe assembly, probe housing, probe coil . . . . . . . . 202

3. Readout Fixture . . . . . . . . . . . . . 203

4. Calibration and Standard Fixture. . . . . . . . . 204

5. Complete Instrumentation System . . . . . . . . . . . 205 
1. Recurring spalling patterns observed on element E255H . . . . 208

2. Oxide films typical of fuel plates of element E255H . . . . 210

3. Two mil diameter bumps in oxide on side plate of element

$\mathrm{E} 462 \mathrm{H}$. . . . . . . . . . . . . . . 2 21

4. Oxide films typical of fuel and side plates of element

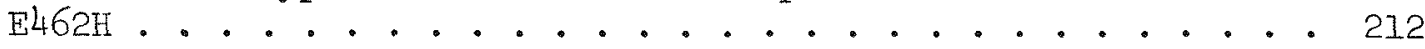

1. Cutaway view of the Nuclear Test Gage (NTG) as modified to irradiate ends of $8 \mathrm{ATR}$ fuel element simultaneously . . . . 216

2. An actual beta autoradiographs of the bare fuel core pieces are shown .................... 217

3. Typical calibration for conversion of film densities to clad thickness. . . . . . . . . . . . . . . 218

4. Thin clad spot width as measured on 66 type $7-F$ ATR fuel elements. . . . . . . . . . . . . . . . 219

5. Thin clad spot length as measured on 66 type 7-F ATR element . . . . . . . . . . . . . . . 220

6. Plot of the valves of minimum clad thickness measured on 66 type 7-F fuel elements. . . . . . . . . . . . 221

7. Plot of the valves of minimum clad thickness measured on 99 standard type ATR fuel elements . . . . . . . . . 222

1. Beryllium irradiated at $100^{\circ} \mathrm{C}$ to fluence of $1.13 \times 10^{22}$ $\mathrm{n} / \mathrm{cm}^{2} \mathrm{E}>1 \mathrm{MeV}$ and compression tested. . . . . . . . 227

2. Density change in irradiated 1100 Al-MTR skirt plate. . . . . 228

3. Strength and ductility of irradiated 1100 Al-MTR skirt plate. . 229

4. Aluminum showing difference in void density at two fluence values (a) at $1.8 \times 10^{22} \mathrm{n} / \mathrm{cm}^{2}(\mathrm{E}>1 \mathrm{MeV})(\mathrm{b})$ at $5.6 \times 10^{22}$ $\mathrm{n} / \mathrm{cm}^{2}(\mathrm{E}>\mathrm{I} \mathrm{MeV}) . . . . . . . . .230$

1. Subpress used for compression testing of this plates. . . . 234

2. Compression stress-strain curve at room temperature comparing ETR UAlX composite clad with 2219 aluminum compared with standard ETR UAIX fuel plates . . . . . . . . . . 235

I. Blister test results. . . . . . . . . . . . . 238

1. ATR delayed neutron count from the exit cooling water during Cycle 9B.................... . 249 
1. Complete industrial neutron radiography facility ready for operation ................. . 2 256

2. Cutaway diagram of the portable neutron radiography . . . . 257

1. Core layout . . . . . . . . . . . . . . 266

1. Gas core nuclear rocket concept . . . . . . . . . . . 268

2. Typical cavity flow tests . . . . . . . . . . . 269

3. Iso-density plot of central gas concentration in the cavity . . 270

1. Diagram of Spherical Gas Core Reactor showing gamma heating measurement detail . . . . . . . . . . . 272

2. Gamma dose rate in beryllium oxide reflector. . . . . . . 273

1. Measurements of Control Concepts for the Gas Core . . . . . 276

I. CFRMF neutron spectrum . . . . . . . . . . . 280

1. The total, fission and capture cross sections of ${ }^{239} \mathrm{Pu} . . . .289$

2. The total, fission and capture cross sections of ${ }^{239} \mathrm{Pu}$. . . . 290

3. The total, fission and capture cross sections of ${ }^{239} \mathrm{Pu} . . . .291$

1. Total, fission, and capture cross sections for U-235. . . . . 296

2. Cross Sections of U-235 from 7 to $15 \mathrm{eV}$. . . . . . . 298

3. Cross sections of U-235 from 14 to $22 \mathrm{eV}$. . . . . . . 300

4. Cross sections of U-235 from 21 to $29 \mathrm{eV}$. . . . . . . 302

1. Sport weld of $\mathrm{Ti}$ to $\mathrm{Zr}-4$ tube . . . . . . . . . 318

2. Junction laser welded to $\mathrm{Zr}-4$ tube........... 318

3. Cross section through two spot welds. . . . . . . . . 319

4. Titanium sheath laser welded to $\mathrm{Zr}-4$ tube . . . . . . . 319

1. Contact neutron-induced autoradiograph showing bands, starproducing aggregates and diffusely distributed fission fragment tracks in the lumbar vertebral body from a dog given $0.93 \mu \mathrm{Ci}$ of 239 Pu.kg and killed 1288 days later .......... 323

2. Detailed neutron-induced autoradiograph of portions of two bone spicules, showing fission fragment tracks distributed mostly upon bone surfaces in the lumbar vertebral body from a dig given 2.6 $\mu \mathrm{Ci}$ of $239 \mathrm{Pu} / \mathrm{kg}$ and $\mathrm{killed} 28$ days later 
1. Simplified experiment loop system. . . . . . . . . . 330

2. Thermal swell volume and flow for maximum conditions. . . . 331

1. Sketch of System Simulated on Hybrid Computer . . . . . . . 340

2. Sketch Defining Flows and Pressures............ 341

1. System I block diagram. . . . . . . . . . . . . 348

2. System II block diagram . . . ........... . . 349

3. System III block diagram. . . . . . . . . . . . 350

4. Reliability -- System I . . . . . . . . . . . 351

5. Reliability -- System II. . . . . . . . . . . . 352

6. Reliability - System III . . . . . . . . . . . . 353

7. Plant availability - System I. . . . . . . . . . . 354

8. Plant availability -- System II . . . . . . . . . . 355

9. Plant availability -- System III. . . . . . . . . . 356

1. Materials Testing Reactor lattice plan. . . . . . . . . 361

I. . . . . . . . . . . . . . . . . 366

2. . . . . . . . . . . . . . . . . . 367

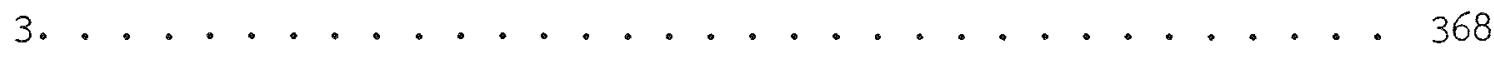

4. . . . . . . . . . . . . . . . . 370

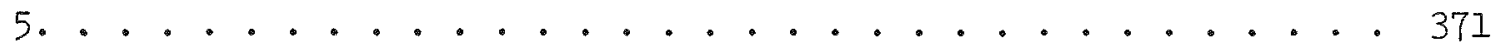

6................................ 371

7.......................... . . . . 372

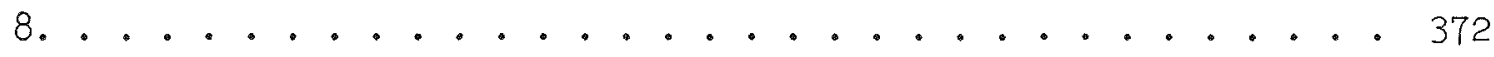

1. Sample of plotter output from telemetered profiling isotopic snow gauge ................. . . 376

1. Exploded view of the ETR pneumatic rabbit shielded irradiation section . . . . . . . . . . . . 382

1. CFRMF neutron spectrum. . . . . . . . . . 384 
1. Diagram of the Nuclear Test Gage showing the neutron radiography collimator diverging from the right side and the californium neutron source positioned in the central tube near the center................... . 386

2. Thermal flux at $40^{\prime \prime}$ image plane from $\mathrm{Cf}-252$ in $\mathrm{NTG}$ and from $\mathrm{Cf}-252$ in $\mathrm{H}_{2} \mathrm{O} I \mathrm{mg} \mathrm{CF}-2.3 \times 109 \mathrm{n} / \mathrm{sec} . . . . . . .387$

1. Split table reactor . . . . . . . . . . . . 390

2. Neutron radiography reactor mockup in the Split Table Reactor . . . . . . . . . . . . . . . 391

1. A photograph of the unshielded sample changer . . . . . . 396

2. An assembly of the ORELA sample changer showing the support table, transport cask, and lead shielding . . . . . . . 397

3. A block diagram showing the electronic circuitry for the ORELA sample changer. . . . . . . . . . . . . . 398

1. Partial level scheme of ${ }^{124} \mathrm{Sb}$ showing transitions used to determine FE-DE peak energy differences . . . . . . . . 407

1. Illustration of the cascade-crossover combinations and energy differences involved in the construction of the two basic gamma-ray energy "sets"................. 414

2. Sample spectrum for the measurement of gammamay energies . . . 415

3. Portion of a gamma-ray spectrum illustrating the resolution of the peaks for the measurement of the $1173(60 \mathrm{Co})-1177$ (160Tb) and the $1271(160 \mathrm{~Tb})-1291(59 \mathrm{Fe})$ energy differences . . . . 415

4. Portion of a spectrum showing the resolution of the peaks for the measurement of the $879(160 \mathrm{~Tb})-884(192 \mathrm{Ir})-889\left(46_{\mathrm{Sc}}\right)$ energy differences. .............. 416

1. A schematic drawing of the experimental arrangement utilized for prompt neutron capture gamma-ray assay with the $2-m g$ $252 \mathrm{Cf}$ source using the internal target geometry . . . . . . . 426

2. Design of a gamma-ray collimator for use with the internal target geometry . . . . . . . . . . . . 426

3. Prompt gamma-ray spectrum obtained with a sample consisting of $100 \mathrm{gm}$ of tungsten using the internal target geometry arrangement ................ . . 427

4. Prompt gamma-ray spectrum obtained with a sample consisting of $42.2 \mathrm{gm}$ of basalt using the internal target geometry arrangement .................. . . 427 
1. Bias curves as a function of count rate for single time coincidences and change-coincidence with $252 \mathrm{Cf}$ in the

chamber ................. . . 430

2. Bias curves as a function of count rate for single, true

coincidence and chance-coincidences with $252 \mathrm{Cf}$ and $244 \mathrm{Cm}$ in

the chamber ............... . 431

1. A comparison of a proton-recoil measurement in a beam of neutrons filtered by iron and an infinite media calculation by slowing down theory ................ 435

1. Grid positions for the five Rocky Flats barrels, and for

the standard. . . . . . . . . . . . . . . 440

1. A comparison of a proton-recoil measurement in a beam of neutrons filtered by iron and an infinite media calculation by slowing down theory. . . . . . . . . . . . 452

1. This scintillation scanning facility is completely automated with complete information on each plate read-out on a teletype .................. 455

2. Photographic plate method . . . . . . . . . . . 456

1. Resonance neutron flux monitoring alloy spectrum. . . . . . 458

2. Fast neutron monitoring alloy spectrum. . . . . . . . . 459

1. Flow Data................... . . 461

1. Pictorial view of sample capsule. . . . . . . . . . 463

1. ARMF with experimental apparatus. . . . . . . . . . 466

2. In-core measuring equipment ... . . . . . . . . . . 467

3. Sample traverse measurement . . . . . . . . . . . 468

1. Forming dies and washer before and after forming. . . . . . 471

1. Photo of the inside of the electron beam vacuum chamber . . . 472

2. Transaucer assembly before welding . . . . . . . . 474

3. Welded transducer assembly . . . . . . . . . . . 474

1. Chopper tester model 0571 block diagram . . . . . . . . 479

2. Dynamic chopper tester model 0671 block diagram . . . . . . 480

3. Chopper Tester Model 0571 . . . . . . . . . . . 481

4. Dynamic Chopper Tester Model 0671 . . . . . . . . . 482 
1. Automatic Underwater Radioactive Wire Scanner Block

Diagram . . . . . . . . . . . . . . . 484

2. Automatic Underwater Radioactive Wire Scanner Control and Data System Modules ............... . 485

3. Wire Scanner Teletypewriter Format. . . . . . . . . 486

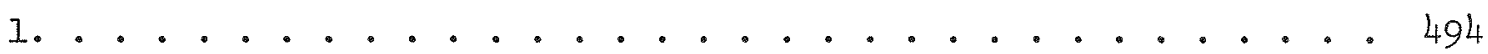

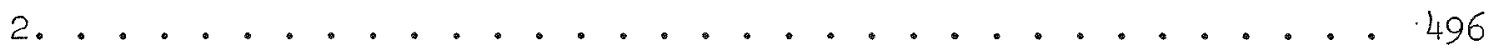

1. Plotting routine for gamma-ray spectra. . . . . . . . . 499

2. Example of a gamma-ray spectrum processed using improved technique . . . . . . . . . . . . . . 4 499

1. Example of the output obtained from the "Best" Level Energy Program; for an adjustment of the 15N levels. . . . . . . 501

1. Much like the classical shape fitting, theoretical cross section data computer using initial guess parameters are first Doppler and resolution broadened, then compared with the input data. . . . . . . . . . . . . 505

2. Input function cards for a short ACSAP run. . . . . . . 506

1. CFRMF- various calculations of the real flux spectrum . . . . 509

1. CFRMF as constructed .................... 515

2. CFRMF - with ${ }^{10}$ B sleeve only............ 517

3. CFRMF - with ${ }^{235}$ U sleeve only ........... 518

4. CFRMF - with both sleeves removed ......... 516

\section{TABLES}

I. Percentage Composition of the Cm Samples. . . . . . . . 6

II. List of Resonance Parameters for the Various Cm Isotopes. . . 7

I. Fission Product Activities for the Thermal Irradiation of

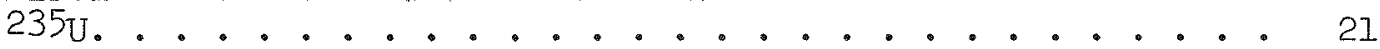

II. Fission-Product Decay . . . . . . . . . . . . 21

III. Fission-Product Decay . . . . . . . . . . . . 22 
I. Intensities of Low-Energy Capture $\gamma$-Ray Transitions Resulting from Thermal- and $2-\mathrm{keV}$ Neutron Capture in

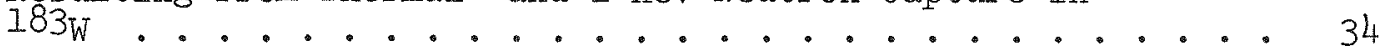

II. Comparison of prompt $\gamma$-Ray Intensities Resulting from $2-\mathrm{keV}$ and Thermal Neutron Capture in 183W . . . . . .

III. Comparison of Prompt $\gamma$-Ray Intensities Resulting from $2-\mathrm{KeV}$ and Thermal Neutron Capture in Tungsten . . . . . . 36

IV. Comparison of the Intensities of the Secondary Capture $\gamma$-Ray Transitions Resulting from Thermal- and 2-keV Neutron Capture in I7IYb. . . . . . . . . . . . . 37

V. Comparison of Prompt $\gamma$-Ray Intensities Resulting from 2-keV and Thermal Neutron Capture in I7IYb. . . . . . . . 38

VI. Comparison of the Intensities of the Secondary Capture $\gamma$-Ray Transitions Resulting from Thermal and 2-keV Neutron Capture in $173_{\mathrm{Tb}}$.................

VII. Comparison of Prompt $\gamma$-ray Intensities Resulting from 2-keV and Thermal Neutron Capture in $173 \mathrm{Yb} . . .2 . . .2 . .40$

I. Energies and Relative Intensities of $\gamma$-Rays Emitted in the Decay of $44 \mathrm{mSc}-44 \mathrm{Sc}$ in Equilibrium ........... 44

II. Energies and Relative Intensities of $\gamma$-Rays Fmitted in the Decay of $4{ }_{T i}{ }^{4}{ }^{S} \mathrm{Sc}$ in Equilibrium ........... 45

III. Measurements of the Conversion Coefficients of the 68- and $78 \mathrm{-keV}$ Transitions Emitted in the Decay of $44 \mathrm{Ti} . . . .245$

I. K Conversion-Electron Data from ${ }^{160_{\mathrm{Tb}}}$......... 51

II. I Subshell Ratios in the Decay of $16 \mathrm{O}_{\mathrm{Tb}} \ldots . . . . . . .51$

I. De-Excitation of States with Energies $>0.9 \mathrm{MeV}$ in $183_{W} . .55$

I. Summary of Lower Excitation States in ${ }^{184} \mathrm{~W}$ and their De-Exciting Transitions . . . . . . . . . . . 60

II. Ratios of Reduced Transition Probabilities for Decay of States in the Gamma-Vibrational Band into the Ground-State Band. . . . . . . . . . . . . . . . . . . .

III. Ratios of Reduced Transition Probabilities for Decay from the $2^{+}$State at $112 \mathrm{lkeV}$.............. 67

IV. Ratios of Reduced Transition Probabilities for Decay of States of the $1130 \mathrm{mkeV} \mathrm{K} \mathrm{K}^{\pi}=2^{-}$Band into the Gamma-Vibrational Band at 903-keV . . . . . . . . . . . . . . . 
I. ${ }^{23} 3_{U}$ Gamma-Ray Energies and Intensities ......... 70

I. Energies and Branching Ratios of Gamma Rays Fmitted in the Decay of $235 \mathrm{U}$.................. 75

I. Some Characteristics of the Decay of ${ }^{192}$ Ir. . . . . . . 80

II. Summary of ${ }^{192}$ Ir Conversion-Electron Line Study . . . . . . 80

III. $I_{1} / I_{3}$ Ratios for 295- and 316-keV Transitions . . . . . . 81

IV. Partial Summary of Preliminary Results of ${ }^{192}$ Ir Conversion-Electron Spectrum .......... 81

I. Identification and History of Specimens Examined. . . . . 99

I. Swelling and Blister Results for INC-16-I Sample Fuel Plates. . . . . . . . . . . . . . . 134

I. Oxide Buildup on Aluminum with ATRC Exposure. . . . . 138

I. Autoclave Test Results. . . . . . . . . . . I4I

I. Summary of Materials Examinations for PWR-FLECHT Tests 8874 and 9573.................. 150

I. Tensile Properties of Type 316 Stainless Steel. . . . . . 153

II. Tensile Properties of Types 304L and 318 Stainless Steel. . 154

I. Measured ETR Power Coefficients of Reactivity . . . . . 163

I. ETR Bulk Water Temperature Coefficient . . . . . . . 165

I. Performance Specifications and Parameter Values . . . . . 193

I. Summary of Certifications . . . . . . . . . . . 197

I. Density Changes in Irradiated 1100 AI MTR Skirt Plate . . . 226

II. Mechanical Properties of Irradiated 1110 AI-MTR Skirt

Plate at Ambient Temperature. . . . . . . . . . 226

I. Operating Parameters of ATR Startup Program Fuel Elements Which Were Destructively Examined . . . . . . . . 237

I. Weight Percent Change in Fuel Powder. . . . . . . . . 246

I. Examples of Neutron Sources . . . . . . . . . 255

I. . . . . . . . . . . . . . . . . 277

I. Spectrum Consistency Comparisons. . . . . . . . . 282 
I. Experimental Operating Conditions. . . . . . . . . . . . 284

II. ${ }^{239} \mathrm{Pu}$ Resonance Parameters ............... 285

III. Total Smooth File. . . . . . . . . . . . 286

I. ${ }^{235}$ U Resonance Parameters. . . . . . . . . . . . 295

I. ${ }^{244} \mathrm{Cm}$ Resonance Parameters Used for ENDF/B Revision. . . . 305

I. Thermal Energy Region Cross Sections of Pu-242 . . . . . . 307

II. Resonance Parameters of Pu-242 . . . . . . . . . 308

I. Configuration and Operating Conditions for Loop Model. . . . 326

II. Thermal Swell Accumulator Performance. . . . . . . . . 327

III. Thermal Swell Design Criteria . . . . . . . . . . 329

I. Failure Rate Information . . . . . . . . . . . 344

I. List of Idaho Wildlife Samples . . . . . . . . . . . 365

II. Analysis of Pheasant Sample \#3I. . . . . . . . . . . 369

III. Elements Detected in Air Filter Sample . . . . . . . . 373

I. Features of the ORELA Radioactive Sample Changer . . . . . 395

I. Camma-Ray Energies from Decay of ${ }^{110 \mathrm{~m}} \mathrm{Ag}$. . . . . . . . . 405

II. Fiela Effect for Ge(Li) Detectors ............ 405

III. Measurement of FE-DE Peak Energy Difference Pre-
liminary Results for $12{ }^{\mathrm{Sb}}$ Gamma Rays . . . . . . . . 406

I. Gamma-Ray Energies Determined Below $400 \mathrm{keV}$. . . . . . 417

II. Summary of Gamma-Ray Energies (Unadjusted) Between
400 and $1300 \mathrm{keV}$. . . . . . . . . . . 418

III. Comparisons of Garma-Ray Sets Above I MeV . . . . . . . . 419

IV. Comparison of Gamma-Ray Energy Sets Below I MeV by
Means of the $646-\mathrm{keV}$ Gamma Ray From 1850s. . . . . . . . 419

V. Final Adjusted Gamma-Ray Energies Between 400 and 1300
keV. . . . . . . . . . . . . . . . . . 420

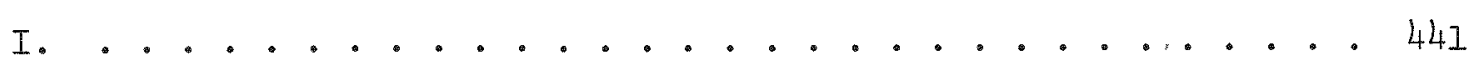

II. . . . . . . . . . . . . . . . 44 
III. . . . . . . . . . . . . . . . 442

IV. . . . . . . . . . . . . . . . 442

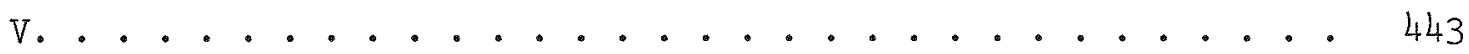

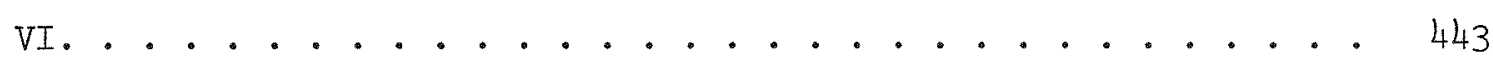

VII. Results of Gamma Ray Counting of Rocky Flats Barrels. . . . . 444

I. . . . . . . . . . . . . . . . . 464

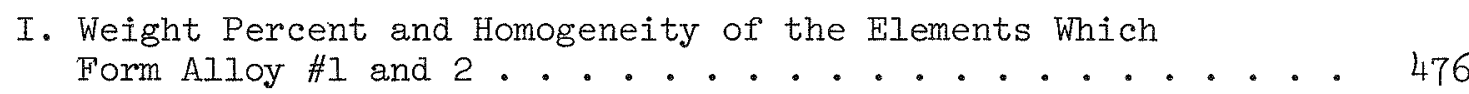

I. Summary of ACSAP Function Cards . . . . . . .... 504

I. A Comparison of Calculated and Measured Reactivities. . . . 512

I. Calculated Reactivities of Samples in the CFRMF - Various

Configurations.............. 514 
A. NEUTRON INTERACTIONS WITH NUCLEI 
-

- 


\author{
J. R. Berreth, F. B. Simpson
}

The curium isotopes are a stepping stone towards the production of Cf-252 which is an intense spontaneous neutron source with considerable application potential. In the reactor production of Cf-252 starting with plutonium, several steps of successive neutron capture through the $\mathrm{Cm}$ isotopes occur. Knowledge of the total neutron cross sections of these isotopes is important to predict Cf-252 buildup. Three different samples of varying $\mathrm{Cm}$ isotopic composition were measured for their total cross sections as a function of neutron energy using the Materials Testing Reactor (MTR) fast chopper. The three different samples all contained large amounts of $\mathrm{Cm}-244(<93 \%)$ and varying amounts of $\mathrm{cm}-243, \mathrm{~cm}-245$, and $\mathrm{cm}-246$.

The Cm samples were prepared by $E$. I. duPont de Nemours \& Company at the Savannah River Plant. All three samples contained small amounts of Pu-240 as a contaminant. After chemical purification the samples were ignited to the oxide and prepared for cross section measurements in a manner similar to that described in a previous paper (1). For these samples an improved version employing a compacting sleeve open in the direction of the neutron beam was used, thus allowing the compaction of pure $\mathrm{CmO}_{2}$ without the addition of aluminum powder. This feature was very important because it allowed sufficient material to be used to make the amounts of $\mathrm{cm}-243$, 245, 246 significant on an absolute basis and also eliminated the need for correcting for the amount of aluminum normally used in samples of this type. Each compact sleeve contained approximately 1.7 grams of oxide material. Two sample sleeves were sealed into a sample holder. Table I shows the isotopic composition of the samples.

The samples were measured on the MTR fast chopper (2) employing the shielded sample changer(3). Measurements were made using the 20 meter flight path. For all measurements the samples were rotated alternately in and out of the neutron beam along with a sample holder blank used as the open beam condition, a standard Au sample, and a closed beam (background). The cycling time was normally 20 minutes, thus smoothing the effects due to reactor flux and background variations. Low energy data from 0.01 to $1 \mathrm{eV}$ was collected at a chopper resolution of $1.5 \mathrm{usec}$ per meter. Intermediate energy data from 1 to $10 \mathrm{eV}$ was measured at 0.1 $\mu \mathrm{sec} / \mathrm{meter}$ and high energy data from 10 to $200 \mathrm{eV}$ was measured at 0.05 usec/meter.

These samples were somewhat unique in that each involved the simultaneous analysis of several unknown isotopes. One had to not only determine parameters of small amounts of material in the presence of a large contaminant ( $293.5 \% \mathrm{Cm}-244$ ) but also assign the resonances as belonging to one of four possible $\mathrm{Cm}$ isotopes. The Pu-240 resonances had been previously measurea $(4)$. 
Figure 1 shows the transmission curves as a function of energy from 1 to $7.7 \mathrm{eV}$, for the three different composition samples as well as a theoretical fit to the data. A repliminary fit to the data over this energy range was reported earlier (5). Parameters have changed somewhat, due to the interference effects from the higher energy resonances which. has not been analyzed or considered at that time and the addition of another resonance at $1.49 \mathrm{eV}$ for $\mathrm{Cm}-243$. In the case of $\mathrm{Cm}-245$ the manner in which the resonances interfere has been changed to be more nearly compatible with the data. The presence of very braod resonance attributable to $\mathrm{Cm}-243$ and $\mathrm{Cm}-245$ indicated large fission components for these isotopes. The Reich-Moore multijevel formula (6) in conjunction with a semi-automatic fitting program $(7)$ was used in determining the resonance parameters by the transmission curves for the three different samples from 7.7 to $33.5 \mathrm{eV}$. The solid lines in Figure 2 represent a theoretical fit to the data. Sample No. 1 is the most complex in that it contains approximately equal amounts of $\mathrm{Cm}-245$ and $\mathrm{Cm}-243$. It was the only sample with a significant amount of $\mathrm{cm}-243$. All three samples showed the presence of $\mathrm{Cm}-245$. Therefore, resonance parameters were determined for $\mathrm{Cm}-245$ by fitting all three percentage compositions for $\mathrm{Cm}-245$ that were determined by mass spectroscopy. Figure 3 shows a theoretical fit to the transmission curve for the purest Cm-244 sample from $33.5 \mathrm{eV}$ to $214 \mathrm{eV}$. Preliminary data were previously reported $(8)$.

Resonance parameters were determined for Cm-244 using the ACSAP Program (7). A level spacing of $14.2 \mathrm{eV}$ was determined resulting in $a_{1} \bar{\Gamma}_{n} \% / D=0.51$. The fit to the total cross section in the low energy region for $\mathrm{Cm}-244$ was determined by postulating a negative energy resonance at $-1.48 \mathrm{eV}$. These results agree with the 23.5 barn measured total cross section at $2200 \mathrm{~m} / \mathrm{sec}$. Thorough knowledge of the shape of the cross section curve in the region from 0.3 to $1 \mathrm{eV}$ is lacking because the large Pu-240 resonance at $1.056 \mathrm{eV}$ hinders accurate determination of the $\mathrm{Cm}-244$ values. Even though $\mathrm{Cm}-245$ is only slightly abundant in the purest $\mathrm{Cm}-244$ sample, its low energy cross section still necessitated a sizable correction to the transmission data.

Shown in Table II are the resonance parameters as determined for $\mathrm{Cm}-243$, 245, 246 up to $30 \mathrm{eV}$, and the $\mathrm{Cm}-244$ resonance parameters to $200 \mathrm{eV}$. It was necessary to postulate two fission channels for the very broad resonances of $\mathrm{Cm}-243$ in order to fit the data. Even though the samples consisted mainly of $\mathrm{Cm}-244$ we were able to isolate and determine resonance parameters for $\mathrm{Cm}-243$, 245,246 up to $30 \mathrm{eV}$ with some degree of confidence.

[1] J. R. Berreth, "Fabrication of Radioactive Samples for Cross-Section Measurements," Nuc. Appl. Vol. 1. p. 230-234 (June 1965).

[2] R. G. Fluharty, F. B. Simpson and O. D. Simpson, "Neutron Resonance Measurements of Ag, Ta, and U-238," Phys. Rev. 103 (1956) pp. 1778-1786.

[3] R. B. Simpson and R. P. Schuman, "Cross Section Measurements on RadioActive Samples," Proc. of the Symp. on Neutron Time of Flight Methods. Saclay, France Session II. (1961) pp. 85-91. 
[4] O. D. Simpson and R. G. Fluharty, "Neutron Resonance Parameters for Pu-239 and Pu-240," Bull. Am. Phys. Soc. Ser II, 2, p. 219 (1957).

[5] J. R. Berreth, et. al., "Total Neutron Cross Section Measurements of Cm-243, 245, 246," Trans. AINS, Vol. 12, No. I (June 1969) pp. 280-281.

[6] C.W. Reich and M. S. Moore, "Multilevel Formula for the Fission Process," Phys. Rey. Vol. III No. 3 (Aug. 1958) pp. 929-933.

[7] N. H. Marsha11, J. W. Codding, O. D. Simpson, and J. R. Smith, "An Automated Cross Section Analysis Program (ACSAP)," AlvS Trans. Topical Meeting on Neutron Cross Sections (March 1971).

[8] J. Berreth and F. B. Simpson, "Total Neutron Cross Sections of Mixed Cm Isotopes Above $10 \mathrm{eV}$," IN-1407 (December 1970) pp. 80-82. 
Table I

Percentage Composition of the Cm Samples

\begin{tabular}{|c|c|c|c|}
\hline Sample No. & $\mathrm{Cm}-244 \%$ & $\mathrm{Cm}-245 \%$ & $\mathrm{Cm}-246 \%$ \\
\hline 1 & 93.16 & 4.03 & 0.66 \\
\hline 2 & 93.33 & 1.60 & 3.46 \\
\hline \multirow[t]{2}{*}{3} & 93.85 & 0.71 & 3.94 \\
\hline & $\mathrm{Cm}-243 \%$ & Pu-240\% & $A m-243 \%$ \\
\hline 1 & 1.51 & 0.63 & - \\
\hline 2 & 0.05 & 1.27 & 0.31 \\
\hline 3 & 0.05 & 1.26 & 0.18 \\
\hline
\end{tabular}


Table II

List of Resonance Parameters for the Various Cm Isotopes

\begin{tabular}{|c|c|c|c|c|c|}
\hline Isotope & $\begin{array}{c}\text { Resonance } \\
\text { Energy } \\
\mathrm{eV} \\
\end{array}$ & $\begin{array}{l}I_{n}{ }^{\circ} \\
m V \\
\end{array}$ & $\begin{array}{c}\Gamma_{\alpha} \\
m V \\
\end{array}$ & $\begin{array}{l}\Gamma_{f 1} \\
m V\end{array}$ & $\begin{array}{l}\Gamma_{f 2} \\
\mathrm{mV}\end{array}$ \\
\hline $\mathrm{Cm}-244$ & $\begin{array}{r}7.66 \\
16.77 \\
22.76 \\
34.90 \\
52.47 \\
69.6 \\
85.4 \\
95.5 \\
131.7 \\
137.3 \\
169.7 \\
179.4 \\
195.0\end{array}$ & $\begin{array}{l}3.370 \\
0.400 \\
0.165 \\
0.500 \\
0.072 \\
0.062 \\
2.390 \\
0.348 \\
0.80 \\
0.12 \\
0.12 \\
0.29 \\
0.66\end{array}$ & $\begin{array}{l}35 \\
40 \\
40 \\
40 \\
40 \\
40 \\
40 \\
40 \\
40 \\
40 \\
40 \\
40 \\
40\end{array}$ & & \\
\hline $\mathrm{Cm}-243^{*}$ & $\begin{array}{r}1.490 \\
2.270 \\
3.086 \\
3.341 \\
5.398 \\
5.964 \\
8.800 \\
10.230 \\
11.073 \\
14.55 \\
15.65 \\
21.68 \\
24.44 \\
25.84\end{array}$ & $\begin{array}{l}0.218 \\
1.261 \\
0.421 \\
0.156 \\
0.354 \\
1.570 \\
0.313 \\
1.554 \\
1.000 \\
0.254 \\
0.435 \\
0.917 \\
0.558 \\
0.560\end{array}$ & $\begin{array}{l}40 \\
40 \\
40 \\
40 \\
40 \\
40 \\
40 \\
40 \\
40 \\
40 \\
40 \\
40 \\
40 \\
40\end{array}$ & $\begin{array}{r}-100 \\
225 \\
-130 \\
80 \\
150 \\
-150 \\
-300 \\
100 \\
-90 \\
250 \\
-250 \\
250 \\
-150 \\
150\end{array}$ & $\begin{array}{r}350 \\
325 \\
1000 \\
1400\end{array}$ \\
\hline $\mathrm{Cm}-245 *$ & $\begin{array}{r}1.955 \\
4.675 \\
9.168 \\
11.340 \\
13.880 \\
16.010 \\
21.397 \\
24.74 \\
27.39 \\
29.35 \\
58.28\end{array}$ & $\begin{array}{l}0.224 \\
0.961 \\
0.221 \\
0.2096 \\
0.093 \\
0.324 \\
0.700 \\
0.78 \\
0.17 \\
0.70 \\
1.98\end{array}$ & $\begin{array}{l}40 \\
40 \\
40 \\
40 \\
40 \\
40 \\
40 \\
40 \\
40 \\
40 \\
40\end{array}$ & $\begin{array}{r}-285 \\
375 \\
-150 \\
130 \\
-170 \\
400 \\
-500 \\
220 \\
230 \\
400 \\
-230\end{array}$ & \\
\hline $\mathrm{Cm}-246$ & $\begin{array}{r}4.308 \\
15.29 \\
83.58 \\
91.60 \\
158.00\end{array}$ & $\begin{array}{l}0.1580 \\
0.140 \\
1.500 \\
1.810 \\
2.540\end{array}$ & $\begin{array}{l}35 \\
35 \\
35 \\
35 \\
35\end{array}$ & & \\
\hline
\end{tabular}

* For the even-odd isotopes $\Gamma_{n}^{\circ}=2 g \Gamma_{n}^{\circ}$ 

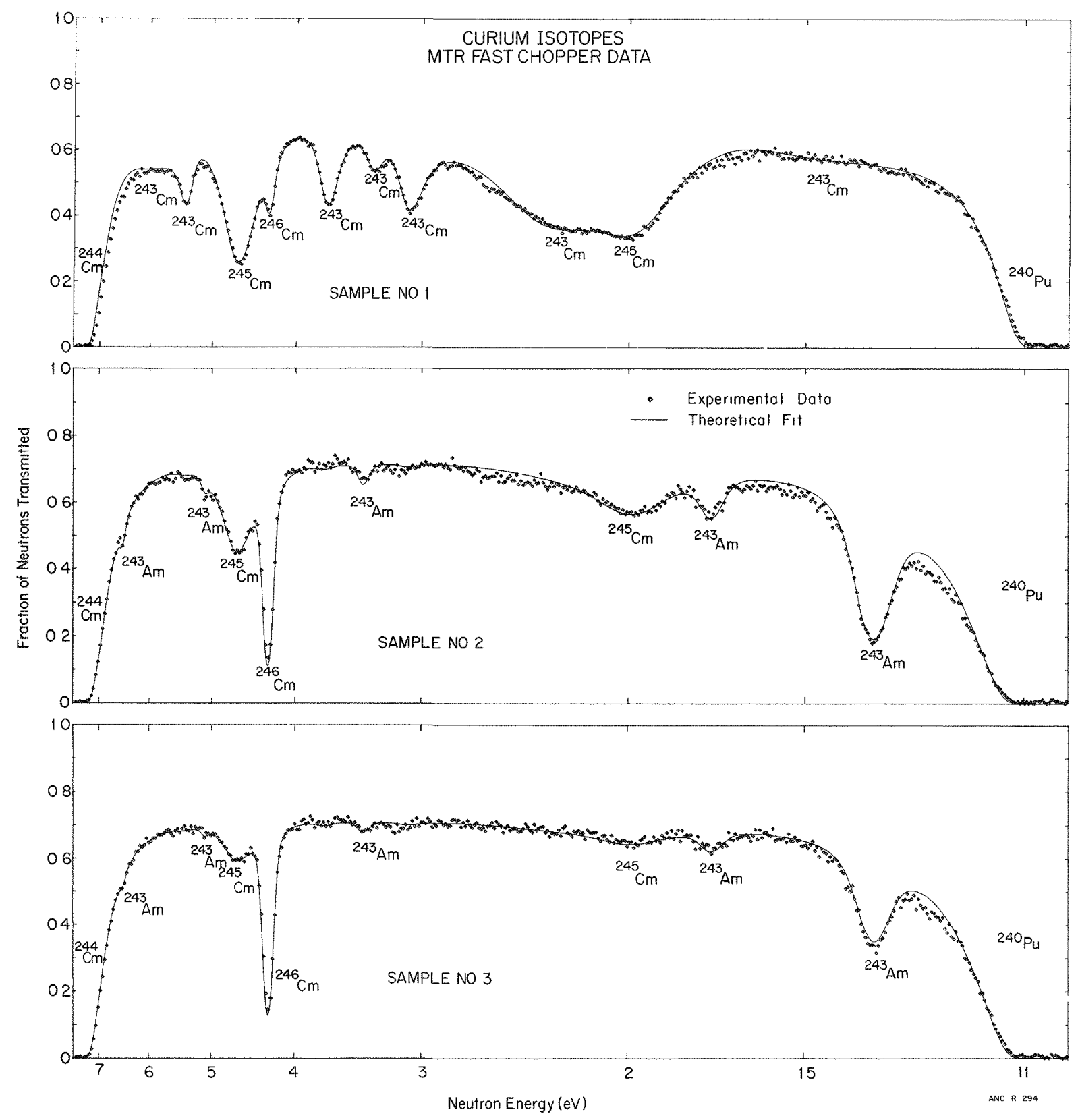

Figure 1. Neutron transmission of the mixed $\mathrm{Cm}$ isotopes as a function of time of flight. The solid line was determined by Doppler and resolution broadening the multilevel theoretical data obtained from the resonance parameters of Table II. The data cover the energy range from 1 to $7.7 \mathrm{eV}$. 

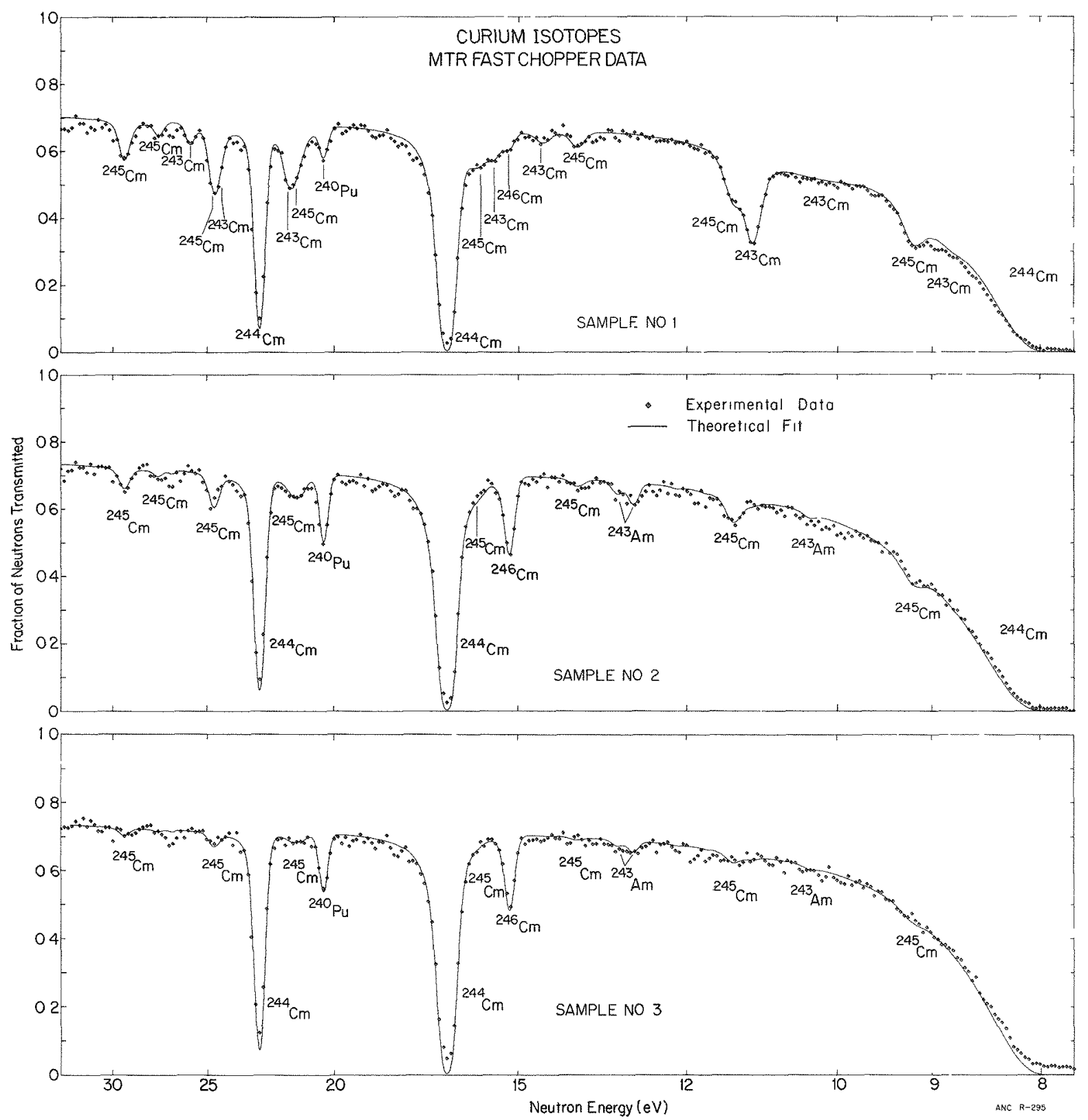

Figure 2. Neutron transmission of the mixed $\mathrm{Cm}$ isotopes as a function of time of flight. The solid line was determined by Doppler and resolution braodening the multilevel theoretical data obtained from the resonance parameters of Table II. The data cover the energy range from 7.7 to $33.5 \mathrm{eV}$. 


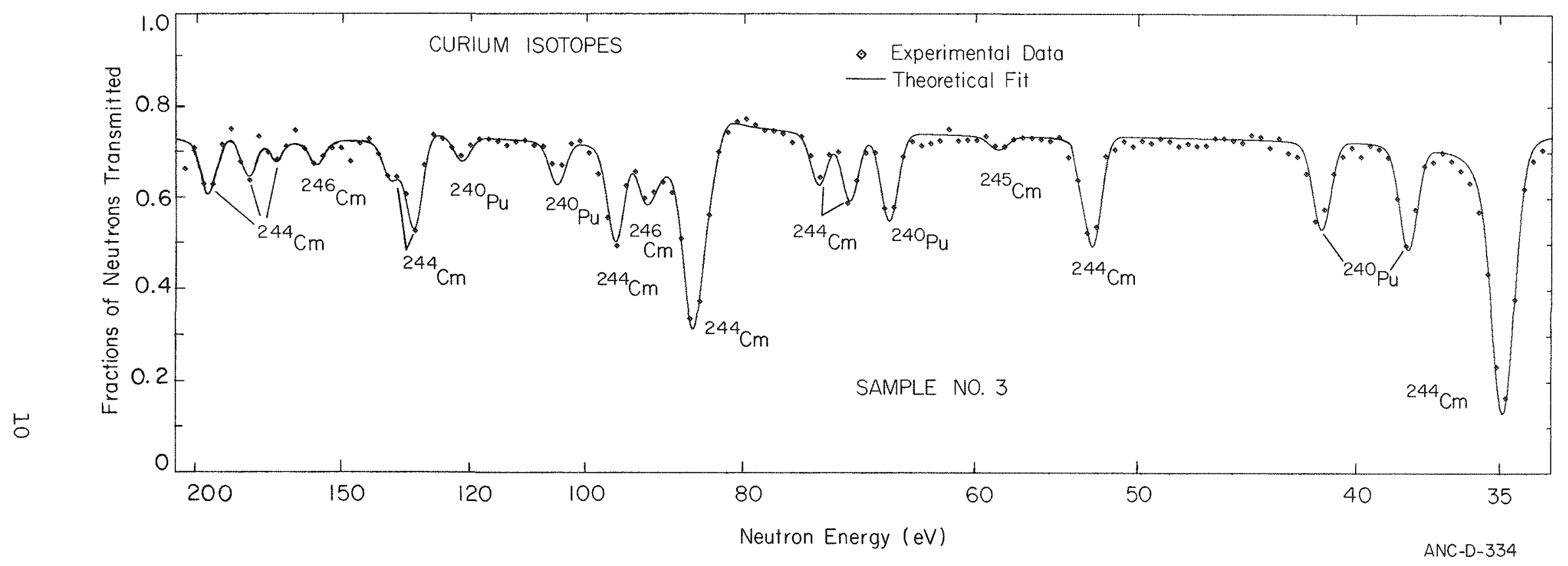

Figure 3. Neutron transmission of the mixed $\mathrm{Cm}$ isotopes as a function of time of flight. The solid line was determined by Doppler and resolution broadening the Breit-Wigner data obtained from the resonance parameters of Table II. The data cover the energy range from 33.5 to $214 \mathrm{eV}$. 
THE TOTAL NEUTRON CROSS SECTION OF $242 \mathrm{Pu}$ BELOW $10 \mathrm{keV}$ MEASURED AT LIQUID NITROGEN TEMPERATURE

F. B. Simpson, O. D. Simpson, H. G. Miller

These measurements were made for the USAEC Production Division to help in evaluating the reactor parameters for the production of ${ }^{252} \mathrm{Cf}$. The Production Division's reactors are quite thermal, and hence their main interest is in the lower energy region. In addition, the data in the keV region are of interest to the LMFBR program. Because of funding limitations the main effort on the analysis of the data will be restricted to the low energy region.

A liquid nitrogen cryostat was adopted to our radioactive sample changer installed on the Oak Ridge Electron Linear Accelerator (ORELA). This facility enables us to make cross section measurements at liquid nitrogen temperatures which reduces the Doppler effect and makes possible measurements at higher neutron energies with good energy resolution. The total neutron cross section of ${ }^{242} \mathrm{Pu}$ has been measured from $15.0-30,000 \mathrm{eV}$ using the ORELA.

Transmission data were taken on three different metal samples at liquid nitrogen temperature $\left(77^{\circ} \mathrm{K}\right)$ having inverse thicknesses of 41.19 , 175.5 and 763.9 barns/atoms. The metal samples were made at Los Alamos and were originally prepared for thermal cross section measurements on the Materials Testing Reactor (MPR) fast chopper. The measurements were made at the 80 meter flight path with a $28 \mathrm{nsec}$ accelerator burst width. Figure $I$ is a plot of the observed neutron levels as a function of neutron energy. From these data the average level spacing, below 1,000 $\mathrm{EV}$, is approximately $16.3 \mathrm{eV}$. The experiment worked exceedingly well and preliminary studies of the raw data indicate that we will be able to analyze resonances below $6-10 \mathrm{keV}$. Extending the resolved energy region above $400 \mathrm{eV}$, as reported in the ENDF/B Version II, to 6-10 keV should be of great interest to the LMFBR program. The average parameters obtained from these new data will predict better cross sections for the unresolved resonance region. 


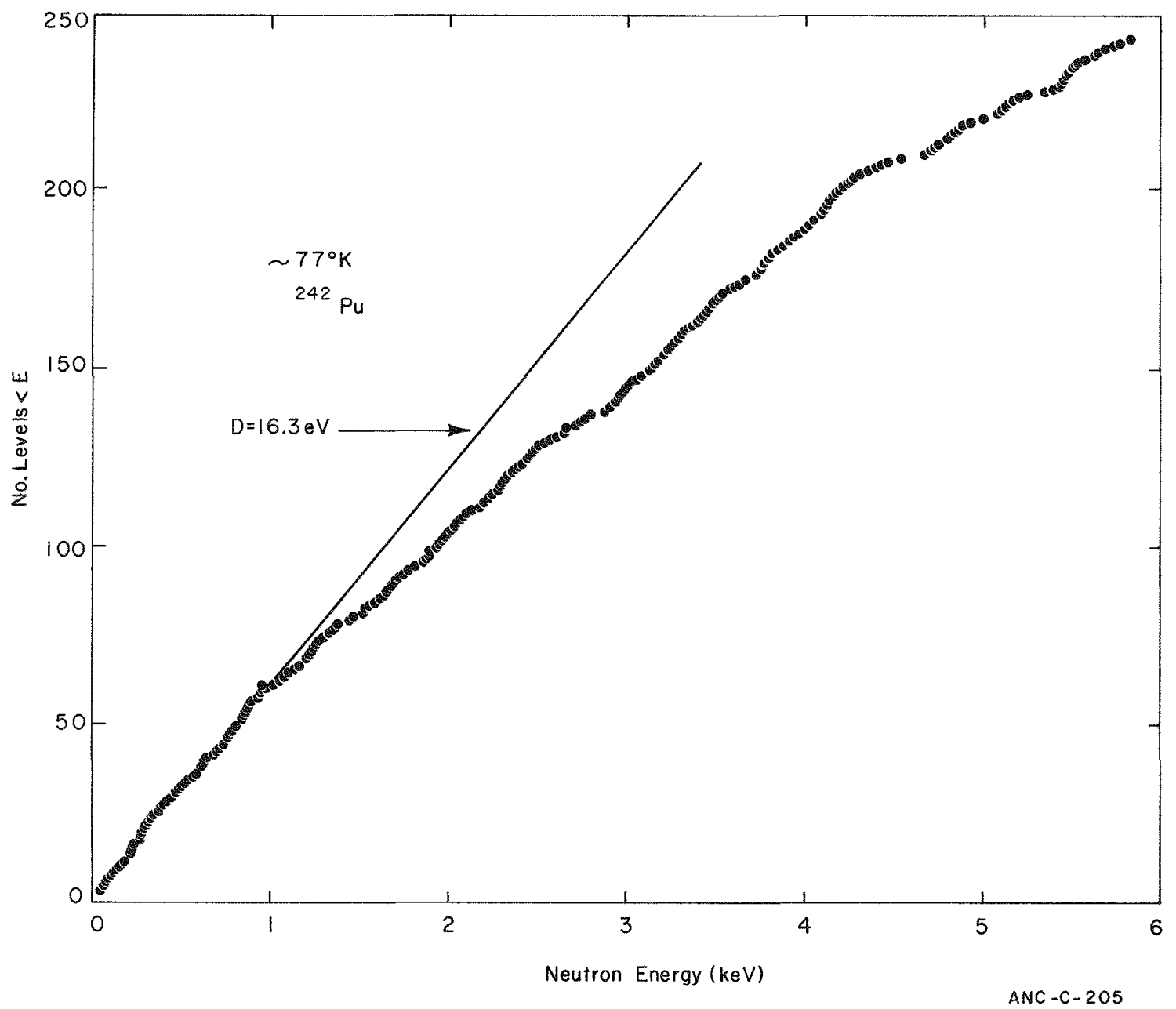

Figure 1 The number of resonances below energy $E$ (keV) as a function of energy for ${ }^{242} \mathrm{Pu}$. 
THE TOTAL NEUTRON CROSS SECTION OF Cm-244 FROM 0.01 TO $1000 \mathrm{eV}$

T. E. Young, O. D. Simpson, F. B. Simpson

Detailed, energy-dependent cross sections of the transplutonium nuclides are needed for accurate prediction of reaction rates in $\mathrm{AEC}$ production reactors. Precise information on $\mathrm{Cm}-244$ neutron cross sections is important because this isotope is a major link in the production chain leading to heavier nuclides having usefulness for both industrial and scientific purposes. Therefore, the total neutron cross section of Cm-244 has been measured from 0.01 to $1000 \mathrm{eV}$ using the Oak Ridge electron linear accelerator. An accelerator pulse width of $40 \mathrm{nsec}$ and an effective combined thickness of $3 \mathrm{~cm}$ for the neutron source and Ii glass detector prom duced a resolution of $4.5 \mathrm{nsec} / \mathrm{m}$ at $1000 \mathrm{eV}$ and $45 \mathrm{nsec} / \mathrm{m}$ at the $7.7 \mathrm{eV}$ resonance in $\mathrm{Cm}-244$.

Transmission measurements were made on oxide samples having inverse sample thicknesses of 1212 and $7413 \mathrm{~b} / \mathrm{a}$ and diameters of $0.3175 \mathrm{~cm}$. An empty holder and the holders containing the samples were alternated in the neutron beam at intervals of 2 to 20 minutes by an automatic switching device. This procedure permitted the recording of sample-in and sample-out counts under essentially identical conditions. Backgrounds were described by a quadratic function of the open counting rate. This function was evaluated by normalization to the background observed at thick resonances in the samples and filters.

The number of observed levels as a function of neutron energy is shown in Figure 1. The inverse slope of a straight line drawn through the data gives an average level spacing of $14.1 \mathrm{eV}$. The analyses of the data are continuing and will be completed during the first part of FY-72. 


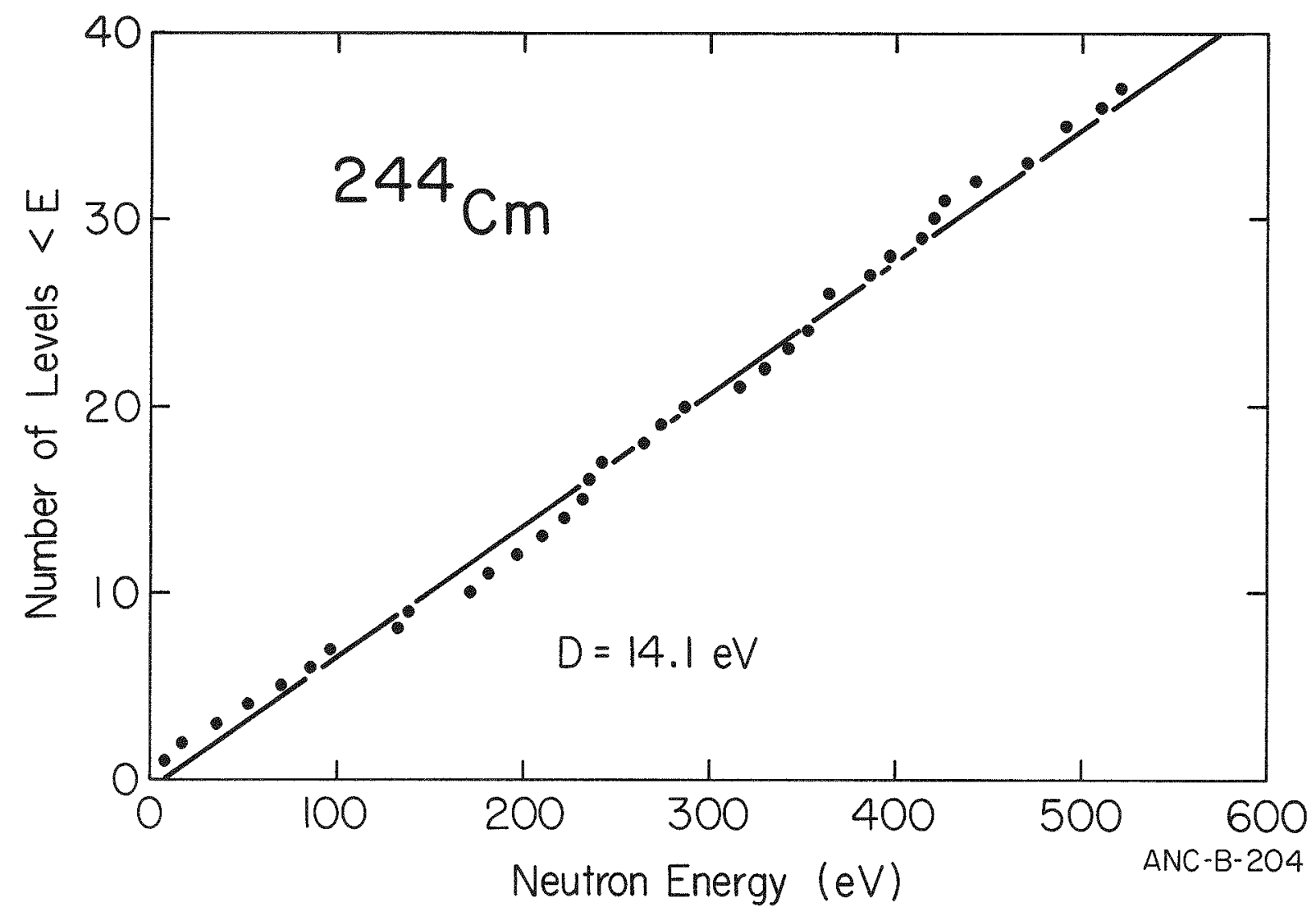

Fíg. I Number of nuclear levels observed in $\mathrm{Cm}-244$ as a function of neutron energy. The solid line represents a visual fit to the data, and corresponds to a level spacing, $D$, of $14.1 \mathrm{eV}$. 
Y. D. Harker, J. W. Rogers, J. J. Scoville

The present interest in fast reactor technology has emphasized a need for more cross section information for fertile, fissile, and fission product materials in the range from $1 \mathrm{keV}$ to $1 \mathrm{MeV}$. As a part of the overall program to obtain this information integral capture cross sections of individual fission products are measured in the CFRMF, whose spectrum approximates that of a typical IMFBR. These integral measurements play an important role in the evaluation of differential cross section data sets that will be used in IMFBR design calculations. For most fission products, experimental differential cross section data in this energy range is either limited to a few energy points or does not exist at all. To gain more information on the whole fission product class, it has been decided that the primary emphasis should be on utilizing semi-emperical nuclear models with parameters determined from lower energy resonance data to predict energy dependent cross section sets and integral measurements such as those performed in the CFRMF to verify these predictions. These measurements therefore are to be used to check or provide normalization for the calculations of Benzi and Reffo[I], Australian fission product data of Cooke[2] and those calculations done by the evaluators of CSEWG. Presently a cooperative effort is proceeding in which these integral measurements are being supplied to evaluators of CSEWG at WALCO and BNL who are using them in the adjustment of the parameters used in their nuclear models.

Reported here are the measured capture cross sections of ${ }^{87} \mathrm{Rb}$, $99 \mathrm{Tc}, 102 \mathrm{Ru}, 104 \mathrm{Ru}, 107 \mathrm{Ag}, 109 \mathrm{Ag}, 115 \mathrm{In}, 121 \mathrm{Sb}, 123 \mathrm{Sb}, 127 \mathrm{I}, 133 \mathrm{Cs}$, $141 \mathrm{Pr}, 165 \mathrm{Ho}$ and $169 \mathrm{Tm}$. Also reported are the spectrum averaged cross sections based on the calculations of Benzi and Reffo[I].

These capture cross sections were determined by the activation technique. A sample is placed in the CFRMF, irradiated for a specified time and then the activity is counted on a Ge( $\mathrm{Ii}$ ) gamma spectrometer. Knowing the decay scheme of the activation product, one can determine the saturated activity of the sample, $\Phi \bar{\sigma}$. Along with the sample, a monitor foil of gold is irradiated and its saturated activity determined. The integral cross section of the sample relative to that of gold is then obtained by dividing the saturated activity of the sample by that of the gold monitor. For the present, gold has been chosen as the standard for several reasons; because of its availability and utility, differential data exist and have been inccrporated in the ENDF/B-II file, and its spectral response is similar to that of most fission products. Other reactions such as $235 \mathrm{U}(\mathrm{n}, \mathrm{f})$ are being considered as possible standards in addition to, orin place of, gold.

The samples are generally in the form of a disk sufficiently thin to reduce self-shielding to less than 3\%. The samples have been in most cases either metal foils or oxide powders. For the stable materials the normal isotopic abundances were assumed in determining the saturated activity. Because of the short half life of the activation products of 
$99 \mathrm{Tc},{ }^{107} \mathrm{Ag}$, and $109 \mathrm{Ag}$ special handling techniques were required to transport the sample from the reactor to the detector in a time sufficiently short to be able to record the product activity before it had decayed below measurable limits. This transport time was approximately 12 seconds.

Listed in Table 1 are the measured cross sections along with some calculated values. The calculated values are arrived at by generating a reaction rate, $\phi(E) \sigma(E)$, as a function of energy and integrating this from $1 \mathrm{keV}$ to $10 \mathrm{MeV}$. These limits were established because of similar limits on the Benzi cross section information. The upper limit is quite adequate but the lower limit should be decreased to at least $100 \mathrm{eV}$ to account for the lower energy reaction contribution[2]. The spectrum used is an evaluated composite of measured and calculated spectra done. in July, 1970; a more recent evaluation has been completed and is described in another section of this report; however, it is felt that still more study is needed on the spectrum below $10 \mathrm{keV}$. Further comparisons between calculated and measured cross sections will be made when this study has proceeded further. The determinations of this study will not effect the measured cross section values but could possibly alter the calculated values derived from differential cross section information.

The calculated gold cross section was determined from the ENDF/B-II data file and was integrated from $116 \mathrm{eV}$ to $10 \mathrm{MeV}$, the results being that the spectrum averaged cross section for gold is $\bar{\sigma}_{\mathrm{Au}}(\mathrm{n}, \gamma)=443 \mathrm{mb}$.

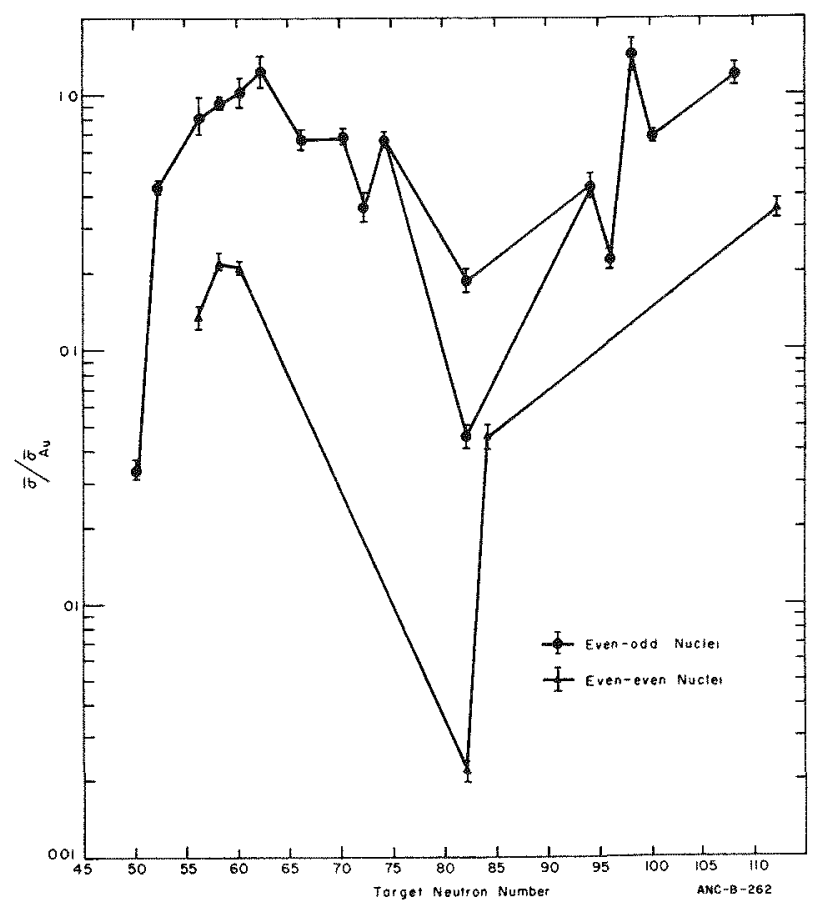

Figure 1. Integral capture cross section relative to that of gold versus target neutron number. The straight lines between points are for clarity only and connect the data points of nuclei which belong to the same class. 
Table 1

Spectrum Averaged Cross Sections

$\bar{\sigma} / \bar{\sigma} A u$

Reaction

${ }^{87} \mathrm{Rb}(n, \gamma){ }^{88} \mathrm{Rb}$

${ }^{99} \mathrm{Tc}(\mathrm{n}, \gamma)^{100} \mathrm{Tc}$

${ }^{102} \mathrm{Ru}(\mathrm{n}, \gamma)^{103} \mathrm{Ru}$

${ }^{104} \mathrm{Ru}(\mathrm{n}, \gamma){ }^{105} \mathrm{Ru}$

${ }^{107} \mathrm{Ag}(\mathrm{n}, \gamma){ }^{108} \mathrm{Ag}$

${ }^{109} \mathrm{Ag}(\mathrm{n}, \gamma)^{110} \mathrm{Ag}$

${ }^{115} \operatorname{In}(n, \gamma)^{116} \operatorname{In}$

${ }^{121} \mathrm{Sb}(\mathrm{n}, \gamma)^{122} \mathrm{Sb}$

${ }^{123} \mathrm{Sb}(\mathrm{n}, \gamma){ }^{124} \mathrm{Sb}$

${ }^{127} I(n, \gamma)^{128} I$

${ }^{133} \mathrm{Cs}(\mathrm{n}, \gamma)^{134 \mathrm{~m} \mathrm{Cs}}$

${ }^{14} \operatorname{Pr}(\mathrm{n}, \gamma)^{142} \mathrm{Pr}$

$165_{\mathrm{Ho}}(\mathrm{n}, \gamma)^{166} \mathrm{gHo}$

${ }^{169} \operatorname{Tm}(n, \gamma)^{170} \operatorname{Tm}$
Measured

$0.0341 \pm 0.0024$

$0.85 \pm 0.14$

$0.225 \pm 0.017$

$0.212 \pm 0.012$

$1.04 \pm 0.13$

$1.25 \pm 0.18$

$0.68 \pm 0.06$

$0.701 \pm 0.055$

$0.372 \pm 0.050$

$0.685 \pm 0.052$

$0.096 \pm 0.006$

0.19 - -

$1.46 \pm 0.21$

$0.70 \pm 0.04$
Calculated

$0.83 \dagger$

- -

- -

$0.90^{1}$

$1.12^{1}$

$0.42^{1}$

$-$

$--$

- -

$0.75^{1}+\dagger$

$0.14^{1} 0.16^{3}$

$2.04^{3}$

+ Value determined from this work based upon calculated cross sections of odd-even nuclei as determined by Benzi and Reffo.

$+\dagger$ Calculation is for general reaction, ${ }^{133} \mathrm{Cs}(\mathrm{n}, \gamma){ }^{134} \mathrm{Cs}$, rather than the reaction to the isomeric state as listed.

These data and data reported earlier[4] are plotted as a function of neutron number in Figure 1. As can be seen from this figure, the overall dependence of the integral cross sections is generally what is expected from simplified nuclear models, i.e., low cross sections at and near the magic numbers and the odd-even nuclei cross sections being greater than those of the even-even nuclei of the same neutron number[5]. A difficulty arises when one endeavors to predict accurately the cross section for an individual isotope, for there are with neutrons of this energy range at least $p$-wave contributions to be considered in addition to the s-wave contributions assumed in most simple models and also the spin state effects contribute to the variations of cross sections from atom to atom. These effects contribute to the scatter of points in Figure 1. For these reasons strict nuclear model calculations are quite impractical. The next best approach appears to be a semi-emperical calculation based upon the nuclear model, available experimental data, 
and systematic studies of the fundamental parameters as a function of mass and neutron number. The model provides the basic functional dependence but various parameters are derived from the available experimental data and from studies of similar nuclei. These integral measurements are a part of the experimental data from which one can determine parameters and also check the final calculations. Examples of checks on calculated cross sections is demonstrated in the comparison of these measurements with the calculations of Benzi and Reffo[1]. From this comparison one can see that the calculation is able to, in most cases, predict the cross sections value close to but generally lower than the measured values. It is felt that the latter effect is the result of the absence of calculational data below $1 \mathrm{keV}$.

The CFRMF has been designed to give a neutron spectrum typical of an LMFBR; consequently the capture integral measurements made in it are important contributions to the overall LMFBR program. This is accomplished in two ways; first these measurements provide a single number from which the capture effect of a particular isotope can be estimated in the performance of an LMFBR, second the measurements are used as an important check in the evaluation of differential capture data in the energy range from $100 \mathrm{eV}$ to $10 \mathrm{MeV}$. The second approach was applied to some of the data reported here. The differential data used were primarily that calculated by Benzi and Reffo. For the most part, the measurements and the calculations are in reasonable agreement with some consistent differences being attributed able to the lack of calculational data below $1 \mathrm{keV}$. The measurements will be compared further with this and other sets of differential data, and in the case of the evaluations being done by members of CSEWG these measurements are being used to adjust the parameters used in the calculational models.

The variations in the measurements of capture integrals of nuclei with very similar characteristics emphasize the need to consider each isotope in the fission product class separately and not rely upon general classifications if the cross sections are to be known accurately.

Although the results reported herein have all been obtained from activation analysis, a further check on differential cross section knowledge is available through reactivity measurement techniques. This procedure requires considerably larger samples of isotopically enriched fission products. Samples of the following isotopes are currently on loan from the Stable Isotopes Pool (ORNL); $91,92,94 \mathrm{Zr}, 95,96,97,98,100 \mathrm{Mo}$, and $152,154 \mathrm{Sm}$. These materials will be measured in the near future, and the results compared to calculations employing the Cooke data currently available on ENDF/B format. It should be noted that the reactivity measurements provide a much more stringent test of cross section data since it involves all neutron reactions that occur in the sample, i.e. elastic and nonelastic scattering. Other fission product isotope samples are currently on order. 
[1] V. Benzi and G. Reffo, CCDN-NE/10, ENEA Neutron Data Compilation Centre (December 1969) p. 6.

[2] J. J. Scoville, "The Effect of Cross-Section Variations on Integrals Measured in the CFRMF" "Third Conference on Neutron Cross Sections and Technology, USAEC Division of Technical Inf., Oak Ridge, Tenn. (March 1971).

[3] M. D. Goldberg, et al., "Neutron Cross Sections", BNI-325, and Rdition. Suppl. No. 2(1966).

[4] J. J. Scoville, et al., Nuclear Technology Branch Annual Progress Report for Period Fnding June 30, 1970, USAEC Report IN-1407, (December 1970) p. 29.

[5] A. M. Weinberg and E. P. Wigner, "The Physical Theory of Neutron Chain Reactors", The University of Chicago Press, Chicago, III. (1958) p. 143. 
ESTIMATES OF FISSION PRODUCT ACTIVITIES FOR

SHORT IRRADIATIOI AND DECAY TIMES

R. G. Nisle

Previous estimates of fission product activities[1] have dealt with irradiations of several days duration and of decay times of comparable magnitudes. Recently, however, the irradiation of small quantities of fuels for short periods at high flux levels has raised the question of fission product activities under such conditions. Extrapolation of Iong term results to shorter times is invalid because short half-life ( $<10$ hours, $\lambda=10^{-5} \mathrm{sec}^{-1}$ ) isotopes do not contribute significantly to long term activities. Therefore, recent fission-product yield data[2] were used to calculate activities for short irradiation and decay times. This reference[2] gives only total mass chain yields whereas the primary yields of the individual isotopes in the chain are needed. The yields of the latter were obtained from estimates given by Weaver, et al.[3] adjusted to the mass chain results of Lisman. Half-lifes were obtained from the U. S. Government Printing Office edition of the former G. $E$. Chart of the Nuclides [4].

The applicable equations are derivable from the Bateman equation and are readily solved by the use of modern computers. In the present work primary yields and first and second daughter products were calculated during irradiation and subsequent decay. Total sample activity in disintegrations per second was then obtained by summing the individual yields. In a number of cases isomeric states are produced. Since no data were available on the relative yields in such cases, the activity was calculated by using that decay constant that would give the largest magnitude of activity.

The Lisman data lacks values for mass chains 107 through 130 . For thermal fission the yields in this mass range are very small and have been ignored in this calculation. The error is partially compensated for by the use of the larger decay constants mentioned above. Tables $1,2,3$ and Figures 1,2 and 3 show the results of these calculations.

The simplified equations developed by Way and Wigner[5] apply to times either shorter or longer than those of interest in this project. To make a comparison of decay activities it would have been necessary to apply the complete equation (Eq. 15a of Ref. 5). Such an effort was not considered to be warranted at this time. 


\section{TABLE 1}

Fission Product Activities for the Thermal Irradiation of ${ }^{235} \mathrm{U}$

$$
\phi=10^{14} \mathrm{n} / \mathrm{cm}^{2} / \mathrm{sec} .
$$

Irradiation

Time (sec)

$1 \mathrm{sec}$.

2

3

5

10

15

30

45

60

90

120
Estimated Activity

Dis/sec/g

$0.6391 \times 10^{11}$

Curies/mg

1. 0418

1.73

2.82

1. 3457

3.64

1.7947

4.85

2.4975

6.75

2.9470

7.96

3.7580

10.16

4.2510

11.49

4.6093

12.46

5.1230

13.85

5.4882

TABLE 2

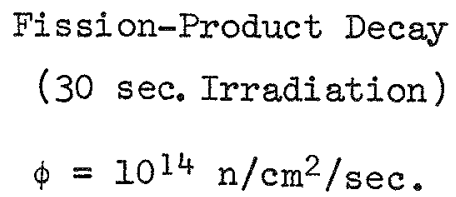

Decay

Time

0

1

3

6

10

15

30

45

60

90

120

$\begin{array}{lc}\text { Dis/sec/mg } & \text { Activity } \\ 3.7580 \times 10^{11} & \frac{\text { Curies/mg }}{10.16} \\ 3.1418 & 8.49 \\ 2.5142 & 6.80 \\ 1.9993 & 5.40 \\ 1.6042 & 4.34 \\ 1.3022 & 3.52 \\ 8.5293 \times 10^{10} & 2.31 \\ 6.4277 & 1.74 \\ 5.1651 & 1.40 \\ 3.6784 & .99 \\ 2.8216 & .76\end{array}$


TABLE 3

Fission-Product Decay

(60 sec. Irradiation)

$$
\phi=10^{14} \mathrm{n} / \mathrm{cm}^{2} / \mathrm{sec} \text {. }
$$

$\begin{array}{clc}\begin{array}{l}\text { Decay } \\ \text { Time } \\$\cline { 1 - 2 } 0\end{array} & $\frac{\text { Dis/sec/mg }}{4.6093 \times 10^{11}} & \frac{\text { Activity }}{12.46} \\ 1 & 3.9912 & 10.79 \\ 2 & 3.6090 & 9.75 \\ 3 & 3.3254 & 8.99 \\ 5 & 2.9162 & 7.88 \\ 10 & 2.3077 & 6.24 \\ 15 & 1.9461 & 5.26 \\ 30 & 1.3681 & 3.70 \\ 45 & 1.0716 & 2.90 \\ 60 & 8.8265 & 2.39 \\ 90 & 6.4846 & 1.75 \\ 120 & 5.0755 & 1.37\end{array}$

[1] R. L. Koontz and A. A. Jarret, "Radioactivity of Irradiated Uranium", Nucleonics 12, 6, p. 26 (June 1954).

[2] F. L. Lisman, et al., "Fission Yields for ${ }^{23}{ }^{3} \mathrm{U},{ }^{235} \mathrm{U},{ }^{239} \mathrm{Pm}$ and $24{ }^{\mathrm{Pu}}$ ", Nucl. Sci. and Eng. 42, pp. 191-214 (1970).

[3] L. E. Weaver, et al., "Estimated Total Chain and Independent Fission Yields for Several Neutron-Induced Fission Processes", USNRDL-TR-633 (March 1963).

[4] Chart of the Nuclides, U. S. Government Printing Office Ed. (1970), $0-389-841$.

[5] K. Way and E. P. Wigner, "The Rate of Decay of Fission Products", Phys. Rev. 13, 1318 (1948). 


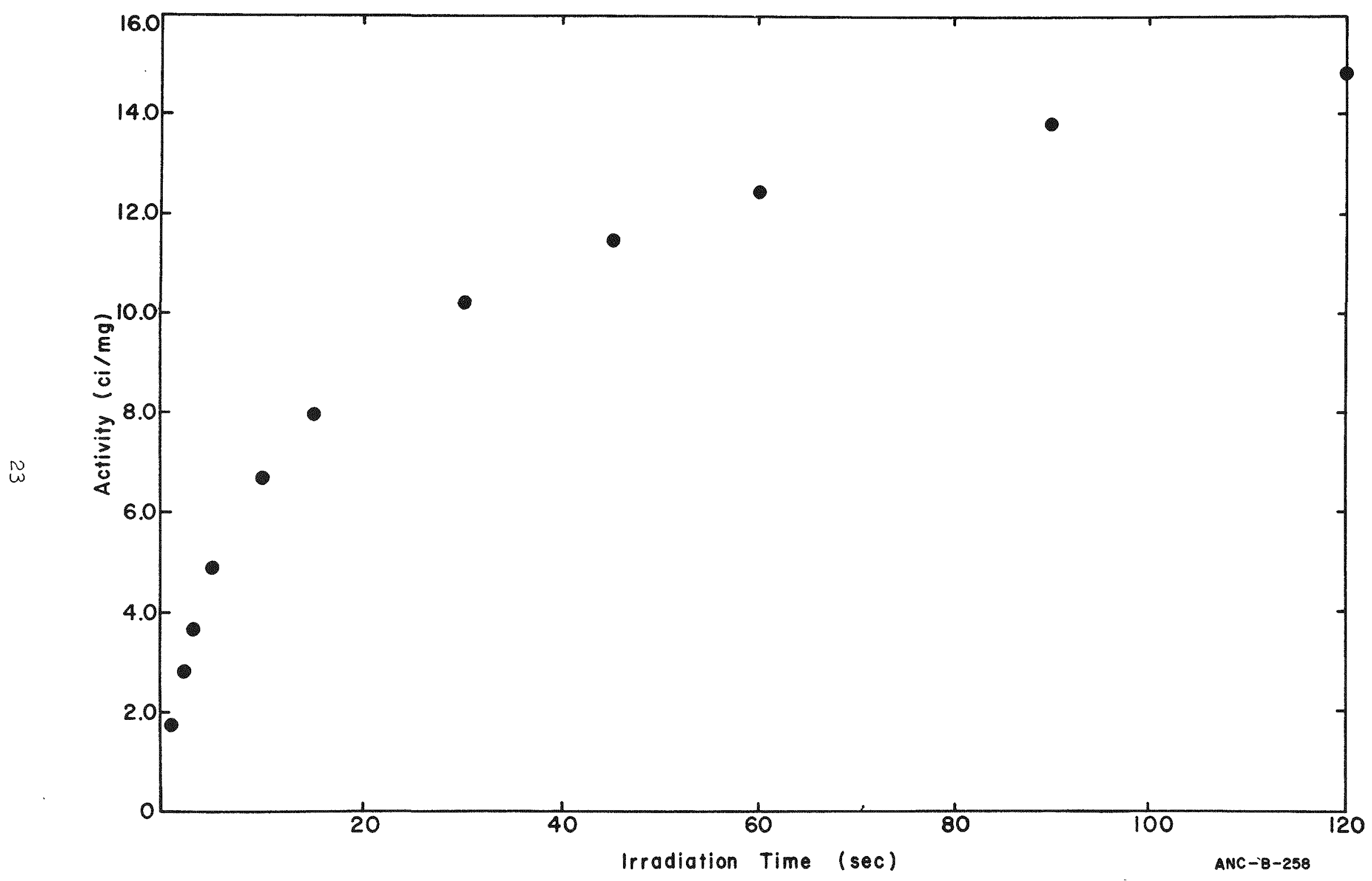

Fig. 1 Fission product activities for the thermal irradiation of ${ }^{235} \mathrm{U}$. 


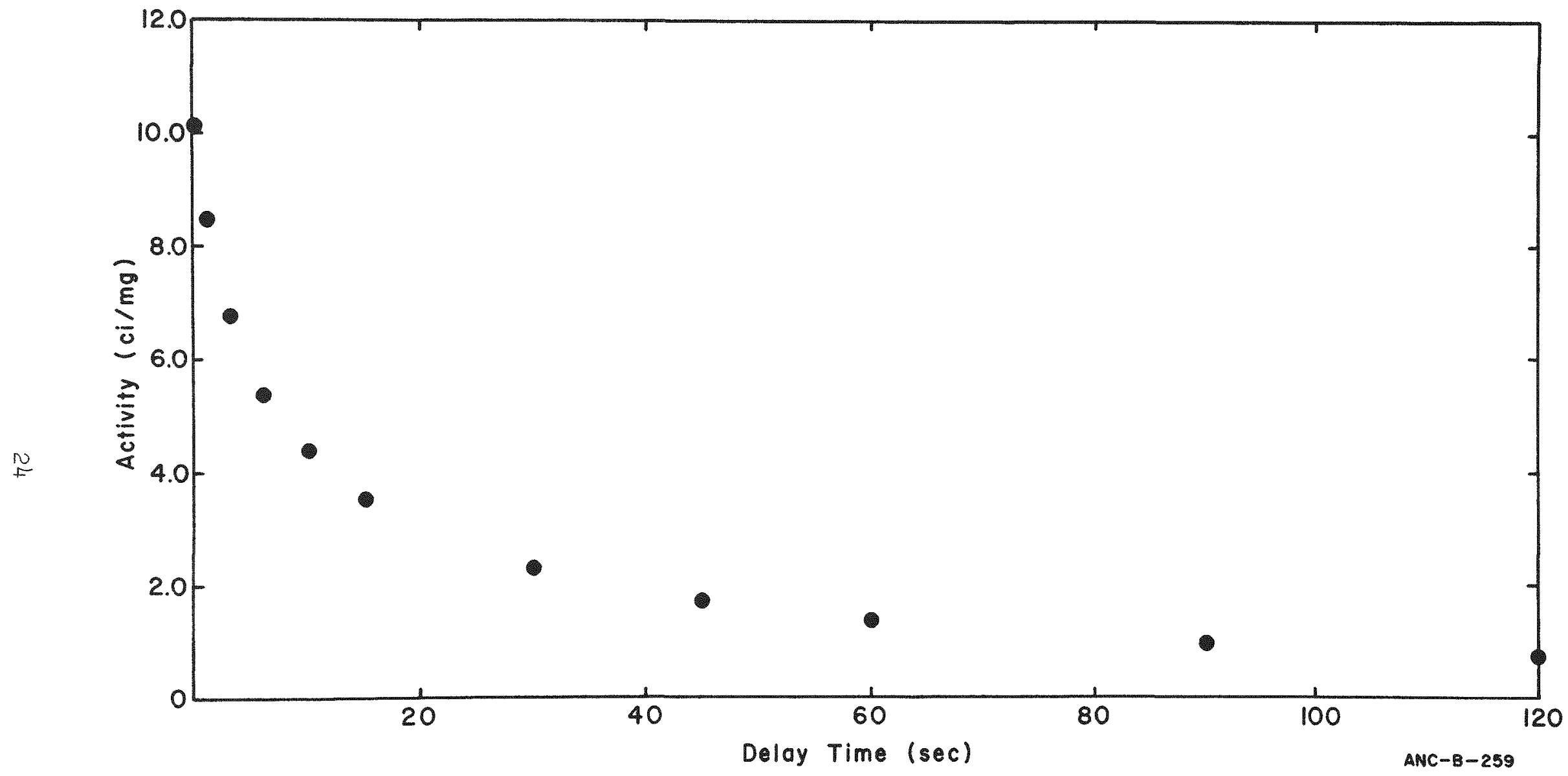

Fig. 2 Fission-product decay (30 sec. irradiation). 
8

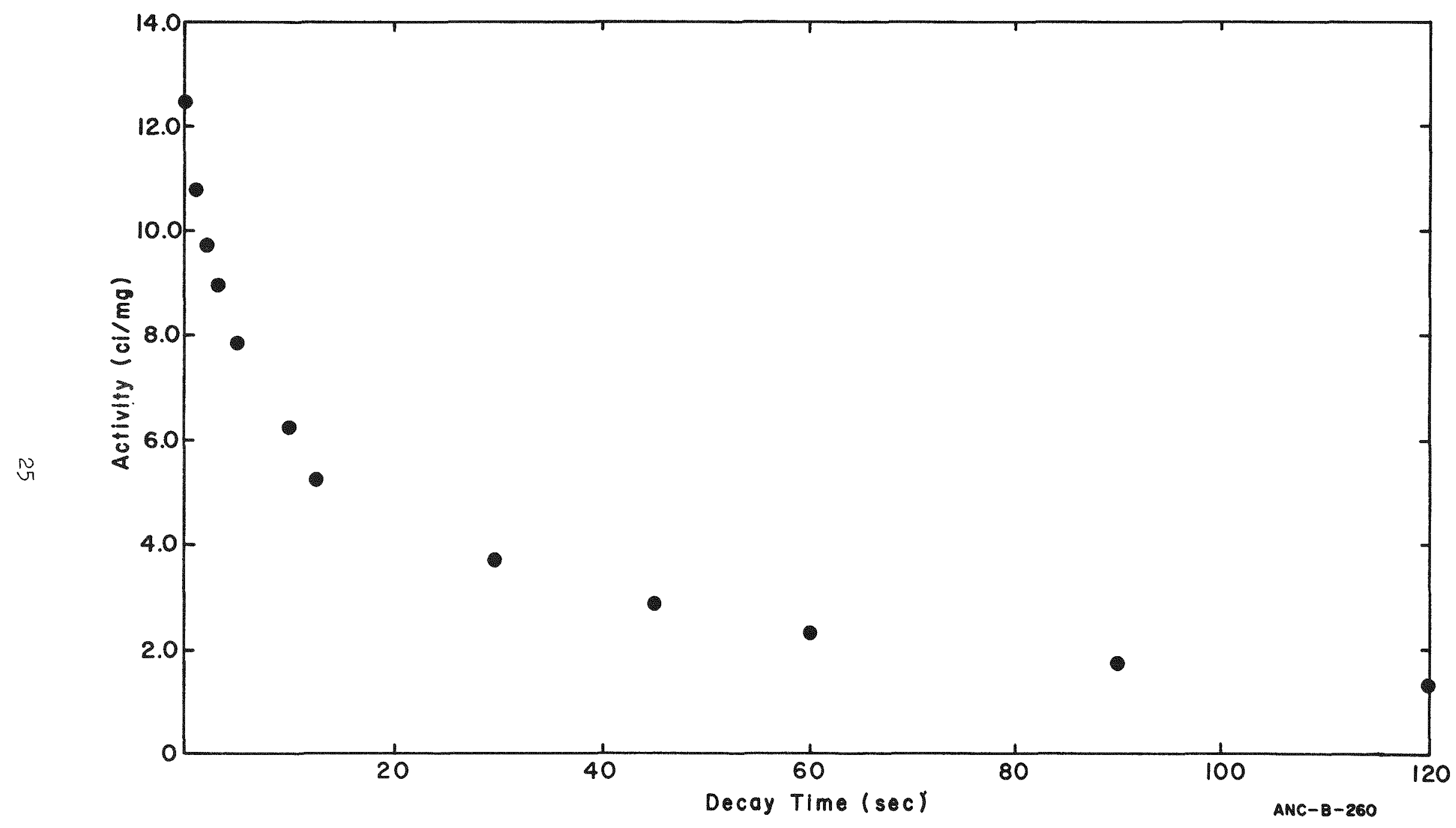

Fig. 3 Fission-product decay (60 $\mathrm{sec}$. irradiation). 
SAMPLE PREPARATIONS

J. R. Berreth, D. A. George

Often the preparation of radioactive and rare samples is the key to the success of an advanced technology research program. During the past year, various types of samples were prepared in support of several Nuclear Technology Division programs. The major programmatic support is for the Fast Breeder Physics Constants Group. Samples prepared for this effort include ten $\mathrm{UO}_{2}$ cylindrical pellets, 0.187 inch in diameter, which were compacted at 110,000 psi using a special die and encapsulated to be used as standards for reactivity measurements. Eighteen $\mathrm{UO}_{2}-\mathrm{PuO}_{2}$ pellets were loaded into special stainless steel cylinders, two or three to a cylinder, and welded under a helium atmosphere. An improved welding technique was used to seal these samples. These special samples were scheduled to be irradiated in EBR-II and measured for reactivity changes after irradiation. Several metal pellet compacts of $\mathrm{Cu}$ and $\mathrm{Au}$ were prepared in a similar fashion as reference materials for the above work.

Other samples of various types were prepared by using the normal processes of electroplating, evaporating, precipitation, etc. among which were ${ }^{99} \mathrm{Tc},{ }^{252} \mathrm{Cf},{ }^{244} \mathrm{Cm}$ and ${ }^{238} \mathrm{U}$. Several of these sources were prepared for use in calibrating a fission chamber used to measure fissions in the presence of high levels of alpha activity. Considerable purification of an irradiated $240 \mathrm{Pu}$ sample was performed for a decay scheme study of the ${ }^{24 l} \mathrm{Pu}$ formed. 
B. NUCLEAR PROPERTIES

 


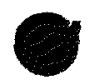

- 
NEUTRON CAPTURE $\gamma$-RAY STUDIES USING THE 2-KeV NEUTRON BEAM FACILITY

R. C. Greenwood, C. W. Reich

In the computation of shielding requirements for fast reactors it will be necessary to understand how the prompt $\gamma$-ray spectrum of component materials varies as a function of neutrcn capturing energy, $\mathrm{E}_{\mathrm{n}}$. The only extensive body of neutron capture $\gamma$-ray data which currently exists is for thermal neutrons. Unfortunately, because the partial radiative widths of the primary capture gamma-ray transitions are governed by Porter-Thomas statistics, these thermal neutron capture spectra are not representative of the spectra resulting from higher energy neutrons. For $E_{n}>1 \mathrm{keV}$ few neutron capture $\gamma$-ray data exist, and those which do are often not of sufficient quality to be useful in shielding calculations. Probably one of the largest bodies of high-quality keV-neutron capture $\gamma$-ray data in existence is the data we have obtained using the $2-\mathrm{keV}$ neutron scandium beam facility on the MTR.

At this time it will not be possible, and it is probably not desirable in any case, to measure prompt $\gamma$-ray spectra for all component materials and all neutron energies of interest in a fast reactor. Instead it will be necessary to use existing data, and to measure selected new data, in order to estimate the variation in the spectral shape of the prompt $\gamma$-ray spectrum [henceforth denoted by $S S(n, \gamma)$ ] as a function of $E_{n}$.

In the case of the heavier elements, not too near closed shells, where the resonance spacings are tens of $\mathrm{eV}$ or less, this is relatively easy to do if a prompt $\gamma$-ray spectrum which is an average over many resonance states has been measured. In such cases, for a single isotope, the $\operatorname{SS}(n, \gamma)$, averaged over groups of $E_{n}$ which are large enough to encompass many resonances, will not change as a function of $E_{n}$ until $E_{n}$ becomes sufficiently large that p-wave capture becomes important. (The energies of the primary prompt $\gamma$ rays in adjacent groups will of course differ in energy by $\Delta \mathrm{E}_{\mathrm{n}} \cdot$ ) For elements containing several stable isotopes the composite elemental $\operatorname{SSE}_{\mathbb{E}}(n, \gamma)$ is simply obtained from the individual isotopic $\operatorname{SS}_{I}(n, \gamma)$ as

$$
\operatorname{SS}_{E}(n, \gamma)=\sum_{I} \frac{\mathrm{SS}_{I}(n, \gamma) A_{I} \sigma_{I}\left(E_{n}\right)}{\sigma_{E}\left(E_{n}\right)}
$$

where the $A_{I}$ are the relative isotopic abundances and the $\sigma_{I}\left(E_{n}\right)$ and $\sigma_{\mathrm{E}}\left(E_{\mathrm{n}}\right)$ are, ${ }^{\mathrm{I}}$ respectively, the isotopic and elemental neutron capture cross sections at energy $E_{n}$.

Since, for many of the single isotopes and natural elements for which we have $2-\mathrm{keV}$ neutron capture $\gamma$-ray spectral data, the resultant $S S(n, \gamma)$ is an average over many resonance states, we have the possibility, from the above considerations, of generating fairly extensive sets of $\operatorname{ss}(n, \gamma)$ versus $E_{n}$ data. 
As a first step towards generating sets of $S S(n, \gamma)$ versus $E_{n}$ data for these heavier elements, we must first obtain a complete analysis of the $2-\mathrm{keV}$ neutron capture $\gamma$-ray spectra to obtain the $I_{\gamma}$ versus $E_{\gamma}$ distribution. This analysis problem is complicated for these heavier nuclides by the fact that, with existing $\gamma$-ray detector systems, only a part of the $\gamma$-ray spectrum is visible as discrete line components, the remainder being contained in an unresolved continuum which is more difficult to analyze quantitatively. Initially then, we have chosen to analyze only the discrete components in the spectra to determine their energies and absolute intensities (in $\gamma / 100$ neutron captures). (The $\gamma$-ray lines which one observed as discrete components in the prompt $\gamma$-ray spectra will be the "harder" $\gamma$-ray components which are difficult to shield against.) Then from the relation

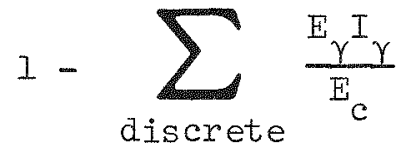

(where $E_{C}$ is the capturing state energy) it is possible to estimate the intensity contained in the continuum part of the spectrum and thus to account for it.

A major accomplishment to date in the analysis of these $2-\mathrm{keV}$ neutron capture $\gamma$-ray spectra has been the development of a method for estimating the absolute transition intensities of discrete lines observed in spectra which are an ayerage over many resonance states (that is, from the higher-Z nuclides which are not near closed shells). Basically, this method utilizes the fact that the lower-lying ( $<3 \mathrm{MeV}$ ) levels in a nucleus are populated by a sufficiently large number of different primary (and secondary) transitions that fluctuations in their relative intensities as a function of $E_{n}$ are not expected to be nearly as great as for the primary transitions. Thus it is reasonable to assume that the sum of the transition intensities of a randomly selected, and large, group of secondary transitions de-exciting these levels will be equal at thermal and $2-\mathrm{keV}$ neutron energies. Using this equality, then, one can determine the absolute transition intensities if there is some information available on the absolute transition intensities from thermal neutron capture, as there usually is. This method has been found to be particularly useful for the even-Z, odd-A target nuclides, since the secondary transitions into the ground-state band of the product nucleus all have energies $>0.5 \mathrm{MeV}$ and are clearly visible in both the 2-keV neutron capture spectrum and the corresponding thermal neutron spectrum. If a large, and randomly selected, group of secondary transitions into the groundstate band is selected, then:

$$
\sum_{\text {many }} \text { rel. } I_{\gamma}^{\text {thermal }} \text { (sec. trans. into g.s. band) }=\text { Constant }=
$$

$$
\sum_{\text {many }} r e l \cdot I_{\gamma}^{2} \mathrm{keV} \text { (sec. trans. into g.s. band). }
$$


Examples of our use of this method for determining absolute transition intensities in the $2-\mathrm{keV}$ neutron capture $\gamma$-ray spectra are given in the following paragraphs.

Absolute Yield of Prompt $\gamma$-Ray Produced by 2-keV Neutron Capture in $183 \mathrm{~W}$ The high-energy portion of the prompt $r$-ray spectrum resulting from capture of $2-\mathrm{keV}$ neutrons in a sample of $183 \mathrm{~W}$ (enriched to $82.5 \%$ in $183 \mathrm{~W}$ ) is shown in Figure 1 in Reference [1]. In order to obtain absolute intensities for the $\gamma$-ray transitions corresponding to the lines observed in this spectrum we first note that, based upon the absolute transition intensity data of Rasmussen et al.[2] for thermal-neutron capture in natural tungsten and our relative intensity data,

for natural $W$

$$
I_{\gamma}^{\text {th }}(7299.9+74 I I .1)=0.536 \mathrm{\gamma} / \text { neut. cap., }
$$

and thus, for $183_{\mathrm{W}}$

$$
I_{\gamma}^{\text {th }}(7299.9+7411.1)=6.75 \gamma / 100 \text { neut. cap. }
$$

Next, in order to utilize these thermal neutron canture data in determining transition intensities for $2-\mathrm{keV}$ neutron capture, we must go via Equation (2). The group of secondary transitions selected for inclusion in this secondary transition intensity balance equation, together with their respective thermal and $2-\mathrm{keV}$ neutron capture intensities is shown in Table I. From Table I we note that (I) $50 \%$ of all the secondary transitions populating the ground-state band are included in the sum, and (2) while there are significant changes in the individual thermal and $2-\mathrm{keV}$ intensities of some of these secondary transitions, the overall intensity pattern is similar for both thermal and $2-\mathrm{keV}$ neutron capture. This suggests that a reasonable conversion factor, from relative to absolute $2-\mathrm{keV}$ neutron capture $\gamma$-ray intensities, can be obtained using this technique. With this conversion factor we obtain, for capture of $2-\mathrm{keV}$ neutrons in $183 \mathrm{~W}$

$$
I_{\gamma}^{2} \mathrm{keV}(7301.9+7413.1)=4.23 \gamma / 100 \text { neut. cap. }
$$

Comparison of the higher energy $(>4.69 \mathrm{MeV})$ prompt $\gamma$-rays resulting from thermal and $2-\mathrm{keV}$ neutron capture in $183 \mathrm{~W}$ is shown in Table II. Primary capture $\gamma$-rays are observed in these spectra down to $* 3.3 \mathrm{MeV}$ and 73.7 $\mathrm{MeV}$, respectively, in thermal and $2-\mathrm{keV}$ neutron capture. It is interesting to note that the primary $\gamma$-rays observed as discrete components in the thermal and $2-\mathrm{keV}$ neutron capture spectra account for $242 \%$ and $237 \%$ of the total primary capture $\gamma$-ray intensities, respectively. 
Absolute Yield of Prompt $\gamma$-Rays Produced by 2-keV Neutron Capture in Natural Tungsten The high-energy portions of the prompt $\gamma$-ray spectra resulting from capture of $2-\mathrm{keV}$ and thermal neutrons in natural tungsten are shown in Figure 1. As is obvious from this figure, there are gross differences in these spectra. A contributing factor to this difference is that neutron capture in the ${ }^{182} \mathrm{~W}$ and ${ }^{186} \mathrm{~W}$ isotopes dominates at thermal energies, while capture in $183_{\mathrm{W}}$ dominates at $2 \mathrm{keV}$. Significant shape differences, however, are also observed between thermal and 2-keV neutron capture in each of the separated isotopes (see, for example, Table II for data on the ${ }^{183} \mathrm{~W}(\mathrm{n}, \gamma)$ reaction).

Absolute values for the intensities of these transitions observed for $2-\mathrm{keV}$ neutron capture in natural tungsten can be obtained from the $2-\mathrm{keV} 183_{\mathrm{W}}(\mathrm{n}, \gamma)$ reaction data (in Table II) by using appropriate 2-keV neutron capture cross sections [3] for ${ }^{183} \mathrm{~W}$ and natural tungsten (taken as $26.0 \mathrm{~b}$ and $1.5 \mathrm{~b}$, respectively). With these cross-section values we obtain a value of $2.42 \gamma / 100$ neutrons captured for the intensity of the $(7301.9+7413.1) \mathrm{keV} \gamma$-ray transitions produced as a result of $2-\mathrm{keV}$ neutron capture in natural tungsten. A comparison of the prompt $\gamma$-ray intensities from the $W(n, \gamma)$ reaction down to an energy of $4.5 \mathrm{MeV}$ is shown in Table III. Primary capture $\gamma$-ray transitions are observed in these spectra down to ${ }_{3} 3.1 \mathrm{MeV}$ and $73.7 \mathrm{MeV}$, respectively, in thermal and $2-\mathrm{keV}$ neutron capture. From these data, the primary $\gamma$-rays observed as discrete components in the thermal and $2-\mathrm{keV}$ neutron capture spectra account for $\approx 38 \%$ and $828 \%$ of the total primary capture $\gamma$-ray intensities, respectively.

Absolute Yield of Prompt y-Rays Produced by 2-keV Neutron Capture in 17 Iy Rasmussen et al. [2I have measured absolute intensities for thermal neutron capture in natural ytterbium and obtained a value of $0.54 \mathrm{r} / 100$ neutrons captured for the intensity of the $5539-\mathrm{keV}$ transition in $172 \mathrm{Yb}$. For thermal neutron capture in $171 \mathrm{Yb}$, then, we compute an intensity of $2.79 \mathrm{\gamma} / 100$ neutrons captured for the $5539-\mathrm{keV} \gamma$-ray transition (using cross-section values of $50 \mathrm{~b}$ and $37 \mathrm{~b}$ for thermal neutron capture in natural ytterbium and ${ }^{17} \mathrm{l} \mathrm{Yb}$, respectively). In order to utilize these thermal-neutron intensity data in determining absolute intensities for the prompt $\gamma$-ray transitions resulting from $2-\mathrm{keV}$ neutron capture, we utilize Equation (2). The group of secondary transitions selected for inclusion in this intensity-balance equation is shown in Table IV. From this Table we note that (I) $42 \%$ of all secondary transitions populating the ground-state band in $172 \mathrm{Yb}$ are included, and (2) while (as for ${ }^{184} \mathrm{~W}$ ) there are significant differences between the intensities of individual transitions from thermal and 2-keV neutron capture (e.g., the $1093-\mathrm{keV}$ line intensity is relatively much stronger in the latter while the $2401-\mathrm{keV}$ line intensity is much stronger in the former), the overall intensity pattern is similar in both spectra. Applying the data shown in Table IV to the intensity-balance equation, we obtain an intensity value of $1.09 \gamma / 100$ neutrons captured for the $8021.9-k e V ~ \gamma-r a y$ transition resulting from $2-\mathrm{keV}$ neutron capture in ${ }^{171} \mathrm{Yb}$. A comparison of the prompt $\gamma$-ray intensities from the $17 l_{Y b}(n, \gamma)$ reaction, down to an energy of $\$ 5.1 \mathrm{MeV}$ is shown in Table V. Primary capture $\gamma$-ray transitions are observed in these spectra down to $\$ 3.6 \mathrm{MeV}$ and $\$ 4.1 \mathrm{MeV}$, respectively, in thermal and $2-\mathrm{keV}$ neutron capture. Comparison of the data (in Table V) 
TABLE I

\begin{tabular}{|c|c|c|c|}
\hline \multirow{2}{*}{$\begin{array}{l}\text { Gamma-Ray } \\
\text { Energy } \\
(\mathrm{keV}) \\
\end{array}$} & \multicolumn{2}{|c|}{ Thermal-neutron Capture } & \multirow{2}{*}{$\begin{array}{c}2-\mathrm{keV} \\
\text { Neutron Capture } \\
\underline{\text { rel. } I_{Y}^{\mathrm{a}}}\end{array}$} \\
\hline & $\begin{array}{c}I_{\gamma} \\
\left(\gamma^{\prime} s / 100 \text { neut. capt. }\right)\end{array}$ & rel. $I_{y}$ & \\
\hline 724 & 1.54 & 14.4 & 17.9 \\
\hline 757 & 2.34 & 21.9 & 17.0 \\
\hline$(763+769)$ & 1.48 & 13.8 & 18.8 \\
\hline 792 & 10.7 & 100 & 100 \\
\hline$(891+894)$ & 11.5 & 107.9 & 81.0 \\
\hline 903 & 11.0 & 103 & 96.2 \\
\hline 1010 & 3.09 & 28.9 & 26.4 \\
\hline 1121 & 1.20 & 11.2 & 8.3 \\
\hline 1275 & 2.74 & 25.6 & 11.2 \\
\hline$(1313+1319)$ & 1.82 & 17.0 & 15.7 \\
\hline 1386 & 2.19 & 20.5 & 8.7 \\
\hline 1430 & 0.91 & 8.5 & 9.5 \\
\hline SUM & 50.5 & 472.7 & $4 \longdiv { 4 1 0 . 7 }$ \\
\hline
\end{tabular}

a) On this intensity scale $I_{\gamma}(7301.9+7413.1)=34.3$. In comparing the intensities of the primary and secondary transitions in the $2-\mathrm{keV}$ neutron capture spectrum it must be remembered that a portion of the secondary transition intensity arises from capture of the $>2-\mathrm{keV}$ neutrons. To account for this, in these data, a correction factor of 0.962 was obtained by convoluting the ${ }^{183} \mathrm{~W}(n, \gamma)$ crosssection curve into the neutron energy distribution in the beam; that is, the intensities of the secondary transitions were multiplied by a factor 0.962 relative to the intensities of the primary transitions. 
TABLE II

COMPARISON OF PROMPT $\gamma$-RAY INTENSITIES RESULTING

FROM 2-keV AND THERMAL NEUTRON CAPTURE IN ${ }^{183} \mathrm{~W}$

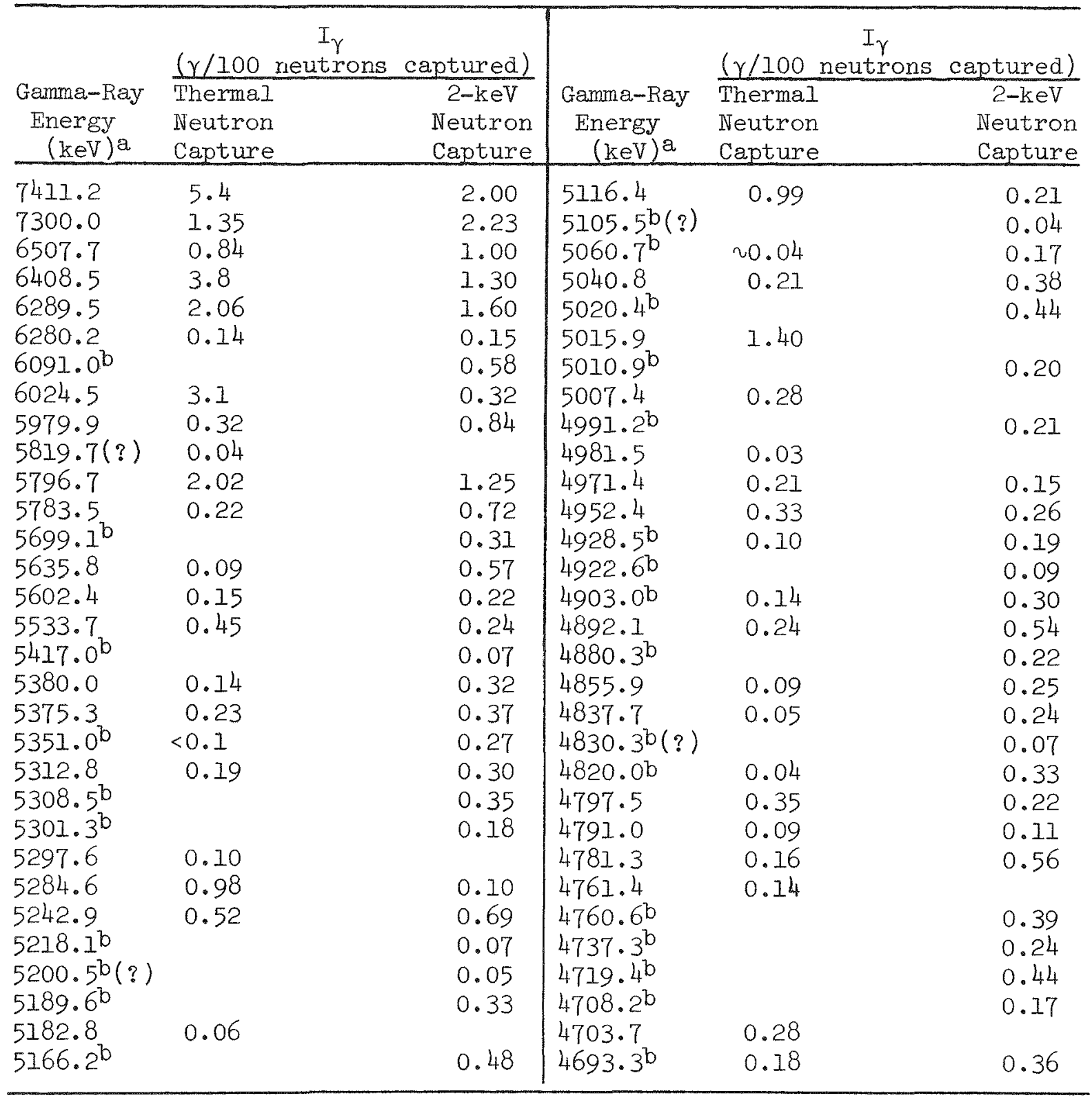

a) The thermal neutron capture gamma-ray energies are listed here, the corresponding neutron capture gamma rays have energies which are $2 \mathrm{keV}$ higher than these.

b) These energies are those determined from the 2-keV neutron capture gamma-ray data alone. 
shows significant differences in the intensity distributions; for example, only $q_{2.2 \%}$ of the primary transitions have energies $>6.0 \mathrm{MeV}$ in the thermal-capture data compared to $66.1 \%$ in the $2-\mathrm{keV}$ neutron capture data. Closer inspection of the modes of decay of the primary transitions indicates that the strongest primary transitions in thermal neutron capture all decay to $\mathrm{I}^{+}$states, which suggests that there is a significant contribution to the thermal neutron capture cross section from resonances with $I^{\pi}=0^{-}$. The lowest-lying $I^{\pi}=I^{+}$state in ${ }^{172} \mathrm{Yb}$ has energy $2010 \mathrm{keV}$ (the $6009.8 \mathrm{keV}$ transition decays to it), thus explaining why the thermal neutron capture $\gamma$-ray spectrum is "softer" than the 2-keV neutron capture spectrum.

The primary $\gamma$-rays observed as discrete compounds in the thermal and $2-\mathrm{keV}$ neutron capture spectra account for $\approx 17 \%$ and $\approx 15 \%$ of the total primary $\gamma$-ray intensity, respectively.

Absolute Yield of Prompt $\gamma$-Rays Produced by 2 -keV Neutron Capture in $173 \mathrm{Yb}$ The $5255 \mathrm{keV}$ transition from the $174 \mathrm{Yb}(n, \gamma)$ reaction is a prominent feature of the thermal neutron capture $\gamma$-ray spectrum measured with our ${ }^{173} \mathrm{Yb}$ target sample (enriched to $82.5 \%$ in $173 \mathrm{Yb}$, with $9.6 \%$ ${ }^{174} \mathrm{Yb}$ ) and thus is used as the primary intensity standard. Rasmussen et al. [2] have obtained an intensity value of $2.86 \mathrm{r} / 100$ neutrons captured for the intensity of the $7387.7 \mathrm{keV}$ transition resulting from thermal neutron capture in the ${ }^{173} \mathrm{Yb}$ isotope. With an absolute intensity scale for thermal neutron capture in ${ }^{173} \mathrm{Yb}$ based upon this value, we are able to obtain, via Equation (2) and utilizing the set of secondary transitions listed in Table VI, a value of $1.33 \mathrm{\gamma} / 100$ neutrons captured for the intensity of the $7389.6 \mathrm{keV}$ transition resulting from $2-\mathrm{keV}$ neutron capture in $173 \mathrm{Yb}$. A comparison of the prompt $\gamma$-ray intensities from the $173 \mathrm{Yb}(n, \gamma)$ reaction, down to an energy $24.5 \mathrm{MeV}$, is shown in Table VII. Primary capture $\gamma$-ray transitions are observed in the spectra resulting from thermal and $2-\mathrm{keV}$ neutron capture down to $\sim 3.5 \mathrm{MeV}$ and $\sim 3.6 \mathrm{MeV}$, respectively. The primary $\gamma$-ray transitions observed as discrete components in the thermal and $2-\mathrm{keV}$ neutron capture spectra account for $\% 25 \%$ and $\approx 19 \%$ of the total primary $r$-ray intensity, respectively.

[1] R. C. Greenwood and C.W. Reich, "Gamma-Ray Transitions De-exciting States near $1 \mathrm{MeV}$ in $183_{\mathrm{W}}$ observed in the $(n, \gamma)$ Reaction", this report.

[2] N. C. Rasmussen, Y. Hukai, T. Inouye and V. J. Orphan, Mass. Inst. of Tech. Report MITNE-85 (1969); and V. J. Orphan, et al., Gulf General Atomic Report GA-10248.

[3] M. D. Goldberg, S. F. Mughabghab, S. N. Purohit, B. A. Magurno and V. M. May, Brookhaven National Laboratory Report BNL-325 (2nd Ed., Suppl. 2), Vol. IIC, $Z=61$ yo 87 (1966). 
TABLE III

COMPARISON OF PROMPT $\gamma$-RAY INTENSITIES

RESULTING FROM 2-keV AND THERMAL NEUTRON CAPTURE IN TUNGSTEN

\begin{tabular}{|c|c|c|c|c|c|c|c|}
\hline $\begin{array}{c}\text { Gamma-Ray } \\
\text { Energy } \\
(\mathrm{keV})^{\mathrm{a}} \\
\end{array}$ & $\begin{array}{l}\text { Mass Number } \\
\text { of Product } \\
\text { Nucleus } \\
\end{array}$ & $\begin{array}{l}\frac{(y / 100 r}{\text { Thermal }} \\
\text { Neutron }\end{array}$ & 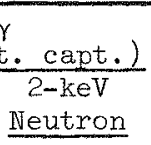 & $\begin{array}{l}\text { Gamma-Ray } \\
\text { Energy } \\
\text { (keV)a } \\
\end{array}$ & $\begin{array}{c}\text { Mass Number } \\
\text { of Product } \\
\text { Nucleus } \\
\end{array}$ & $\begin{array}{l}\frac{(x / 100}{\text { Thermal }} \\
\text { Neutron }\end{array}$ & $\frac{t \cdot \text { capt.) }}{2-\mathrm{keV}}$ \\
\hline 7411.1 & 184 & 0.43 & 1.13 & 5.124 .1 & & & 0.09 \\
\hline 7299.9 & 184 & 0.10 & 1.29 & 5116.6 & 184 & 0.10 & $0.1 \overline{4}$ \\
\hline 6508.4 & 184 & 0.07 & 0.52 & 5089.0 & 185 & 0.09 & 0.14 \\
\hline 6408.5 & 184 & 0.32 & 0.63 & 5042.9 & 183,4 & & 0.33 \\
\hline 6289.8 & 184 & 0.16 & 0.97 & 5023.1 & 184 & & 0.24 \\
\hline 6190.6 & 183 & 4.35 & 0.48 & 5016.0 & 184 & 0.12 & 0.15 \\
\hline 6144.1 & 183 & 1.57 & 1.11 & 4994.3 & 184 & & 0.18 \\
\hline 6091.5 & 184 & & 0.41 & 4986.8 & 185 & & 0.29 \\
\hline 6024.3 & 184 & 0.27 & 0.16 & 4973.0 & 184 & & 0.10 \\
\hline 5982.4 & 183,4 & & 0.92 & 4952.9 & 184 & & 0.11 \\
\hline 5796.8 & 184 & 0.14 & 0.72 & 4930.3 & 185 & 0.08 & \\
\hline 5784.8 & 184 & & 0.46 & 4928.1 & 185 & & 0.73 \\
\hline 5753.8 & 185 & 0.05 & 0.26 & 4902.3 & 183,4 & & 0.28 \\
\hline 5731.5 & 185 & & 0.29 & 4893.8 & 184 & & 0.31 \\
\hline 5700.3 & 184 & & 0.24 & 4880.3 & 184 & 0.07 & 0.12 \\
\hline 5661.6 & 185 & & 0.58 & 4858.2 & 183,4 & & 0.26 \\
\hline 5637.9 & 184 & & 0.33 & 4837.8 & & & 0.10 \\
\hline 5604.0 & 184 & & 0.20 & $4817 \cdot 3$ & & & 0.07 \\
\hline $5551.7(?)$ & & & 0.13 & 4797.5 & 184 & & 0.07 \\
\hline 5534.3 & 184 & & 0.16 & 4782.4 & 184 & & 0.25 \\
\hline 5517.1 & & & 0.13 & 4757.6 & 183,4 & & 0.24 \\
\hline 5465.8 & 187 & 0.11 & 0.13 & 4747.2 & 187 & 0.12 & 0.30 \\
\hline 5400.4 & 184 & & 0.15 & 4719.6 & 183,4 & 0.20 & 0.39 \\
\hline 5382.3 & 184 & & 0.25 & 4706.0 & 183,4 & 0.04 & 0.24 \\
\hline 5375.9 & 184 & & 0.18 & 4609.7 & 184,7 & & 0.29 \\
\hline 5353.8 & & 0.03 & & 4684.5 & 187 & 1.29 & \\
\hline 5349.9 & 184 & & 0.08 & 4650.2 & $184,5,7$ & 0.36 & 0.46 \\
\hline 5320.8 & 187 & 2.77 & 0.49 & 4645.2 & $184^{\circ}$ & & 0.23 \\
\hline 5315.4 & 184 & & 0.30 & 4635.4 & 183 & 0.12 & 0.31 \\
\hline 5308.4 & 184 & & 0.17 & 4626.7 & 187 & 1.05 & \\
\hline 5302.3 & 184 & & 0.17 & 4611 & & 0.04 & 0.25 \\
\hline 5284.9 & 184 & 0.09 & 0.18 & 4597.7 & 184 & & 0.20 \\
\hline 5261.8 & 183,7 & 3.93 & 0.29 & 4574.5 & 187 & 0.81 & \\
\hline 5256.3 & 183 & & 0.21 & 4569.3 & 183,4 & & 0.14 \\
\hline 5243.3 & 184 & 0.06 & 0.35 & 4563.4 & & 0.25 & \\
\hline 5227.1 & $183(?)$ & 0.03 & & 4557.9 & 183 & 0.23 & \\
\hline 5219.6 & 184 & & 0.07 & 4534.2 & 183,5 & 0.11 & 0.24 \\
\hline 5191.2 & 184 & & 0.22 & 4525.9 & & 0.05 & \\
\hline 5164.5 & 183,4 & 1.80 & 0.46 & 4518.0 & 183,4 & 0.38 & 0.28 \\
\hline 5142.2 & & 0.04 & 0.12 & 4509.1 & & 0.09 & \\
\hline
\end{tabular}

a) In cases where the line is seen in both thermal and $2-\mathrm{keV}$ neutron capture the thermal neutron capture line energy is given here, the 2-keV neutron capture Iines have energies which are $2-\mathrm{keV}$ higher than these. 
TABLE IV

COMPARISON OF THE INTENSITIES OF THE SECONDARY CAPTURE $\gamma$-RAY TRANSITIONS RESULTING FROM THERMAL- AIN 2-keV NEUTRON CAPTURE IN ${ }^{171} \mathrm{Yb}$

\begin{tabular}{|c|c|c|c|c|}
\hline \multirow{2}{*}{$\begin{array}{c}\text { Gamma-Ray } \\
\text { Energy } \\
(\mathrm{keV}) \\
\end{array}$} & & \multicolumn{2}{|l|}{ Thermal Neutrons } & \multirow{2}{*}{$\begin{array}{l}\frac{2-\mathrm{keV} \text { Neutrons }}{\text { Relative }} \\
\text { Intensity }\end{array}$} \\
\hline & & $\begin{array}{c}\text { Absolute Intensity } \\
(\gamma / 100 \text { neutrons captured }) \\
\end{array}$ & $\begin{array}{l}\text { Relative } \\
\text { Intensity } \\
\end{array}$ & \\
\hline$(855+857)$ & & 2.51 & 46.5 & 204 \\
\hline 911 & & 0.45 & 8.4 & 101 \\
\hline$(961+964)$ & & 3.89 & 72.1 & 351 \\
\hline 1039 & & 2.29 & 42.4 & 211 \\
\hline$(1070+1076)$ & & 5.7 & 104.6 & 476 \\
\hline 1093 & & 2.59 & 48.0 & 423 \\
\hline$(1117+1119)$ & & 5.9 & 109.4 & 500 \\
\hline 1143 & & 1.12 & 20.7 & 114 \\
\hline$(1152+1155)$ & & I.3I & 24.3 & 86 \\
\hline 1387 & & 1.49 & 27.6 & 177 \\
\hline 1397 & & 1.40 & 26.0 & 167 \\
\hline 1521 & & 2.04 & 37.7 & 205 \\
\hline 1529 & & 1.03 & 19.1 & 101 \\
\hline 1599 & & 1.31 & 24.3 & 136 \\
\hline 1608 & & 0.85 & 15.8 & 65 \\
\hline 1715 & & 0.89 & 16.4 & 59 \\
\hline 1931 & & 0.75 & 13.8 & 86 \\
\hline 1968 & & 0.73 & 13.6 & 107 \\
\hline 2010 & & 0.98 & 15.9 & 76 \\
\hline$(2019+2024)$ & & 1.36 & 25.1 & 88 \\
\hline 2135 & & 0.48 & 8.9 & 63 \\
\hline 2326 & & 1.12 & 20.8 & 77 \\
\hline 2401 & & 2.19 & 40.5 & $<25$ \\
\hline & SUM & $\overline{42.3}$ & $7 \overline{781.9}$ & $\sqrt{3887}$ \\
\hline
\end{tabular}

a) Based upon a scale for which the $8021.9 \mathrm{keV}$ primary $\gamma$-ray has an intensity of 100. These secondary capture $\gamma$-ray intensity values have been corrected for the contribution of the $>2-\mathrm{keV}$ neutrons. A correction factor of fy0.92 was obtained by convoluting the ${ }^{17} \mathrm{Yb}(\mathrm{n}, \gamma)$ cross-section curve (approximated as being similar to the cross-section curve for natural Ytterbium in Ref. 3) with the neutron energy distribution in the beam. 
TABLE V

COMPARISON OF PROMPT $\gamma$-RAY INTENSITIES RESULTING

FROM 2-keV AND THERMAL NEUTRON CAPTURE IN $171 \mathrm{Yb}$

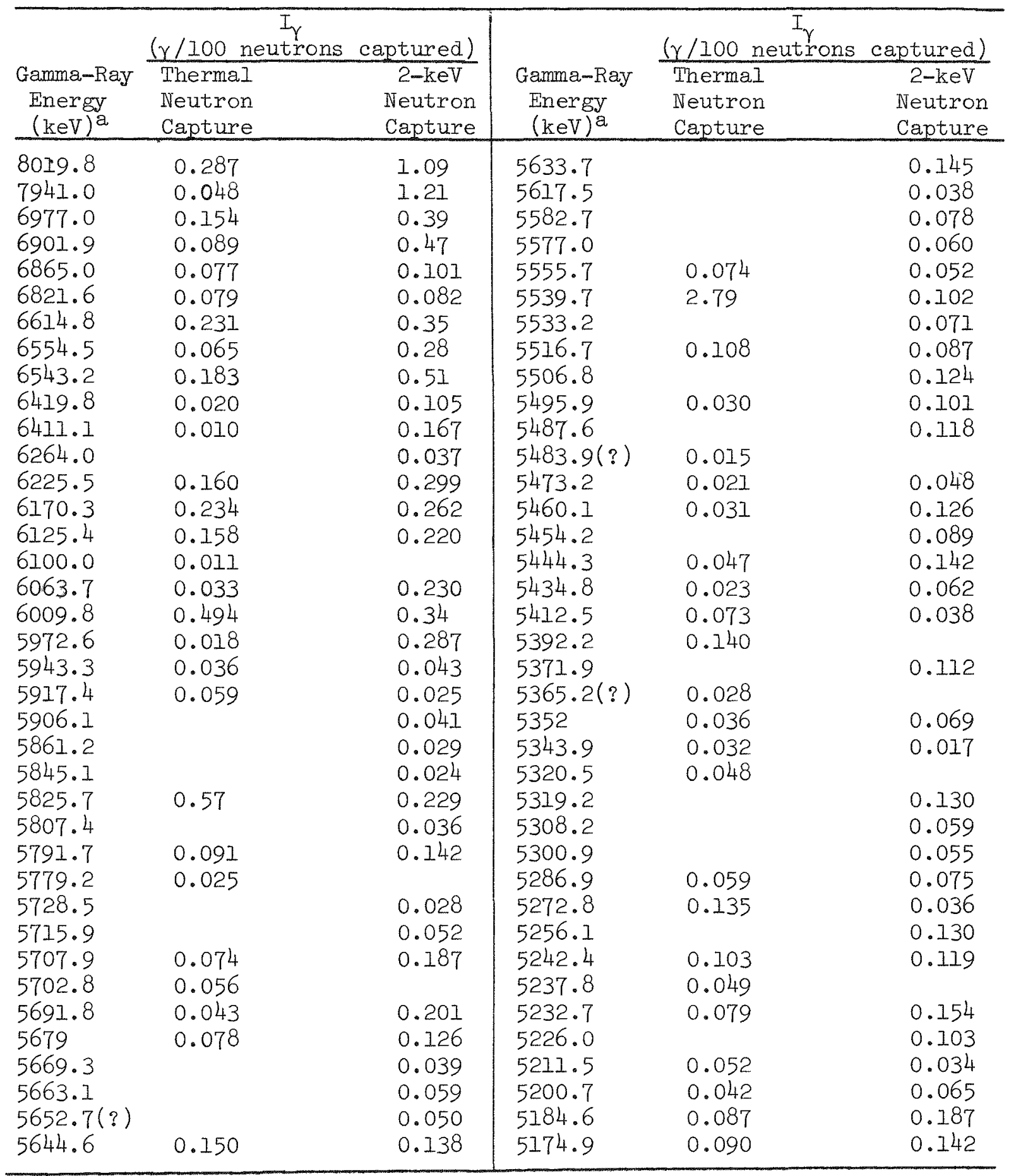

a) In cases where the line is seen in both thermal and $2-\mathrm{keV}$ neutron capture, the thermal neutron capture line energy is given here, the $2-\mathrm{keV}$ neutron capture lines have energies which are $2 \mathrm{keV}$ higher than these. 
TABLE VI

COMPARISON OF THE INTENSITIES OF THE SECONDARY CAPTURE $\gamma$-RAY TRANSITIONS RESULTING FROM THERMAL AND $2-\mathrm{keV}$ NEUTRON CAPTURE IN $173 \mathrm{Yb}$

\begin{tabular}{|c|c|c|c|c|}
\hline \multirow[b]{2}{*}{$\begin{array}{c}\text { Gamma-Ray Energy } \\
(\mathrm{keV})\end{array}$} & \multicolumn{3}{|c|}{ Thermal Neutrons } & 2-keV Neutrons \\
\hline & $\begin{array}{r}\mathrm{Abs} \\
(\gamma / 100 \\
\end{array}$ & $\begin{array}{l}\text { olute Intensity } \\
\text { neutrons captured) }\end{array}$ & $\begin{array}{l}\text { Relative } \\
\text { Intensity }\end{array}$ & $\begin{array}{c}\text { Relative } \\
\text { Intensity } \\
\end{array}$ \\
\hline $\begin{array}{c}992 \\
1128 \\
1215 \\
1241 \\
(1305+1307) \\
1318 \\
\left(1525^{b}+1529+1532\right) \\
(1543+1548+1552) \\
1557 \\
1598 \\
1633 \\
1657 \\
1674 \\
(1709+1710) \\
2025 \\
2043 \\
2198 \\
(2284+2287)\end{array}$ & SUM & $\begin{array}{c}0.75 \\
1.29 \\
1.74 \\
10.7 \\
7.3 \\
0.78 \\
2.86 \\
3.07 \\
2.58 \\
0.85 \\
4.91 \\
1.34 \\
1.28 \\
2.27 \\
1.41 \\
0.37 \\
0.65 \\
2.23 \\
46.4\end{array}$ & $\begin{array}{r}7.0 \\
12.1 \\
16.3 \\
100 \\
68.3 \\
7.3 \\
26.7 \\
28.7 \\
24.1 \\
7.9 \\
45.9 \\
12.5 \\
12.0 \\
21.2 \\
13.2 \\
3.5 \\
6.1 \\
20.8 \\
433.6\end{array}$ & $\begin{array}{c}77.4 \\
100.4 \\
152.7 \\
906 \\
630 \\
88.1 \\
211 \\
252 \\
198 \\
69.9 \\
432 \\
144 \\
112 \\
136 \\
97.9 \\
31.1 \\
61.6 \\
84.4 \\
3785\end{array}$ \\
\hline
\end{tabular}

a) Based upon a scale for which $I_{Y}(7313.0+7389.6)=177.0$. These secondary capture $\gamma$-ray intensities have been corrected for the contribution of the $>2 \mathrm{keV}$ neutrons. A correction factor of $\simeq 0.92$ was obtained by convoluting the ${ }^{173} \mathrm{Yb}(n, y)$ cross section curve (considered to be approximately the same as the cross section curve for natural ytterbium given in Ref. 3) into the neutron energy distribution in the beam.

b) Double-escape peak. 
TABLE VII

COMPARISON OF PROMPT $\gamma$-RAY INTENSITIES RESULTING FROM 2-keV AND THERMAL NEUTRON CAPTURE IN $173_{\mathrm{Yb}}$

\begin{tabular}{|c|c|c|c|c|c|}
\hline \multirow[b]{2}{*}{$\begin{array}{c}\text { Gamma-Ray } \\
\text { Energy } \\
(\mathrm{keV})^{a} \\
\end{array}$} & \multicolumn{2}{|c|}{$\begin{array}{c}I_{\gamma} \\
(\gamma / 100 \text { neutrons captured })\end{array}$} & \multirow[b]{2}{*}{$\begin{array}{c}\text { Gamma-Ray } \\
\text { Energy } \\
(\mathrm{keV})^{\mathrm{a}} \\
\end{array}$} & \multicolumn{2}{|c|}{ (y/100 neutrons captured) } \\
\hline & $\begin{array}{l}\text { Thermal } \\
\text { Neutron } \\
\text { Capture }\end{array}$ & $\begin{array}{c}\text { 2-keV } \\
\text { Neutron } \\
\text { Capture } \\
\end{array}$ & & $\begin{array}{l}\text { Thermal } \\
\text { Neutron } \\
\text { Capture }\end{array}$ & $\begin{array}{c}\text { 2-keV } \\
\text { Neutron } \\
\text { Capture } \\
\end{array}$ \\
\hline 7387.7 & 1.21 & 1.33 & 5129.8 & & 0.105 \\
\hline 7211.1 & 0.81 & 0.84 & 5104 & 0.186 & 0.146 \\
\hline 6145.9 & 0.038 & 0.128 & 5080.0 & 0.099 & 0.085 \\
\hline 6081.7 & 0.028 & 0.129 & 5060.0 & 0.022 & 0.069 \\
\hline 5995.6 & 0.021 & 0.06 & $5029 \cdot 3$ & & 0.094 \\
\hline 5903.6 & 0.96 & 0.45 & 5015.3 & & 0.060 \\
\hline 5858.3 & 0.48 & 0.51 & 5000.1 & 0.087 & 0.27 \\
\hline 5842.5 & & 0.201 & 4963.8 & 0.073 & 0.055 \\
\hline 5830.6 & 1.32 & 0.57 & 4949.9 & 0.094 & 0.130 \\
\hline 5789.7 & 0.033 & 0.55 & 4944.9 & 0.186 & 0.193 \\
\hline 5762.6 & 0.033 & 0.28 & 4937.3 & 0.123 & 0.116 \\
\hline 5755.0 & 0.195 & 0.51 & 4926.0 & & 0.080 \\
\hline 5749.6 & 0.184 & 0.29 & $4917(?)$ & 0.076 & \\
\hline 5732.8 & & 0.56 & 4882 & 0.35 & 0.30 \\
\hline 5678.4 & 0.028 & 0.089 & 4876.6 & 0.226 & 0.217 \\
\hline 5659.3 & 0.122 & 0.28 & 4863 & 0.35 & 0.223 \\
\hline 5613.5 & 0.086 & 0.077 & 4841.3 & 0.237 & 0.193 \\
\hline 5605.7 & 0.40 & 0.209 & 4822.5 & 0.142 & 0.094 \\
\hline 5530.9 & & 0.084 & 4819.5 & & 0.144 \\
\hline 5517.1 & & 0.060 & $4807 \cdot 3$ & 0.106 & 0.207 \\
\hline 5505.4 & 0.056 & 0.30 & 4801.2 & 0.186 & 0.174 \\
\hline 5495.3 & & 0.048 & 4784.1 & 0.36 & 0.170 \\
\hline 5448.7 & 0.30 & 0.36 & 4760.2 & 0.35 & 0.064 \\
\hline 5425.9 & 0.031 & & 4752.2 & 0.86 & 0.138 \\
\hline 5416.9 & & 0.069 & 4738.4 & & 0.100 \\
\hline 5410.5 & 0.021 & & 4732.6 & 0.37 & \\
\hline 5398.0 & & 0.14 & 4715.3 & 0.128 & 0.193 \\
\hline $5377 \cdot 3$ & & 0.070 & $4697.7(?)$ & 0.074 & \\
\hline 5363.5 & 0.60 & 0.166 & $4790.2(?)$ & 0.133 & \\
\hline 5355.3 & & 0.074 & 4780.4 & 0.040 & 0.065 \\
\hline 5341.5 & 0.255 & 0.234 & 4671.2 & 0.212 & \\
\hline 5301.5 & 0.72 & 0.38 & 4667.4 & & 0.055 \\
\hline 5293.0 & 0.60 & 0.34 & 4655.0 & 0.073 & \\
\hline 5276 & 0.076 & 0.064 & 4652.7 & & 0.064 \\
\hline 5268.2 & & 0.055 & $4 6 \longdiv { 4 6 . 2 }$ & 0.227 & \\
\hline 5227.4 & 0.249 & 0.200 & 4640.2 & 0.137 & 0.092 \\
\hline 5218.1 & 0.47 & 0.27 & 4619.1 & 0.238 & 0.096 \\
\hline 5208.5 & 0.44 & 0.221 & 4581.6 & 0.121 & 0.125 \\
\hline 5170.8 & & 0.32 & 4569.0 & 0.31 & 0.178 \\
\hline
\end{tabular}

a) In cases where the line is seen in both thermal and 2-keV neutron capture the thermal neutron capture line energy is given here, the 2-keV neutron capture lines have energies which are $2 \mathrm{keV}$ higher than these. 


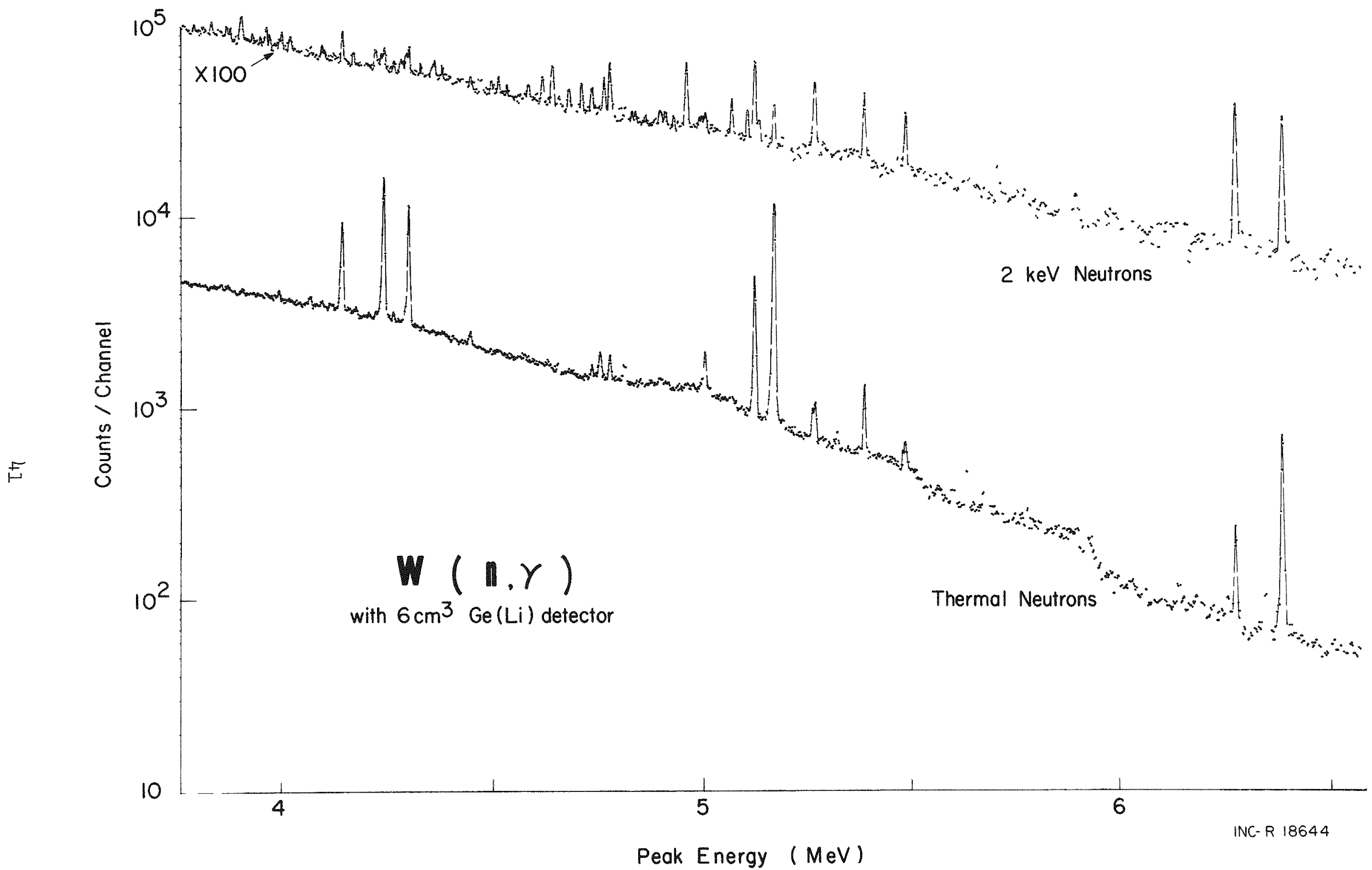

Figure 1 The high-energy portion of the prompt $\gamma$-ray spectra resulting from the capture of thermal and $2-\mathrm{keV}$ neutrons in natural tungsten. The peaks observed in this spectrum are principally doubleescape peaks and, hence, the prompt $\gamma$-ray energies are $1.022 \mathrm{MeV}$ higher. 
A SURVEY OF NONROTATIOHAL STATES OF

DEFORMED ODD-A NUCLEI $(150<A<190)$

M. E. Bunker ${ }^{[1]}$, C. W. Reich

The survey and critical evaluation of the properties of the nonrotational energy states of the deformed odd-mass nuclei in the "rareearth" region have been completed. The results of this study were incorporated into a review paper which has been accepted for publication in Reviews of Modern Physics and will appear in the July 1971 issue of this journal. The following is the abstract of this paper:

The configuration assignments for the known energy levels of the odd-A deformed nuclei in the mass range $150<\mathrm{A}<190$ have been systematically reviewed. For each nucleus, a summary is given of the intrinsic states that are considered well identified, and the main arguments that support particular assignments are outlined. A discussion is presented of the theoretical framework used in the analysis, which is basically the Nilsson singleparticle model with pairing correlations and Coriolis coupling taken into account, and with appropriate consideration given to vibrational excitations and their mixing with the one-quasiparticle states. Energy systematics are shown for the one-quasiparticle excitations and for the gamma-vibrational excitations (most of which have significant one-quasi-particle admixtures), and these systematics are discussed in terms of current theory.

[1] Los Alamos Scientific Laboratory, Los Alamos, New Mexico. 
Accurate relative intensity measurements of the 271- and $1156-\mathrm{keV}$

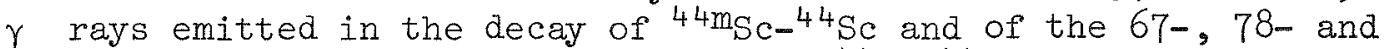
$1156-\mathrm{keV} \gamma$ rays emitted in the decay of ${ }^{44} \mathrm{Ti}-{ }^{44} \mathrm{Sc}$ have not been reported in the literature. As a byproduct of our programmatic effort in studying neutron deficient nuclides, we have measured the energies and relative intensities of the $\gamma$ rays emitted in the decays of ${ }^{44 \mathrm{~m}_{\mathrm{Sc}}{ }^{44} \mathrm{Sc}}$ in transient equilibrium and of ${ }^{44} \mathrm{Ti}-{ }^{4} 4 \mathrm{Sc}$ in secular equilibrium and their values are listed in Tables I and II, respectively. These values are compared in the tables to those reported from other investigations [2-6]. The relative intensities we report were consistent to within $2 \%$ from measurement to measurement, and the assigned uncertainties are the accuracies to which we believe these measurements have been made. The relative intensity of the $1157-\mathrm{keV} \gamma$ ray in the decay chain of $44 \mathrm{~m}_{\mathrm{Sc}-}{ }^{44} \mathrm{Sc}$ has been normalized to 100 . In the decay chain of ${ }^{44} \mathrm{Ti}-{ }^{44} \mathrm{Sc}$, however, the relative intensity of the $67-\mathrm{keV} \gamma$ ray has been normalized to 100 in order to facilitate comparison of the present relative intensity measurements with the other reported measurements listed in Table II.

From our relative intensities in Table $I_{\text {ine }}$ thalf lives of $44 \mathrm{~m}_{\mathrm{Sc}}$ $(58.6 \pm 0.1 \mathrm{~h})^{[8]}$ and ${ }^{44} \mathrm{Sc}(3.927 \pm 0.008 \mathrm{~h})^{[8]}$ and the decay scheme of Okano et al.[7] (see Figure l), the total conversion coefficient of the $271-\mathrm{keV}$ isomeric transition $\left(\alpha_{T}(271)\right)$ was determined when the decaying radionuclides ${ }^{4} \mathrm{~m}_{\mathrm{Sc}}{ }^{44} \mathrm{Sc}$ are in transient equilibrium. We report a value for the total conversion coefficient of the 27l-keV isomeric transition of $0.17 \pm 0.06$ which compares favorably with the theoretical value for an $E 4$ transition $[9]\left(\alpha_{T}(271)=0.131\right)$ and with the measured value of Blue and Bleuler[10] $\left(\alpha_{\mathrm{T}}(271)=0.14\right)$. The branching ratios of the isomeric transition and of the electron capture and $\beta^{+}$branches resulting from the decay of ${ }^{44} \mathrm{~m} \mathrm{Sc}$ and from the decay of ${ }^{44} \mathrm{Sc}$ were also determined and are listed in parenthesis in Figure 1 next to the measured values of Okano et et al [7].

From the ${ }^{44} \mathrm{Ti}$ decay scheme of Ristinen and Sunyar[3] (see Figure 2) and the relative $\gamma$-ray intensities listed in Table II, the total conversion coefficients $\left(\alpha_{\mathrm{T}}\right)$ of the $67-$ and $78 \mathrm{-keV}$ transitions were determined to be $0.11+0.06$ and $0.028 \pm 0.067$, respectively. These measured values for $\alpha_{T}(67)$ and $\alpha_{T}(78)$ are listed in Table III and are compared to the results of other investigations and to the theoretical MI and E2 values for the conversion coefficients of the $67-$ and $78-\mathrm{keV}$ transitions [9].

[1] Physics Department, University of Nevada, Reno, Nevada.

[2] J. K. Kliwer, J. J. Kraushaar, R. A. Ristinen, J. R. Keith, and A. A. Bartlett, Nuclear Physics 49 (1963) 328.

[3] R. A. Ristinen and A. W. Sunyar, Phys. Rev. 153 (1967) 1209 


\section{TABLE I}

ENERGIES AND RELATIVE INTENSITIES OF $\gamma$-RAYS EMITTED

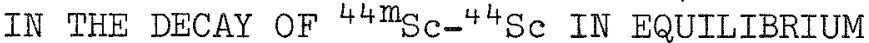

\begin{tabular}{|c|c|c|c|c|c|c|c|}
\hline \multicolumn{4}{|c|}{ King et al. [4] } & \multicolumn{2}{|c|}{$\begin{array}{l}\text { Levkovskii } \\
\text { et al. [6] }\end{array}$} & \multicolumn{2}{|c|}{ K. Okano et al. [7] } \\
\hline \multicolumn{3}{|c|}{$\mathrm{E}(\mathrm{keV})$} & $I(r e I)$ & $\mathrm{E}(\mathrm{keV})$ & $I($ reI $)$ & $\mathrm{E}(\mathrm{keV})$ & $I(r e l)$ \\
\hline $\begin{array}{l}271.1 \\
511.1\end{array}$ & $\begin{array}{l} \pm \\
\pm\end{array}$ & $\begin{array}{l}0.2 \\
0.2\end{array}$ & $\begin{array}{r}\left.98^{a}\right) \\
\left.>156^{b}\right)\end{array}$ & $271 \pm 1$ & $100^{a)}$ & 271 & $98.73^{a)}$ \\
\hline 1002 & \pm & 1 & 1.1 & $1000 \pm 3$ & $\sim 1.8$ & $1001.9 \pm 0.6$ & $1.27 \pm 0.06$ \\
\hline 1127 & \pm & 1 & 1.2 & $1127 \pm 2$ & 1.8 & $1125.6 \pm 0.6$ & $1.27 \pm 0.06$ \\
\hline 1157.9 & \pm & 0.3 & 100 & $1157 \pm 1$ & 100 & $1156.9 \pm 0.5$ & 100 \\
\hline 1499 & \pm & 1 & 0.76 & $1499 \pm 1$ & 0.9 & $1499.6 \pm 0.5$ & $0.94 \pm 0.04$ \\
\hline 1666 & \pm & 2 & 0.25 & Not obser & & & \\
\hline 2655 & \pm & $\left.2^{c}\right)$ & 0.3 & $2656 \pm 1$ & 0.15 & $2656.4 \pm 0.5$ & $0.136 \pm 0.011$ \\
\hline
\end{tabular}

a) Isomeric transition intensity.

b) Annihilation radiation: intensity not corrected for the loss of positrons from the source material.

c) Energy determined from double-escape peak at $1633 \pm 1 \mathrm{keV}$. 


\section{TABLE II}

ENERGIES AND REIATIVE INTENSITIES OF $\gamma$-RAYS EMITTED IN THE DECAY OF ${ }^{44} \mathrm{Ti}-{ }^{44} \mathrm{SC}$ IN EQUILIBRIUM

\begin{tabular}{|c|c|c|c|c|c|}
\hline \multicolumn{2}{|c|}{ Present Experiment } & \multicolumn{2}{|c|}{ Ristinen and Sunyar [3] } & \multicolumn{2}{|c|}{ Kliwer et al. [2] } \\
\hline $\mathrm{E}(\mathrm{keV})$ & $I(r e I)$ & $\mathrm{E}(\mathrm{keV})$ & $I(r e I)$ & $\mathrm{E}(\mathrm{keV})$ & $I(r e l)$ \\
\hline $\begin{array}{l}67.868 \pm 0.005 \\
78.335 \pm 0.010\end{array}$ & $\begin{array}{l}100 \\
106 \pm 2\end{array}$ & $\begin{aligned} 67.85 & \pm 0.04 \\
78.38 & \pm 0.04 \\
147 & \pm 1.5\end{aligned}$ & $\begin{array}{l}100 \\
106.2 \\
0.10\end{array}$ & $\begin{array}{l}67.85 \pm 0.07 \\
78.44 \pm 0.07\end{array}$ & $\begin{array}{l}100 \\
141 \pm 28\end{array}$ \\
\hline $\begin{array}{ll}1156.92 & \pm 0.15 \\
1499.50 & \pm 0.20 \\
2657 & \end{array}$ & $\begin{aligned} 110.9 & \pm 7 \\
1.0 & \pm 0.1 \\
0.16 & \pm 0.06\end{aligned}$ & & & $1156.1 \pm 1.0$ & $180 \pm 60$ \\
\hline
\end{tabular}

TABLE III

MEASUREMENTS OF THE CONVERSION COEFFICIENTS OF THE 68- AND 78-keV TRANSITIONS EMITTED IN THE DECAY OF ${ }^{44} \mathrm{Ti}$

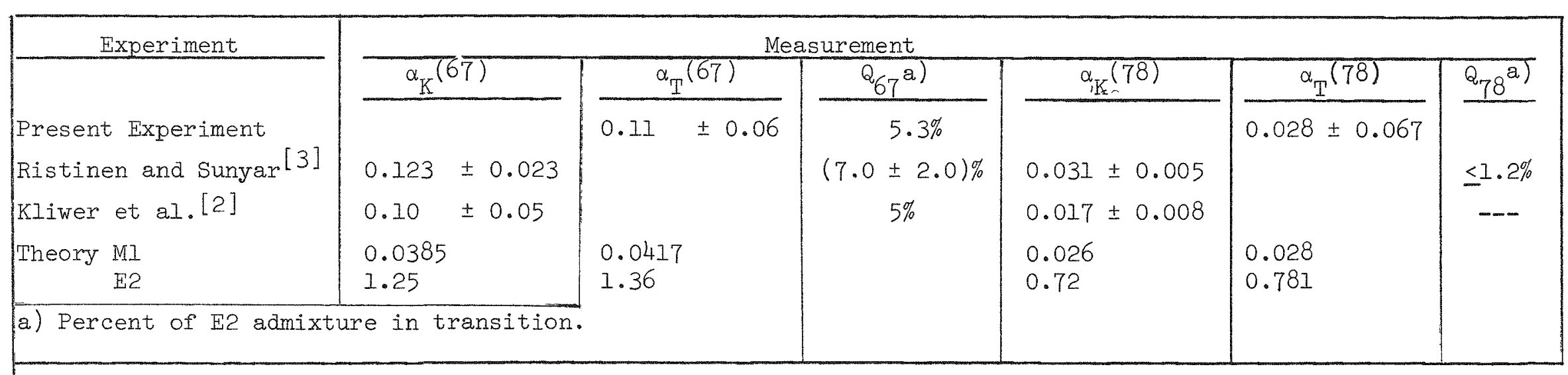




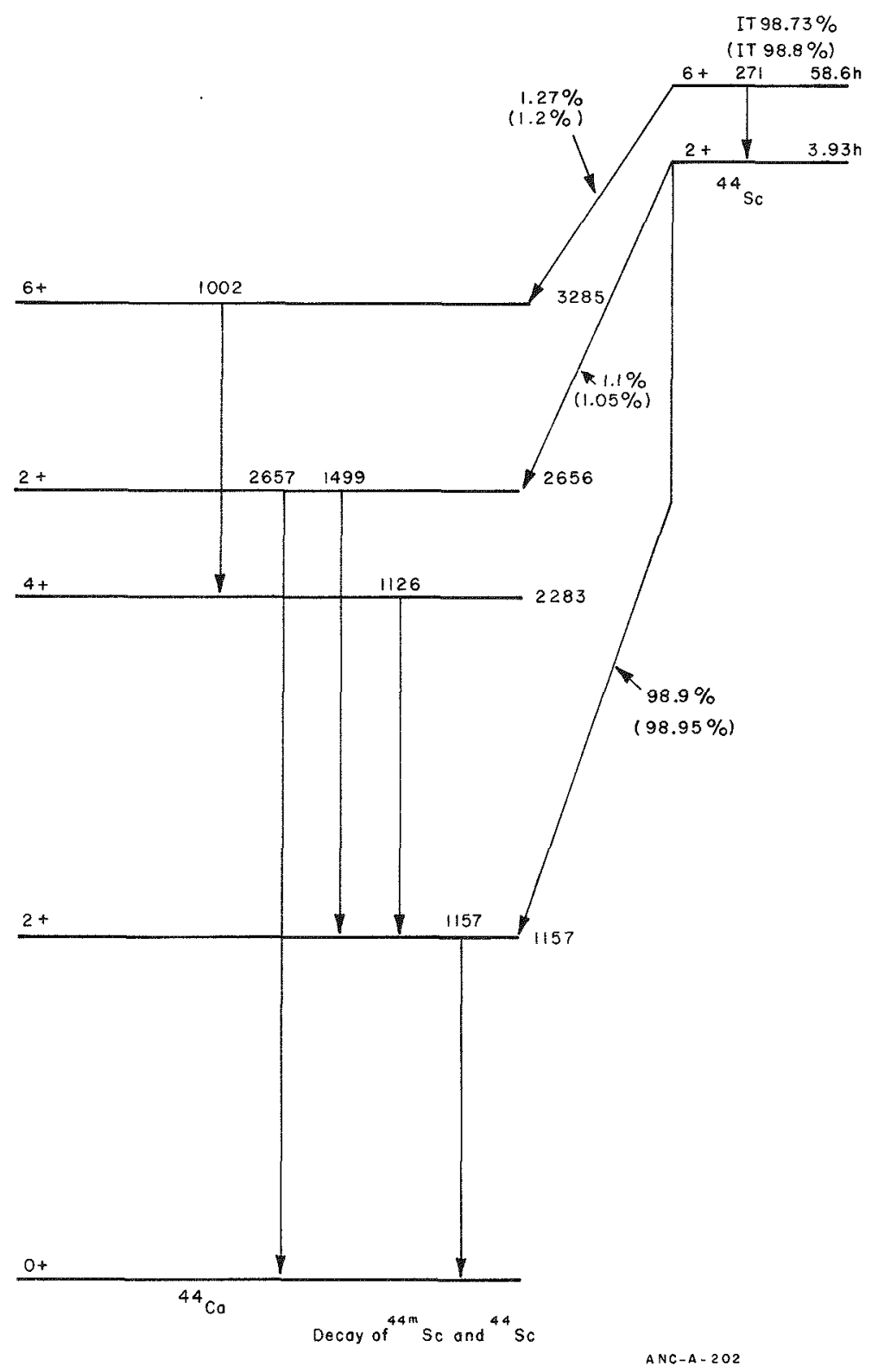

Figure I Decay scheme of ${ }^{44 \mathrm{~m}} \mathrm{Sc}$ and ${ }^{44} \mathrm{Sc}$ as proposed by Okano et al [10].

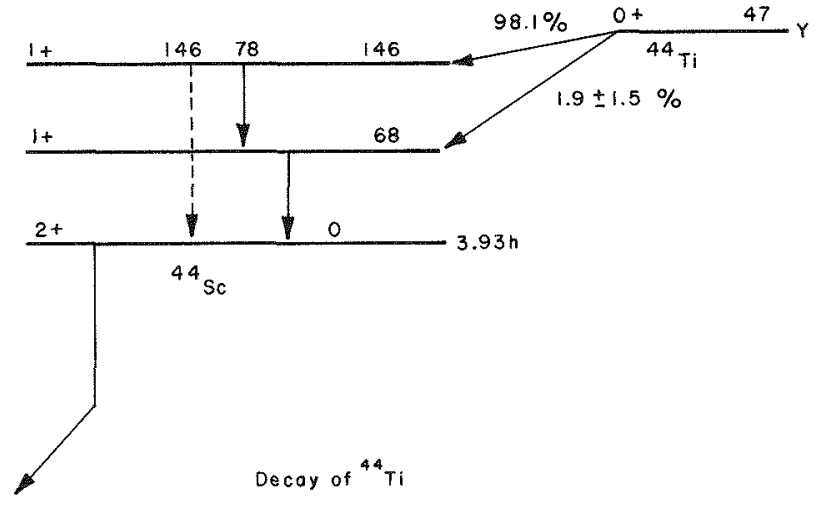

Figure 2 Decay scheme of $44 \mathrm{Ti}$ as proposed by Ristinen and Sunyar [3] 
[4] J. D. King, N. Neff and H. W. Taylor, Canadian Journal of Physics 45 (1967) 2446.

[5] J. D. King, B. Lalovic and H. W. Taylor, Canadian Journal of Physics 46 (1968) 2119.

[6] V. N. Levkovskii and I. V. Kazachevskii, Society Journal of Nuclear Physics 11 (1970) 271.

[7] Kotoyuki Okano, Yoichi Kawase and Shinichi Vehara, Journal of the Physical Society of Japan 30 (1971) 1231.

[8] J. Raun, J. Inorg. Nucl. Chem. 3I (1969) 1883.

[9] I. A. Sliv and I. M. Band, in Gamma Luchi, Academy of Sciences of the USSR (Moscow, Leningrad, 1961) p. 487, et seq.

[10] J.W. BIue and E. Bleuler, Phys. Rev. 100 (1955) 1324. 
DECAY SCHEME STUDIES OF $18 \mathrm{~min} 141 \mathrm{Ba}$ AND $11 \mathrm{~min} 142 \mathrm{Ba}$

L. D. McIsaac, E. L. Murri [I]

The decay schemes of ${ }^{141} \mathrm{Ba}$ and ${ }^{142} \mathrm{Ba}$ have been studied using high resolution $\mathrm{Ge}(\mathrm{Li})$ and $\mathrm{Si}(\mathrm{Li})$ detectors. Coincidence measurements were made using $\mathrm{NaI}(\mathrm{TI})$ detectors and a $256 \times 256$-channel analyzer. We have

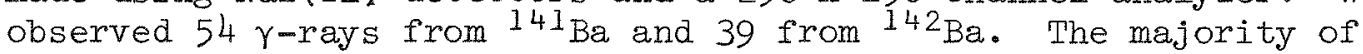
these are previously unreported. These data establish the existence of levels in the daughter nucleus, ${ }^{141} \mathrm{La}$, at the following energies: $190.33,304.18,467.26,580.1,647.89,826.3,831.6,929.4,1066.4$, $1501.4,1740.8$, and $1872.7 \mathrm{keV}$. Energy levels proposed for ${ }^{142} \mathrm{La}$ occur at $77.59,255.29,309.16,334.97,363.94,424.97,432.22,599.71$, $1078.6,1204.2$ and $1457.7 \mathrm{keV}$. The $\mathrm{K}$-conversion coefficient of the $77.59 \mathrm{keV}$ transition deexciting the first excited state in ${ }^{142} \mathrm{La}$ was measured to be $2.0 \pm 0.4$.

[1] Present address: Consumer's Power Company, Jackson, Michigan. 
INTERNAL CONVERSION COEFFICIENTS IN THE DECAY OF $160 \mathrm{~Tb}$

R. G. Helmer

The internal conversion electrons in the decay of $160 \mathrm{~Tb}$ for the gamma-ray transitions from 298 to $1312 \mathrm{keV}$ have been studied with the $\pi \sqrt{2}$ electron spectrometer. This study was initiated to remove conflict as to the multipolarity of the 682-keV transition and to improve the multipolarity data for the weak transitions. For the study, several samples were made by vacuum evaporation. A block of terbium metal was heated in a vacuum by means of the bean from an electron gun. The sublimed terbium metal was collected on a group of aluminum foils $(0.0007 \mathrm{~cm}$ thick) with masks to define the source area. X-ray fluorescence techniques were used to measure the amount of terbium collected on each foil. One of these samples $(0.060 \mathrm{~cm} \mathrm{x} 2 \mathrm{~cm}$ with $60 \mu \mathrm{gm}$ of terbium) was irradiated in the ATR in a flux of $\sim 6 \times 10^{14} \mathrm{n} / \mathrm{cm}^{2} \mathrm{sec}$ for 15 days.

After alignment of the source in the spectrometer, the observed resolution was $0.055 \%$ for lines above $700 \mathrm{keV}$. This is the value expected for this source width $(0.060 \mathrm{~cm})$. At $\sim 200 \mathrm{keV}$, the observed resolution is $0.11 \%$ which indicates that at this energy some of the electrons are being scattered in the source. This is not unexpected for a source this thick $\left(300 \mu \mathrm{g} / \mathrm{cm}^{2}\right)$.

Portions of the observed conversion-electron spectrum are shown in Figure 1. The relative line intensities were determined by analysis with a computer program which fits an empirical line shape to the data for each peak. (For the high energies and the moderate resolution involved here, the natural widths of the atomic shells did not need to be taken into account.) Two line shapes were used: one below $400 \mathrm{keV}$ and the second above $600 \mathrm{keV}$. The computer program provides the area of the line shape used, so that the results of the two sections can be normalized.

As shown in Figure 1, the three $L$ lines from a transition are not resolved for the transitions above $600 \mathrm{keV}$. This complexity results in a rather large uncertainty in the intensities of these lines. Since the $\mathrm{L}_{2}$ lines are the least well-defined, the results are quoted here only for the ratio of the $I_{1}$ and $I_{3}$ intensities.

In order to compute the $K$ conversion coefficients, it is necessary to also have the gamma-ray intensities. Five gamma-ray spectra were measured with two $\mathrm{Ge}(\mathrm{Li})$ detector systems. A sample spectrum is shown in Figure 2. It should be noted that several of the peaks of interest are quite weak and one is on a Compton edge. In several cases, the gamma-ray intensities are the limiting factor in the accuracy of the conversion coefficient.

The relative gamma-ray and $K$-conversion-electron intensities are given in Table $I$. For the determination of the conversion coefficients these two sets of values were normalized to give the theoretical EI conversion coefficient for the $1177-\mathrm{keV}$ transition. For comparison the 
theoretical EI, MI and $E 2$ coefficients are also listed. The uncertainties quoted for the gamma-ray and conversion-electron intensities are estimates of both the systematic and statistical contributions. The latter come from the various analysis programs. The former are taken to be 5\% for both sets of data except for a few strong, closely spaced lines. For the electron data this 5\% includes errors in the detector efficiency values and in unaccounted for variation in line shape (due to electron scattering in the source). The dominant multipolarities deduced from these data are given in the last column.

The ratio of the $L_{1}$ and $L_{3}$ conversion line intensities for four transitions are given in Table II along with the theoretical values. Since these lines are closely spaced only the error from the peak analysis is given. However, due to the complexity of the analysis, these error estimates may be low. The dominant multipolarity and a limit on the intensity of the secondary multipolarity for these data are given.

The results in Tables I and II agree with the accepted decay scheme[2,3] for $160 \mathrm{~Tb}$. The data are, however, more extensive than these works $[2,3]$. (There are no reported values for these I subshell ratios or the $337 \mathrm{~K}$ line, and the $682 \mathrm{~K}$ intensity is much improved.)

[1] R. S. Hager and E. C. Seltzer, Nucl. Data A4, I (1968).

[2] G. R. Ewan, R. L. Graham and J. S. Geiger, Nucl. Phys. 22, 610 (1961).

[3] C. Gunther, H. Ryde and K. Krien, Nucl. Phys. Al22, 401 (1968). 
TABLE I

K CONVERSION-ELECTRON DATA FROM 160 Tb

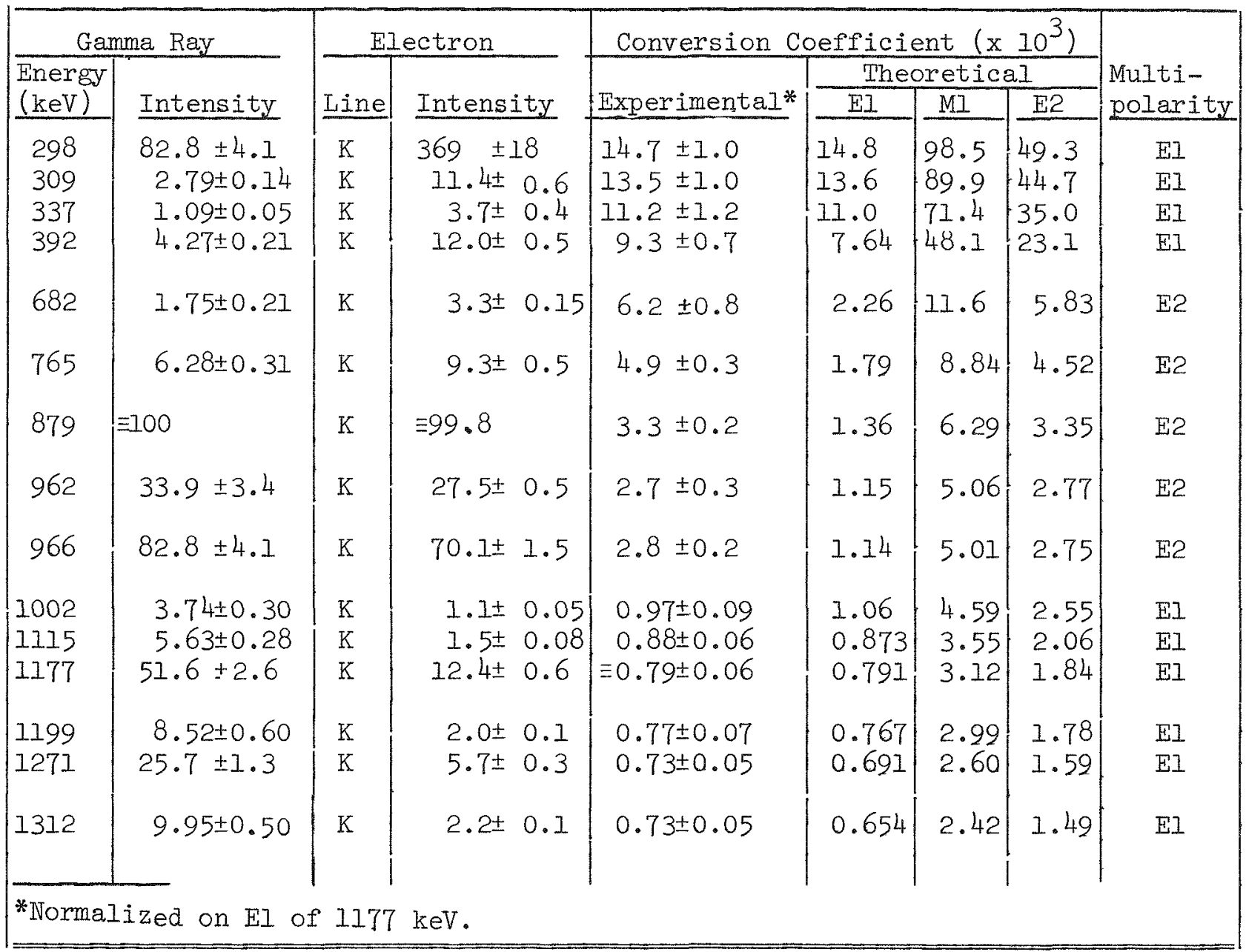

\section{TABLE II}

L SUBSHELI RATIOS IN THE DECAY OF $160 \mathrm{~Tb}$

\begin{tabular}{|c|c|c|c|c|}
\hline \multirow{3}{*}{$\begin{array}{c}\text { Transition } \\
\text { Energy } \\
(\mathrm{keV}) \\
\end{array}$} & \multicolumn{3}{|c|}{ L3/LI Ratio } & \multirow[b]{3}{*}{ Multipolarity } \\
\hline & \multirow{3}{*}{$\frac{\text { Experimental }}{0.101 \pm 0.025}$} & \multicolumn{2}{|c|}{ Theoretical } & \\
\hline & & & $\mathrm{E2}$ & \\
\hline 765 & & 0.009 & 0.091 & $\mathrm{E} 2,<10 \% \mathrm{MI}$ \\
\hline 879 & $0.065 \pm 0.004$ & 0.009 & 0.072 & $\mathrm{E} 2,<10 \% \mathrm{MI}$ \\
\hline 962 & $0.057 \pm 0.006$ & 0.009 & 0.063 & $\mathrm{E2},<12 \% \mathrm{MI}$ \\
\hline 966 & $0.049 \pm 0.007$ & 0.009 & 0.062 & $\mathrm{E} 2,<20 \% \mathrm{MI} *$ \\
\hline
\end{tabular}

*From decay scheme, this is a pure E2 transition. 

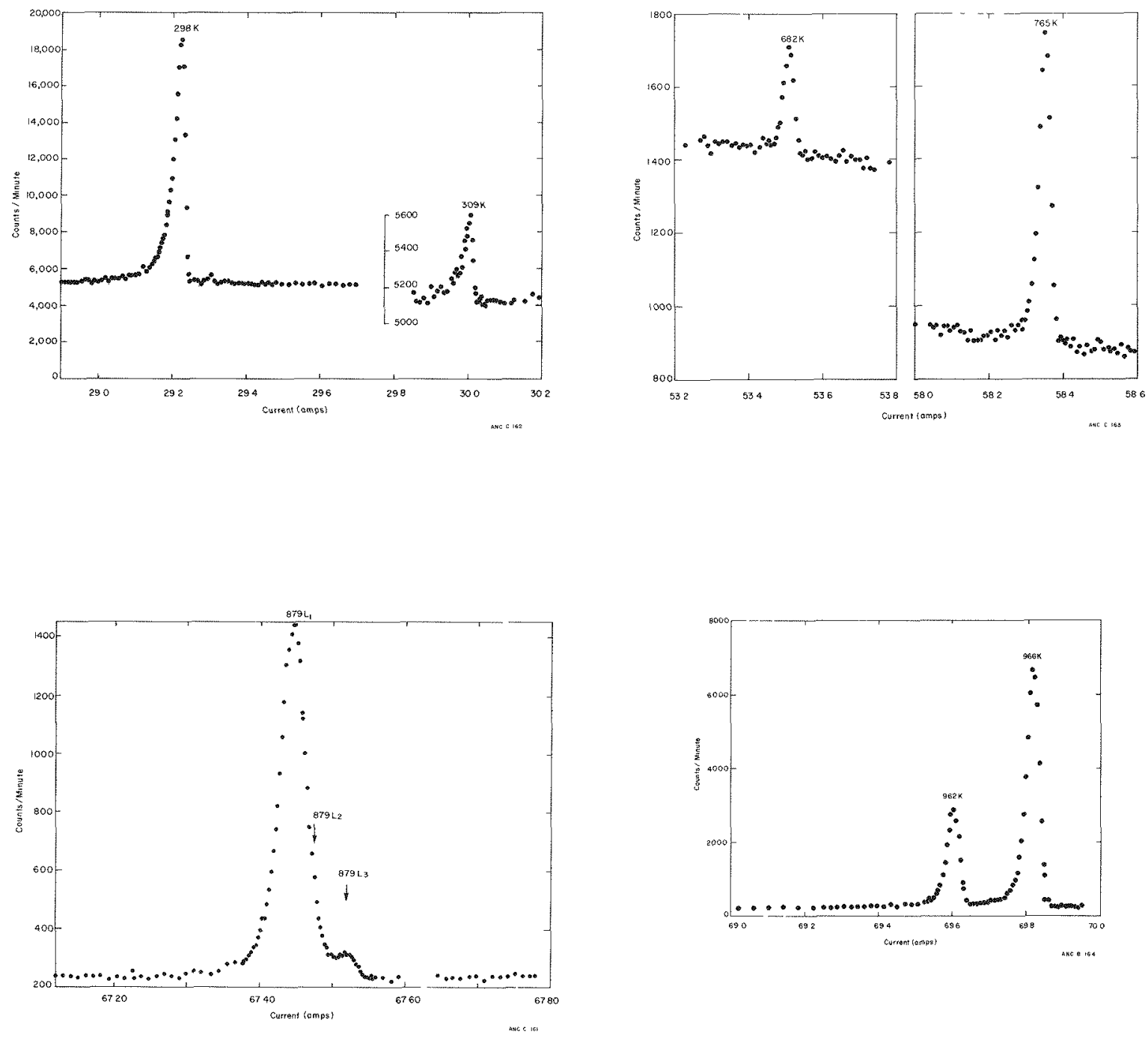

Figure 1 Portions of internal-conversion electron spectrum from $160 \mathrm{~Tb}$. 


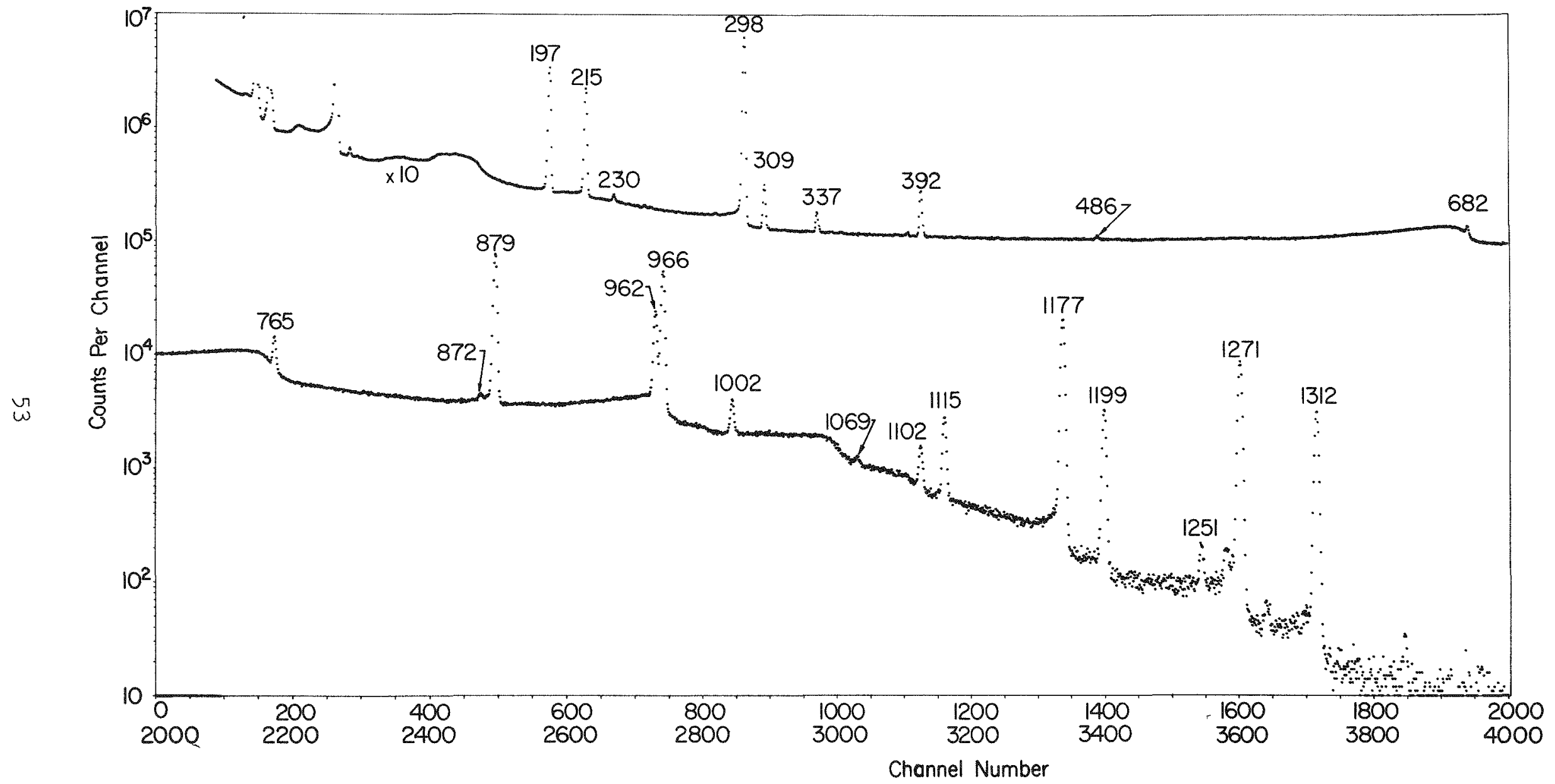

Figure 2 Gamma-ray spectrum of $160 \mathrm{~Tb}$. 


\section{GAMMA-RAY TRANSITIONS DE-EXCITING STATES NEAR I MeV}

IN IO3 W OBSERVED IN THE $(n, y)$ REACTION

R. C. Greenwood, C.W. Reich

In the course of our studies of the level structure of ${ }^{184} \mathrm{~W}$ utilizing the ${ }^{183} \mathrm{~W}(n, \gamma)$ reaction [1], it/ was necessary to make some measurements of the prompt $\gamma$-ray spectra from both thermal and $2-\mathrm{keV}$ neutron capture in ${ }^{18} \mathrm{~W}$ in order to fully determine the background contributions to the $183_{W}(n, \gamma)$ reaction spectra. Although extensive measurements of the $182 \mathrm{~W}(n, \gamma)$ spectra were not made, the data which were obtained did provide some information on the location of states having spins of $1 / 2$ and $3 / 2$ in $183_{\mathrm{W}}$, together with the de-excitation modes of many of these states. Of particular interest here, in view of recent measurements of the ${ }^{184} \mathrm{~W}(\mathrm{~d}, t)$ reaction spectra[2], are the states at $\sim \mathrm{I} \mathrm{MeV}$.

The high-energy portion of the primary $\gamma$-ray spectrum resulting from $2-\mathrm{keV}$ neutron capture in ${ }^{182} \mathrm{~W}$ is shown in Figure 1 . The states at 934 and $1026 \mathrm{keV}$ which are noted in this figure have been identified [2] as the $\frac{1^{-}}{2}$ and $\frac{3^{-}}{2}$ members of the $\frac{1}{2}^{-}[521]$ band. The level at $1026 \mathrm{keV}$ is very strongly populated by a $5164-\mathrm{keV}$ primary transition in thermal neutron capture and hence, the $\gamma$-rays de-exciting this level are observed as prominent lines in the thermal-neutron capture $\gamma-r$ ay spectrum.

The modes of de-excitation of states at $v 1 \mathrm{MeV}$ in $183_{\mathrm{W}}$ are summarized in Table I. While the level at $903 \mathrm{keV}$ noted in this Table is not populated by a primary transition, the $\gamma$-rays de-exciting this level are also prominent in the thermal-neutron $\gamma$-ray spectrum. The branching ratios for $\gamma$-decay from the 903,934 and $1026 \mathrm{keV}$ levels are of particular interest in this study and are being interpreted in terms of a Coriolis band-mixing picture.

[I] R. C. Greenwood, C. W. Reich, Levels in ${ }^{184} \mathrm{~W}$ from ${ }^{183} \mathrm{~W}(\mathrm{n}, \gamma)$, this report.

[2] R. F. Casten, private communication. 
TABLE I

DE-EXCITATION OF STATES WITH ENERGIES $>0.9 \mathrm{MeV}$ IN ${ }^{183} \mathrm{~W}$

\begin{tabular}{|c|c|c|c|c|c|}
\hline \multicolumn{2}{|c|}{ Initial State } & \multirow{2}{*}{$\begin{array}{c}\gamma \text {-Ray }[\mathrm{a}] \\
\text { Transition } \\
(\mathrm{keV})\end{array}$} & \multirow{2}{*}{$\begin{array}{l}\text { Relative } \\
\gamma \text {-Ray } \\
\text { Intensity }\end{array}$} & \multicolumn{2}{|c|}{ Final State } \\
\hline $\begin{array}{l}\text { Energy } \\
(\mathrm{keV}) \\
\end{array}$ & $I, K^{\pi}$ & & & $\begin{array}{l}\text { Energy } \\
(\mathrm{keV}) \\
\end{array}$ & $I, K^{\pi}$ \\
\hline \multirow[t]{4}{*}{903.45} & $\frac{5}{2}, \frac{5}{2}$ & {$[857.67(15)]$} & $10.0(15)$ & 46.48 & $\frac{3}{2}, \frac{1}{2}$ \\
\hline & & {$[696.76(6)]$} & $18.2(18)$ & 207.01 & $\frac{7}{2}, \frac{1^{-}}{2}$ \\
\hline & & $694.62(6)$ & $20.8(21)$ & 208.81 & $\frac{3}{2}, \frac{3}{2}^{-}$ \\
\hline & & $611.73(9)$ & $8.0(12)$ & 291.72 & $\frac{5}{2}, \frac{3}{2}$ \\
\hline 934.56 & $\frac{1}{2}, \frac{1}{2}$ & $888.08 \quad(6)$ & $39 \quad(3)$ & 46.48 & $\frac{3}{2}, \frac{1^{-}}{2}$ \\
\hline \multirow[t]{4}{*}{1026.28} & $\frac{3}{2}, \frac{7}{2}$ & $1026.30(5)$ & $100 \quad(5)$ & 0 & $\frac{1}{2}, \frac{1}{2}$ \\
\hline & & $979.82(6)$ & $61 \quad(3)$ & 46.48 & $\frac{3}{2}, \frac{1}{2}$ \\
\hline & & $927.17(12)$ & $17.4(26)$ & 99.08 & $\frac{5}{2}, \frac{1}{2}$ \\
\hline & & $817.44(12)$ & $16.4(25)$ & 208.81 & $\frac{3}{2}, \frac{3}{2}$ \\
\hline \multirow[t]{2}{*}{1291.7} & $\left(\frac{1}{2}, \frac{3}{2}\right)$ & $1192.7 \quad(2)$ & $14.2(28)$ & 99.08 & $\frac{5}{2}, \frac{1}{2}$ \\
\hline & & $999.7 \quad(4)$ & $3.4(13)$ & 291.72 & $\frac{5}{2}, \frac{3}{2}$ \\
\hline \multirow[t]{2}{*}{1471.0} & $\left(\frac{1}{2}, \frac{3}{2}\right)$ & $1424.1 \quad(2)$ & $16.5(33)$ & 46.48 & $\frac{3}{2}, \frac{1}{2}$ \\
\hline & & $1262.7 \quad(2)$ & $12.4(25)$ & 208.81 & $\frac{3}{2} \cdot \frac{3}{2}$ \\
\hline 1555.61 & $\left(\frac{3}{2}\right)$ & $652.16(7)$ & $17.3(12)$ & 903.45 & $\frac{5}{2}, \frac{5}{2}$ \\
\hline
\end{tabular}

[a] Brackets around a $\gamma$-ray transition energy indicates a tentative assignment. 


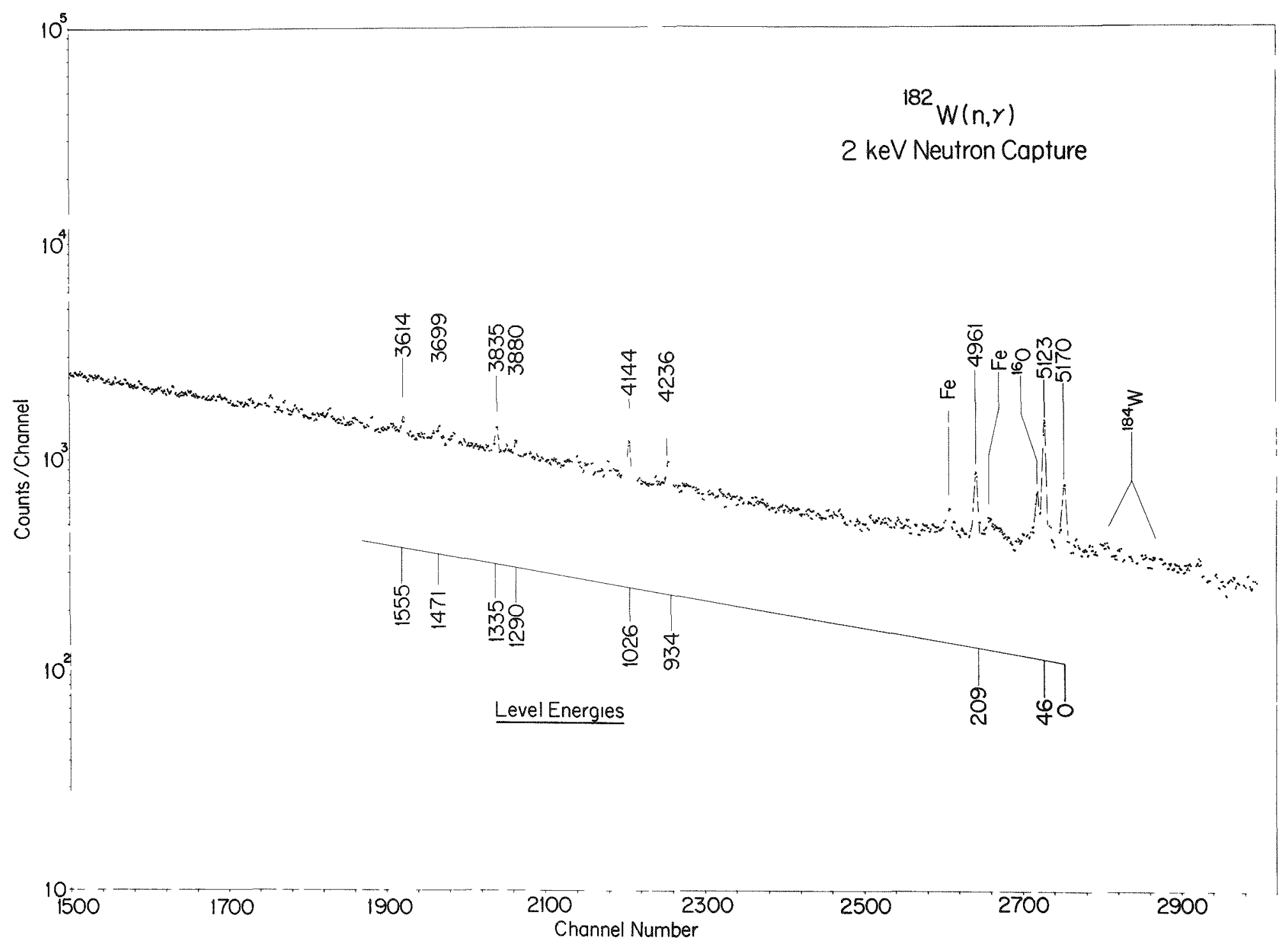

Figure I A portion of the prompt $\gamma$-ray spectrum resulting from $2-\mathrm{keV}$ neutron capture in ${ }^{182} \mathrm{~W}$. 
A. G. Taylor $\frac{\text { THE DECAY OF } 183 \mathrm{Re}}{[1], \text { R. G. Helmer, C. W. Reich }}$

The gamma radiation and internal-conversion electrons associated with the electron-capture decay of 183 Re have been studied using Ge(Li) detectors and the $35-\mathrm{cm}$ air-cored $\pi \sqrt{2}$ conversion-electron spectrometer. The objectives of this study were twofold:

First, to obtain precise values for the gamma-ray intensities and mixing ratios. These data would form the basis of a Coriolismixing analysis of the low-lying $(<0.5 \mathrm{MeV})$ negative-parity states in the daughter nucleus, $183_{\mathrm{W}}$. These levels have been well studied experimentally from the decay of ${ }^{183} \mathrm{Ta}$ (see, e.g., ref. [2]), and theoretically in terms of Coriolis mixing[3]. However, the decay scheme studies of ${ }^{183} \mathrm{Re}$ are much less extensive, the only conversionelectron studies being those of Harmatz et al.[4], using permanentmagnet spectrographs. Furthermore, recent single-nucleon-transfer reaction experiments [5] have provided valuable information on the location and properties of the negative-parity states above w0.5 MeV in $183_{\mathrm{W}}$, so that it is now possible to include their effect on the low-lying states in a more satisfactory manner than was possible in the previous Coriolis-mixing studies [3], since these data were unavailable at the time they were being carried out.

Second, to resolve the question of the feeding of the 5.3-sec. isomeric state at $309.5 \mathrm{keV}$ in the ${ }^{183^{2}}$ e decay. In the conversionelectron data[4] on $183_{\mathrm{Re}}$, it was reported that conversion lines corresponding to the transitions de-exciting this isomer were observed, In the ${ }^{183} \mathrm{Ta}$ decay, the isomer $\left(I \pi=I I / 2^{+}\right)$is observed [6] to be fed entirely by a gamma-ray transition from an $I^{\pi}=9 / 2^{+}$state at $622.8 \mathrm{keV}$ which is itself directly fed by a $\beta^{-}$ transition from the ${ }^{183} \mathrm{Ta}$ ground state $\left(I^{\pi}=7 / 2^{+}\right)$. However, since the energy available in the ${ }^{18} 3^{3}$ e electron-capture decay is not sufficient to excite this $9 / 2^{+}$state and since direct electroncapture feeding of the state is not reasonable $\left(I^{\pi}=5 / 2^{+}\right.$for the $1{ }^{3} \mathrm{Re}$ ground state), it seemed desirable to reinvestigate this decay scheme in order to resolve this apparent discrepancy.

The conversion-electron data and the gamma-ray data [the latter consisting also of extensive gamma-gamma coincidence data taken using two large-volume $\mathrm{Ge}(\mathrm{Li})$ detectors coupled into a two-parameter ADC interfaced to a PDP-8 computer] are currently being analyzed to provide the gamma-ray intensities and internal-conversion coefficients.

These data provide no evidence for the feeding of the $309.5-\mathrm{keV}$ isomeric state, in that the principal transition ( $102.5 \mathrm{keV}$ ) in its de-excitation is not seen. From the conversion-electron spectrum, it has been possible to set an upper limit on the intensity of the $\mathrm{K}$ conversion line of the $102.5 \mathrm{keV}$ transition some 2.5 times lower than the intensity value reported for it by Harmatz et al. [4]. This conclusion, i.e., the nonpopulation of the 5.3-sec. isomeric state, was further confirmed by an additional experiment. In this, ${ }^{183^{3}}$ e was placed 
on an ion-exchange column and the ${ }^{183} \mathrm{~W}$ was eluted off as it was formed

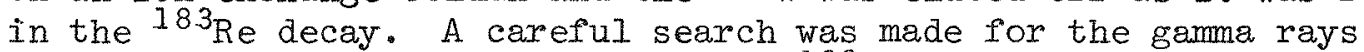
associated with the decay of $5.3-\mathrm{sec} .183 \mathrm{~m}_{\mathrm{W}}$ in the elutrient, but no evidence was found for theix presence.

II] Graduate student, Utah State University, Logan, Utah.

[2] P. Alexander and R. S. Hager, Phys. Rev. 139, B288 (1965).

[3] A. K. Kerman, Mat. Fys. Medd. Dan. Vid. Selsk. 30, No. 15 (1956); R. T. Brockmeier, S. Wahlborn, E. J. Seppi and F. Boehm, Nucl. Phys. 63, $102(1965)$.

[4] B. Harmatz, T. H. Handley and J. W. Mihelich, Phys. Rev. 128, 1186 (1962).

[5] R. F. Casten, P. Kleinheinz, P.J. Daly and B. Elbek, to be published; R. F. Casten, private communication.

[6] E. Moll and U. Gruber, Z. Physik 197, 113 (1966); U. Gruber, R. Koch, P. P. Maier and O. W. B. Schult, Z. Naturforsch. 20a, 929 (1965). 


\section{LEVELS IN $184 \mathrm{~W}$ FROM $183_{\mathrm{W}}(\mathrm{n}, \mathrm{y})$}

R. C. Greenwood, C. W. Reich

Measurement of the prompt $\gamma$-rays emitted as a result of neutron capture in $183^{\mathrm{W}}$ have provided an opportunity for detailed study of the low-spin states of ${ }^{18 \frac{1}{W}} \mathrm{~W}$. The ${ }^{183} \mathrm{~W}(n, \gamma)$ reaction is similar to the ${ }^{171} \mathrm{Yb}(\mathrm{n}, \gamma)$ reaction [1] in that s-wave neutron capture results in compound nucleus states having $I^{\pi}=0^{-}$and $I^{-}$and hence, subsequent deexcitation of these states via primary El transitions will excite final states with $I^{\pi}=(0-2)^{+}$.

In order to determine the location and distribution of the $(0-2)^{+}$ states, a spectrum of prompt $\gamma$-rays resulting from $2-k e V$ neutron capture in $183 \mathrm{~W}$ was measured. The high-energy portion of this spectrum is illustrated in Figure 1 . We estimate that because of the finite width of the energy distribution in the $2-\mathrm{keV}$ neutron beam (approximately Gaussian with a FWHM $0.7 \mathrm{keV}$ ) this spectrum represents an average over ¿56 compound-nucleus resonances. Hence the Porter-Thomas fluctuations in the primary $\gamma$-ray intensities will be averaged out and the El transitions, which are intrinsically stronger than the Ml transitions, should be easily distinguishable from the MI transitions. This is illustrated by the plot of reduced transition probabilities $\left(I_{\gamma} E_{\gamma}{ }^{-5}\right)$ versus transition energy in Figure 2. Furthermore, lines corresponding to all El transitions to final $(0-2)^{+}$states should be visible in Figure $l$.

While the $2-\mathrm{keV}$ neutron capture data allow us to locate all $(0-2)^{+}$ states in ${ }^{184} \mathrm{~W}$, more definitive information about the properties of these levels is obtained from their de-excitation characteristics. Hence, detailed measurements of the low-energy ( $<3 \mathrm{MeV}$ ) prompt $\gamma$-ray spectra were also made using both a $6 \mathrm{~cm}^{2} \times 8 \mathrm{~mm}$ planar Ge(Li) detector and a $35 \mathrm{~cm}^{3}$ coaxial $\mathrm{Ge}(\mathrm{Li})$ detector.

By combining these low-energy capture $\gamma$-ray data with the primary capture $\gamma$-ray data we are in the process of constructing a fairly complete level scheme for the lower-lying low-spin states in $184 \mathrm{~W}$. In constructing this level scheme we have also utilized presently existing data on the $184 \mathrm{~W}$ level scheme obtained from decay studies of $184 \mathrm{Re}[2-4]$ and $184 \mathrm{Ta}[5]$ and from resonance neutron capture in $183 \mathrm{~W}[6-10]$. A preliminary scheme of levels in $184 \mathrm{~W}$ which are observed to be populated in the $183 \mathrm{~W}(\mathrm{n}, \gamma)$ reaction is shown in Figure 3 and the de-excitation modes from these levels are summarized in Table $I$.

Some specific features of this level scheme thus far deduced are discussed below:

$K^{\pi}=2^{+} \gamma$-Vibrational Band The principal features of this bapd have previously been discussed in detail in decay studies of $184 \mathrm{Re}^{12-4]}$. The $I^{\pi}=2^{+}$member of this band is populated strongly in Coulomb excitation[11]. The relative $\gamma$-ray branching ratios for decay from each of the levels are in excellent agreement with those measured by McMillan et al. [2] from the decay of ${ }^{184} \mathrm{Re}$. As noted in Table II, the experimental transition probabilities can be fitted using a band-mixing parameter $z_{2} \sim 0.040$. 
TABLE I

SUMMARY OF LOWER EXCITATION STATES IN $184 \mathrm{~W}$ AND THEIR DE-EXCITING TRANSITIONS

\begin{tabular}{|c|c|c|c|c|c|c|}
\hline \multirow{2}{*}{$\begin{array}{l}\text { Initial } \\
\begin{array}{l}\text { Energy } \\
(\mathrm{keV})\end{array} \\
\end{array}$} & \multirow{2}{*}{$\begin{array}{l}\text { State } \\
I, K^{\pi} \\
\end{array}$} & \multirow{2}{*}{$\begin{array}{l}\gamma \text {-Ray } \\
\text { Transition }[\mathrm{a}] \\
(\mathrm{keV}) \\
\end{array}$} & \multirow{2}{*}{\multicolumn{2}{|c|}{$\begin{array}{c}\text { Relative } \\
\gamma \text {-Ray } \\
\text { Intensity }[b] \\
\end{array}$}} & \multirow{2}{*}{$\begin{array}{l}\text { Final } \\
\text { Energy } \\
\text { (keV) }\end{array}$} & \multirow{2}{*}{$\begin{array}{l}\text { State } \\
-\quad I, K^{\pi}\end{array}$} \\
\hline & & & & & & \\
\hline $111.218(10)$ & $2,0^{+}$ & $111.218(10)$ & 163 & (II) & 0 & $0,0^{+}$ \\
\hline $364.068(16)$ & $4,0^{+}$ & $252.850(12)$ & 60 & (3) & 111.218 & $2,0^{+}$ \\
\hline $903.26 \quad(3)$ & $2,2^{+}$ & $\begin{array}{l}903.262(35) \\
792.056(30) \\
539.38(25)\end{array}$ & $\begin{array}{l}103 \\
100 \\
1.3\end{array}$ & $\begin{array}{l}(5) \\
(5) \\
(3)\end{array}$ & $\begin{array}{c}0 \\
111.218 \\
364.068\end{array}$ & $\begin{array}{l}0,0^{+} \\
2,0^{+} \\
4,0^{+}\end{array}$ \\
\hline $1002.49 \quad(4)$ & $0,0^{+}$ & $891.271(35)$ & 51 & (5) & 111.218 & $2,0^{+}$ \\
\hline $1005.96 \quad$ (3) & $3,2^{+}$ & $\begin{array}{l}894.748(35) \\
641.874(30)\end{array}$ & $\begin{array}{l}57 \\
6.9\end{array}$ & $\begin{array}{l}(3) \\
(3)\end{array}$ & $\begin{array}{l}111.218 \\
364.068\end{array}$ & $\begin{array}{l}2,0^{+} \\
4,0^{+}\end{array}$ \\
\hline $1121.41 \quad$ (4) & $2,0^{+}$ & $\begin{array}{r}1121.390(35) \\
1010.247(30) \\
757.314(30)\end{array}$ & $\begin{array}{l}11 \cdot 2 \\
28 \cdot 9 \\
21.9\end{array}$ & $\begin{array}{l}(8) \\
(14) \\
(11)\end{array}$ & $\begin{array}{c}0 \\
111.218 \\
364.06\end{array}$ & $\begin{array}{l}0,0^{+} \\
2,0^{+} \\
4,0^{+}\end{array}$ \\
\hline $1130.00 \quad$ (4) & $2,2^{-}$ & $\begin{array}{r}1018.63(8) \\
226.743(12) \\
124.071(15)\end{array}$ & $\begin{array}{l}2.9 \\
59 \\
5.7\end{array}$ & $\begin{array}{l}(3) \\
(3) \\
(6)\end{array}$ & $\begin{array}{c}111.218 \\
903.26 \\
1005.96\end{array}$ & $\begin{array}{l}2,0^{+} \\
2,2^{+} \\
3,2^{+}\end{array}$ \\
\hline $1133.79(10)$ & $4,2^{+}$ & $\begin{aligned} 1022.57 & (5) \\
769.44 & (4)\end{aligned}$ & $\begin{array}{l}4.7 \\
8.1\end{array}$ & $\begin{array}{l}(5) \\
(6)\end{array}$ & $\begin{array}{l}111.218 \\
364.068\end{array}$ & $\begin{array}{l}2,0^{+} \\
4,0^{+}\end{array}$ \\
\hline $1221.29 \quad(4)$ & $3,2^{-}$ & $\begin{array}{l}1109.88(20) \\
318.017(15) \\
215.332(12) \\
91.31 \quad(5)\end{array}$ & $\begin{array}{r}1.5 \\
16.7 \\
8.7 \\
0.55\end{array}$ & $\begin{array}{r}(2) \\
(8) \\
(6) \\
(8)\end{array}$ & $\begin{array}{l}111.218 \\
903.26 \\
1005.96 \\
1130.00\end{array}$ & $\begin{array}{l}2,0^{+} \\
2,2^{+} \\
3,2^{+} \\
2,2^{-}\end{array}$ \\
\hline 1321.9 & $(0,2),+$ & $\begin{array}{c}418.847(20) \\
{[315.59(5)]}\end{array}$ & $\begin{array}{l}7.1 \\
1.3\end{array}$ & $\begin{array}{l}(5) \\
(3)\end{array}$ & $\begin{array}{r}903.26 \\
1005.96\end{array}$ & $\begin{array}{l}2,2^{+} \\
3,2^{+}\end{array}$ \\
\hline $1345.35(10)$ & $4,2^{-}$ & $\begin{array}{ll}339.34 & (4) \\
211.63 & (5)\end{array}$ & $\begin{array}{l}3.0 \\
0.8\end{array}$ & $\begin{array}{l}(6) \\
(2)\end{array}$ & $\begin{array}{l}1005.96 \\
1133.79\end{array}$ & $\begin{array}{l}3,2^{+} \\
4,2^{+}\end{array}$ \\
\hline $1386.28 \quad(6)$ & $2,+$ & $\begin{array}{lr}1386.36 & (5) \\
1275.07 & (5) \\
482.925(30) \\
380.24 \quad(9)\end{array}$ & $\begin{array}{r}20.5 \\
25.6 \\
5.0 \\
1.0\end{array}$ & $\begin{array}{r}(10) \\
(13) \\
(5) \\
(3)\end{array}$ & $\begin{array}{c}0 \\
111.218 \\
903.26 \\
1005.96\end{array}$ & $\begin{array}{l}0,0^{+} \\
2,0^{+} \\
2,2^{+} \\
3,2^{+}\end{array}$ \\
\hline $1424.95 \quad(6)$ & 3 & 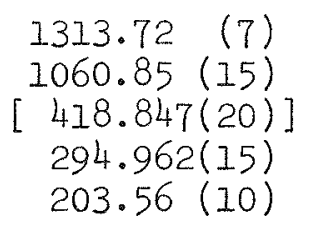 & $\begin{array}{l}6.2 \\
2.0 \\
7.1 \\
8.4 \\
1.1\end{array}$ & $\begin{array}{l}(6) \\
(3) \\
(5) \\
(4) \\
(2)\end{array}$ & $\begin{array}{l}111.218 \\
364.068 \\
1005.96 \\
1130.00 \\
1221.29\end{array}$ & $\begin{array}{l}2,0^{+} \\
4,0^{+} \\
3,2^{+} \\
2,2^{-} \\
3,2^{-}\end{array}$ \\
\hline
\end{tabular}


TABLE I (Contd.)

\begin{tabular}{|c|c|c|c|c|c|c|}
\hline \multirow{2}{*}{$\begin{array}{l}\text { Initial } \\
\begin{array}{l}\text { Energy } \\
(\mathrm{keV})\end{array} \\
\end{array}$} & \multirow{2}{*}{$\begin{array}{l}I, K^{\pi} \\
\end{array}$} & \multirow{2}{*}{$\begin{array}{c}\gamma \text {-Ray } \\
\text { Transition }[\mathrm{a}] \\
(\mathrm{keV}) \\
\end{array}$} & \multirow{2}{*}{\multicolumn{2}{|c|}{$\begin{array}{l}\text { Relative } \\
\quad \gamma \text {-Ray } \\
\text { Intensity }[\mathrm{b}] \\
\end{array}$}} & \multicolumn{2}{|c|}{ Final State } \\
\hline & & & & & $\begin{array}{l}\text { Energy } \\
(\mathrm{keV})\end{array}$ & \multirow{2}{*}{$\begin{array}{l}I, K^{\pi} \\
0,0^{+} \\
2,0^{+}\end{array}$} \\
\hline $1431.06(10)$ & $(2),+$ & $\begin{array}{ll}1430.97 & (6) \\
1319.84 & (6)\end{array}$ & $\begin{array}{r}8.5 \\
10.8\end{array}$ & $\begin{array}{l}(6) \\
(7)\end{array}$ & $\begin{array}{c}0 \\
111.218\end{array}$ & \\
\hline $1614.90 \quad(8)$ & (I), + & $\begin{array}{r}1503.74(15) \\
711.58(6) \\
{[607.620(25)]}\end{array}$ & $\begin{array}{l}6.9 \\
8.8 \\
9.0\end{array}$ & $\begin{array}{l}(5) \\
(9) \\
(5)\end{array}$ & $\begin{array}{l}111.218 \\
903.26 \\
1005.96\end{array}$ & $\begin{array}{l}2,0^{+} \\
2,2^{+} \\
3,2^{+}\end{array}$ \\
\hline $1627.69 \quad(6)$ & $(0-2),+$ & $\begin{array}{l}724.388(30) \\
241.46 \quad(6)\end{array}$ & $\begin{array}{r}14.4 \\
0.7\end{array}$ & $\begin{array}{l}(7) \\
(2)\end{array}$ & $\begin{array}{r}903.26 \\
1386.28\end{array}$ & $\begin{array}{l}2,2^{+} \\
2,+\end{array}$ \\
\hline
\end{tabular}

[a] Parentheses around a $\gamma$-ray transition energy indicates a tentative assignment.

[b] 100 units of relative intensity are equivalent to $10.7 \mathrm{r} / 100$ neutrons captured.

TABLE II

RATIOS OF REDUCED TRANSITION PROBABILITIES FOR DECAY OF STATES

IN THE GAMMA-VIBRATIONAL BAND INTO THE GROUND-STATE BAND

\begin{tabular}{|c|c|c|c|c|}
\hline $\begin{array}{l}\text { Initial } \\
\text { State } \\
\text { Energy } \\
\text { (keV) } \\
\end{array}$ & $\begin{array}{l}\text { Transition Ratio } \\
\left(I_{i} 2 \rightarrow I_{f} 0\right) /\left(I_{i} 2 \rightarrow I_{f} 0\right) \\
\end{array}$ & $\begin{array}{l}\text { Experimental } \\
\text { Ratio[a] } \\
\end{array}$ & $\begin{array}{l}\text { Alaga Rules }[12] \\
\text { (without band } \\
\text { mixing) } \\
\end{array}$ & $\begin{array}{r}{ }^{2} \\
\left(\times 10^{3}\right) \\
\end{array}$ \\
\hline \multirow[t]{2}{*}{903} & $\frac{B(2 ; 22 \rightarrow 00)}{B(2 ; 22 \rightarrow 20}$ & $0.54 \quad(4)$ & 0.70 & $43(12)$ \\
\hline & $\frac{B(2 ; 22 \rightarrow 40)}{B(2 ; 22 \rightarrow 20)}$ & $0.089(22)$ & 0.052 & $48(28)$ \\
\hline 1005 & $\frac{B(2 ; 32 \rightarrow 40)}{B(2 ; 32 \rightarrow 20)}$ & $0.63 \quad(4)$ & 0.40 & $35(5)$ \\
\hline 1133 & $\frac{B(2 ; 42 \rightarrow 20)}{B(2 ; 42 \rightarrow 40)}$ & $0.18 \quad(2)$ & 0.34 & $42(7)$ \\
\hline
\end{tabular}

[a] Values quoted assuming pure E2 transitions. 


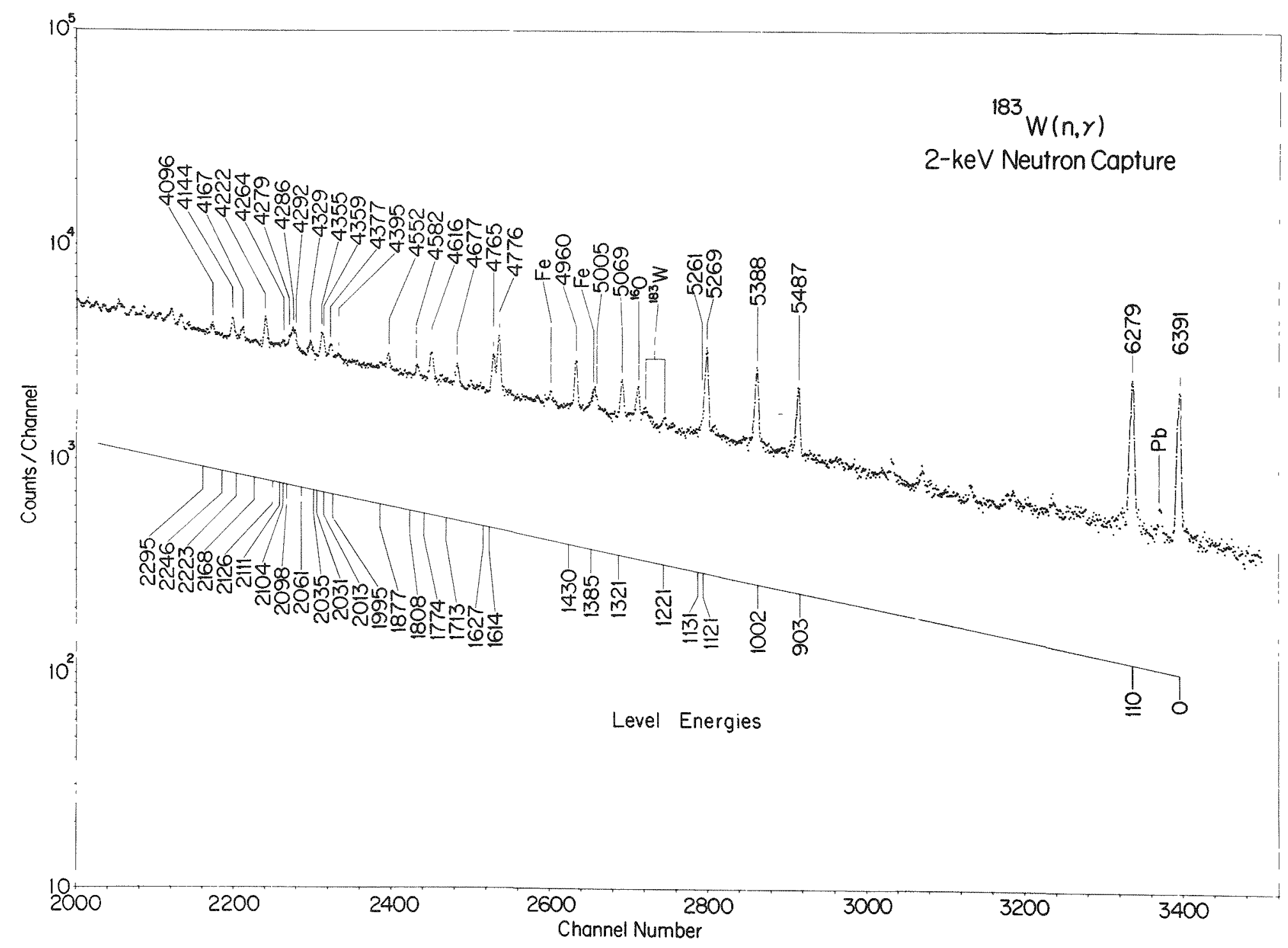

Fig. I A portion of the prompt $\gamma$-ray spectrum resulting from $2-\mathrm{keV}$ neutron capture in ${ }^{183_{\mathrm{W}}}$. 


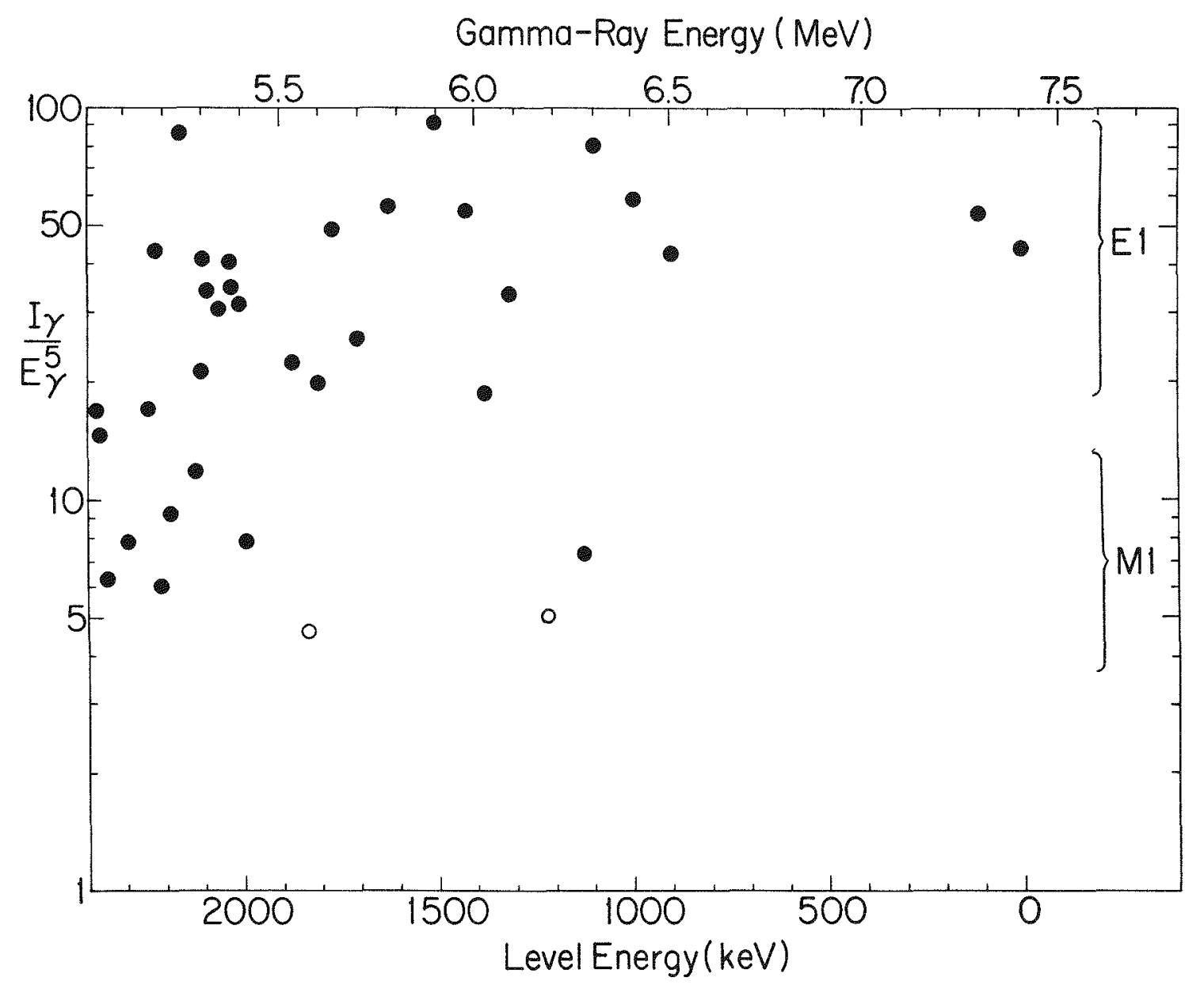

Fig. 2 A plot of $I_{\gamma} E^{-5}$ versus the energy, $E_{\gamma}$, of the prompt $\gamma$-ray transitions observed from 2-keV neutron capture in ${ }^{183_{W}}$. 


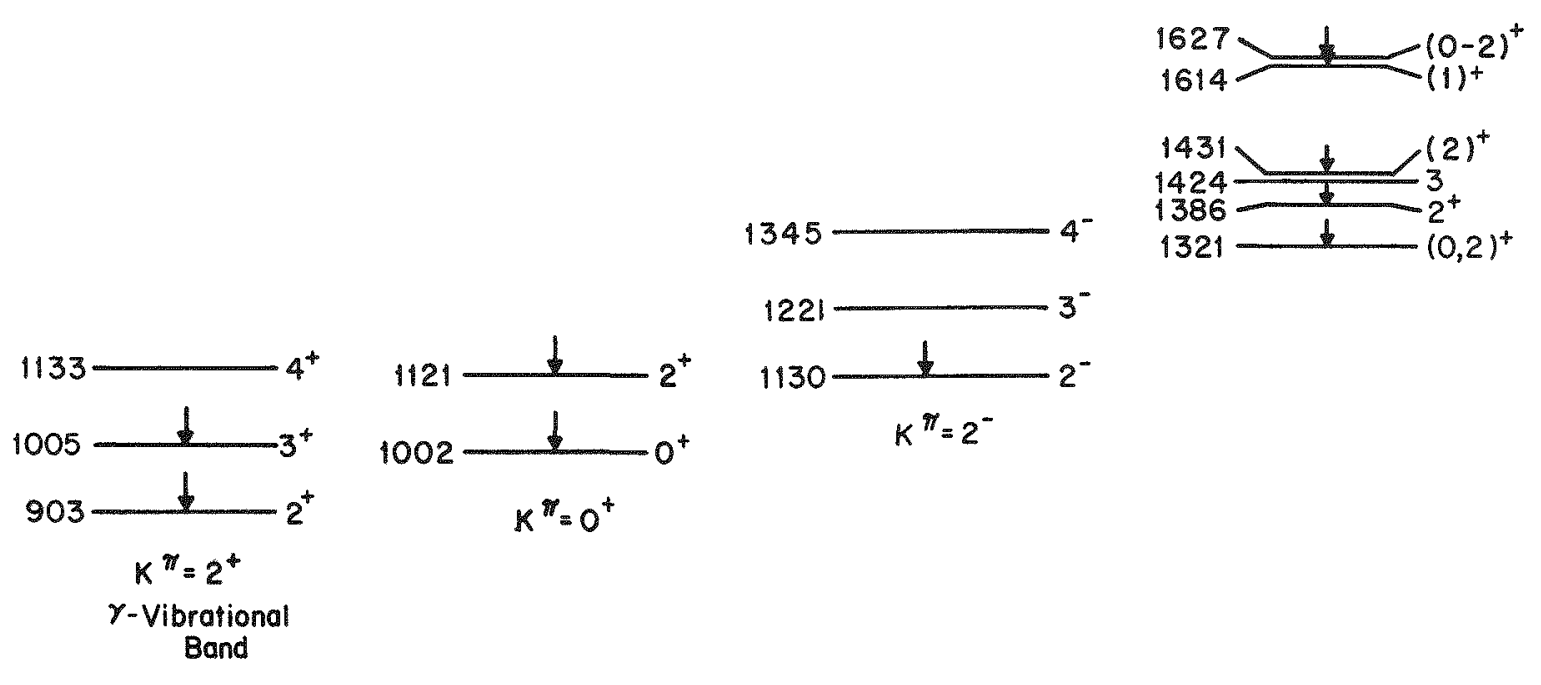

$364 \longrightarrow 4,0^{+}$

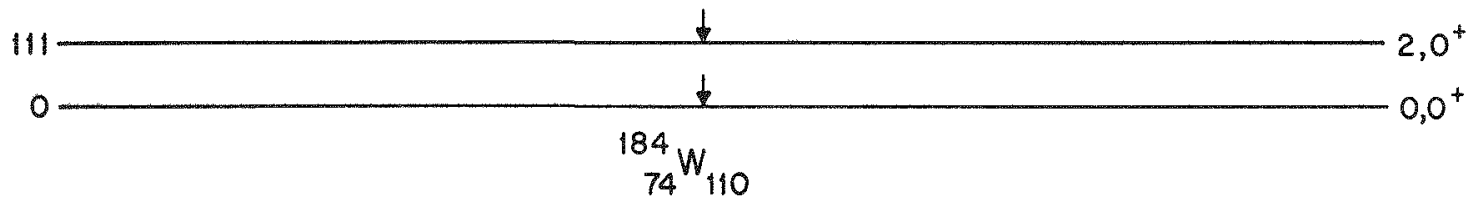

Fig. 3 Levels in ${ }^{184} \mathrm{~W}$ populated by the ${ }^{183} \mathrm{~W}(\mathrm{n}, \mathrm{\gamma})$ reaction. An arrow into a level indicates that the level is populated by primary neutron capture $\gamma$-ray transitions. 
$\mathrm{K}^{\pi}=\mathrm{O}^{+}$Band It was first suggested by Faler et al. [7] that the levels at $1002 \mathrm{keV}$ and $1121 \mathrm{keV}$ were the $0^{+}$and $2^{+}$members of a $\mathrm{K}^{\pi}=0^{+}$ band. The state at $1121 \mathrm{keV}$ is also populated in the decay of ${ }^{184} \mathrm{Re}$. The relative $\gamma$-ray branching ratios for decay from this state are in good agreement with those measured by McMillan et al.[2] and Kukoc et al.[3] from the decay of ${ }^{184} \mathrm{Re}$ and by Faler et al.[7] following capture of $7.6-\mathrm{eV}$ neutrons by $183 \mathrm{~W}$. If the assumption that the $\Delta I=0$ transition is pure $\mathrm{E} 2$ is valid, then we see from Table III that the experimental branching ratios from the $1121 \mathrm{keV}$ level cannot be described by a single band mixing parameter, $\mathrm{Z}_{\mathrm{O}}$.

$\mathrm{K}^{\pi}=2^{-}$Band The existence of this band is established in this present work, although the existence of states at $1130 \mathrm{keV}$ and $1221 \mathrm{keV}$ had previously been established from studies of the decay of ${ }^{184} \mathrm{Re}$. The salient feature of this band is the predominant decay of levels in the band to levels in the $\gamma$-vibrational band. We note from Table IV that the relative $\gamma$-ray branching ratios from this band into the $\gamma$-vibrational band appear to be reasonably well described by the Alaga rules[12].

Other states in $184 \mathrm{~W}$ There are three other states below $1.6 \mathrm{MeV}$ excitation energy in $184 \mathrm{~W}$ which are populated by primary EI transitions in the $183 \mathrm{~W}(\mathrm{n}, \mathrm{\gamma})$ reaction. One of these, at $1386 \mathrm{keV}$, has been established from the decay of $18{ }^{4}$ Re to have $I^{\pi}=2^{+}$. The most probable spin for the $1431 \mathrm{keV}$ state is also 2. The $1321 \mathrm{keV}$ state also probably has spin 2, although $I^{\pi}=0^{+}$cannot be completely ruled out for this level.

In adaition to these states, we note that Kukoč, et al. tentatively identify a level at $1424 \mathrm{keV}$ which they suggest has spin 3 or 4 . The existence of a level at $1424 \mathrm{keV}$ is established in this present work. Based upon the decay modes of this state and the fact that it is relatively strongly populated in the ${ }^{183} \mathrm{~W}(\mathrm{n}, \mathrm{\gamma})$ reaction we can assign it a spin value of 3 with the parity as yet undetermined.

[1] R. C. Greenwood, C. W. Reich and S. H. Vegors, Jr., USAEC Report IN-1407, p. 114 (1970).

[2] D. J. McMillan, R. G. Helmer, L. D. McIsaac and C. W. Reich, ibid p. $144(1970)$.

[3] A. H. Kukoč, B. Singh, J. D. King and H. W. Taylor, Nucl. Phys. Al43, p. 545 (1970).

[4] J. Glatz, K. E. G. Lobner, F. Oppermann, Z. Physik 227, 83 (1969).

[5] N. R. Johnson, N. K. Aras, ORNL-3994, p. 19 (1966).

[6] R. R. Spencer and K. T. Faler, Phys. Rev. 155, 1368 (1967).

[7] K. T. Faler, R. R. Spencer and R. A. Harlan, Phys. Rev. 175, 1495 (1968). 
[8] M. Beer, M. Bhat, R. E. Chrien, M. A. Lone and O. A. Wasson, Proc. Conf. on Slow Neutron-Capture Gamma-Ray Spectroscopy, ANL, 1966, USAEC Report ANL-7282, p. 459 (1968).

[9] C. Samour, J. Julien, R. N. Alves, S. deBarros and J. Morgenstern, Nucl. Phys. Al23, 581 (1969).

[10] E. R. Rae, W. R. Moyer, R. R. Fullwood and J. L. Andrews, Phys. Rev. 155, 1301 (1967).

[1]] W. R. Milner, F. K. McGowan, R. I. Robinson and P. H. Stelson, Bull. Am. Phys. Soc. 14, 1204 (1969).

[12] G. Alaga, K. Alder, A. Bohr and B. R. Mottleson, Mat. Fys. Med. Dan. Via. Selk., No. 9 (1955). 
TABLE III

RATIOS OF REDUCED TRANSITION PROBABILITIES FOR DECAY

FROM THE $2^{+}$STATE AT $1121 \mathrm{keV}$

\begin{tabular}{|c|c|c|c|c|}
\hline $\begin{array}{l}\text { Initial } \\
\text { State } \\
\text { Energy } \\
\text { (keV) } \\
\end{array}$ & $\begin{array}{l}\text { Transition Ratio } \\
\left(20 \rightarrow I_{f} 0\right) /\left(20 \rightarrow I_{f} 0\right)\end{array}$ & $\begin{array}{l}\text { Experimental } \\
\text { Ratio[a] }\end{array}$ & $\begin{array}{l}\text { Alaga Rules [12] } \\
\text { (without band } \\
\text { mixing) } \\
\end{array}$ & $\begin{array}{l}\mathrm{Z}_{0} \\
\left(\times 10^{3}\right) \\
\end{array}$ \\
\hline \multirow[t]{3}{*}{1121} & $\frac{B(2 ; 20 \rightarrow 00)}{B(2 ; 20 \rightarrow 20)}$ & $0.229(20)$ & 0.70 & $71(4)$ \\
\hline & $\frac{B(2 ; 20 \rightarrow 40)}{B(2 ; 20 \rightarrow 20)}$ & $3.22(23)$ & 1.80 & $24(4)$ \\
\hline & $\frac{B(2 ; 20 \rightarrow 00)}{B(2 ; 20 \rightarrow 40)}$ & $0.071(6)$ & 0.39 & $48(3)$ \\
\hline
\end{tabular}

[a] Values quoted assuming pure E2 multipolarity for the $2^{+} 2^{+}$transition.

TABLE IV

RATIOS OF REDUCED TRANSITION PROBABILITIES FOR DECAY OF STATES

OF THE $1130-\mathrm{keV} \mathrm{K}^{\mathrm{T}}=2^{-}$BAIND INTO THE GAMMA-VIBRATIONAL BAND AT 903-keV

\begin{tabular}{|c|c|c|c|}
\hline $\begin{array}{l}\text { Initial } \\
\text { State } \\
\text { Energy } \\
(\mathrm{keV}) \\
\end{array}$ & $\begin{array}{l}\text { Transition Ratio } \\
\left(I_{i} 2 \rightarrow I_{f} 2\right) /\left(I_{i} 2 \rightarrow I_{i} 2\right)\end{array}$ & $\begin{array}{l}\text { Experimental } \\
\text { Ratio } \\
\end{array}$ & $\begin{array}{c}\text { Prediction } \text { of }^{\circ} \\
\text { Alaga Rules } 12]\end{array}$ \\
\hline 1130 & $\frac{B(1 ; 22+32)}{B(1 ; 22 \rightarrow 22)}$ & $0.59(7)$ & 0.50 \\
\hline 1221 & $\frac{B(1 ; 32 \rightarrow 32)}{B(1 ; 32 \rightarrow 22)}$ & $1.68(14)$ & 1.40 \\
\hline 1345 & $\frac{B(1 ; 42 \rightarrow 42)}{B(1 ; 42 \rightarrow 32)}$ & $1.1(4)$ & 0.60 \\
\hline
\end{tabular}


LEVELS IN 229 Th EXCITED IN THE $\alpha$-DECAY OF $233 \mathrm{U}$

L. A. Kroger $[1]$, C. W. Reich, J. E. Cline

The gamma radiation emitted following the $\alpha$-decay of ${ }^{23}{ }^{3} \mathrm{U}$ has been studied with high-resolution Ge(Li) spectrometers. The study was undertaken to provide a more complete picture of the ${ }^{229} \mathrm{Th}$ level scheme, with two special emphases:

1. To attempt to elucidate the nature of the reported first excited state (at $\mathrm{k} 29 \mathrm{keV}$ ) in ${ }^{229} \mathrm{Th}$. The most reasonable spin-parity assignment for this state is $5 / 2^{+}$, and it has been assumed in a previous study[2] to have $K=5 / 2$. This is quite surprising, since the ground state of $229 \mathrm{Th}$ also has $K=5 / 2$ (the NiIsson state $5 / 2^{+}[633]$ ), and no other known $\mathrm{K}^{\pi}=5 / 2^{+}$states are predicted to occur at this low an excitation energy.

2. To search for negative-parity states in $229 \mathrm{Th}$. These states will arise from the $j_{15 / 2}$ spherical shell-model state and should be quite strongly coupled via the Coriolis interaction.

In addition to the gamma-ray "singles" data, taken using a variety of $\mathrm{Ge}(\mathrm{Li})$ detectors and a $\mathrm{Si}(\mathrm{Li})$ detector, extensive gamma-gamma coincidence measurements have been made. These latter have been carried out using two large volume $\mathrm{Ge}(\mathrm{Li})$ detectors coupled into a two-parameter $A D C$ interfaced to a PDP-8 computer. The energies and intensities obtained for the gamma rays from $233 \mathrm{U}$ decay are listed in Table I. The proposed decay scheme is shown in Figure 1 .

Although the level-scheme data are still in the process of analysis and interpretation, the following two features which have thus far emerged are worthy of note:

First, we prefer to interpret the $5 / 2^{+}$state at $29.16 \mathrm{keV}$ as a rotational state of a $K^{\pi}=3 / 2^{+}$band rather than as a band head. of $a K^{\pi}=5 / 2^{+}$band. (We feel that the tentative $3 / 2^{+} \operatorname{spin}$ assignment suggested[3] for this state is not likely on the basis of our data.) The principal reason for this is simply that there are no $\mathrm{K}^{\pi}=5 / 2^{+}$bands expected this close to $5 / 2^{+}[633]$ in $229 \mathrm{Th}$. While this argument is admittedly entirely model dependent, we have not yet found any evidence that seriously contradicts it and alI the currently available data tend to support it. For example, the relative magnitudes of the hindrance factors [4] of the $\alpha$-transitions into various members of the band are more consistent with a $K=3 / 2$ assignment than with a $K=5 / 2$ assignment. One obvious problem with this proposal is the apparent absence of any state which is readily identifiable as the $3 / 2^{+}$band head. Based on the energy spacings of the other band members, the energy of this state is expected to be quite near ( $\lesssim 1 \mathrm{keV}$ ) that of the ground state. A careful examination of the line shapes and energy differences of gamma rays from various levels into the ground state and proposed 
$3 / 2^{+}$state has been carried out. No systematic differences in either line shape or energy differences were found which could be used as proof of the existence of a doublet structure near the ground state. The limit of precision of this is $\approx 0.1 \mathrm{keV}$, that is, the proposed $3 / 2^{+}$band head must lie within $\approx 0.1 \mathrm{keV}$ of the 229 Th ground state. The proposed Nilsson orbital assignment for the $3 / 2^{+}$band is $3 / 2^{+}[63 I]$, which orbital is expected to occur close in energy to $5 / 2^{+}[633]$ in this region of deformation.

Second, the pattern of negative-parity states is quite interesting. At first glance, there is no apparent association of these states with a rotational-band structure. However, since these states are most likely associated with Nilsson states originating from the $j 15 / 2$ spherical shell-model state, strong Coriolis mixing among them is expected. We have carried out a

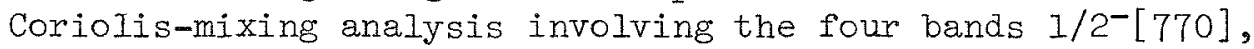
$3 / 2^{-}[761], 5 / 2^{-}[762]$ and $7 / 2^{-}[742]$ which has, with quite simple and reasonable assumptions concerning unperturbed rotational energylevel spacings and coupling-strength attenuation factors, been able to do a rather good job of reproducing the observed energy level structure. This fit is illustrated in Figure 2.

The proposed existence of a $3 / 2^{+}$state essentially degenerate with the ground state "explains" a puzzling feature of the gamma-ray transitions from the $5 / 2^{-}$states. The absence of transitions from them to the $7 / 2^{+}$ member of the ground state band was somewhat surprising, since initially it appeared that the bandhead (ground state) as well as the $7 / 2^{+}$state at $71.8 \mathrm{keV}$ was fed. However, if one postulates that the observed gamma rays in fact proceed at least predominantly to the $3 / 2^{+}$state and not the $5 / 2^{+}$ground state, then the observed gamma-branching pattern can be interpreted simply as a preference for exciting the members of the $3 / 2^{+}$ [633] band. This pattern is exhibited by all the negative-parity states observed. Perhaps some evidence of the relative El strength into the two bands is provided by the gamma-ray branching from the $7 / 2^{-}$state at $148.16 \mathrm{keV}$. Here, the $148.14-\mathrm{keV}$ gamma ray must go to $5 / 2^{+}$[633] rather than to $3 / 2^{+}[631]$, since to do the latter would require that it have M2 multipolarity. In fact, this gamma ray is seen to be considerably weaker than the others de-exciting this state, which go to various members of $3 / 2^{+}[631]$.

[1] Graduate student, University of Wyoming, Laramie, Wyoming.

[2] I. Ahmad, Ph.D. Thesis, U.S.A.F.C. Report UCRI-I6888 (1966).

[3] C. M. Lederer, J. M. Hollander and I. Perlman, "Table of Isotopes", (John Wiley and Sons, New York, 1967) Sixth edition.

[4] S. A. Baranov, M. K. Gadzhiev, V. M. Kulakov and V. M. Shatinskii, Sov. J. of Nucl. Phys. 5, 365 (1967). 
TABIE I

$23{ }^{3} \mathrm{U}$ GAMMA-RAY ENERGIES AND INTENSITIES

\begin{tabular}{|c|c|c|c|}
\hline $\begin{array}{c}\text { Gamma-ray Energy } \\
\text { (keV) }\end{array}$ & $\begin{array}{r}\text { In } \\
\text { (gamma } \\
\text { disin } \\
\end{array}$ & $\begin{array}{l}\text { nsity } \\
\text { ys per } 10^{5} \\
\text { grations) }\end{array}$ & $\begin{array}{l}\text { Assignment } \\
\text { Designation }\end{array}$ \\
\hline $25.30 \pm 0.06$ & 2.4 & & $E$ \\
\hline $29.16 \pm 0.06$ & 12.0 & & $\mathrm{E}$ \\
\hline $32.2 \pm 0.2$ & $-\infty-$ & & $E$ \\
\hline $37.8 \pm 0.1$ & 0.5 & & $\mathrm{E}$ \\
\hline $42.44 \pm 0.02$ & 62.0 & \pm 5.0 & $\mathrm{E}$ \\
\hline 52.6 & $-m-\infty$ & & $C D$ \\
\hline $53.59 \pm 0.05$ & 4.1 & \pm 0.3 & $E$ \\
\hline $54.69 \pm 0.2$ & 15.0 & \pm 1.0 & $\mathrm{E}$ \\
\hline $63.88 \pm 0.15$ & 0.03 & & $\mathrm{C}$ \\
\hline 65.7 & $--\cdots$ & & $C D$ \\
\hline $66.11 \pm 0.04$ & 0.87 & \pm 0.05 & $\mathrm{C}$ \\
\hline $67.98 \pm 0.05$ & 0.2 & & $\mathrm{E}$ \\
\hline $70.33 \pm 0.05$ & 0.59 & & $\mathrm{C}$ \\
\hline $71.84 \pm 0.02$ & 2.9 & \pm 0.25 & $\mathrm{C}$ \\
\hline $74.59 \pm 0.05$ & 1.6 & \pm 0.08 & $\mathrm{C}$ \\
\hline $76.41 \pm 0.08$ & 0.52 & \pm 0.03 & $\mathrm{C}$ \\
\hline $78.20 \pm 0.10$ & 0.1 & & $\mathrm{E}$ \\
\hline $83.08 \pm 0.05$ & 0.14 & & $\mathrm{E}$ \\
\hline $84.75 \pm 0.20$ & 0.07 & & NA \\
\hline $91.0 \pm 0.5$ & 0.2 & \pm 0.05 & $\mathrm{C}$ \\
\hline $96.28 \pm 0.10$ & 3.0 & \pm 0.5 & $E$ \\
\hline $97.14 \pm 0.02$ & 22.0 & \pm 1.1 & E \\
\hline $100.03 \pm 0.15$ & 0.05 & & $\mathrm{C}$ \\
\hline $101.75 \pm 0.10$ & 0.07 & & C \\
\hline 103.4 & $-\infty-$ & & $C D$ \\
\hline $109.5 \pm 0.1$ & 0.03 & & $E$ \\
\hline $112.0 \pm 0.1$ & 0.45 & & C \\
\hline $117.16 \pm 0.02$ & 2.6 & \pm 0.13 & C \\
\hline $118.98 \pm 0.05$ & 3.7 & \pm 0.2 & $\mathrm{C}$ \\
\hline $120.82 \pm 0.05$ & 3.1 & \pm 0.15 & $\mathrm{E}$ \\
\hline $123.93 \pm 0.05$ & 0.73 & \pm 0.04 & $\mathrm{C}$ \\
\hline $125.4 \pm 0.1$ & 0.07 & & $E$ \\
\hline $129.4 \pm 0.1$ & 0.1 & & $\mathrm{C}$ \\
\hline $131.05 \pm 0.15$ & 0.03 & & $\mathrm{E}$ \\
\hline $135.34 \pm 0.05$ & 2.3 & \pm 0.12 & $\mathrm{C}$ \\
\hline $139.72 \pm 0.08$ & 0.1 & & C \\
\hline 141.6 & $-\cdots$ & & $C D$ \\
\hline $144.7 \pm 0.5$ & 0.9 & \pm 0.3 & $\mathrm{E}$ \\
\hline $145.37 \pm 0.1$ & 1.5 & \pm 0.2 & C \\
\hline $146.34 \pm 0.05$ & 6.4 & \pm 0.32 & $\mathrm{C}$ \\
\hline $148.14 \pm 0.05$ & 0.42 & & $\mathrm{C}$ \\
\hline $149.86 \pm 0.1$ & 0.13 & & NA \\
\hline $153.2 \pm 0.2$ & 0.14 & & $\mathrm{C}$ \\
\hline $154.85 \pm 0.1$ & 0.17 & & C \\
\hline $156.1 \pm 0.14$ & 0.07 & & $\mathrm{C}$ \\
\hline $162.6 \pm 0.1$ & 0.24 & & $\mathrm{E}$ \\
\hline
\end{tabular}




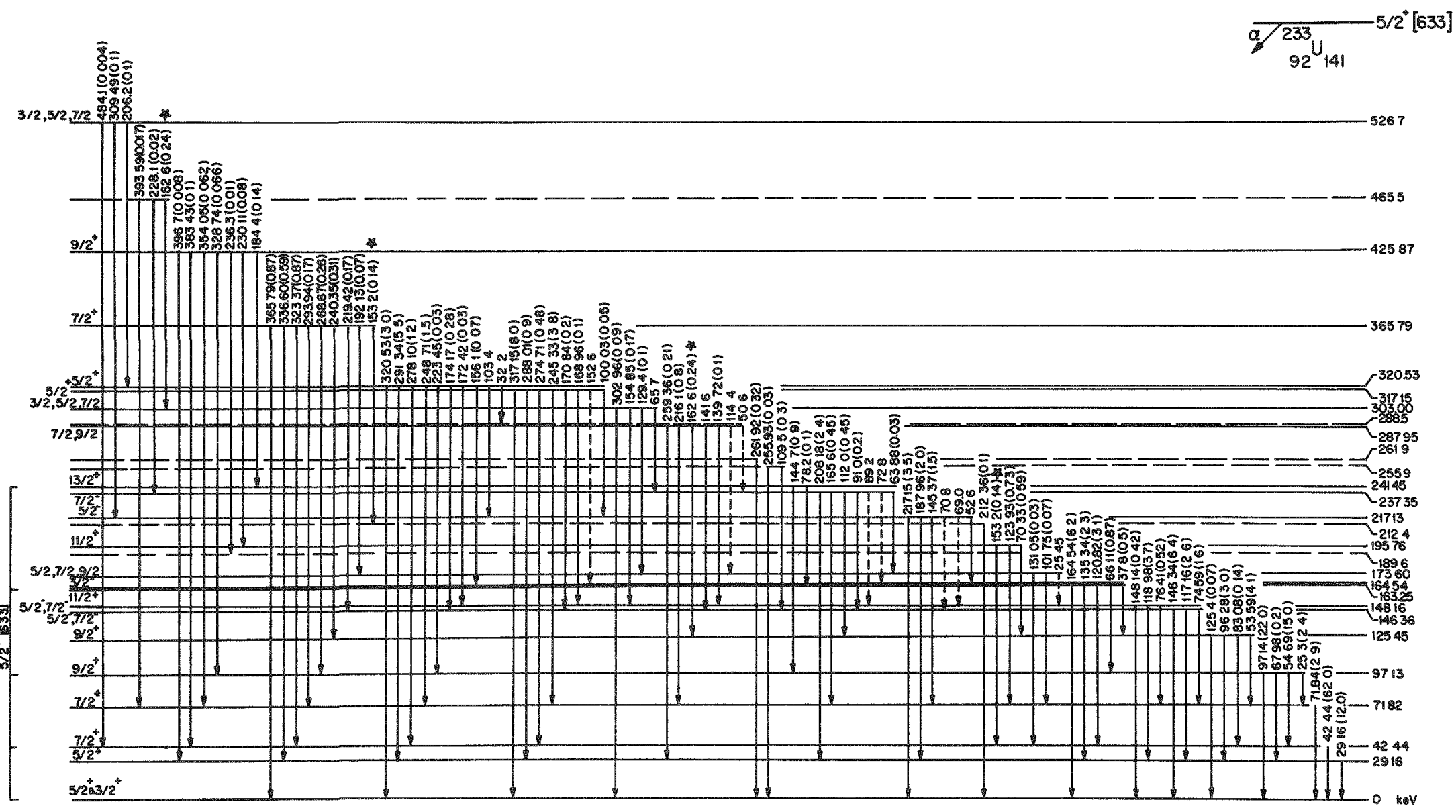

$$
{ }_{90}^{229} \mathrm{Th}
$$

Figure 1 Proposed decay scheme for ${ }^{23} \mathrm{U}$. The number in parentheses following the energy values labeling the gamma-ray transitions are the gamma-ray intensities. Gamma-ray transitions shown dotted have been suggested from the coincidence data and are not observed in the singles data. Levels shown as dotted lines are those for which some evidence (generally from other types of experimental data) exists and for which our gamma-ray data provide some, but not conclusive, support. The $3 / 2^{+}$and $5 / 2^{+}$notation at the ground state is not meant to imply an uncertainty in the ground-state spin but rather that there is presumed to exist a $3 / 2^{+}$state that is essentially degenerate with the $5 / 2^{+}$ground state. 


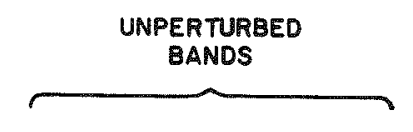

CORIOLIS - COUPLED

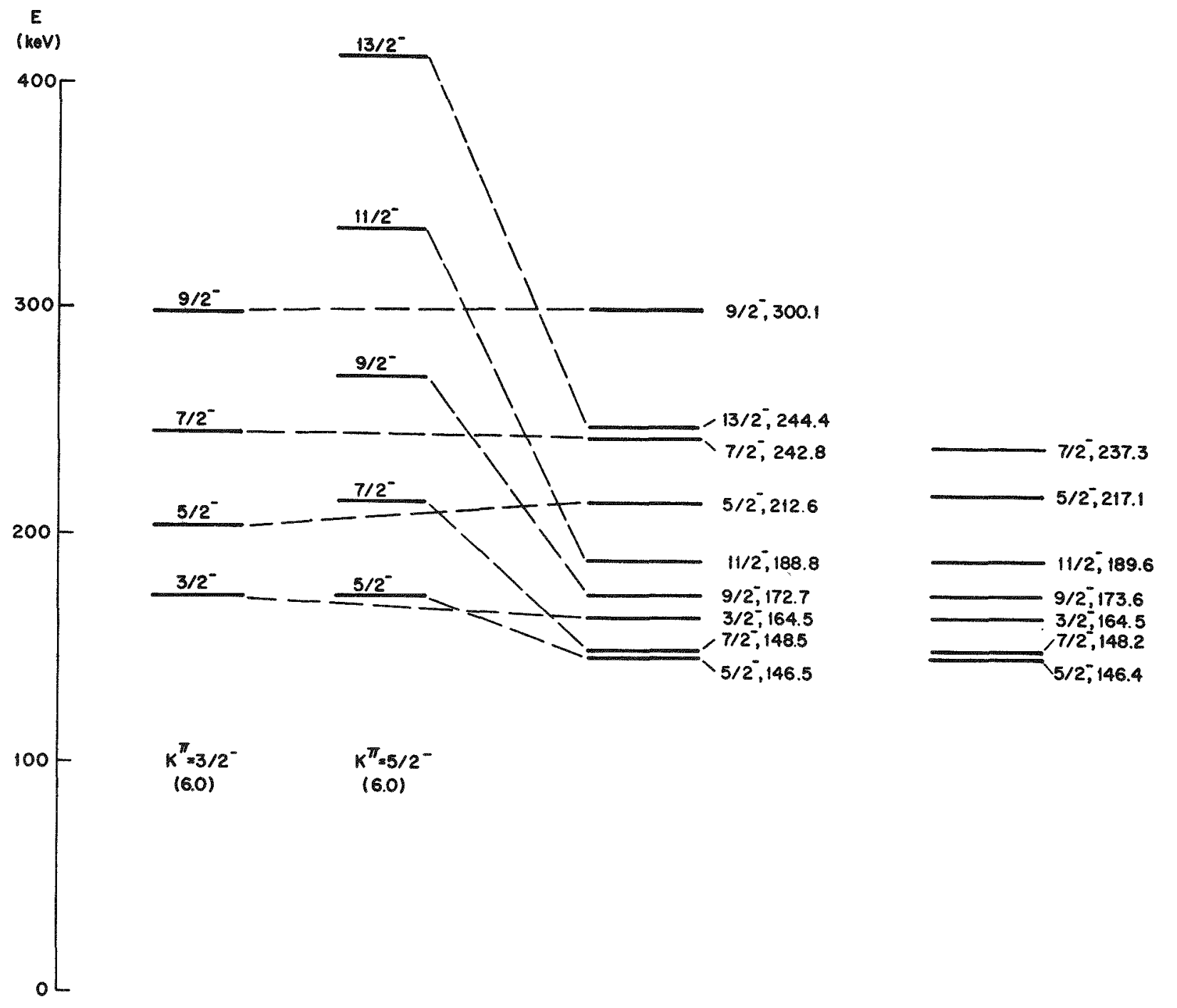

Figure 2 Coriolis-mixing analysis of the energy-level structure of the negative-parity states in $229 \mathrm{Th}$. At the left is shown the assumed unperturbed $\mathrm{K}^{\pi}=3 / 2^{-}$and $5 / 2^{-}$bands (the asymptotic quantum numbers being [761] and [752], respectively). In the center is shown the Coriolis-coupled energy spectrum, and at the right is shown the observed energy levels. The numbers in parentheses beneath the unperturbed bands are the $\mathrm{h}^{2} / 2$ values (in $\mathrm{keV}$ ) assumed for the two bands. (Not shown in the figure, but included in the calculation, are the Nilsson states $1 / 2^{-}[770]$ and $7 / 2^{-[743]}$, which exert a significant influence on the energy-level spectrum.) 
LEVELS IN 231 Th EXCITED IN THE $\alpha$-DECAY OF $235 \mathrm{U}$

I. A. Kroger $[I], C$. W. Reich, J. E. Cline

The gamma radiation emitted following the alpha decay of ${ }^{235} \mathrm{U}$ has been studied using high-resolution Ge( $\mathrm{Li}$ ) spectrometers. Until now, the ${ }^{235} \mathrm{U}$ decay has not been studied using these detectors, the decay data consisting primarily of an alpha-particle energy spectra and gammaray spectra taken using $\mathrm{NaI}(\mathrm{Tl})$ scintillation spectrometers. The present study was undertaken to provide a more complete decay scheme for ${ }^{235} \mathrm{U}$, from which it was hoped that additional information concerning the Coriolis coupling among the low-lying negative-parity states in ${ }^{231}$ Th would emerge. These negative-parity states originate from the $j_{15 / 2}$ spherical-shell-model state, and the Coriolis matrix elements among these states are expected to be exceptionally large $(|<j+| \sim 7)$.

In addition to singles gamma-ray spectroscopy utilizing a Si(Li) detector and a variety of $\mathrm{Ge}(\mathrm{Li})$ spectrometers, extensive gamma-gamma coincidence studies have been made using two large-volume Ge( Li) detectors coupled to a PDP-8 digital data processor. These studies have established the existence of 34 gamma-ray transitions, 30 of which have been placed unambiguously in the ${ }^{231}$ Th level scheme. The gammaray energy and intensity data are summarized in Table I. The level scheme proposed for 231 Th is shown in Figure I. Theoretical analysis of the level scheme data is currently in progress.

TABLE I

Energies and branching ratios of gamma rays emitted in the decay of $235 \mathrm{U}$. Unless otherwise indicated, the uncertainities in the gamma-ray intensities are $\approx \pm 10 \%$.

\begin{tabular}{|c|c|c|c|c|}
\hline $\begin{array}{c}\text { Gamma-Ray Energy } \\
(\mathrm{keV})\end{array}$ & $\begin{array}{r}\text { Gamma-Ray } \\
(\% \text { per }\end{array}$ & $\begin{array}{l}\text { Intensity } \\
\text { decay) }\end{array}$ & $\begin{array}{c}\text { Gamma-Ray Energy } \\
(\mathrm{keV})\end{array}$ & $\begin{array}{c}\text { Gamma-Ray Intensity } \\
\text { (\% per decay) }\end{array}$ \\
\hline $31.6 \pm 0.1$ & & -- & $215.31 \pm 0.05$ & 0.4 \\
\hline \pm 0.2 & $\approx 0.05$ & & $221.37 \pm 0.03$ & 0.075 \\
\hline 51.2 & - & & 225.7 & 0.06 \\
\hline \pm 0.1 & $\approx 0.13$ & & 228.8 & 0.008 \\
\hline $109.12 \pm 0.05$ & 1.5 & \pm 0.2 & $233.53 \pm 0.04$ & 20.02 \\
\hline $115.2 \pm 0.5$ & 0.11 & \pm 0.03 & $240.93 \pm 0.04$ & 0.003 \\
\hline $140.75 \pm 0.10$ & 0.17 & \pm 0.02 & $246.83 \pm 0.04$ & $\approx 0.02$ \\
\hline $143.78 \pm 0.02$ & 9.7 & \pm 0.5 & $266.44 \pm 0.08$ & 0.005 \\
\hline $150.96 \pm 0.03$ & 0.11 & \pm 0.03 & 275.4 & $<0.04 \mathrm{~b}$ \\
\hline $163.36 \pm 0.02$ & 4.6 & \pm 0.3 & \pm 0.2 & 0.003 \\
\hline $173.4 \pm 0.1$ & $\approx 0.015$ & & \pm 0.1 & $<0.02^{\bar{b}}$ \\
\hline $182.72 \pm 0.20$ & 0.40 & \pm 0.04 & 311.6 & 0.0016 \\
\hline $185.72 \pm 0.02$ & $54 a$ & & 345.91 & 0.0016 \\
\hline $194.94 \pm 0.02$ & 0.65 & \pm 0.05 & 356.0 & \\
\hline $198.91 \pm 0.06$ & 0.03 & & 387.85 & \\
\hline $202.13 \pm 0.03$ & 1.0 & \pm 0.5 & $410.2 \pm 0.2$ & \\
\hline $205.31 \pm 0.02$ & 4.9 & \pm 0.3 & $447.5 \pm 0.2$ & \\
\hline
\end{tabular}

a. Normalization point.

b. Observed peak contains significant contribution from coincidence summing. 
[1] Graduate student, University of Wyoming, Laramie, Wyoming.

[2] R. C. Pilger, F. S. Stephens, F. Asaro and I. Perlman, BuII. Am. Phys. Soc., Sec. II, 2, 394 (1957).

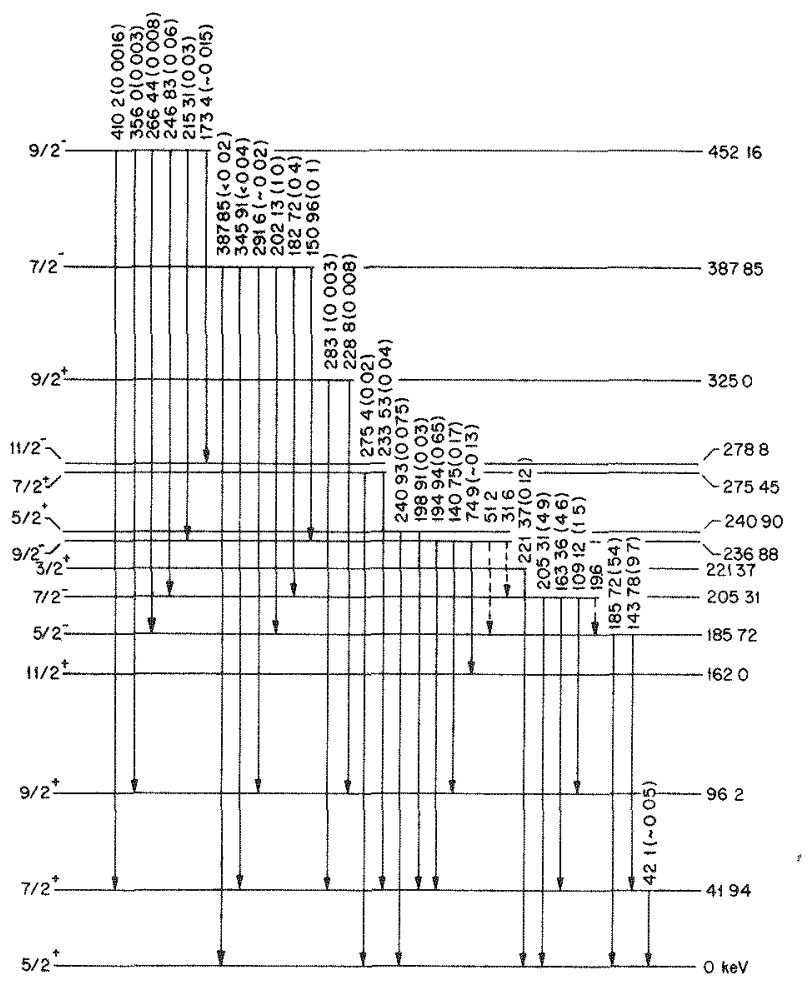

${ }_{90}^{231} \mathrm{Th}_{141}$ $\underbrace{235 U}_{92+143} 7 / 2[743]$

$9 / 2^{-}$

$7 / 2-[743]^{-}$

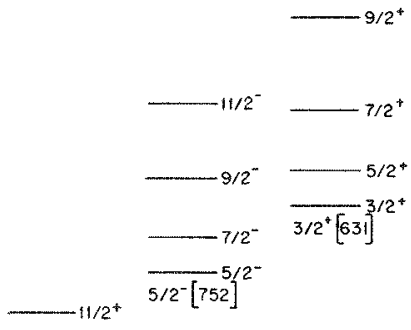
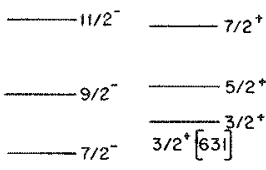
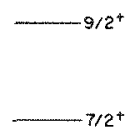

$\overline{5 / 2}^{+}[633]^{+}$

ANC C 251

Figure I Proposed decay scheme for ${ }^{235} \mathrm{U}$. The numbers in parentheses associated with the gamma-ray energies represent the gamma-ray intensities. At the right is shown the grouping of the energy levels into rotational bands with the proposed Nilsson-state assignments. 
LIMITS ON THE PRECISION OF MEASURED RELATIVE INTERNAL-CONVERSION COEFFICIENTS - 192 Ir

$$
\text { R. G. Helmer, R. A. Lokken }[1]
$$

A study of several of the internal-conversion-electron lines from the decay of ${ }^{192}$ Ir has been carried out. This study was undertaken primarily to provide a comparison of the experimental and theoretical $I$ and $M$ subshell intensities for the pure E2 transitions at 205 and 316 $\mathrm{keV}$. In addition, data were obtained for other transitions and other shells. In the course of the analysis of these data, it became necessary to study the limiting factors on the accuracy of the measured intensities. Measurements of internal-conversion coefficients (both absolute and relative) with a precision of about $1 \%$ are needed in order to test the current theories upon which the calculation of internalconversion coefficients is based.

The decay of ${ }^{192}$ Ir populates states in the even-even nuclei ${ }^{192} \mathrm{Pt}$ and ${ }^{192}$ Os. Since the ground states and first excited states of each of these nuclei are $0^{+}$and $2^{+}$, respectively, pure E2 transitions occur between these states in both cases. The other transitions may have mixed multipolarities. The transitions observed in this study are listed in Table I along with the spins and parities of the associated initial and final states and the resulting multipolarities of the gammaray transitions.

A sample of iridium was made by vacuum evaporation of the metal from a heated iridium wire. The material was collected on an aluminum foil $(0.0006 \mathrm{~cm}$ thick) with the source area $(0.015 \mathrm{~cm} \times 1 \mathrm{~cm})$ defined by a mask. By $X$-ray fluorescence methods, it was determined that the source contained about 2-1/2 $\mu \mathrm{g}$ of iridium which corresponds to a thickness of $150 \mu \mathrm{g} / \mathrm{cm}^{2}$. This sample was irradiated in the ATR in a flux of $27 \times 10^{14} \mathrm{n} / \mathrm{cm}^{2} \mathrm{sec}$ for 215 days. At the end of the irradiation, about 10\% of the sample is calculated to be active ${ }^{192} \mathrm{Ir}$. (This is about the maximum specific activity possible for $(n, \gamma)$ production of ${ }^{192}$ Ir with normal iridium and without isotope separation.)

After mounting and aligning the source, a resolution of $0.016 \%$ was obtained, which corresponds to the value expected for a source of this width $(0.015 \mathrm{~cm})$. Table II gives a summary of the conversion lines measured for this source. Two types of runs were made. In the first ("unmonitored") group, the spectrometer ran automatically with no check of the coil currents, so the current for each data point was assumed to be equal to the value set up by the controller. For the other four measurements (316I, 205I, 316M, 295I), the current was monitored with a potentiometer (sensitivity $\sim 1$ ppm) several times during each counting period, so that all drifts in the coil currents were observed. The spectra for several measurements are shown in Figures 1-3.

The relative intensities of the lines in a group (e.g., $I_{1}, L_{2}$ ard $\mathrm{I}_{3}$ ) were determined by means of a computer program written for this purpose[2]. This program uses an empirical line shape to generate a component for each observed Iine. Each component is shifted in small 
steps to determine the optimum position, and the intensities are computed by a least-squares fit. The background under the peaks is assumed to be a straight line, which is determined in the fit. The program also provides for the convolution of the line shape for any component with a Lorentzian function of specified width $\Gamma$ in order to account for the difference in widths of the various atomic levels.

An estimate of the natural level widths has been obtained from the calculated probability for filling an electron hole together with the $\mathrm{K}$ shell and $I$ subshell fluorescence yields. For levels in Pt, these values give $50 \mathrm{eV}$ for the $K$ shell and 10,5 and $5 \mathrm{eV}$ for the $I$ subshells. For the I subshells, the uncertainties in the fluorescence yields contribute an uncertainty of $220 \%$ to these values. This means the difference in the $I_{1}$ and $I_{3}$ widths could vary from 2 to $8 \mathrm{eV}$. Therefore, the first part of the analysis of the ${ }^{192}$ Ir data involved a study of the quality of fit parameter $\left(\varepsilon^{2}\right)$ for different assumed Lorentzian widths for the L subshells. The data on the L lines for the 295-, 308- and 316-keV transitions were used for this test. For these lines, the total number of counts in a single peak varied from 32,000 (for the $308 \mathrm{~L}_{3}$ ) up to $1,550,000$ (for the $316 \mathrm{~L}_{2}$ ).

In the analysis of these I line groups, the empirical line shape was derived from an $L_{2}$ or $L_{3}$ line. An example is shape $L 5$ in Figure 4. Therefore, it includes $55 \mathrm{eV}$ of natural level width in addition to the spectrometer contribution to the observed line width. Only the difference in the line width is then apparent in the analysis. The computed values of the $L_{1} / L_{3}$ relative intensities are given in Table III for the 295-, 308- and 316-keV transitions for four different sets of Lorentzian widths $\Gamma_{L_{i}}$. These results indicate that:

1. The quality of fit improves (i.e., $\varepsilon^{2}$ decreases) when $\Gamma_{L_{1}}$ is 5 or $10 \mathrm{eV}$ greater than $\Gamma_{\mathrm{L}_{3}}$, in agreement with the above calculated widths.

2. An increase in $\Gamma_{\mathrm{L}_{2}}$ of $5 \mathrm{eV}$ causes a negligible change in the $\mathrm{L}_{1} / \mathrm{L}_{3}$ ratio. (But it does give a significant change in the $\mathrm{L}_{1} / \mathrm{L}_{2}$ or $\mathrm{L}_{2} / \mathrm{L}_{3}$ ratios.)

3. For the $295 \mathrm{~L}$ and $308 \mathrm{I}$ data, a change in the $\mathrm{L}_{1}$ Lorentzian width from 5 to $10 \mathrm{eV}$ does not change the quality of fit, significantly.

4. For all three sets of data, the $L_{1} / L_{3}$ ratio changes as a function of $\Gamma_{L_{1}}$ at a rate of $20.5 \%$ per $\mathrm{eV}$.

From these last two results it is concluded that the $L_{1} / L_{3}$ ratio cannot be determined to $1 \%$ by this fitting method unless the difference in natural level widths is known to $22 \mathrm{eV}$. At least for the $295 \mathrm{I}$ and $308 \mathrm{I}$ groups, the uncertainty in the $I_{1} / I_{3}$ ratio will be $\omega 5 \%$ if the choice of Lorentzian widths is based on the $\varepsilon^{2}$ value. It is of interest to compare the size of these error estimates with those obtained from the area of the peaks. For the $316 \mathrm{~L}$ lines, the areas of $1 \times 10^{6}$ and $6 \times 10^{5}$ would imply an uncertainty of $0.15 \%$ in the $L_{1} / L_{3}$ ratio, whereas the values in Table III are about $0.8 \%$. 
The analysis of the $K$ Iines of the 295-, 308-, 316- and 468-keV transitions (which have electron energies from 217 to $390 \mathrm{keV}$ ) indicates the effect of electron scattering in the source. Of the two shapes used, $K 5$ and $K 7$ in Figure 4, the narrower (K7) gives the better fits to the $468 \mathrm{~K}$ line, while the wider gives the better fit to the other $\mathrm{K}$ lines. (The $\mathrm{K} 7$ shape is still too wide for, the $468 \mathrm{~K}$, since its best fits are for a Lorentzian width of $\approx 38 \mathrm{eV}$, while the best fits to the other lines are for a width of $\approx 48 \mathrm{eV}$.$) It is of interest to note that for the$ $295 \mathrm{~K}$, the spectrometer resolution for this source is $\approx 37 \mathrm{eV}$ while the natural level width is $\approx 50 \mathrm{eV}$. Although the analyses that have been carried out are satisfactory for getting accurate relative intensities for the three lower energy $K$ lines, a more extensive analysis will be necessary to the best possible $468 \mathrm{~K}$ intensity. This work suggests that it would be useful to try to account for the electron scattering in the source in a systematic manner. The results of the analyses of the $\mathrm{K}$ lines are:

1. An increase of $5 \mathrm{eV}$ in the assumed Lorentzian width gives a significant change in $\varepsilon^{2}$ and a $1.0-1.5 \%$ change in the line intensities.

2. For the ratio of the $295 \mathrm{~K}, 308 \mathrm{~K}$ and $316 \mathrm{~K}$ intensities an increase in $\Gamma_{K}$ of $5 \mathrm{eV}$ produces a change of $0.2-0.5 \%$.

Therefore, where the scattering in the source can be taken into account, accurate values of the relative $K$ line intensities can be determined without prior knowledge of the natural level width.

For the analysis of the $M$ and $N$ shell data the line shape $I_{1}$ in Figure 4 (as well as others) has been used. It is assumed that there is no essential difference in the natural widths of any of the levels.

In conclusion, Table IV gives a summary of some of the $I_{1} / I_{3}$, $M_{1} / M_{3}$ and $N_{1} / N_{3}$ results that have been obtained. For the $L$ subshells, the results for $\Gamma_{L_{1}}=\Gamma_{L_{3}}+5 \mathrm{eV}$ and $\Gamma_{L_{2}}=\Gamma_{L_{3}}$ have been used. For comparison, the corresponding theoretical values are given.

[1] Graduate student, Utah State University, Logan, Utah.

[2] R. G. Helmer, R. A. Lokken and D. J. McMillan, "Best' Level Energy Program", this report.

[3] R. S. Hager and E. C. Seltzer, Nuclear Data A 4, Nos. 1 and 2, I (1968). 
TABLE I

SOME CHARACTERISTICS OF THE DECAY OF ${ }^{192} \mathrm{Ir}$

\begin{tabular}{|c|c|c|c|c|}
\hline \multicolumn{2}{|l|}{ Gamma-Ray } & \multicolumn{3}{|c|}{ Level Spin \& Parity } \\
\hline Energy (keV) & Nucleus & Initial & Final & Multipolarity \\
\hline 205 & $192 \mathrm{Os}$ & $2^{+}$ & $0^{+}$ & $\mathrm{E2}$ \\
\hline 316 & ${ }^{192} \mathrm{Pt}$ & $2^{+}$ & $\mathrm{O}^{+}$ & $\mathrm{E2}$ \\
\hline 295 & $192 \mathrm{Pt}$ & $2^{+}$ & $2^{+}$ & $\mathrm{EO}, \mathrm{MI}, \mathrm{E} 2,$. \\
\hline 308 & $192 \mathrm{Pt}$ & $3^{+}$ & $2^{+}$ & $\mathrm{MI}, \mathrm{E} 2,$. \\
\hline 468 & ${ }^{192} \mathrm{Pt}$ & $4^{+}$ & $2^{+}$ & E2, M3, ... \\
\hline
\end{tabular}

TABLE II

SUMMARY OF ${ }^{192}$ Ir CONVERSION-ELECTRON IINE STUDY

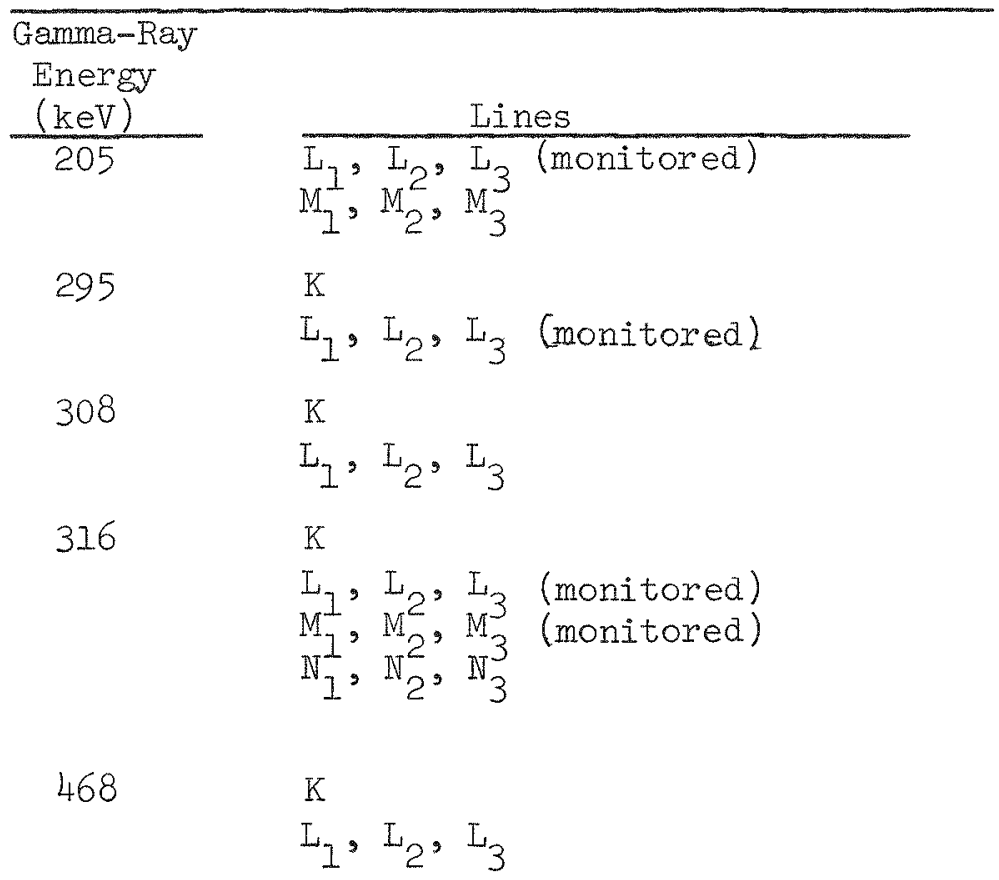


TABLE III

$\mathrm{I}_{1} / \mathrm{L}_{3}$ RATIOS FOR 295- AND 316-keV TRANSITIONS

\begin{tabular}{|c|c|c|c|c|c|c|}
\hline \multirow{2}{*}{$\begin{array}{l}\text { Gamma-Ray } \\
\text { Energy } \\
\text { (keV) } \\
\end{array}$} & \multicolumn{5}{|c|}{ Lorentzian Wiath $(\mathrm{eV})$} & \multirow[b]{2}{*}{$\begin{array}{c}\text { Change } \\
(\%)\end{array}$} \\
\hline & $I_{1}$ & $\underline{\mathrm{L}_{2}}$ & $I_{3}$ & $\varepsilon^{2}$ & $\mathrm{I}_{1} / \mathrm{I}_{3}$ & \\
\hline \multirow[t]{4}{*}{295} & 0 & 0 & 0 & 2.5 & $1.270 \pm 0.017$ & -2.8 \\
\hline & 5 & 0 & 0 & 2.0 & $1.307 \pm 0.015$ & $\equiv 0.0$ \\
\hline & 10 & 0 & 0 & 1.9 & $1.340 \pm 0.016$ & 2.5 \\
\hline & 5 & 5 & 0 & 2.1 & $1.308 \pm 0.016$ & 0.1 \\
\hline \multirow[t]{4}{*}{316} & 0 & 0 & 0 & $3 \cdot 3$ & $1.302 \pm 0.013$ & $-2 \cdot 3$ \\
\hline & 5 & 0 & 0 & 2.5 & $1.332 \pm 0.011$ & $\equiv 0.0$ \\
\hline & 10 & 0 & 0 & 2.9 & $1.359 \pm 0.012$ & 2.0 \\
\hline & 5 & 5 & 0 & 1.9 & $1.333 \pm 0.010$ & 0.1 \\
\hline \multirow[t]{4}{*}{308} & 0 & 0 & 0 & 2.2 & $1.364 \pm 0.019$ & $-2 \cdot 3$ \\
\hline & 5 & 0 & 0 & 1.8 & $1.396 \pm 0.018$ & $\equiv 0.0$ \\
\hline & 10 & 0 & 0 & 1.7 & $1.432 \pm 0.018$ & 2.6 \\
\hline & 5 & 5 & 0 & 1.7 & $1.398 \pm 0.017$ & 0.1 \\
\hline
\end{tabular}

TABLE IV

PARTIAL SUMMARY OF PRELIMINARY RESULIS OF ${ }^{192}$ Ir CONVERSION-EIECTRON SPECTRUM

\begin{tabular}{|c|c|c|c|c|c|c|}
\hline \multirow{3}{*}{$\begin{array}{l}\text { Gamma-Ray } \\
\text { Energy } \\
\text { (keV) } \\
\end{array}$} & \multirow{3}{*}{ Lines } & \multirow{3}{*}{ Shape } & \multirow{3}{*}{$\underline{\varepsilon^{2}}$} & \multicolumn{3}{|c|}{ Relative Conversion Coefficients } \\
\hline & & & & Experimental & \multicolumn{2}{|c|}{ Theoretical $^{a}$} \\
\hline & & & & & & MI \\
\hline 205 & $\mathrm{M}_{1}$ & LI & 1.3 & $0.386 \pm 0.035$ & 0.443 & -- \\
\hline 295 & $I_{1}$ & L5 & 2.0 & $1.307 \pm 0.015$ & 1.11 & 121 \\
\hline 308 & $I_{I} \prime$ & L5 & 1.8 & $1.396 \pm 0.018$ & 1.22 & 122 \\
\hline \multirow[t]{2}{*}{316} & $\mathrm{I}_{1} /$ & L5 & 2.5 & $1.332 \pm 0.011$ & 1.29 & 123 \\
\hline & $N_{1} / N_{3}$ & LI & 1.4 & $1.055 \pm 0.032$ & - & -- \\
\hline
\end{tabular}

a) See Ref. 3 . 


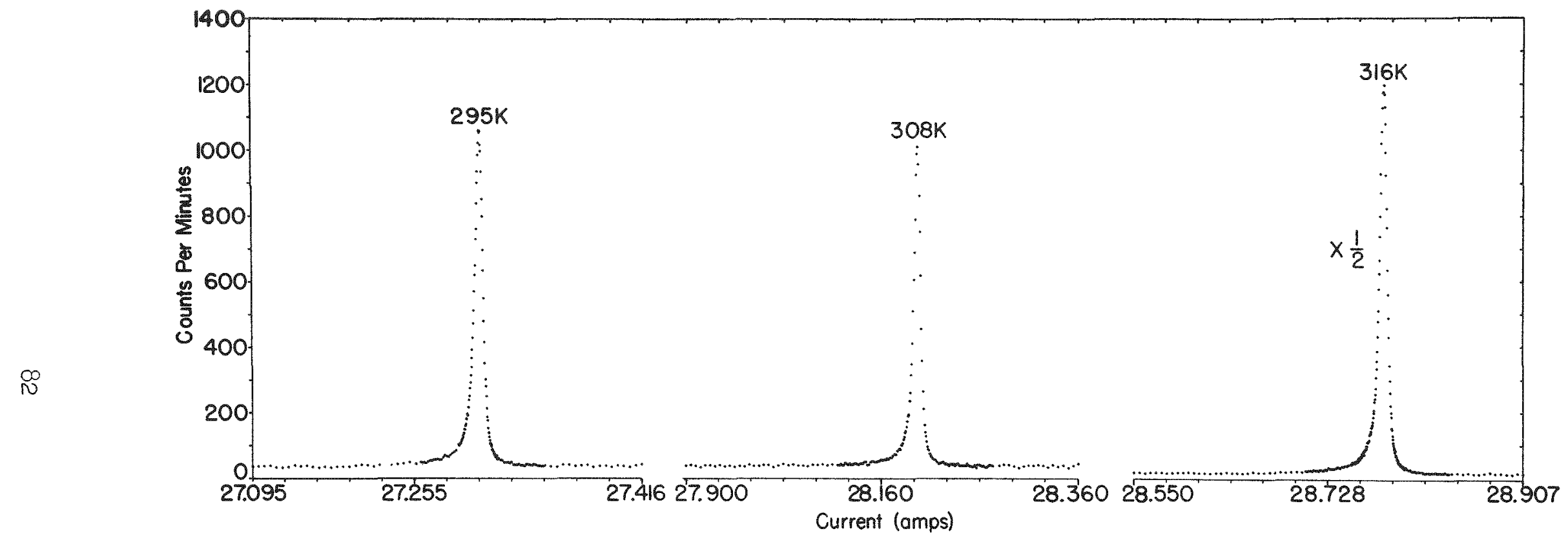

Fig. I Three $K$ conversion electron lines from the decay of ${ }^{192}$ Ir measured at $0.016 \%$ resolution. 


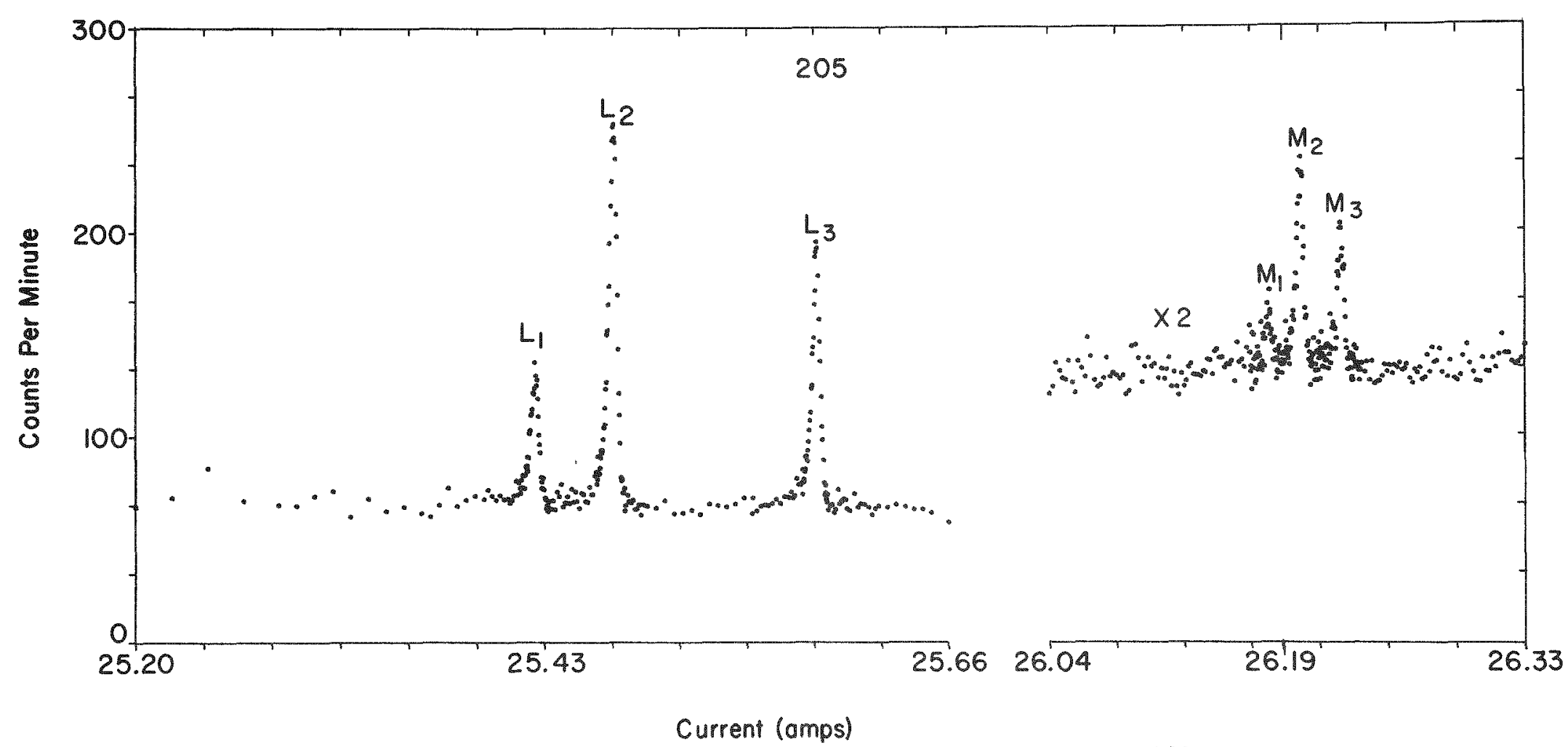

Fig. 2 I and M conversion electron lines from the $205-\mathrm{keV}$ transition in ${ }^{192}$ Os. 


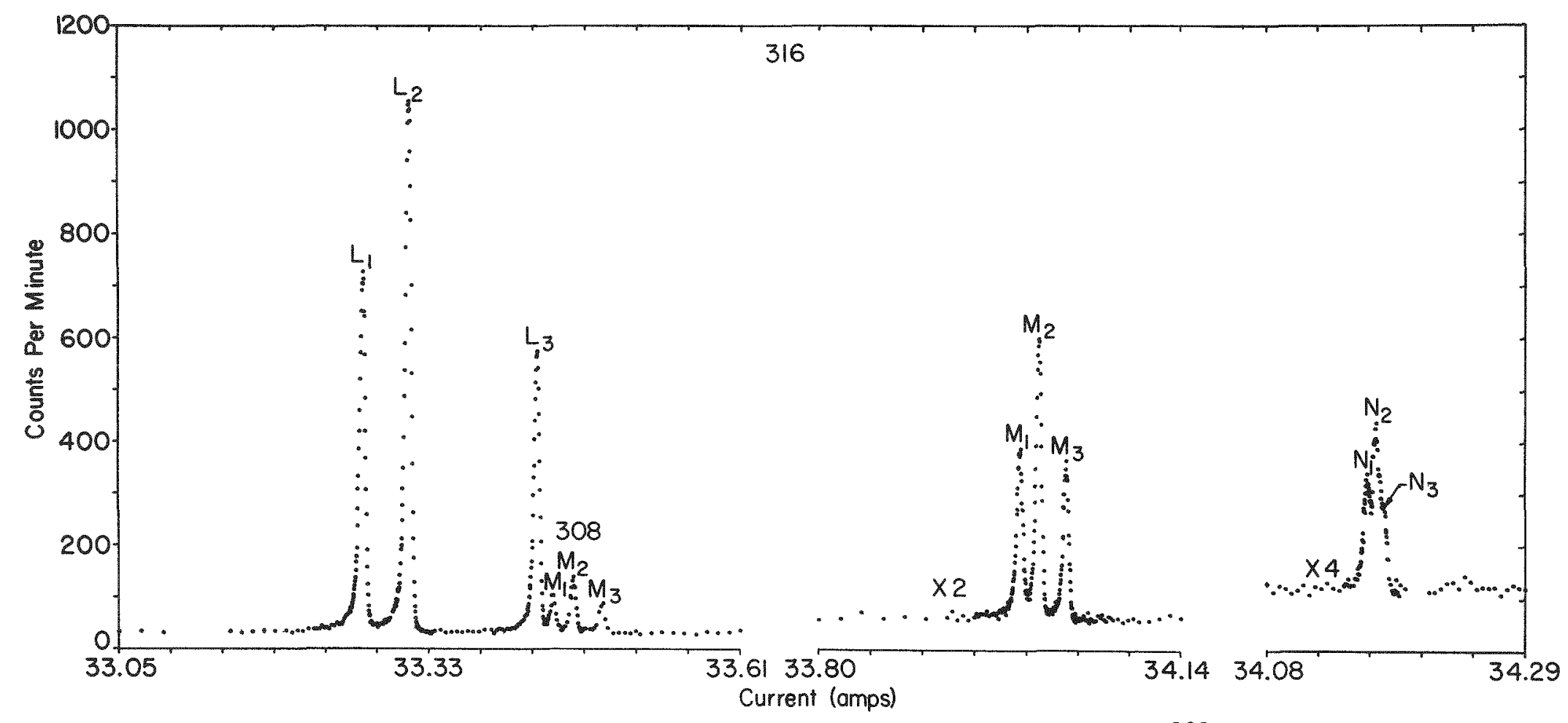

Fig. $3 \mathrm{~L}, \mathrm{M}$ and $\mathbb{N}$ conversion electron lines from the $316-\mathrm{keV}$ transition in ${ }^{192} \mathrm{Pt}$. 


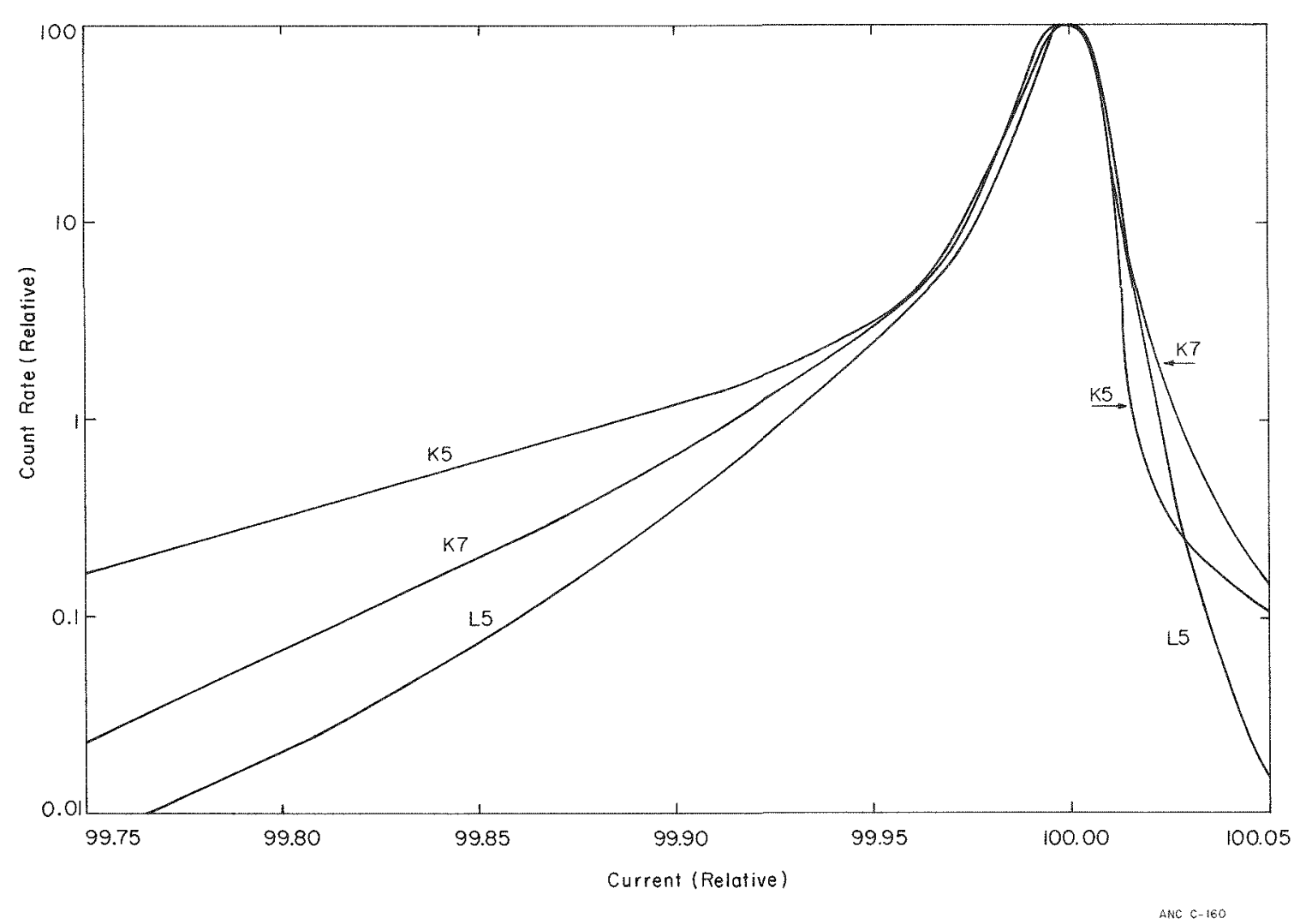

Figure 4 Line shapes used in the analysis of the ${ }^{192}$ Ir conversion electron spectra. 
GAMMA RAYS EMITTED BY THE FISSIONABLE NUCLIDES AND ASSOCIATED ISOTOPES [I]

\section{J. E. Cline}

To provide reference material on the gamma-ray spectra of nuclides of importance to the application of gamma-ray spectrometry to the nuclear fuel safeguards program, a collection of nuclear data has been compiled and issued as a laboratory report. Data on energies and intensities of gamma rays emitted by the nuclides present in nuclear fuel materials are presented. The compilation contains data and gammaray pulse-height spectra for 13 nuclides. These energies and intensities have been measured at this laboratory using high-resolution $\mathrm{Ge}(\mathrm{Li})$ spectrometers.

[I] Idaho Nuclear Corporation report IN-1448 (January 197I). 
SPALIATION REACTION PRODUCTS FROM IRRADIATION OF GOLD BY 600-MeV PROTONS

R. A. Lokken $[1]$

A study has been carried out to determine the production yields of various isotopes resulting from the bombardment of gold with $600 \mathrm{MeV}$ protons. This information is of value as a check on theoretical estimates of spallation yields as well as for use in estimating the yield of specific isotopes produced in this and similar spallation reactions.

In this experiment, two gold foils were bombarded with $600 \mathrm{MeV}$ protons in the internal beam of the syncrocyclotron at the Space Effects Radiation Laboratory. The gamma-ray spectrum from one of these foils was measured over a period of six months. The time variations in these spectra, and their complexity are illustrated in Figures 1 and 2. From these spectra, it was desired to identify the isotopes present and to compute their "initial" intensities. For some isotopes these "initial" intensities give the cross section for the direct production of the particular isotope. However, in most cases the initial intensities represent the sum of the direct production cross sections for all of the short-lived isotopes which decay into the final radioisotope observed, the so-called chain yields.

The analysis of the $u 30$ gamma-ray spectra proceeded in the following manner. For a few spectra taken at different decay times, tentative isotopic assignments were made for several peaks. From the energies of the peaks, the energy calibration function was determined and the energies of the remaining lines were computed. From the energies and intensities of the observed peaks, the tentative isotopic assignments were verified. Also from the relative gamma-ray intensities and the decay of the peaks, the purity of the various peaks was determined. In most cases, it was found that the peaks contained contributions from more than one isotope.

In the initial analysis of a spectrum, the automatic peak location and analysis features of GAUSS-V were used. Subsequent analyses of the same spectrum, however, were done with more user control. First, the weak peaks were analyzed more successfully by fixing the width of the Gaussian function in each peak fit. Also, the peaks with more than one component were analyzed by both fixing the widths of the component Gaussians and providing initial positions for each component.

Given this information, six of the spectra were completely analyzed. This complete analysis included (I) making an isotopic assignment for each observed peak, (2) making an approximate allotment of the intensity to various isotopes for each complex peak, (3) verifying, for each isotopic assignment, that all the gamma rays of that isotope were present in the proper amount, and (4) that each peak decayed with the half-life or lives that agreed with the assignment(s). 
From the results of the complete analysis of these six spectra, one can determine the optimum time at which to use a peak area in order to compute the initial yield. Even though a peak may contain contributions from, say, three isotopes, at different times it may be $\mathrm{f}$ sibie to use it to compute each of the three yields. For isotopes that exhibit several gamma rays, data from these six spectra indicate which peaks should be used in the computation of yields.

The analysis of these data is continuing.

[1] Graduate student, Utah State University, Logan, Utah. 


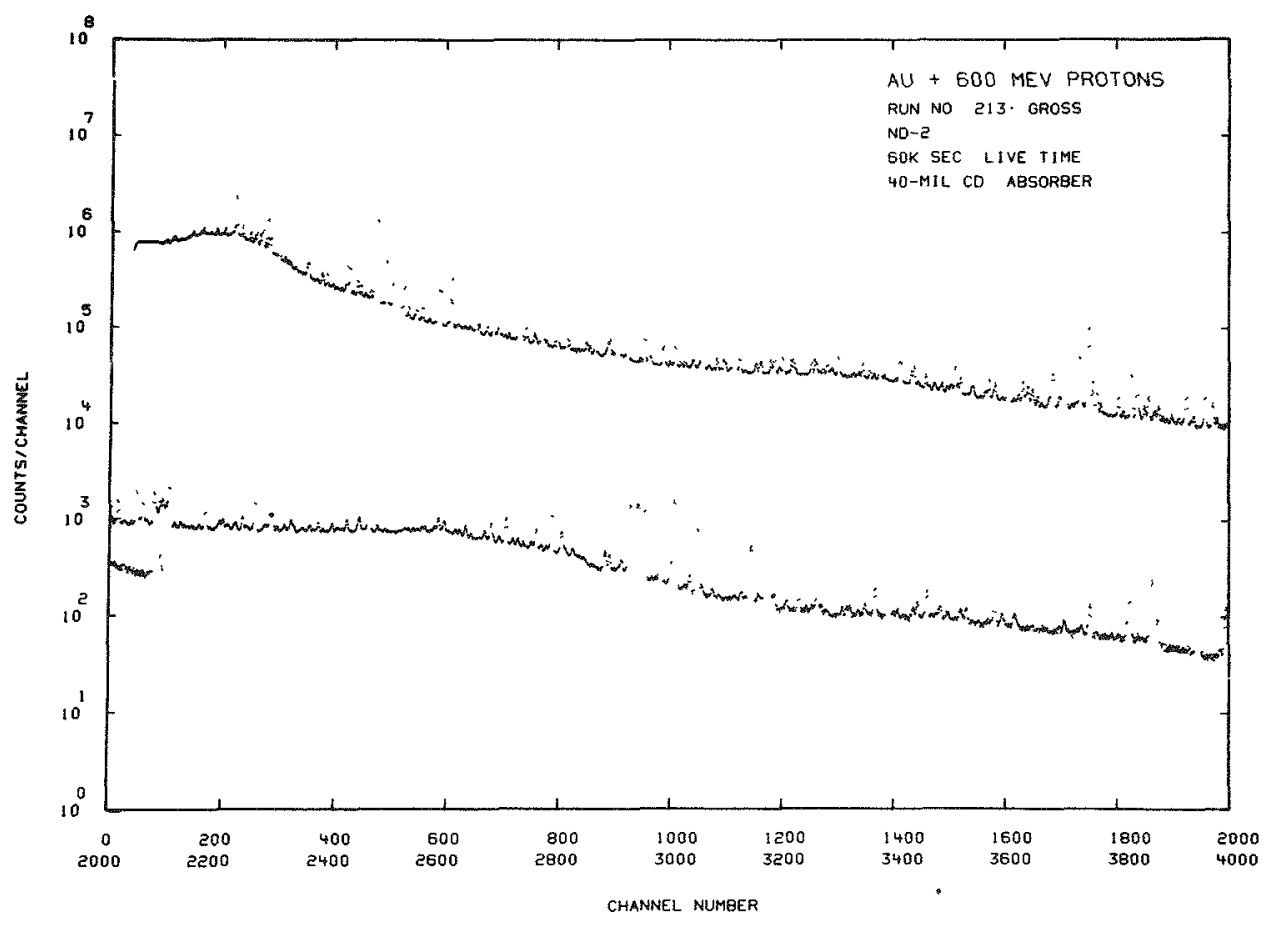

Figure I Gamma-ray spectrum from gold sample about four days after irradiation with 600-MeV protons.

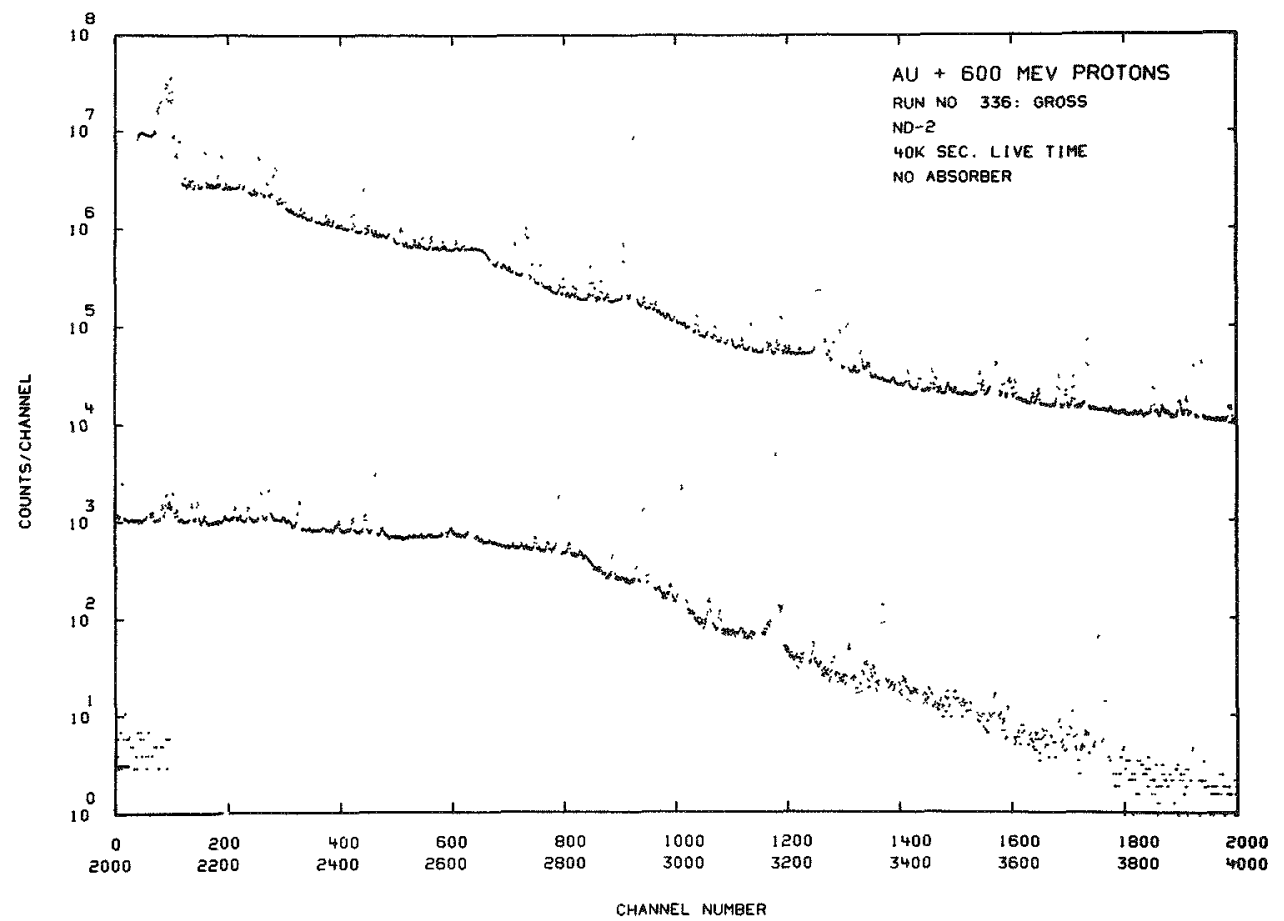

Figure 2 Gamma-ray spectrum from gold sample about sixty-five days after irradiation with $600-\mathrm{MeV}$ protons. 
-

$\bullet$ 
C. MATERIALS

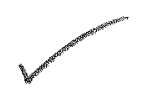

91 
-

$\bullet$ 
SHORT AND INTERMEDIATE RANGE CRYSTAL POTENTIALS

IN TERMS OF VALENCE SYMMETRY COORDINATES

H. L. McMurry

This work aims at a method for calculating crystal vibrations which gives good normal modes as well as correct calculated frequencies. Among the potential benefits is the ability to calculate the neutron moderating properties of crystalline materials more accurately. In addition, any crystalline properties which are sensitive to the normal mode patterns would be more amenable to inference from calculation. Among these are predictions about phase changes which might occur when vibration amplitudes are increased at elevated temperatures. There is also the possibility that empirically determined force constants and polarizability constants might reveal important characteristics of the electronic structures of the crystals.

The method is developed for valence bonded crystals in which the short range forces are important and the bonds between near neighbor atoms in adjacent primitive cells are comparable to those between atoms within the cells. The work extends earlier work in molecules[1] and molecular crystals [2] in which coordinates possessing the symmetry of local atom groups (like $\mathrm{CH}_{3}$ ) were used to define the coordinates for calculating the vibrations. As in the work on molecular crystals, the secular equation for the vibrations is in two parts. One arises from short range forces arising from distortions of the molecular orbitals bonding the atoms within a primitive cell, and in adjacent primitive cells. The second term gives the contribution of the long range interaction which develops between dipoles induced in the primitive cells in the lattice during distortion induced by the vibrations. The final equations are like those from the molecular crystals[2], except for exhibiting features arising from the strong intercell bonding.

The procedure parallels that given for molecular crystals[2]. However, in this work the symmetry coordinates (called Valence symmetry Coordinates, VSC $[1,2]$ ) are defined in terms of displacements of the atoms within a cell, and the near neighbor atoms in the adjacent cells. The inclusion of displacements of the near neighbor atoms takes account of the strong forces extending between atoms in adjacent cells. These were unimportant for molecular crystals. The coordinates are chosen to belong to symmetry representations of the group of atoms. They then possess symmetry properties like those of the crystal and of the electron wave functions which are suited to describing the ground state electron configuration of the crystal[3]. The force constants in the potential function are then potentially more amenable to calculation (or at least interpretation) in terms of distortion in the electron configuration than are force constants in potential functions where the crystal symmetry is not used in defining the coordinates.

The ultimate aim is to calculate the force constants for the potential function employing these VSC directly from changes in the ground state electron energy. In the interim applications, it is hoped that the theory may show how to choose the dominant force constants 
(especially interaction constants) so that the calculated normal modes are correct, as well as the frequencies. If successful, the work will represent a step toward removing the ambiguity posed when a choice must be made among different sets of force constants which give comparable calculated frequencies but different normal modes. This work is described in a forthcoming report [4].

[1] H. I. McMurry, Molecular Potential Functions in Terms of Group Valence Symmetry Coordinates, IN-1421 (April 1971).

[2] H. L. McMurry, Application of Solbrig's Cell-Dipole Method to Molecular Crystals, IN-1410 (October 1970).

[3] H. I. McMurry, Electron Ground States in Molecular and Valence Bonded Crystals, IN-1420 (October 1970).

[4] H. L. McMurry, Short and Intermediate Range Crystal Potentials for Valence Bonded Crystals Using Valence Symmetry Coordinates, ANCR-1017 (in preparation). 


\section{CALCULATION OF FORCE CONSTANTS IN MOLECULES AND CRYSTALS}

H. L. McMurry

This work is a step toward providing a theoretical basis for calculating force constants from changes in the ground state electron energy of a molecule or crystal arising from vibrationally induced distortions. If the method proves valid and is amenable to practical calculations the way will be open to obtaining force constants which yield physically accurate normal modes as well as frequencies. This enlarges the usefulness of calculating the vibrational properties of materials. Not only are the neutron scattering properties given more accurately, but other material properties such as polarizabilities and possibly phase changes become amenable to calculation.

This method aims at exploiting ideas for choosing coordinates for expressing the vibrational potentials so as to make the force constants more amenable to direct calculation $[1,2]$. A theoretical prescription for calculating the equilibrium ground state electron energy[3] is modified to account for energy changes arising when the atoms are displaced from their equilibrium positions. The method is developed for valence bonded crystals but can be specialized to molecular crystals or molecules.

The change in electron energy is expressed to quadratic approximation in the changes in the valence symmetry coordinates $[1,2]$. The linear terms must vanish and this provides constraint relations which help to determine how the coefficients in the ICAO MO's (Iinear combination of atomic orbital-molecular orbitals) are altered when the VSC are excited. It becomes possible, ultimately, to express the energy changes in terms of changes in matrix elements which are coefficients in an energy expansion in terms of the valence symmetry coordinates.

The difficulties which will attend actually calculating the force constants from these relations remains to be assessed, as does the general validity of the procedure.

The work will be reported in an ANCR report ${ }^{[4]}$.

[1] H. L. McMurry, Molecular Potential Functions in Terms of Group Valence Symmetry Coordinates, IN-1421 (Apri1 1971).

[2] H. L. McMurry, Application of Solbrig's Cell-Dipole Method to Molecular Crystals, IN-1410 (April 1970).

[3] H. I. McMurry, Electron Ground States in Valence Bonded CrystaIs, IN-1420 (October 1970).

[4] H. I. McMurry, Calculation of Force Constants in Molecules and Crystals from Changes in Ground State Electron Energy with Atom Displacements, ANCR-1018 (to be published). 
TRANSMISSION ELECTRON MICROSCOPY SURVEILLANCE OF FATIGUE AND IRRADIATION DAMAGE INTERACTION

$$
\text { G. E. Korth }
$$

Transmission electxon microscopy (TEM) is being utilized to characterize microsctuctural changes that occur in irradiated specimens resulting from the elevated temperature inelastic processes. Examination of samples of 304, 304L ( $\mathrm{Ti}$ modified), 316 stainless and Incoloy 800 taken from specimens that were irradiated at $\sim 700-750^{\circ} \mathrm{C}$ (to fluences of $1-4 \times 10^{22} \mathrm{c} / \mathrm{cm}^{2} \mathrm{E}>$ $.1 \mathrm{MeV}$ and tested at $700^{\circ} \mathrm{C}$ ) is currently under way. A summary of specimens examined to date is shown in Table I. It is felt that a better understanding of complex material behavior and its influence on the time and cycle dependent processes leading to elevated temperature material failure can be obtained through TEM surveillance of the fatigued specimens.

The relative ease with which dislocations are generated, move, and assume equilibrium arrangements depending on time and temperature is important in both nucleation and crack growth kinetics. Extensive plastic flow at the tip of an advancing fatigue crack is beneficial in that the strain concentration factor is reduced; on the other hand extensive subgrain formation resulting from such time-temperature deformation processes may be important in that transgranular cracking can occur along subgrain boundaries. Further, irradiation effects may influence the relative ease with which crack nucleation can occur particularly in material containing helium or irradiation induced voids at large angle grain boundaries.

Substructure resulting from fatigue deformation contains dislocations which form into a diffuse or well defined cellular structure of subgrains depending on strain, time, temperature, and the relative ease with which dislocations can climb and glide. Irradiation-produced defects do have a definite effect on the formation of these dislocation cells and thereby could affect the overall fatigue damage mechanisms. Helium filled or vacancy voids, faulted and unfaulted dislocation loops, and point defect clusters are some of the irradiation-produced defects that have been observed.

The effect of irradiation on dislocation cell formation is quite evident in that cells of irradiated specimens averaged 1.2 to 2.0 times as large and the walls are much more diffuse, as illustrated in Figure 1. Also the initial formation of the dislocation cells appears to be retarded by irradiation. Both of these effects have been seen by other investigators 1]. Cells of irradiated $304 \mathrm{SS}$ (52E) and $304 \mathrm{~L}$ (Ti) (31F) specimens fatigued at relatively low total strain range were found only by considerable searching and then only in the initial stages of formation while their unirradiated counterparts displayed typical dislocation cells as shown in Figure 2. It was also noted in these same 
irradiated specimens ( $52 \mathrm{E}$ and $31 \mathrm{~F}$ ) that partial dislocations with stacking faults in between, on the average, were separated by an order of magnitude more than in the control specimens. Figure 3 illustrates this point for the 304 material. The longer distance between the extended dislocations indicates the possibility that irradiation decreases the stacking fault energy which would be beneficial in terms of increasing material fatigue resistance. Another concurrence of this same possibility was observed in the Incoloy 800 in which annealing twins were much more prevelant in the irradiated specimen, indicating a lower stacking fault energy [2].

The void or helium bubble size and distribution is of considerable interest in addition to swelling, due to their potential for dislocation pinning and crack nucleation and propagation. The distribution of voids (which may be helium bubbles) appears to be related to the type alloy and second phase precipitation. Voids in type 304L(Ti) agglomerate preferentially at the grain boundaries and only at the grain boundaries in Incoloy 800 , but no voids at all were observed at boundaries of 304 and only occasionally in 316. Al1 types of material examined except Incoloy 800 showed voids within the grains with a denuded area adjacent to grain or twin boundaries as shown in Figure 4. As can be seen from this figure the $304 \mathrm{~L}$ (Ti) SS and Incoloy 800 are relatively free of grain boundary precipitates whereas the 304 and 316 grain and twin boundaries are profusely 1ined with precipitates. It is speculated that the grain boundary-precipitate combination acts as sinks for the voids. The matrix precipitates, identified by electron diffraction as coherent $\mathrm{M}_{23} \mathrm{C}_{6}$ carbides, would not provide the same type of sinks as the boundary carbides due to the loss of coherency with at least part of the particle due to the very nature of a grain or twin boundary. The boundaries of the Incoloy 800 and $304 \mathrm{~L}(\mathrm{Ti})$ SS would not provide the same type of sinks since the boundary-carbide combination is not continuously present.

Two other observations worthy of note concerning voicis involve the rows of voids seen within grains of $304 \mathrm{~L}(\mathrm{Ti})$ and the complete absence of voids within the Incoloy 800 grains (see Figure 5). Evidently voids formed on a grain boundary of the 304L(Ti) during irradiation and then the boundary moved during testing leaving the string of voids behind. The voids in the Incoloy 800 are on the average only $1 / 4$ to $1 / 2$ the size of those observed in the $304,304 \mathrm{~L}(\mathrm{Ti})$, and $316 \mathrm{SS}$. In addition to being smaller the void density of the Incoloy is approximately two orders of magnitude less and considering the gas analysis of similar samples[3], it appears that the voids in Incoloy 800 are helium bubbles while those in $304 \mathrm{~L}$ (Ti) as well as the 304 and 316 SS are probably vacancy voids. Also calculations show that the cavity volume of the 304 and 316 SS (assuming equilibrium between gas pressure and surface tension) is two to three orders of magnitude larger than required to contain the amount of helium measured. However, voids are not normally seen in stainless steels irradiated above $\sim 650^{\circ} \mathrm{C}$. Work is continuing to resolve this inconsistency. 
Total strain range $\left(\Delta \varepsilon_{t}\right)$ of the fatigue tests also had an obvious effect on the observance of voids. Samples taken from the gage section of the specimen showed a drastic reduction of voids with respect to the head area when the strain range was greater than $0.3 \%$. No voids were observed at all in the gage section of $55 \mathrm{E}\left(\Delta \varepsilon_{t}=2.4 \%\right)$ and considerable searching was required to find any voids in the gage section of $53 \mathrm{E}(\Delta \varepsilon=$ $1.04 \%$ ), but 1ittle difference, if any, was noted between the void density of the gage and head sections of the low strain range specimens $52 \mathrm{E}, 31 \mathrm{~F}$, and $34 \mathrm{G}$. The $316 \mathrm{SS}$ specimen $63 \mathrm{D}(\Delta \varepsilon=2.4 \%)$ showed the same effect as the 304 SS but not as extensive, i.e. void density in the gage section was approximately $1 / 3$ to $1 / 2$ of that seen in the head. Just what happens to the voids in the strained region is not clear but evidence indicates that at some critical $\Delta \varepsilon_{t}$ the voids migrate due to applied stress and diffuse up the gradient to the external surface or to the propagating crack as predicted by models recently developed $[4,5]$. No evidence of dislocation channeling sweeping up of the voids was seen.

[1] R. C. Rau, G. R. Anderson, and F. T. Williams, Jr., Eighth Annual Report - AEC Fuels and Materials Development Program, GEMP-1012-1 (March 31, 1969).

[2] D. McLean, Mechanical Properties of Metals, Published by John Wiley \& Sons (1967).

[3] Unpublished data, Idaho Nuclear Corporation.

[4] S. H. Leiden and M. J. Trinko, Pure Motion in Solids Resulting from App1ied Stress, WAPD-TM-983 (February 1971.

[5] I. A. Nichels, "Movement of Pores in Solids," J. of Meta1s, 21 , p. 19 (1969). 
TABLE I

IDENTIFICATION AND HISTORY OF SPECIMENS EXAMINED

\begin{tabular}{|c|c|c|c|}
\hline $\begin{array}{l}\text { Specimen } \\
\text { Number } \\
\end{array}$ & Material & $\begin{array}{c}\text { Fluence, } \Phi \\
\mathrm{n} / \mathrm{cm}^{2}(\mathrm{E}>0.1 \mathrm{MeV}) \\
\end{array}$ & $\begin{array}{l}\text { Total Strain } \\
\text { Range, } \Delta \varepsilon_{t}[\text { a }]\end{array}$ \\
\hline $55 E$ & 304 SS & $2.9 \times 10^{22}$ & $2.45 \%$ \\
\hline $20 E$ & 304 SS & 0 & $2.41 \%$ \\
\hline $52 \mathrm{E}$ & 304 SS & 3.0 & 0.35 \\
\hline $30 E$ & $304 \mathrm{SS}$ & 0 & 0.35 \\
\hline $31 \mathrm{~F}$ & $304 \mathrm{~L}(\mathrm{Ti})$ & 2.0 & 0.36 \\
\hline $14 \mathrm{~F}$ & $304 \mathrm{~L}(\mathrm{Ti})$ & 0 & 0.35 \\
\hline $53 \mathrm{E}$ & 304 SS & 1.5 & 1.04 \\
\hline $18 \mathrm{E}$ & 304 SS & 0 & 1.01 \\
\hline $63 D$ & 316 SS & 2.4 & 2.44 \\
\hline $24 \mathrm{D}$ & $316 \mathrm{SS}$ & 0 & 2.40 \\
\hline $34 G$ & Incoloy 800 & 3.4 & 0.32 \\
\hline $14 \mathrm{G}$ & Incoloy 800 & 0 & 0.33 \\
\hline
\end{tabular}




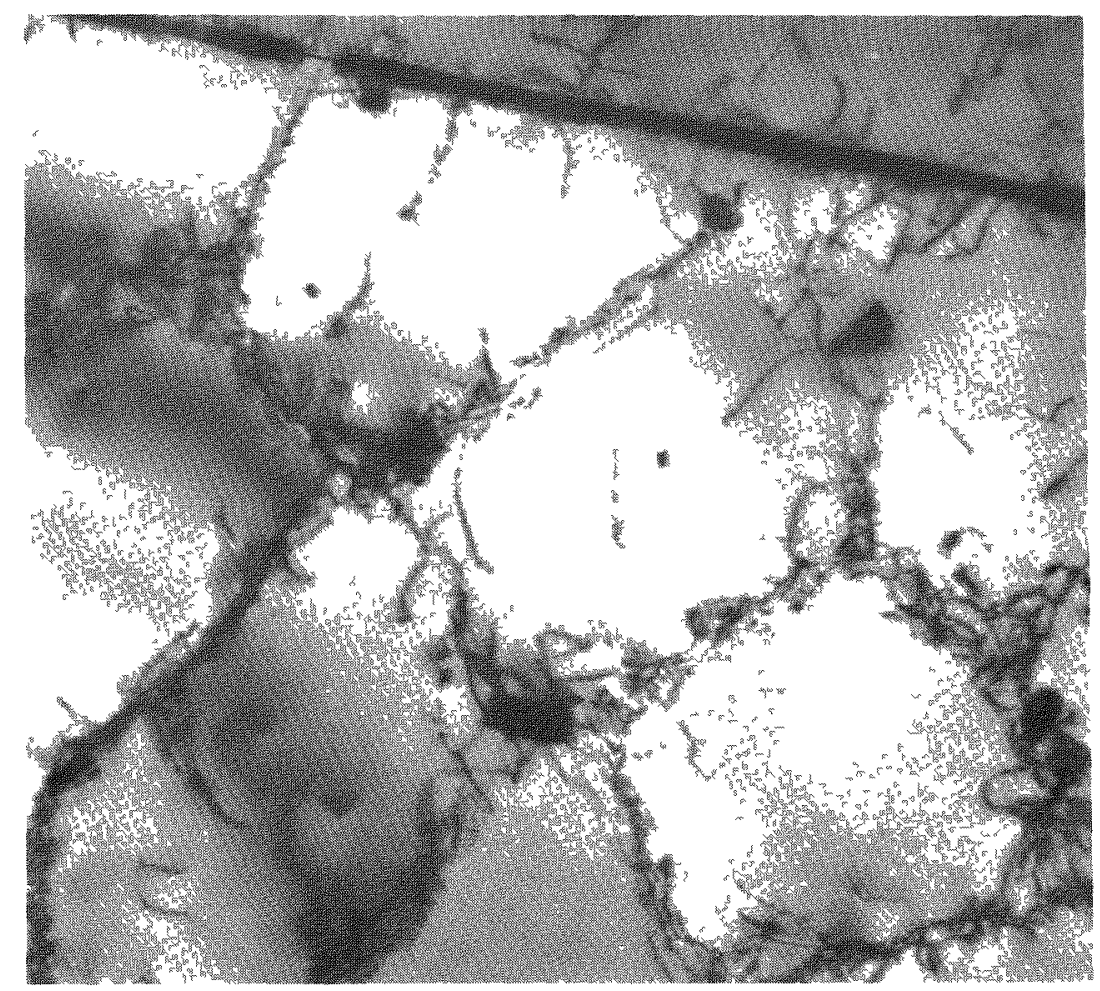

$6-71-233$

$30,000 x$

(a) Unirradiated Control

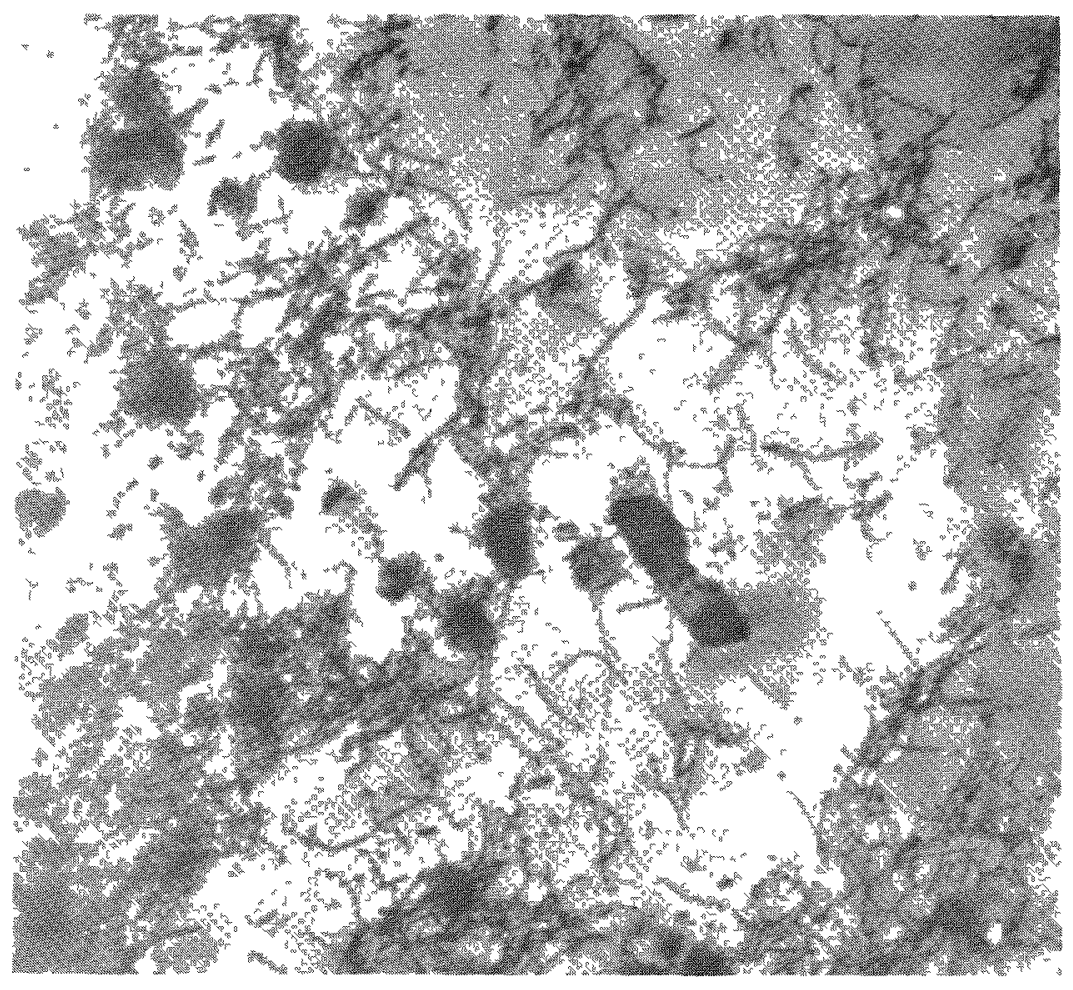

$6-71-267$

$30,000 x$

(b) Irradiated at $700-750^{\circ} \mathrm{C}$ to

$2.4 \times 10^{22} \mathrm{n} / \mathrm{cm}^{2}(E>0.1 \mathrm{meV})$

Figure 1 - Micrographs showing difference in dislocation cell formation between unirradiated (a) and irradiated (b) 316 SS fatigue specimens. Both specimens were tested at $2.4 \%$ total strain range at $704^{\circ} \mathrm{C}$. 


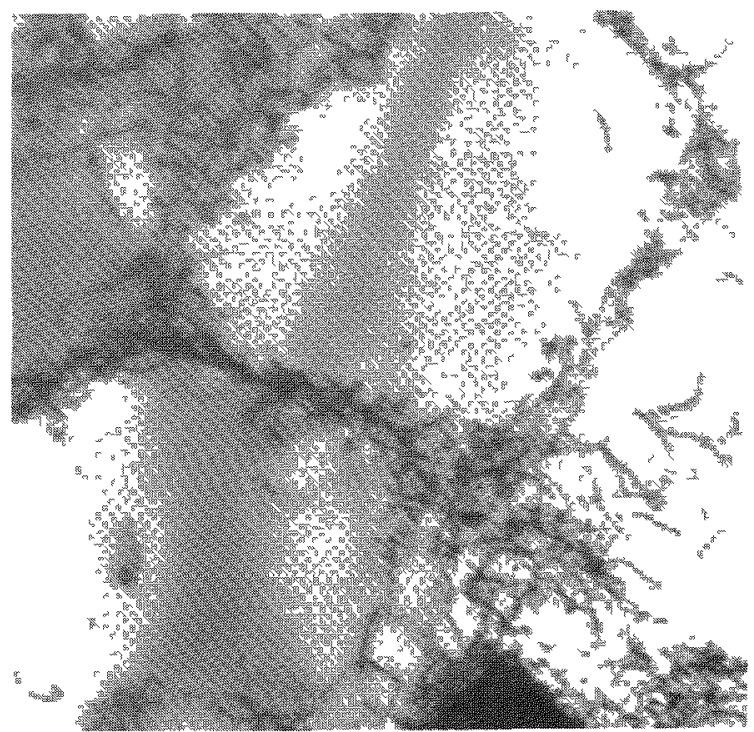

$5-71-140$

$25,600 x$

(a) Unirradiated Control

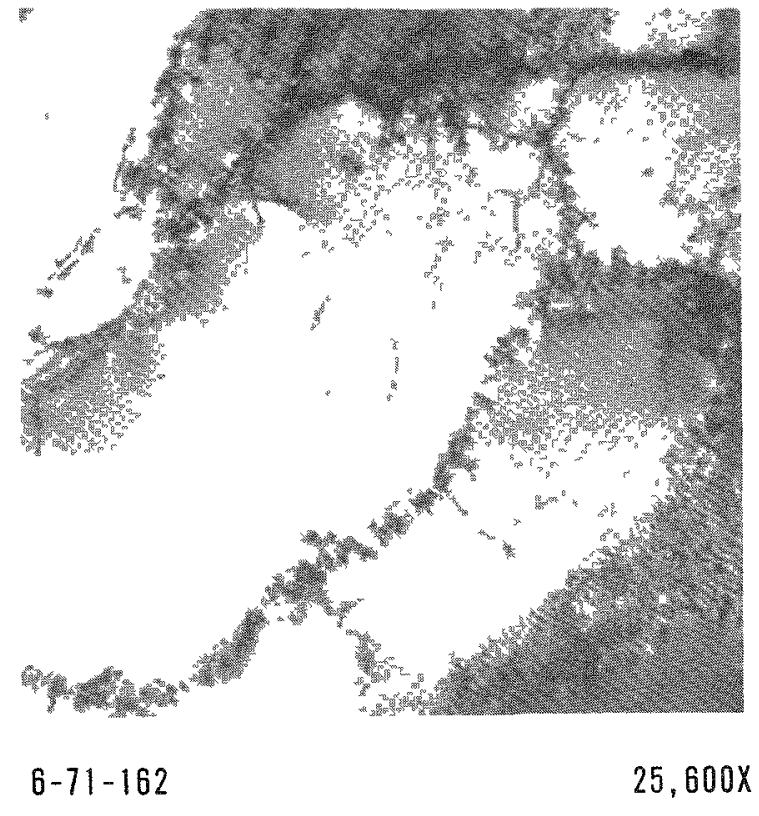

(c) Unirradiated Control

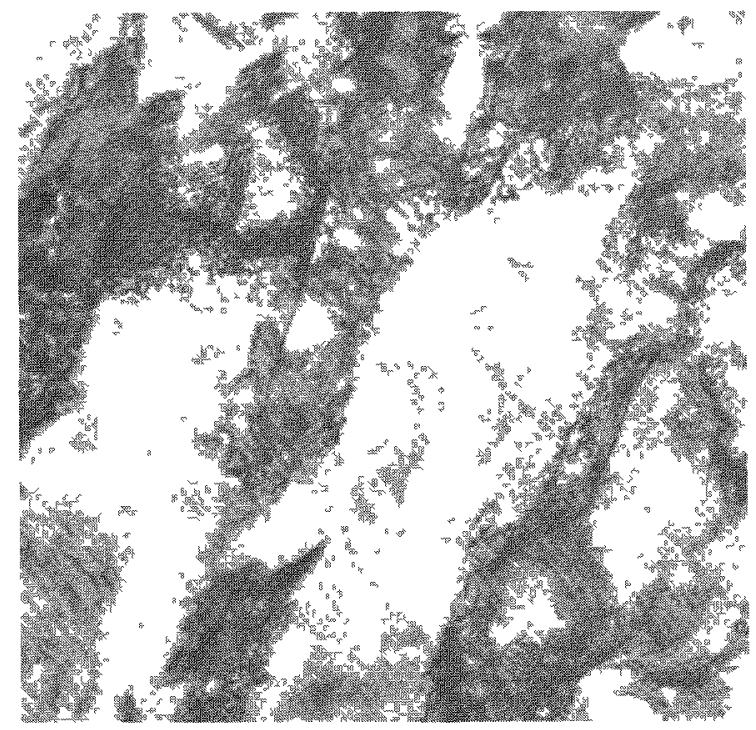

$3-71-32$

$25,600 x$

(b) Irradiated at $700-750^{\circ} \mathrm{C}$ to $3.0 \times 10^{22} \mathrm{n} / \mathrm{cm}^{2}(E>0.1 \mathrm{MeV})$

304

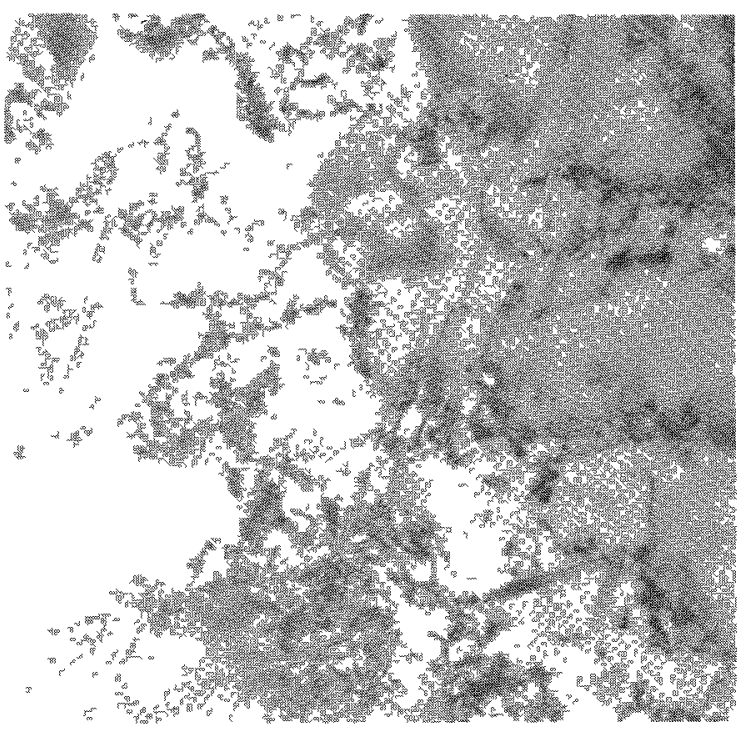

$6-71-249$

$25,600 x$

(d) Irradiated at $700-750^{\circ} \mathrm{C}$ to $2.0 \times 10^{22 \mathrm{n} / \mathrm{cm}^{2}}(\mathrm{E}>0.1 \mathrm{MeV})$

$304(\mathrm{TI})$

Figure 2 - Micrographs showing effect of irradiation retarding dislocation cell formation of 304 SS (a) and (b) tested at a total strain range of $0.35 \%$ and $304 \mathrm{~L}$ ( $\mathrm{Ti})$ SS (c) and (d) tested at a total strain range of $0.36 \%$ 


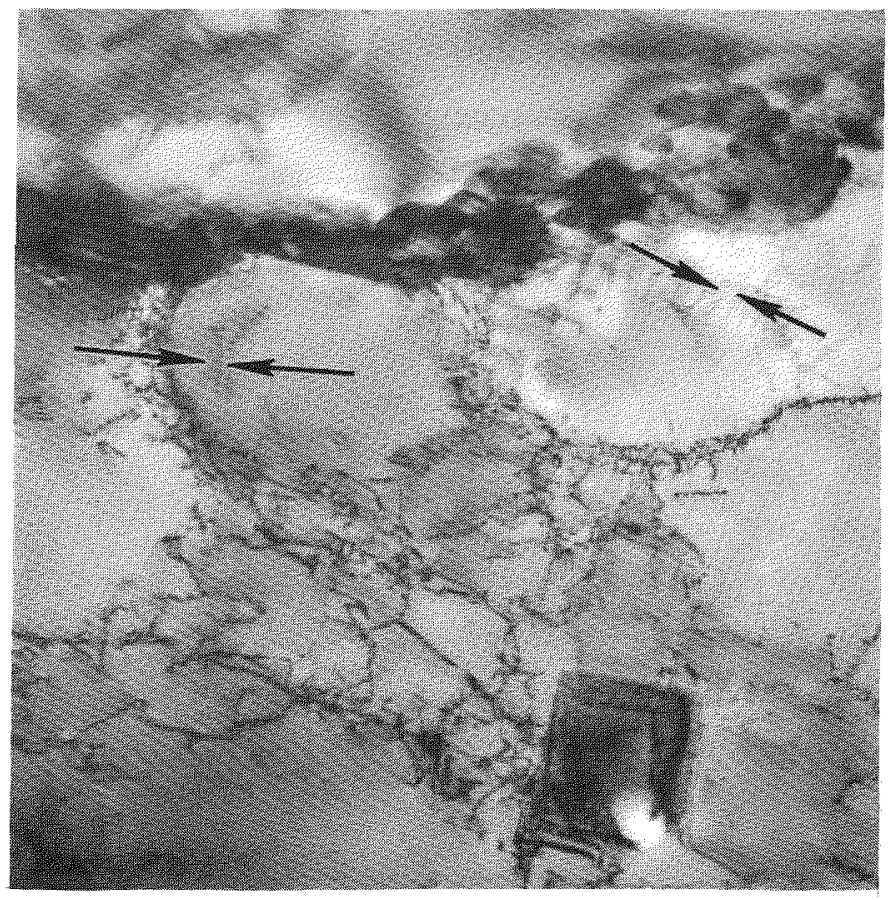

$5-71-143$

$30,000 x$

(a) Unirradiated Control

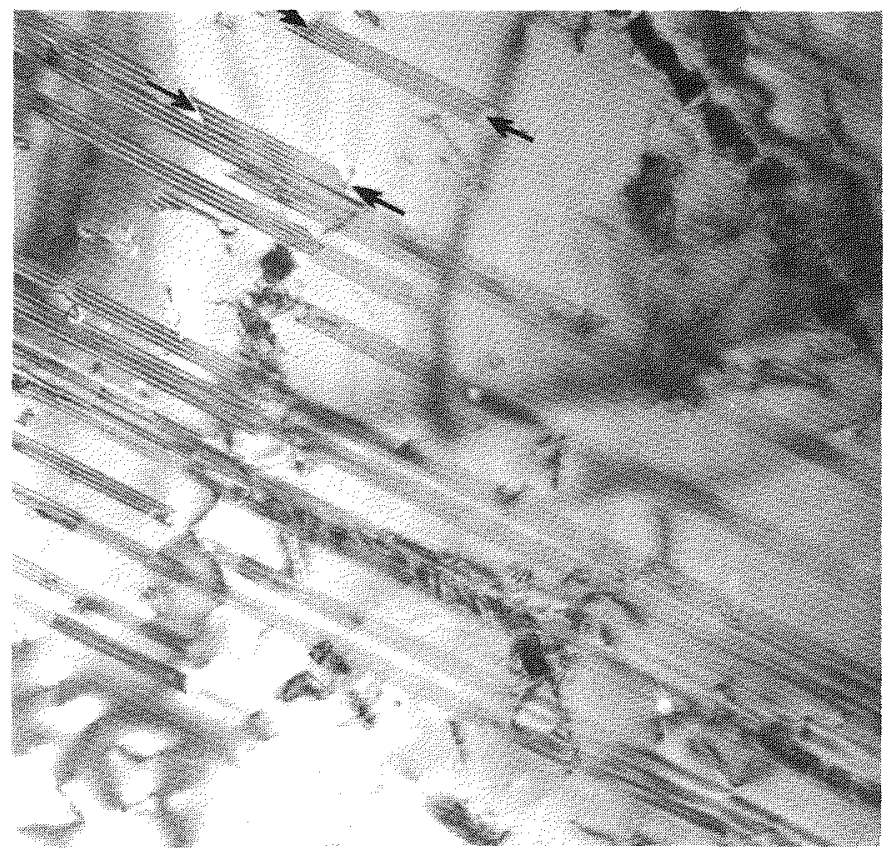

$3-71-26$

$30,000 x$

(b) Irradiated at $700-750^{\circ} \mathrm{C}$ to

$3.0 \times 10^{22} \mathrm{n} / \mathrm{cm}^{2}(E>0.1 \mathrm{MeV})$

Figure 3 - Micrographs illustrating the difference in distance between extended dislocations between unirradiated (a) and irradiated (b) 304 SS fatigue specimens tested at $0.35 \%$ total strain range. 


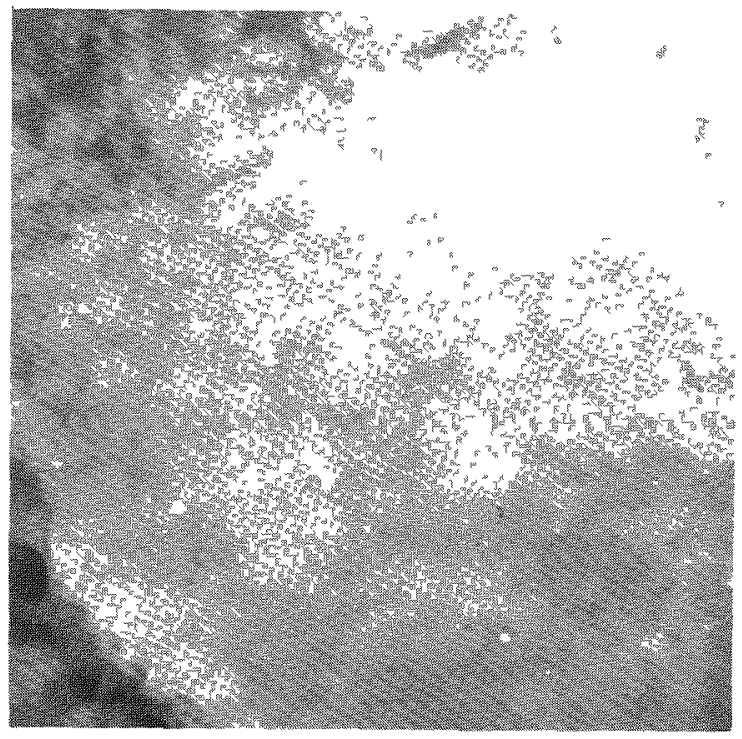

$15,000 x$

$7-71-295$

(a) $3045 S \Phi=1.5 \times 10^{22} \mathrm{n} / \mathrm{cm}^{2}$

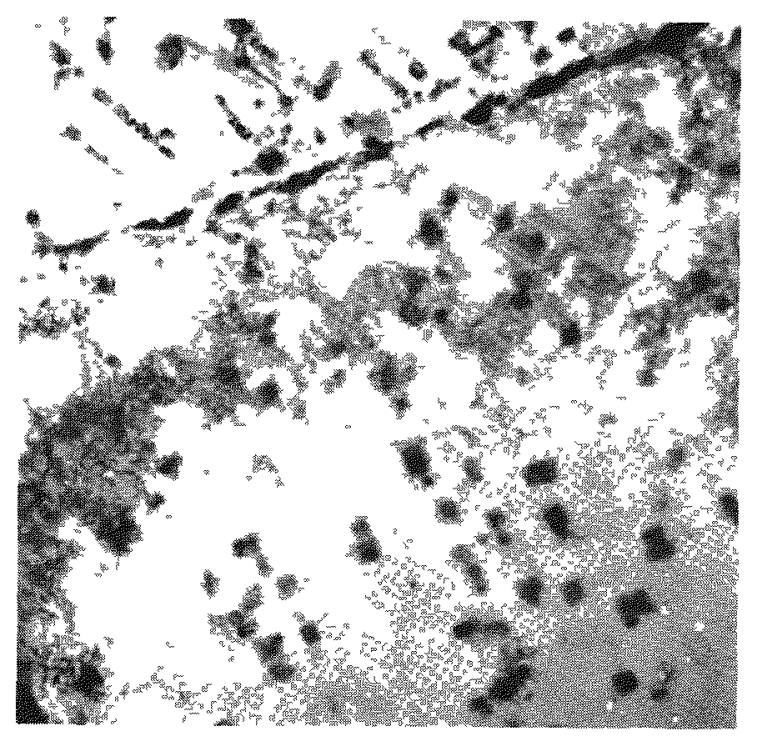

$15,000 x$

$6-71-279$

(c) $316 \mathrm{Ss} \Phi=2.4 \times 10^{22} \mathrm{n} / \mathrm{cm}^{2}$

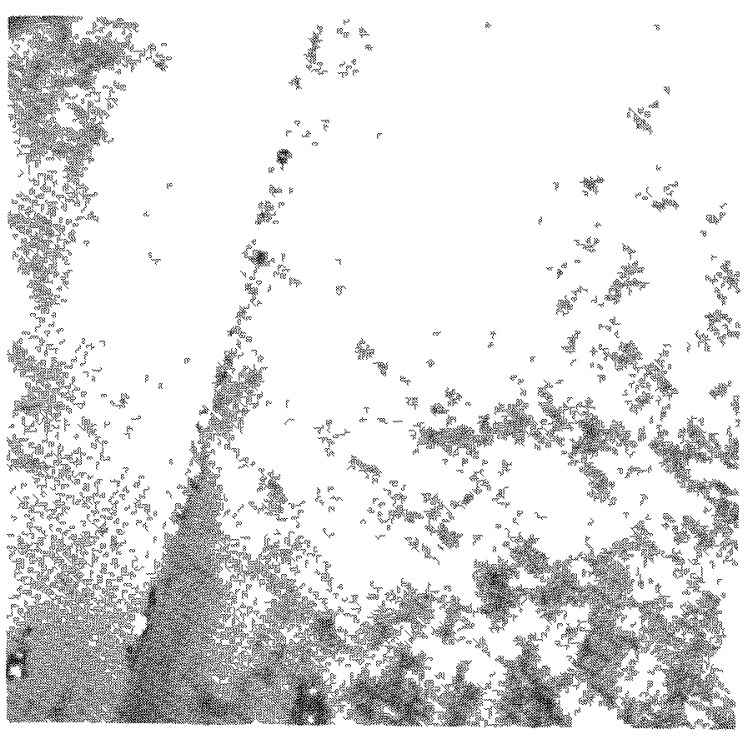

$15,000 x$

$6-71-253$

(b) $304 \mathrm{~L}$ (TI) SS $\Phi=2.0 \times 10^{22} \mathrm{n} / \mathrm{cm}^{2}$

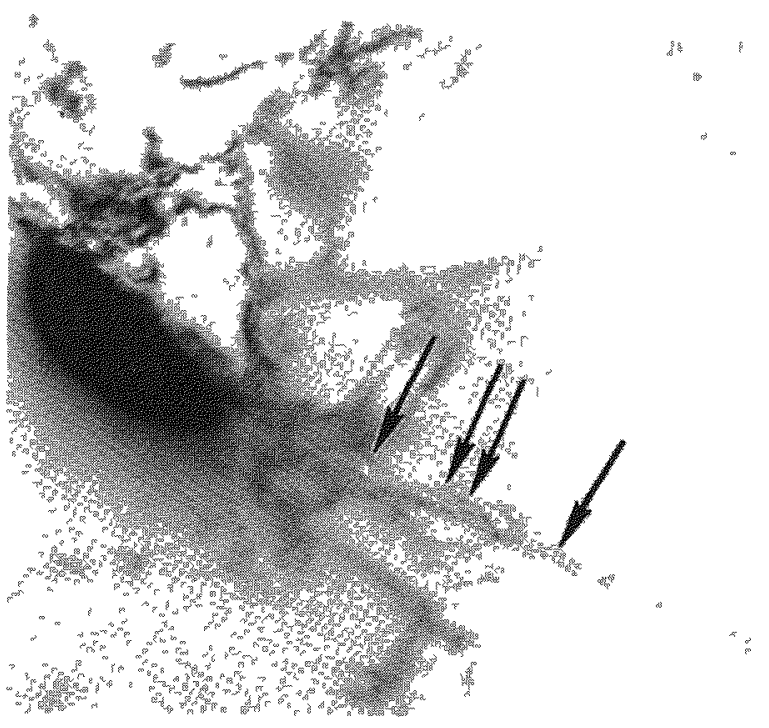

$30,000 x$

$4-71-94$

(d) Incoloy $800 \Phi=3.4 \times 10^{22} \mathrm{n} / \mathrm{cm}^{2}$

Figure 4 - Micrographs showing distribution of voids and/or hellum bubbles in 304 SS (a), $304 \mathrm{~L}$ (Ti) SS (b), $316 \mathrm{SS}$ (c), and Incoloy 800 (d). All specimens irradiated at $700-750^{\circ} \mathrm{C}$ to fluences shown ( $\mathrm{E}>0.1 \mathrm{MeV}$ ) 


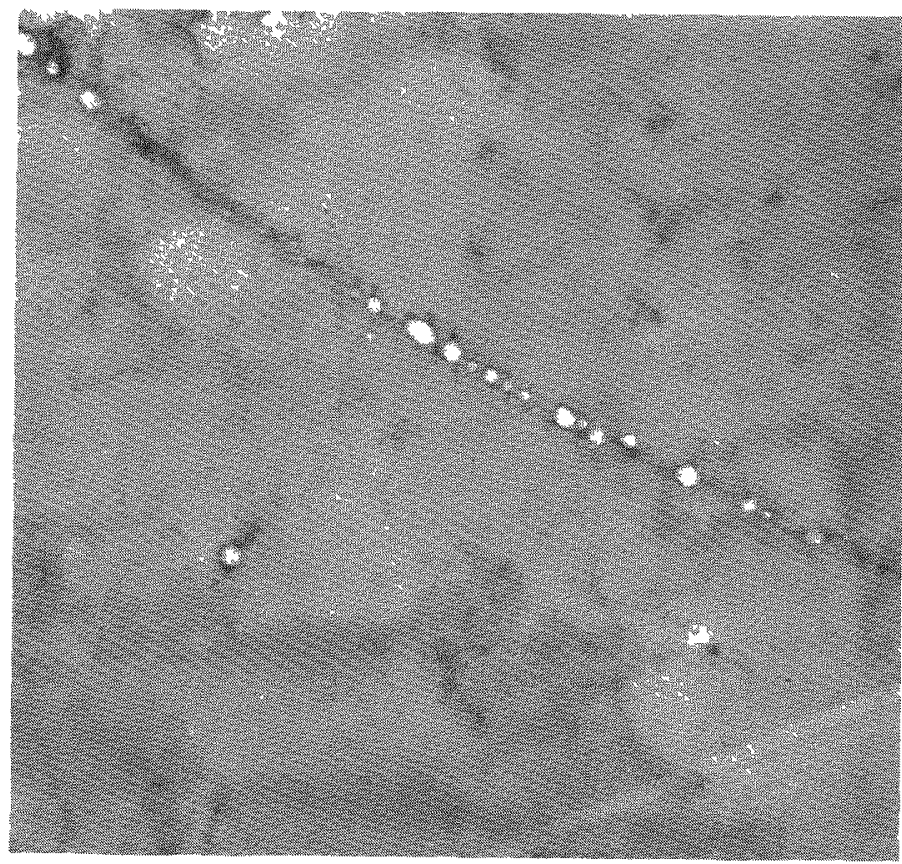

$6-71-295$

$30,000 x$

(a) $\Phi=2.0 \times 10^{22} \mathrm{n} / \mathrm{cm}^{2}$

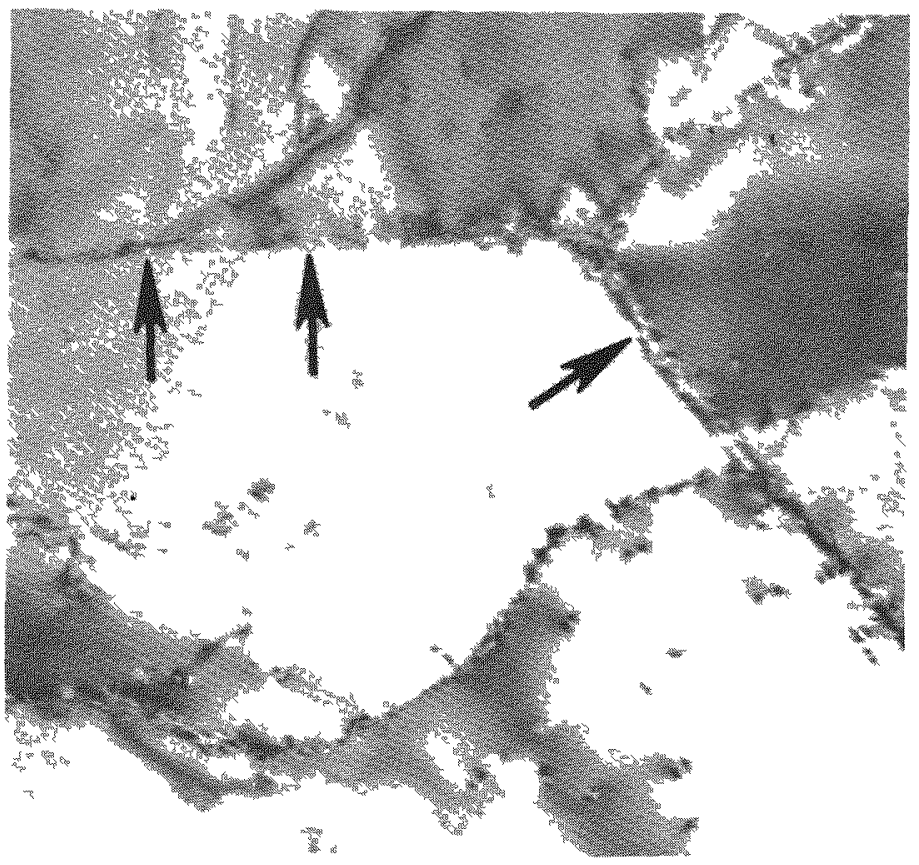

$4-71-95$

(b) $\Phi=3.4 \times 10^{22} \mathrm{n} / \mathrm{cm}^{2}$

$30,000 x$

Figure 5 - Micrographs showing strings of volds probably left behind from moving grain boundary (a) in $304 \mathrm{~L}(\mathrm{Tl}$ ) and bubbles occur at major grain boundaries in incoloy 800 (b). Both specimens irradiated at $700-750^{\circ} \mathrm{C}$ at fluences shown ( $E>0.1 \mathrm{MeV}$ ). 
ELEVATED TEMPERATURE FATIGUE

\author{
C. R. Brinkman, G. E. Korth, J. M. Beeston
}

The utilization of materials in the Liquid Metal Fast Breeder Reactor (LMFBR) Program poses many new problems. To overcome some of these problems, Aerojet Nuclear Company is currently conducting elevated temperature fatigue tests on a number of reactor cladding and structural materials including stainless types 304, 316, 304L(Ti mod.), and Incoloy 800 . These materials are prime candidates for usage as cladding and structural materials in the LMFBR Program.

The utilization of materials is limited because of a number of important factors: (1) High operating temperatures such that structural components will be exposed to temperatures in the range of 425 to $650^{\circ} \mathrm{C}$, while fuel clad may reach spot temperatures of 650 to $750^{\circ} \mathrm{C}$. (2) Prolonged exposure $(20-40$ years) under load at these temperatures may mean that both creep as well as material changes (aging effects) are possibilities. (3) Irradiation induced changes in material mechanical properties can occur. (4) Severe thermal induced variable elastic and inelastic load histories will occur during service lifetime necessitating consideration of elevated temperature fatigue behavior in design.

This test program has the following objectives:

(1) To determine the effects of cyclic and time-dependent loading on the lifetime of irradiated and unirradiated LMFBR cladding and structural materials in the temperature range of $425-700^{\circ} \mathrm{C}$. (2) To establish relationships for predicting fatigue, creep-fatigue interaction, and cumulative damage behavior over times, temperatures, and stress-strain levels appropriate to reactor design. (3) To characterize changes in substructure of these materials resulting from the combined effects of irradiation defects, time at temperature (aging), and fatigue or creep induced plasticity. Microscopic changes in surface topography as seen on the fatigue fractured surfaces is also important and is being investigated. (4) To determine the influence of irradiation and time at temperature on the fatigue behavior of prototypical heavy section stainless weldments.

Progress made towards accomplishment of the above objectives will be discussed in the following sections.

Elevated Temperature Tensile and Fatigue Test Results. During the past year the fatigue test facility (two test frames) was modified in several important ways: (1) An axial strain computer was built and installed to permit test control of either axial or diametral strain range. (2) A constant strain rate tensile testing function generator was added to the system to permit constant strain-rate to failure tensile testing of hour glass-shaped fatigue specimens at elevated temperatures.

(3) Hold-time function 
generators were installed to permit introduction of either tensile or compressive dwell periods into the otherwise triangular straintime wave form.

Examples of the tensile data obtained from the constant strain rate tensile tests are shown in Figures 1 and 2. Engineering stress-strain curves obtained from these tests are compared in Figure 1 for several stainless steels tested at $700^{\circ} \mathrm{C}$. The tensile properties show the characteristic increase in yield strength and decrease in non-uniform elongation (ductility) normally found in stainless steels irradiated to the indicated fluences at elevated temperatures. Alternately, because diametral measurements were made during the test, plots of true stress-strain can be made as shown in Figure 2. Figure 2 shows that irradiation results in a decrease in the strain hardening exponent $(m)$ and a substantial decrease in the instability strain.

Fatigue data obtained at $700^{\circ} \mathrm{C}$ is plotted as total strain range, $\Delta \varepsilon_{t}$ versus cycles to failure in Figures 3 to 5 . Fatigue life is generally ductility dependent in the low cycle range (plastic strain range dominant) and strength dependent in the high cycle range (elasfic strain range dominant). Several semiempirical expressions $[1,2,3]$ have been developed relating tensile properties to fatigue life. Two of these expressions are as follows:

Universal Slopes Equation:

$$
\Delta \varepsilon_{t}=\frac{3.5 \sigma}{\mathrm{Ets}} \mathrm{N}_{\mathrm{f}}^{-0.12}+\mathrm{D}^{0.6} \mathrm{~N}^{0.6}
$$

Characteristic Slopes Equation:

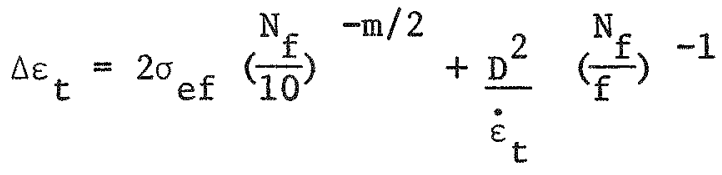

$$
\begin{aligned}
& \text { where } \dot{\varepsilon}_{t}=2 \mathrm{f} \Delta \varepsilon_{t} \\
& \Delta \varepsilon_{t}=\text { total strain range } \\
& \sigma_{\text {uts }}=\text { ultimate strength } \\
& E=\text { Young 's modulus } \\
& D=\text { fracture ductility }=\ln 100 / 100-\mathrm{R} \cdot \mathrm{A}(\%) \\
& \mathrm{N}_{\mathrm{f}}=\text { cycles to failure } \\
& \sigma_{\text {ef }}=\text { elastic strain at fracture }=\text { true fracture stress divided }
\end{aligned}
$$




$$
f=\text { cyclic frequency. }
$$

Predictions of fatigue life based on the above equations, using tensile data, are compared with the experimentally determined fatigue behavior in Figures 3, 4, and 5. For the stainless steels the universal slopes equation (upper bound) gives a better estimate of the expected fatigue life than the equation of characteristic slopes. Both equations reflect the ductility decreases induced by irradiation damage.

In Figure 6, comparisons between irradiated fatigue behavior of the stainless steels and the unirradiated controls are made. Type $304 \mathrm{~L}$ with the slightly higher pre- post-irradiation ductility was found to have the superior fatigue life at $700^{\circ} \mathrm{C}$, while from the limited data, no difference in the fatigue life of types 304 or 316 is evident. Similar results [4] were found in comparing the fatigue behavior of types 304 and $304 \mathrm{~L}$ irradiated to fluences of $2-5 \times 10^{21} \mathrm{n} / \mathrm{cm}^{2}>0.1 \mathrm{MeV}$ at approximately $750^{\circ} \mathrm{C}$ and tested at $500^{\circ} \mathrm{C}$.

The fatigue behavior of Incoloy 800, Figure 5, unlike the stainless steels, was found to be fluence dependent which accounts for the data scatter shown in Figure 5. Irradiation induced embrittlement in the highly irradiated materials resulted in rather large decreases in fatigue life. Equations (1) and (2) gave rather high values for the unirradiated predicted fatigue lives, however, the universal slopes line shown is an upper bound line. When an average life line is plotted, i.e. $0.2 \mathrm{~N}_{f}$, good agreement is found at the higher strain ranges. For the irradiated material, comparisons of the methods of estimating fatigue lives are complicated by the data scatter (fluence variation). However, in the absence of fatigue data these equations appear to be effective as a first approximation for predicting the fatigue behavior of irradiated materials when reliable tensile data is available. Work will continue in attempting to quantitatively relate tensile and creep properties to the cyclic behavior of irradiated materials.

It was mentioned above that metallurgical reactions which are time and temperature dependent (aging) can occur in austenitic stainless steels. These reactions may be either deleterious or beneficial to the fatigue resistance of the material in service. An example of the latter effect is shown for unirradiated 316 stainless in Figure 7 . The reasons for the increase in fatigue life with aging are not readily apparent and are still being investigated.

Creep-Fatigue Tests. Hold times or frequency effects become progressively more important in influencing fatigue failure as the temperature is increased into the range where creep processes begin to occur. Reactor components in service will not be subject to simple triangular strain-time wave forms; but will 
see prolonged periods of either constant stress or strain between cycles. In order to study the effects of these hold times, strain controlled tests with tensile hold times are employed. During periods of constant strain the stress relaxes in the characteristic form shown in Figure 8. An increase in the plastic strain range occurs at the expense of the elastic strain range. Figure 8 also shows actual hysteresis loops for strain controlled tests and the effects of cyclic hardening and macrocrack growth on the shape of the loop. Creep damage also results in other subtle changes in that voids or cavities are formed at the large angle grain boundaries. These voids weaken the grain boundaries and result in a change in the mode of fatigue crack propagation as shown in Figure 9. Figure 9 indicates that when creep damage is allowed to occur a definite "path of least resistance" occurs at the large angle grain boundaries. Additional information concerning this transition in mode of crack growth can be obtained by techniques of scanning electron microscopy (SEM). SEM photomicrographs of specimen surfaces are shown in Figure 10. Figure 10 also shows fatigue striations which can be used to locate the origin of crack initiation on the specimen surface as well as to determine the number of cycles required for crack initiation.

A number of fully reversed strain controlled fatigue tests containing tensile only hold times ranging from .01 to 1.0 hour have been conducted on 316 stainless specimens irradiated to fluences in excess of $10^{22} \mathrm{n} / \mathrm{cm}^{2} \mathrm{E}>.1 \mathrm{MeV}$. The data are compared with fatigue data from tests without hold periods in Figure 11. The data plotted in Figure 11 show that irradiation under the indicated conditions results in a pronounced reduction in cyclic life when a tensile hold period is incorporated as a part of every cycle. While a reduction in cyclic life also occurred for the control specimens the effect is still more marked for the irradiated materials.

The mechanism by which irradiation damage decreases the cyclic life of specimens subjected to hold periods during strain controlled tests is not altogether clear. Creep damage, i.e. wedge or cavitation type void formation at grain boundaries transverse to the applied stress, occurring during tensile hold periods results in increased intergranular cracking, Figures 9-10, with resultant decrease in ductility in the unirradiated as well as the irradiated material, thus decreasing the time required for both fatigue crack initiation and growth. Helium bubbles, resulting from transmutation reactions at grain boundaries, could serve to accelerate formation of wedge or cavitation voids in the irradiated material and stress gradients set-up in the vicinity of these voids or microcracks could further serve as a driving force for bubble migration to the vicinity of these cracks, thus further facilitating the intergranular crack growth process. Work to further elucidate the effects of irradiation on creep-fatigue interaction is continuing. 
Attempts are also being made to formulate cummulative damage relations which will be valid for summing both fatigue (cyclic) and creep (static) damage that occurs over several strain amplitudes. Creep and fatigue damage are presently being separated and summed linearly according to the linear damage law:

Creep damage + Fatigue damage $=\sum_{i}^{z} \frac{t_{i}}{t_{I}}+\sum_{i}^{z} \frac{n_{i}}{n_{f}}=1$

where

$t_{i}=$ time spent at some strain level $i$

$t_{r}=$ time to rupture at strain level i

$\mathrm{n}_{\mathbf{i}}=$ number of cycles in service at strain level $i$

$\mathrm{n}_{\mathrm{f}}=$ number of cycles to failure from a fatigue curve.

Accordingly, a simple computer program was developed to allow calculation of creep damage occurring during elevated temperature hold time tests. The program utilizes an equation which fits the stress relaxation curves and is expressed as follows:

$$
\ln \frac{\sigma_{\max }}{\sigma_{\min }}=\frac{A}{1+m} t^{1+m}
$$

where $\sigma_{\max }$ is the peak stress at the start of a hold period and $\sigma_{\mathrm{min}}$ is the melaxed stress at the end of the hold time $t, A$ and $m$ are $m$ constants. Values of the constants $K$ and $n$ from the stress rupture equation

$$
t_{r}=(K / \sigma)^{n}
$$

obtained from the literature are used along with values of $\sigma_{\max }$ and $\sigma_{\text {min }}$ in the program and the results are a plot of the stress relaxation equation (3) along with the creep damage $t / t_{r}$ that occurred during the hold time cycle. An example of the creep-fatigue damage analysis presently being conducted on these materials is given in Figure 12. Figure 12 shows a number of stress relaxation plots obtained during various tensile hold periods of 0.01 hour in duration. The specimen of type 304 stainless was fatigue tested at $538^{\circ} \mathrm{C}$ at a strain range of $0.5 \%$ and required 3089 cycles for failure. In the stress versus hold time plots the specimen strain hardened for the first few cycles (10 to 20) after which the relaxation curve was fairly consistent from one cycle to the next. Creep damage calculations were done via the computer program on cycle 1600 and the creep damage for this cycle was found to be $3.24 \times 10^{-6}$. Assuming that the relaxation cycles are repetitious from one cycle to the next during most of the fatigue life, the total damage, i.e. $\Sigma t / t r$, accumulated throughout the test is found by multiplying the creep damage per cycle 
by the total cycles to failure. The total fatigue damage is simply the number of cycles to failure with hold $(N)$ divided by the cycles to failure without a hold time $\left(\mathrm{N}_{f}\right)$ for a given temperature and strain range.

[1] G. R. Halford and S. S. Manson, Applications of a Method of Estimating High Temperature Low Cycle Fatigue Behavior of Materials, NASA TMX-52357.

[2] J. B. Conway, Evaluation of Plastic Fatigue Properties of Heat-Resistant Alloys, GEMP-740.

[3] L. F. Coffin, A Generalized Equation for Predicting HighTemperature, Low Cycle Fatigue, Including Hold Times, GE R\&D Center, Schenectady, New York, Report 69C 401 (1970).

[4] J. M. Beeston and C. R. Brinkman, "Axial Fatigue of Irradiated Stainless Steels Tested at Elevated Temperatures," ASTM STP-484 pp. 419-450 (1970). 


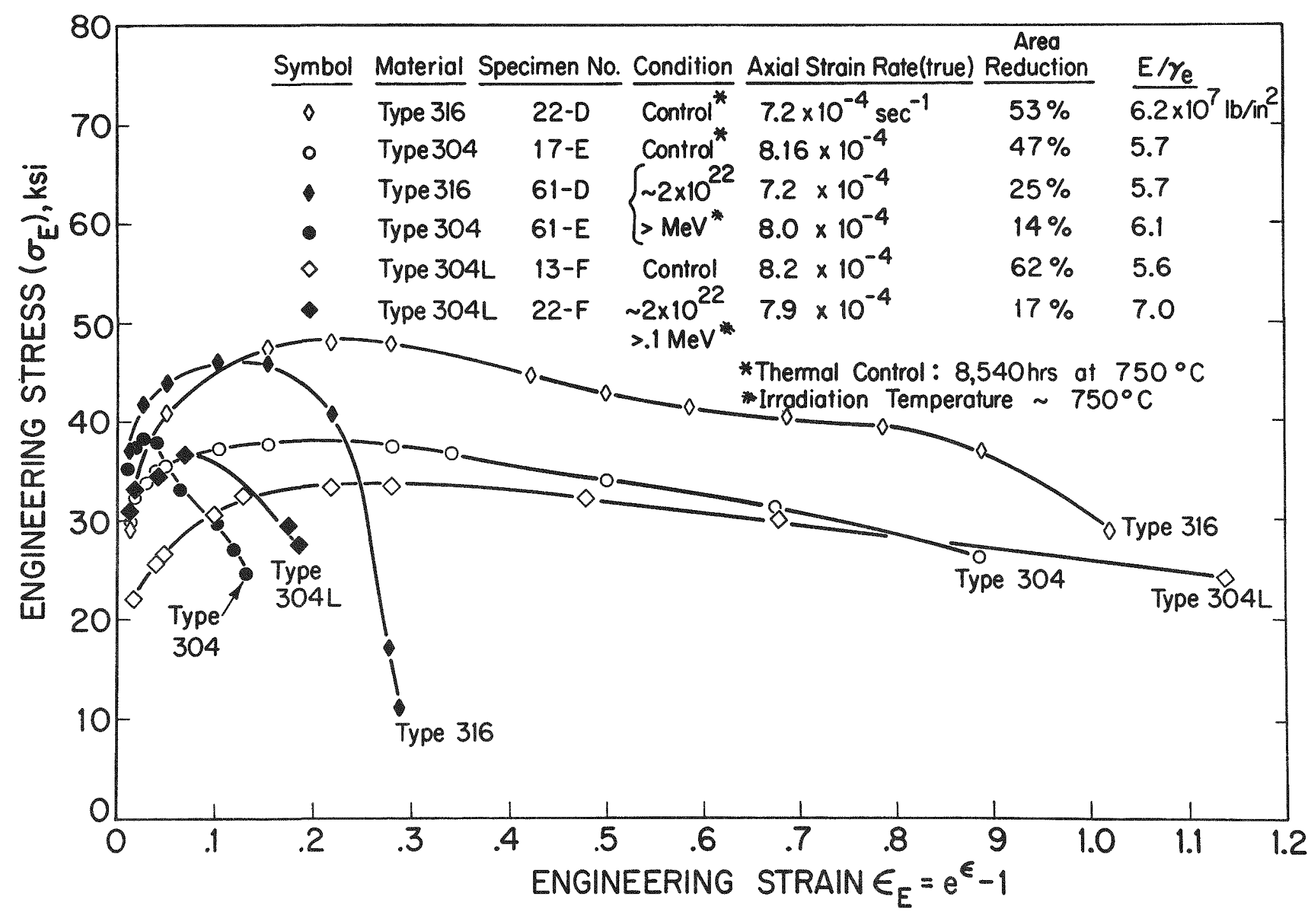

Fig. 1 Engineering stress-strain behavior of irradiated and unirradiated stainless steel obtained from constant strain rate testing of hourglass shaped specimens at $700^{\circ} \mathrm{C}$. 


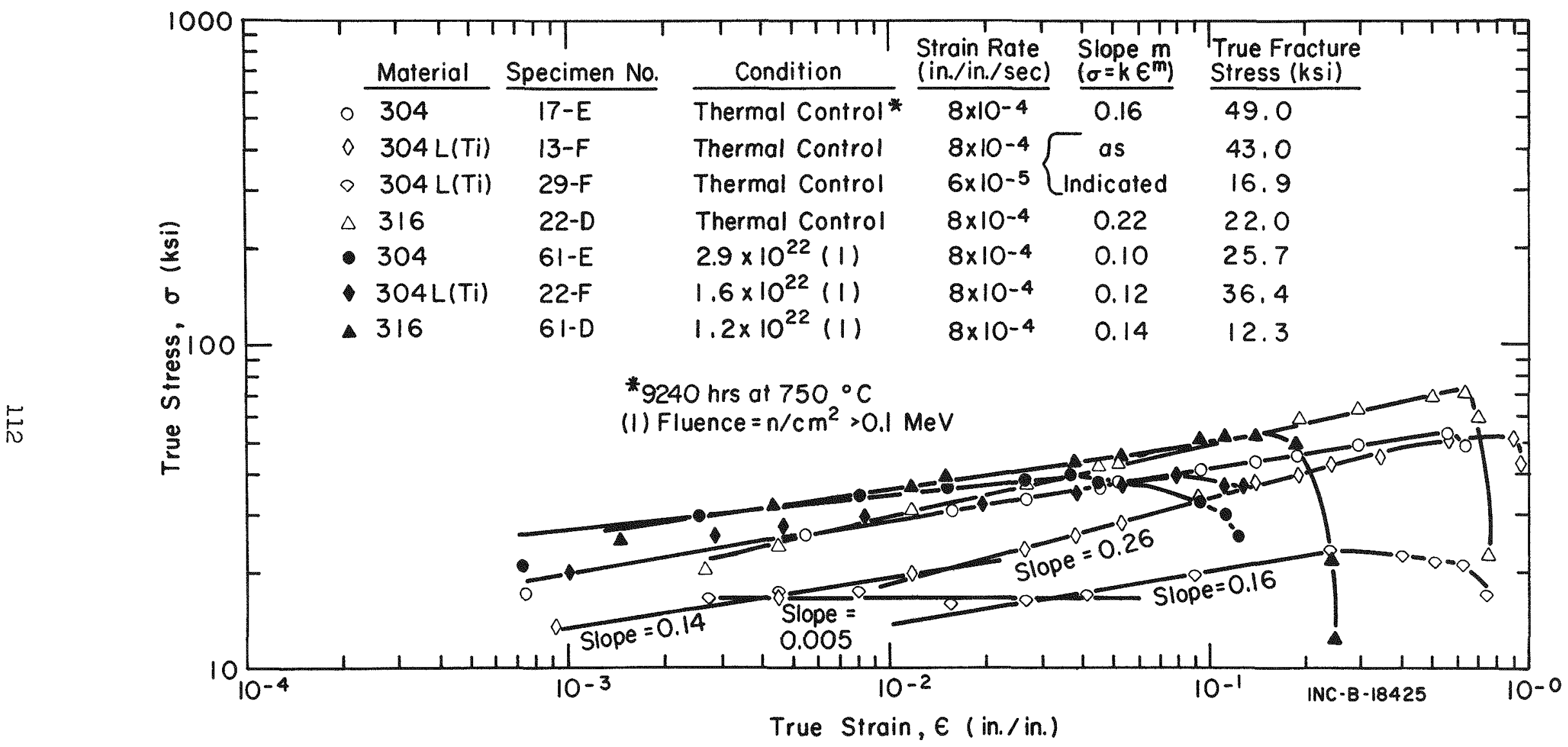

Fig. 2 True stress-strain curves for irradiated and unirradiäted stainless steels tested at $700^{\circ} \mathrm{C}$. 


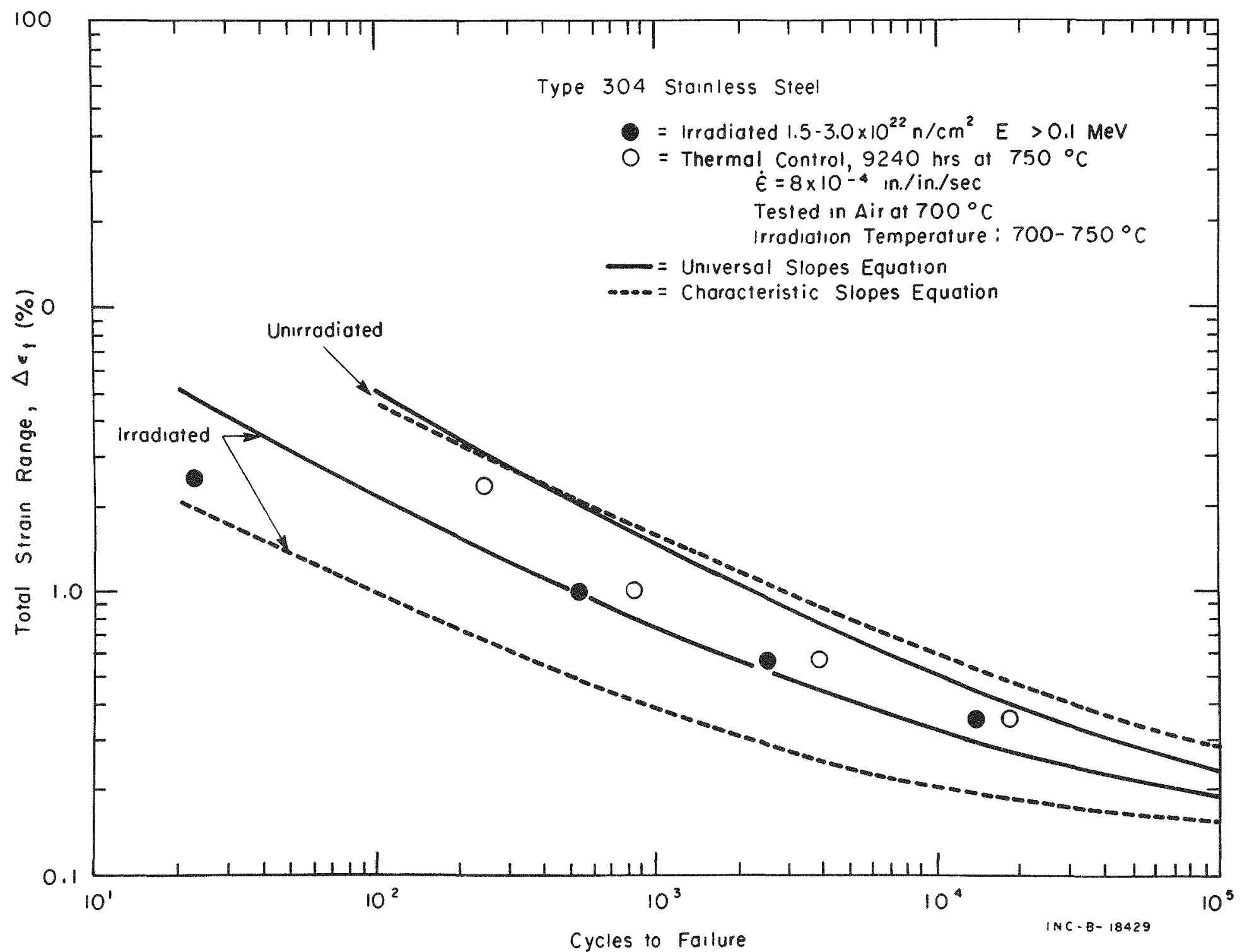

Fig. 3 Irradiated and unirradiated fatigue behavior of Type 304 stainless steel tested at $700^{\circ} \mathrm{C}$ compared with predictions based on tensile data. 


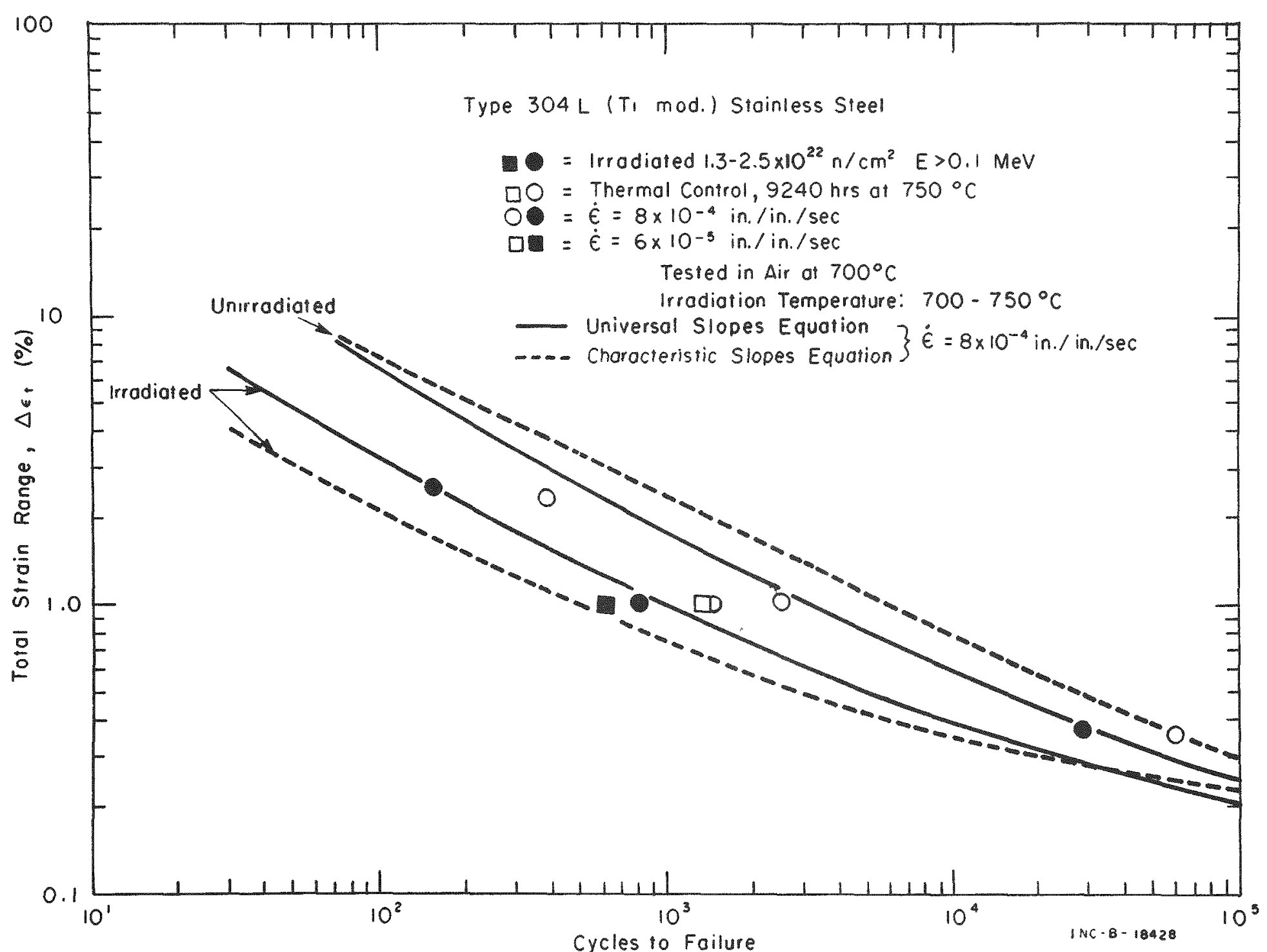

Fig. 4 Irradiated and unirradiated fatigue behavior of Type $304 \mathrm{~L}$ (Ti mod.) stainless stee1 tested at $700^{\circ} \mathrm{C}$ compared with predictions based on tensile data. 


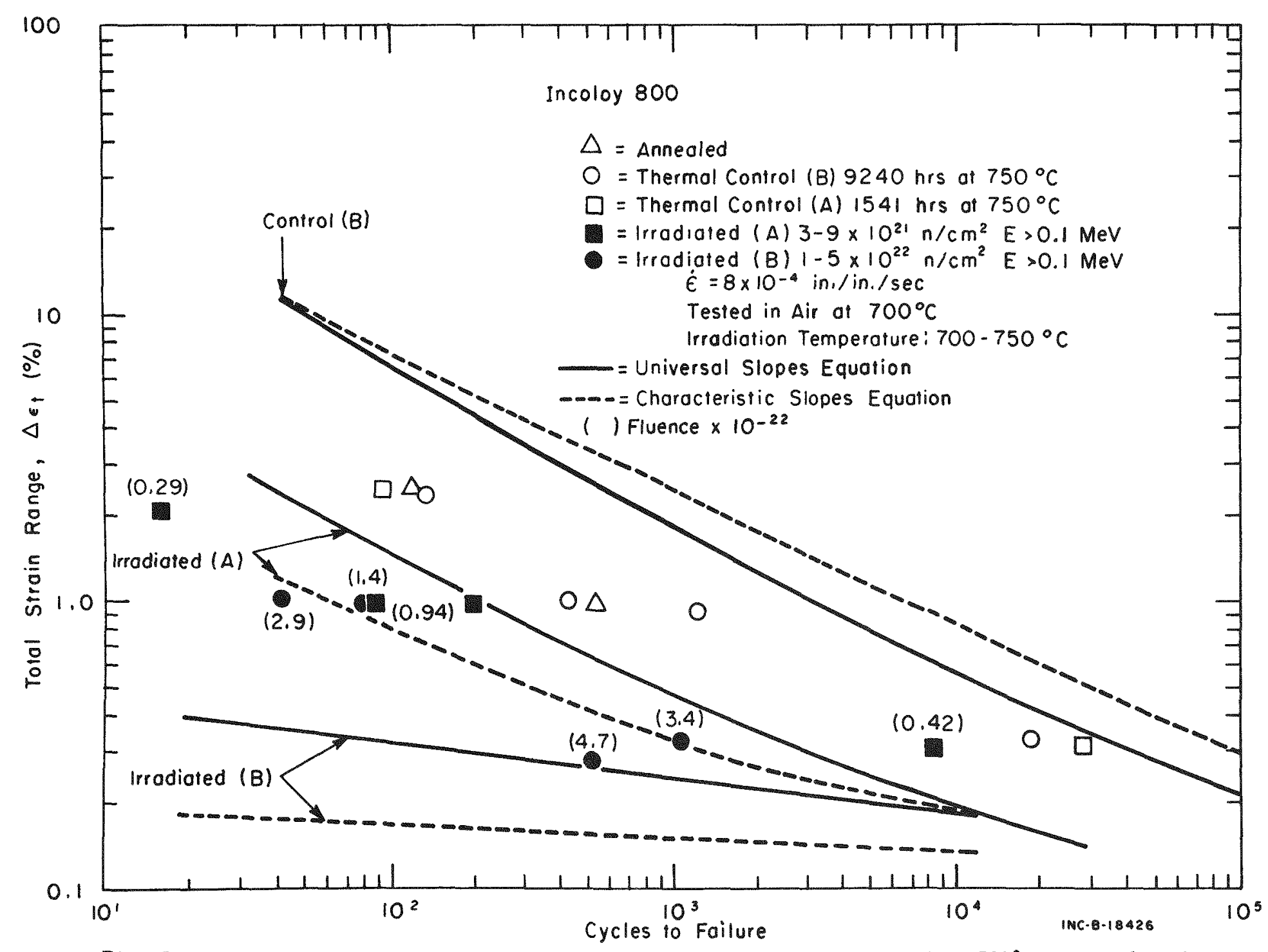

Fig. 5 Irradiated and unirradiated fatigue behavior of Incoloy 800 tested at $700^{\circ} \mathrm{C}$ compared with predictions based on tensile data. 


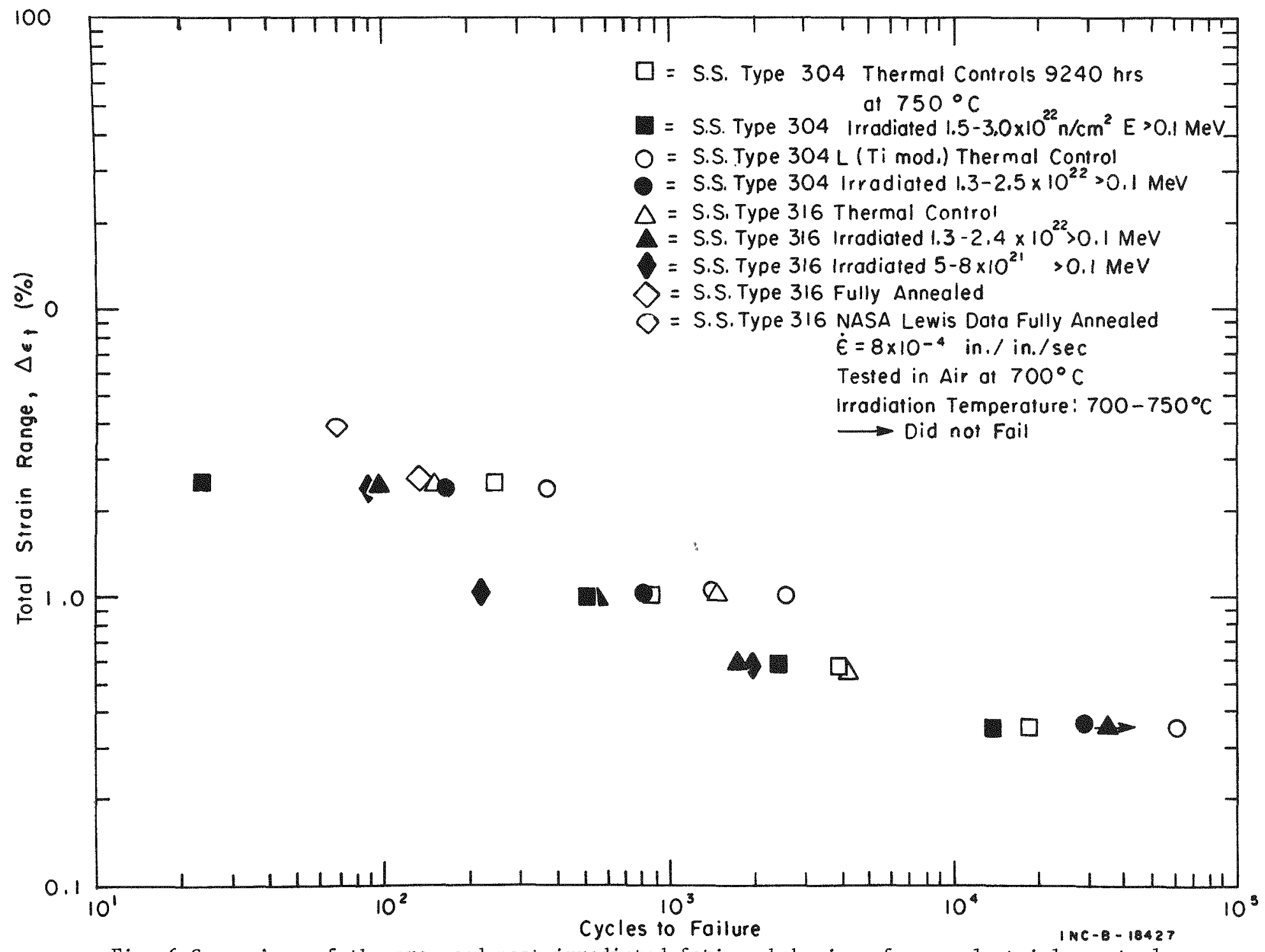

Fig. 6 Comparison of the pre- and post-irradiated fatigue behavior of several stainless steels tested at $700^{\circ} \mathrm{C}$. 


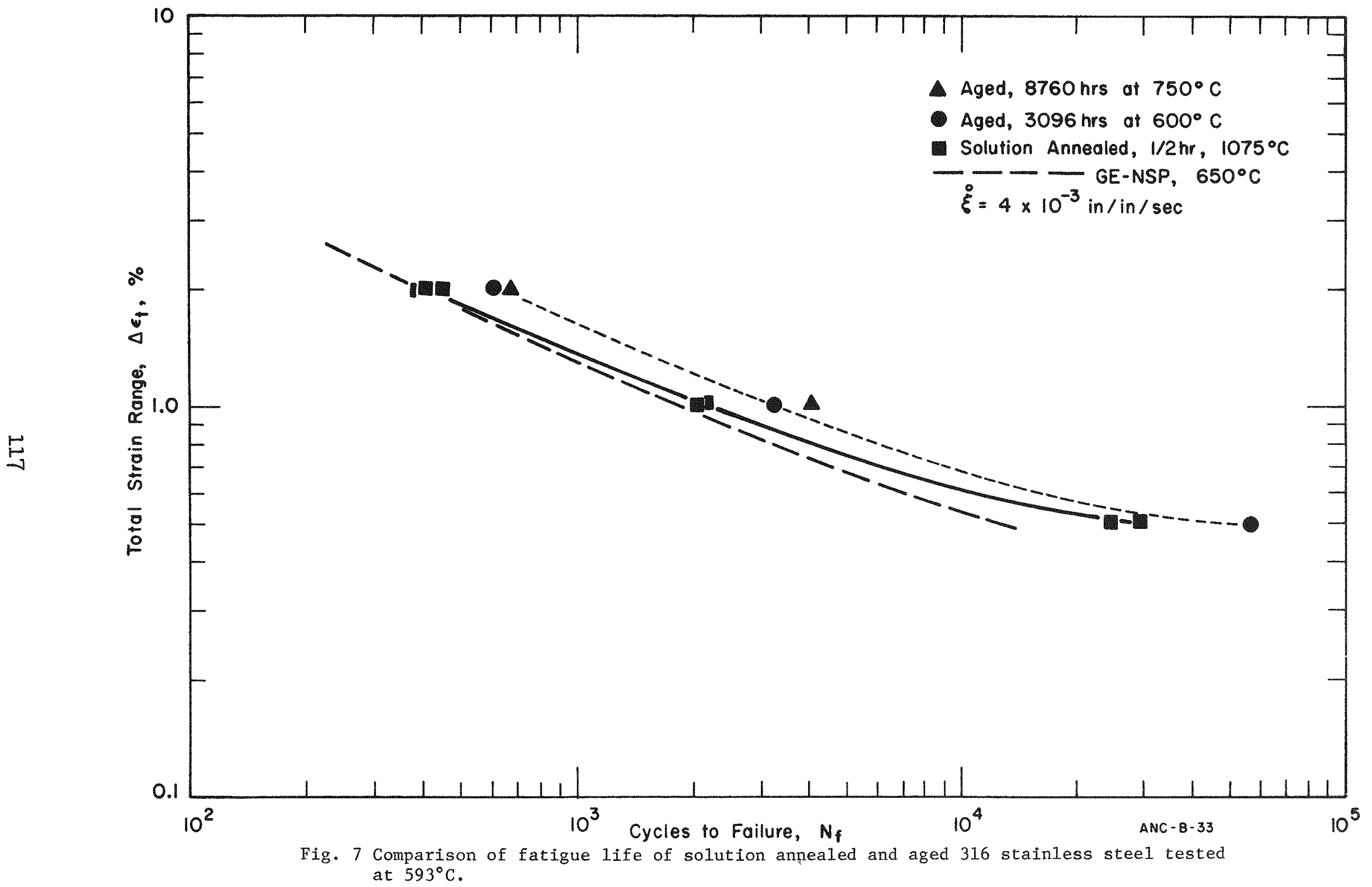



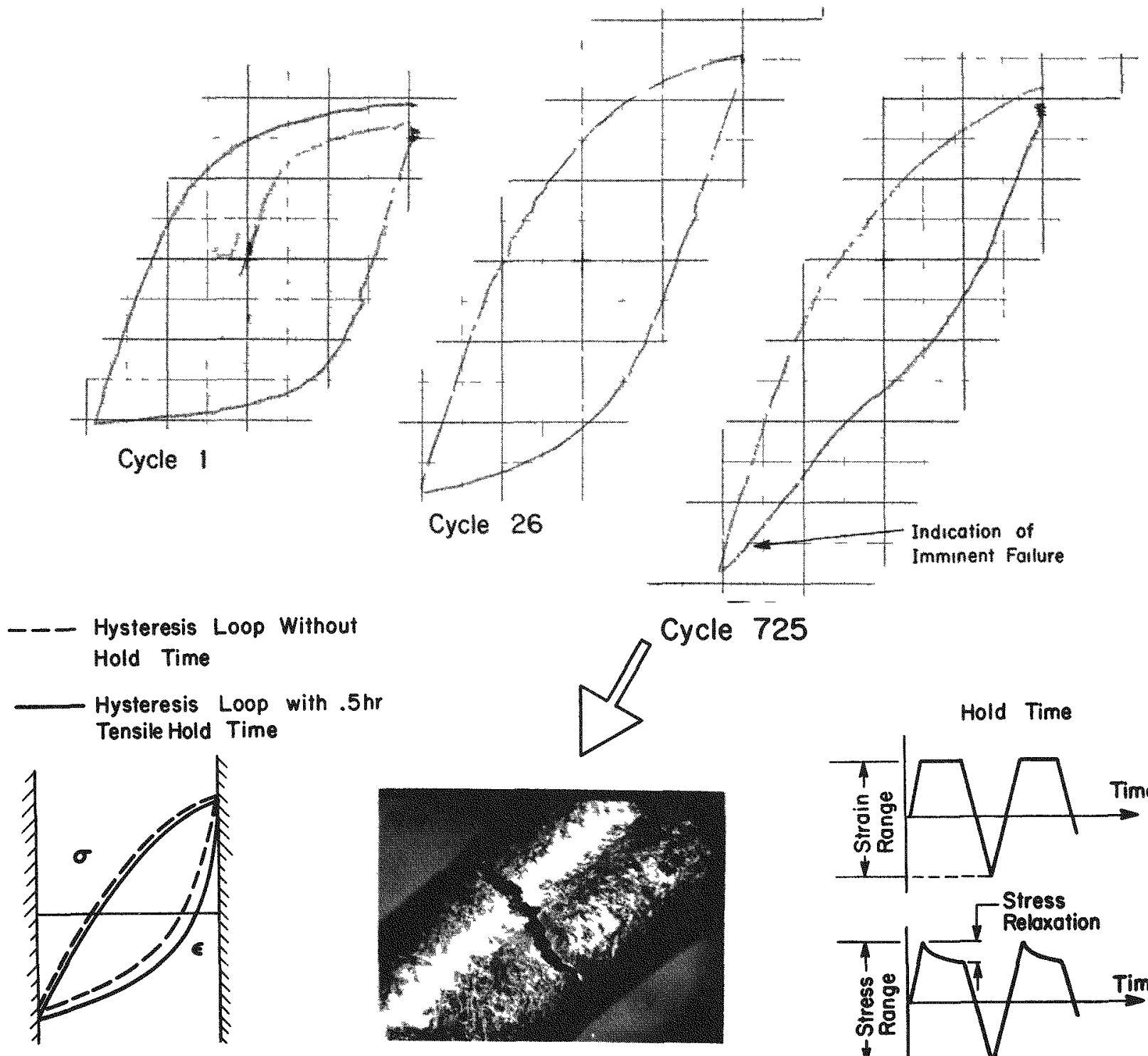

Hysteresis

Hysteresis Loop with .5 hr

Test Stopped Prior to Failure
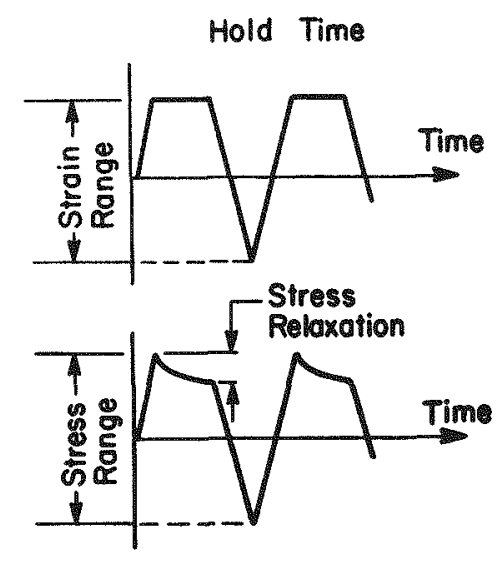

Fig. 8 Cyclic hold time testing of unirradiated stainless steel at $1100^{\circ} \mathrm{F} \cdot \Delta \varepsilon_{t}=.5 \%$ 


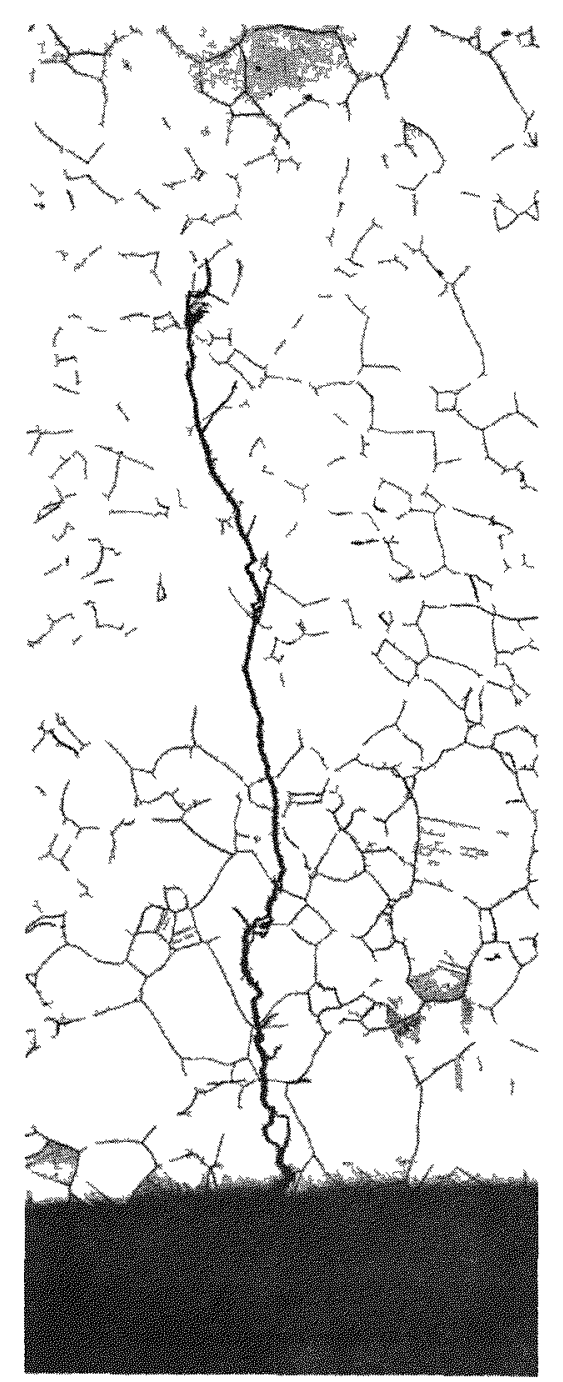

a. Without Tensile Hold Time

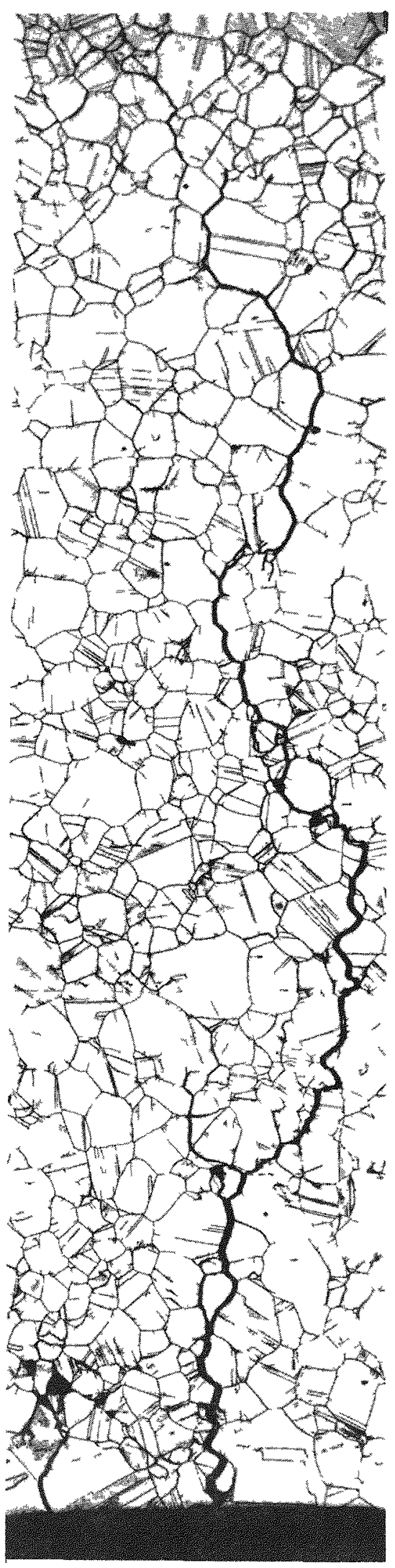

b. With a $.1 \mathrm{hr}$ Tensile Hold Time

Fig. 9 Comparison of the mode of fatigue crack propagation in two specimens of unirradiated 316 stainless stee1. $\Delta \varepsilon_{t}=.5 \%, T=1100^{\circ} \mathrm{F}$ 
Surface

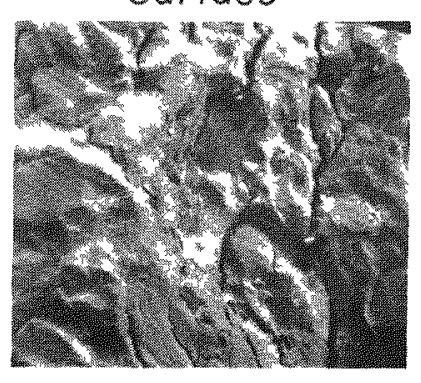

a. Transcrystalline Failure Tensile Hold Time $=.005$ hr. $200 \mathrm{X}$

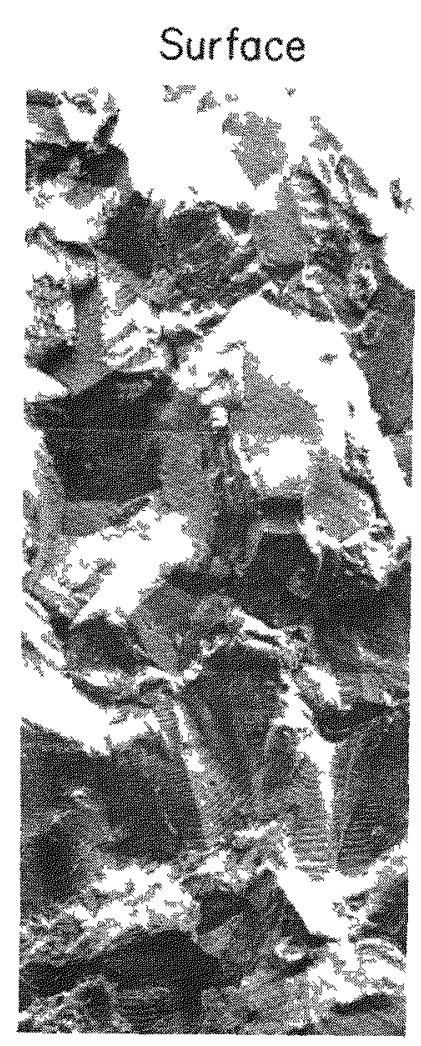

b. Mixed Mode Failure

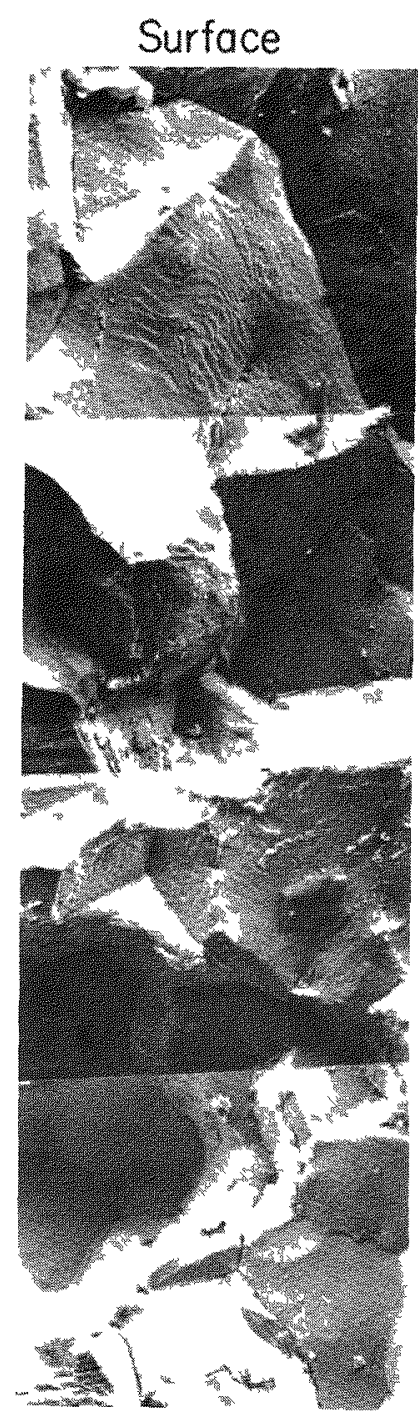

c.Intercrystalline Failure

Tensile Hold Time $=.5 \mathrm{hr}$. $500 x$

F1g. 10 Scanning electron microscope fractographs showing the fatigued surfaces of 316 stainless steel tested at a total strain range of $1.0 \%$ at $1100^{\circ} \mathrm{F}$. 


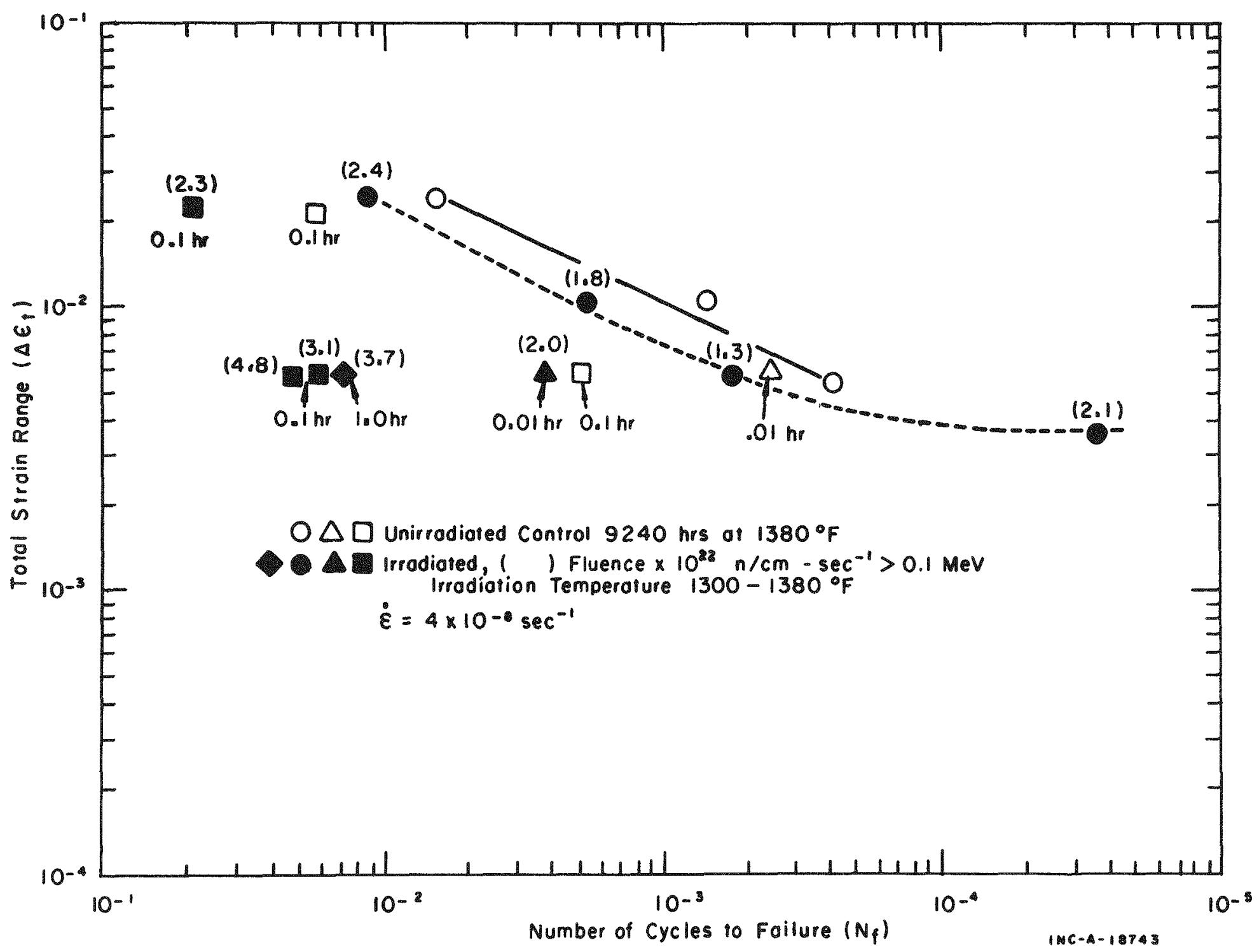

Fig. 11 Influence of tensile hold periods on the fatigue life of pre- and post-irradiated 316 stainless steel tested at $700^{\circ} \mathrm{C}\left(1292^{\circ} \mathrm{F}\right)$. 


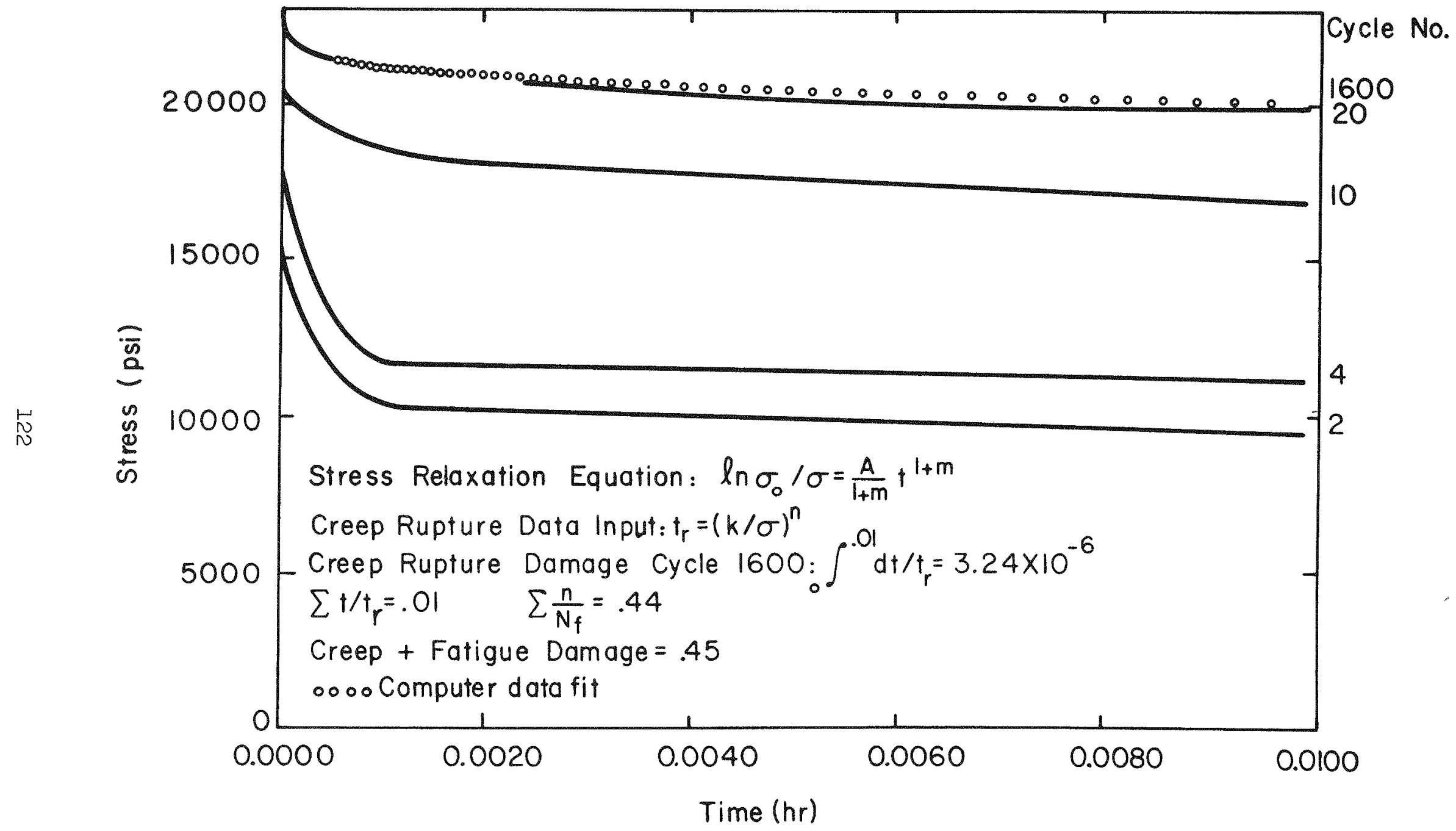

Fig. 12 Stress relaxation at various fatigue cycles during hold time period. 


\section{CALCULATION OF CREEP STRESS IN POWER BURST FACILITY (PBE)}

FUEL GRAINS

R. E. Schmunk

Utilizing Folweiler's equation [1], calculations have been made to evaluate the expected creep stresses in PBF fuel grains and to determine whether or not the adjustment of grain size in fuel pellets could be used to control these stresses. Folweiler's equation is based on the Nabarro and Herring model which assumes that deformation is controlled by a diffusional creep process rather than by basal slip. This equation predicts a linear

relationship between stress $\sigma$ and strain rate $\dot{\varepsilon}$ given by $\sigma=\frac{k T R^{2}}{10 D \Omega} \dot{\varepsilon}$

where $k$ is Boltzmann's constant; $T$, absolute temperature; $R$, grain radius; $D$, diffusion coefficient; and $\Omega$, atomic volume of oxygen.

Strain rates were determined using the temperature profile data (vs position and time) from Wadkin's Engineering Report A-35 [2] along with the thermal expansion data of Feith et. al. contained in GESP-4I[3]. According to Wadkins' data the fuel temperature rises rapidly starting $56 \mathrm{msec}$ after the initiation of a $1.3 \mathrm{msec}$ power burst. Fuel temperature is uniform across the pellet at first, but a definite temperature gradient develops near the fuel surface as heat is lost to the volume around the fuel. This gradient, which is initially confined to a depth of $20 \mathrm{mils}$ from the surface, results in thermal strains being produced over the same region. Strain rates determined from Wadkins' data for three different cases (different values assumed by Wadkins for the thermal conductivity of the insulator surrounding the fuel) are shown as a function of time in Figure 1. Creep stress as a function of strain rate is then shown in Figure 2 for one particular case and three different fuel grain diameters which are indicated in the figure.

The dominant factor in the creep stress for this model is the diffusion constant $D$ which has a low value at low temperature and hence has much more effect than factors such as grain size, strain rate, and temperature, per se. One could not expect to control the creep stress through grain size because of insufficient sensitivity to this factor. Because the rupture strength for PBF fuel pellets is much lower than the calculated creep stress it is expected that cracking of the fuel grains will result from a power burst.

The high temperatures reached by the fuel following the power burst results in an increase in the equilibrium concentration of vacancies and interstitials. The local shear stresses which are generated serve as a driving force to increase the diffusion of vacancies which can contribute to the formation of microcracks within grains, the formation of voids, and a partial relief of the stresses. 
[1] R. C. Folweiler, J. App1. Phys. 32, 773 (1961).

[2] R. P. Wadkins, Phillips Petroleum Company, Atomic Energy Division, Engineering Task Report 非-35.

[3] A. D. Feith, et. al., "Thermal Property Data for $62 \mathrm{ZrO}_{2}$ $30 \mathrm{UO}_{2}-8 \mathrm{CaO}$ at High Temperatures," General Electric Company Report GESP-41 (undated). 


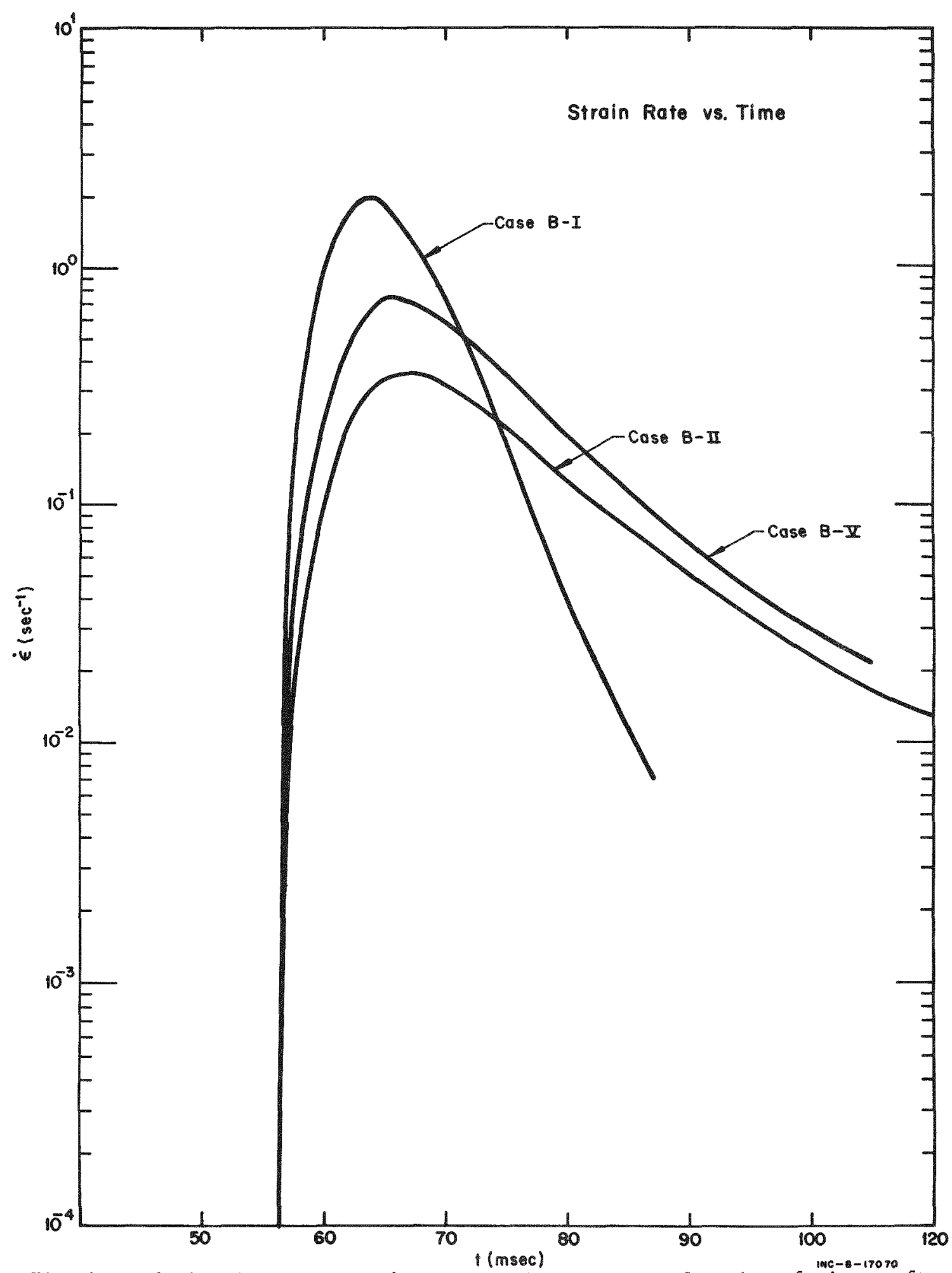

Fig. 1 Calculated strain rate $\dot{\varepsilon}$ in $\mathrm{PBF}$ fuel grains as a function of time $t$ after the initiation of a $1.3 \mathrm{msec}$ power burst for three cases assuming different fuel insulator contact coefficients and heat capacities. 


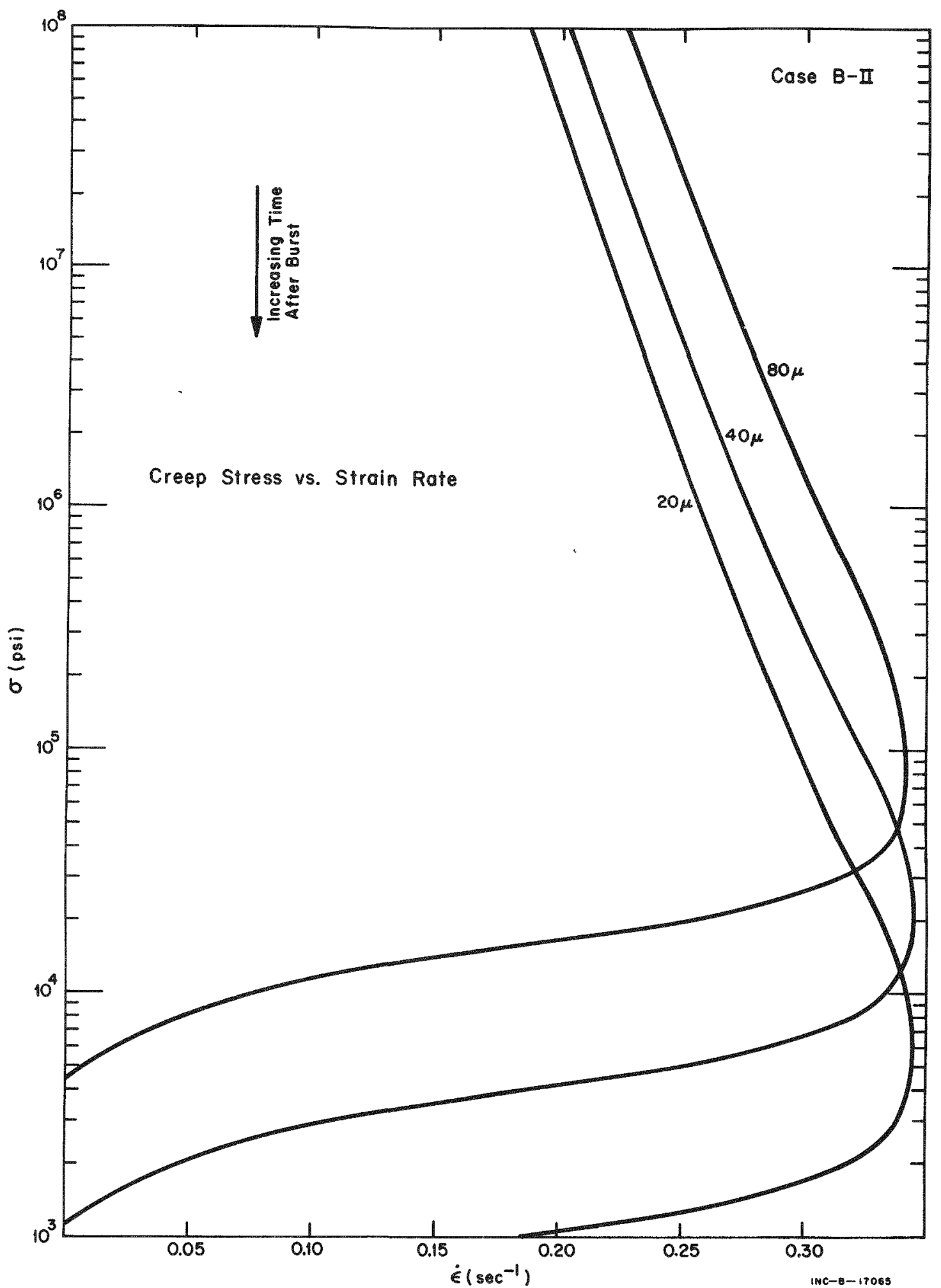

Fig. 2 Creep stress $\sigma$ vs strain rate $\dot{\varepsilon}$ for three different grain sizes calculated for PBF fuel using Folweiler's equation. 
EXPERIMENTAL BREEDER REACTOR (EBR-II) MARK II FUEL EXAMTNATION BY MICROPROBE

\section{R. E. Schmunk}

Argonne National Laboratory has been performing irradiation tests of an improved EBR-II driver fuel (designated Mark II) to improve operating efficiency of the reactor. During the past year, the ANC shielded microprobe has been used to examine samples of this fuel irradiated to 3.5 at. percent and 6.0 at. percent burnup in order to evaluate fuel-cladding compatibility. Material used in the samples was originally composed of 93 percent enriched uranium-fissium alloy clad in 304 stainless steel tubing having a nominal I.D. of 0.150 in. and wall thickness of 0.012 in. Examination of the two specimens included line scans, areal scans, and point counting for $\mathrm{Fe}, \mathrm{Cr}, \mathrm{Ni}, \mathrm{U}, \mathrm{Cs}, \mathrm{Mo}$, and $\mathrm{Ru}$.

Line scans for the 3.5 at. percent burnup sample showed an increased intensity for both iron and chromium in the cladding adjacent to the fuel-cladding interface with a decrease in nickel content over the same area. Iron and chromium were not found in the fuel from these same scans, however, point counting for nickel revealed a diffusion zone extending approximately 100 microns into the fuel. The fission products, molybdenum and ruthenium, were found to be uniformly distributed throughout the fuel, whereas cesium showed some agglomeration in the fuel.

Examination of the 6.0 at. percent burnup sample showed a diffusion zone at the O.D. of the fuel extending into the fuel 150 microns and containing both iron and nickel. The cladding adjacent to the fuel revealed a 10 micron band in which iron and nickel were partially depleted resulting in an enhancement of the remaining chromium. Although it was expected that there would be some diffusion of fission products from the fuel into the cladding, none was detected. 


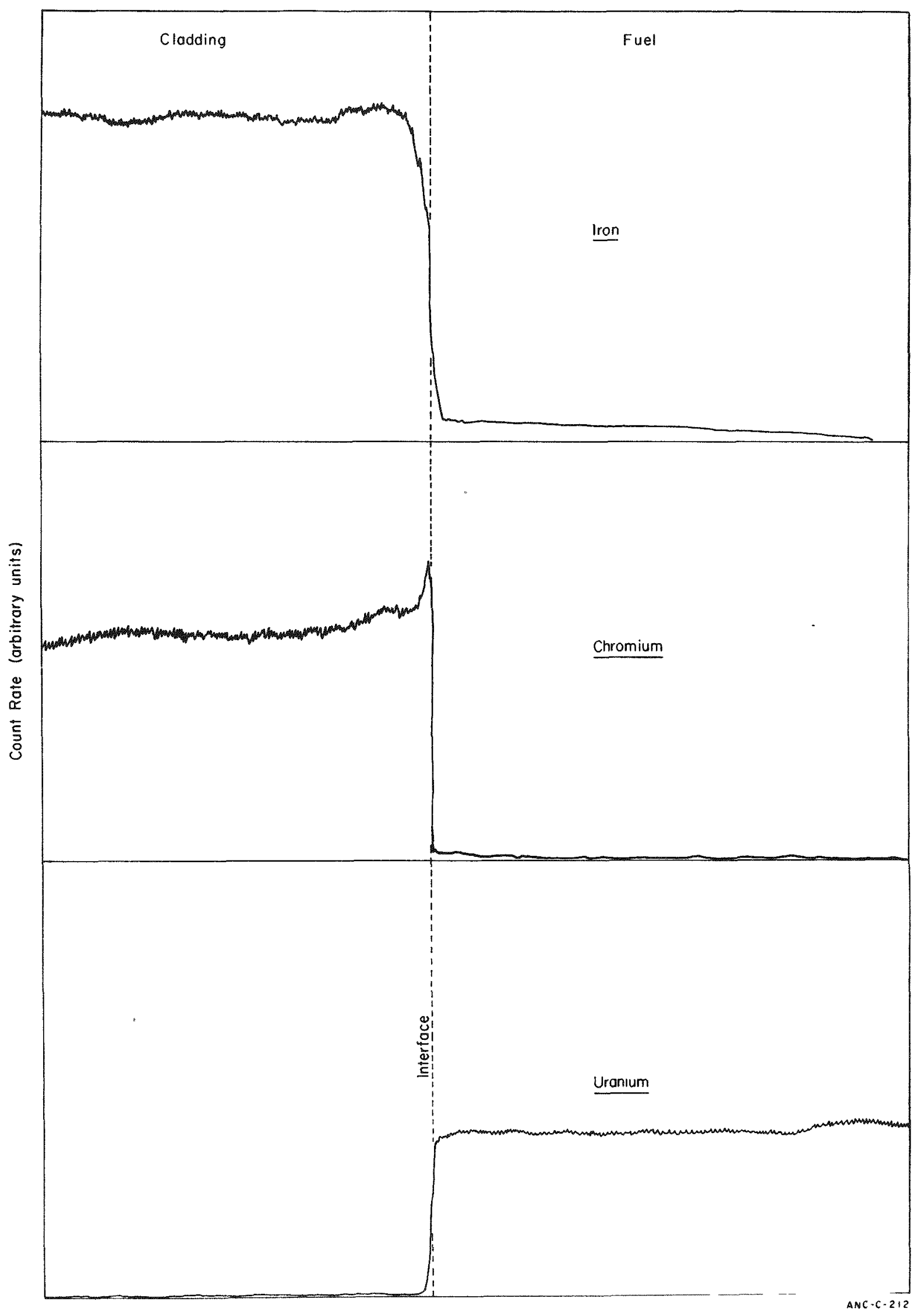

Fig. 1 Line scans data for uranium, iron, and chromium across the fuel-cladding interface revealing the chromium enhancement at the interface and iron diffusion into the fuel region. 


\section{R. E. Schmunk}

The ANC microprobe has been used to examine fuel nozzle caps removed from the waste calciner facility. Three of these caps, A, $B$, and $C$, had been in service for 3700 hours while a fourth cap, $D$, had failed after only 11 hours service. Caps $A, B$, and $C$ of type 17-4PH stainless steel appeared to be the same when viewed optically on the metallograph. Therefore, we chose cap A as typical of the three and examined it in detail with the microprobe. In addition, cap D of type 303 stainless steel was examined with the microprobe. Both caps $A$ and $D$ were examined for the presence and distribution of elements iron, chromium, nickel, carbon, and oxygen. Since we have the capability of looking for three elements simultaneously and since both carbon and oxygen require the use of the light element kit, repeated scans were made looking for iron, nickel, and oxygen and then iron, chromium, and carbon. While some line scanning was done, the most useful information was obtained from point counting.

Nozzle cap A displayed a very high carbon content in the region around the cap orifice. Curiously, the high carbon density was limited entirely to one side of the orifice with a strong concentration gradient perpendicular to the orifice. Point counting for carbon revealed concentrations adjacent to the orifice that were 10 times higher than the carbon content in areas well removed from the orifice. Areas which showed a high carbon content were generally found to be partially deficient in iron, chromium and nickel, the reduction in the point count for these elements corresponding directly to the increase in carbon content. Regions which were found to be high in carbon content also showed some increase in oxygen although on a smaller scale. While it is difficult to quote a percentage composition because of the effects of other elements present, the following table of point count data dramatizes the carburization that has taken place. All counts are quoted for a ten second counting period.

\begin{tabular}{|c|c|c|c|c|c|}
\hline & $\mathrm{Fe}$ & $\mathrm{Cr}$ & $\mathrm{Ni}$ & $\mathrm{C}$ & $0_{2}$ \\
\hline On Standard & --- & --- & --- & $110 \mathrm{~K}$ & $4.4 \mathrm{~K}\left(\mathrm{on} \mathrm{SiO}_{2}\right)$ \\
\hline On Back of Cap $A$ & $92.5 \mathrm{~K}$ & $34.7 \mathrm{~K}$ & $3.7 \mathrm{~K}$ & $2.8 \mathrm{~K}$ & 75 \\
\hline Near Orifice of Cap $A$ & $16.5 \mathrm{~K}$ & $13.5 \mathrm{~K}$ & $1.2 \mathrm{~K}$ & $29 \mathrm{~K}$ & 227 \\
\hline
\end{tabular}

The values given on the bottom line above represent the maximum point counts obtained for carbon and oxygen and the minimum point counts obtained for iron, chromium, and nickel. 
Nozzle cap $D$, in contrast, had a carbon content which was much more uniform than for cap $A$ with increases by $21 / 2$ times in regions around the orifice. Also, the region over which carburization had taken place on cap $D$ was much smaller than for cap A. Oxygen was barely detectable on this cap and elements $\mathrm{Fe}, \mathrm{Cr}$, and $\mathrm{Ni}$ again showed a decrease in regions where the carbon content was up. The visual appearance of cap $D$ indicated melting and recrystallization had taken place in a band $50-100$ microns deep around the cap orifice.

The carburization process apparently involves the diffusion of carbon from the fuel into the nozzle caps with a diffusion of the stainless steel constituents in the reverse direction. 


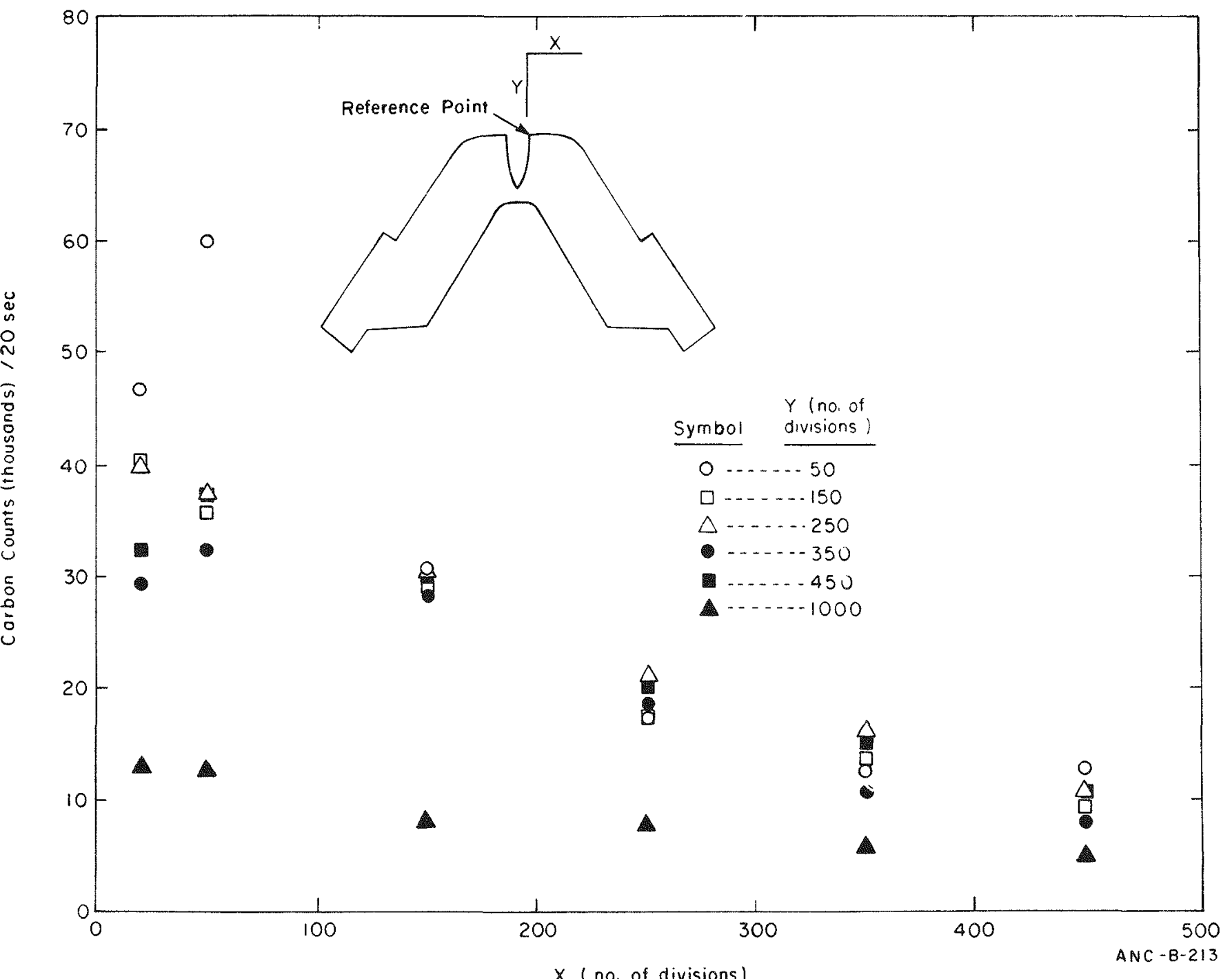

Fig. I Carbon $K \alpha$ X-ray intensities observed during microprobe point counting on waste calciner fuel nozzle caps. Intensities are plotted as a function of distance (microscope divisions where 1 div $\simeq 1.3$ micron) away from the cap orifice and for selected depths below the cap surface. 
IRRADIATION TESTING OF SAMPLE FUEL PLATES TO VERY HIGH BURNUPS (INC-16-1)

M. J. Graber, R. R. Hobbins, G. O. Hayner, J. K. Crandal1, G. W. Gibson, E. H. Porter

Sample fuel plates of a variety of aluminum dispersion types were irradiated to study fuel stability and performance at high burnups with the aim of extending test reactor fuel cycle life. The sample plates measured 2.312 in. $x 2.875$ in. $x 0.050$ in. in overa11 dimensions. With one exception the plates had 20 mil cores and $15 \mathrm{mils}$ of cladding. In the exceptional case, a 30 mil core and 10 mils of cladding were used. Two plates, 157-38 and 158-65, were removed after 9434 MWd; the rest of the plates received 18,667 MWd of exposure in the ETR.

The post-irradiation examination of INC-16-1 samples is underway. Eddy current oxide thickness measurements, gamma scanning, plate thickness measurements, plate densities before and after stripping, and blister testing have all been performed. In addition, samples for chemical burnup analysis and metallographic examination have been selected. At this point, only the blister data and the core swelling results have been evaluated. Table I contains these results.

The blister testing was carried out in $100^{\circ} \mathrm{F}$ increments from 400 to $600^{\circ} \mathrm{F}$ and $50^{\circ} \mathrm{F}$ increments from $600^{\circ} \mathrm{F}$ to failure. The blister temperatures in Table $\mathrm{I}$ are the temperatures at which blisters first appeared on the plates. The results for INC-16-1 ATR composition samples are plotted with previously obtained data in Figure 1. It is notable that the slope of the failure-nofailure line decreases at higher burnups.

In Table I, core swelling is presented in terms of the percent increase in core volume as a result of irradiation. The expected core swelling is derived by subtracting the fabrication void content from the net volume increase due to the formation of solid fission products. The latter quantity has been calculated as a function of fission density ${ }^{2}$. The results in Table I show that measured core swelling generally is in reasonable agreement with expectations, i.e., fabrication voids have been effective in accommodating volume increases due to the formation of fission products.

[1] M. J. Graber, M. F. Marchbanks, "Blister Resistance of Various Aluminum Dispersion Fuel Systems," in Annual Progress Report on Reactor Fuels and Materials Development for FY-1967, IN-1131, pp 43-47 (February 1968).

[2] M. J. Graber, W. F. Zelezny, and R. A. Moen, "Volume Changes in Sample Fuel Plates," in Annual Progress Report on Reactor Fuels and Materials Development for FY-1965, pp 7-11 (February 1966). 


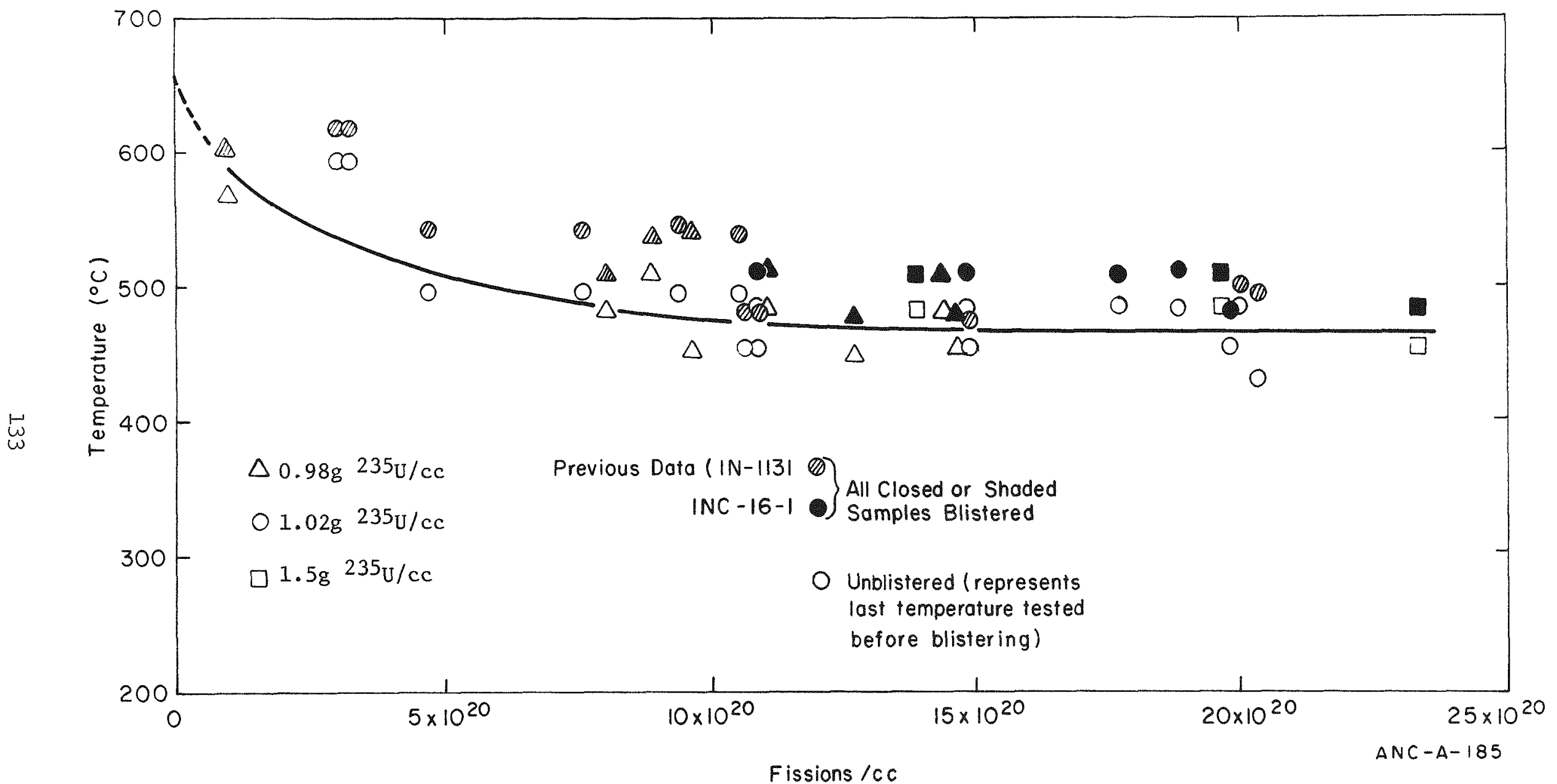

Fig. 1 Post-irradiation blister failure-no-failure curve for UA1 fuels. 
TABLE I

SWELLING AND BLISTER RESULTS FOR INC-16-1 SAMPLE FUEL PLATES

\begin{tabular}{|c|c|c|c|c|c|c|c|}
\hline Composition & Plate No. & $\begin{array}{r}\mathrm{Ca} l \mathrm{c} \\
\text { Fiss/ce } \\
\end{array}$ & $\begin{array}{l}\text { BIister } \\
\text { Temp. }{ }^{\circ} \mathrm{C}\end{array}$ & $\begin{array}{l}\text { Core Swel } \\
\text { Measured } \\
\end{array}$ & $\begin{array}{l}\text { ing, } \% \Delta V / V \\
\text { Expected }[a]\end{array}$ & $\begin{array}{l}\% \Delta v / V \\
\text { Fission } \\
\text { Growth[b] }\end{array}$ & $\begin{array}{l}\text { Fabrication } \\
\text { Voids, } \% \\
\end{array}$ \\
\hline ETR UAI $x$ & $\begin{array}{l}154-50 \\
154-47\end{array}$ & $\begin{array}{l}1.59 \times 10^{21} \\
1.36\end{array}$ & $\begin{array}{l}455 \\
260\end{array}$ & $\begin{array}{l}5.4 \\
4.2\end{array}$ & $\begin{array}{l}4.2 \\
3.1\end{array}$ & $\begin{array}{r}10.2 \\
8.7\end{array}$ & $\begin{array}{l}6.0 \\
5.6\end{array}$ \\
\hline ETR $B_{4} \mathrm{C} \underset{" \text { Foil }}{ }, 3.8 \mathrm{gB} / \mathrm{el}$ & $\begin{array}{l}155-11 \\
155-23\end{array}$ & $\begin{array}{l}1.78 \\
1.60\end{array}$ & $\begin{array}{l}425 \\
425\end{array}$ & $\begin{array}{l}9.1 \\
8.6\end{array}$ & $\begin{array}{l}11.4 \\
10.2\end{array}$ & $\begin{array}{l}11.4 \\
10.2\end{array}$ & $\begin{array}{l}0.0 \\
0.0\end{array}$ \\
\hline $\operatorname{ETR} \mathrm{B}_{4} \mathrm{C} \underset{\|}{\mathrm{F} O \mathrm{i} 1,}, 4.6 \mathrm{gB} / \mathrm{el}$ & $\begin{array}{l}156-45 \\
156-64\end{array}$ & $\begin{array}{l}1.79 \\
1.63\end{array}$ & $\begin{array}{l}425 \\
425\end{array}$ & $\begin{array}{l}9.2 \\
8.7\end{array}$ & $\begin{array}{l}11.5 \\
10.4\end{array}$ & $\begin{array}{l}11.5 \\
10.4\end{array}$ & $\begin{array}{l}0.0 \\
0.0\end{array}$ \\
\hline $\begin{array}{l}\text { ATR Standard Loading } \\
" 1 \\
" 1\end{array}$ & $\begin{array}{l}157-30 \\
157-31 \\
157-38\end{array}$ & $\begin{array}{l}1.98 \\
1.77 \\
1.48\end{array}$ & $\begin{array}{l}480 \\
510 \\
510\end{array}$ & $\begin{array}{l}5.1 \\
5.9 \\
4.4\end{array}$ & $\begin{array}{l}7.5 \\
5.5 \\
4.4\end{array}$ & $\begin{array}{r}12.7 \\
11.3 \\
9.5\end{array}$ & $\begin{array}{l}5.2 \\
5.8 \\
5.1\end{array}$ \\
\hline $\begin{array}{c}\text { ATR } 7 \mathrm{~F} \text { High Loading } \\
" 1\end{array}$ & $\begin{array}{l}158-54 \\
158-67 \\
158-65\end{array}$ & $\begin{array}{l}2.33 \\
1.96 \\
1.39\end{array}$ & $\begin{array}{l}480 \\
510 \\
510\end{array}$ & $\begin{array}{l}6.9 \\
5.2 \\
6.4\end{array}$ & $\begin{array}{l}8.2 \\
5.8 \\
2.7\end{array}$ & $\begin{array}{r}14.9 \\
12.5 \\
8.9\end{array}$ & $\begin{array}{l}6.7 \\
6.7 \\
6.2\end{array}$ \\
\hline ATR 7F High 30 Mil Core & $\begin{array}{l}159-14 \\
159-24\end{array}$ & $\begin{array}{l}1.45 \\
1.38\end{array}$ & $\begin{array}{l}510 \\
510\end{array}$ & $\begin{array}{l}5.1 \\
5.1\end{array}$ & $\begin{array}{l}5.6 \\
5.3\end{array}$ & $\begin{array}{l}9.3 \\
8.7\end{array}$ & $\begin{array}{l}3.7 \\
3.4\end{array}$ \\
\hline ATR $\underset{" 7 F}{70}$ Low Loading & $\begin{array}{l}160-37 \\
160-34\end{array}$ & $\begin{array}{l}1.47 \\
1.27\end{array}$ & $\begin{array}{l}480 \\
480\end{array}$ & $\begin{array}{l}6.6 \\
6.4\end{array}$ & $\begin{array}{l}5.7 \\
4.9\end{array}$ & $\begin{array}{l}9.4 \\
8.1\end{array}$ & $\begin{array}{l}3.7 \\
3.2\end{array}$ \\
\hline 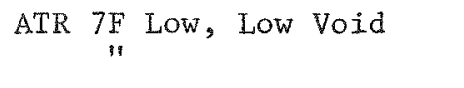 & $\begin{array}{l}162-1 \\
162-4\end{array}$ & $\begin{array}{l}1.44 \\
1.09\end{array}$ & $\begin{array}{l}510 \\
510\end{array}$ & $\begin{array}{l}6.1 \\
4.8\end{array}$ & $\begin{array}{l}6.0 \\
3.3\end{array}$ & $\begin{array}{l}9.2 \\
7.0\end{array}$ & $\begin{array}{l}3.2 \\
3.7\end{array}$ \\
\hline ATR Regular, Low Void & $\begin{array}{l}163-4 \\
163-16\end{array}$ & $\begin{array}{l}1.88 \\
1.08\end{array}$ & $\begin{array}{l}510 \\
510\end{array}$ & $\begin{array}{l}6.8 \\
3.3\end{array}$ & $\begin{array}{l}7.5 \\
2.3\end{array}$ & $\begin{array}{r}12.0 \\
6.9\end{array}$ & $\begin{array}{l}4.5 \\
4.6\end{array}$ \\
\hline
\end{tabular}

[a]Expected for $100 \%$ utilization of voids for fission product accommodation.

$[b]_{\text {Growth }}$ due to fissioning of ${ }^{235} \mathrm{U}$ on a core volume basis. 
R. R. Hobbins, E. H. Porter*, J. K. Cranda11, M. W. E11ingford

Post-irradiation testing many types of sample fuel plates has established failure-no-failure $[1,2]$ curves for fuel plate blistering as a function of fission density and plate temperature. No in-pile blister failures have occurred in the region predicted as safe from blistering. However, because of dynamic irradiation effects such as the re-solution 3$]$ of fission gas bubbles, inpile blister behavior may differ from that observed in postirradiation tests.

In order to relate in-pile blister behavior to postirradiation failure-no-failure curves a capsule irradiation program has been instituted to irradiate sample fuel plates at temperatures near to, above, and below the blister curve established for UAl fuels. This curve is shown in Figure 1. The experiment has been designed to produce irradiation temperatures ranging from 350 to $550^{\circ} \mathrm{C}$ and fission densities in the range 1.8 to $2.3 \times 10^{21} \mathrm{fiss} / \mathrm{cc}$.

Six ATR standard, two ATR $7 F$ high, and one ATR 7F low loaded sample fuel plates have been encapsulated in three Na-filled, double-contained, 347 stainless steel capsules. The three capsules are arranged one on top another in a vertical stack as are the three fuel plates within each capsule. The fuel plates measure 0.625 in. $x 3.200$ in. $x 0.050$ in. in overa11 dimensions. The cores are 20 mils thick and are clad with 15 mils of 6061 aluminum.

Two thermocouples are located in the $\mathrm{Na}$ adjacent to, and on either side of, each of the top two fuel plates in the assembly. With the data from these thermocouples, temperatures in the cores of the sample fuel plates can be calculated using the two dimensional heat transfer computer code SIMIR.

This experiment is scheduled to be inserted in the ETR during the 112 mid-cycle shutdown (July 1971).

* Member of the Engineering Division.

[1] M. J. Graber, M. F. Marchbanks, "B1ister Resistance of Various Aluminum Dispersion Fuel Systems," in Annual Progress Report on Reactor Fuels and Materials Development for FY-1967, IN-1131, Pp. 43-47 (February 1968).

[2] M. F. Marchbanks, W. C. Francis, M. L. Griebenow, "Characterization of the Reactor Performance of Sample Fuel Plates with MTR, ETR and ATR Conditions," in Reactor Engineering Branch Annual Report Fiscal Year 1969, IN-1335, pp. 49-53 (November 1969).

[3] J. A. Turnbu11 and R. M. Corne11, J. Nuc1. Mat. 37, 355 (1970). 


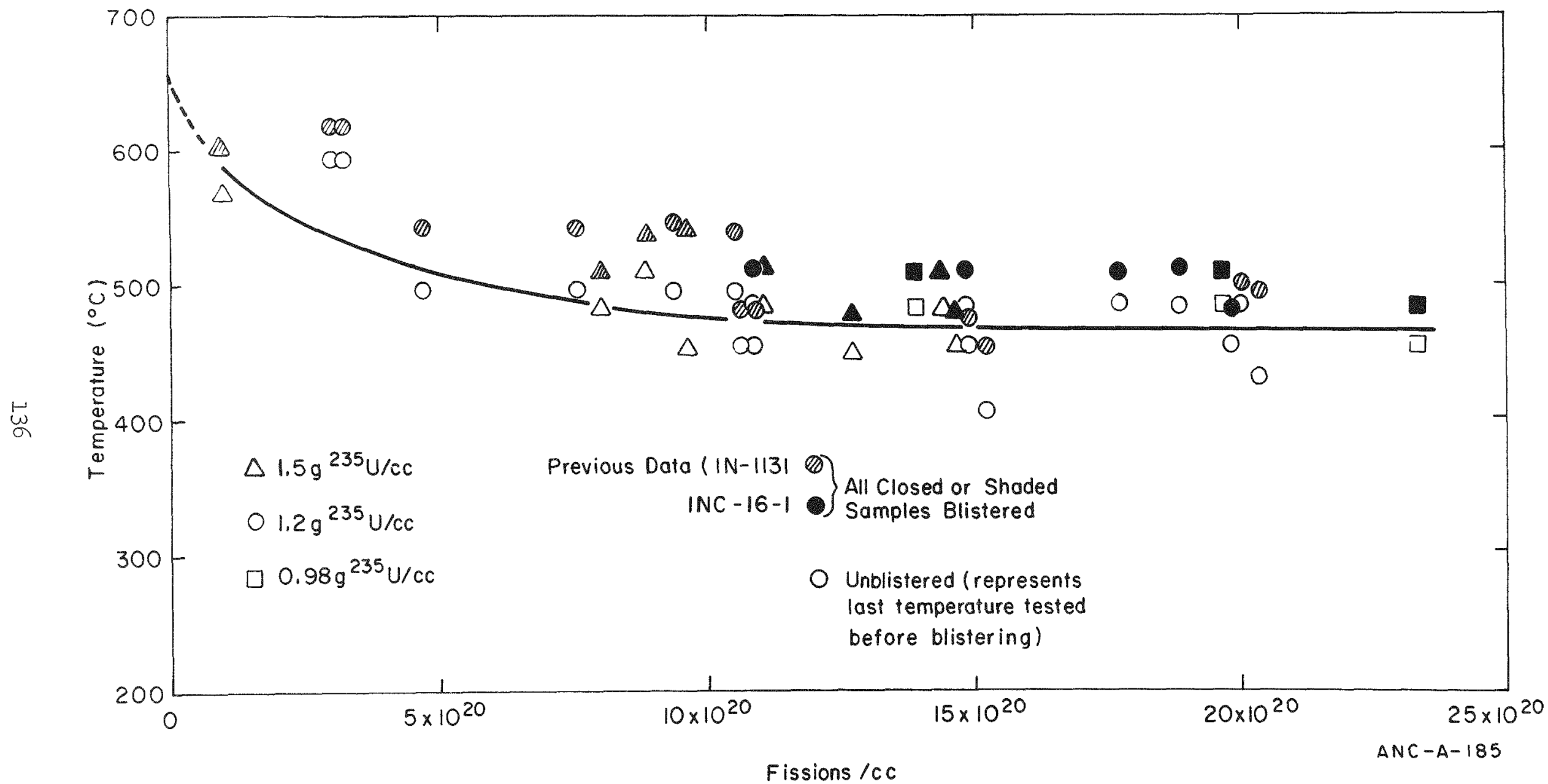

Fig. 1 Post-irradiation blister failure-no-failure curve for UA1 fuels. 


\title{
CORROSION OF ALUMINUM IN CRITICAL FACILITIES AND CANALS
}

\author{
R. R. Hobbins
}

The purpose of this work was to investigate the corrosion behavior of the aluminum components of ATR and ETR fuel elements in the critical facilities and canals. Experiments were designed to measure the oxide buildup that new, clean elements would experience and to observe what changes, if any, would occur in the oxide of used, corroded elements upon extended storage in these facilities.

Samples of fuel plate cladding material [6061-0 (ATR), 1100H14 (ETR as-fabricated), and 1100-0 (ETR annealed)] and side plate material [6061-T6 (both ATR and ETR)] were placed in rows in test fixtures. Figure $I$ is a photograph of the corrosion test fixture. The sample plates are separated by $0.10 \mathrm{in.} \mathrm{water}$ channels.

Samples both bare and precoated with bayerite $\left(\mathrm{Al}_{2} \mathrm{O}_{3} \cdot 3 \mathrm{H}_{2} \mathrm{O}\right)$ or boehmite $\left(\mathrm{Al}_{2} \mathrm{O}_{3} \cdot \mathrm{H}_{2} \mathrm{O}\right)$ were exposed in the ATR and ETR canals and the ATRC and ETRC. Changes in the oxides were followed by eddy current measurements of film thickness and $\mathrm{X}$-ray determination of crystal structure.

Prefilmed samples with up to three months exposure show no changes in crystal structure, i.e., there has been no detectable transformation from boehmite to bayerite or vice versa. With one exception no measurable changes in prefilm oxide thickness were observed. The exception occurred with bayerite prefilmed 6061-0 and 6061-T6 plates exposed for three months in the ETR and ATR canals. These film thicknesses increased from $0.23 \pm$ 0.01 to $0.26 \pm 0.01 \mathrm{mil}$, on the average. Samples with six months exposure remain to be analyzed.

All of the bare samples begin to corrode at the 6061-Al washer separating the plates. Evidently, crevice corrosion is the initial mechanism here. The oxide then spreads over the rest of the plate surface. Typical corrosion patterns are shown in Figure 2. It is interesting to note that the plate orientation can be reversed (washer at bottom) without affecting the corrosion pattern. Bayerite was the only oxide found in corrosion films on initially bare sample plates.

In one experiment, $0.50 \mathrm{in}$. channels were used in addition to the $0.10 \mathrm{in}$. channels. Surfaces forming the $0.50 \mathrm{in}$. channels exhibited very little corrosion compared to those of the $0.10 \mathrm{in}$. 
channels. Maximum oxide thicknesses are given in Table I for exposures in the ATRC. It is evident that the films are thicker on the 6061 than the $1100 \mathrm{Al}$ and that the different tempers have little effect on film thickness.

Some additional work will be necessary to complete this project. The six month exposures will be analysed and the film buildup data for bare plates will be evaluated for all experiments.

TABLE I

OXIDE BUILDUP ON ALUMINUM WITH ATRC EXPOSURE

\begin{tabular}{|c|c|c|c|c|c|}
\hline Exposure & $\begin{array}{l}\text { Channel } \\
\text { Spacing }\end{array}$ & $1100-0$ & $1100-\mathrm{H} 14$ & 6061-0 & $6061-\mathrm{T} 6$ \\
\hline 4 weeks & $0.10 \mathrm{in.}$ & 0.30[] & 0.30 & 0.39 & 0.40 \\
\hline 4 weeks & 0.50 in. & 0.03 & 0.03 & 0.04 & 0.02 \\
\hline 8 weeks & $0.10 \mathrm{in.}$ & 0.34 & 0.34 & 0.44 & 0.42 \\
\hline 8 weeks & $0.50 \mathrm{in.}$ & 0.01 & $0.21[\mathrm{~b}]$ & 0.10 & 0.01 \\
\hline
\end{tabular}

[a]Oxide thicknesses in mils. [b]Probable sample mixup. 


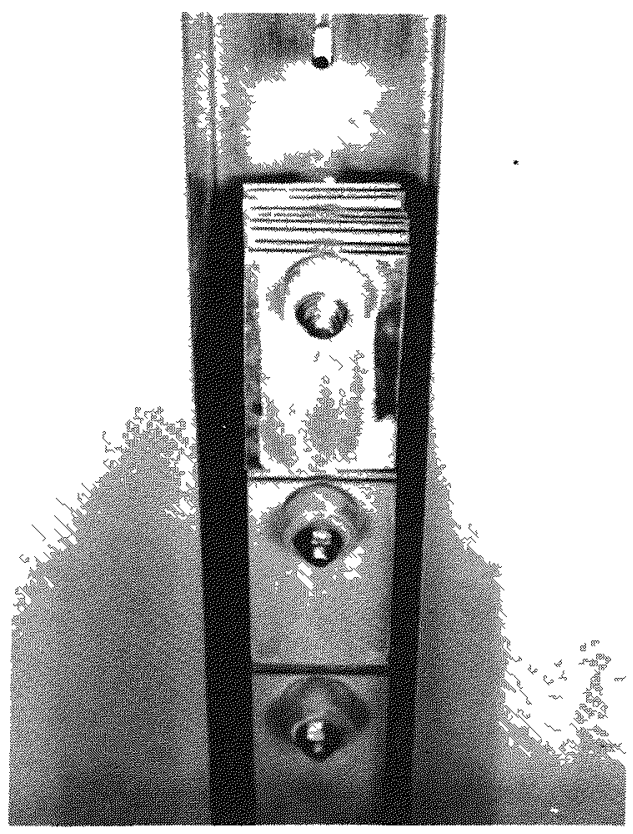

Fig. 1 Plates assembled in corrosion test fixture.

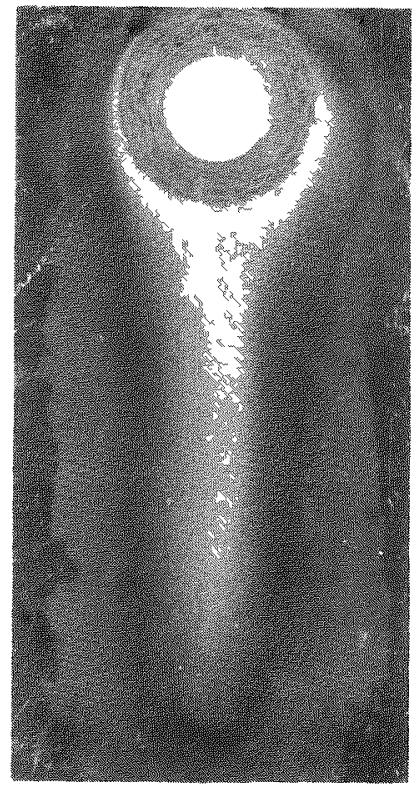

A. Norma1

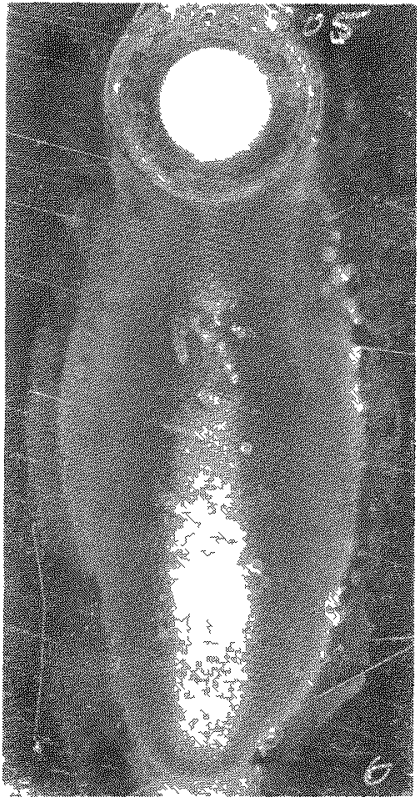

B. Inverse

Fig. 2 Typica1 corrosion patterns - normal and inverse plate orientations. 
ETR FUEL OXIDE INVESTIGATION - BULK TRANSFORMATION

OF ALUMINUM OXIDE

G. E. Korth

The investigation of the anomalous oxide buildup on ETR fuel plates was supported through bulk transformation tests in a stirred autoclave. It previously had been observed that spent plates stored in the canal as well as newly discharged plates from the reactor had an unusually thick oxide layer often analyzed as bayerite $\left(\mathrm{Al}_{2} \mathrm{O}_{3} \cdot 3 \mathrm{H}_{2} \mathrm{O}\right)$, which had the potential of decreasing thermal conductivity. Kinetically one would expect a thinner layer of boehmite $\left(\mathrm{Ai}_{2} \mathrm{O}_{3} \cdot \mathrm{H}_{2} \mathrm{O}\right.$ ) when considering the $250-300^{\circ} \mathrm{F}$ fuel plate surface temperature. It was speculated that the nitrate ion might have an effect on the extent of oxide formation since no problem had been observed in the ETR when $\mathrm{HNO}_{3}$ was added through the G-12 loop experiment, and ATR used $\mathrm{HNO}_{3}$ for $\mathrm{pH}$ control and had experienced no anomalous oxide buildup. To resolve the kinetic discrepancy and the other speculation, autoclave tests were performed to investigate (1) which oxide was stable at fuel plate temperatures, (2) whether the oxide would transform in the canal environment, and (3) if the nitrate ion had any effect on the transformations.

The initial bayerite sample was prepared by precipitating $\mathrm{Al}(\mathrm{OH})_{3}$ from an $\mathrm{Al}\left(\mathrm{NO}_{3}\right)_{3}$ solution with $\mathrm{NH}_{4} \mathrm{OH}$. The precipitate was washed with flowing distilled water for three days at room temperature. After drying, $\mathrm{X}$-ray analysis showed the fine powdered sample to be very pure bayerite. Distilled water was degassed in a one gallon, $316 \mathrm{SS}$ autoclave by heating to $85^{\circ} \mathrm{C}$ and bubbling nitrogen through it for five hours. The pH after this treatment ranged between 7.8 and 8.5 . A dilute solution of distilled water and $\mathrm{HNO}_{3}$ was added to the autoclave until the $\mathrm{pH}$ was stabilized at 5.5. The sample was then added; the autoclave was sealed, pressurized to 280 psig with argon, stirring motor started, and heated to $290^{\circ} \mathrm{F}$. Samples were periodically extracted through a small valved tube without opening the system. Product analysis and $\mathrm{pH}$ were analyzed on each sample using electron and X-ray diffraction and a Beckman Model G pH meter respectively.

After 70 hours the temperature was lowered to $130^{\circ} \mathrm{F}$ and the test was continued another ten days. The same procedure was then repeated using ETR primary water extracted from the outlet at $10: 30 \mathrm{a} \cdot \mathrm{m}$. on December $11,1970$.

The results of the tests (Table I) showed that bulk samples will transform completely to boehmite at $2900 \mathrm{~F}$ within two days. The boehmite once formed was found to be stable and further runs at $130^{\circ} \mathrm{F}$ (temperature conducive to bayerite formation) for up to ten days indicated no transformation back to bayerite. The presence of the nitrate ion had no observable effect on the transformations. 
TABLE I

AUTOCLAVE TEST RESULTS

\begin{tabular}{|c|c|c|c|c|c|c|}
\hline Sample & Hours & $\operatorname{Temp}\left({ }^{\circ} F\right)$ & Pressure (psig) & $\mathrm{pH}$ & Water & $\begin{array}{l}\text { Product } \\
\text { Analysis }\end{array}$ \\
\hline $1 C-1$ & 0 & 290 & 280 & 5.5 & $D^{[a]}$ & Pure Bayerite \\
\hline $1 C-2$ & 14 & 290 & 280 & 5.5 & $\mathrm{D}$ & $\begin{array}{l}\text { Bayerite-Major } \\
\text { Boehmite-Minor }\end{array}$ \\
\hline $1 C-3$ & 38 & 290 & 250 & 5.5 & $\mathrm{D}$ & $\begin{array}{l}\text { Boehmite-Major } \\
\text { Bayerite-Minor }\end{array}$ \\
\hline $1 C-4$ & 62 & 290 & 250 & 5.5 & $\mathrm{D}$ & A11 Boehmite \\
\hline $1 C-5$ & 70 & 290 & 250 & 5.5 & $\mathrm{D}$ & A11 Boehmite \\
\hline $1 \mathrm{CA}-1$ & 18 & 130 & 250 & 5.7 & $\mathrm{D}$ & A11 Boehmite \\
\hline $1 \mathrm{CA}-2$ & 92 & 130 & 250 & 6.2 & D & All Boehmite \\
\hline $1 \mathrm{CA}-3$ & 116 & 130 & 250 & 6.4 & $\mathrm{D}$ & A11 Boehmite \\
\hline $1 \mathrm{CA}-4$ & 259 & 130 & 250 & 6.5 & $\mathrm{D}$ & A11 Boehmite \\
\hline $1 \mathrm{CP}-1$ & 0 & 290 & 200 & 5.5 & $\operatorname{ETR}^{\lfloor b\rfloor}$ & Pure Bayerite \\
\hline $1 \mathrm{CP}-2$ & 4 & 290 & 200 & 5.0 & ETR & Bayerite-Major \\
\hline $1 C P-3$ & 27 & 290 & 200 & 5.4 & ETR & Boehmite-Major \\
\hline $1 C P-4$ & 51 & 290 & 200 & 5.4 & ETR & Boehmite-Major \\
\hline $1 \mathrm{CP}-5$ & 73 & 290 & 200 & 5.3 & ETR & Al1 Boehmite \\
\hline $1 \mathrm{CPA}-1$ & 26 & 130 & 200 & 5.4 & ETR & Al1 Boehmite \\
\hline $1 \mathrm{CPA}-2$ & 98 & 130 & 200 & 5.6 & ETR & A11 Boehmite \\
\hline $1 \mathrm{CPA}-3$ & 194 & 130 & 200 & 5.9 & ETR & A11 Boehmite \\
\hline $1 \mathrm{CPA}-4$ & 285 & 130 & 200 & 5.8 & ETR & A11 Boehmite \\
\hline
\end{tabular}

[a] D designates distilled water adjusted to $\mathrm{pH} 5.5$ with $\mathrm{HNO}_{3}$.

[b] ETR designates ETR primary water extracted Dec. 11, 1970. 


\section{METALLURGICAL SUPPORT FOR BWR-FLECHT}

\section{J. Graber}

The Full Length Emergency Cooling Heat Transfer Project (FLECHT) is directed toward experimental investigation of the thermal and hydraulic aspects of termination of the thermal transient of the Loss of Cooling Accident (LOCA) by emergency core cooling $[1,2]$. In support of Boiling Water Reactors (BWR), bundles containing 49 Zircaloy-clad, electrically heated, simulated fuel rods were tested using spray cooling under LOCA conditions. Three tests were run in which the predicted bundle initial maximum temperatures were to be 2100 to $2400^{\circ} \mathrm{F}$. The simulated fuel rods used Zircaloy-2 as cladding, alumina as an internal insulator filler material and molybdenum inner-coil heating elements. Under the test conditions, unexpected temperature excursions up to $2940^{\circ} \mathrm{F}$ were encountered.

Samples were selected for metallurgical evaluation to determine the relationships between prior history, microstructure, properties, and performance. It was found that in the high temperature regions of the tests, the alumina was reduced by zirconium producing aluminum metal which in turn formed a low melting point alloy, $2400^{\circ} \mathrm{F}$, with zirconium[3], Figure 1 . Thermochemical calculations and Differential Thermal Analysis indicate that the zirconium-alumina reaction is endothermic. But, when the tubing was perforated because of this reaction, or for any other reason, liquid metal was available to produce an uncontrollable reaction with the steam.

Attack by the alumina on the inside of the tubing in areas which had not perforated varied in intensity from wall thinning and intergranular oxidation to the production of a brittle $\alpha$ zirconium layer on the inside surface (as well as the $\alpha$ layer produced on the outside surface by the steam[4], Figure 2) any of which increased the amount of embrittlement compared to tubes exposed to steam alone. In order to show the embrittling effect in these samples, a "ring compression" test was developed $[5,6]$. A standard $1 / 2$ in. Iong section of the tube was diametrically compressed at ambient temperature and a plot of the load-deflection curve was made. Failure in the specimens was defined as the point where there is a sharp load reduction which was not recoverable. A decrease in the amount of deflection to produce failure is an indication of brittle behavior. It was found by electron microscopy that the oxygen diffusion-produced a layers on the tube wall surfaces always fractured in a brittle manner while the interior part of the tube wall with a prior $\beta$ structure may remain ductile, Figure 3 . In severely attacked samples, the $\beta$ structure which exhibited intergranular oxidation, also failed in a brittle manner, Figure 4 . These results show that embrittlement of the Zixcaloy is dependent on oxygen distribution as well as total oxygen content. 
One type of tube perforation resulted from incompatibility caused by the use of Inconel "Lantern Springs" as spacers in the rod bundles, Figure 5. Although this is understandable since the major components of Inconel, $\mathrm{Cr}$ and $\mathrm{Ni}$, form eutectics at $2370^{\circ} \mathrm{F}$ and $1760^{\circ} \mathrm{F}$ respectively with zirconium, it was not expected because these simulated fuel rods were made from production tubing which had the standard pre-oxidation protective coating.

[1] J. C. Haire, G. F. Brockett, Boiling Water Reactor Full Length Emergency Cooling Heat Transfer (BWR-FLECHT) Tests Project, IN-1385 (June 1970).

[2] J. E. Leonard, J. D. Duncan, Preliminary Results of the BWRFLECHT Internally Pressurized Zircaloy-Clad Bundle Spray Cooling Test, NEDG-13064 (January 1970).

[3] M. Hansen and K. Anderko, Constitution of Binary Alloys, McGraw Hill Book Company (1958).

[4] A. W. Lemmon, Studies Relating to the Reaction Between Zirconium and Water at High Temperatures, BMI-1154 (January 1957).

[5] M. J. Graber, W. F. Zelezny, "Metallurgical Evaluation of Zircaloy Exposed to Emergency-Core-Cooling Conditions," Trans. Am. Nucl. Soc. 12, 356 (1969).

[6] R. Herze1, R. H. Meservey, "The Brittle Behavior of Zircaloy Under Emergency-Core-Cooling Conditions," Trans. Am. Nucl. Soc. 12, 355 (1969). 
$2 \mathrm{Al}_{2} \mathrm{O}_{3}+3 \mathrm{Zr} \rightarrow 3 \mathrm{ZrO}_{2}+4 \mathrm{Al}$

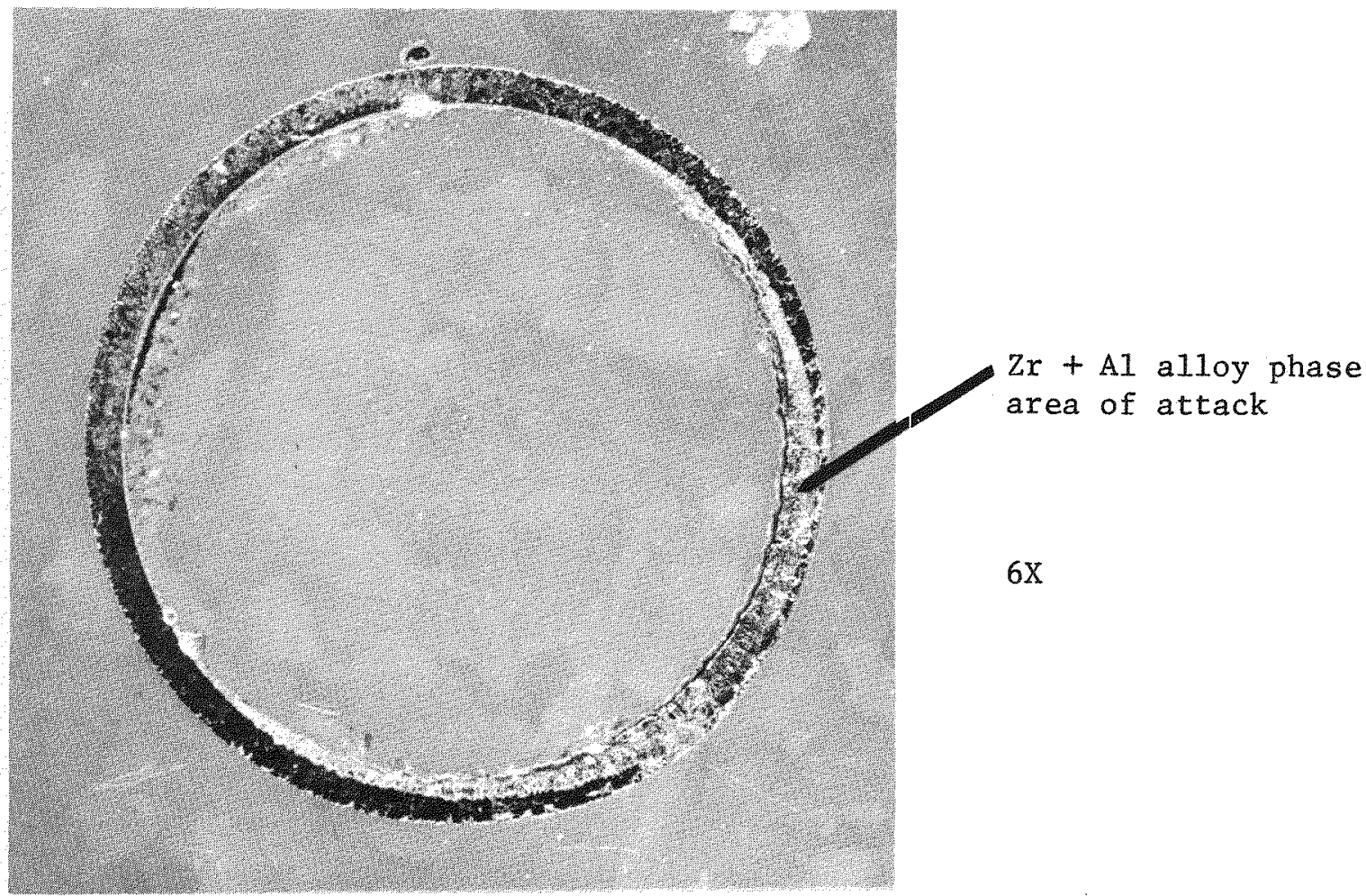

Fig. 1 A cross section macrograph of Tube 11 at the $8 \mathrm{ft}$ elevation from Test $\mathrm{Zr}-3$. 
Outside

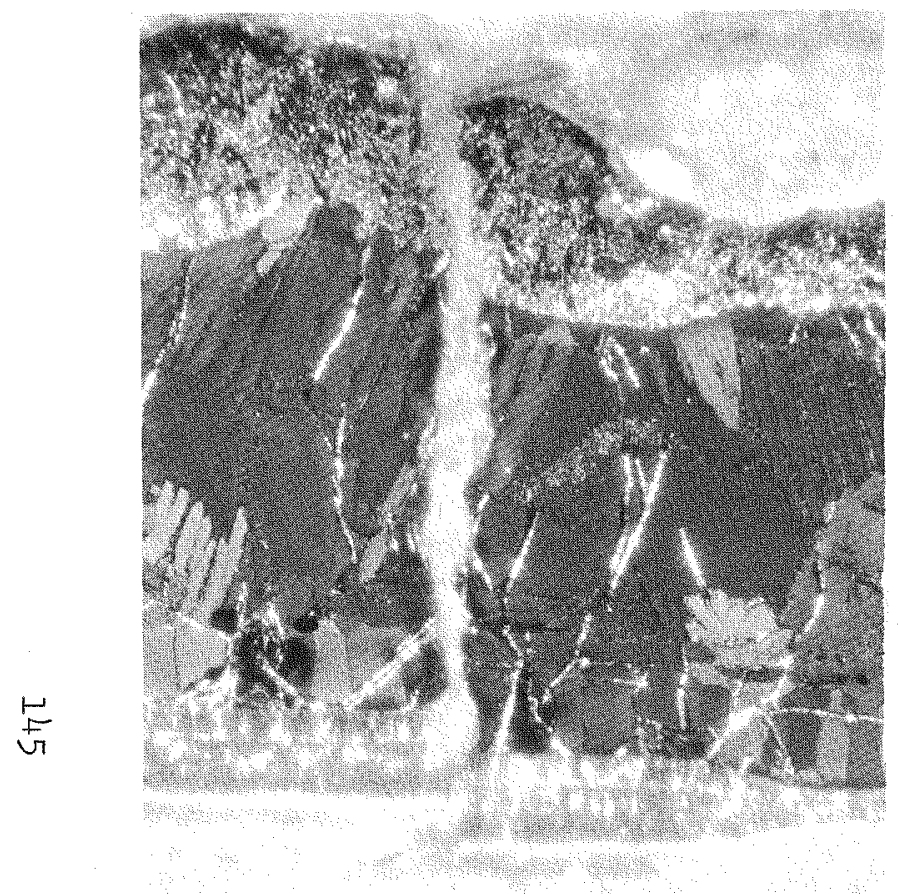

Chemical Polish

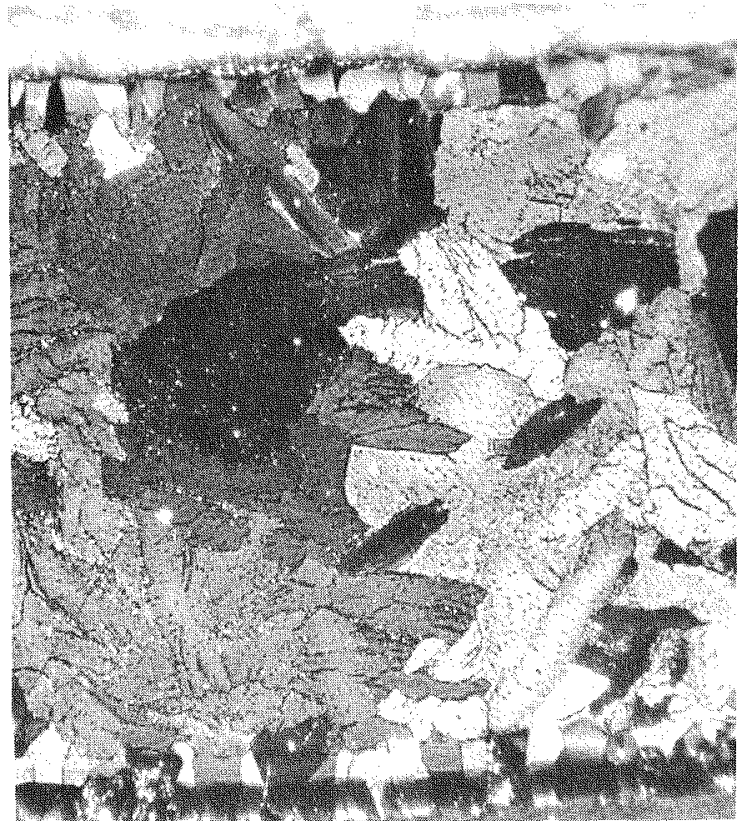

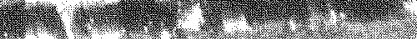

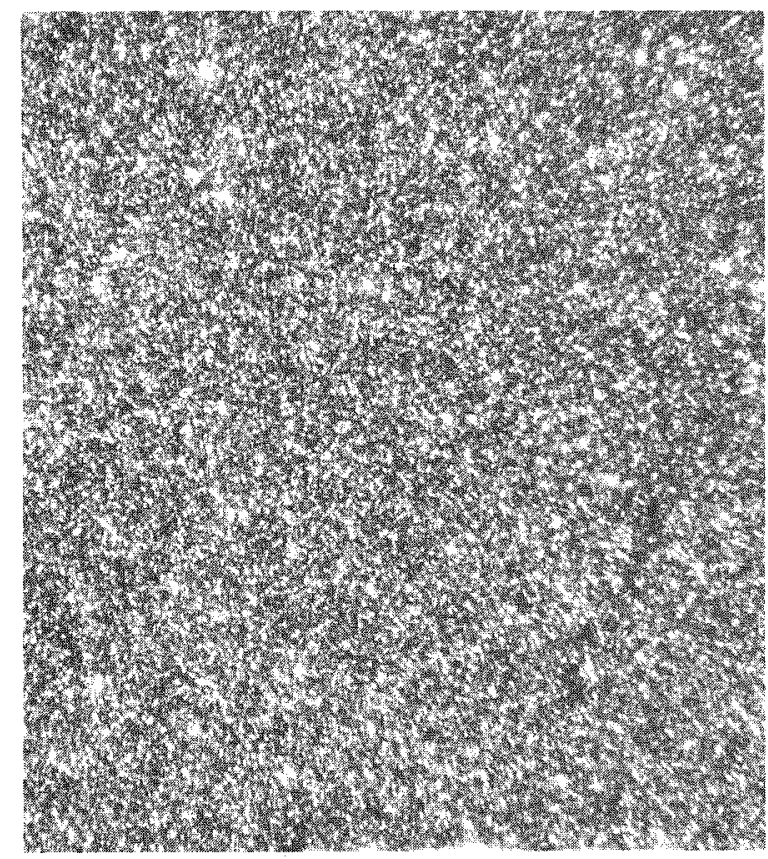

Inside

$100 \mathrm{X}$

(c) Severe Attack

(a) Control

(b) Intermediate Attack

Fig. 2 Comparison of control sample (a) to posttest samples with intermediate attack (b) and severe attack (c). 

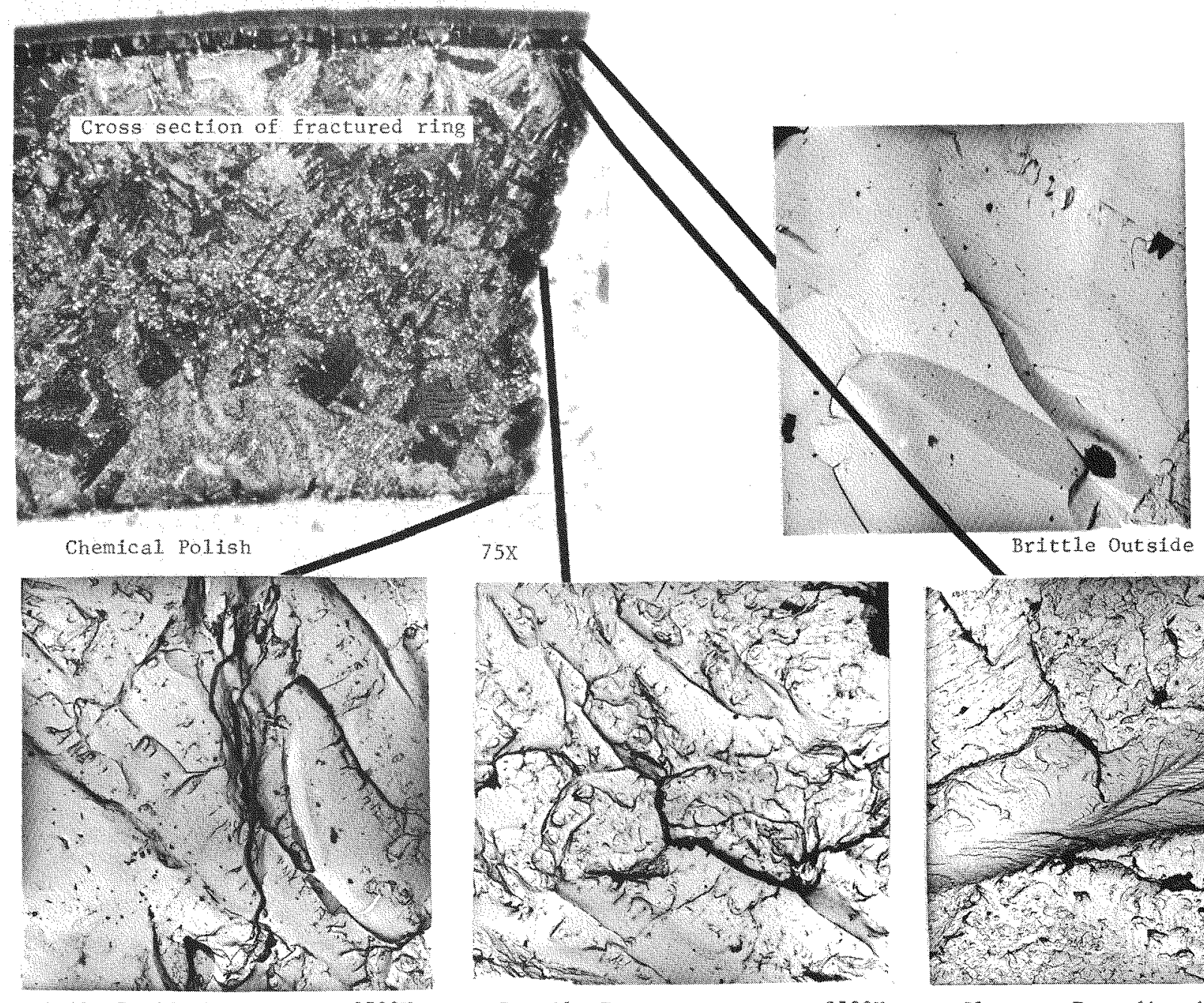

Brittle Inside Zone

$2500 \mathrm{x}$

Ductile zone

$2500 \mathrm{x}$

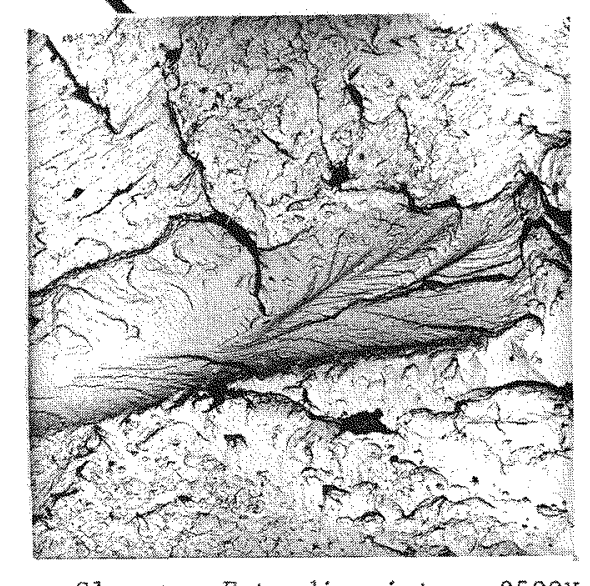

Cleavage Extending into

$2500 \mathrm{x}$

Fig. 3 Electron microscove fractography of the ring compression sample from Rod 12 at the $6 \mathrm{ft}$ elevation. This location had a 7 to $19 \%$ ring compression to fatiure and a $\sqrt{D_{\alpha} t}$ of 2.99 $10^{-3}$. 


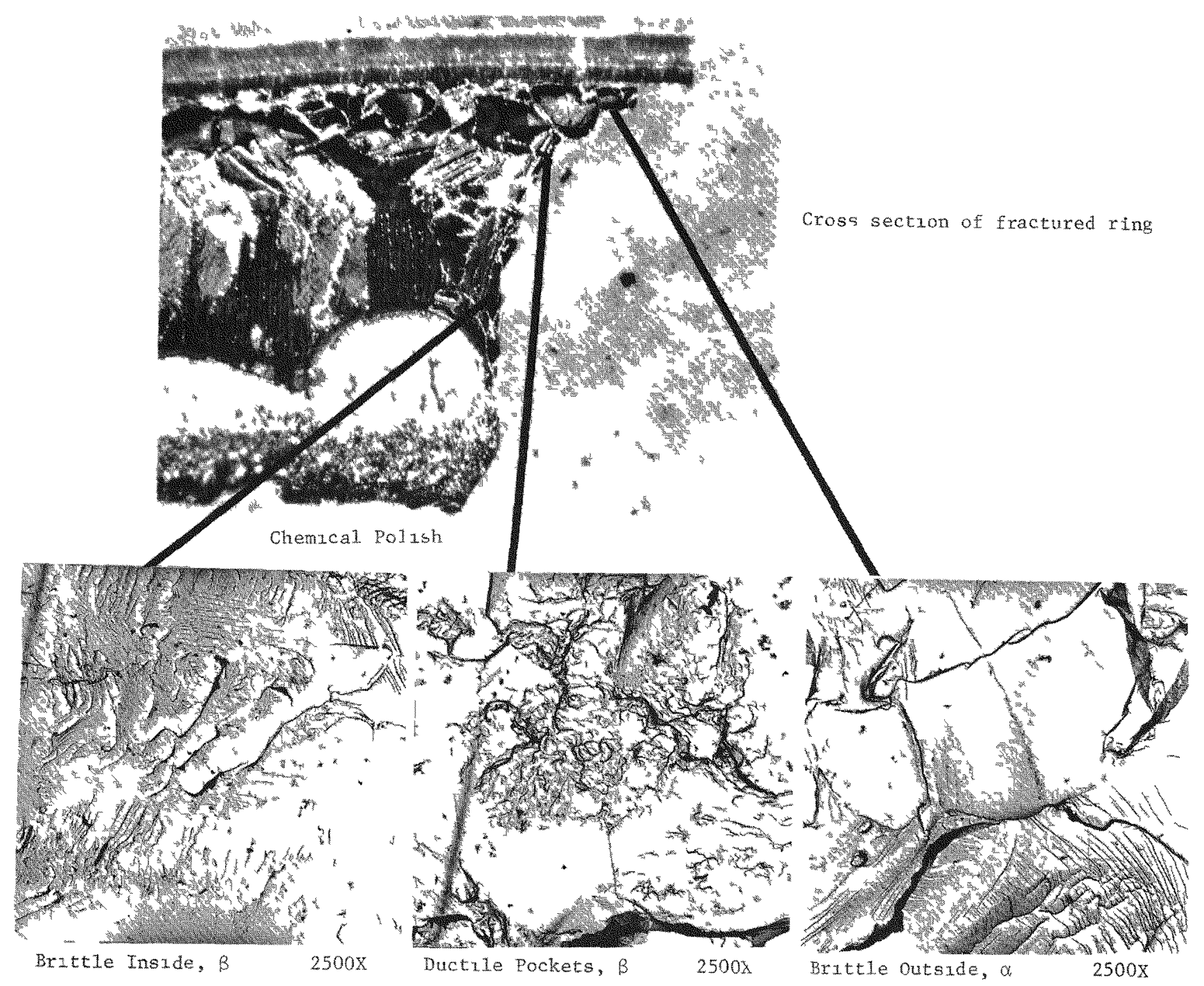

Fig. 4 Flectron microscope fractography of the rung compression sample from Rod 27 at the 6 ft elevation. This location had a $3 \%$ ring compression to fallure and a $\sqrt{D}$ t of $477 \times 10^{-3}$ (rod was perforated). 


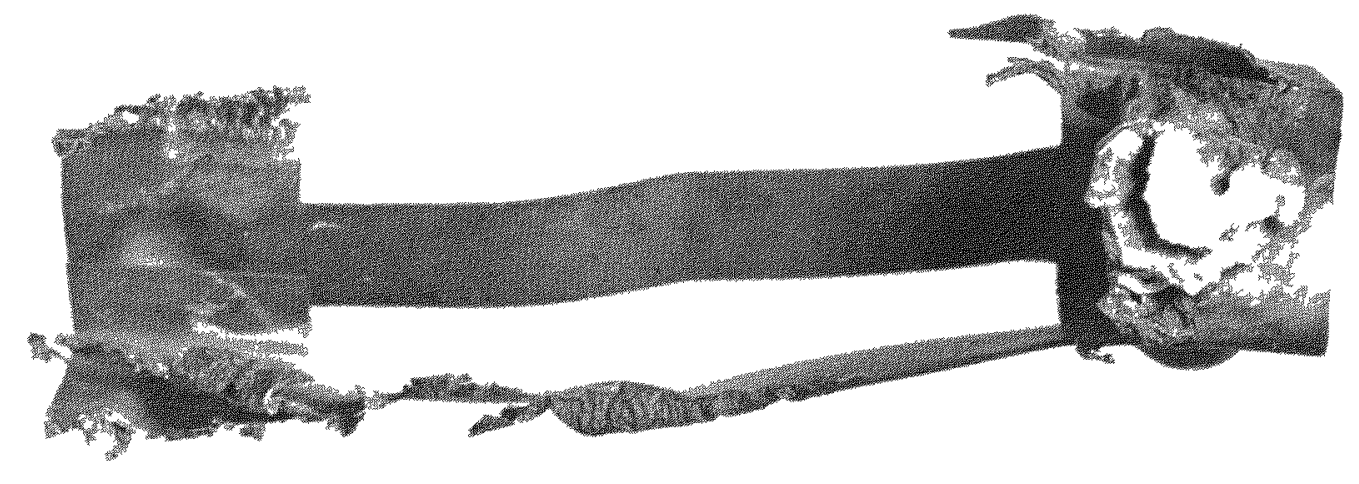

Fig. 5 Photograph of "1antern spring" after Test $\mathrm{Zr}-3$. 


\section{METALLURGICAL SUPPORT FOR PWR-FLECHT}

\section{J. Graber}

A metallurgical examination was performed on fuel rods from two test runs ( 8874 and 9573 ) of the PWR-FLECHT tests. These fuel rods were Zircaloy-4 rods with boron nitride insulation and an inner heating coil of Kanthal A-1 (5.5\% A1, $22 \% \mathrm{Cr}$, and $0.5 \%$ cobalt with a M.P. of $2700^{\circ} \mathrm{F}$ ). The attached chromel-alume1 wire thermocouples were sheathed with 40 mil stainless steel containing $\mathrm{MgO}$ insulation. It was required to determine if the $\mathrm{BN}$ is compatible with the Zircaloy from test 8874 , to determine oxide thickness at three TC locations from test 9573 and to characterize a crystalline slag formation.

A summary of the scope and results of the work is given in Table I. It is concluded from this limited examination that (1) BN did not present an incompatibility problem with Zircaloy-4 during this test, (2) the melting point of Zircaloy-4 may have been reached, (3) large amounts of oxygen were not present in the $\alpha$ zirconium layer, and (4) there is no evidence that Inconel (possible spacer material) or Kanthal contributed to the Zircaloy damage. 
TAB LE I

SUMMARY OF MATERIALS EXAMINATION FOR PWR-FLECHT TESTS 8874 AND 9573

\begin{tabular}{|c|c|c|c|c|c|c|c|}
\hline Test & $\underline{\text { Rod }}$ & Mount & $\begin{array}{c}\text { Sample } \\
\text { Location } \\
\end{array}$ & $\begin{array}{c}\text { Sample } \\
\text { Description }\end{array}$ & $\begin{array}{l}\text { Purpose of } \\
\text { Examination } \\
\end{array}$ & Results in Brief & $\begin{array}{l}\text { Mils-0xide } \\
\text { Thickness } \\
\end{array}$ \\
\hline 8874 & $5 B$ & 419 & Fragmented End & $\begin{array}{l}\text { Inside } \\
\text { Erosion }\end{array}$ & $\begin{array}{l}\text { Zircaloy-BN } \\
\text { Compatibility }\end{array}$ & $\begin{array}{l}\text { No } B \text { or } N \text { Found - } \\
\text { Sma11. Inside a layer }\end{array}$ & \\
\hline 8874 & $5 B$ & 420 & $\begin{array}{l}1 / 4^{\prime \prime} \text { from } \\
\text { Fragmented } \\
\text { End }\end{array}$ & $\begin{array}{l}\text { BN in con- } \\
\text { tact with } \\
\text { Zircaloy }\end{array}$ & $\begin{array}{l}\text { Zircaloy-BN } \\
\text { Compatibility }\end{array}$ & $\begin{array}{l}\text { No } B \text { or } N \text { Found - } \\
\text { No } \alpha \text { Layer Found on ID } \\
\text { Low O in OD } \alpha\end{array}$ & \\
\hline 9573 & $4 \mathrm{E}$ & 424 & $\mathrm{TC}$ & $\begin{array}{l}\text { One Layer } \\
\text { Oxide } \\
\text { Peeled }\end{array}$ & $\begin{array}{l}\text { Oxide Thick- } \\
\text { ness }\end{array}$ & & 0.75 \\
\hline 9573 & $4 E$ & 421 & $2^{\prime \prime}$ Above TC & Oxide \& S1ag & $\begin{array}{l}\text { Oxide Thick- } \\
\text { ness, Slag } \\
\text { Characteri- } \\
\text { zation }\end{array}$ & $\begin{array}{l}\text { No Kanthal constituents } \\
\text { or } \mathrm{Ni} \text { Found in Slag, } \mathrm{Zr} \\
\& \mathrm{O} \text { Were Found. } \alpha \text { has } \\
\text { Low } \mathrm{O} \text { content. }\end{array}$ & 2.25 \\
\hline 9573 & $2 \mathrm{E}$ & 422 & TC & & $\begin{array}{l}\text { Oxide Thick- } \\
\text { ness }\end{array}$ & & 1.75 \\
\hline 9573 & $4 F$ & 423 & TC & & $\begin{array}{l}\text { Oxide Thick- } \\
\text { ness }\end{array}$ & & 1.75 \\
\hline
\end{tabular}


METALLURGICAL EXAMINATIONS OF STAINLESS STEEL SAMPLES FROM THE WASTE CALCINING FACILITY

R. R. Hobbins, B. G. Carlson, N. A. DePue, J. K. Cranda11

The Waste Calcining Facility at the Idaho Chemical Processing Plant is a fluidized bed calciner for converting high radioactive aqueous waste into granular solids, Heat is supplied to this calciner by circulating $\mathrm{NaK}$ at $760^{\circ} \mathrm{C}$ through a bayonet heat exchanger. As part of an over-all surveillance program on this loop, service tests have been conducted on stainless steel components to determine the effects of the extended time, high temperature exposure to Nak. Such an examination had been made and reported[1] earlier for a service time of 16,300 hours. More recently, metallurgical tests were performed in support of a similar examination after a total exposure of 32,600 hours. These metallurgical results have been included in a paper presented at the 1971 High Temperature Corrosion Symposium [2], and will only be summarized here.

Table I contains tensile test data on 316 stainless steel from the NaK loop of the Waste Calciner. These data show that the 316 stainless steel has lost considerable ductility after 16,300 hours exposure, but doubling the exposure caused little further change in tensile properties.

Tensile data for 304L stainless steel are presented in Table II. Comparison with published data for unexposed material indicates that the tensile properties of the $304 \mathrm{~L}$ have been changed very little by 15,600 hours exposure to $\mathrm{NaK}$ at $760^{\circ} \mathrm{C}$.

The 318 stainless steel data are also presented in Table II. In this material, 32,600 hours service in the Waste Calciner caused strength increases of about $40 \%$ and elongation decreases up to $90 \%$.

The embrittlement displayed by the 316 and 318 stainless steels is attributed to the presence of sigma phase (up to 15\% observed metallographically).

It has been concluded ${ }^{[2]}$ that types $304 \mathrm{~L}, 316$, and 318 stainless steel alloys performed in a satisfactory manner as components of the Waste Calcining Facility.

[1] T. L. Hoffman, R. A. Moen, and K. C. Sumpter, "How Sodium Potassium Eutectic Alloy Affects Type 316 Stainless Stee1," Materials Protection 7, 35 (1968). 
[2] T. L. Hoffman, "Performance of Type 316 Stainless Steel in Sodium-Potassium Eutectic Alloy During 32,600 Hours Service," 1971 High Temperature Corrosion Symposium, National Association of Corrosion Engineering (March 1971). 


\section{TABLE I}

TENSILE PROPERTIES OF TYPE 316 STAINLESS STEEL

\begin{tabular}{ccc} 
Tensile Strength & Yield Strength & Elongation \\
(ksi) & $0.2 \%$ offset & in 2 in. \\
& $(\mathrm{ksi})$ & $(\%)$ \\
\hline
\end{tabular}

Published Values[a]

$\begin{array}{rrrr}22{ }^{\circ} \mathrm{C} & 90 & 40 & 60 \\ 500^{\circ} \mathrm{C} & 70 & 30 & 50 \\ 760^{\circ} \mathrm{C} & 35 & 25 & 40\end{array}$

First $16,300 \mathrm{Hr}$

NaK Service[b]

$\begin{array}{rrrl}22^{\circ} \mathrm{C} & 96 & 51 & 30 \\ 760^{\circ} \mathrm{C} & 36 & 15 & 14-16\end{array}$

Last $16,300 \mathrm{Hr}$

NaK Service

$\begin{array}{rrrr}22^{\circ} \mathrm{C} & 107 & 58 & 23 \\ 500^{\circ} \mathrm{C} & 76 & 48 & 11\end{array}$

$36,600 \mathrm{Hr}$

NaK Service

$\begin{array}{rrrr}22^{\circ} \mathrm{C} & 98 & 56 & 25 \\ 500^{\circ} \mathrm{C} & 70 & 45 & 11\end{array}$

[a] ASM Handbook, Vo1. 1, 8th Ed. pp. 503-504.

[b] T. L. Hoffman, R. A. Moen, and K. C. Sumpter, "How Sodium Potasium Eutectic Alloy Affects Type 316 Stainless Stee1," Materials Portection 7, p. 35 (1968). 


\section{TABLE II}

TENSILE PROPERTIES OF TYPES 304L AND 318 STAINLESS STEEL

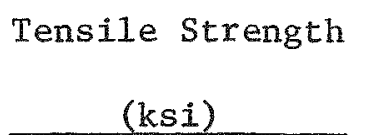

[a]

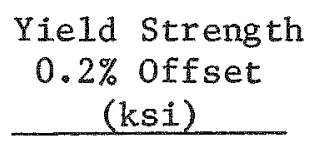

Elongation

in 2 in.

(\%)

Published Values

$304 \mathrm{~L}$

$$
760^{\circ} \mathrm{C}
$$

27

18

35

318

$$
22{ }^{\circ} \mathrm{C}
$$

87

70

50

50

47

40

$15,600 \mathrm{Hr}$

NaK Serwice

$304 \mathrm{~L}$

$$
760^{\circ} \mathrm{C}
$$

23

17

43

$32,600 \mathrm{Hr}$

NaK Service

318

$$
\begin{aligned}
& 22^{\circ} \mathrm{C} \\
& 500^{\circ} \mathrm{C}
\end{aligned}
$$

116

70

5

99

70

5

[a] ASM Handbook, Vo1. 1, 8th Ed. pp. 503-504. 
METALLOGRAPHIC EXAMINATION OF BORATED STAINLESS STEEL CORROSION COUPONS

B. G. Carlson

Two coupons made of type 304 stainless steel containing boron had been immersed in the H-130 thermo-syphon evaporator for 27.2 months for purposes of comparing the corrosion rate of the grid with unexposed material. This is a uranium concentrating process evaporator containing a uranium nitrate-nitric acid solution operating at $185^{\circ} \mathrm{F}$. Metal loss during the last seven months of operation was $1.5 \mathrm{mils} / \mathrm{month}$. The boron addition was needed for criticality control in the vessel so it was desirable to know if the metal loss was uniform or a preferential leaching away of the boron.

Cross sections from the two coupons were compared with a control specimen which had not seen any service. Micrographs of the three sections (Figures 1, 2, and 3) when compared did not reveal any localized corrosion effects on the boron. 


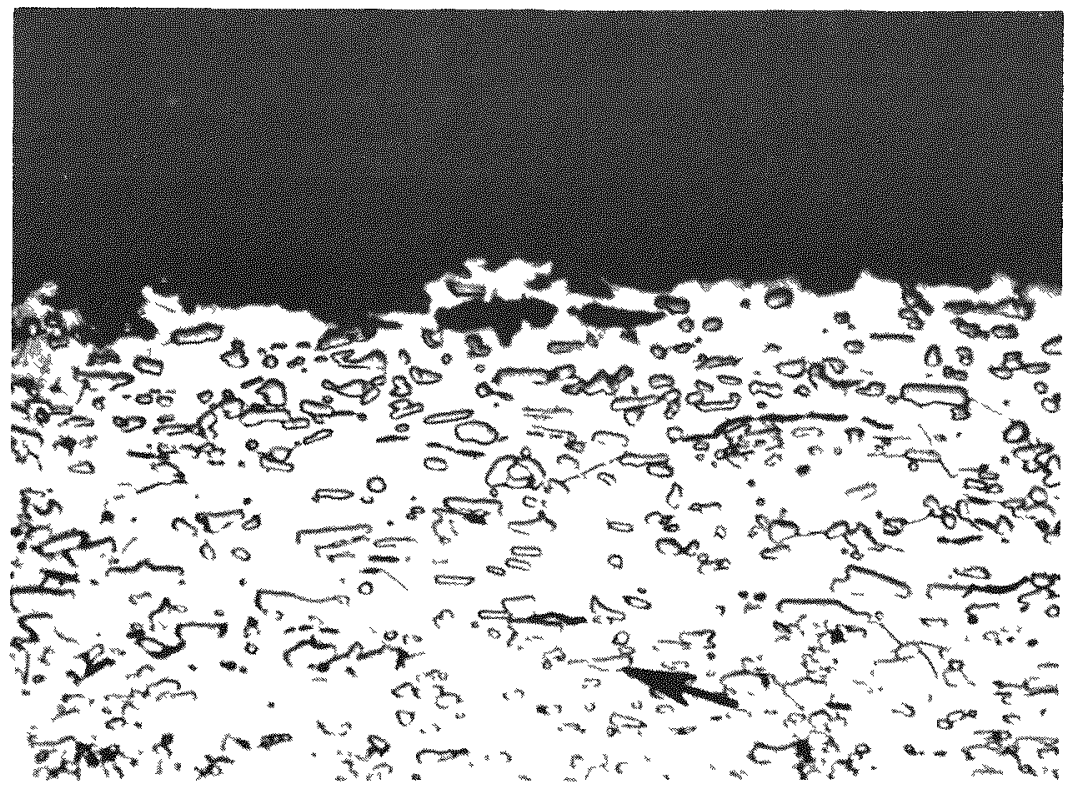

Oxalic Etch

$500 \mathrm{x}$

Fig. 1 Coupon $444-27.2$ months exposure. Arrow indicates boron bearing compound.

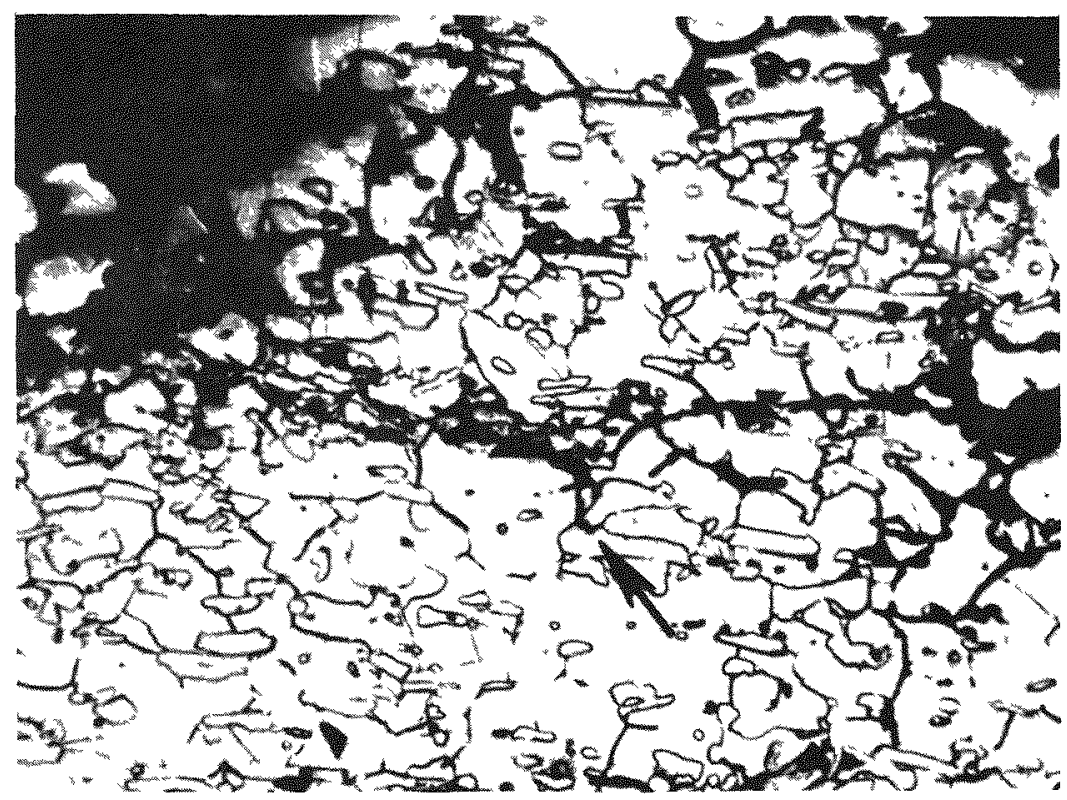

Oxalic Etch

$500 \mathrm{x}$

Fig. 2 Coupon 446 - 27.2 months exposure in uranium nitrate nitric acid solution. 


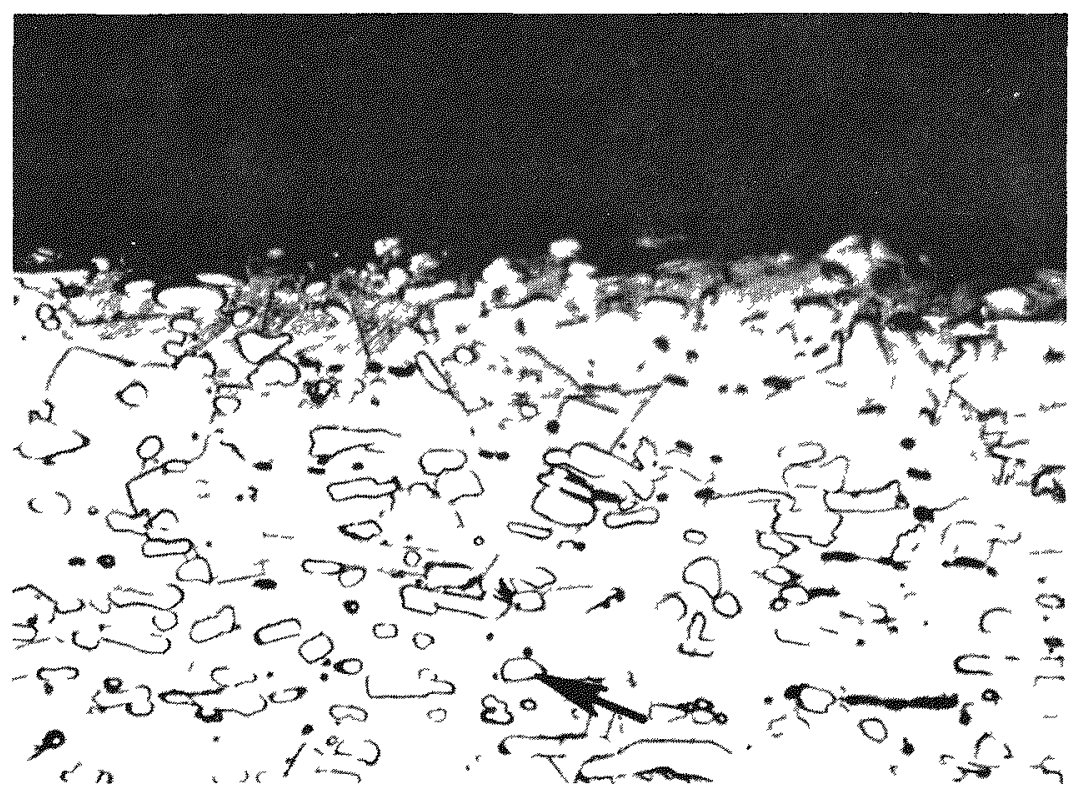

Oxalic Etch

$500 \mathrm{x}$

Fig. 3 Control 非690. Typical surface and structure from unexposed boron type 304 stainless steel. 
•

- 
D. TEST REACTOR OPERATIONS SUPPORT 
-

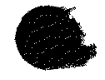




\section{MEASUREMENT OF $\beta / \ell$ IN THE MTR PHOENIX CORE}

J.A. Close

The cross power spectral density (CPSD) of neutron noise in the MTR was measured several times during the Phoenix. Fuel Experiment to detect changes in $B / \ell$ (the effective delayed neutron fraction divided by the prompt neutron lifetime) with conversion and burnup of the plutonium fuel. It was important to be aware of any variation of this ratio since an increase in $\beta / \ell$ may allow a greater energy release during a power excursion.

The signals from two neutron detectors were recorded on magnetic tape and were subsequently processed on a Time Data 100 Signal Analyzer. At the beginning of core life $\mathrm{B} / \mathrm{l}$ was $9.23 \pm 0.1 \mathrm{~Hz}$. During the experiment several other measurements were made, but resonances (attributed. to vibrating flux wands) in the neighborhood of the $\beta / l$ break point masked the desired data. There was, however, sufficient information to determine that $\beta / \ell$ was not increasing significantly.

The end-of-core-life measurement was different in that it occurred in a "dirty" core. Since the high gamma field tended to saturate the high efficiency ${ }^{3} \mathrm{He}$ chambers used in earlier measurements, less efficient fission chambers were employed as neutron detectors. Also, the large inherent neutron source in the core caused the effective multiplication constant $(\mathrm{k})$ of the reactor to be less than unity at low operating power. Operation at a higher power would have obviated this effect but would also have caused greater departure from the zero-power spectrum. As a compromise, the reactor power chosen for the measurement was $10 \mathrm{~kW}$.

After 921 MWd of operation (the end of core life) the break frequency of the CPSD did not coincide with $\beta / \ell$ due to the subcritical condition of the reactor. The relationship between break frequency and $\beta / \ell$ obtained from Uhrig[1] is:

$$
\omega_{b}=\frac{I-k(1-\beta)}{\ell}
$$

where $\omega_{b}$ is the measured break frequency. The ratio $\beta / \ell$ can be found from the equation if good estimates of $\mathrm{k}$ and $l$ are available. The $\mathrm{k}$ of the reactor was interpolated from experimental data to be 0.99986 at $10 \mathrm{~kW}$ and the effective delayed neutron fraction $B$ was calculated for 1000 MWd burnup to be 0.0029 . Using these values for $k$ and $\beta$ and using the break frequency from the CPSD, the above equation yields an estimate of the prompt neutron lifetime. Having now obtained values for $k$ and $l$, the equation can be solved for $\beta / l$. The estimated error ( $1 \sigma$ ) in determining the difference between the break frequency and $\beta / l$ is \pm 0.13 percent. This is small compared to the estimated experimental error of \pm 5.8 percent in determining $\omega_{b}$. The end-of-core-life $\beta / l$ was found to be $8.8 \pm 0.5 \mathrm{~Hz}$. 
Although the errors were larger than desired, the determination of the end-of-core-life $B / \ell$ by the CPSD technique was effective for our application. It should be noted that the major contribution to the uncertainty was due to poor resolution of the break frequency of the CPSD and not due to the estimation of $l$.

REFERENCES

[1] Uhrig, Robert E., "Measurement of Reactor Shutdown Margin by Noise Analysis", AEC Symposium Series, No. 2 (TID-7662), pp 1-26, 1964 . 
One of the prime considerations in the recent updating of the ETR safety analysis was the feedback reactivity due to water displaced by thermal expansion during a power excursion. To check the validity of feedback calculations, the feedback was measured for a simple step change in power at four different times in two cores during ETR Cycle 110. The results are presented in Table I below.

\section{TABLE I}

MEASURED ETR POWER COEFFICIENTS OF REACTIVITY

\begin{tabular}{lllll} 
Date & $1-15-71$ & $2-3-71$ & $2-19-71$ & $3-11-71$ \\
MWd equivalent of fuel depletion & 350 & 3000 & 5650 & 4470 \\
Average withdrawal of rods 7, & 18.0 & 13.0 & 23.5 & 24.5 \\
$\quad$ 9, and 14 (in.) & & & & \\
Inlet water temperature ( $\left.{ }^{\circ} \mathrm{F}\right)$ & 110 & 110 & 110 & 111 \\
Initial power (MW) & 175 & 171 & 171 & 175 \\
Power change (MW) & -17.5 & -24.5 & -22.8 & -21.0 \\
Power coefficient ( $\mu \mathrm{k} / \mathrm{MW})$ & 14.5 & 22.7 & 19.1 & 19.9 \\
\hline
\end{tabular}

Reactor power was under automatic control throughout the tests. Power was decreased by reducing the reference voltage supplied to the ETR servo controller. The power was reduced in an approximately linear ramp requiring about 5 to 10 seconds to execute. The servo system then maintained the power constant at the new power.

The reactor power was measured by two special neutron sensitive channels placed in spare thermopile thimbles, one on the north side and one on the south side of the core. A reactivity meter was attached to a third special neutron sensitive channel installed in another spare thermopile thimble on the north side of the core. The reactivity meter computed reactivity from changes in neutron flux by an inverse analog solution of the basic neutron balance equation with six delayed neutron groups. An auxilliary regulating rod position recorder with high chart speed was used to provide time resolution of the regulating rod movements. Inlet water temperature was monitored by the standard bulkwater temperature recorder.

The basic equation describing the experiment is the reactivity balance equation

$$
\rho(\text { net })=\rho(\text { rod })+\rho(\text { xenon })+\rho(\text { inlet })+\rho(\text { power }),
$$

where $\rho$ (net) is the net reactivity

$\rho$ (rods) is the reactivity worth of the regulating rod movement from its initial position 
$\rho(x e n o n)$ is the reactivity worth of the xenon change from its initial, equilibrium value

$\rho$ (inlet) is the reactivity introduced by the change in inlet water temperature from its initial value

$\rho$ (power) is the reactivity introduced by the power change. This is the reactivity value being sought in the test.

The feedback reactivities, $\rho$ (xenon), $\rho$ (inlet), and $\rho$ (power), depend upon the power history, which was in turn controlled by $\rho$ (rod). Within less than one second after the power was stabilized, $\rho$ (power) was a constant whose value could be determined by evaluating the other reactivities at any convenient time. The time chosen was when the regulating rod returned to its initial position, at which time $\rho$ (rod) became zero.

The value of $p$ (net) was provided by the reactivity meter. The value of $\rho$ (inlet) was determined by multiplying the previously measured bulk water temperature coefficient, $-25.8 \mu \mathrm{k} / \mathrm{F}$, by the change in inlet bulk water temperature observed at the time when the regulating rod returned to its initial position. The value of $\rho$ (xenon) was calculated using the equation

$$
\rho(\text { xenon })=A \frac{\left(P_{0}-P_{1}\right) T \phi^{3} u d v}{\phi u d v}
$$

where $T$ is the elapsed time from the midpoint of the power reduction until the regulating rod returned to its initial position

Po is the initial steady-state power

$P_{1}$ is the ending steady-state power

$u$ is the burnup corrected ${ }^{235} \mathrm{U}$ density

$A$ is a constant

$\phi$ is neutron flux as a function of space.

The measured power coefficients were somewhat larger than power coefficients calculated by the model used in the ETR safety analysis, indicating a comfortable conservatism in the analysis. The variation of the power coefficient with rod withdrawal observed in the measured results was predicted by the calculational model and results from the vertical flux skewing that accompanies partial withdrawal of a rod gang. 


\section{ETR BULK WATER TEMPERATURE COEFF ICIENT}

$$
\text { D. W. Knight }
$$

The ETR bulk water temperature coefficient (BWTC) has been measured to provide data to analyze a "cold water accident" at the ETR.

The BWTC is a function of the temperature of the primary coolant. At a water temperature of $100^{\circ} \mathrm{F}$ the $B W^{T C C}$ is approximately $-0.0024 \Delta \mathrm{k} / \mathrm{k} /{ }^{\circ} \mathrm{F}$. The BWTC was measured by increasing the temperature of the ETR primary coolant and measuring the resultant reactivity change. The heat generated from the primary coolant pumps was used to increase the temperature from $61.5^{\circ} \mathrm{F}$ to $120^{\circ} \mathrm{F}$. The reactor was operated in servo control during the temperature increase. The reactivity effect of increasing the temperature was determined from changes in the position of the regulating rod which was previously calibrated in terms of reactivity by using standard period in hour techniques. Figure 1 shows the regulating rod calibration curve, while Figure 2 shows the measured change in the compensating reactivity (introduced by the regulating rod) as the temperature was increased.

The change in reactivity from a reference temperature (100 F) may be described as:

$$
\begin{aligned}
\rho(\%)=-2.5773 \times 10^{-3} & (\mathrm{~T}-100)-3.7677 \times 10^{-5}(\mathrm{~T}-100)^{2} \\
& +1.9784 \times 10^{-7}(\mathrm{~T}-100)^{3}+7.0207 \times 10^{-9}(\mathrm{~T}-100)^{4}
\end{aligned}
$$

where $T=$ the inlet water temperature $\left({ }^{\circ} F\right)$

$$
\rho=\text { reactivity change (in } \% \text { ) resulting from changing the tempera- }
$$
ture from $100^{\circ} \mathrm{F}$ to $\mathrm{T}$.

This function fits the measured data points with a standard deviation of $0.001177 \rho(\%)$. The bulk water temperature coefficient which is the derivative with respect to temperature of the above function may be described as:

$$
\begin{aligned}
\frac{d \rho}{d T}=\text { BWTC }-2.5773 \times 10^{-3}-7.5354 \times 10^{-5}(\mathrm{~T}-100)+ & 5.9352 \times 10^{-7}(\mathrm{~T}-100)^{2} \\
& +28.08 \times 10^{-9}(\mathrm{~T}-100)^{3}
\end{aligned}
$$

where $\mathrm{T}=$ the inlet water temperatore $\left({ }^{\circ} \mathrm{F}\right)$.

The BWTCS of the ETR at three temperatures are shown in Table I.

$$
\text { TABLE I }
$$

ETR BULK WATER TEMPERATURE COEFFICIENT

\begin{tabular}{ccc}
\hline Primary Coolant Temperature & $\begin{array}{c}\text { Temperature Coefficient } \\
(\%)\end{array}$ \\
\cline { 1 - 2 }$(\%)$ & & $-0.54 \times 10^{-3}$ \\
100 & $-2.58 \times 10^{-3}$ \\
120 & $-3.60 \times 10^{-3}$ \\
\hline
\end{tabular}




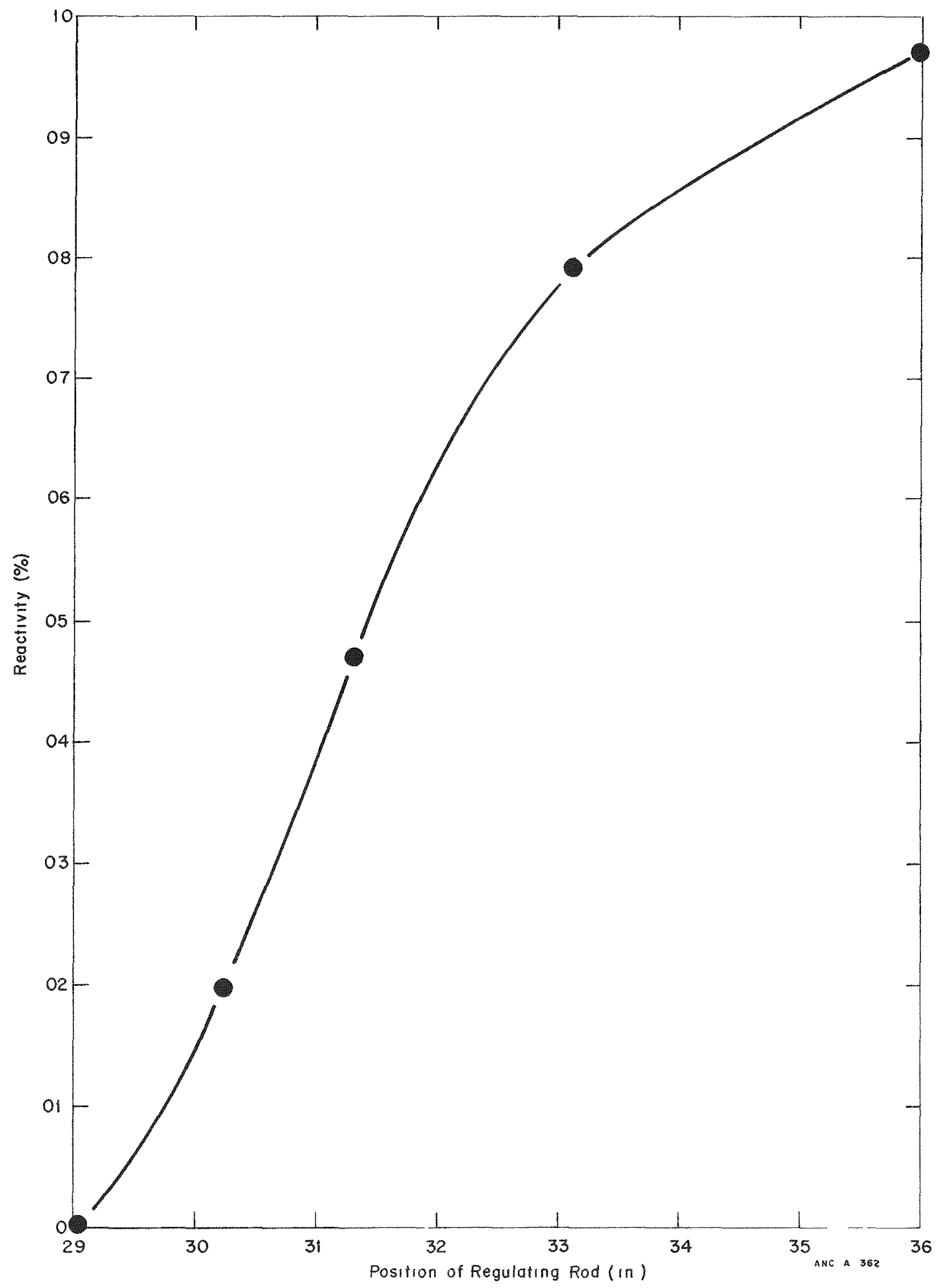

Figure 1 Calibration curve of ETR regulating rod (shim rod No. 10). 


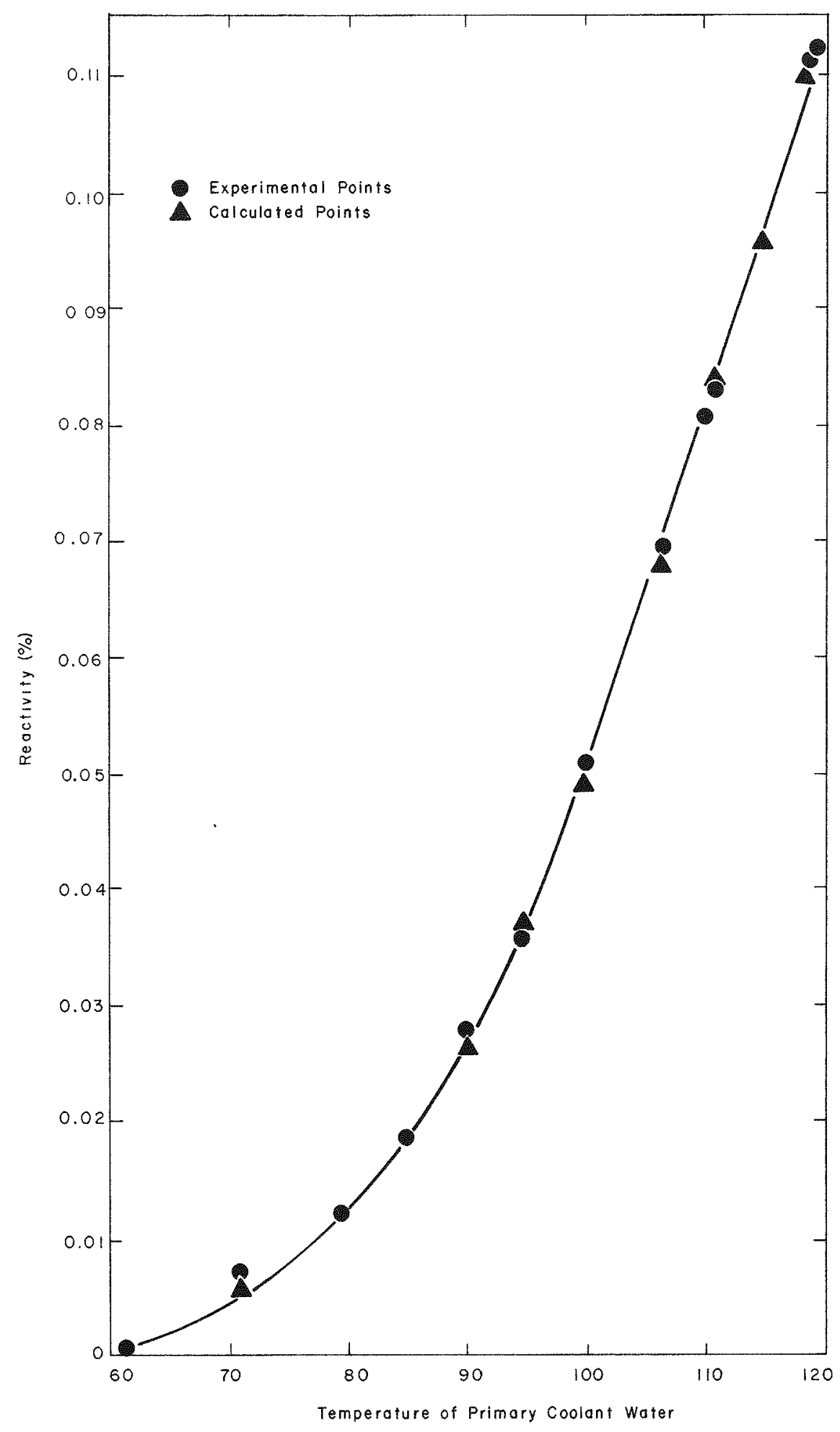

$A N C-B-333$

Figure 2 Reactivity change as a function of the temperature of ETR primary coolant water. 


\section{ATR AND ETR INSTRUMENTATION UPGRADE}

N. C. Kaufman, E. E. Burdick

During FY 171 a concerted effort was begun to upgrade the control and protective instrumentation at the ETR and ATR. This effort, involving personnel from several INC organizations, was directed by the Reactor Technology Branch. The goals of this effort were fourfold: (a) to reduce reactor lost time due to spurious power reductions, (b) to reduce required instrument maintenance time, (c) to eliminate operating problems caused by instrument readjustment, and (d) to correct known system deficiencies. The greatest emphasis was placed on the reduction of lost time at ATR due to spurious scrams, which during the first quarter of FY'7l caused an average loss of $630 \mathrm{MWd} / \mathrm{mo}$. As significant evidence of the success of this program, the average lost-time rate at ATR for the last quarter of FY' 71 had been reduced to $7 \mathrm{MWa} / \mathrm{mo}$.

During FY 171 one major system modification was completed, a second, major modification was partially completed, and several major modifications were begun. The systems upon which these major efforts were performed were those most troublesome with respect to all four of the goals of the upgrade effort. As illustration of such efforts, two of the major changes will be considered.

A substantial modification of the ATR neutron level channels was completed and another begun in FY '71. This modification was needed because of excessive maintenance and spurious signals resulting from ion chamber connector and cable damage. That in turn was due to high radiation fields into which frequent repositioning placed the detector. Moreover, the potential existed to melt all cables simultaneously and was a major system design defect. To correct these problems, it was necessary to fix the detector position, to retain channel adjustability, to minimize channel response-time dependence on cable type, and to procure and install radiation and temperature resistant cables. The first three requirements were achieved by redesign of the level channel preamplifier (see "ATR Level Channel Modification", this report) and the fourth effort was begun with completion expected in FY 172.

A second major system modification, involving the ATR Lobe Power Monitoring Subsystem, was partially completed in FY '71. This modification was necessary due to a number of spurious power reductions due to noise pickup and difficulties in performing maintenance during reactor operation. Additionally, excessive system drift and recalibration problems were experienced, effecting system accuracy and requiring frequent maintenance and adjustment. To correct these problems the methods of recording signals, setting setpoints, and transmitting signals were significantly changed. With the required modifications only partially complete in FY' 71 , a significant reduction of spurious power reductions has already occurred. 
By far the greatest impact in terms of reducing spurious power reductions was made by the results of many small system changes. These were made as the result of intensive studies of problem channel modules and environmental testing of them. Several circuit deficiencies were found with respect to stability under verying temperature and input power conditions. Additionally, the intensive studies revealed several minor hardware failures not detected during routine maintenance. One additional outcome of these study efforts was an increased level of instrument surveillance which contributed to reduction of lost time by detecting incipient troubles before they caused operating problems.

The upgrade efforts described above will continue in FY '72. The major modifications begun in FY ' 71 will be completed, and new ones deriving from the studies thus far conducted will be begun. Moreover, the effort is expected to be expanded to improve standard maintenance methods and to develop proposals for upgrade of systems to current codes and standards. 


\section{ATR LEVEL CHANNEL MODIFICATION}

$$
\text { R. I. Copyak }
$$

Functional and electronic changes have been made in the preamplifiers in the neutron level channels which are a part of the ATR nuclear safety subsystem. Each neutron level channel consists of an ion chamber, an ion chamber power supply, a level preamplifier, and a sigma amplifier. Each sigma amplifier drives the sigma bus which in turn provides a common signal for the magnet current amplifiers for the safety rod electromagnets. Many functions were added to the level preamplifiers, including modification such that they will serve as ion chamber power supplies. To reduce costs, the modified units use the major modules of the original preamplifier units.

The modifications primarily provided the means (a) to perform all level channel adjustments presently performed by vertical movement of ion chambers and (b) to maintain the ion chambers and attached cabling near their full-power positions and thus in relatively low-radiation environments. Formerly the ion chambers were frequently inserted or withdrawn to maintain recorder indications at $\mathbb{N}_{F}$ at full power. These adjustments had been required because the channels are sensitive to regional power changes (eg, lobe power and fuel element power redistribution within lobes) and to position changes of the adjacent outer shims. Additionally, the ion chambers were often required to be inserted for scram recovery operations, and the required insertion for one channel was often quite different from another (during unbalanced power operations). This repositioning was required to maintain the setpoint at or below 1.2 times nominal full total power and 1.2 times nominal full lobe power. The required ion chamber adjustments in turn made excessive demands of the chamber drive systems relative to their original design bases. The new preamplifier system, capable of performing the required channel adjustments, will thus eliminate the need for an expensive revamp of the chamber drives. Also, by maintaining the ion chambers and cabling near their full-power positions, the neutron and gamma environment is much less severe than for positions into which the chambers and cabling were formerly placed. Thus, the radiation damage to the connectors and cables, which was previously experienced, has been reduced with cost savings relative to replacement frequency.

The essential operational functions of the modified preamplifiers are listed below:

1. Three different preamplifier gains were included: high (for startup), intermediate, and normal (for full power).

a. High gain is always 100 times normal gain.

b. The ratio of intermediate gain to normal gain is adjustable from 1.0 to 2.0 . 
c. Transfer from high to intermediate gain (when the reactor is pressurized) and from intermediate to high gain occurs automatically at $10^{-3} \mathrm{~N}_{\mathrm{F}}$. (The 11-sec delay feature has been retained, )

d. In each channel, transfer from intermediate to normal gain can be made manually when the level channel reads greater than $0.70 \mathrm{~N}_{\mathrm{F}}$.

e. Transfer from normal to intermediate gain occurs automatically whenever the level recorder indication decreases to $0.35 \mathrm{~N}_{\mathrm{F}}$.

2. All three gains are simultaneously adjustable by $\pm 50 \%$ (exclusive of the variable ratio between intermediate and normal gains).

On an instrument basis, the design of the modified preamplifier is a considerable improvement over the design of the former preamplifier, although the power supply and operational amplifiers used and the layout of the mechanical chassis are the same. The new preamplifier was designed to be the same as or out-perform the original level preamplifiers and to serve as the ion chamber power supply. Significantly, the preamplifier response time was improved by increasing the overall frequency response of the unit and by providing a zero-input impedance. Because zero-input impedance was included, the operation of the preamplifier is independent of length or type of input signal cable, an important item when considering cable replacement.

The original requirement for a continuity monitor of the ion chamber signal and power cables is met in the new design by introducing a pulse at the output connector of the de power supplied to the ion chamber. This pulse, superimposed on the ion chamber signal, must return to and be detected within the electronics of the preamplifier. If this returning cable monitoring pulse is not detected, a reactor control room annunciator alarms. This type of cable monitoring has two advantages over the original type (which monitored only the connection of the cable shields): (a) it assures that the signal conductors are connected, and (b) it minimizes ground circuits, which can produce undesirable false signals.

The modified preamplifier is also superior to the existing unit in two other ways. The original type of low-voltage monitor of the ion chamber power supply is replaced by a comparator circuit which actuates the control room annunciator if a chamber power supply voltage becomes less than $270 \mathrm{Vdc}$. Additionally, protection has been added in the preamplifier circuits such that if the input signal shorts to the cable shell or to ground, the preamplifier will develop a voltage larger than any scram setpoint. This protection was accomplished by installing a thermocouple at the input of the first operational amplifier within the amplifier feedback loop. During normal operation, the thermocouple output is insignificant and cannot be detected. However, if the input shorts to ground or to the cable shell, the amplifier feedback is disabled and the amplifier must saturate to give a scram condition. 
In summary, the redesign of the preamplifier (made in accordance with applicable codes and standards) has improved electronic and functional operation of the preamplifier. This in turn has improved the operation of the level channels significantly. 


\section{EFFECTS OF INSTALIING A LARGE}

IN-PILE TUBE IN THE ATR

J. A. Close

A series of measurements was completed at the ATRC to evaluate the effects on core physics of installing a large in-pile tube (approximately 5 inches $0 . D_{.}$) in the ATR NE flux trap. The reactivity effect of inserting the large in-pile tube (IIPT) and its experiment varied from $-1.7 \$$ to $-3.0 \$$ (depending on shroud and fuel content of the experiment train) relative to the present flux trap loading. This relatively large negative reactivity effect severely depresses the fission rate in the fuel adjacent to the large test and shortens core life to the extent that xenon equilibrium cannot be obtained in a flat power division if the experiment is heavily shrouded. Core life can be extended by operating the NE lobe at reduced power; however, outer shims around other lobes must be withdrawn to compensate for the large negative reactivity of. the LIPT and core life is still reactivity limited, though not as severely.

Measurements of the void coefficient of reactivity in the LIPT were also performed for various shroud and fuel conditions. The effect of increasing the shroud poison density was to increase the void coefficient. For all cases considered the void coefficient was of the order of $+0.2 \phi /$ in $^{3}$. 
GAMMA HEAT MEASUREMENTS IN THE

LARGE IN-PILE TUBE EXPERIMENT MOCK-UP AT THE ATRC

J. C. Tappendorf

In support of the proposed large in-pile tube experimental program in ATR, gamma heat measurements were made in a mock-up of the large inpile tube experiment in the NE flux trap of the ATRC. These measurements were made using the thermoluminescent phosphor, $\mathrm{CaF}_{2}: \mathrm{Mn}$. These data showed that (1) the relative gamma heat distribution around the periphery of the in-pile tube was similar to the fuel element power distribution around that lobe, and (2) the absolute values of the gamma heat data around the in-pile tube after correction to equivalent ATR full power conditions are well corroborated by gamma heat values obtained during a. gamma heating test with thermocouples in ATR.

The technique used to measure gamma heat in the ATRC was to irradiate the CaF : Min glass-encapsulated dosimeters during a 20-minute flux run. These dosimeters were then read out on a thermoluminescent detector analyzer. The digital representation of the absorbed dose in the dosimeters was converted to watts/gram of aluminum based on calibration data, duration of the irradiation and energy absorption relationships for air and aluminum.

Figure 1 shows the relative distribution of the measured gamma heat values around the NE large in-pile tube and the relative fuel element power distribution around the NE Iobe. The agreement between these distributions was as expected because the primary gamma source for the in-pile tube is fissioning in the fuel elements.

A gamma heat value measured using CaF : Mn in the ATRC and corrected to ATR full-power conditions compared within $2 \%$ of an earlier gamma heat determination made in the ATR using a thermocouple. These gamma heat values in the ATR and ATRC were both obtained for the same position in the reactor with nearly identical environments. This comparison indicates the validity of the results of the gamma heating measurements in the ATRC using $\mathrm{CaF}_{2}: \mathrm{Mn}$. 


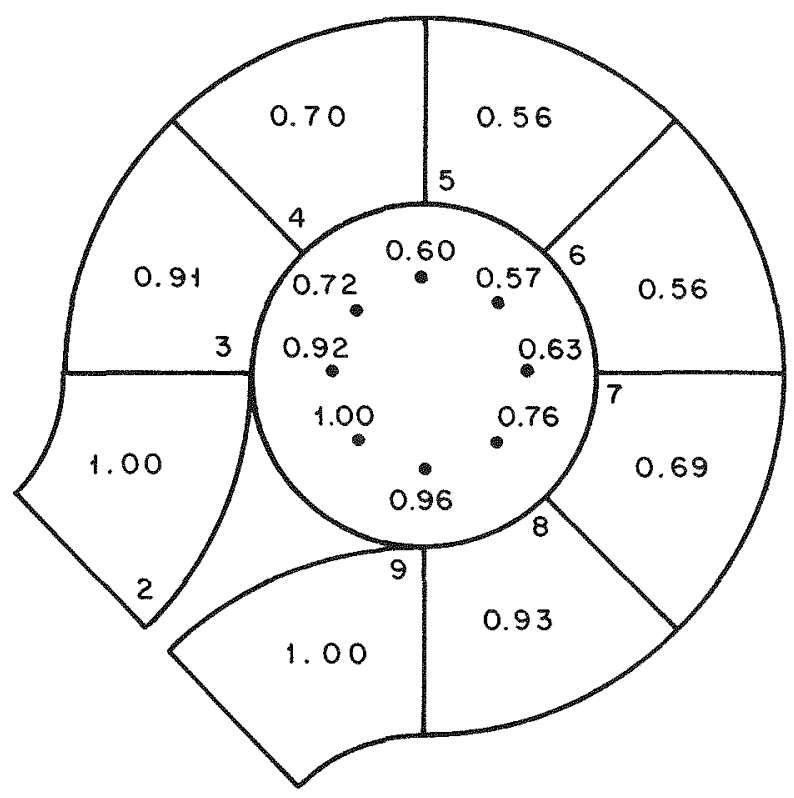

ANC-A- 209

Figure 1. Distribution of the measured relative gamma heating values around the periphery of the NE large in-pile tube (numbers in the center of the flux trap) and the relative fuel element power distribution around the $\mathbb{N E}$ lobe (numbers in the fuel element positions). 
K. A. Strong

The 100p temperature reactivity effect at ATR has been investigated and found to be a slowly varying function of the loop metal-to-water ratio as illustrated in Figure 1. Knowing the 100 p metal-to-water ratio and the anticipated loop temperature rise, the loop temperature reactivity effect for a given core loading can be predicted within an estimated 5 percent using the curves in Figure 1 .

During ATR Cycle $4 \mathrm{~A}$ startup, shim positions at criticality were noted with the six experiment loops at temperatures ranging from $103^{\circ} \mathrm{F}$ to $115^{\circ} \mathrm{F}$. A second set of shim positions at criticality was noted with the loops at operating temperatures ranging from $358^{\circ} \mathrm{F}$ to $573^{\circ} \mathrm{F}$. From the difference in the shim positions, a total loop temperature reactivity effect was determined. Using this measured total effect and data obtained during ATR startup testing for water-filled loops[1], the curves in Figure 1 were developed. As a test of the applicability of these curves, a total loop temperature effect was calculated for Cycle $2 \mathrm{C}$ for which a measured reactivity value had also been obtained. For this test case there was less than 2 percent difference between calculated and measured values.

The relationships expressed in Figure 1 provide a method of estimating the loop temperature reactivity effects which is based on actual measurements. This method is now being used routinely by ATRC personnel in providing support information for ATR core loadings.

\section{REFERENCES}

[1] N. C. Kaufman, et al, Reactor Physics Results From Low-Power Measurements In The Advanced Test Reactor, IN-1260 (February 1969) p 7 . 


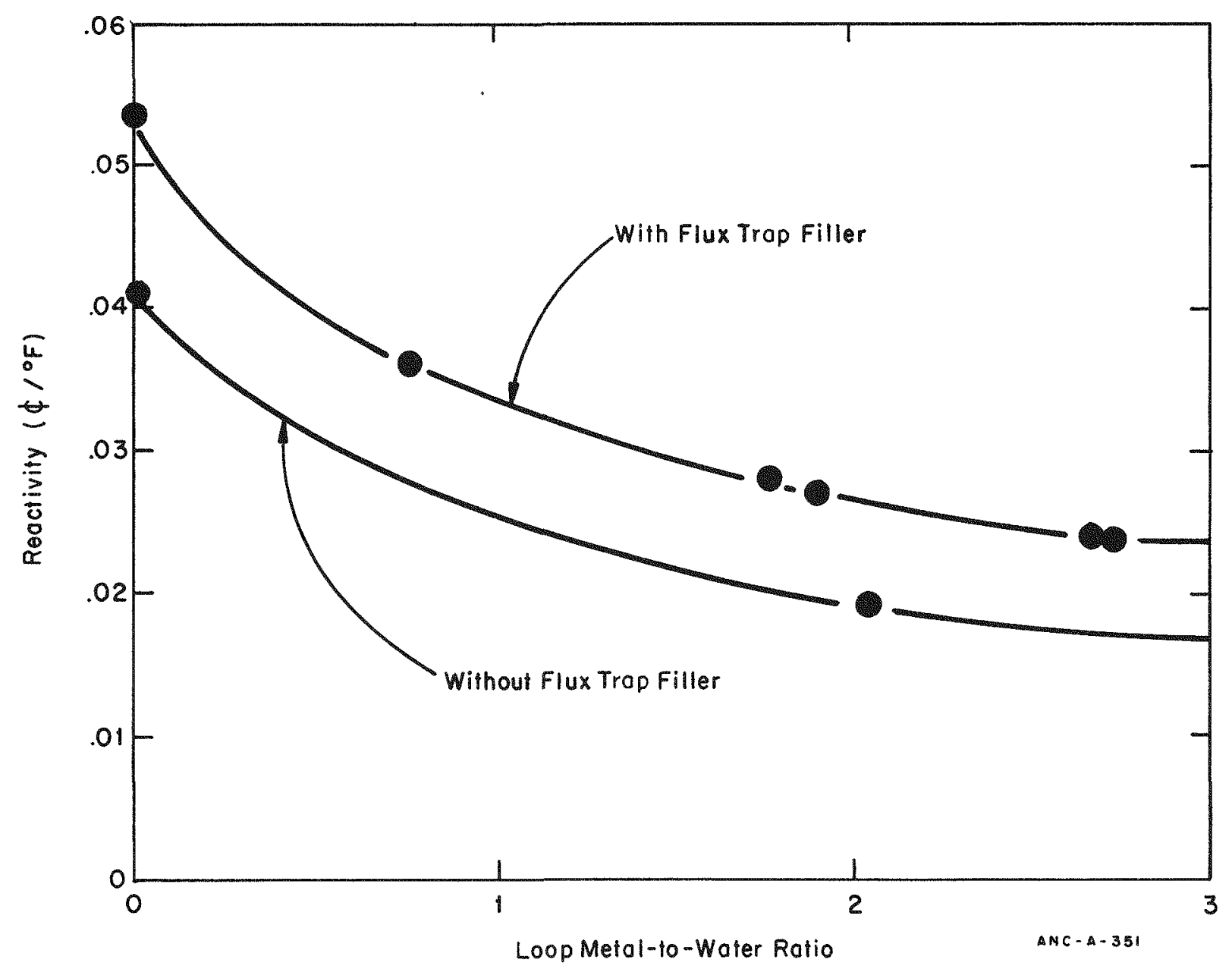

Figure 1 ATR loop temperature reactivity effect as a function of loop metal-to-water ratio. 


\section{PREDICTION OF SHIM POSITIONS \\ FOR CRITICAIITY FOR ATIB STABTTIPS}

P. R. Napper

Predicted shim positions for criticality are supplied to ATR Operations by ATRC personnel for all startups following changes or manipulations of the ATR core loading. These predicted shim positions are checked against actual shim positions for criticality as a separate check on the proper experiment and fuel element loading. The calculation of these predicted shim positions is based on a mock-up in the ATRC and/or previous operating history in ATR. Since some of the reactivity differences between the startup in question and the chosen base are time dependent, the ATR mid-cycle shim prediction program has been developed to facilitate calculations.

When the ATR loading contains partially spent fuel elements, shim positions from a previous ATR startup are used as the base for the calculation and the ATR Mid-Cycle Shim Prediction Program is used to calculate the differences. This program calculates xenon-samarium number densities on a lobe basis for each time increment specified and in the particular power division for the specified time increment. These number densities are then converted to reactivities, power-square weighted for the particular power division, and summed over the core. The reactivity effects of hafnium burnup and beryllium poisoning are then calculated assuming a fixed rate of change per MWd. These calculated reactivity values are then summed along with the reactivity effect of temperature changes resulting from inlet water and power level changes. This sum is then added to the initial shim positions specified in dollars of reactivity. This value, representing the new shim position in dollars, is then converted to degrees of outer shim rotation. This process is repeated for each specified time step to give a time dependent output of xenon poisoning, samarium poisoning, dollar value of calculated outer shim position and calculated outer shim position in degrees. This prediction program has been used to determine ATR shim positions for criticality for several cycles and has proven to be more accurate and faster than previous methods.

When the ATR loading consists of new fuel, the time-dependent computer calculation is not needed. Both the ATRC mock-up and a previous ATR startup are used as bases for two shim position calculations and the results are averaged. Corrections are made for temperature reactivity effects, power reactivity effects, hafnium burnup, beryllium poisoning and basic reactivity differences between the ATRC mock-up and the ATR loading. 
EFFECTS OF INSTALIING THE ATR

BEAM TUBE-RABBIT FACILITY

J. A. Close

During the past year it was proposed to install a combined beam tube-rabbit facility in the ATR west flux trap; consequently, measurements were performed in the ATRC to determine what effects, if any, the installation would have. A schematic representation of the beam tube mock-up used for the ATRC measurements is shown in Figure 1. The main tube of the mock-up was 4-3/8 inches in $O$. D. and had a 0.175-inch-thick 304 stainless steel wall. The compartments were separated by 1/4-inch steel plates. The upper compartment, the water gate, was the mechanism for "opening" and "closing" the beam tube.

The net reactivity effect of removing the gas loop dummy (now residing in the $A T R$ ) and installing the beam tube mock-up in the west flux trap was $-0.85 \$$. This had the effect of depressing the peak fission rate in fuel elements adjacent to the mock-up and also reducing the hot spots in fuel positions $27,28,33$ and 34 . Hence, the limiting hot spot of the core does not occur in this vicinity.

A principal concern was that the installation would significantly perturb the axial flux profile in adjacent flux traps. This was not the case, however, as the peak flux was displaced only an inch above core midplane. As far as detrimental effects on the reactor are concerned, the only apparent problem was brought about by the steel plate located six inches above core midplane. This plate along with the separation of air and water it causes, induces a relatively severe local axial fission rate gradient in plates $I$ and 2 of those fuel elements adjacent to the mock-up. This effect could probably be reduced by replacing the plate with a section of a paraboloid shell, installed with the concave surface down.

Another concern was the reactivity effect of displacing the air in the water gate with water during reactor operation. This action was shown to produce a reactivity change of $-0.01 \$$, a value well within the control capabilities of the ATR manual and automatic control systems. 


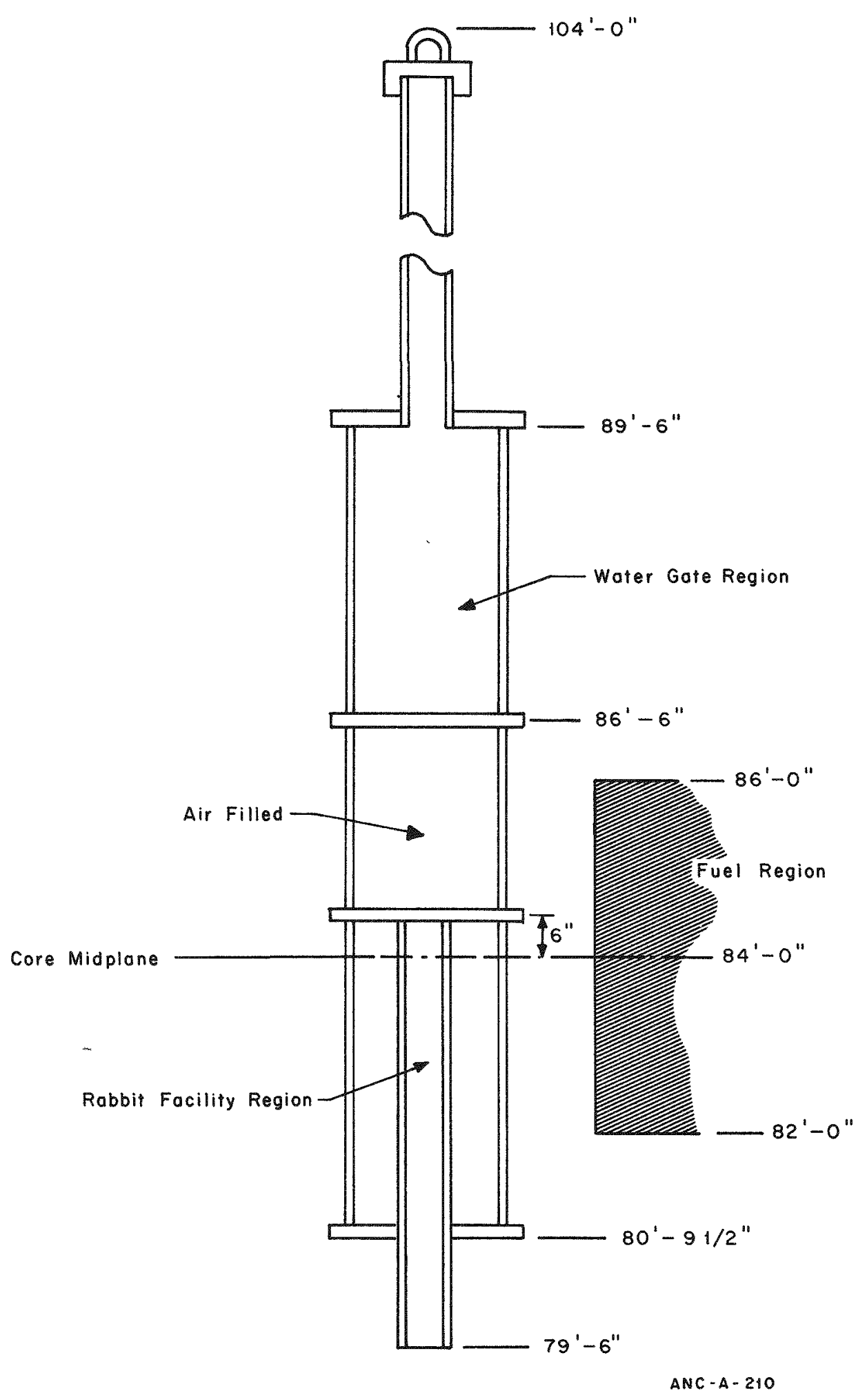

Fig. I Effects of Installing the ATR Beam Tube-Rabbit Facility. 
EXPANDED ATR DATA SYSTEM

J. C. Tappendorf

The Advanced Test Reactor Data System is an on-line computer system which monitors analog signals and the aspects of two-position switches and relays which describe the operation of the reactor and the plant. Storage of these signal values during reactor operation for future reference is provided by a magnetic tape unit. The current value of any analog signal or aspect of any of the two-position switches or relays is available by request from any one of a number of peripheral data terminals. The Data System also uses a number of these analog signals as input parameters in nuclear and reactor calculations (eg, fission product concentrations and reactor lobe power distribution). The Data System also monitors its own operation and provides diagnostic messages on the data terminals when an abnormality is detected.

A PDP-I5 computer has been recently installed and tied to the original CDC-636 to expand the capabilities of the ATR Data System. In addition three Syner-Data printers have been acquired to replace and augment the slower IBM typewriters as data terminals for experiment sponsor output. Three paper tape punches have also been added to the Data System in order to provide the experiment sponsors a faster means of transmitting information to their home offices. A block diagram of the components of the expanded. ATR Data System is shown in Figure 1.

In the near future a visual display system will be added to the ATR Data System. The visual display will be located in the ATR reactor console and will provide the operator with a more immediate and accessible link with the conditions of the reactor and plant. Presently proposed displays include (1) a display of the values of the reactor power parameters, (2) a display of the variation of parameters as a function of time, (3) a display of suggestions for shim movement to achieve a desired power division, and (4) a display of requested Standard Practices, Operating Limits, and Operating Procedures. 


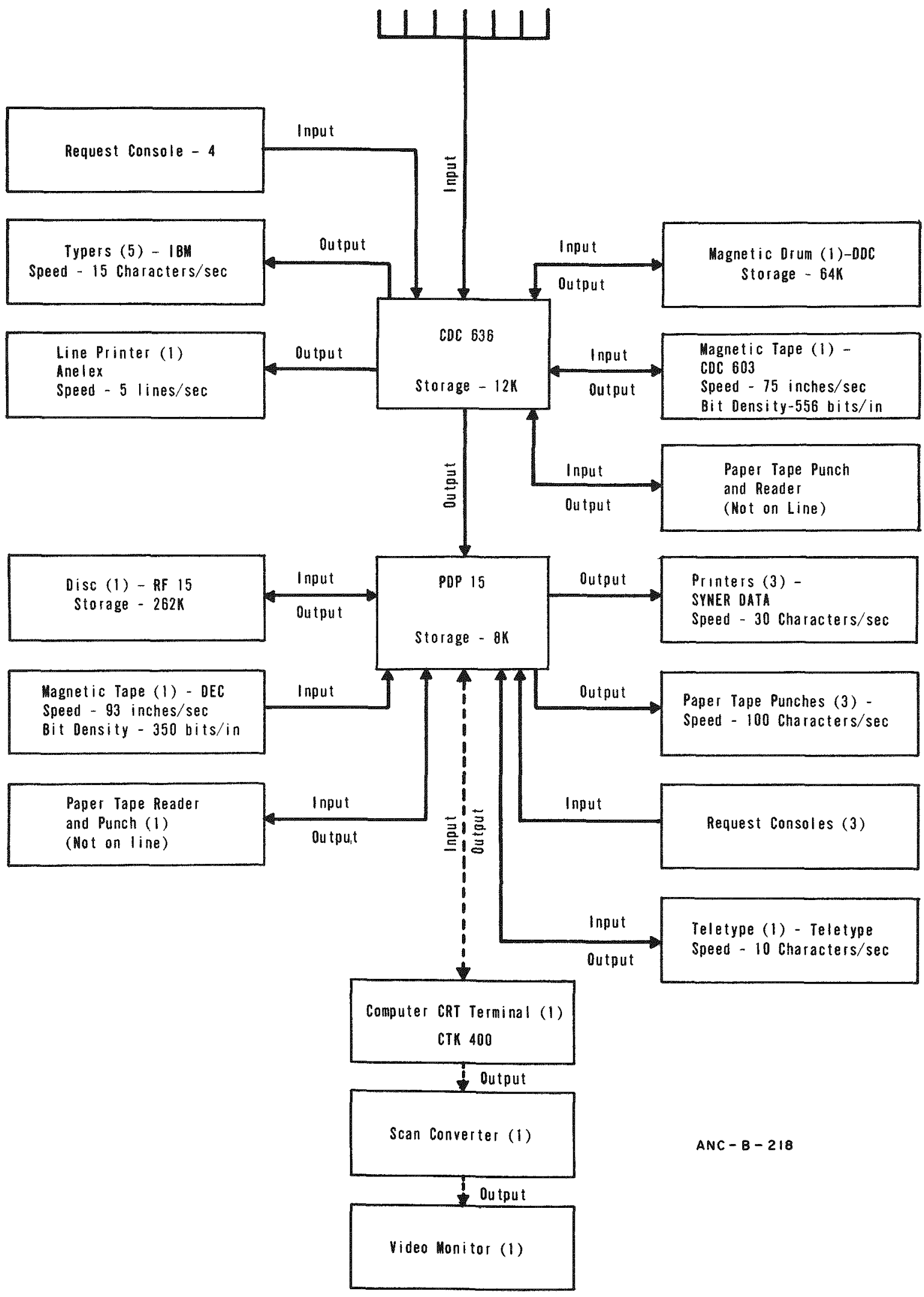

Figure 1. Block diagram of the components of the expanded ATR Data System. 
K. A. Strong

The reactivity meter developed by the Dynaruics Analysis and Reactor Simulation Section was evaluated at the ATRC and subsequently employed in several reactivity measurement tasks in support of test reactor operations. This instrument which calculates reactivity directly through an analog solution to the reactor kinetics equations, eliminates the need for period measurements in the measurement of reactivity.

The evaluation with respect to accuracy consisted of some thirty direct comparisons with the conventional period-inhour method of reactivity measurement at the ATRC. In these tests the reactivity meter measurement was made simultaneously with a period measurement after a change in reactivity. The average reactivity calculated from the two log-N recorder traces was then compared with the reactivity obtained from the reactivity meter. The agreement was within \pm 1 percent (one sigma).

Practical applications of the reactivity meter have included calibration of the regulating rods at ATR, shim calibration and experiment reactivity studies at ATRC, boron assaying of ETR fuel elements at ETRC, and reactor diagnostics relative to power oscillation problems at ETR. The regulating rod calibration at ATR is an example of the speed and convenience of using the reactivity meter. In roughly ten minutes of data acquisition time the ATR regulating rods were calibrated over their usable range. This task would have required about two hours by conventional period-inhour methods. The reactivity output trace obtained during this calibration is shown in Figure 1 . The resultant regulating rod worth curves (uncorrected for power feedback) are shown in Figure 2.

Future uses of the reactivity meter at the test reactors will include studies of power feedback effects, on-line reactivity measurements relative to spurious power spikes and noise problems, and periodic worth measurements of safety rods and shims to track hafnium burnup. At the critical facilities, use of the reactivity meter will result in substantial time savings in the performance of most support tasks which require the measurement of reactivity.

At the present time the use of the reactivity meter is on a priority basis due to the many possible applications at both test reactors and both critical facilities. However, two more reactivity meters are being built which will allow most of the desired reactivity measurements to be made routinely. 


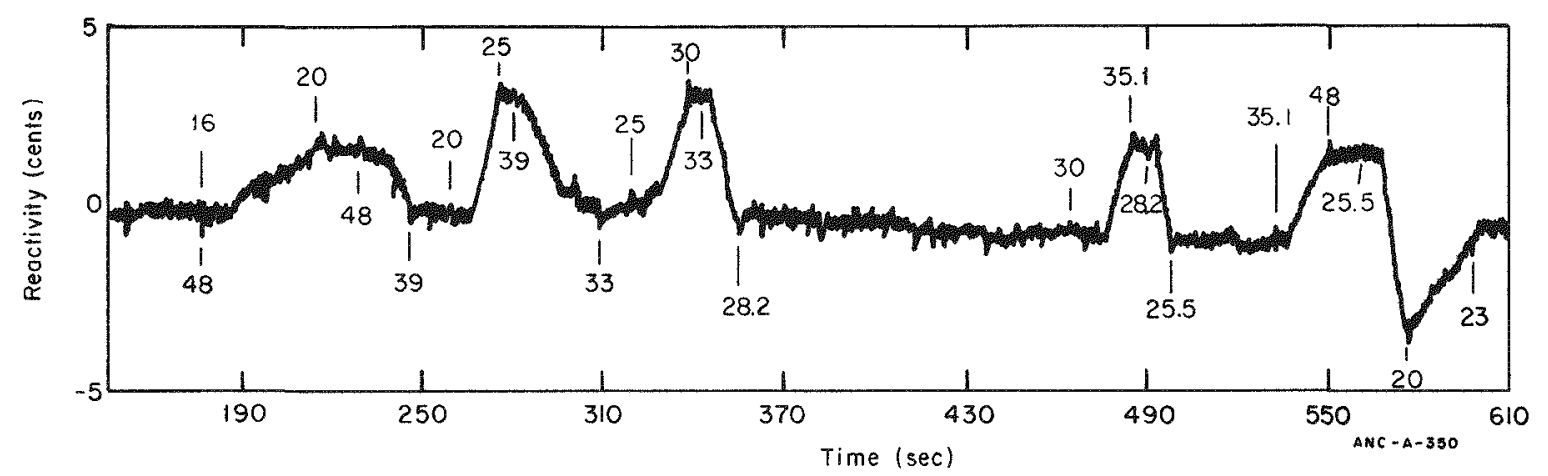

Figure 1 Reactivity as a function of time during the ATR regulating rod calibration. This is a reproduction of the actual recorder chart trace obtained during the calibration. The numbers above the trace are regulating rod No. 2 ( $\mathrm{SW}-4$ ) position in inches at the indicated points in time, and the numbers below the trace are regulating rod No. I (NE-4) positions. 


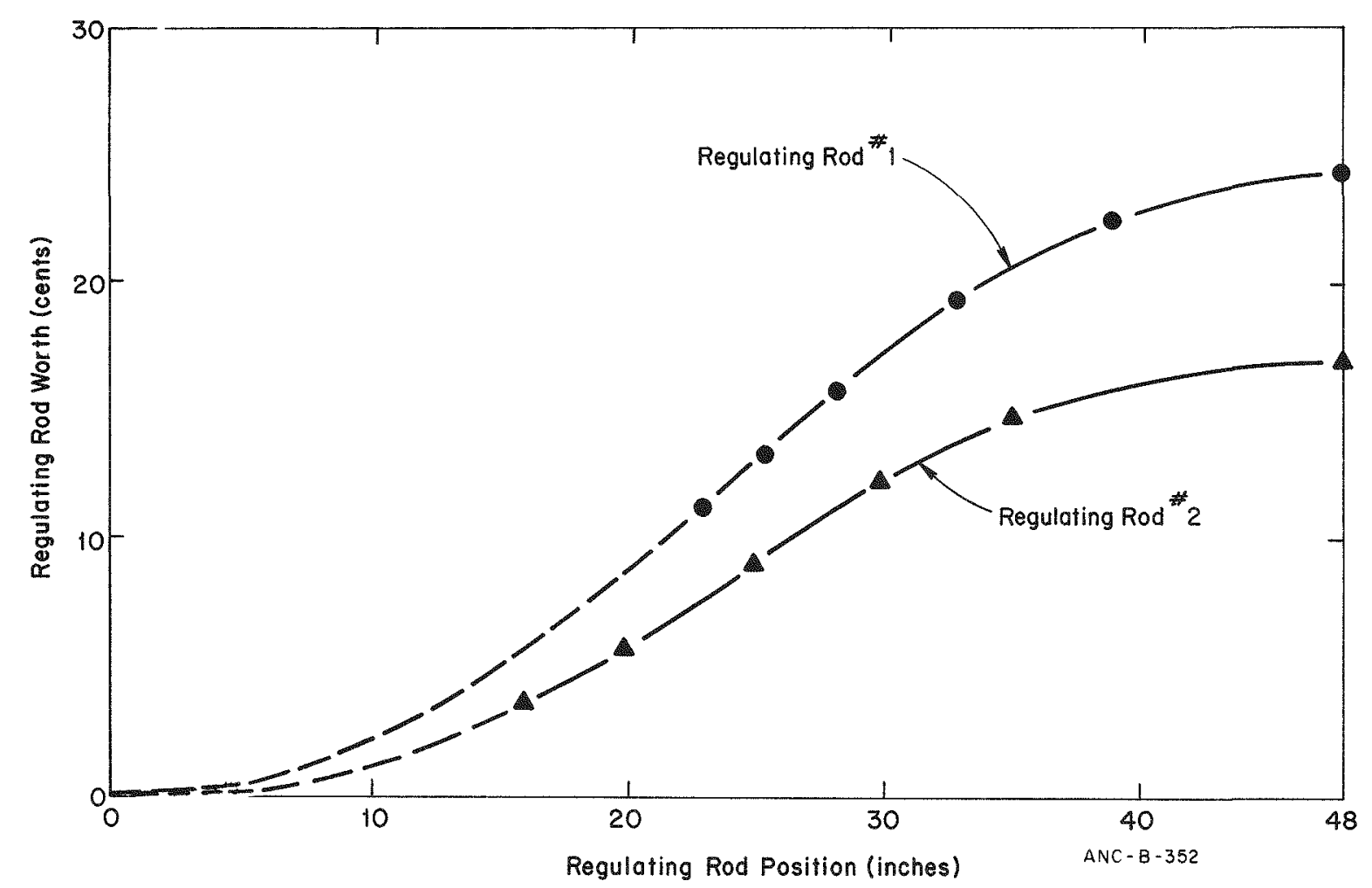

Figure 2. Regulating rod worth as a function of position. The solid line is a hand fit to the data points, and the dashed portions of the curves are extrapolations. 
REACTIYITY METER THEORY AIND DESIGN

S. R. Gossmann

Introduction

A prototype analog reactivity meter was designed and fabricated by the Dynamics Analysis and Reactor Simulation Section. The design of the unit combines techniques from automatic control theory with reactor kinetics in accomplishing instantaneous and continuous measurement of reactivity given a signal proportional to neutron flux.

Tests, designed to evaluate the performance of the reactivity meter and to identify experimental applications for the unit, were conducted at the ATRC and ETRC[I]. The results of these tests justified the design and fabrication of two more reactivity meters incorporating improvements such as extending the input range of ion chamber current, increasing the range for negative reactivity measurements and simplification of the controls and set-up procedure. Work on the new units commenced in July 1971.

The reactivity meter is designed for use with the ATR, ETR, ATRC and ETRC. These reactors are all light water cooled and moderated and use highly enriched. $235_{U}$ fuel. The similarity of these reactors, from a neutron kinetics point of view, simplifies the design in that the same delayed neutron models and constants can be assumed for all four reactors. The delayed neutron data used in the design are given in Table I, item 3.

The unit has provisions for changing the kinetics parameter, $\beta / \ell$. This provides the operator a means to distinguish between the different reactors or between different operating conditions for the same reactor under which $\beta / \ell$ might be different.

The reactivity meter gives the operator three options for reactor operating conditions. These options are:

(1) Subcritical reactor operations with equivalent, extraneous neutron source strength up to $1.0 \$$.

(2) Low power, critical reactor operation. (This assumes negligible extraneous neutron source and no inherent power or temperature feedback processes.)

(3) High power operation with power feedback.

These three options are discussed in greater detail in the following sections.

$[1]_{K}$. A. Strong, "Reactivity Meters". 


\section{Reactor Kinetics}

The principle of operation of this system is explained in the following sections, but first, reactor kinetics relationships will be reviewed. The basic reactor kinetics model utilized is described by:

$$
\begin{aligned}
& \frac{l}{\beta} \dot{N}=\rho N-N+\sum_{i=1}^{6} a_{i} C_{i}+\frac{l}{\beta} Q, \\
& \dot{C}_{i}=\lambda_{i} N-\lambda_{i} C_{i} ; i=1,2--6, \\
& \rho=\Delta \rho+\rho_{f}+\rho_{Q}, \\
& \rho_{Q}=-\frac{\ell}{\beta} \frac{Q}{N_{0}} ;
\end{aligned}
$$

Where:

$$
\begin{aligned}
\mathbb{N} & =\text { neutron density or neutron flux } \\
C_{1} & =\text { delayed neutron variables } \\
\beta, a_{i}, \lambda_{i} & =\text { delayed neutron constants } \\
\ell & =\text { prompt neutron generation time } \\
\beta / \ell & =\text { Prompt neutron kinetics parameter, in sec-1 } \\
\rho & =\text { net reactivity, in } \$ \\
\Delta \rho & =\text { reactivity change to be measured, in } \$ \\
Q & =\text { equivalent neutron source strength } \\
\rho_{Q} & =\text { equivalent reactivity worth of source } \\
\rho_{f} & =\text { reactivity effect of inherent feedback processes at high } \\
\mathbb{N}_{0} & =\text { initial, equilibrium value of } \mathbb{N}
\end{aligned}
$$

The zero-power kinetics equations are obtained from Eq!s. (1) - (4) by setting $Q=\rho_{Q}=\rho_{f}=0$. The zero-power equations are:

$$
\begin{aligned}
& \frac{\ell}{\beta} \dot{N}=\Delta \rho N-N+\sum_{i=1}^{6} a_{i} C_{i} \\
& \dot{C}_{i}=-\lambda_{i} C_{i}+\lambda_{i} N ; i=1,2--6
\end{aligned}
$$


Eq's. (5) and (6) are commonly used to predict the changes in $N$ due to some assumed reactivity disturbance, $\Delta \rho$. In such cases $\Delta \rho$ is considered as the input and $\mathrm{N}$ as the output reactor variable. For reactivity computer systems $\mathbb{N}$ is measured and considered the input variable. $\Delta p$ is computed from Eq's. (5) and (6) and considered the system output.

\section{Principles of Operation of Reactivity Meter}

As previously stated, the reactivity computer system with which this report is concerned, utilizes techniques from automatic control theory. The basic concept underlying this system is the concept of a high performance position control system or, equivalently, of a neutron level tracking system. Let the measured neutron level or flux be designated by $\mathbb{N}^{*}$ and the output of the neutron level tracking system be designated by $\mathbb{N}$, also let the quantity $(\Delta \rho N)$ in $\mathrm{Eq}$. (5) be considered as a single input variable. Considering $(\Delta p \mathbb{N})$ as a variable in effect linearizes Eq's. (5) and (6) and a system transfer function can be derived:

$$
\frac{\Delta N(S)}{(\Delta \rho N)(S)}=\frac{1}{S\left[\frac{\ell}{\beta}+\sum_{i=1}^{6} \frac{a_{i}}{s+\lambda_{i}}\right]}=G(S)
$$

where $\Delta \mathbb{N}$ is the change in $\mathbb{N}$ relative to $\mathbb{N}_{0}$. This is, of course, the zero-power reactor transfer function.

A block diagram of a simple neutron level tracking system is shown in Figure $I[1]$. The functional $f\left(\mathbb{N}^{*}, \mathbb{N}\right)$ shown in this figure represents a suitable operator, operating on the error signal ( $\mathbb{N}^{*}-\mathbb{N}$ ) to generate the signal $(\Delta \rho N)$. The functional must be selected with the following considerations in mind: (a) $\mathbb{N}$ must track $\mathbb{N}^{*}$ with negligible lag (b) zero static error must be provided and (c) the closed-loop feedback system must be stable and well damped.

The simplest possible choice for $f\left(\mathbb{N}^{*}, \mathbb{N}\right)$ is the frequency and amplitude-independent, linear operator $K$. In this case $K$, is simply a gain. Having made this choice, the system configuration resulting is shown in Figure 2 .

The three considerations listed above will now be investigated to show that this system is consistent with those considerations. The closed-1oop transfer function for Figure 2 is

$$
\frac{\Delta N(S)}{\Delta N^{*}(S)}=\frac{K G(S)}{I+K G(S)}=H(S)
$$

where, $\Delta \mathbb{N}$ and $\Delta N^{*}$ are the changes in $\mathbb{N}$ and $N^{*}$ relative to $\mathbb{N}_{O}$.

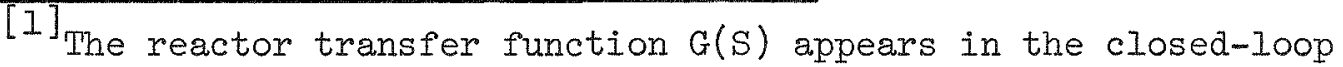
system because a signal must exist in the system that is in some direct or obvious manner related to reactivity.
} 
The error transfer function is

$$
\frac{\left(\mathbb{N}^{*}-N\right)(S)}{\Delta \mathbb{N}^{*}(S)}=\frac{I}{1+K G(S)}
$$

The stability of the system is analyzed by studying the root locus of the closed loop characteristic equation $1+K G=0$ in the $S-p l a n e$.

The root locus shows that the closed-loop system has absolute stability for all values of $K$. All closed-loop system poles are real and negative, thus the system will not "ring" or oscillate. The root locus also shows that for large values of $\mathrm{K}$, the system closed-loop response is characterized by a single pole at $S \simeq-\mathrm{K} / \mathrm{L}$. For large $\mathrm{K}$, the closed-loop transfer function, Eq. (8), becomes approximately

$$
\frac{\Delta N(S)}{\Delta N^{*}(S)} \simeq \frac{K \beta / \ell}{S+K \beta / l}
$$

If $K \geq 1000$ and $\frac{\beta}{l} \simeq 100$ (a reasonable value for evaluation purposes), it is seen that the system has very high band width--in the order of $10^{5} \mathrm{rad} / \mathrm{sec}$. This means that the speed of response of the system is very high so that $N$ is able to follow $\mathbb{N}^{*}$ with negligible lag. Application of the final value theorem to $\mathrm{Eq}$. (9) for constant $\mathrm{N}^{*}$ shows that zero static error is obtained. Thus, the choice of $f\left(\mathbb{N}^{*}, \mathbb{N}\right)=K$ results in a tracking system meeting all three stated requirements if $\mathrm{K}$ is large $(\geq 1000)$.

The remaining problem is to determine how the reactivity $\Delta \rho$ is to be extracted from this system. First, from Figure 2 it is seen that a variable or signal $(\Delta \rho \mathbb{N})$ is generated at the output of the block $K$. If this signal is divided by $\mathbb{N}$, a signal proportional to $\Delta \rho$ is obtained. Figure 3 shows the overall system block diagram. From this figure it can be seen that the signal $(\Delta \rho N)$ is given by

$$
(\Delta \rho \mathbb{N})=K\left(N^{*}-\mathbb{N}\right)
$$

Combining $\mathrm{Eq}$. (11) and (8) results in

$$
(\Delta \rho \mathbb{N})(S)=\frac{K}{I+K G(S)} \quad \Delta N^{*}(S)=\frac{H(S)}{G(S)} \Delta N^{*}(S)
$$

The mathematical basis for the operation of this reactivity meter can be shown most simply by considering a reactivity step, $\rho^{*}$, applied to the reactor producing a change in neutron level $\Delta \mathrm{N}^{*}$. It is not difficult to show that the Laplace transform of the neutron level change is given by

$$
\Delta \mathbb{N}^{*}(S)=\rho^{*} G(S) \mathbb{N}^{*}(S) \text {. }
$$

Substituting Eq. (13) in Eq. (12) results in

$$
(\Delta \rho N)(S)=\rho * H(S) N *(S) \text {. }
$$


The time response for the signal $(\Delta \rho N)$ is obtained from Eq. (14) by application of the convolution integral,

$$
(\Delta \rho \mathbb{N})(t)=\Delta \rho(t) \mathbb{N}(t)=\rho^{*} \int_{0}^{t} H(\tau) \mathbb{N}^{*}(t-\tau) d \tau
$$

For the case $K \geq 1000$, it can be shown that the function $H(t)$ approaches very closely the properties of the unit impulse function, $\delta(t)$. In particular,

$$
\int_{0}^{t} H(t) d t \simeq \int_{0}^{\infty} \delta(t) d t=1
$$

This property of $H(t)$ permits the following approximation for the integral of Eq. (15),

$$
\Delta \rho(t) \mathbb{N}(t) \simeq \rho^{*} \mathbb{N}^{*}(t) \text {. }
$$

It has previously been shown that for $K \geq 1000, \mathbb{N}(t) \simeq \mathbb{N}^{*}(t)$ at all times. Utilizing this result in Eq. (IT) finally yields,

$$
\Delta \rho(t) \simeq \rho^{*}
$$

Error analysis for the approximations leading to Eq. (18) has shown that dynamic or transient errors in the approximation of $\mathrm{Eq}$. (18) do not exceed $0.1 \%$. There is no static error, thus the result of Eq. (I8) converges to the correct value. While the above discussion has assumed a step reactivity $p *$, for simplicity, the results are equally valid for time varying reactivities $p^{*}(t)$. Thus $E q$. (18) may be written as

$$
\Delta p(t) \simeq p^{*}(t)
$$

This equation simply states that the measured reactivity $\Delta p(t)$ is approximately equal to the reactivity $\rho^{*}(t)$ disturbing the reactor. As before, the error is less than $0.1 \%$.

Analog Circuit Requirements

The discussions in the preceding paragraphs have established a basis for the reactivity meter. Figure 3 is a block diagram of the system showing the system functional requirements. Implementation of the various requirements is accomplished using dc operational amplifiers, synthesis of the transfer function $G(S)$ using RC circuits, and an analog division circuit. Figure 4 is the simplified circuit diagram of the system.

Ainplifier \#l converts the ion chamber current to a voltage $N^{*}$. The feedback resistor in amplifier \#1 can be varied to adjust the voltage $\mathbb{N}^{*}$ to any value between 0 and -1.0 volts. 
Amplifier \#2 subtracts the measured voltage $\mathbb{N}^{*}$ from the calculated voltage $\mathbb{N}$ and provides the required gain $\mathrm{K}$.

Amplifier \#3 provides range changes in reactivity. With $A=1$, the full scale value of reactivity is $I \$$. With $A=10$, the full scale value is $0.1 \$$.

Amplifier \#4, together with the RC circuits shown, constitutes an active circuit with transfer function $G(S)$ as required.

Amplifier \#5, provides a necessary sign change for stability and a reference voltage, $N_{0}$, for the initial conditions.

The analog divider shown provides the necessary division of $(\Delta p \mathbb{N})$ by $\mathbb{N}$ producing an output voltage $10 \mathrm{~A} \Delta \rho$. Analog division by electronic circuits is difficult to perform with high accuracy. The accuracy of the reactivity computer at low reactor power levels will ultimately depend on the accuracy of the divider circuit. The divider used performs division with errors held to about $0.2 \%$ of full scale. This corresponds to about $0.2 \phi$ in reactivity in the $I \$$ range.

In general, the following design requirements have been met:

(1) The dc operational amplifiers shall be high quality, drift stabilized and the open-loop gain shall exceed $10^{6}$.

(2) The resistors and capacitors shall be computer grade components.

(3) Circuit arrangement and wiring practices shall follow methods used in high precision analog computers to obtain maximum performance from the system.

(4) The system shall have provisions for balancing amplifiers, calibration of the divider, and control circuits for rapidly and accurately establishing initial conditions (charging capacitors) consistent with reactor operating conditions.

(5) The system shall be portable and operate from a 110 Vac source.

(6) The output from an ionization chamber shall be the system input. No additional signal conditioning shall be required at the input.

(7) The system shall contain a meter read out for on-line monitoring. Additional outputs for connection to recorders or external meters shall be provided.

Performance specifications are given in Table $I$.

Source Range and High Power Range Operation

Up to this point, the reactivity meter design is based on the zero power reactor kinetics model (Eq's. 5 and 6 ) in which the neutron 
source $Q$ is neglected as well as high power reactivity feedback processes. Under certain conditions it would be desirable to include the effects of a neutron source at low power and at least a first order approximation of high power effects at high power.

Addition of circuitry to include the effects of an extraneous neutron source $Q$ is a fairly simple and straightforward matter. The problem is one of determining a good value for the source strength $Q$ or its equivalent reactivity worth $P Q$ (Eq. 4$)$.

Operation at high power levels makes the reactivity computation more difficult because the linear zero power kinetics model no longer suffices to describe the reactor dynamics. A possible, first order approximation for the high power range could be based on the following considerations. If power level changes are slow so that the reactor can be considered to be in thermal equilibrium at all times, the feedback reactivity effects can be "lumped" into one overall reactivity feedback and related to power level changes by means of the reactor power coefficient. In this case, $\rho_{f}$ in $\mathrm{Eq}$. (3) is given by

$$
\rho_{f}=-\left|\alpha_{f}\right|\left(N-N_{0}\right) ;
$$

Where $\alpha_{f}$ is the negative power coefficient.

Figure 5 shows the required changes to include the source effect and reactivity feedback as discussed above. The other parts of the circuit not shown in this figure are the same as in Figure 4. 


\section{TABLE I}

\section{PERFORMANCE SPECIFICATIONS AND PARAMETER VALUES}

I. Input Specifications

Input to the unit is an ionization chamber output. Two fixed current ranges are provided.

$$
\begin{aligned}
& 0.1 \mu a \text { to } 3.0 \mu a \\
& 1.0 \mu a \text { to } 30.0 \mu a
\end{aligned}
$$

The input amplifier has variable gain for continuous and overlapping adjustment of the input signal over the two ranges.

2. Output Specifications

The output is a voltage proportional to reactivity. Three reactivity ranges are provided:

$$
\begin{aligned}
& \pm 0.1 \$ \text { Full Scale } \\
& \pm 1.0 \$ \text { Full Scale } \\
& \pm 10.0 \$ \text { Full Scale }
\end{aligned}
$$

The output voltage varies between $t 10$ volts. The reactivity error

\begin{tabular}{|c|c|c|}
\hline $\begin{array}{c}\text { Group } \\
i \\
\end{array}$ & $\begin{array}{c}\text { Decay Constant } \\
\lambda_{i}\left(\mathrm{sec}^{-1}\right) \\
\end{array}$ & $\begin{array}{c}\text { Relative Abundance } \\
a_{i} \\
\end{array}$ \\
\hline 1 & 0.0127 & 0.038 \\
\hline 2 & 0.0317 & 0.213 \\
\hline 3 & 0.115 & 0.188 \\
\hline 4 & 0.311 & 0.407 \\
\hline 5 & 1.40 & 0.128 \\
\hline 6 & 3.87 & 0.026 \\
\hline
\end{tabular}
for all three ranges is $\pm 0.2 \%$ of Full scale.

\section{Kinetics Parameters}

$\beta / \ell$ is adjustable and can be set to within $10 \%$ of the desired. value. Delayed Neutron Constants are listed below: 


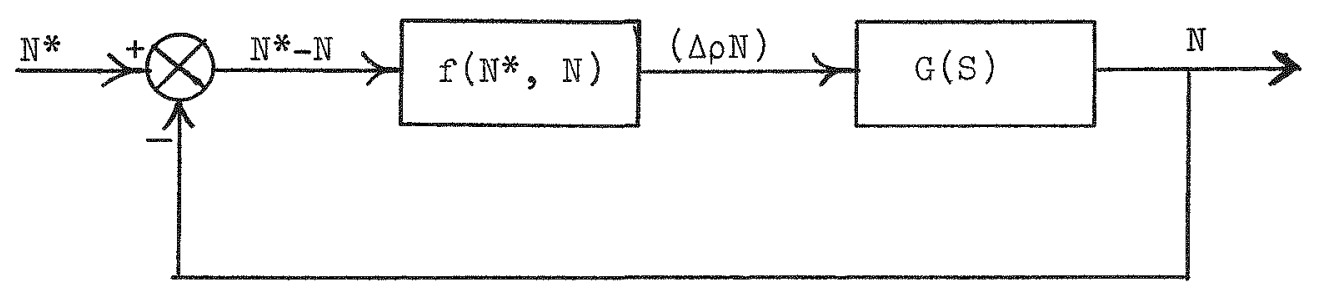

Fig. I. Block diagram of Neutron Level Tracking System.

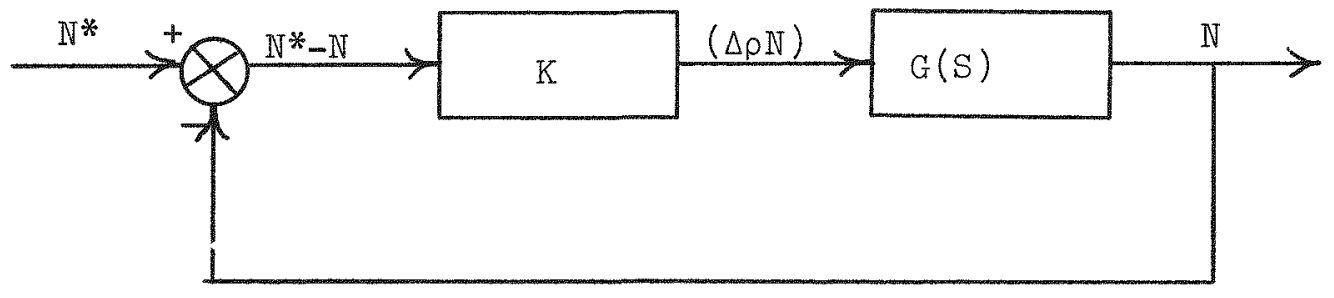

Fig. 2 Block diagram of Neutron Level Tracking System for reactivity meter.

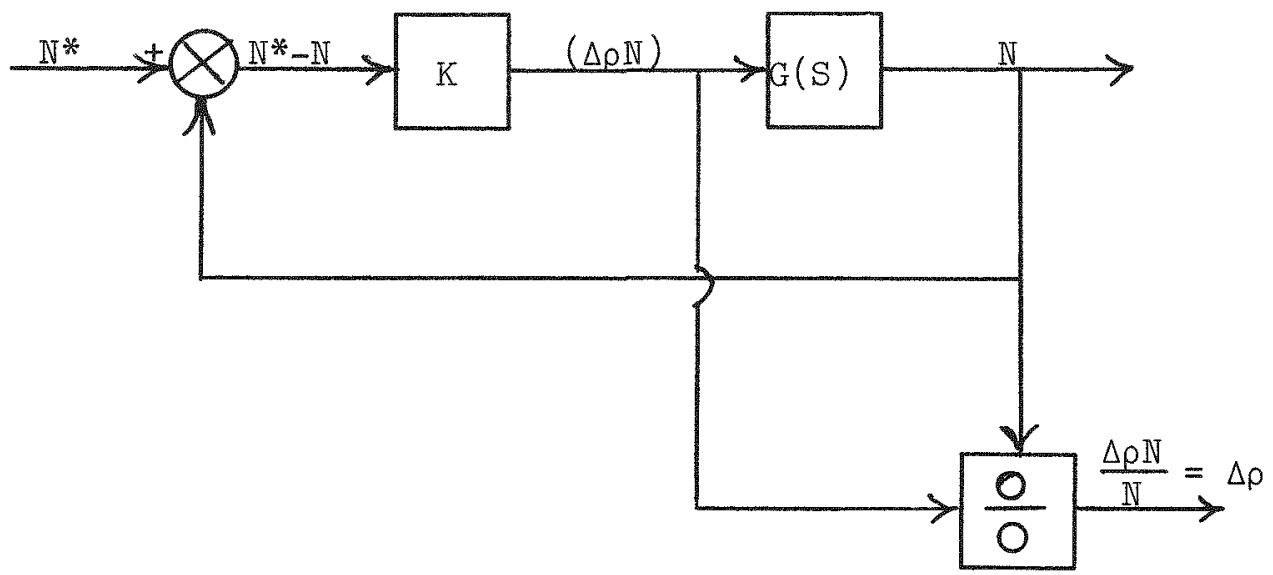

Fig. 3. Block diagram of reactivity meter. 


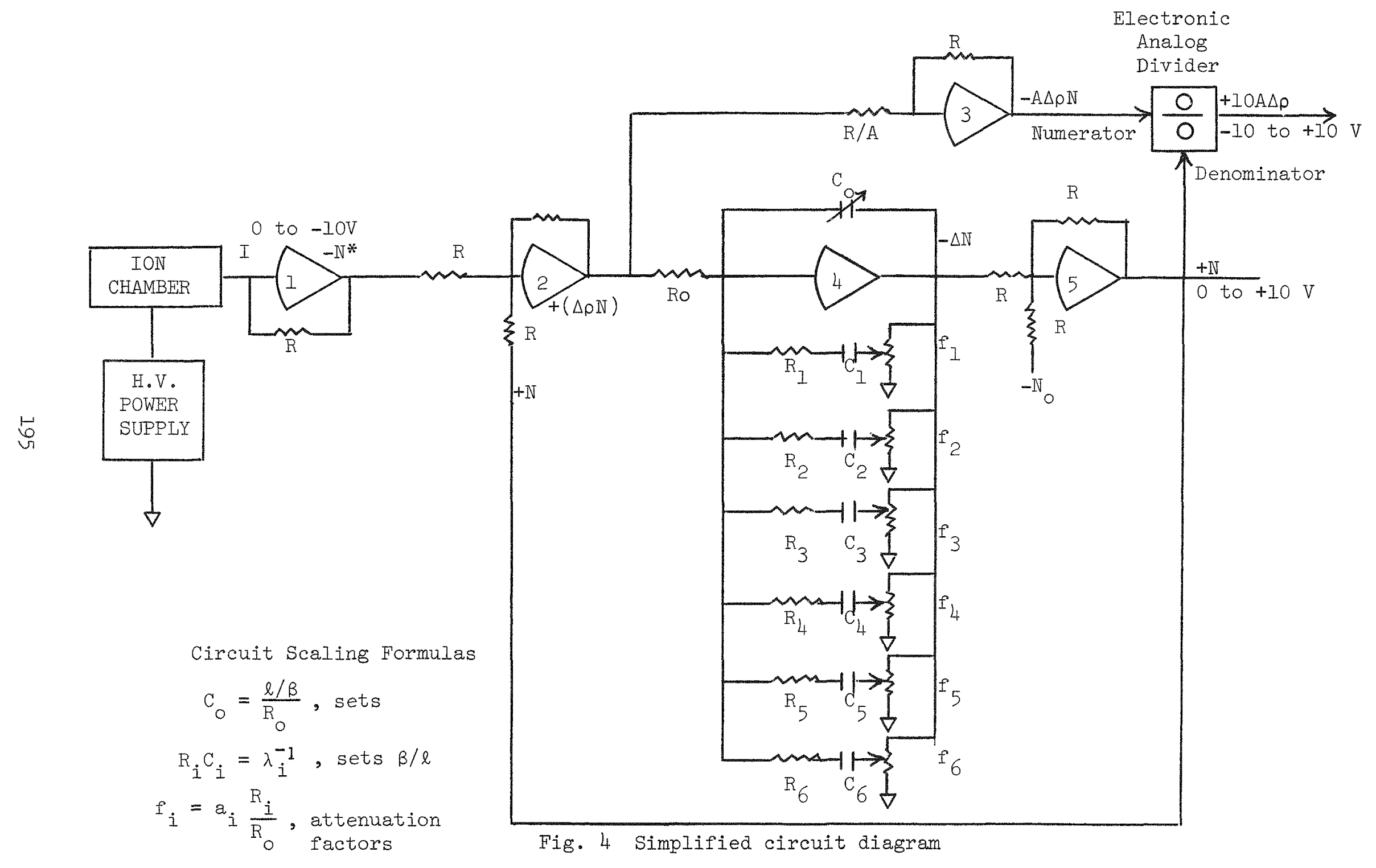




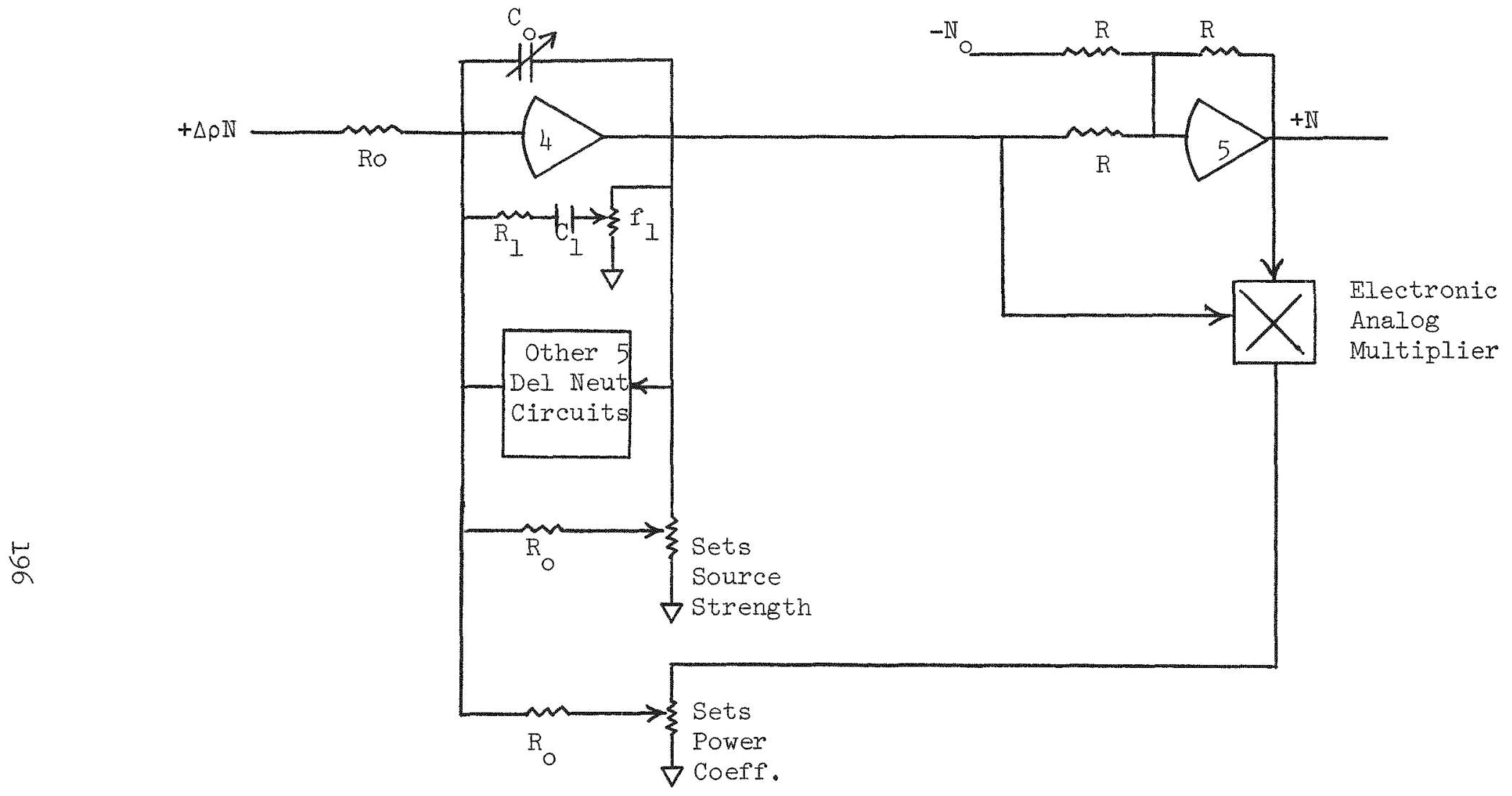

Fig. 5 Simplified circuit diagram for source and power feedback 


\section{REACTOR OPERATOR CERTIFICATION PROGRAM}

D. W. Knight

To comply with AEC Immediate Action Directive 8401-6, an operator training and certification program has been established in the Nuclear Technology Division for personnel working at the ATR Critical Facility, the Advanced Reactivity Measurement Facilities I and II, the ETR Critical Facility, the Nuclear Test Gauge, and the Split Table Reactor.

Minimum standards have been established for Reactor Operators, Senior Reactor Operators, and Reactor Supervisors. In order to be certified, an operator-candidate must successfully pass a medical examination which is given by an AEC physician and written, oral, and operating examinations which are administered by the Division Examination and Certification Board.

Table I shows the number of Division personnel that have been certified at the different reactors. Each person must be recertified at the end of a two-year period. Additionally, an examination covering emergency procedures is given every year.

\section{TABLE I}

SUMMARY OF CERTIFICATIONS

\begin{tabular}{lccc}
\hline & \multicolumn{3}{c}{ Number of Persons Certified* } \\
\cline { 2 - 4 } Reactor & $\begin{array}{c}\text { Reactor } \\
\text { Supervisor }\end{array}$ & $\begin{array}{c}\text { Senior } \\
\text { Reactor Operator }\end{array}$ & $\begin{array}{c}\text { Reactor } \\
\text { Operator }\end{array}$ \\
ATRC & 5 & 2 & 1 \\
ARMF I \& II & 2 & 1 & 1 \\
ETRC & 6 & 1 & 1 \\
NTG & 2 & 1 & 1 \\
STR & 1 & 2 & \\
*The total number of persons certified is shown. Since the examinations \\
were given, some persons have qualified for another position and others \\
have terminated.
\end{tabular}

A complete record of all examinations is maintained. In addition, a record of all class work and special training is kept.

The membership of the Board is as follows:

1. A person appointed by the Nuclear Technology Division Manager to act as a permanent member of the Board and also as Board Chairman. (Generally the Division Training and Certification Coordinator.)

2. The candidate's immediate supervisor. 
3. A representative from the Nuclear and Operational Safety Division, as appointed by the N\&OS Division Manager.

4. Another supervisor from the Reactor Development or Reactor Technology Branches as requested by the Board Chairman. 
NON-DESTRUCTIVE MEASUREMENT OF OXIDE FILM BUILDUP

ON REACTOR FUEL ELEMENTS

T. J. Boland, E. G. Grafwallner, R. L. Kynaston

Oxide film buildup on reactor fuel elements is measured routinely at ETR and ATR using eddy current instrumentation. [1] These measurements are an important part of the reactor fuel surveillance and control program which has resulted in extended fuel cycle lifetime and consequent substantial fuel cost reduction. Continued use of the instrumentation has led to some improvements and refinements in the probe sensors, readout fixture and the electronics package.

Sensor Probe Development. A 0.25 " diameter probe was designed and has been placed in service. Optimization of the coil shape improved the sensitivity of this miniature probe. The probe housing is "necked down" from $0.5^{\prime \prime}$ diameter to $0.25^{\prime \prime}$ diameter. The $0.5^{\prime \prime}$ section allows space for attaching the connecting probe cable to the coil leads. The active face of the transducer is a stainless steel window $0.25^{\prime \prime}$ diameter and $0.001^{\prime \prime}$ thick.

Work on compensating the instrument for gamma heating continued. Most of the drift with temperature is due to the temperature coefficient of coil resistance. Coils were wound with wire of near zero coefficient. This essentially eliminated the drift in the $0.5^{\prime \prime}$ diameter probe coils but resulted in a loss of sensitivity. This loss of sensitivity did not degrade the performance with the $0.5^{\prime \prime}$ diameter probe. However, the sensitivity of the $0.25^{\prime \prime}$ probe, already less than the $0.5^{\prime \prime}$ probe, was lowered to the point that performance became unacceptable. Since the smaller diameter probe is now the standard for oxide measurements, another approach to the temperature compensation is necessary. A DC current flowing through the probe coil produces an IR voltage drop which is used to correct the null meter for resistance vs. temperature changes. The DC currents in the two probes are of opposite polarity so that when the probes are at the same temperature, the net correction voltage is zero. Figure 1 shows the compensation circuit applied to the $4 \mathrm{arm}$ bridge which includes the search and reference probes as two of the bridge arms.

Readout Fixture. The readout fixture has been modified to reduce gear wear. A spring was also installed to preload the gears to remove backlash. The main support piece was changed from ordinary aluminum to a special stress-relieved aluminum to minimize dimensional changes caused by variations in temperature.

1. T. J. Boland, E. G. Grafwallner, E. E. Owen, "Measurement of Oxide Coating Thickness on Reacotr Fuel Elements by Eddy Current Sensor", Nuclear Tech. Branch Annual Progress Report for Perios Ending June 30, 1969, IN-1317 (Jan. 1970). 
Electronics Unit. The electronics unit was redesigned utilizing solid state operational amplifiers to replace cascaded transistors and several transformers. A new phase comparator using a differential amplifier circuit has proven considerably more stable with temperature than the original ring diode demodulator. The improved stability is the result of having the entire active circuit built on a single chip. Ten turn potentiometers and dials were installed to replace the simple control knobs used previously. This permits the recording of dial readings and locking the shaft in position to guard against accidental bumping of the control and upsetting the null balance.

It is planned to design an in-line electrical readout and automatic null balance when time and manpower are available.

The photographs of Figures 2, 3, 4, and 5 illustrate the 0.25 inch diameter probe, the readout fixture with reference probe, the calibration and standard fixture, and the complete instrumentation system respectively.

Conclusion. The accuracy and repeatability of the oxide measurements with this instrumentation has proven to be better than the specified requirements. Despite the high use factor there has been no failure or malfunction of the electronics in more than two years of use. Careful handling has minimized the number of replacements of the probe sensor and the mechanical readout. 


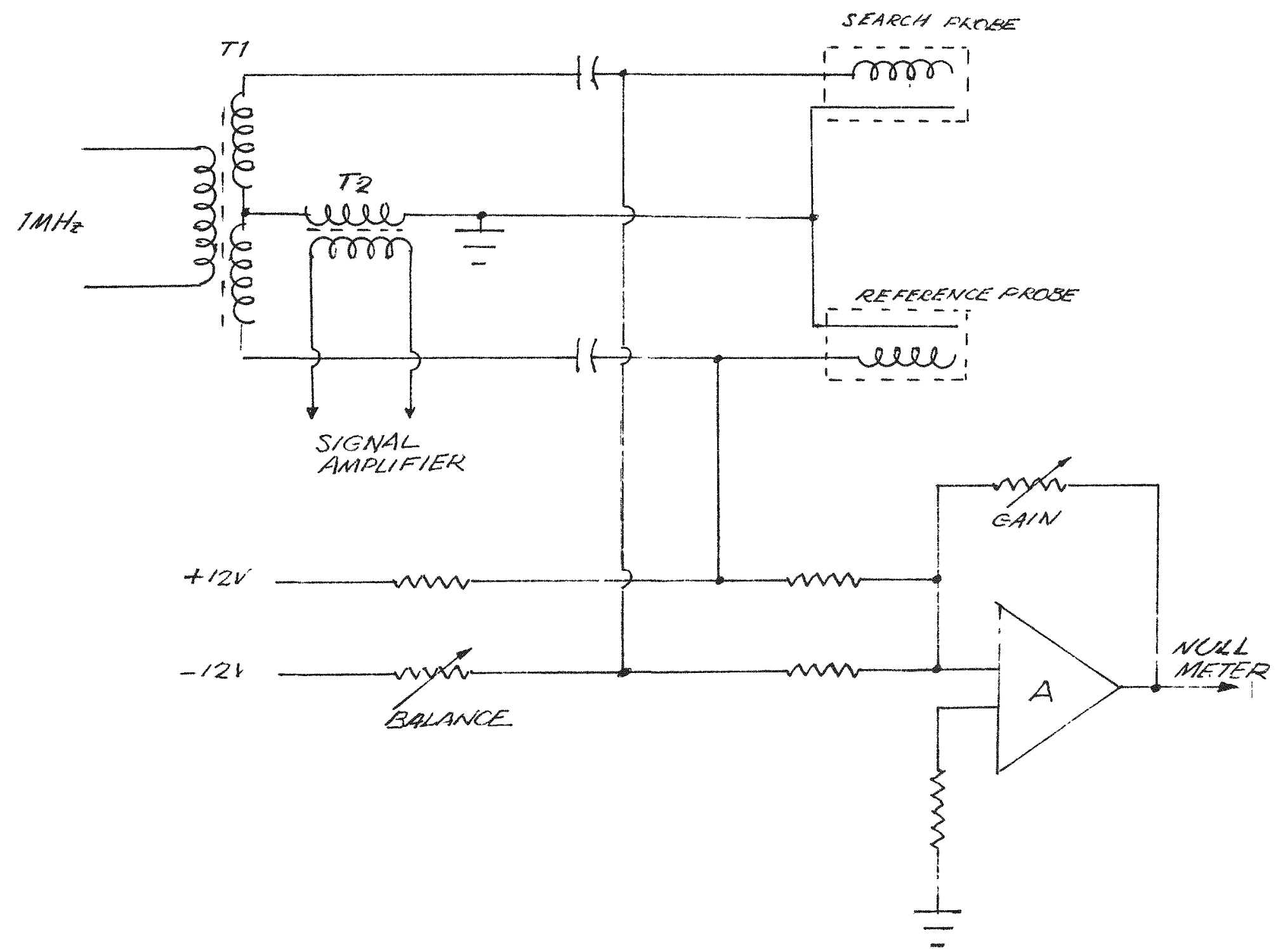

Fig. I Probe drift compensation circuit. 


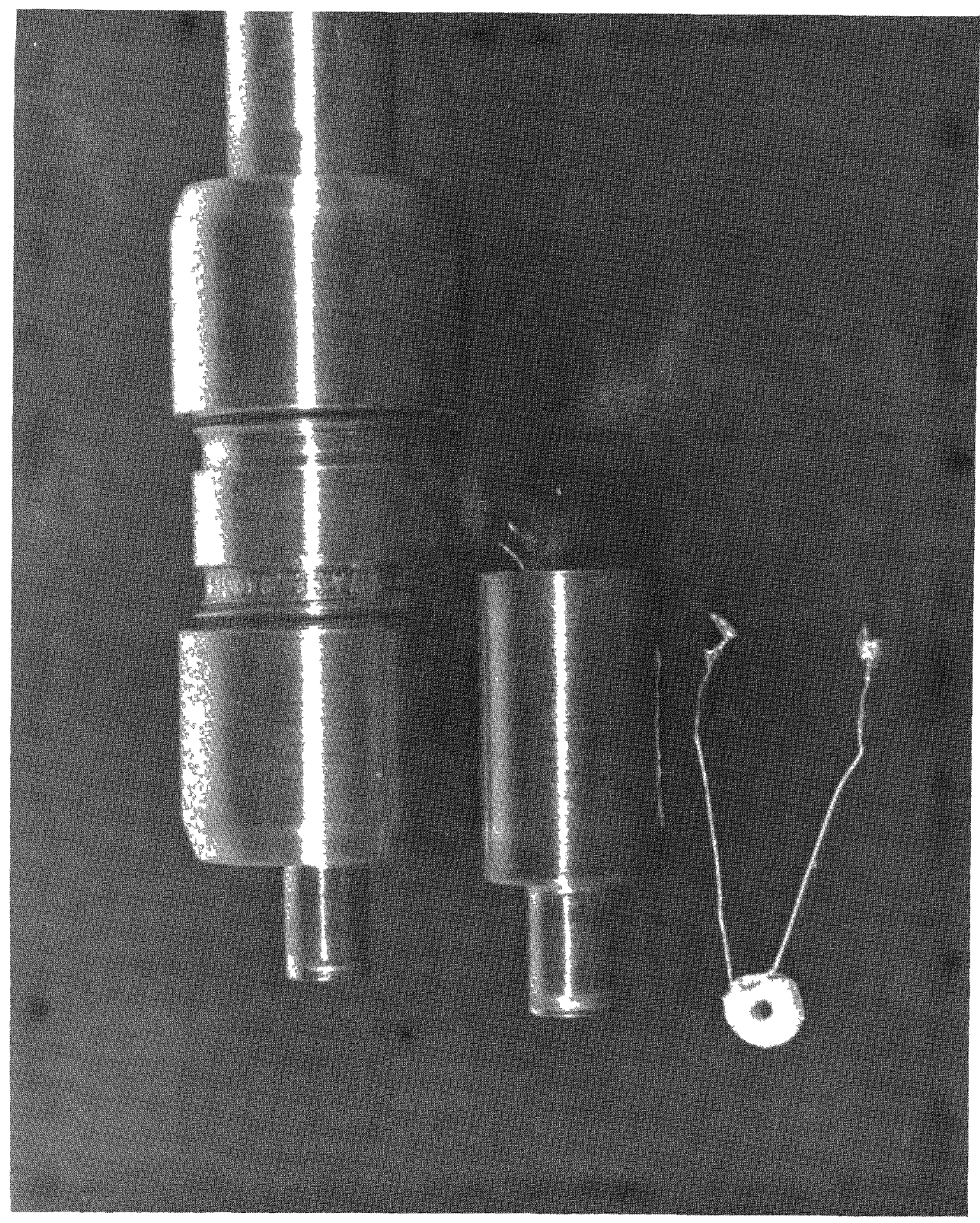

Figure 2. (2 $1 / 2$ times actual size) Probe assembly, probe housing, probe coil. 


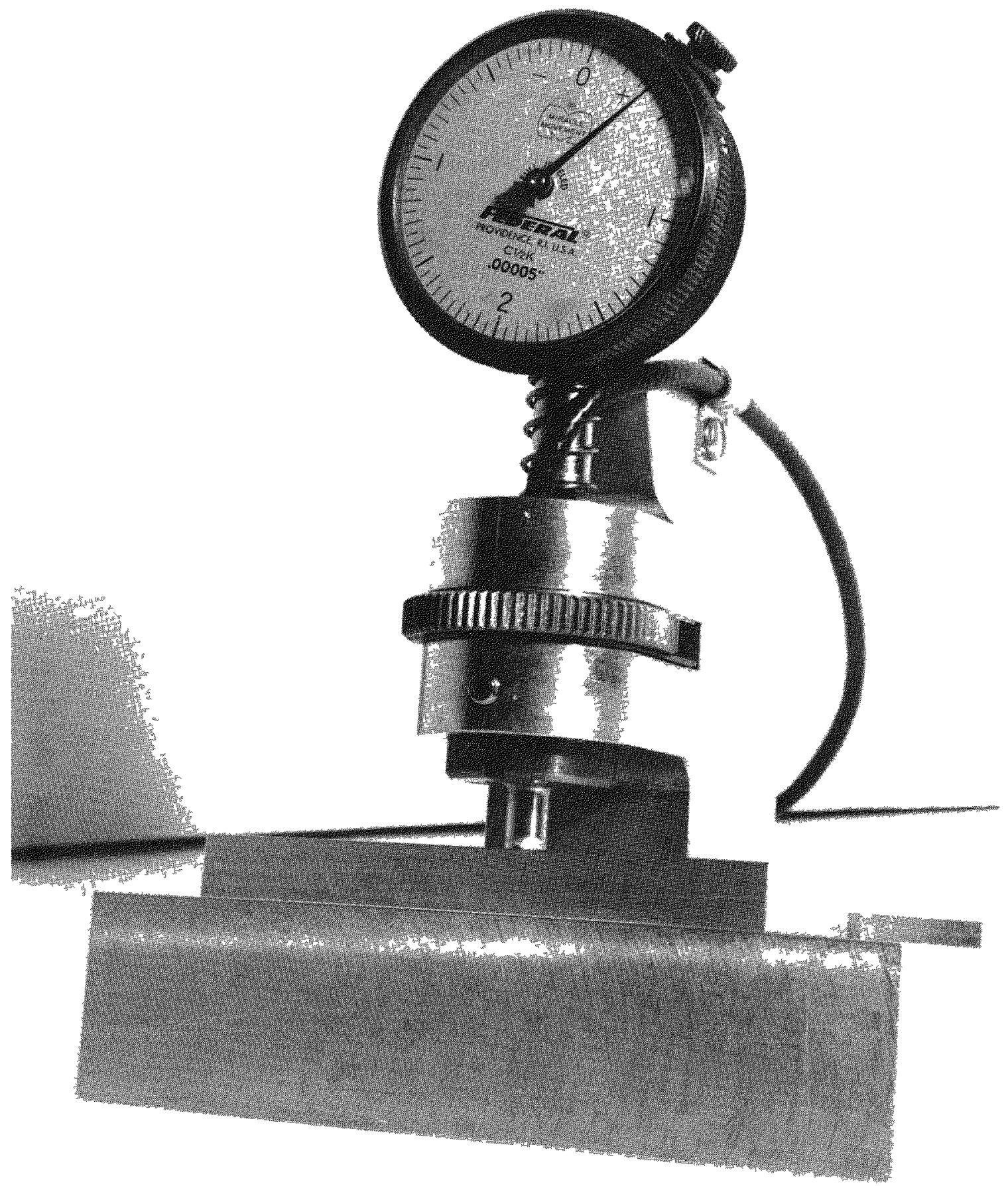

Figure 3. Readout Fixture 


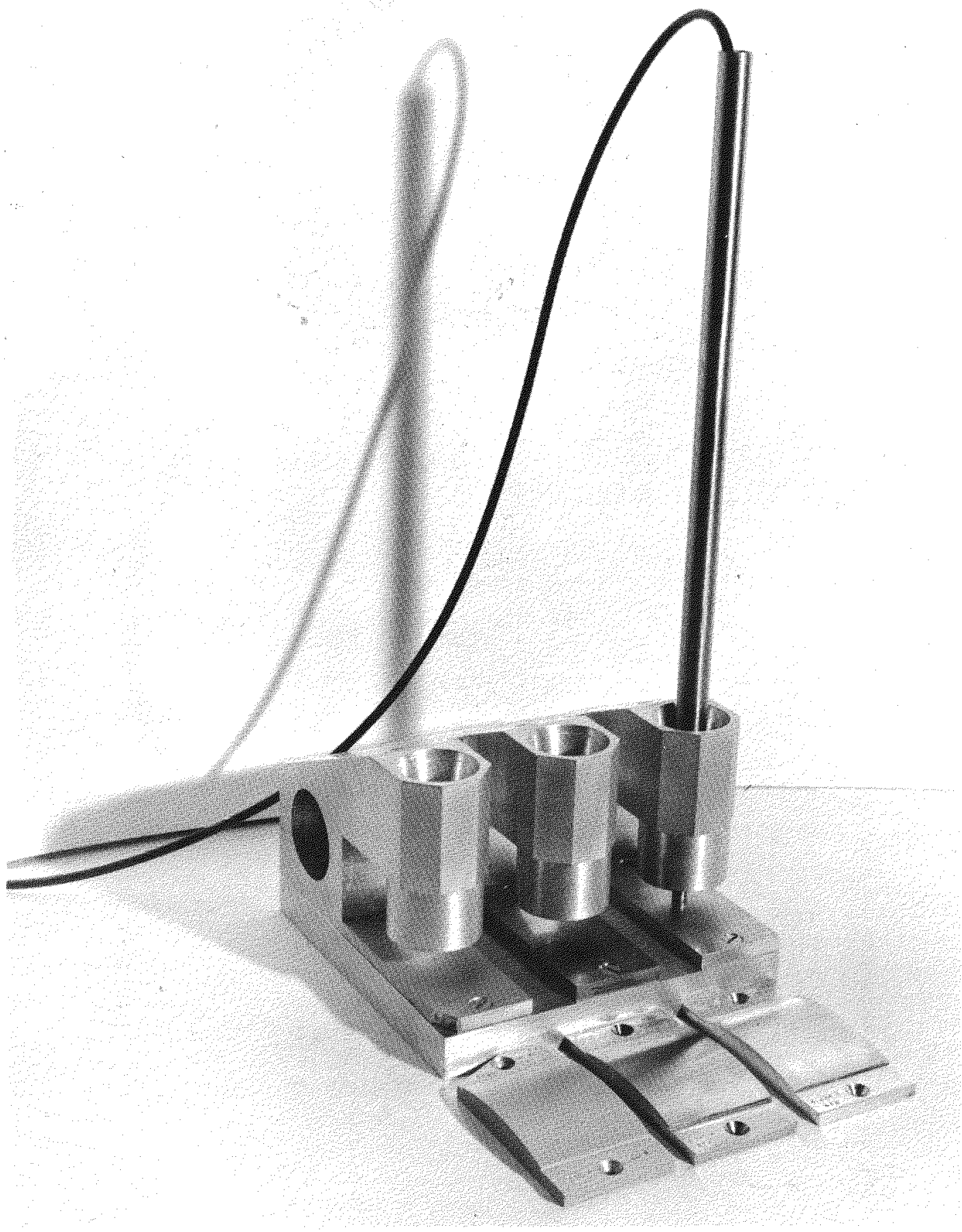

Figure 4. Calibration and Standard Fixture 


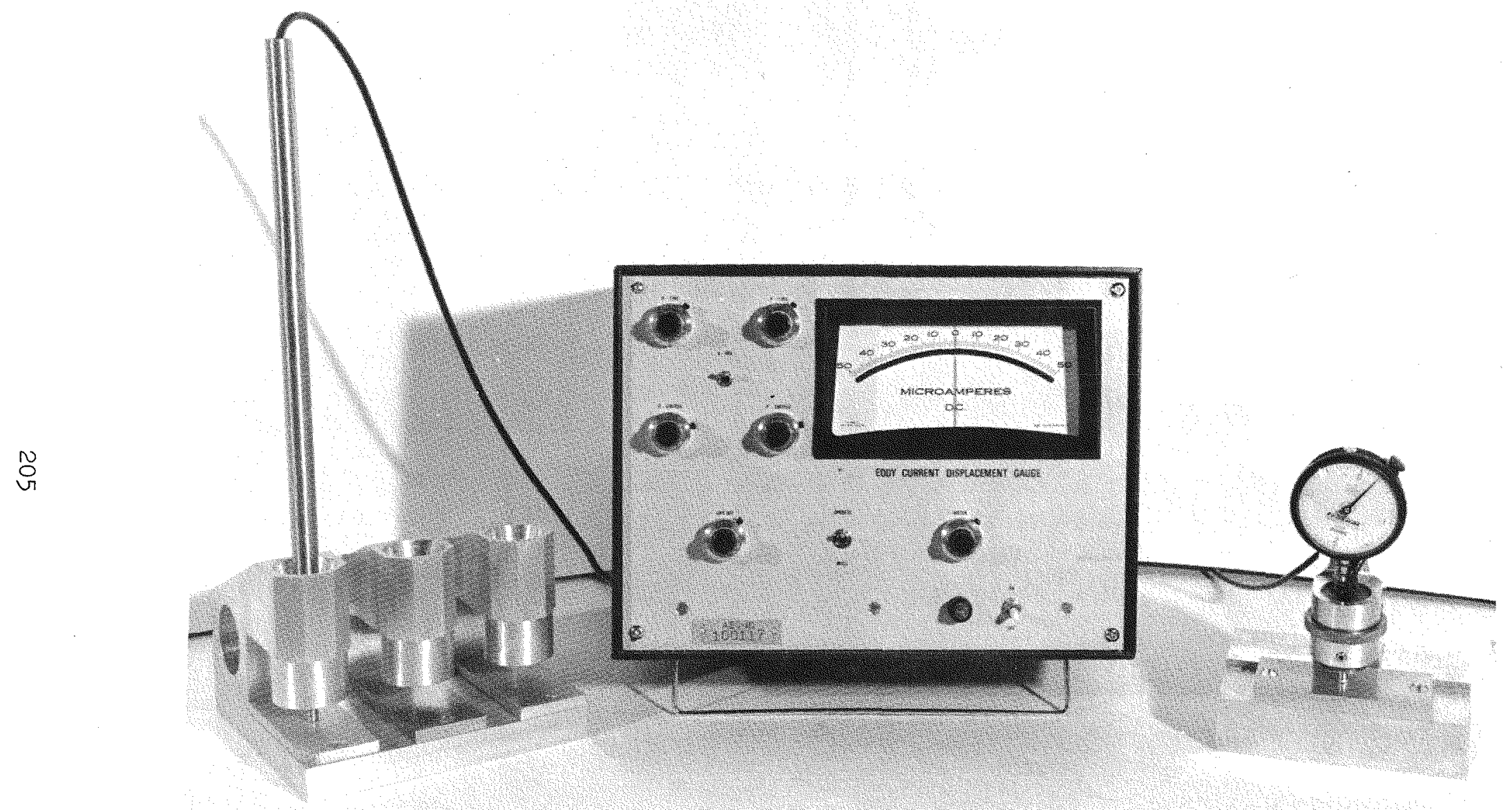

Figure 5. Complete Instrumentation System 
POST-IRRADIATION EXAMINATION OF OXIDE FILMS ON ETR FUEL ELEMENTS

\author{
R. R. Hobbins
}

During ETR Cycle 108, oxide films up to 3 mils thick were formed on fuel plates. These films caused increases in fuel plate operating temperatures which, in turn, resulted in measurable plate buckling, If the oxide films were formed by corrosion of the cladding, cladding penetration up to 3 mils could be possible. In order to determine whether significant cladding penetration had occurred, a program of hot cell examinations was undertaken. Visual inspection, metallographic examination, eddy current oxide thickness measurement, and oxide scrapings for chemical and $x$-ray analysis are some of the techniques which have been employed to characterize the corrosion buildup on fuel elements. Two ETR elements underwent examination in FY-71: E255H from Cycle 108 and E462H from Cycle 110.

Element E255H exhibited very thick oxide (up to 3.0 mil) and significant channel distortion due to plate buckling. Visual examination revealed patterns of oxide spalling which recurred regularly on all the fuel plates of the element. Examples of these spalling patterns are shown in Figure 1. The photographs are orientated such that the top of the element is up to the left. The patterns are characteristic of the locations measured relative to the top of the element and are repeated on every fuel plate. Eddy current measurements of oxide thickness did not correlate well with similar measurements taken in the canal. Most likely, this circumstance resulted from the fact that, due to the extensive spalling, small discrepancies in positioning the eddy current probe could lead to large differences in oxide thickness measurements.

Metallography revealed the oxide to be present in two or more layers. The outside layers are thicker than the inside layers, often accounting for about $70 \%$ of the total oxide. Photomicrographs of oxide films are presented in Figure 2. In Figure 2B, three layers appear to be present. $\mathrm{X}$-ray analyses of scrapings from spalled and undisturbed oxide failed to reveal a systematic difference in the crystal structures of the layers. Generally, boehmite was the principal oxide constituent found over the fuel core while bayerite predominated over the cooler picture frame.

Past experience with aluminum fuels has led to the rule of thumb that the depth of cladding consumed by corrosion is roughly equivalent to the thickness of the oxide film produced. Thus, in the present case, with oxide films in the 2 to 3 mil range, one might expect cladding thicknesses from 2 to 3 mils thinner than the nominal 15 mils. However, clad penetration of this order of magnitude was not observed. It must be concluded therefore that the bulk of the oxide present did not result from the corrosion of the fuel plate cladding. 
In FY-71, post-irradiation examination was begun on element E462H. This element was chosen as being representative of Cycle 110 in which more acceptable oxide thicknesses were achieved. Visual examination under a 10X stereoscope revealed what appeared to be small bumps ( $\sim 2$ mils diameter) in the oxide on the side plates. A photograph of these bumps appears in Figure 3. Similar bumps, but less prominent, were also observed in fuel plate oxides. Metallographic examination failed to reveal any bumps in cross section. Metallography did reveal that the oxide was sometimes present as two layers. In Figure 4, photomicrographs of oxide films typical of the side plates and fuel plates are presented. Additional work to investigate more thoroughly the small bumps in the oxide and to aid the correlation of metallography to canal eddy current oxide thickness measurements is being carried out. 


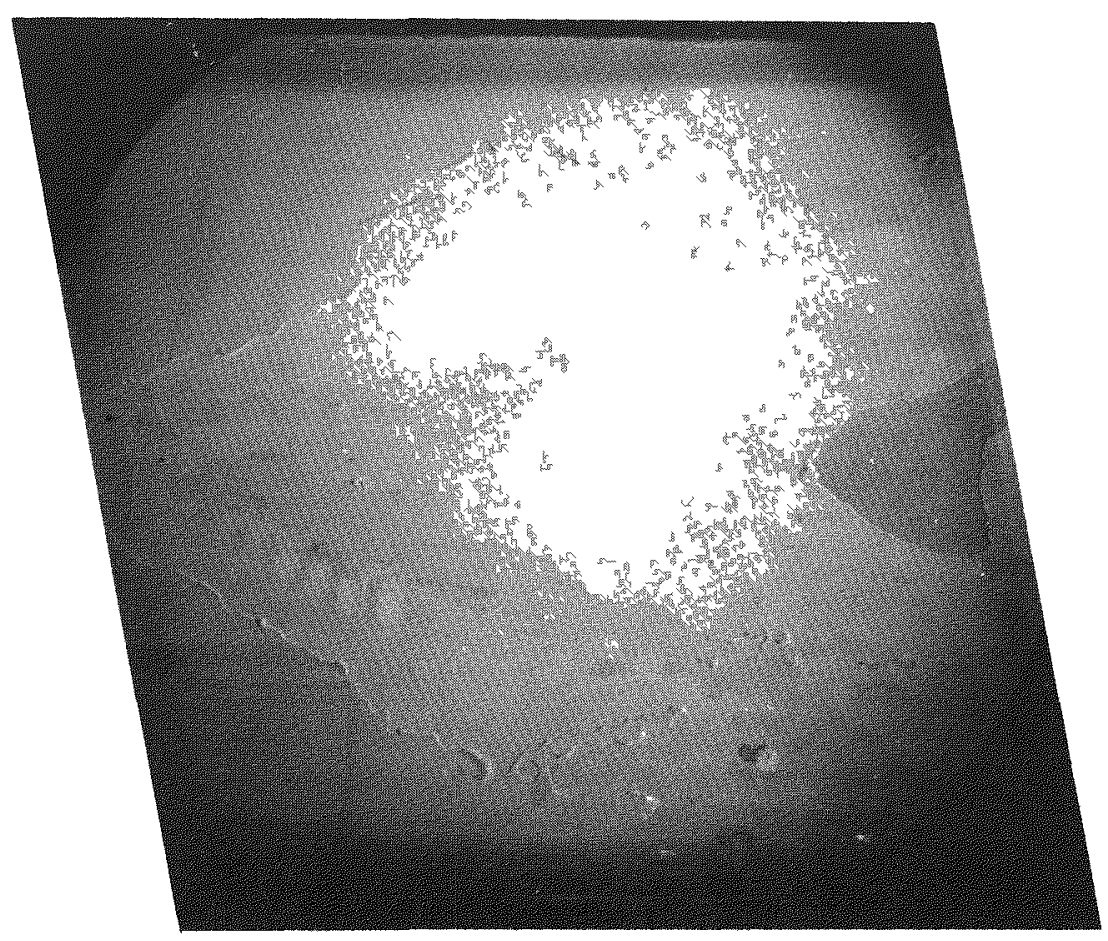

$1.3 \mathrm{X}$

A. Ten in. from top of element.

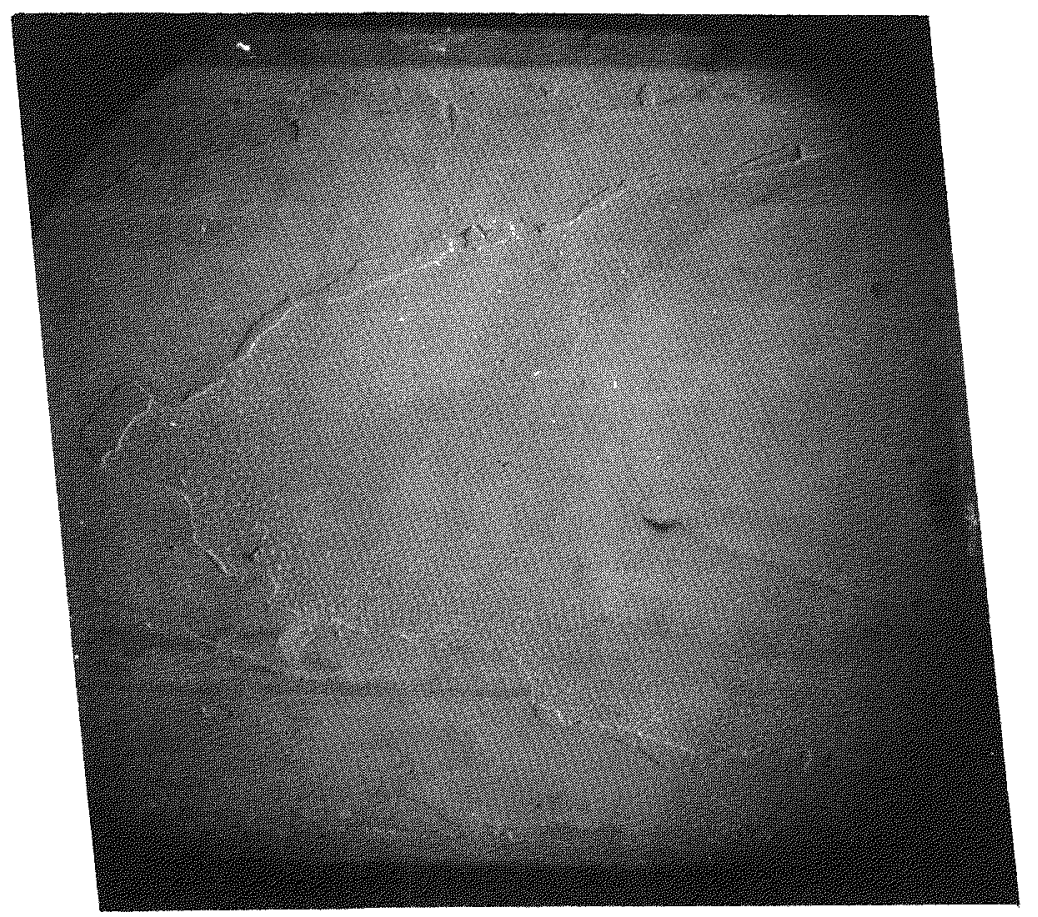

$1.3 \mathrm{X}$

B. Sixteen in. from top of element.

Top of element toward left.

Fig. 1 Recurring spalling patterns observed on element E255H. 


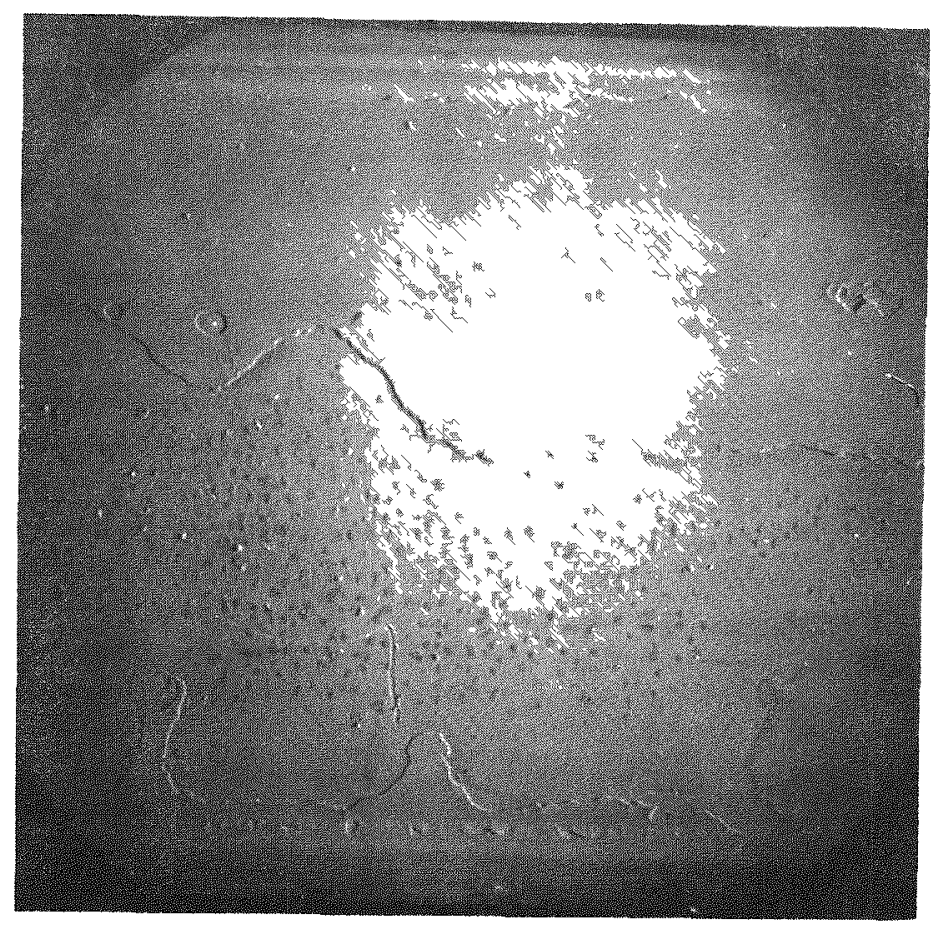

$1.3 \mathrm{X}$

C. Twenty-four in. from top of element.

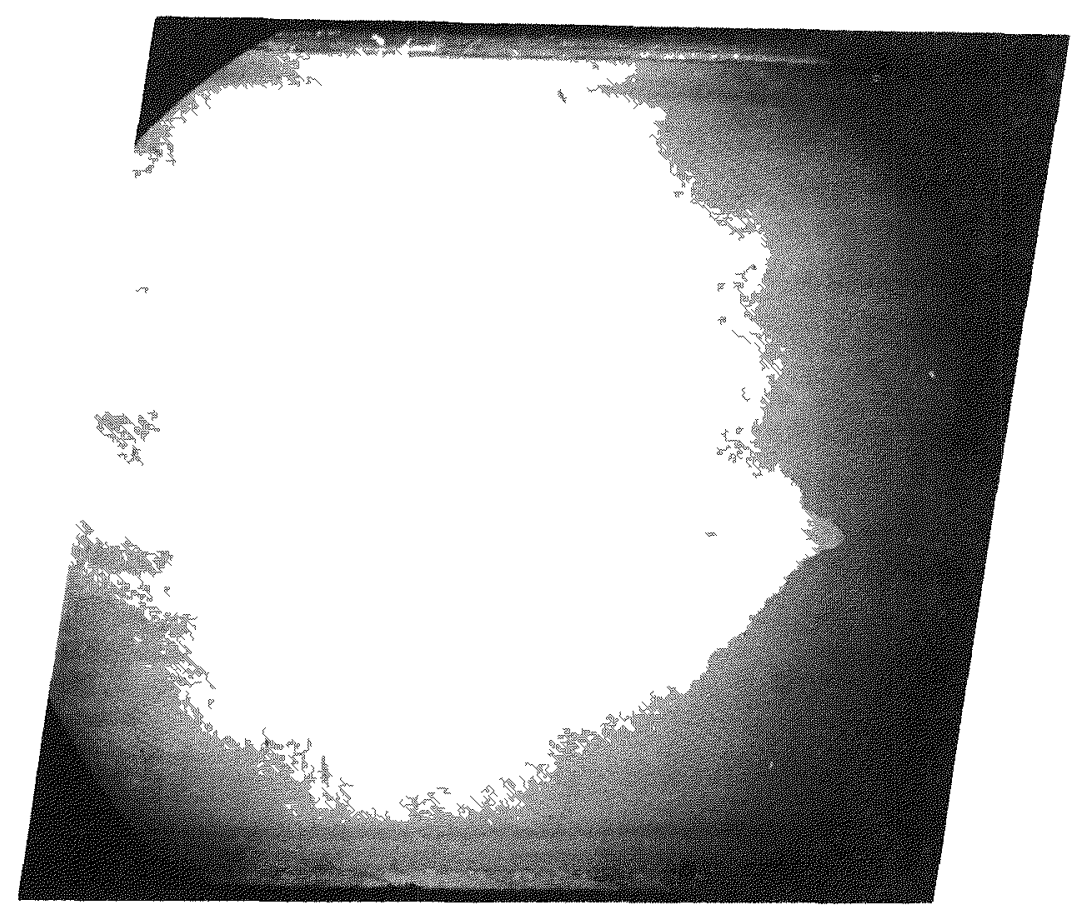

$1.3 \mathrm{X}$

D. Thirty-five in. from top of element.

Top of element toward left.

Fig. 1 Continued. 
Mounting Material

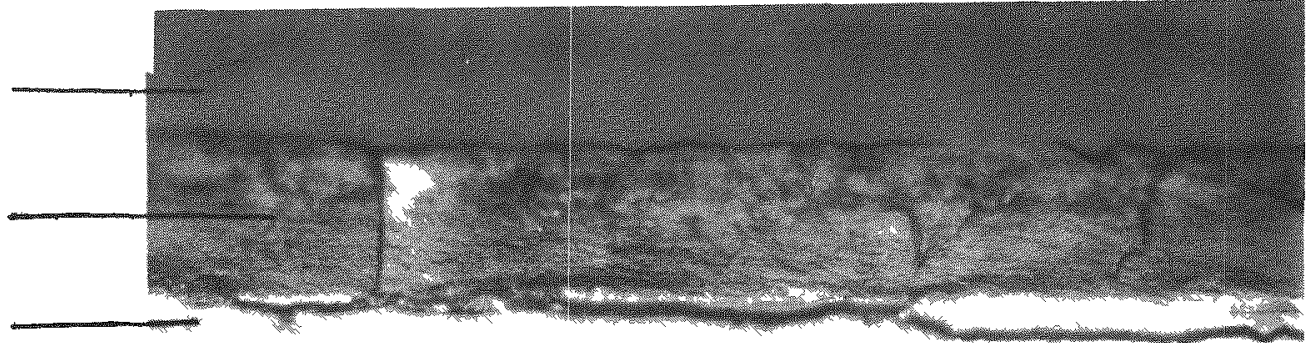

Inner Layer Oxide

Outer Layer Oxide

Inner Layer Oxide

Cladding

$500 \mathrm{x}$

A. Typical two layer oxide film structure.

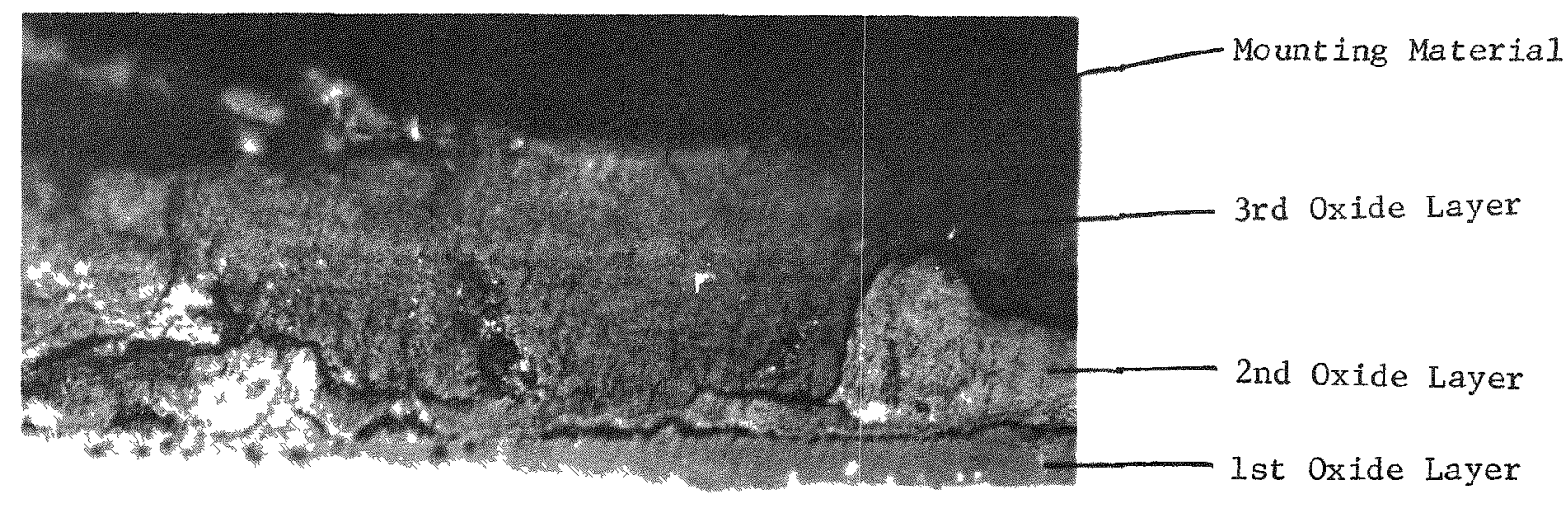

Cladding

$500 x$

B. Three layer oxide film structure.

Fig. 2 Oxide films typical of fuel plates of element E255H. 


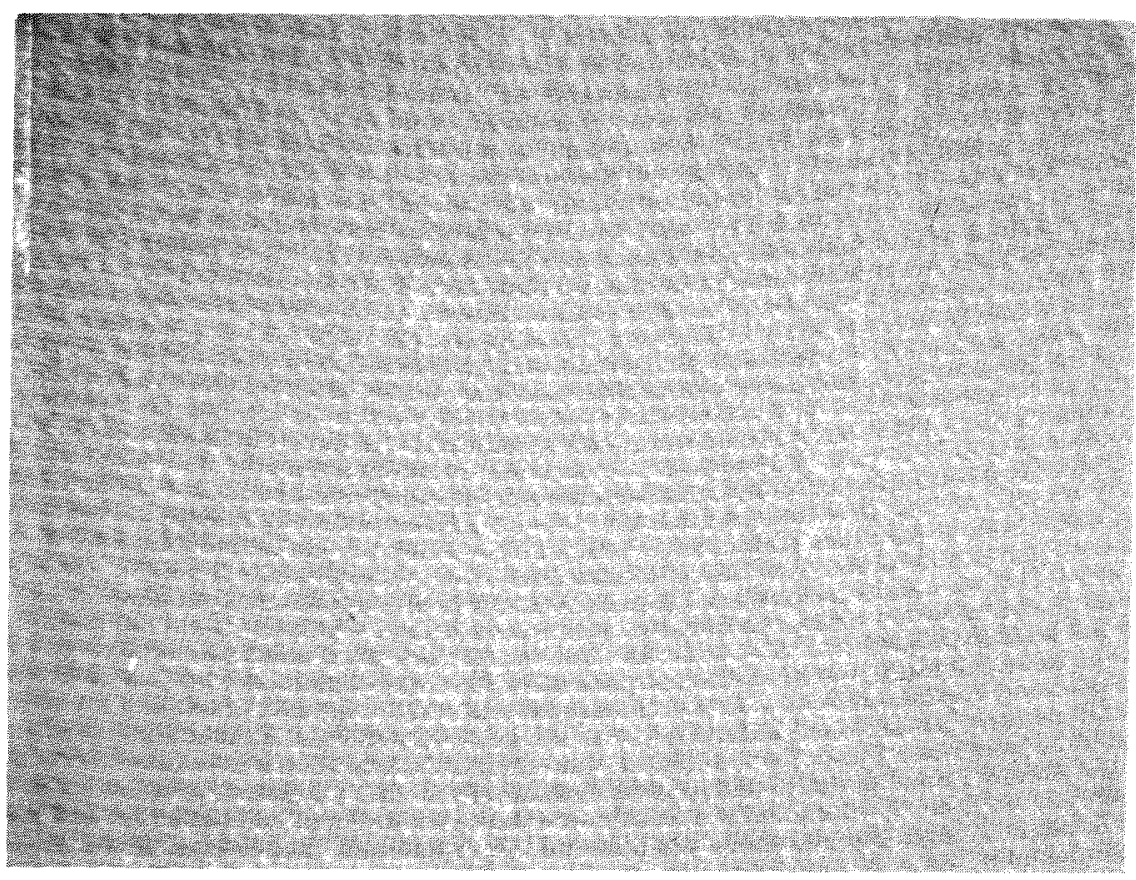

$10 \mathrm{X}$

Fig. 3 Two mil diameter bumps in oxide on side plate of element $\mathrm{E} 462 \mathrm{H}$. 


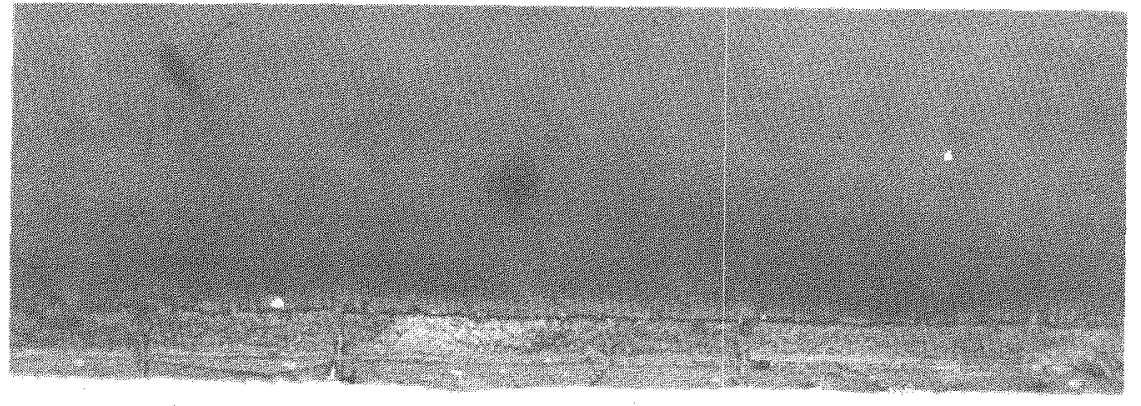

$500 x$

A. Fuel plate oxide.

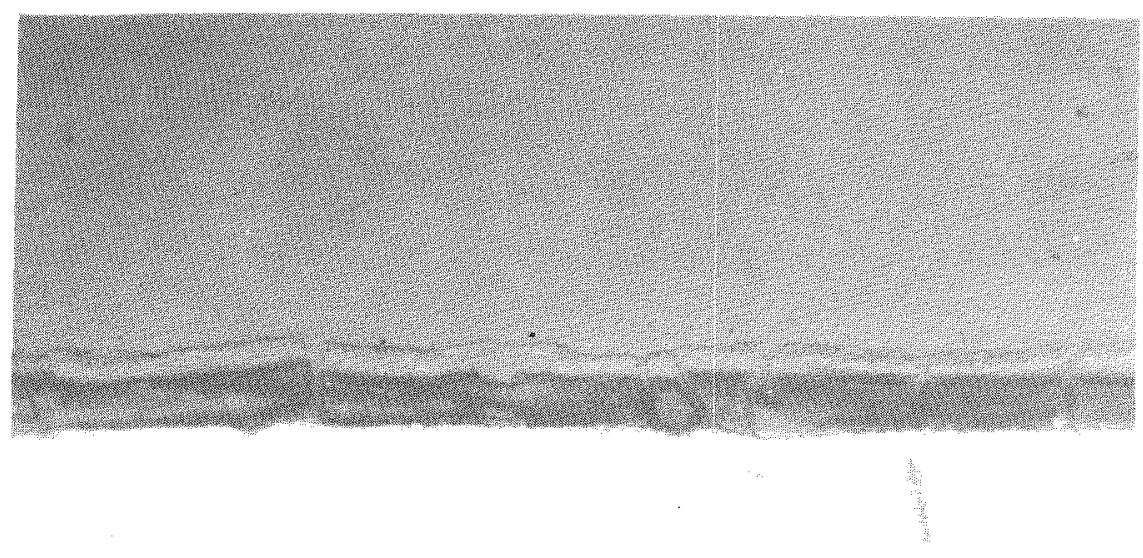

$500 \mathrm{x}$

B. Side plate oxide

Fig. 4 Oxide films typical of fuel and side plates of element E462H. 


\section{CLAD THICKNESS MEASUREMENTS OF ATR FUEL ELEMENTSS}

$$
\text { I. G. Miller, R. R. Jones, H. G. Miller }
$$

Considerable difficulty has been experienced with fission product leaks through the aluminum cladding of fuel plates in the Advanced Test Reactor (ATR). The breaks usually occurred near the ends of the fuel meat, where the rolling process occasionally caused the fuel meat to lump into a "dog-bone," consequently reducing the cladding thickness on one or both sides of the plate. A method of non-destructively examining the plates of assembled elements for cladding thickness has been placed into operation as a quality assurance operation of ATR fuel elements. The technique is sufficiently accurate to detect variations of \pm 0.001 inch in cladding thickness. With modest additional equipment the fuel can be scanned for an assay of fissile material.

The technique for measuring cladding thickness has been named BetaAutoradiography, whereby fission product decay betas are used to measure the thickness of the cladding. The range of fission product betas is such that claddings between 0.03 and $0.12 \mathrm{gm} / \mathrm{cm}^{2}$ can be measured. These thickness densities are in the range of $A I$, Zr and stainless steel claddings used on plate fuel reactors. The length of exposure of the fuel to neutron flux and the length of decay after exposure is a convenient way of tailoring the resulting fission product decay energy spectrum so as to make it most sensitive to the cladding thickness. Typically the fuel meat will be exposed to a thermal flux so as to give $10^{5}$ or more fission per $\mathrm{cm}^{2}$ of fuel meat surface. The beta range in the fuel meat is substantially shorter than the thickness of most fuels, and hence, non-uniformities in fuel meat are not critical to the success of this technique. The fuel is allowed to decay from 4 to 24 hours after the irradiation, principally to reduce the penetrating gamma and long-range (high energy) beta component. $X$-ray type film is placed adjacent to the fuel plate for a period of time to allow exposure to the beta activity. Film is then developed and read. with a densitometer. The relative transmission of beta particles is converted to clading thickness by use of a calibration curve.

Several elements were irradiated in an MTR neutron beam to test the capability of measuring clad thickness on an assembled fuel element. Tests were successful although it soon became evident that a thermal beam would be attenuated considerably passing through the element causing nonuniform irradiation due to the shadowing effect of one plate on the next. Fuel elements irradiated in the MTR thermal beam required a correction for irradiation nonuniformity and as a result the clad thickness measurements were less accurate.

The shutdown of the MPR started a search for a substitute epicadmium neutron beam of sufficient intensity to produce requixed fission product activity. An irradiation test in ATRC indicated a nomuniform irradiation exposure and complications relating to reactor safety. TREAT had an insufficient epicadmium flux. The SPHRT IV reactor was found satisfactory but approval to operate the reactor was denied. 
The Nuclear Test Gage (NTG), used as a californium-252 neutron multiplier produced almost sufficient flux with one mg $\mathrm{Cf}$. The NTG center core was then modified to optimize the epicadmium beam from each end. Three $\mathrm{mg}$ of $\mathrm{Cf}$ was loaded in a storage tank adjacent to the NTIG with an automatic drive system for insertion into the subcritical assembly. The reason for operating it with $C f$ was because the device was approved only for subcritical operation. A cutaway view of the facility is shown in Figure 1 . Sufficient neutron flux was obtained to irradiate ends of $8 \mathrm{ATR}$ fuel elements simultaneously when the effective $\mathrm{k}$ was raised to 0.9995. During a typical one hour irradiation, $k_{\text {eff }}$ dropped to 0.9992 caused by the negative temperature coefficient dropping the flux by a factor of two. The NTG was then used for irradiation throughout the project.

To correlate clad thickness with filn density, a calibration method was devised using bare fuel core material of differing uranium weight densities corresponding to ATR core material. These were irradiated in positions normally occupied by ATR fuel elements on the NTG. Decay time, film and film backings were used to simulate actual conditions. Varying thicknesses of aluminum strips were placed between film and bare fuel core to simulate clad thickness. Figure 2 is a typical calibration film. Several calibration films are made for differing irradiation times as shown in Figure 3 . This would be a calibration curve for a specific irradiation time, decay time, film type and film exposure time.

To expose the film to the beta activity it is necessary to hold the film against the plate surfaces. To prevent beta activity from one plate exposing the film on adjacent plates, a sheet of lead 0.035 inch thick was placed between two pieces of films as a packet. A dense material was used because of the little space available to stop the higher energy beta activity. Beta backscattering from the lead was observed causing higher film exposure. The exposure from backscattered betas produces an image of slightly less resolution but the combined image has sufficient resolution for clading measurement. Exposed film was developed in an automatic film processor and scanned with a manual densitometer.

During the fuel clad thickness measurement program, 165 ATR fuel elements fabricated by Atomics International have been examined for minimum clad thickness. In addition, several National Lead (NL) fabricated elements were also tested for special purposes.

Sixty-six type $7 \mathrm{~F}$ elements were examined for clad thickness. A minimum clad thickness was determined for each element and for all areas where the measured clad thickness was less than 0.005 inch, the thin clad area was measured. The area in each case is that of a rectangle placed around the limits of the thin spot, i.e., points at which the clad thickness dropped below 0.005 inch. The width and length plots Figures 4 and 5 give an extent of the areas under 0.005 inch. Many are of small dimensions but 38 of these are 1 inch or longer with two being greater than 2 inches Iong. The direction of the length is that of the width of the plate so that some of these thin clad areas are nearly the width of the fuel. The minimum clad thickness in Figure 6 indicates that the average value for the element minimum clad thickness is about 0.0045 inch. 
Four National Lead (NL) type $7 F$ elements were examined for clad thickness. The dog bone in a $\mathbb{N L}$ plate is considerably finer in width than those in AI elements with the minimum clad thickness varying from 0.0065 to 0.008 inch

Ninety-nine standard type elements have been examined and a minimum element clad thickness plotted in Figure 7. Average clad thickness is about 0.006 inch, Only 13 elements or 13\% of the standard elements have clad thickness less than 0.005 inch. No thin clad areas will be measured on standard elements unless the need arises.

Since the element $\mathrm{XA}-42-\mathrm{G}$ with its very small thin-clad areas did not fail in the reactor, the importance of measuring the area of thin clad as well as clad thickness should be stressed. An arbitrary limit of 0.005 in. clad thickness irrespective of area had been set as the initial basis for acceptance of elements for use in the ATR.

With the arbitrary 0.005 inch minimum clad thickness limit, three cores of standard elements, most of which were examined elements, have been used in the ATR without a known fission break. Thus, the clad thickness measurement program is paying off especially in critical cycles such as in the extended burnup program. The remaining $7 F$ type elements under 0.005 inch clad thickness may now be segregated as to area and clad thickness and utilized in core loadings for maximum fuel economy and minimum fuel element failure probability. 
1 Neutron Source Drive

Colifornium-252 Neutron

Source Shield Tank

3 Control Rod Drive

4 Conirol Rods

5 Californium Neutron Source

6 Neutron Beam Collimators

7 Flux Trop

8 Reflector

9 Fuel

10 ATR Fuel Elements

II Lead Shielding

12 Collimator

13 Vocuum Cassette

14 Object Being Neutron Rodiogrophed

\section{is Concrete Wall}

16 Neutron Shielding

17 Experiment Room

18 Control Room

19 Water

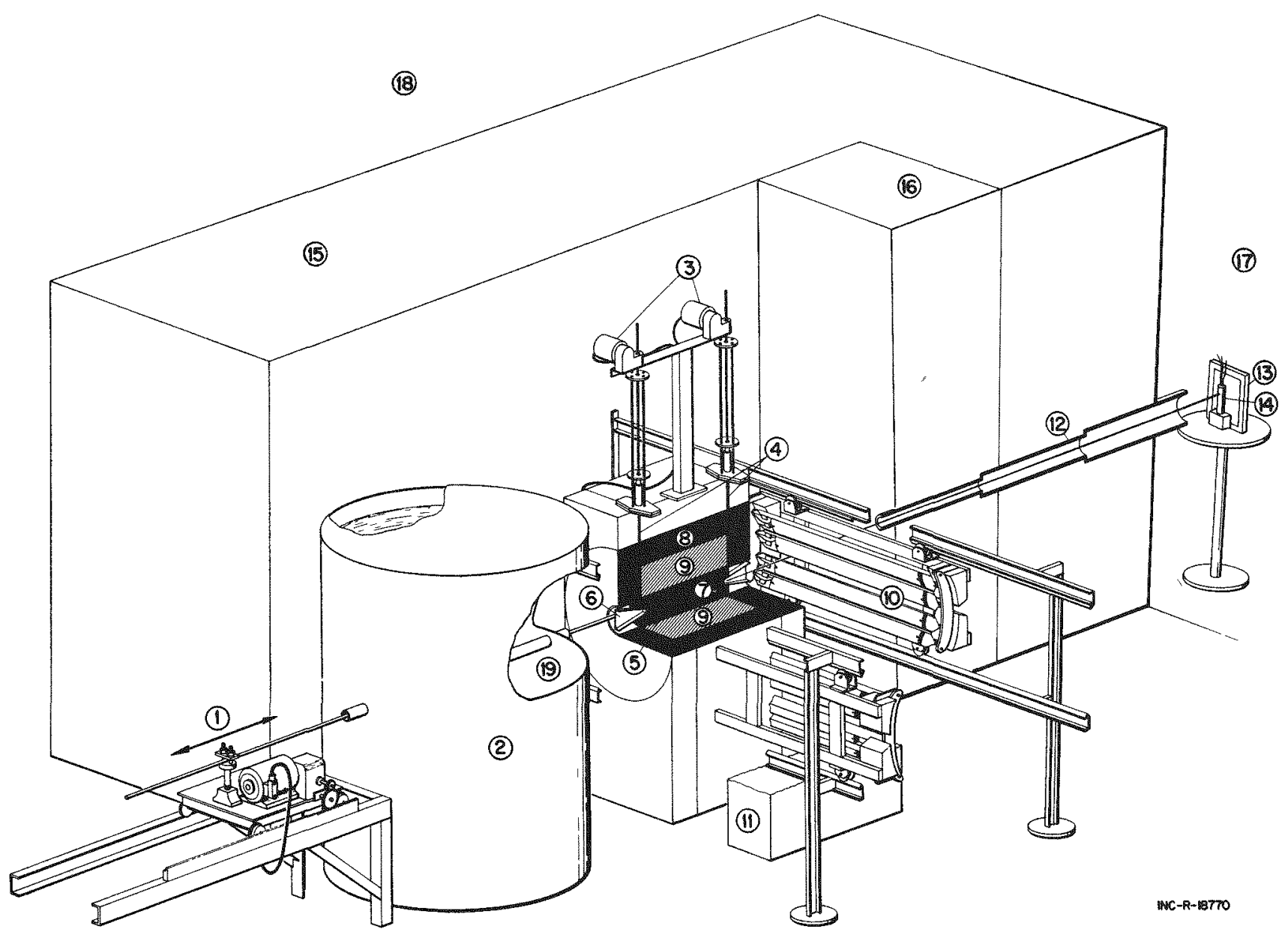

Figure 1. Cutaway view of the Nuclear Test Gage (NTG) as modified to irradiate ends of 8 ATR fuel element

simultaneously. A hole through the wall allows a beam out of the facility for neutron radiography applications. 


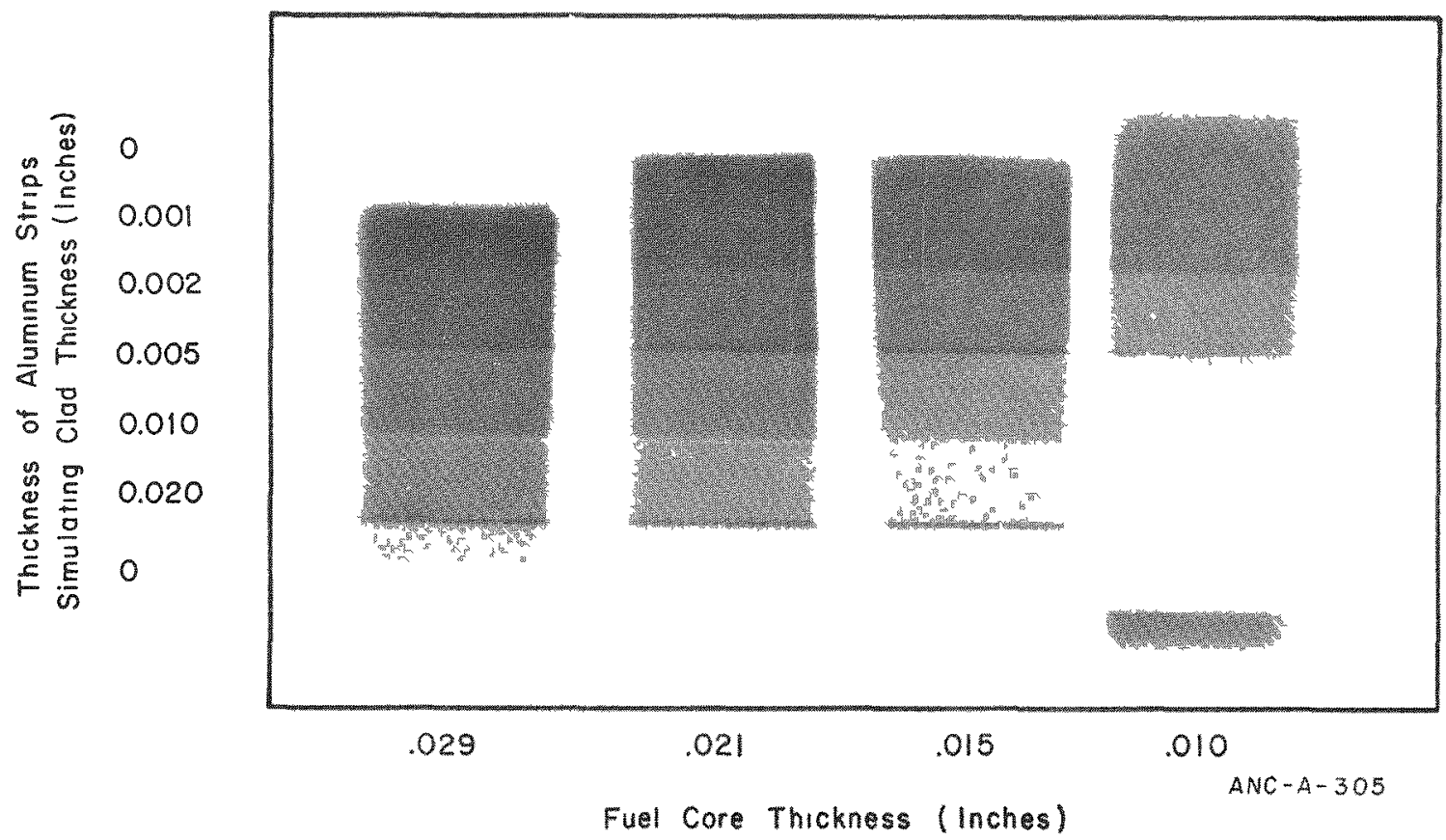

Figure 2. An actual beta autoradiographs of the bare fuel core pieces are shown. The bands across the plates are the various thickness of aluminum strips covering the fuel. 


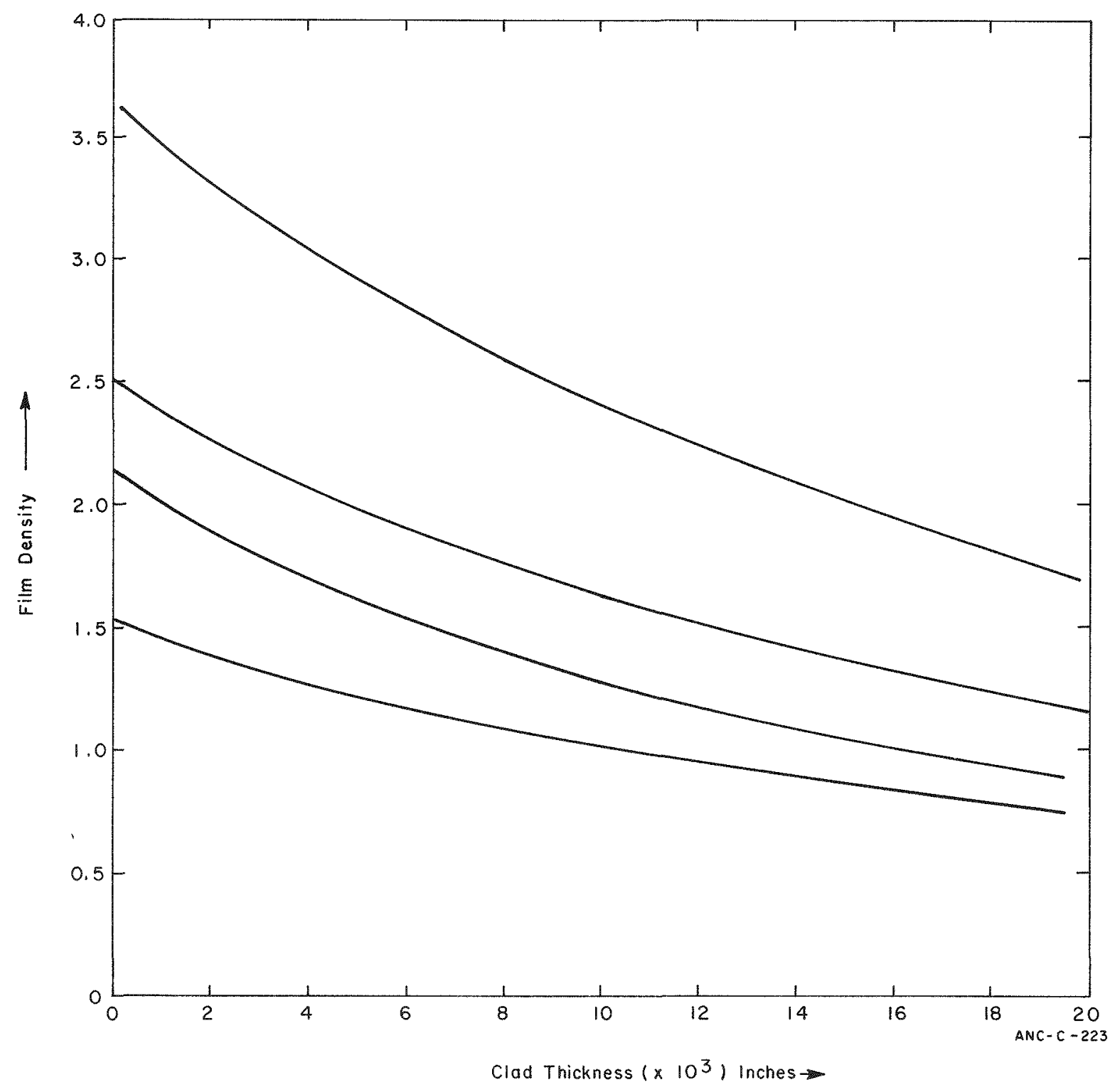

Figure 3. Typical calibration for conversion of film densities to clad thickness. 


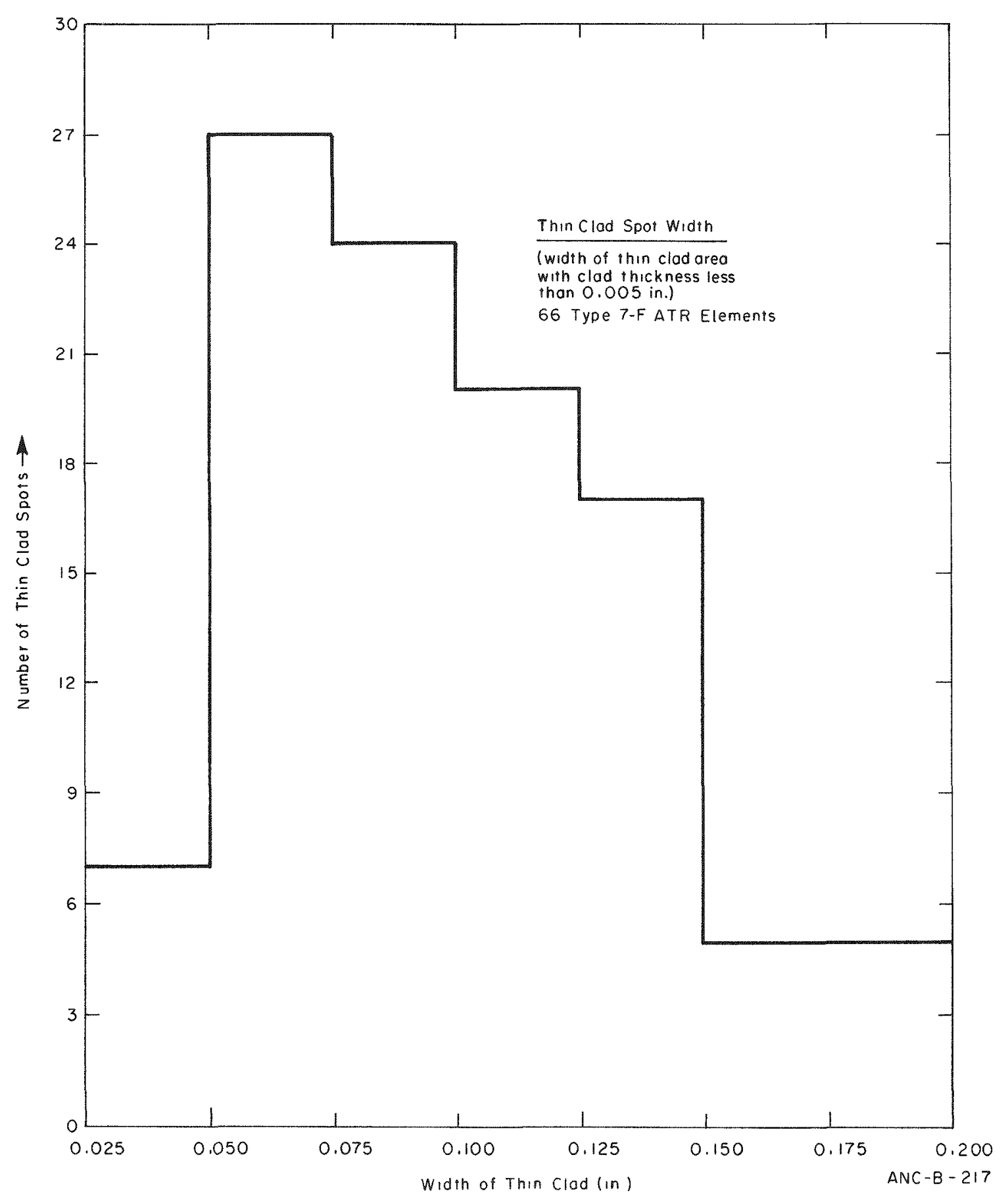

Figure 4. Thin clad spot width as measured on 66 type 7-F ATR fuel elements. 


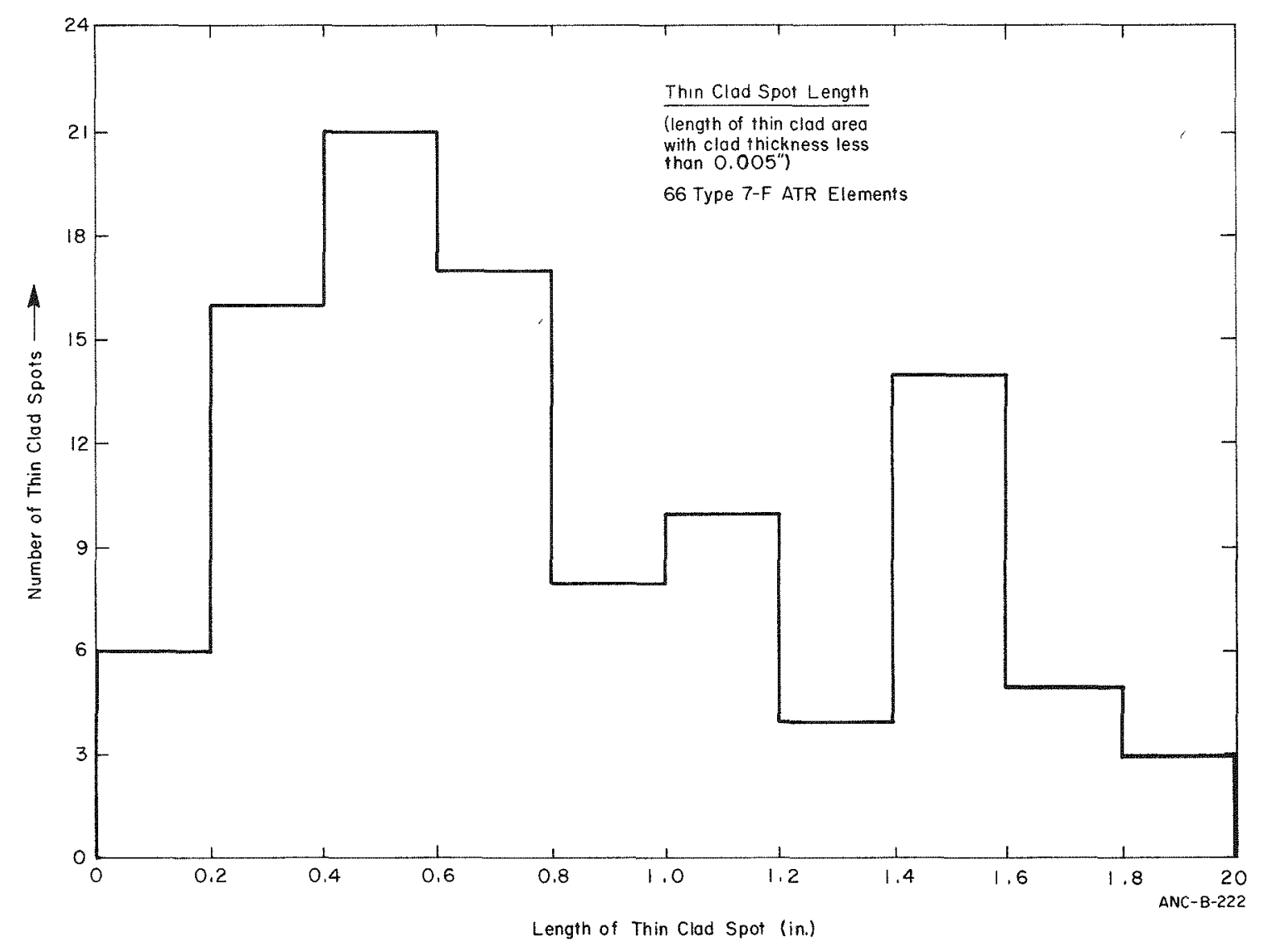

Figure 5. Thin clad spot length as measured on 66 type 7-F ATR element. 


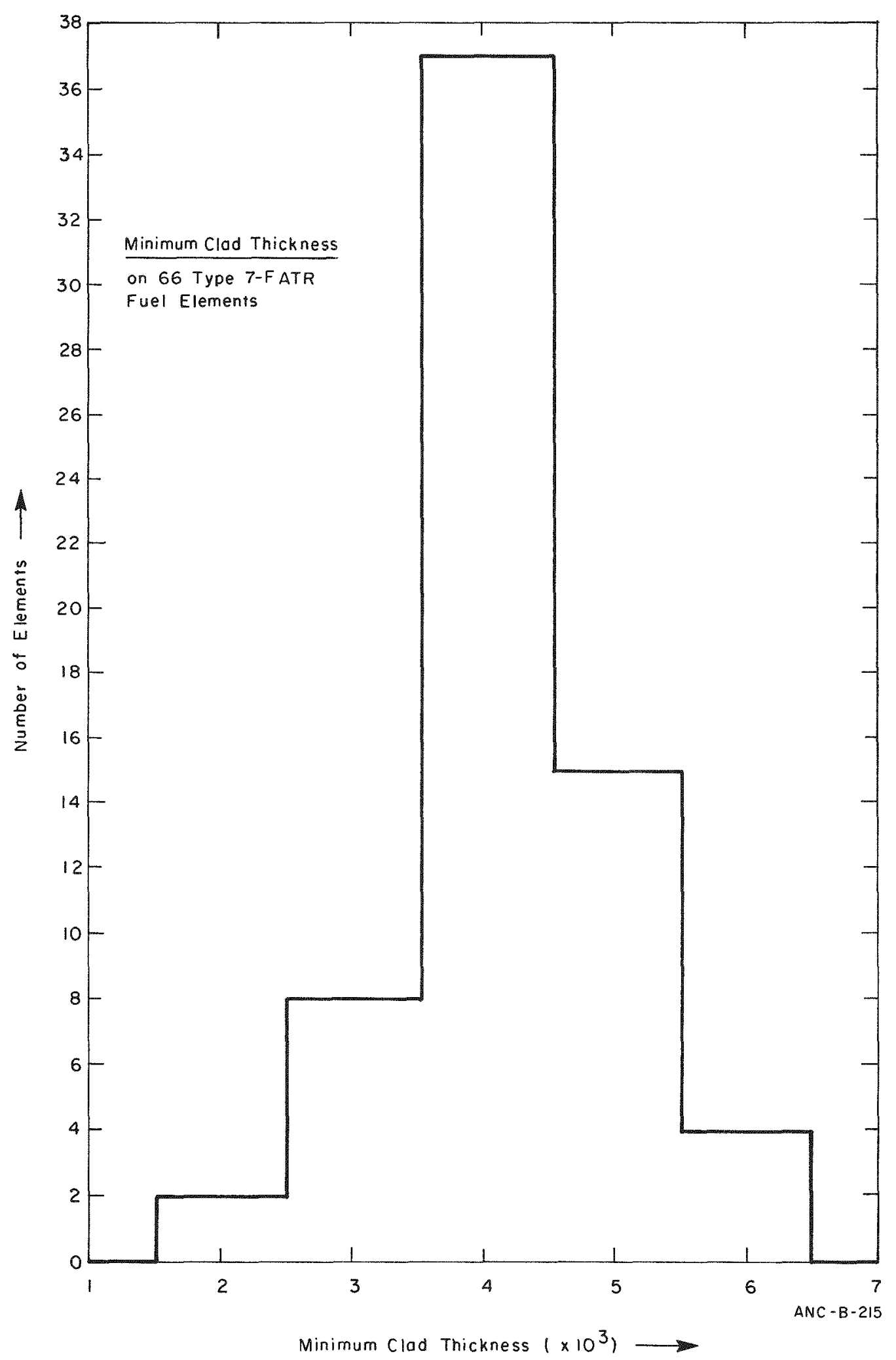

Figure 6. Plot of the valves of minimum clad thickness measured on 66 type $7-F$ fuel elements. 


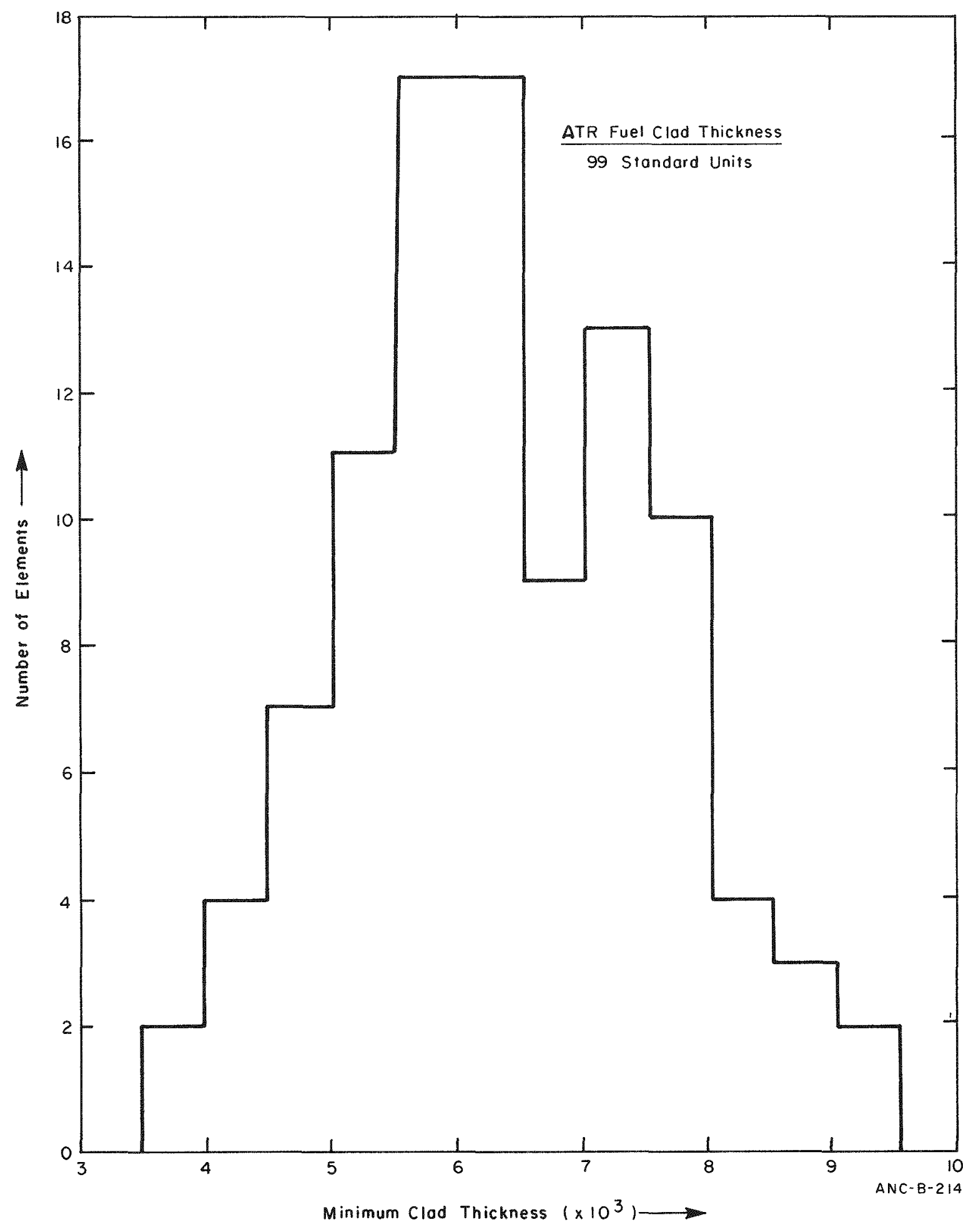

Figure 7. Plot of the valves of minimum clad thickness measured on 99 standard type ATR fuel elements. 


\section{SURVEILLANCE OF ATR REACTOR MATERIALS}

G. E. Korth, M. W. E1lingford, J. M. Beeston

The objective of the surveillance program is to establish the service life of critical ATR structural core components and to relate irradiation damage to operating criteria so that these components may be utilized most effectively and with adequate safety.

The approach has been to establish initial damage rationale with service life predictions based on state-of-the-art and to update these through specimen irradiation and examination, through component monitoring, and through other sources. For beryllium the damage criteria was the fluence at which cracking and spalling would occur and was predicted at $1.8 \times 1022 \mathrm{n} / \mathrm{cm}^{2}$ $\mathrm{E}>1 \mathrm{MeV}$. For hafnium the irradiation damage criteria was a residual ductility (uniform elongation) of $1 \%$, and by extrapolation, a predicted fluence value of $1.2 \times 10^{22} \mathrm{n} / \mathrm{cm}^{2} \mathrm{E}>1 \mathrm{MeV}$ was obtained. A growth damage criteria for aluminum and stainless steel components was considered pertinent because of their dimensional interaction as structural components, while mechanical property changes would be available from other sources. As an example of this dimensional interaction the outer shim control cylinder assemb1y contains a beryllium cylinder held in position with a center 6061 aluminum bolt which is pre-loaded an amount depending on the differential growth of the aluminum and the beryl1ium.

Beryllium. Examination of 65 beryllium specimens has extended mechanical property data up to neutron fluences of $1.3 \times 10^{22} \mathrm{n} / \mathrm{cm}^{2} \mathrm{E}>1 \mathrm{MeV}$. Compression strength is still increasing with maximums in the $200 \mathrm{ksi}$ range with nil ductility above $5 \times 10^{21} \mathrm{n} / \mathrm{cm}^{2}$. The yield strengths were the same as the fracture strengths with little plastic deformation evident. The fracture stress of the extruded material (1.2\% Be0) and the two hot-pressed materials $(1.5 \% \mathrm{BeO}$ and $2.0 \% \mathrm{Be})$ ranged between 130 and $200 \mathrm{ksi}$, while the fracture stress of the vacuum cast beryllium ( $1.0 \% \mathrm{Be} 0$ ) ranged from 20 to $60 \mathrm{ksi}$. These results show that the fracture stress is still increasing with increased irradiation indicating that the service life as predicted is beyond the fluence of these specimens. Impact resistance was shown to decrease sharply after exposure of $5-6 \times 10^{21} \mathrm{n} / \mathrm{cm}^{2}$. Hot-pressed bery11ium specimens irradiated to fluences of $2-5 \mathrm{x}$ $10^{21} \mathrm{n} / \mathrm{cm}^{2}$ accepted 300 to 500 blows at $8.9 \mathrm{~kg}-\mathrm{m}(64.6 \mathrm{ft}-1 \mathrm{bs})$ per blow before failure, while hot-pressed specimens irradiated to $6-11.7 \times 10^{21 \mathrm{n} / \mathrm{cm}^{2}}$ failed after two or three blows. Vacuumcast and extruded beryllium impact resistance was below that of the hot-pressed material. 
Growth rates of $0.2 \%$ per $10^{22} \mathrm{n} / \mathrm{cm}^{2} \mathrm{E}>1 \mathrm{MeV}$ were generally obtained and runaway swelling temperatures (temperatures at which helium rapidly agglomerates into bubbles) is still above $500^{\circ} \mathrm{C}$ and is dependent upon grain size. Of the four types of beryllium examined the type with the smallest grain size had the highest threshold temperature and the lowest total swelling. The presence of the helium which is the principal constituent believed to produce the increase in fracture strength and growth at ambient temperatures is still not resolved at $40,000 \mathrm{X}$ in a specimen irradiated to $1.13 \times 10^{22} \mathrm{n} / \mathrm{cm}^{2}$, Figure 1 . The helium is evidently only effective as a hardener while dispersed in an essentially atomic form, since after annealing above threshold temperatures to agglomerate the helium into bubbles, the ductility returns to a value very near the pre-irradiation value, even though considerable swelling has occurred.

Capsules from ATR position A-11 with fluences ranging from 1.2 to $2.1 \times 1022 \mathrm{n} / \mathrm{cm}^{2}$ have been removed from the reactor for testing.

Hafnium. Hafnium specimens irradiated to $1.4 \times 10^{21} \mathrm{n} / \mathrm{cm}^{2}$ $E>1 \mathrm{MeV}$ were examined for mechanical property changes and dimensional stability; The results of this investigation were reported in IN-1440[1], and indicate that the damage criteria is not approached at these neutron fluences. One of the hafnium neck shims is scheduled for removal and testing after Cycle $10 \mathrm{~A}$ at a fluence of $9.8 \times 1021 \mathrm{n} / \mathrm{cm}^{2}$. Irradiation damage or reactivity changes from burnup comprise the limiting criteria for replacement of these control components. A dimensional measurement performed on one of the fixed shim rods irradiated to an average fluence

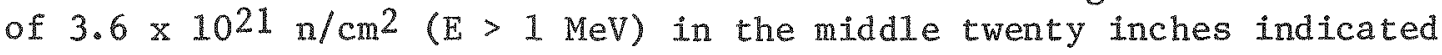
that the growth was 1 ess than $0.01 \%$ which was within the experimental accuracy of the measurement. Reactivity measurements performed on this same rod showed a change of $20 \%$ when compared to one of the spare unirradiated fixed shims.

Aluminum. The aluminum alloy in the ATR is 6061 and 16 specimens of this alloy are undergoing irradiation in capsules from which accurate growth measurements can be made and compression yield strengths determined. The specimens have about reached

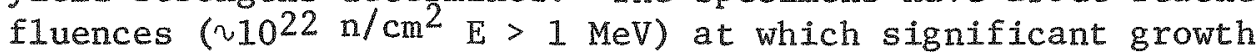
occurs. During this interim time, examination of aluminum (1100 alloy) which had received high fluence as a skirt plate in the MTR was undertaken. A report is in progress. Results of growth and strength measurements are summarized at this time.

Upon irradiation at less than threshold temperatures $\left(<300^{\circ} \mathrm{C}\right)$ the density of aluminum decreases due to formation of voids. An induction period is generally observed during which no voids are seen and no significant growth occurs. This behavior evidently also occurred in the MTR skirt plate. The data for the immersion density measurements on pieces cut from the plate (all oxide removed) is given in Table $\mathbb{I}$. The density change by comparison 
with the 1100 aluminum standard density is given in Figure 2. According to the data a fluence of approximately $10^{22} \mathrm{n} / \mathrm{cm}^{2}$ is required before significant growth occurs. After reaching this fluence growth occurs according to the relationship, $\frac{\Delta V}{V} \simeq-\frac{\Delta \rho}{\rho}=$ $a(\phi t)^{3}$. The swelling exponent of 3 is at the high end of the range generally encountered for the swelling of aluminum alloys.

The mechanical property measurements were made on rectangular specimens with 1 in. gage length cut from the skirt plate and pulled on an Instron at a crosshead speed of $0.02 \mathrm{in} / \mathrm{min}$. The data is given in Table II. Although as generally seen the strength has increased and the ductility decreased upon irradiation, the highest fluence has not produced the highest strength nor greatest loss in ductility, Figure 3. The data indicate that a saturation in strength properties results at fluences of about $1 \times 10^{22} \mathrm{n} / \mathrm{cm}^{2}$. A saturation effect has been noted[2] although data beyond $1.2 \times 10^{22}$ $\mathrm{n} / \mathrm{cm}^{2}$ for this alloy were not reported.

Transmission electron micrographs, Figure 4, indicate the reason for the decrease in density is due to the increase in void volume wherein the higher fluence specimen has a much greater void volume. From electron microscopy measurements the diameter of the voids is $200-1500 \AA$ and the number density is 1015 voids $/ \mathrm{cm}^{3}$. The presence of voids evidently has less effect as a hardener than the reduction of mass at the test cross section which can account for the reduction in strength with the apparent saturation effect on the ductility.

[1] G. E. Korth, C. R. Brinkman, J. M. Beeston, The Effect of Fast Neutron Irradiation on the Mechanical Properties of Hafnium, IN-1440 (Oct. 1970).

[2] J. R. Weir, Jr. and R. T. King, Fuels and Materials Quarterly p. 246 (Sept. 1970). 
TABLE I

DENSITY CHANGES IN IRRADIATED 1100 AI MTR SKIRT PLATE

\begin{tabular}{|c|c|c|c|c|}
\hline Spec. & $\begin{array}{l}\Phi \text { fast } \mathrm{n} / \mathrm{cm}^{2} \\
\mathrm{E}>1 \mathrm{MeV} \times 10^{22} \\
\end{array}$ & $\begin{array}{c}\Phi \mathrm{th} \mathrm{n} / \mathrm{cm}^{2} \\
\times 10^{22} \\
\end{array}$ & $\begin{array}{l}\text { Density } \\
\mathrm{g} / \mathrm{cm}^{3} \\
\end{array}$ & $-\frac{\Delta \rho}{\rho} \%$ \\
\hline $1 \mathrm{MHD}$ & 5.6 & 6.6 & 2.501 & 7.7 \\
\hline$D-1$ & 4.1 & 5.0 & 2.5967 & 4.2 \\
\hline$D-3$ & 4.0 & 5.4 & 2.6088 & 3.7 \\
\hline$D-5$ & 2.8 & 5.1 & 2.6680 & 1.6 \\
\hline$D-4$ & 1.8 & 3.9 & 2.7091 & nil \\
\hline$D-2$ & 1.8 & 3.4 & 2.7039 & 0.2 \\
\hline$D-6$ & 1.7 & 3.7 & 2.6977 & 0.4 \\
\hline$D-7$ & 0.9 & 2.6 & 2.7081 & nil \\
\hline Al std. & -- & -- & 2.71 & -- \\
\hline
\end{tabular}

TABLE II

MECHANICAL PROPERTIES OF IRRADIATED 1100 AI-MTR SKIRT PLATE AT AMBIENT TEMPERATURE

\begin{tabular}{|c|c|c|c|c|c|c|}
\hline $\begin{array}{c}\Phi \text { Fast } \\
\text { E>1MEVx10 } \\
\end{array}$ & $\begin{array}{c}\text { बth } \\
\times 10^{22} \\
\end{array}$ & $\begin{array}{l}\text { U.T.S. } \\
\text { ksi }\end{array}$ & $\begin{array}{l}\text { Y.S. } \\
\mathrm{ksi}\end{array}$ & $\begin{array}{l}\text { Uniform } \\
\text { E1. } \%\end{array}$ & R.A. $\%$ & $\begin{array}{c}\text { Total } \\
\text { El in/in } \\
\% \\
\end{array}$ \\
\hline 4.5 & 5.6 & 23.0 & 18.7 & 7.0 & 45.7 & 24 \\
\hline 4.5 & 5.6 & 19.5 & 13.8 & 9.5 & 53.2 & 16 \\
\hline 2.7 & 4.1 & 25.3 & 19.1 & 6.5 & 49.5 & 18 \\
\hline 1.1 & $4 \cdot 6$ & 32.8 & 28.9 & 8.0 & 48.5 & 25 [a] \\
\hline 1.0 & 4.2 & 31.2 & 27.5 & 7.1 & 46.4 & -- \\
\hline 1.5 & 3.0 & 33.6 & 28.5 & 9.0 & 55.9 & 21 \\
\hline$\cdots$ & $-\infty$ & 13.0 & 5.0 & $-\infty$ & $-\cdots$ & $40^{[C]}$ \\
\hline
\end{tabular}

[a] Specimen broke outside gage marks.

[b] Values from "Aluminum Standards and Data" 2nd Edition (December 1969), The Aluminum Association, New York, New York.

[c] Elongation in 2 in. gage length. 


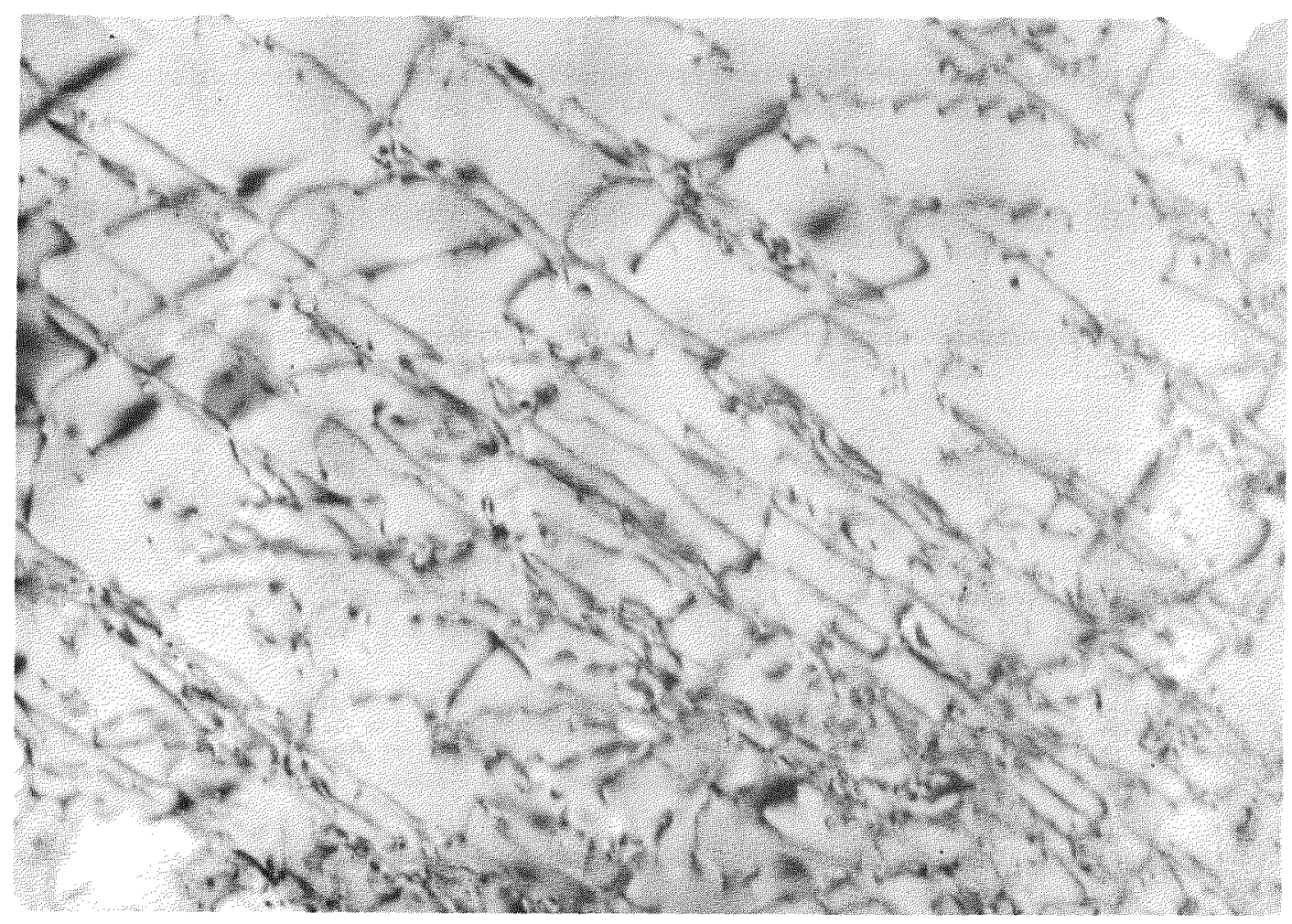

$40,000 x$

Fig. 1 Bery 11 ium irradiated at $100^{\circ} \mathrm{C}$ to fluence of $1.13 \mathrm{x}$ $1022 \mathrm{n} / \mathrm{cm}^{2} \mathrm{E}>1 \mathrm{MeV}$ and compression tested. Note presence of dislocations but absence of voids or bubbles after room temperature tests. 


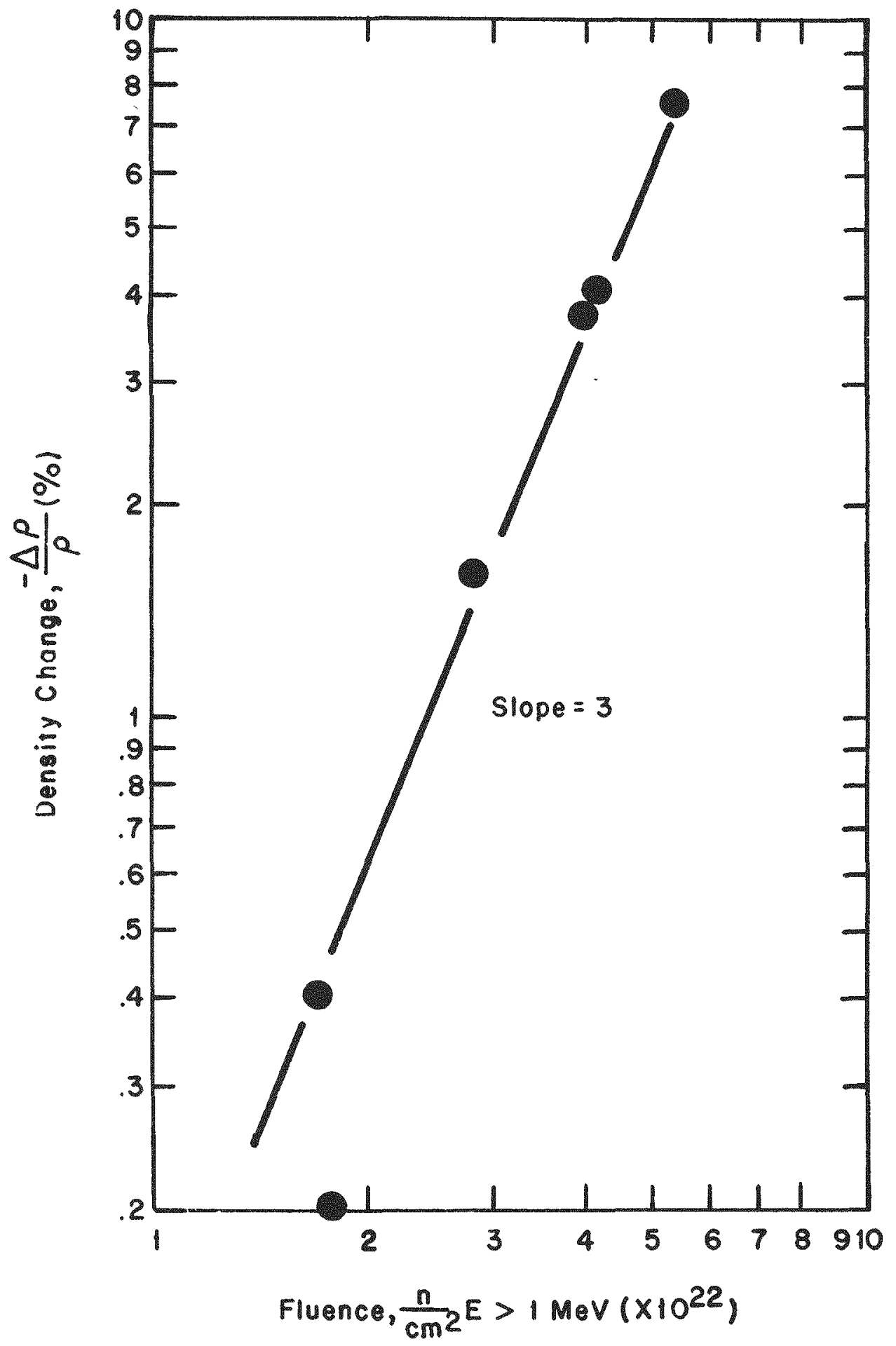

Fig. 2 Density change in irradiated 1100 Al-MTR skirt plate. 

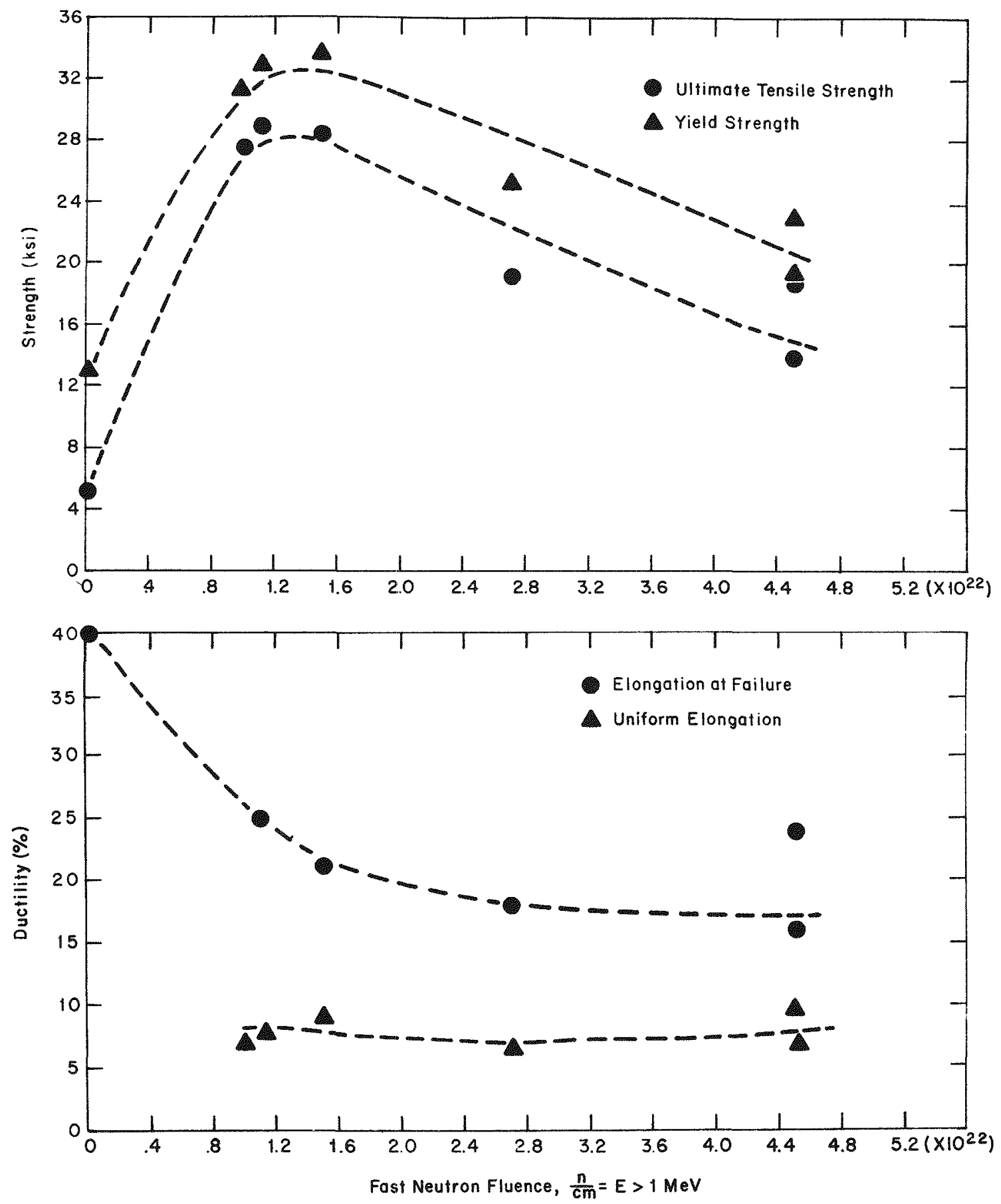

Fig. 3 Strength and ductility of irradiated 1100 Al-MTR skirt plate. 

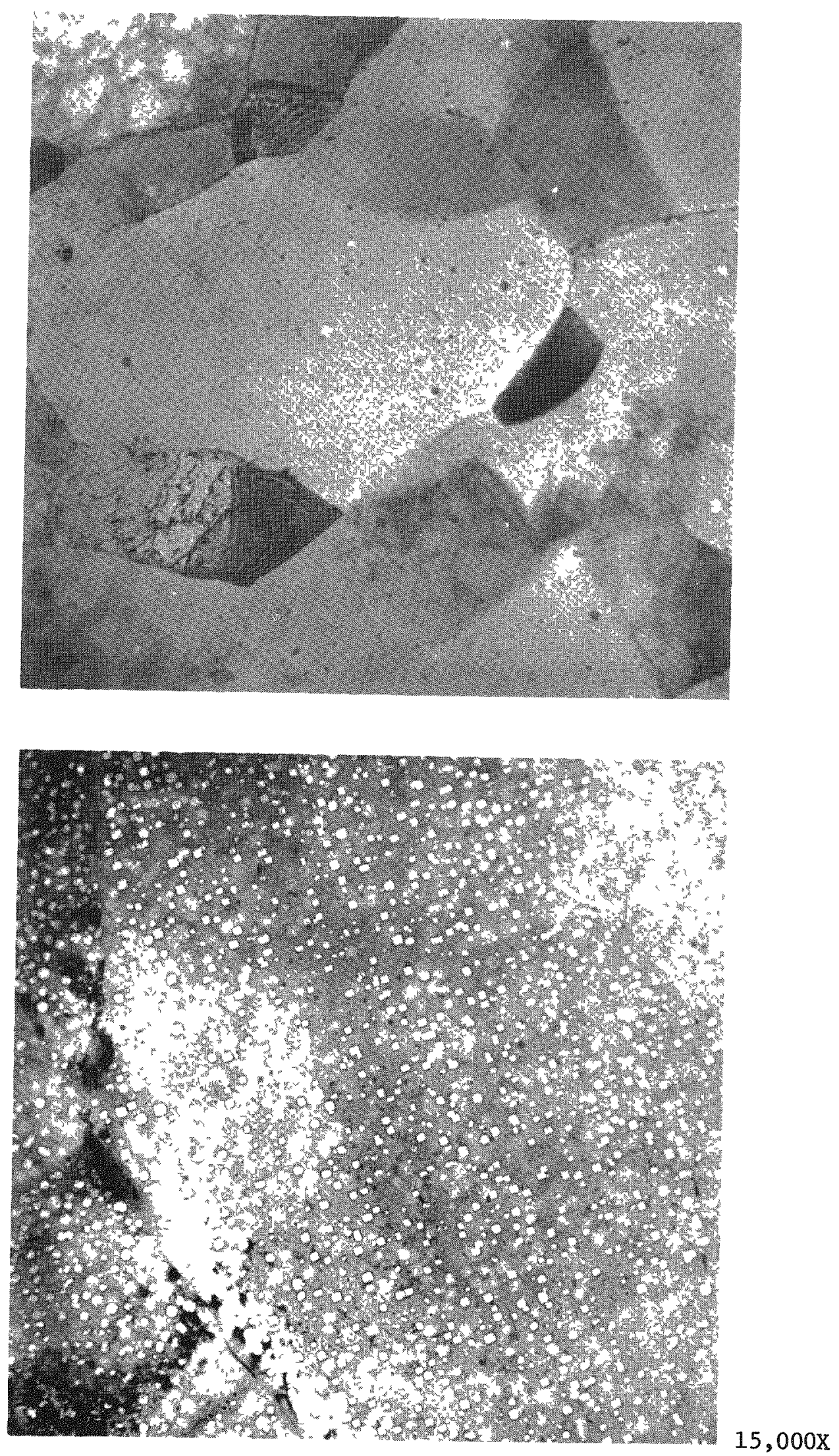

Fig. 4 Aluminum showing difference in void density at two fluence values (a) at $1.8 \times 10^{22} \mathrm{n} / \mathrm{cm}^{2}(\mathrm{E}>1 \mathrm{MeV})(\mathrm{b})$ at $5.6 \times 10^{22} \mathrm{n} / \mathrm{cm}^{2}$ $(E>1 \mathrm{MeV})$. 


\title{
PREPARATION OF TEST REACTOR FUEL PLATES CONTAINING FISSIUM
}

\author{
M. J. Graber, R. R. Hobbins, G. O. Hayner, B. G. Carlson
}

The mechanical stability of fuel elements under irradiation is a matter of prime importance in the operation of a nuclear reactor. Factors unique to a reactor environment which can influence the mechanical and physical properties of a nuclear fuel are radiation damage and fission-produced composition changes. Under the operating conditions of the ATR and ETR the composition changes may well be the more important influence on the properties of the fuel systems in these reactors.

A program was developed to measure mechanical and physical properties of uranium aluminide dispersions containing inactive fission products (fissium) designed to simulate irradiated ATR fuels. The plan included measurements of elastic modulus, yield strength, creep, thermal expansion, and fuel-matrix reaction rate as a function of burnup. The results of this study could then be compared to pre-irradiation and post-irradiation measurements to determine the relative importance of composition changes and radiation damage. Another facet of the plan called for the inpile measurement of creep in order to identify dynamic irradiation effects. If certain properties of the simulated fuels proved to be similar to those measured in-pile or by post-irradiation testing, further characterization of these properties would be carried out on simulated fuels at a substantial cost savings.

Following the lead of $F$. Schmitz ${ }^{[1]}$ the simulated fuel was to be prepared from powders of the fission products, either metallic or, if necessary, hydrides. The procedure calls for the fission products to be blended in the proper proportions to make up the fissium. The fissium, in turn, is blended with $U$ and $A 1$ powders in the proportions needed to simulate fuel compositions at various $U$ burnups. The synthetic fuel is compacted and then sintered at elevated temperature in a sealed Ir tube. During sintering the hydrides decompose and the hydrogen diffuses through the wall of the Ir tube. The sintered fuel is powdered, blended with powdered Al matrix material, and compacted to dense cores. The cores are put into aluminum picture frames and rolled to one eighth inch thick specimens. Alternatively, cover plates may be added to the picture frame before rolling and 50 mil thick clad fuel plates produced by the roll-bonding technique. Thus, both clad and unclad simulated fuel plates would be produced. The materials to make-up the fissium were purchased and powdered hydrides of $\mathrm{Pr}, \mathrm{Nd}, \mathrm{La}, \mathrm{Ce}, \mathrm{Ba}$, and $\mathrm{Y}$ were prepared. 
Because man power was re-directed from this work to the ETR corrosion task force, progress on the simulated fuel studies was limited in FY-71. Due to funding 1imitations, the simulated fuel work has been discontinued in FY-72.

[1] F. Schmitz, Structure and Properties of (U,Pu) 0 Containing Inactive Fission Products. A Simulation of Irradiated Nuclear Fuel, Translated by B. R. Appleby, BNWL-TR-43 (September 1969). 
COMPRESSIVE YIELD STRENGTH DETERMINATIONS ON ETR FUEL PLATES

CLAD WITH 2219 ALUMINUM

G. O. Hayner

In order to evaluate the mechanical properties of 2219 clad fuel plates, a compressive stress-strain curve at room temperature has been determined for unirradiated, 2219 aluminum clad $\mathrm{UAl}_{\mathrm{X}}$ fueled composite samples. The samples which were tested were machined from nine ETR fuel plates fabricated by powder metallurgy techniques. Other than the cladding, these plates were manufactured to the same contract specifications as standard ETR fuel plates. All of the specimens tested were rectangular and of 3.000 in. \pm 0.001 in. $x 1.250$ in. \pm 0.001 in. external dimensions. The specimens tested generally conform to the requirements of ASTM-E-209-65.

The testing was carried out at a cross head speed of $0.02 \mathrm{in} / \mathrm{min}$ in a special subpress designed specifically for compression testing. The subpress is pictured in Figure 1. This design enables the subpress to be used in the hot cell on irradiated specimens with constant restraining platen force against the specimen during testing to prevent buckling in thin section specimens. The results of the testing are shown in Figure 2. In this figure, the compressive stress-strain curve at room temperature for the 2219 clad composite is compared with the standard $\mathrm{UAl}_{\mathrm{X}}$ composite fuel plate (1100 aluminum clad. The following data at room temperature was tabulated for 2219 clad and standard ETR UA1 ${ }_{X}$ fuel plates:

1. The average measured modulus of elasticity for 2219 Al clad ETR fuel plates $=E_{c} 2219=8.7 \times 106$ psi.

2. The average modulus of elasticity for $1100 \mathrm{Al}$ clad ETR fuel plates $=E_{c 1100}=8.3 \times 10^{6}$ psi.

3. The average yield strengths of the 2219 and 1100 composite plates, respectively, are 36,000 psi and 20,000 psi.

Therefore, the yield strength of a composite fuel plate clad with $2219 \mathrm{~A} 1$ is almost twice as great as a normal ETR plate clad with 1100 aluminum at room temperature. Then as a first approximation, a 2219 clad plate would show greater resistance to buckling and yielding from thermal and flow stresses during reactor operation. 


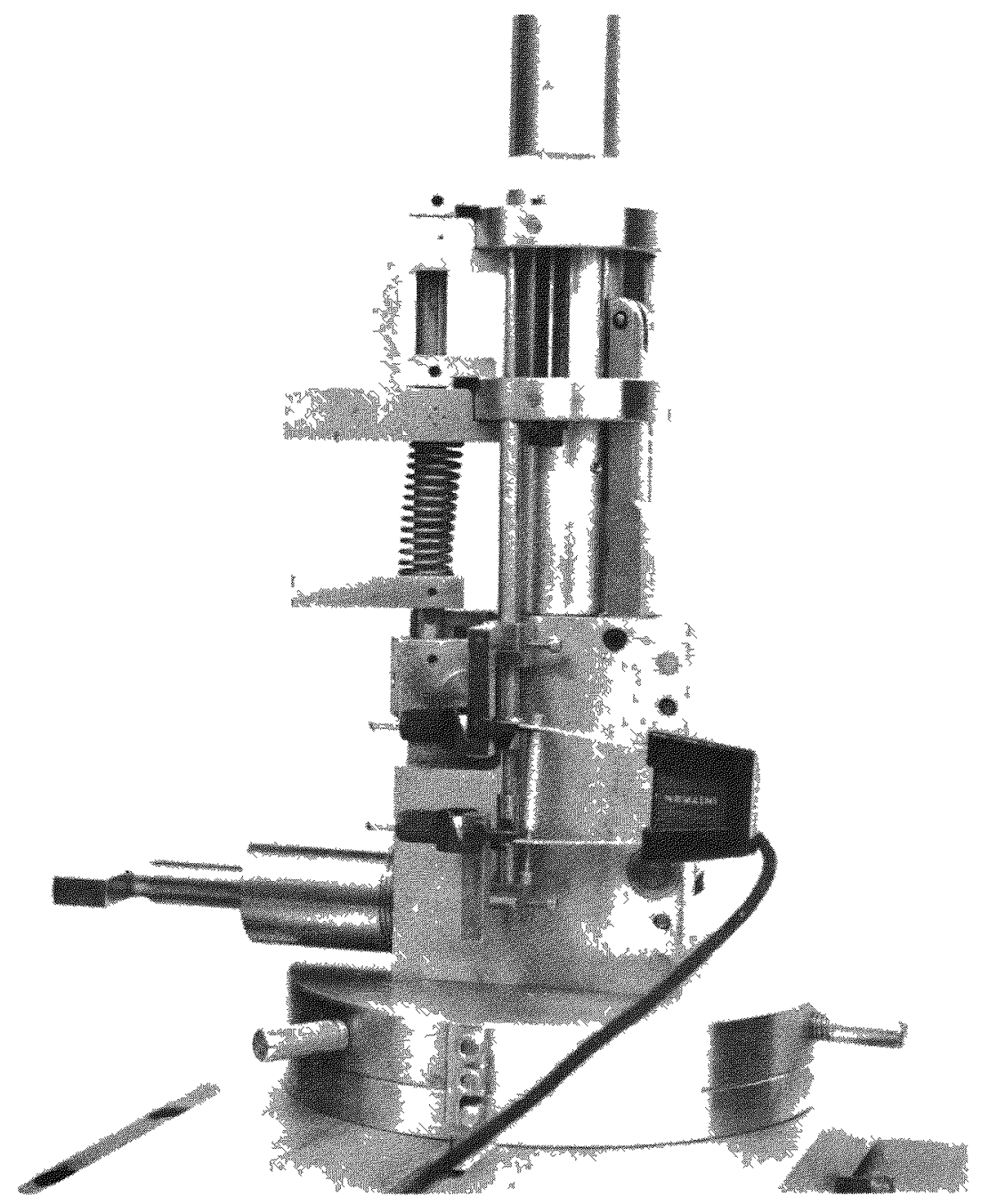

Fig. 1 Subpress used for compression testing of thin plates. 


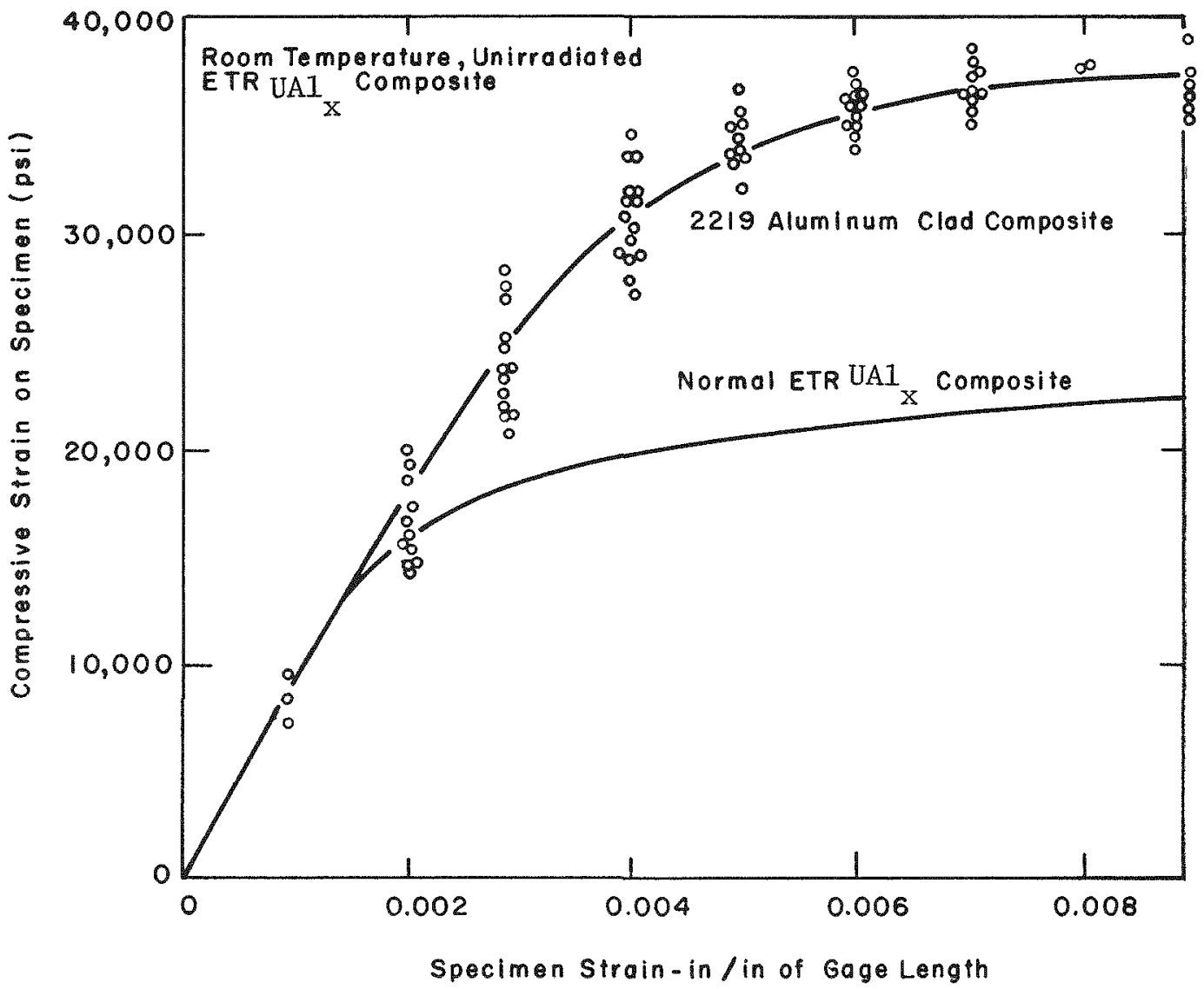

ANC-A -186

Fig. 2 Compression stress-strain curve at room temperature comparing ETR UA1 composite clad with 2219 aluminum compared with standard ETR UA1 fuel plates. 


\section{PERFORMANCE EVALUATION OF FUEL ELEMENTS USED IN STARTUP OF ATR}

M. J. Graber, R. R. Hobbins, G. O. Hayner, G. W. Gibson

Post-irradiation examinations have been performed on fuel. elements from startup cores of the ATR to evaluate the performance of commercially produced fuel elements by making comparisons with laboratory produced samples previously tested. The main performance evaluation effort has been concentrated on the Core II and III elements 1isted in Table I. In addition, some work was performed on HP-4, an instrumented element from Core $I$, an intermediate power thermal-hydraulic test run. The performance evaluation of the core II and III elements is being assembled into a topical report to be published in early FY-72.

The elements were metallurgically examined, dimensioned, analyzed for $235 \mathrm{U}$ depletion, and blister anneal tested. The post-irradiation blister anneal testing was conducted on samples removed from the plates of these elements. As can be seen in Figure 1, these tests indicated blister resistance at least as good as had been experienced on the laboratory produced samples. Plates fueled with $U A I_{X}$ powder continued to out-perform those fueled with $U_{3} 0_{8}$. Extensive gamma scanning and chemical burnup determinations were performed on the plates in order to determine the locations and magnitudes of the peak fuel burnup areas. As had been predicted, the peak burnups were found to be $1.5 \times 10^{21} \mathrm{fiss} / \mathrm{cc}$ of plate core.

The original design basis was for a fuel life of 17 days of full power operation ( $4250 \mathrm{MWd}$ ). This would have resulted in a peak fuel burnup of $7 \times 10^{20}$ fiss/cc of fuel plate core. However, because of the favorable results obtained from developmental efforts, it was possible from the second full power reactor cycle onward to extend the fuel element life to over twice this exposure, $(1.5 \times 1021 \mathrm{fiss} / \mathrm{cc})$. The post-test examinations demonstrated that this extension of the fuel element life was warranted. In these elements, a few of the plates containing $U A I_{X}$ were found to have small cladding ruptures. These were located at the ends of the fuel plate cores and were attributed to thin cladding. 


\section{TABLE I}

OPERATING PARAMETERS OF ATR STARTUP PROGRAM FUEL ELEMENTS WHICH WERE DESTRUCTIVELY EXAMINED

\begin{tabular}{|c|c|c|c|c|c|}
\hline $\begin{array}{c}\text { Fuel Element } \\
\text { Number } \\
\end{array}$ & $\begin{array}{l}\text { Used in } \\
\text { Core }\end{array}$ & $\begin{array}{c}\text { Days of } \\
\text { Operation }\end{array}$ & $\begin{array}{l}\text { Mode of } \\
\text { Operation }\end{array}$ & $\begin{array}{c}\text { Type } \\
\text { Fuel Element }\end{array}$ & $\begin{array}{l}\text { Irradiation } \\
\text { Position } \\
\end{array}$ \\
\hline $\mathrm{A} 172 \mathrm{C}$ & II & 35 & $\begin{array}{l}250 \mathrm{MW} \\
\text { Balanced }\end{array}$ & Std. $\mathrm{U}_{3} \mathrm{O}_{8}$ & $\mathrm{~F} 11$ \\
\hline XA3G & II & 35 & Balanced & $" 7 F " U A 1_{X}$ & $\mathrm{~F} 13$ \\
\hline $\mathrm{XA} 8 \mathrm{G}$ & II & 35 & Balanced & $" 7 F^{\prime \prime} U A 1_{x}$ & F14 \\
\hline A16D & III & 27 & $\begin{array}{l}250 \mathrm{MW} \\
40-50-60 \\
\text { Unbalanced }\end{array}$ & Std. $U A I_{X}$ & $F 11$ \\
\hline $\mathrm{XA} 20 \mathrm{G}$ & III & 27 & Unbalanced & $" 7 F " \mathrm{UA} 1_{x}$ & F13 \\
\hline
\end{tabular}




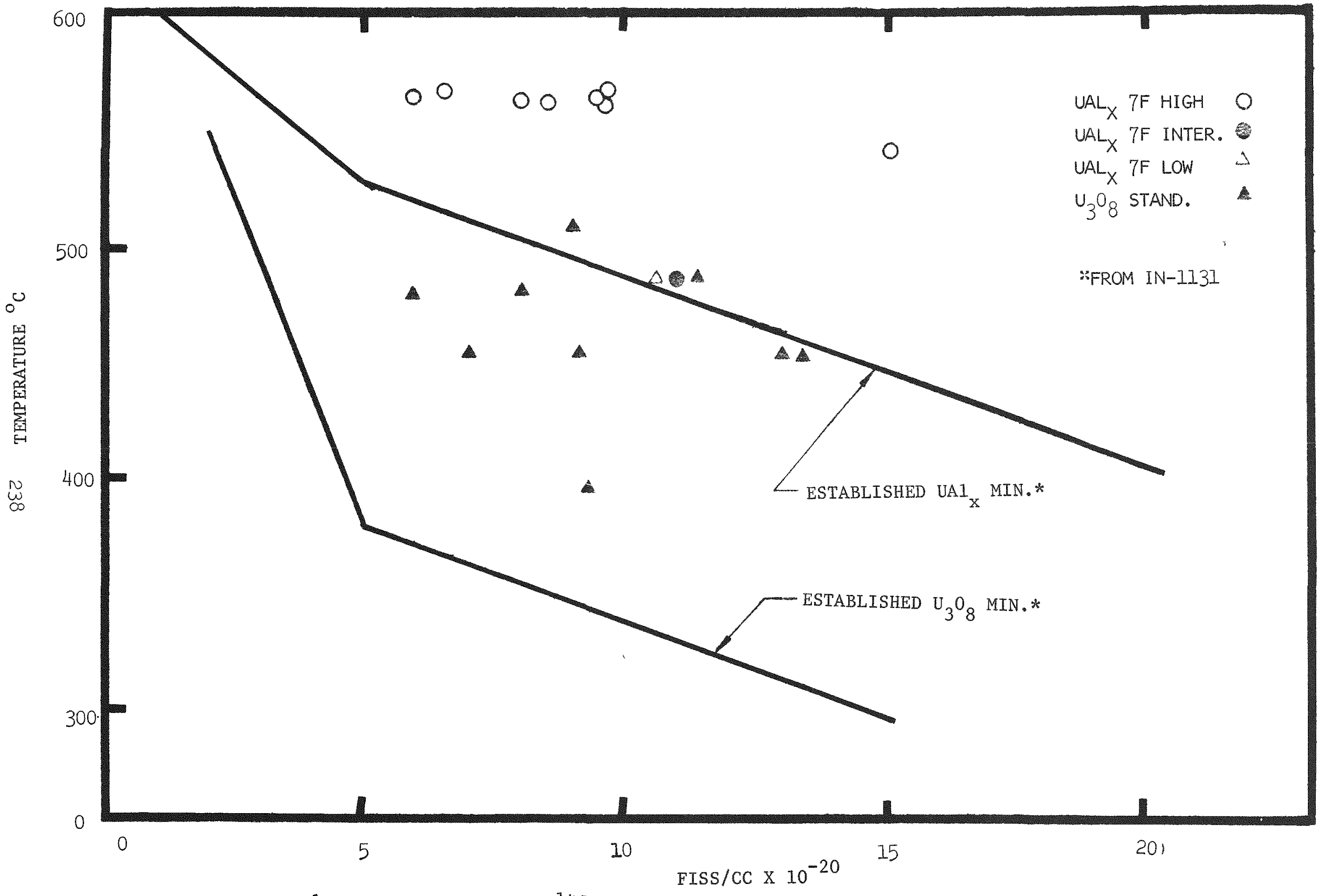

Fig. 1 B1ister test results. 
PHASE I REPORT FOR THE SAFETY AND WASTE MANAGEMENT STUDIES PRIORITT NUMBER 22, STUDY 15 ,

HEALTH PHYSICS INSTRUMENTATION RELIABILITY AND PERFORMANCE STUDY

$$
\text { F. A. Meichle, E. B. Nieschmidt }
$$

Introduction. Radiation exposure is the focus of one of the sharpest controversies concerning the nation's environment. Allowed radiation doses have been cut by orders of magnitude. Given the paucity of data on low exposure, resolution of the problem relies on the acquisition of sufficient exposure data to build a case for the public acceptance of nuclear energy sources.

Radiation released by nuclear power plants was subjected to new federal regulation in 1970. The Atomic Energy Commission (AEC) announced new $A E C$ regulations requiring industry to take advantage of new advances in technology to minimize the level of radiation released by nuclear power plants. Nuclear plants operated for the $A E C$, by $A E C$ contractors, are also required to meet the new regulations. Potential nuclear power thermal pollution is another environmental controversy, but is not the subject of this report.

Radiation monitoring is that function that deals with detecting and measuring of nuclear radiation which might be hazardous to the public health and environmental quality. This puts radiation monitoring into the area of industrial hygiene or health physics. Radiation monitoring instrumentation is used to indicate when radiation levels exceed predetermined limits, measure, and to provide a record of released radioactivity or the absence of it. As a result of the controversy over radiation exposure data, health physics (radiation monitoring) instrumentation has become a very important factor in the resolution of the controversy.

The present state of the health physics radiation monitoring instrumentation is far from satisfactory with out-dated equipment and techniques being widely used. It is vital that modern instrumental techniques find their way rapidly into this important field, and support be given to the further development of instrumentation and techniques for quality health physics monitoring.

New instrumentation and techniques have been developed for research that should be widely applied for radiation monitoring. Investigation of the research area can prevent the unnecessary duplication and expense in health physics monitoring development and point out monitoring requirements that cannot be met by any current technology.

Aerojet Nuclear Company, operating nuclear plants and facilities for the $A E C$, initiated a study of their health physics instrumentation and measurement techniques in light of the new $A E C$ radioactive release to the environment regulations. The study covers the health physics instrumentation and measurement techniques used in personnel safety monitoring, area monitoring and waste effluent management. Major aspects to be considered in the instrumentation area are reliability and maintenance. 
The major objectives of the study are:

1. To provide Aerojet Nuclear Company with radiation monitoring and/or measurement instrument systems for the proper operation of the various plants and facilities to meet the requirements of the new AEC regulations.

2. That the radiation monitoring and/or measurement instrument systems be reliable.

3. That the instrument systems require a minimum of maintenance.

4. That the instrument systems be modular and interchangeable.

5. To provide instrument systems that do not require a demanding degree of proficiency of the operator.

6. To provide calibration standards and clearly written calibration procedures.

7. To provide fully documented instrument systems, operating procedures, and maintenance procedures.

8. That the instrument systems use modern data-handling techniques for data reduction and documentation.

Because of the broad scope of this study a variety of disciplines are necessarily involved. The study is centered around an instrumentation engineer, research nuclear physicist, and a health physicist operating as task leaders. The wide spectrum of scientific and technical expertise available within the company will support the task leaders.

Study Description. The study is organized in two parts: Phase I consisting of 6 months, January to June, 1971 and Phase II, starting July, 1971, continuing for approximately 3 years. Phase $I$ is devoted to a survey of the present health physics instrumentation and measurement techniques and to establish the scope of work to be accomplished in Phase II.

The following is the program plan for Phase I:

1. A survey of the present health physics instrumentation, maintenance history, measurement techniques, and operational problems.

2. A survey of the present commercial health physics instrumentation.

3. A survey of the present instrumentation and measurement techniques used in the research areas for application to health physics requirements.

4. Investigate health physics instrumentation for application of standardization and interchangeability.

5. Develop a program plan for the first 12 months of Phase II. 
The survey covers the present health physics instrumentation in service at the following plants and facilities: ATR, ETR, CPP, and TAN. The instrumentation surveyed were portal monitors, remote area monitors, constant air monitors, hand and foot counters, alpha hand monitors, smear counters, and stack monitors. Hand operated radiation monitoring instruments and dosimeters were not included in this phase of the study.

Phase I Recommendations. The following are the Phase I survey recommendations to meet the major objectives.

1. Adopt the NIM system for al1 health physics instrumentation surveyed in this study and investigate the application to other health physics instruments, such as hand operated instruments and dosimeters.

2. Investigate the application of the CAMAC system to the automatic data-handling of health physics instrumentation.

3. Establish a "clearing house" for the health physics instrumentation and measurements activities. These activities to consist of the following.

3.1 Documentation - The documentation to consist of information on the vendors instrumentation, such as, manuals, system and instrument drawings (not the originals), maintenance procedures, calibration procedures, maintenance histories, modifications (including purpose and justification of the modification), instrumentation inventory, and instrumentation locations. Also included in the documentation are manufacturers' catalogs, reports, and papers relating to health physics activities.

3.2 Record Keeping - Maintain records of data required for the evaluation of monitoring systems and AEC reporting requirements.

3.3 Instrumentation Spares - Maintain a stock of spare instrument modules.

3.4 Instrumentation Maintenance - All instrumentation maintenance, preventive maintenance, and calibrations will be handled through this "clearing house".

3.5 Develop health physics system requirements.

3.6 Procurement - All procurement of health physics instrumentation shall be approved by this "clearing house".

4. Establish in Maintenance a group trained in the maintenance of health physics instrumentation and detectors.

5. Establish a mechanism to provide technical support for maintenance and the "clearing house". The technical support to consist of training, calibration techniques, applications of new devices, components, instruments, systems, to meet health physics requirements, programming, instrumentation specifications, etc. 
6. Review and rewrite the instrumentation maintenance and calibration procedures.

7. Establish performance requalification and preventive maintenance intervals.

8. Recommend changes in the maintenance history documentation format to system, module, and component evaluations.

9. Formulate a list of standardized and interchangeable basic instrument modules which can be used to build health physics instrumentation system. The list is to contain both commercial and in-house developed instrument modules.

10. Investigate and standardize on an air flow and filtering system for both the fixed and moving filter systems. The standardization to include the flow rates, type of filter material, and flow control system if required.

11. Investigate and standardize on two recorder (strip chart) types to be used for general recording and high accuracy recording when required.

12. Establish specifications for a high resolution gamma-ray spectrometry system for isotopic identification in health physics applications.

13. Initiate the development of software, counting techniques, sample collection and preparation techniques to implement high resolution spectrometry systems.

14. Establish dialogue between groups at the NRTS and other laboratories to aid in the dissemination of information pertaining to advances in instrumentation and techniques.

15. Investigate and demonstrate the potential of the small digital processor as a continuous effluent and waste monitor and inventory device. 
ATR EXTENDED BURNUP PROGRAM SUPPORT

E. F. Aber, A. W. Brown

This program is part of an effort to cut ATR fuel costs. The ATR maximum cycle life has been limited to a peak burnup of $1.5 \times 10^{21}$ fiss/cc. This results in 6830-8750 MWD cycles, depending on utilization of the $7 \mathrm{~F}$ graded fuel elements. Extension of the burnup limit to $1.8 \times 10^{21}$ fiss/cc would permit ATR operation to the reactivity limit and could provide a $\$ 500,000$ annual savings in fuel fabrication costs. The ATR extended burnup program[1] was developed to demonstrate the feasibility of operation to the elevated burnup and a part of the program provides for irradiation of test elements to $1.8 \times 10^{21}$ fiss/ce peak burnup. Calculational procedures were required to determine the irradiation time for the test elements to reach $1.8 \times 10^{21}$ fiss/cc peak burnup and to predict power distribution during depletion.

The PDQ $-7^{[2]}$, a neutron diffusion depletion code, has been utilized for such calculations, and a comparison with measured power distribution and burnup has been made for the ATR Core II and Core III operation. The comparison with the measured power distribution indicates good agreement over most of the core. However, at the ends of some of the channels between fuel plates the calculated power was as much as $40 \%$ low. The discrepancy was shown to arise mainly from an error in the description of the nuclear properties in these regions of the core. Comparison of calculated with measured burnup gave an uncertainty at the $95 \%$ confidence level of 18\%. However, for the position of peak burnout the calculated fission density was within experimental exror.

During more than a year of operation of the ATR a considerable burnup of the hafnium shims and a buildup of poison in the beryllium reflector has occurred. Neither of these effects is adequately modeled in the calculational procedures. One result of this deficiency is a mismatch in shim control positions between the calculation and the actual ATR operation. This is due primarily to the greater burnup in those shims within or adjacent to the center lobe. An investigation of the effects of error in shim position has indicated that the power peaking could be changed by as much as $35 \%$ in those elements most affected by shim movement.

A comparison of peak burnup calculated for a variety of conditions of core power distribution indicated that the burnup at the peak position was little affected by the power distribution in the core, or by the control shim movement. Based on these results, it was possible to determine how to schedule test elements to achieve irradiation to $1.8 \times 10^{21}$ fiss/ce during Cycle 8 operation.

[1] M. L. Griebenow, et al., ATR Extended Burnup Program, ANCR-1015 (to be published).

[2] W. R. Cadwell, PDQ-7 Reference Manual, WAPD-TM-678 (January 1967). 


\title{
FUEL PROCUREMENT SUPPORT
}

\author{
G. W. Gibson, G. O. Hayner
}

During the year, technical support was provided the Fuel Management office in connection with procurement of fuel for the test reactors. As part of this effort the preparation of fuel element specifications was undertaken. Visits were made to the vendor"s plants to review the procedures used in the fabrication of fuel elements. Requests from the vendors for deviations from the specifications were reviewed.

A large batch of $\mathrm{UAl}_{\mathrm{x}}$ fuel powder was received from a vendor as a result of the termination of a fuel element supply contract. This powder was sampled and physical property determinations were made. It was found that some batches of fuel powder would not flow through the funnel used for the flow test. The orifice used in the funnel is $0.10 \mathrm{in}$. in diameter while the maximum diameter of the fuel particles is $0.006 \mathrm{in}$. The powders are dry and free from oil. Thus, it was most puzzling as to why the powders did not pass through the flowmeter. Samples of both types of powder were sent to the oak Ridge Nationa1 Laboratory for examination on a scanning electron microscope. This examination indicated that the non-flowing powder contained a much larger fraction of -400 mesh particles than the flowing material. The very fine particles tended to agglomerate. The clumps did not break down during screening. Thus, on a conventional screen analysis, the -400 mesh fraction would be reported as +325 mesh. No explanation has been developed for the cause of agglomeration.

Calibration standards were prepared for use in developing instrumentation for measuring cladding thickness on fuel plates and the per cent voids in the cores of these plates.

Support was also given during FY-1971 on subcontracts S-203 (ETR fuel elements) and $\mathrm{S}-204$ (ETR control rods). This support included: the review of thirteen $\mathrm{S}-203$ approval requests, fourteen $S-204$ approval requests, the review of one deviation request from each subcontract, a compliance review was made for $\mathrm{S}-204, \mathrm{~S}-203$ fabrication procedures were reviewed, S-203 requalification procedures were reviewed, and a specification review was made for IN-F-4-TRA, IN-F-5-TRA, and IN-F-6-TRA. 


\title{
SHELF LIFE OF UA1.
}

\author{
G. O. Hayner
}

The analysis technique employed to determine the enrichment (wt\% 235U) in $U A 1_{X}$ fuel powders supplied to certain vendors during fuel plate production is very dependent on knowing a precise sample weight on which the analysis is being performed. It is thought that this sample weight (and hence the weight of the entire batch) could change due to the exposure of the powder to air of varying moisture contents over a limited period of time. A limited study was performed to establish the extent of any weight changes which took place in $\mathrm{UAl}_{\mathrm{X}}$ fuel powder exposed to room temperature air within a humidity chamber and within a glove box.

The fuel powder used for the testing was from lot number 983560-1 and was manufactured by a commercial vendor. Ten 5 gram samples of this fuel powder were carefully weighed and put in glass containers. Five of these samples were put in the environment of a humidity chamber and five were held inside a glove box. Also, a control sample containing no fuel was included in each environment. With the caps removed from the glass bottles containing the fuel powder, weight changes (presumably caused because of the change in moisture content in the fuel) were recorded on a daily basis. The results of this study, normalized to the change in weight of the standard, are reported as either plus or inus cumulative weight percent changes in Table I. As indicated by this data, the weight percent changes that occurred were generally very small and the variation between replicates was large in most cases. The data does establish that weight changes can and probably do take place upon arrival of fuel material at a different area or if fuel is left exposed to the atmosphere, however, the absolute magnitude of a change which takes place in an actual fuel powder lot after shipment cannot be established with the data obtained to date. 
TABLE I

WEIGHT PERCENT CHANGE IN FUEL POWDER [a]

Glove Box Environment - $77^{\circ} \mathrm{F}-81^{\circ} \mathrm{F}, 62-66 \%$ Relative Humidity - Results in wt\%

\begin{tabular}{|c|c|c|c|c|}
\hline & Day 1 & Day 2 & Day 5 & Day 6 \\
\hline Sample Ic & +0.012 & +0.016 & -0.008 & -0.004 \\
\hline Sample $2 c$ & +0.024 & +0.016 & -0.002 & -0.010 \\
\hline Sample $3 c$ & +0.004 & +0.008 & -0.010 & -0.016 \\
\hline Sample $4 \mathrm{c}$ & +0.028 & +0.016 & -0.008 & 0.000 \\
\hline Sample $5 c$ & +0.016 & +0.014 & -0.040 & -0.036 \\
\hline Average & +0.017 & +0.012 & -0.014 & -0.013 \\
\hline
\end{tabular}

Humidity Chamber Environment

\begin{tabular}{|c|c|c|c|c|}
\hline Temp. $-{ }^{o} F$ & $\frac{\text { Day } 1}{89^{\circ} \mathrm{F}}$ & $\frac{\text { Day } 2}{81^{\circ} \mathrm{F}}$ & $\frac{\text { Day } 5}{82^{6} \mathrm{~F}}$ & $\frac{\text { Day } 6}{86^{\circ} \mathrm{F}}$ \\
\hline \% Humidity & $86 \%$ & $85 \%$ & $78 \%$ & $84 \%$ \\
\hline Sample ld & +0.010 & +0.014 & +0.004 & +0.020 \\
\hline Sample $2 d$ & +0.034 & +0.040 & +0.040 & 0.000 \\
\hline Sample $3 d$ & +0.026 & +0.030 & +0.016 & +0.032 \\
\hline Sample $4 \mathrm{~d}$ & +0.034 & +0.038 & +0.032 & +0.046 \\
\hline Sample $5 d$ & +0.030 & +0.038 & +0.034 & +0.046 \\
\hline Average & +0.027 & +0.032 & +0.025 & +0.029 \\
\hline
\end{tabular}

[a] The moisture content determined in a vacuum for a sample from this powder lot was 0.124 wt\%. 


\section{DELAYED NEUTRON FISSION BREAK MONITOR}

L. G. Miller, T. E. Young, F. B. Simpson

At the ATR a neutron counter of the $\mathrm{BF}_{3}$ type has been installed to monitor the delayed neutron activity in the four different quadrants of the reactor. This detector not only senses a fission break but can identify which quadrant contains the failed fuel element. The system was originally set up to determine whether a fission break which had been identified by iodine tests in Cycle $6 \mathrm{~A}$ could be isolated to a particular quadrant. The data indicated that there were fission breaks in two of the quadrants, the one in the northwest lobe being by far the largest. After shutdown, visual tests of the fuel elements confirmed these results.

The delayed neutrons in the ATR primary coolant have been monitored more or less daily throughout Cycles $7 \mathrm{~B} / \mathrm{C}$ and $8 \mathrm{~A} / \mathrm{B}$. This monitoring consisted of bleeding off samples from the quadrant discharge pipes and the total discharge pipe after the quadrant pipes join, and then counting the neutrons as the water passes through the long counter.

The monitor readings were constant throughout Cycles $7 \mathrm{~B} / \mathrm{C}$ and $8 \mathrm{~A}$. Quadrant readings varied between 60,000 and 80,000 counts/min., and the "total" reading was 16,000 counts/minute. After the ATR started on Cycle 8B all readings began to rise, until at the end of $9 B$ the quadrant readings were 130,000 to 200,000 counts/minute and the total reading was 60,000 counts/ minute. This rise in activity was probably a result of a change in the resin beds in the bypass demineralizer and a reduced flow by a factor of 2 through the demineralizer. The iodine activity and radiation gases readings increased at about the same rate as the delayed neutron counts.

If a break occurs in a fuel element, two types of undesired release of material are possible. First, fission product gases may escape from the element. Second, fissionable material may escape into the primary coolant. (It is also possible for fissionable material to enter the primary coolant from slightly contaminated materials in the system, e.g., the surfaces of the fuel elements.)

The delayed neutron monitor differentiates between activity caused by the release of fission product gases and that caused by contamination of the system by fissionable material. If the fission product activity in the coolant is due primarily to one break which releases only fission products it is to be expected that the delayed neutron monitors of only one or two quadrants will show a large increase in count rate. This effect was observed. in a previous cycle. Due to the short half-life of the delayed neutron precursors, the increase in activity observed in the quadrants opposite the fission break should be very small compared to that observed in the quadrant in which the break occurred.

On the other hand, if the increase in fission product activity is due to irradiation (within the core) of a uranium contaminant in the water or on the surfaces of many fuel elements, the activity from a quadrant will 
be proportional to the power in that quadrant. This was the effect observed during Cycle 8B. Therefore, the delayed neutron monitor indicates that the increase in fission product activity observed during Cycle $8 B$ was due to fissionable material distributed quite uniformly throughout the core.

Figure $I$ is a plot of the delayed neutron activity from the different quadrants as a function of time in days for Cycle 9B. Also included for comparison is the iodine activities which has been a standard method of testing for fission breaks. These data show that the two methods of testing for fission breaks agree very well. The delayed neutron technique could be automated at a normal cost so that the activity could be continuously monitored at the reactor console. 


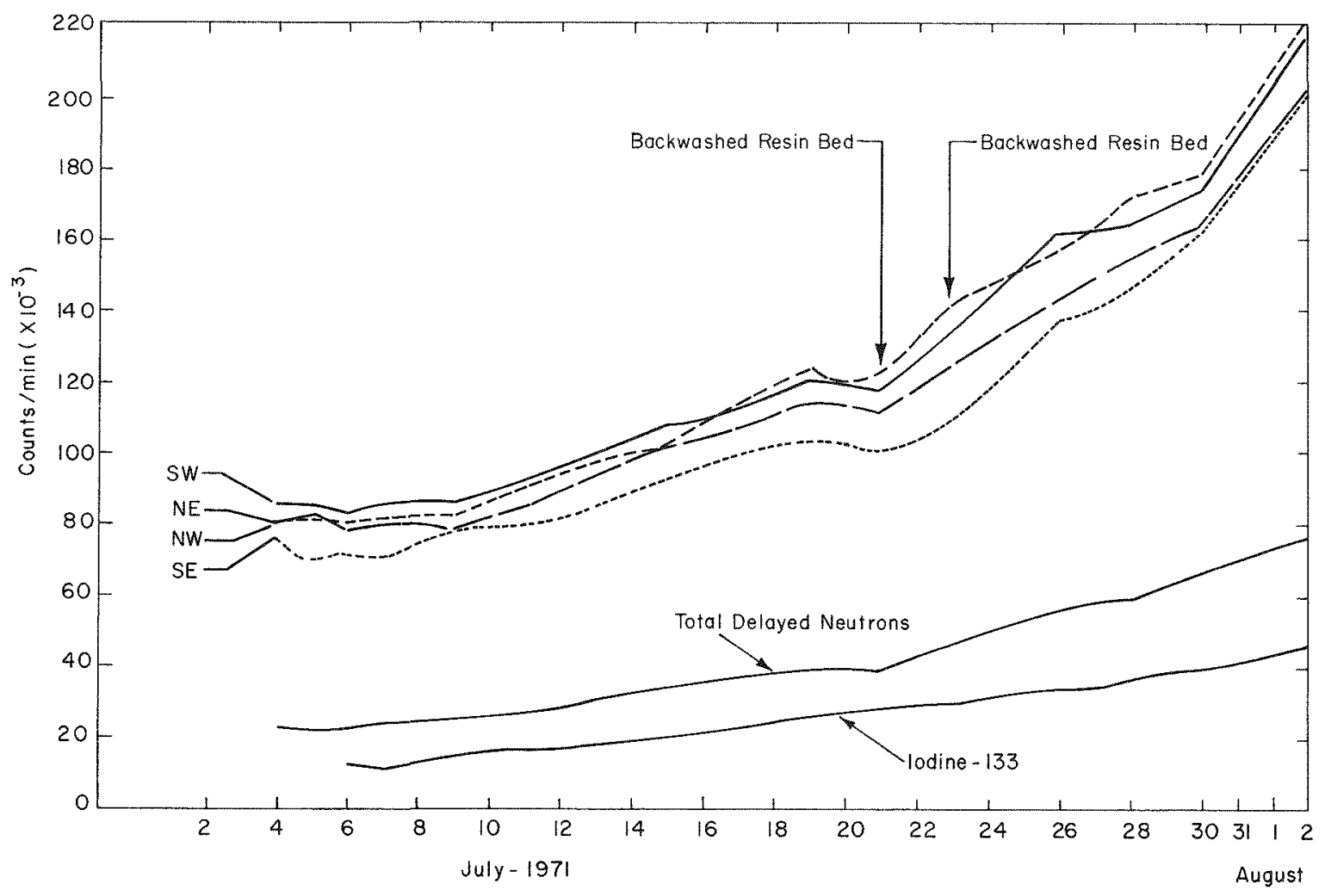

Fig. I ATR delayed neutron count from the exit cooling water during Cycle 9B. SW, $N E, N W$, and $S E$ refer to the reactor quadrants. Total is the delayed neutron count from the mixed quadrant exit cooling water. 


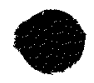


E. REACTOR DEVELOPMENT 
-

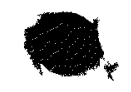


It has been a desire of man from the beginnjrg of time to be able to see through objects to observe the presence of other objects. With the advent of modern technology some real breakthroughs toward this goal have been realized through $\mathrm{X}$-rays and other methods such as radar and sonar. The ultimate means, clairvoyance, has yet to be harnessed by man. Recently neutron radiography has joined the list of usable methods, providing a valuable complement to X-rays and the others. Because of the unique properties of the neutrons, neutron radiography is a valuable addition because it can observe materials unobservable by the other methods.

Neutron radiography is not as well developed by X-radiography because of the neutrons' relative late identification and appreciation by scientists. The sensoring methods for neutrons are still less efficient and the sources are more complicated and weaker than for example, X-rays. However, the neutron source described in the following makes advances over existing sources to provide an effective and convenient portable source for neutron radiography.

An optimized source for neutron radiography would be:
a) inexpensive
the cost of neutrons would be a small fraction of the cost per picture,
b) intense
deliver at least $10^{6} \mathrm{n} / \mathrm{cm}^{2}$ on the image plane at a resolution of $\mathrm{L} / \mathrm{D}>100$,
c) portable moveable to a user's factor and able to examine large objects, i.e. airplanes or reactor vessel,

a) safe the maximum credible incident does not limits its use or portability,
e) adjustable energy of neutron flux

fluxes of thermal, epithermal or fast neutrons are available.

Unfortunately, no single source of neutrons meets these conditions. Table I lists examples of possible sources and their characteristics. Experience has established a threshold of intensity of $10^{6} \mathrm{n} / \mathrm{cm}^{2} \mathrm{sec}$ at the image plane for effective neutron radiography. One notes that the first 3 sources of Table I do not reach this threshold. While the first and third are being used for neutron radiography through longer exposure or poorer resolution, the success of such efforts seems limited.

If one accepts the limit of $10^{6}$ thermal $\mathrm{n} / \mathrm{cm}^{2} \mathrm{sec}$ on the image plane as the lowest acceptable flux with the required collimation to be a satisfactory and successful neutron radiographic source, it becomes obvious that a fission source that is at least delayed critical is needed. We accepted this condition and then designed for portability, 
economy and safety. The neutron source described in the following paragraphs and depicted in Figure $I$ is our conceptual design; which is an effective, economical, safe, portable neutron source.

The design of the neutron radiographic reactor was based on basic understanding of reactor concepts and on specific experience with fuels and flux traps of the Research and Engineering Division of Aerojet Nuclear Company. This experience was gained during design, startup and operation of the test reactors, safety reactors and critical facilities.

Figure 2 is a cutaway diagram of the reactor. It is based on the flux trap concept to deliver maximum thermal flux per kilowatt of power. The beam hole for radiography enters the flux trap area and has a source area of one inch diameter. Maximum flux peaking is obtained with a water trap 5 inches in diameter. The fuel is intermetallic, 235 $\mathrm{U}$ enriched, AI clad. The plates are canted into a toroid to produce a constant metal-to-water ratio core. The core is reflected by beryllium and shielded with water and lead, the total tank will be less than 8 feet in diameter. The flux trap is sized to give maximum thermal peaking. The fuel, beryllium and water-lead shielding will be optimized so that the source area delivers $10^{12} \mathrm{n} / \mathrm{cm}^{2} \mathrm{sec}$ to the beam, that the power is minimized, and that the total weight is minimized. The reactor is designed with enough excessive reactivity to be delayed critical for safety. Calculation shows that a maximum of $1.4 \mathrm{~kg}$ of uranium are needed for delayed criticality. The 13 kilowatts of power are necessary to obtain $10^{12} \mathrm{n} / \mathrm{cm}^{2} \mathrm{sec}$ and are easily removed by water circulation. Control of the reactor is through rotating rods for convenience. The exterior of the tank is as unencumbered as possible to allow versatility of the portable device. The tank is mounted on gimbles on a truck so that the reactor can be moved to the beam and can be pointed in all directions. The optimization of parameters will be made in the final design.

[1] Assistant Manager, Research and Engineering Division, Aerojet Nuclear Company. 


\section{TABLE I}

Examples of Nieutron Sources

\begin{tabular}{|c|c|c|c|c|c|c|}
\hline Type & $\begin{array}{c}\text { Source } \\
\text { Intensity }\end{array}$ & $\begin{array}{l}\text { Source Flux } \\
\text { Thermal } \\
\end{array}$ & $\begin{array}{c}\text { Image } \mathrm{P} \text { lane } \\
\text { Flux at } \mathrm{L} / \mathrm{D}=100 \\
\end{array}$ & Portable & $\begin{array}{l}\text { Slow and/or } \\
\text { Fast Neutrons } \\
\end{array}$ & $\begin{array}{c}\text { Safety } \\
\text { Precautions }\end{array}$ \\
\hline${ }^{252} \mathrm{Cf} \quad 1 \mathrm{mg}$ & $2 \times 10^{9}$ & $\begin{array}{l}9 \times 10^{6} \\
(\mathrm{H}, \mathrm{O} \text { mod })\end{array}$ & $2 \times 10^{2}$ & yes & yes & Iimited \\
\hline $\begin{array}{l}2 \mathrm{~b} 2 \mathrm{Cf} \text { boosted } \\
(1 \mathrm{mg}) \\
\mathrm{k}_{\text {eff }}=0.988\end{array}$ & $2 \times 10^{9}$ & $\begin{array}{l}6 \times 10^{7} \\
(\text { Be mod) }\end{array}$ & $0.3 \times 10^{4}$ & yes & slow & L \\
\hline $\mathrm{D}-\mathrm{T}$ & $10^{12}$ & $10^{9}$ & $10^{4}$ & yes & yes & L \\
\hline $\begin{array}{l}235 \mathrm{U} \text { delayed } \\
\text { critical }\end{array}$ & & $10^{12}$ & $10^{7}$ & yes & yes & moderate \\
\hline Triga I MW & & $10^{13}$ & $10^{8}$ & no & yes & $\mathrm{M}$ to $\mathrm{E}$ \\
\hline MTR $40 \mathrm{MW}$ & & $2 \times 10^{14}$ & $2 \times 10^{9}$ & no & yes & extensive \\
\hline
\end{tabular}




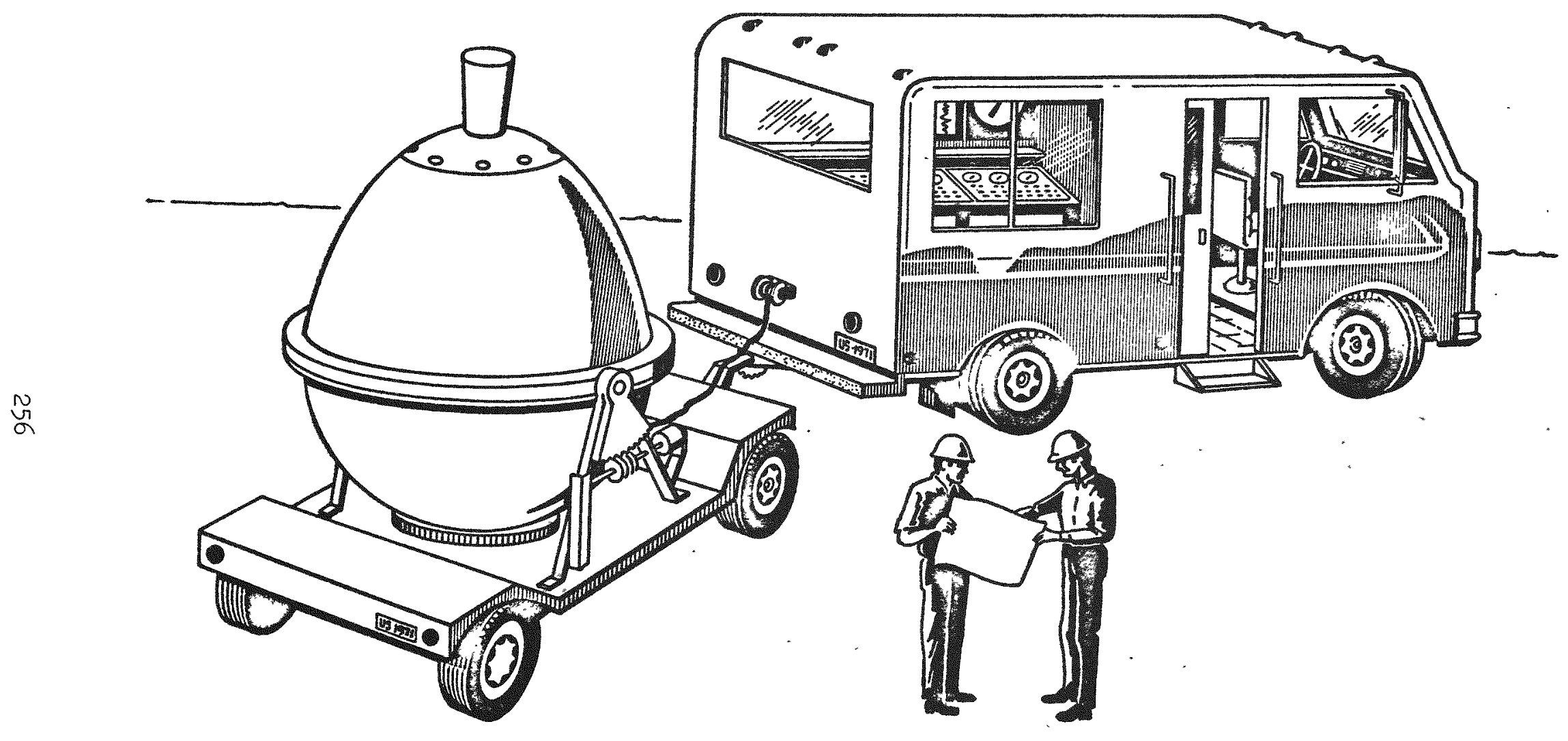

Figure I Complete industrial neutron radiography facility ready for operation. Facility can be moved to any location and be in operation within minutes. 
1. SOURCE

2. FUEL

3. FLUX TRAP

4. BEAM TUBE

5. DRUM TYPE CONTROL ROD

6. CONTROL ROD DRIVE

7. REFLECTOR

8. PRIMARY CIRCULATING PUMP

9. LEAD SHIELDING

10. PRIMARY PRESSURE VESSEL TOP HEAD

11. PRIMARY PRESSURE VESSEL

12. SECONDARY PRESSURE VESSEL TOP HEAD

13. SECONDARY PRESSURE VESSEL
14. FLOW SHROUD

15. INSTRUMENT CHAMBER

16. INSTRUHENT THIMBLE

17. SURGE TANK

18. BORATED POLYETHYLENE

19. SECONDARY CIRCULATING PUMP

20. LOG N \& PERIOD CHAMBERS

21. FISSION \& POWER LEVEL CHAMBERS

22. POWER LEVEL CHAMBER

23. ROTARY TABLE

24. FIXED MOUNTING STRUCTURE

25. WATER

26. TILTING MECHANISM

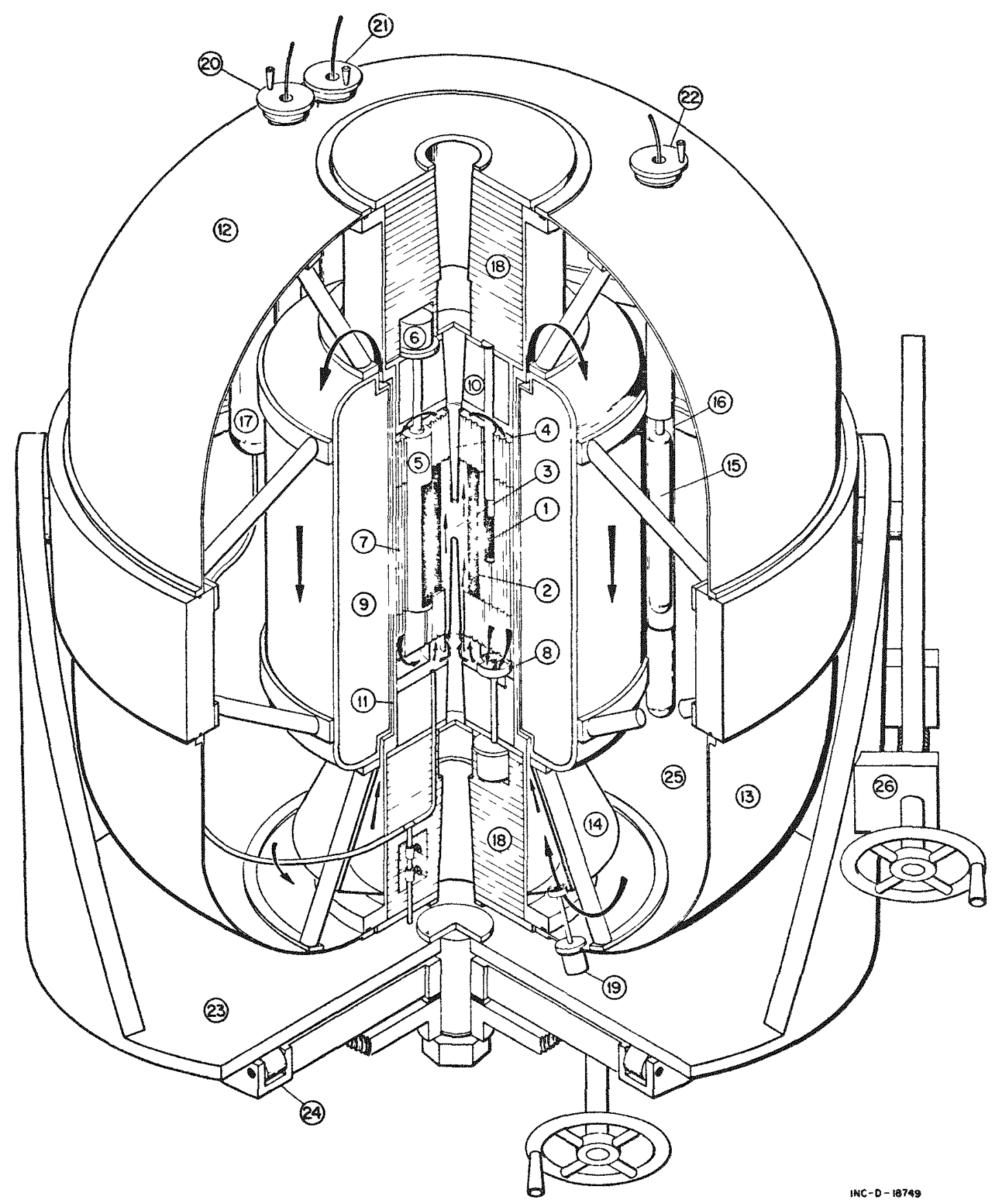

Figure 2 Cutaway diagram of the portable neutron radiography. 
TRANSFER CASK DESIGNS FOR THE FUEL ELEMENT FAILURE PROPAGATION LOOP (FEFPL)

G. K. Wachs

The FEFPL project is a joint effort by Argonne National Laboratory and Aerojet Nuclear Company to develop an irradiation facility to conduct failure propagation experiments of fast fuel elements. The facility will be installed in a $6 \times 6$ region of the core lattice of the ETR. The sodium-package-type loop is a self-contained facility and following irradiation will be transferred to the Hot Fuel Examination Facility (HFEF) for post-irradiation disassembly and inspection.

The engineering design of the loop is not firm at this time. As new engineering concepts are being developed and the loop size and shape change, so does the conceptual preliminary design of the transfer cask. Several cask designs have been made to limit the after-shutdown dose rates to acceptable levels. Depleted uranium metal is used as the shielding material to minimize the total shield weight of the cask.

The major after-shutdown radiation sources are from sodium activation and from fission product decay gammas. These time dependent sources were calculated and input to the point-kernel general shield design code QAD-P5A. The complete package type loop and shield material was described. in three dimensions and dose rates were calculated at l-foot intervals along the entire height of the cask (426 feet). In the event a fuel rupture occurs, fission products are dispersed throughout the loop by the circulating sodium coolant and in.this case become the major contributor to after-shutdown radiation levels. 
FUEL ELEMENT FAILURE PROPAGATION

LOOP INSTRUMENTATION

F. A. Meichle

The fuel element failure propagation loop (FEFPL) project is a joint effort by Argonne National Laboratory and Aerojet Nuclear Co. (formerly Idaho Nuclear Corp.) to develop a sodium irradiation facility in the Engineering Test Reactor. Project objectives are to study the circumstances, rates, and extent to which failures of a LMFBR type fuel element propagate, or do not propagate to neighboring elements.

Progress in implementing the instrumentation system has been slowed due to funding problems. Instrumentation system specifications are being developed based on the proposed instrumentation system described in the Nuclear Technology Branch Annual Progress Report[1]. sys tem.

The following is the present status of the FEFPL instrumentation

1. A PDP-15/20[2] computer has been delivered for the system. The computer has been checked, debugged and is operational. The PDP-15/20 computer consists of central processor, 8,192 18-bit words of core memory, two DECtape[2] magnetic tape transports, extended arithmetic element, KSR-35 teletype, high-speed paper tape reader/punch, and DECdisk[1] magnetic bulk storage system.

2. The PDP-15 ADVANCED software is operational within the limits of the equipment available. The ADVANCED software system is a complete integrated system of programs for the preparation, compilation, assembly, debugging, and operation of user programs.

3. Implemented the PDP-15/20 monitor program systems which simplify the handling of input/output functions and facilitate the creation, debugging, and use of USER programs. This program system allows overlapped input/output, computations, simultaneous operation of a number of asynchronous peripheral devices, and device-independent programing, while freeing the user from the need to create device handling subroutines.

4. A low level multichannel multiplexer and analog-to-digital converter unit has been received. The multiplexer and converter unit consists of 128 channels, random and sequential address, programmable gain amplifier, $20 \mathrm{KHz}$ scan rate multiplexer and 12 bit analog-to-digital converter. A manual control panel was also provided with the unit.

1. "Nuclear Technology Branch Annual Progress Report", Ending June 30, 1970, UC-80, page 379.

2. Digita1 Equipment Corporation. 
5. The multiplexer and $A / D$ converter interface design is complete.

6. A preliminary PDP-15 centra1 processing unit and magnetic disk checking algorithms. The algorithm may be interleaved with other computer programs such that the computer checks itself while performing at normal operation. 


\section{PRELIMINARY DESIGN FOR LOFT 5-I/2 FOOT CORE I}

E. C. Anderson, A. J., Scott, and G. K. Wa,chs

\section{INTRODUCTION}

The Loss of Fluid Test (IOFT) is one of the major safety verifications of the AEC. This 5-I/2 foot core is to be the first of a series which will:

1. Evaluate the adequacy of analytical methods to predict ( 1 ) the accident response of large power reactors, (2) the performance of engineered safety systems, and (3) the margins of safety inherent in that performance.

2. Identify any unexpected event(s) or threshold(s) that is exhibited in the response of either the plant or the engineered safety systems and develop analytical techniques that adequately describe and account for the unexpected behavior.

3. Provide experience in the development of standards and in the application of RDT standards and other standards and codes generally applicable to pressurized water reactors by their development and use on LOFT.

\section{CORE DESIGN OBJECTIVES}

With the LOFT mission in mind, the LOFN reactor must resemble an operating PWR in all respects except size and must necessarily be heavily instrumented. That is, the reactor must have similar plant systems hardware and layout, similar peak power densities, and similar response characteristics. Consistent with plant capacity constraints, a power level of $55 \mathrm{MW}(t)$ was specified. To insure test objectives can be met the core must have sufficient excess reactivity to produce the design power level for 2000 effective full power hours without exceeding control capacity or shutdown margins. The maximum power density must be equivalent to the maximum of that found in a typical PWR or about $19 \mathrm{~kW} / \mathrm{ft}$. The reactor will be controlled by a combined use of control rods and boron concentration control in the reactor coolant (so-called chemical shim). The LOFT core must simulate the lower, center section (non-control rod region of the core) of an LPWR. At "Ioss-of-Coolant-Experiment" test conditions the rods are high in the LOFT core ( $54 \pm 3 \mathrm{in}$. withdrawn). Rods will be maintained in this bank by repositioning with boron compensation. Sufficient rod worth must be available to shut the reactor down $\left(k_{\text {eff }} \leq 0.99\right)$ from any operating condition at any time in life with the most reactive rod stuck in the fully withdrawn position. The design that evolved and the analysis that was performed insures that LOFI meets the above objectives.

PHYSICAL DESCRIPTION OF THE CORE

The core layout is shown in Figure 1. The core is made up of five typical $15 \times 15$ PWR fuel assemblies arranged to form a cross. The corners 
of the cross are filled with triangular fuel assemblies, outside of which are stainless steel filler blocks. Each 15 x 15 element contains 204 fuel pins and 21 zirconium support tubes. The corner elements each contain 70 fuel pins and 8 support tubes. The zirconium clad fuel pins are 0.422 in O.D. and the active fuel length is $5.5 \mathrm{ft}$. The fuel pins are held in radial position in each canless assembly by four axially spaced grids.

The reactor is controlled by 4 rod-cluster control assemblies, one in each of the outer $15 \times 15$ assemblies, in combination with boron concentration control. The rods (or boron concentration control) are used in the control band to control reactor temperature, or compensate for reactivity changes due to normal planned power changes. If the rods reach the edge of the control band, they are repositioned into the band by boron concentration change. Each control assembly consists of $I I$ stainless steel clad $B_{4} C$ control pins in the inner spider positions and hollow stainless steel tubes in the outer " 9 positions. The central spider position is not used. Each control pin is hung from an assembly spider and upon insertion fits inside the appropriate open fuel assembly support tubes. Each control assembly or spider is driven by a separate drive.

Surrounding the core is a stainless steel octagonal flow skirt 0.75 in. thick. The stainless steel core support barrel is 30.0 in. i.d., and is $1.5 \mathrm{in}$. thick. The vessel filler is 35.0 in I.D. and is 13.75 in. thick*. SUMMARY OF CALCULATIONAL RESULTS

The calculations summarized here were made at various stages of the design evolution. Therefore, the calculations must be repeated for the final "as built" design in order to more precisely predict performance.

1. Fuel Enrichment and Boron Requirements

Design specifications require that the reactor be capable of operating for at least 2000 hours at a power level of $55 \mathrm{MW}$ thermal. Additionally, at 2000 hours the rods may not be above the upper maneuvering limit (about 9 in. insertion) and, the soluble boron concentration must be at least $100 \mathrm{ppm}$. The results of several depletion iterations indicate that an enrichment near 4.05 atom percent U-235 is required. This enrichment yields a zero-boron, rods-out excess reactivity of $11.1 \% \Delta \rho$ at beginning of life.

\section{Control Rod Worth}

The rods are maintained in the rod control band by boron concentration adjustments. In addition boron concentration control is used to compensate for the reactivity losses associated with fission product buildup, fuel depletion, and large temperature changes. The control rods may be used only for normal, planned power changes and fine temperature control adjustments. The rods are normally positioned. near the top of the core and remain in a rather narrow maneuvering band-nominally 12 in. \pm 3 in. insertion. The rod worth must be

* These dimensions have since been changed to $35.4-13.35$. 
sufficient to bring quickly the reactor from the hot operating condition to the hot shutdown condition at any time in core life with the most reactive rod stuck fully withdrawn. The control system meets these criterion. Four rods are worth about $21.8 \% \Delta p$ while three rods are worth $12.6 \% \Delta p$. The maximum power defect is about $3.6 \% \Delta p$. The control rod system worth is very satisfactory even after allowing for a reason shutdown margin and for calculational uncertainty. Total and differential rod worths for many various temperatures and boron concentrations were also calculated.

\section{Power Distributions}

As noted earlier, the power distribution constraints on the LOFT core are quite severe. Several XY and RZ power distributions have been calculated with and without the perturbing temperature distribution influence. Whereas the radial distribution changes only about $5 \%$ with core life, the axial shape flattens considerably. Thus, to maintain the desired $19 \mathrm{~kW} / \mathrm{ft}$ at the hot spot throughout Iife and still keep the rods in the maneuvering band, the reactor power must necessarily be adjusted at various times in life.

4. Boron Requirements

A large number of calculations have been made to determine the boron requirements for several plant conditions. The rods out boron requirem ment varies from near $1 \mathrm{y} 1800 \mathrm{ppm}$ at cold standby to about $1400 \mathrm{ppm}$ hot at power at beginning of life. The boron requirements diminish throughout the life of the core.

5. Moderator Temperature Coefficients

Moderator temperature coefficients are necessary for both operational and safety analysis considerations. In a borated reactor the coefficient can be positive in some temperature regimes; $i . e .$, an increase in temperature can cause an increase in core reactivity. After severaI calculations for various reactor conditions it was verified that this undesirable situation does not occur in this high leakage LOFT reactor. At the hot operating condition a representative moderator coefficient is in the order of $-2.5 \times 10^{-2} \% \Delta \rho /{ }^{\circ} \mathrm{F}$.

6. Fuel Temperature Coefficients

The fuel temperature, or Doppler, coefficient" is an inherently rapid and negative reactivity feedback coefficient necessary for safety of fast transients. Doppler coefficients were calculated for the reactor in a cold critical condition for startup accident analysis and for the hot operating reactor for a variety of accident situation analyses. Many calculations were made for various boron concentrations but representative values for the IOFT Doppler coefficient are $-1.4 \times 10^{3} \% \Delta p /{ }^{\circ} \mathrm{F}$ for the cold reactor and $-1.0 \times 10^{-3} \% \Delta \rho /{ }^{\circ} \mathrm{F}$ for the operating reactor.

* Included is the effect of fuel pin expansion. 
7. Void and Pressure Coefficients

Uniform vold and pressure coefficient calculations were made for the reactor in a hot operating condition. These calculations were also primarily made for safety analysis purposes. For small voids the void coefficient was about $-0.2 \% \Delta p / \%$ void. Neutronically, a reduction in pressure at constant temperature affects reactivity through a water density change just as vold formation does. Thus, void information may be converted to pressure coefficient information. The pressure coefficient for LOFT is about $+5.0 \times 10^{-4} \% \Delta \rho / \mathrm{psi}$. These coefficients are functions of boron concentration and therefore change with core life.

8. Generation Time $(\Lambda)$ and Delayed Neutron Fraction ( $\bar{\beta}_{\text {eff }}$ )

These parameters are of fundamental importance in determining the reactors response to any given reactivity perturbation. Using methods outlined in Reference 1 the following representative values were obtained: $\Lambda=18.6 \mathrm{\mu sec}, \bar{\beta}_{\text {eff }}=0.0073$. Both values are slow functions of boron concentration and thus core life.

9. Xenon Stability Analysis

A preliminary study was made of the stability of the LOFT core to induced xenon oscillations. This analysis, made using conservative assumptions, showed that the reactor is stable. Significant analysis remains to be done, however, for although the core is stable and the oscillations die out after only one long term cycle, the effect that the power distributions, attained throughout the cycle will have on heat transfer safety margins must be determined.

10. Neutron Fluxes at Power Range Instruments

Calculations were made to determine the neutron flux level at the power and intermediate range detectors for LOFT Core No. 1 at full power operation. The detectors are located in the primary shield tank at a radial distance of 52 inches from the core axial centerline. These data were used to establish the power range of the nuclear instruments which supply level information as part of the plant operating and protective system. Data were also used to determine secondary gamma ray sources as a result of neutron capture on the radial structural components surrounding the core.

11. Gamma Ray Heating Rate Calculations

Axial and radial gamma ray heating rates were calculated in the lower support structure, core filler pieces, flow skirt, core barrel, and core filler piece for the LOFT Core No. 1. The principal sources of gamma radiation considered in these heating calculations during power operation are from core fission gammas, secondary gammas from the core and all external regions, and inelastic scattered gammas from the core and all external regions. These data are required since the engineering design is usually dictated by the temperature and stress limitations 
of these components, The thicknesses of the radial components were adjusted to limit heating rates to acceptable levels.

A complete report describing these design results and presenting the calculational methods is in preparation(2).

[1] B. I. Rushton, "Physics Methods Used to Compute Reactivity, $\Lambda / \bar{B}_{\text {eff }}$ and Rod Worth for the PBF Critical Experiments, "IDO-17276 (October 1968).

[2] E. C, Anderson, A. J. Scott and B. I. Rushton, "Reactor Physics Analysis for the Preliminary Design of the 5.5 ft LOFT Core I," ANCR-1021 (in preparation). 


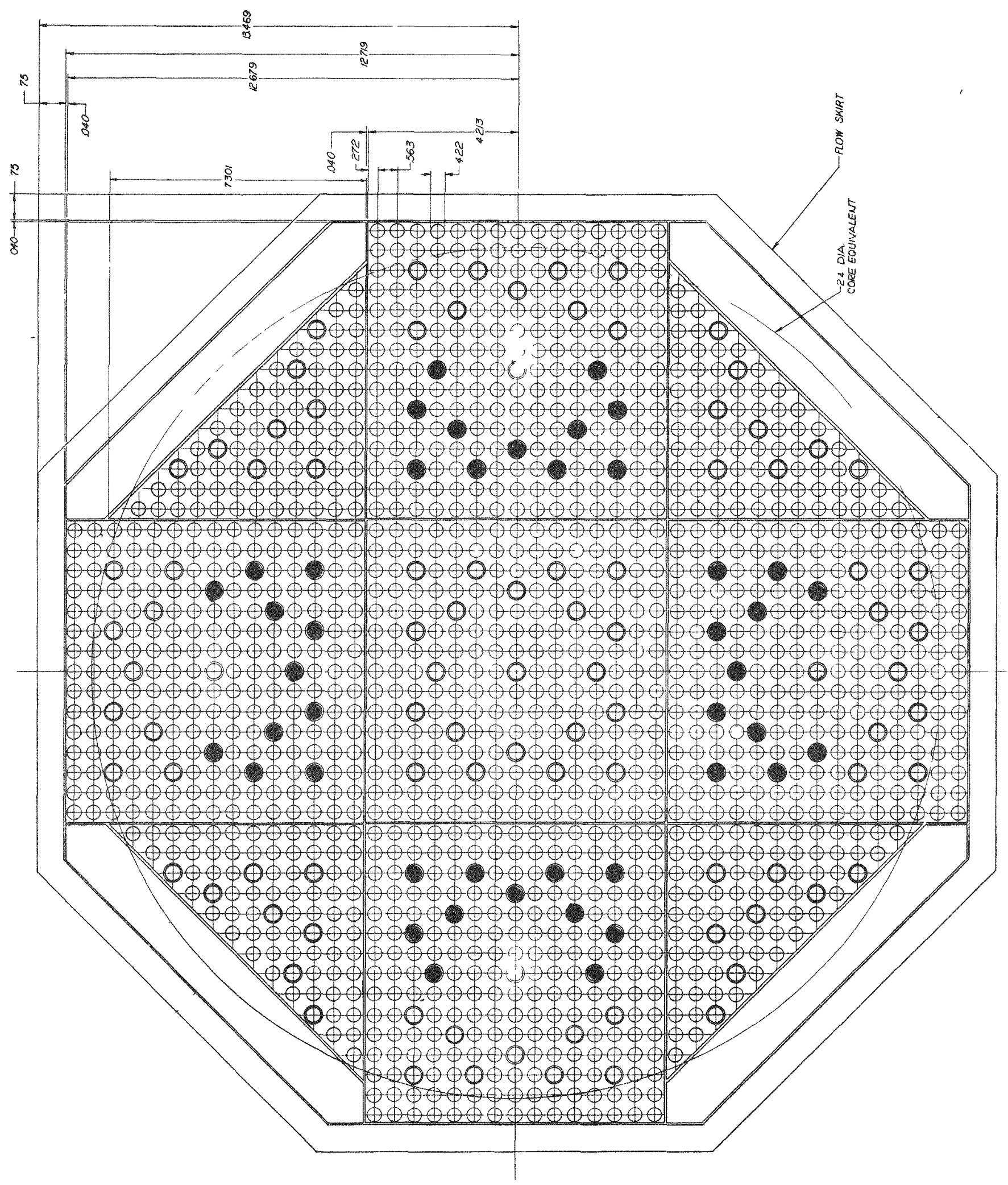

- Control Rod

\section{CORE LAYOUT}

Figure 1 
FLOWING GAS TESTS FOR THE GAS CORE REACTOR CONCEPT

J. F. Kunze, J. H. Lofthouse, D. H. Suckling

The gas core reactor is a relatively new concept, proposed principally as an ultra-high specific impulse rocket for manned or unmanned deepspace penetration space missions. The concept also has other applications, such as to supply the energy and fluid for an MHD electric generator. Fundamentally, the concept is one in which the central reactor core consists of very high temperature gaseous uranium, surrounded by the flowing coolantpropellant, which is hydrogen in the case of the rocket application(I). Figure 1 shows the general concept. The cavity containing the uranium fuel and the hydrogen coolant is surrounded by a moderator with high moderating ratio. Heavy water is the most effective such moderator, but $\mathrm{Be}$ and Beo will serve if solid moderator's or high temperature moderator capability is needed. Theory indicates that this concept will provide specific impuises (pounds of thrust per unit weight flow rate of propellant) of at least 1500 , and perhaps as high as 4000 seconds. This compares with a maximum of about 450 seconds attainable with chemical rocket systems, and 800 seconds for the NERVA solid core nuclear rocket concept.

The principal problem with the gas core concept is the creation and maintenance of a large enough and dense enough volume of $235 \mathrm{~J}$ in the center of the cavity for the reactor to be critical. And furthermore, flow rate of the propellant gas must be orders of magnitude ( 30 to 100 or more) times greater than the flow rate (hence loss rate) of the $235 \mathrm{U}$ fuel for the concept to be economically feasible.

Tests have been run on 18 -inch cavities and on 42 -inch cavities having two flat sides (effectively two dimensional "spheres"). Air and smoke (in air, argon, or freon) have been used to simulate the propellant and fissile fuel, respectively(2). Cavity designs have been repeatedly altered to obtain optimum conditions of large inner gas volume density for high flow rate ratios of outer to inner gas. A typical successful test is shown in Figure 2, where the flow rate ratio is 50 to $I$. In Figure 3 can be seen the results of densitometer traces showing iso-density lines for the gases within the cavity. These "two-dimensional," small scale tests (the real cavity must be at least $4 \mathrm{ft}$, and preferably in the range of $8 \mathrm{ft}$ in diameter) have definitely shown feasibility for the concept both from the cold flow and nuclear criticality aspects.

[1] R. G. Ragsdale and E. A. Willis, Jr., "Gas-Core Rocket Reactors A New Look", Lewis Research Center, NASA TM X-67832 (June 1971)

[2] J. F. Kunze, J. H. Lofthouse, D. H. Suckling and R. E. Hyland, "Flow-Mixing, Reactivity Effects in the Gas Core Reactor", American Nuclear Society Meeting, Boston, Mass. (June 1971). 


\section{GAS-CORE NUCLEAR ROCKET CONCEPT}

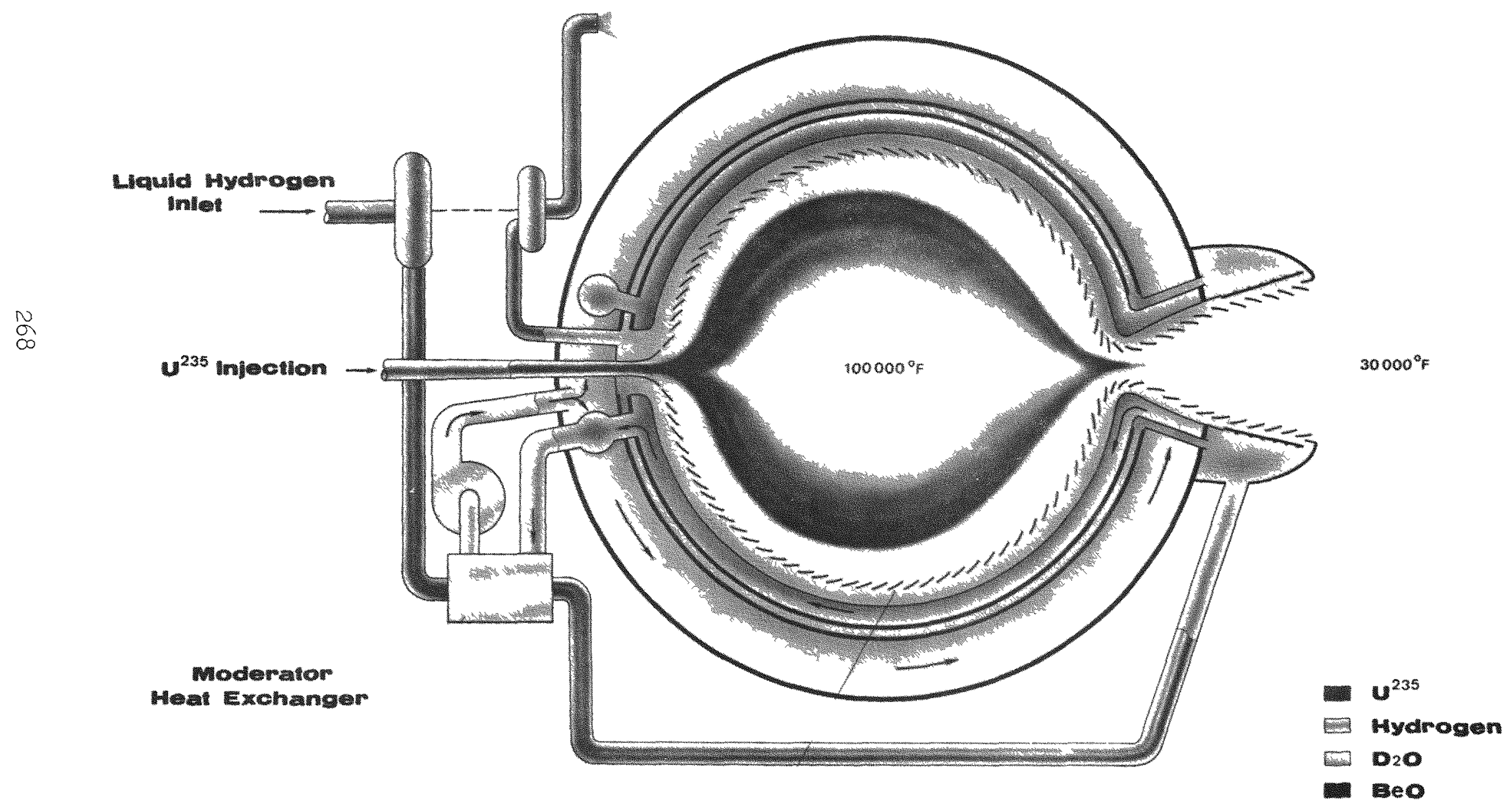

Dirrecllonal Porous Mall

FIg. I Gas core nuclear rocket concept. 

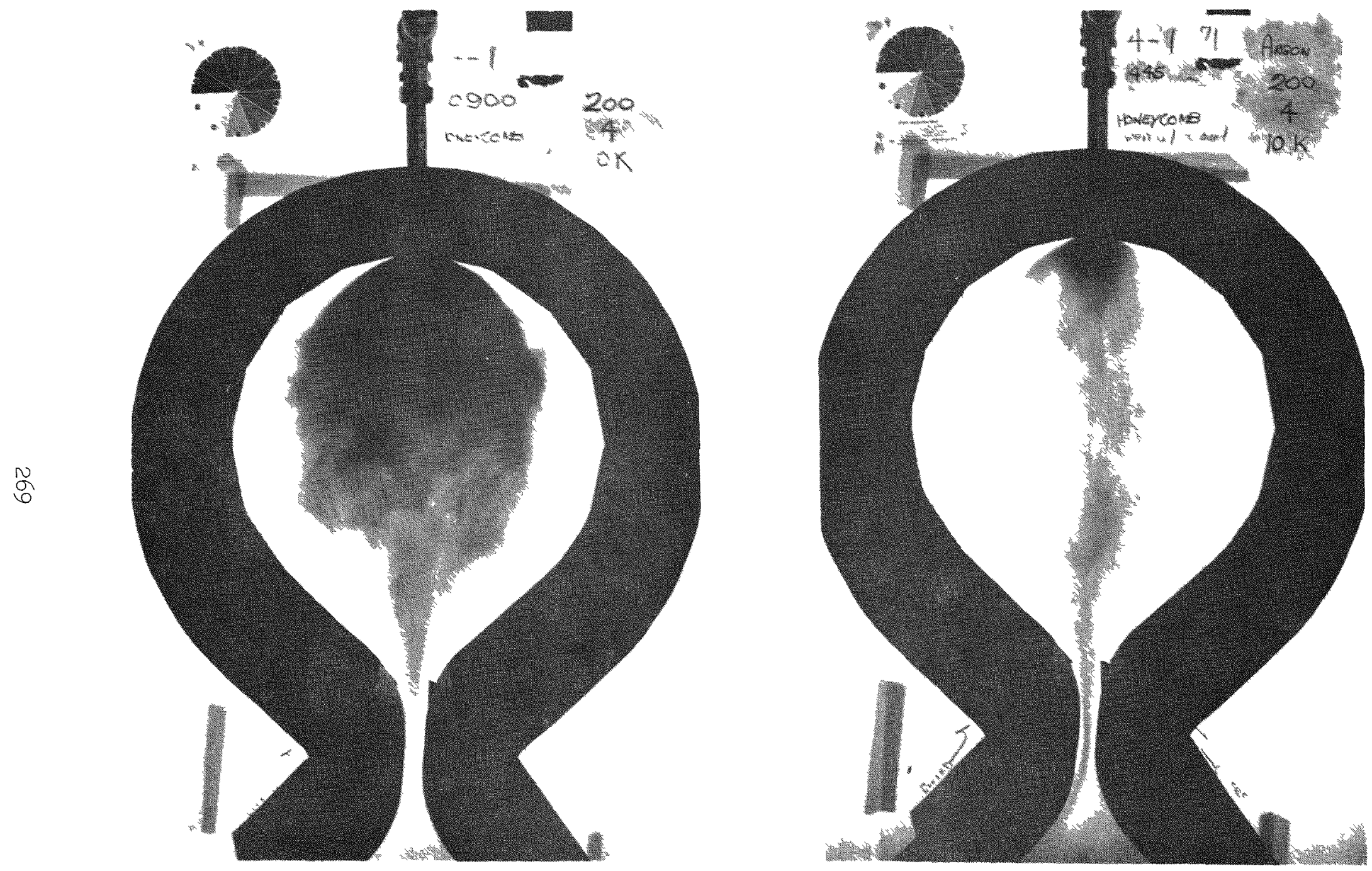

Fig. 2 Typical cavity flow tests. Honeycomb walls direct outside gas (coolant) flow tangentially around cavity. Inner gas (fuel) is smoke colored. Picture on the left is air/air with outer flow to inner flow (argon) ratio of 50. 

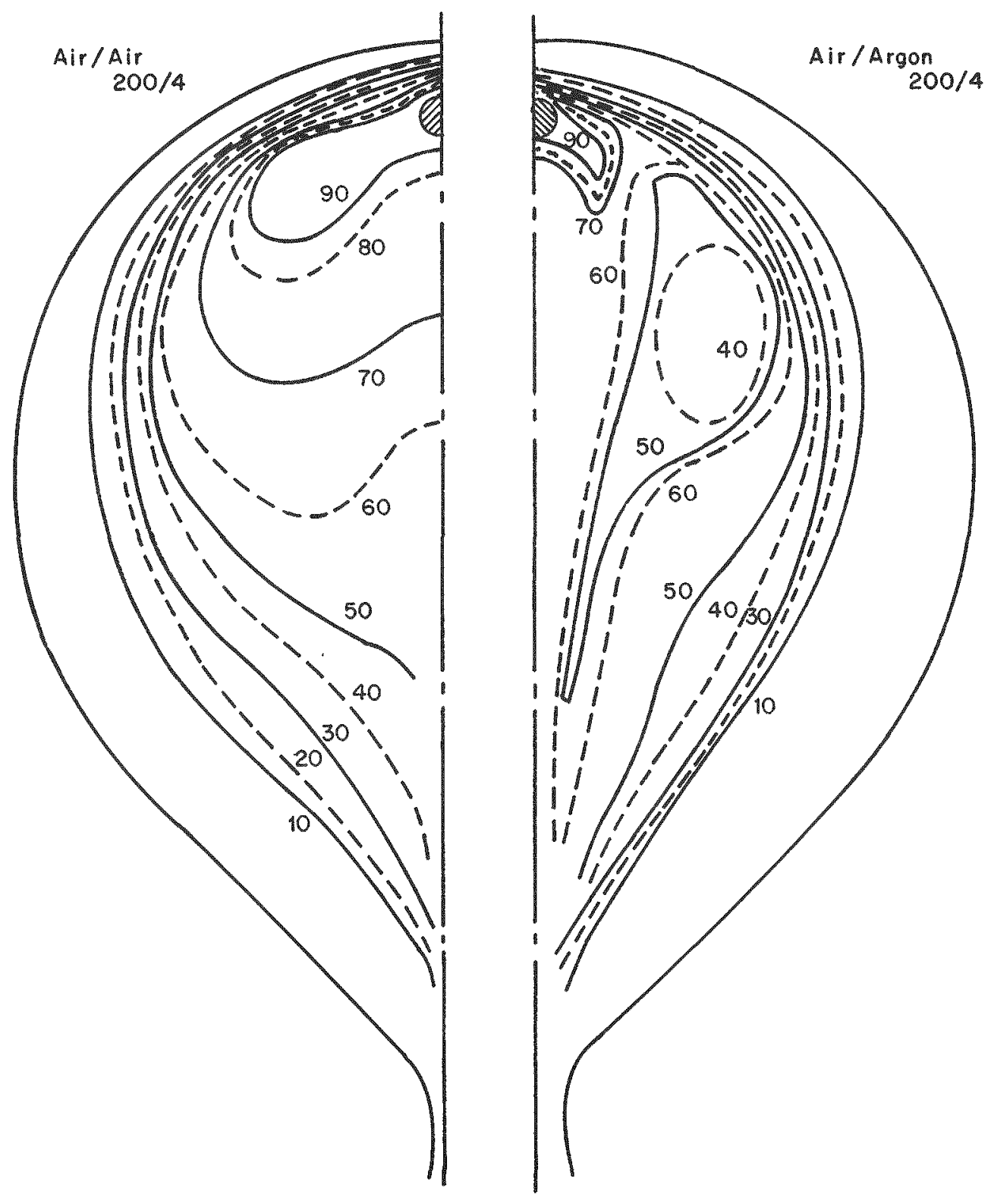

$A N C-A-306$

Figure 3. Iso-density plot of central gas concentration in the cavity. Honeycomb wall with no flow tailoring. Injection nozzle $1-1 / 2$ inches from top. 
GAMMA HEATING RATE MEASUREMENTS IN THE SPHERICAL CAVTTY REACTOR

\section{CRITICAL EXXPERIMENT}

J. H. Lofthouse, J. F. Kunze, C. G. Cooper

The gas core rocket reactor ${ }^{[1]}$ will pre-heat the propellant by running it through the reflector. An excess heat in the reflector must be removed by radiators. Therefore, it is important to know precisely the reflector heating rate so as to size properly the radiator. Gamma heating rates in a berylfium oxide reflector-moderator annulus of a spherical gas core reactor (1) were measured by mocking up a segment of the annulus with rectangular BeO slabs $70 \mathrm{~cm}$ square and of varying thicknesses in the Spherical Cavity Reactor Critical Experiment. The slab was constructed from $1 / 2$ hexagonal bars (cut across corners) stacked in an aluminum tray with $1 / 4$ inch thick walls and floor. The general arrangement is shown in Figure 1.

The bulk of the measurements in the mockup were made with LiF TLD's (thermoluminescent dosimeters) and TLD measurements were correlated with a beryllium Bragg-Gray chamber at selected locations in the sensor tube that penetrated through the slab. Some of these Bragg-Gray measurements were made with the sensor tube section through the slab packed with small Beo pieces to more nearly correspond to the gamma ray spectra seen by the bulk of the TLD locations which were 12 inches off the slab center as shown in Figure 1 .

The results of this benchmark experiment are shown in Figure 2 in units of rads per watt-hour of operation versus the TID location in the slab, for three different slab thicknesses. These data have been corrected for the $\mathrm{Li}^{6}$ response to thermal neutrons where the manufacturers quoted $\mathrm{Li} / \mathrm{Li}$ isotopic ratio is $0.005 \%$. The $\mathrm{Li}(\mathrm{n}, \mathrm{a})$ reaction at thermal energies releases $4.8 \mathrm{Mev}$ of energy to the triton and alpha particle. This correction is not insignificant in the high thermal flux region of the reflectormoderator. A value of $95 \mathrm{mrad}$ per $I \times 10^{9}$ nvt was used to make the correction.

The penetration data from the various shield thicknesses coincide, as would be expected; the shape is a gradually decreasing exponential as the low energy $\gamma$ rays disappear from the spectrum. These absolute doses compare to within $2 \%$ with the Bragg-Gray chamber measurements. This agreement can be theoretically justified by comparing the total gamma energy absorption of LiF and BeO using a reactor prompt and delayed gamma spectrum. The two materials have identical responses to within $0.5 \%$.

These results will next be calculated using existing reactor physics codes in order to determine how accurate these codes are - for they will ultimately provide the basis for the sizing of the radiator design.

[1] J.F. Kunze, J.H. Lofthouse and D. H. Suckling, "Flowing Gas Tests for the Gas Core Reactor Concept," this report. 


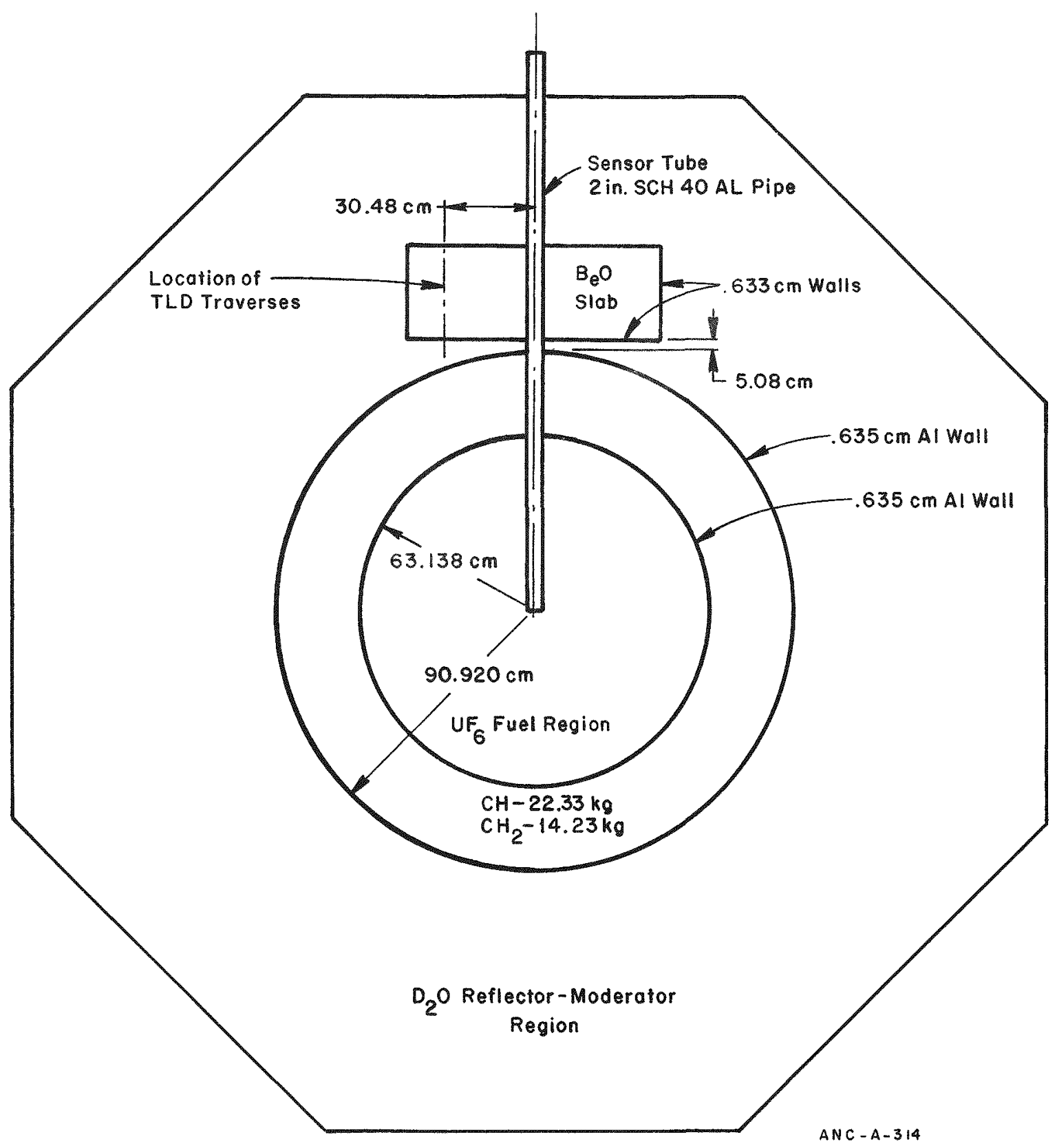

Figure 1. Diagram of Spherical Gas Core Reactor showing gamma heating measurement detail. 


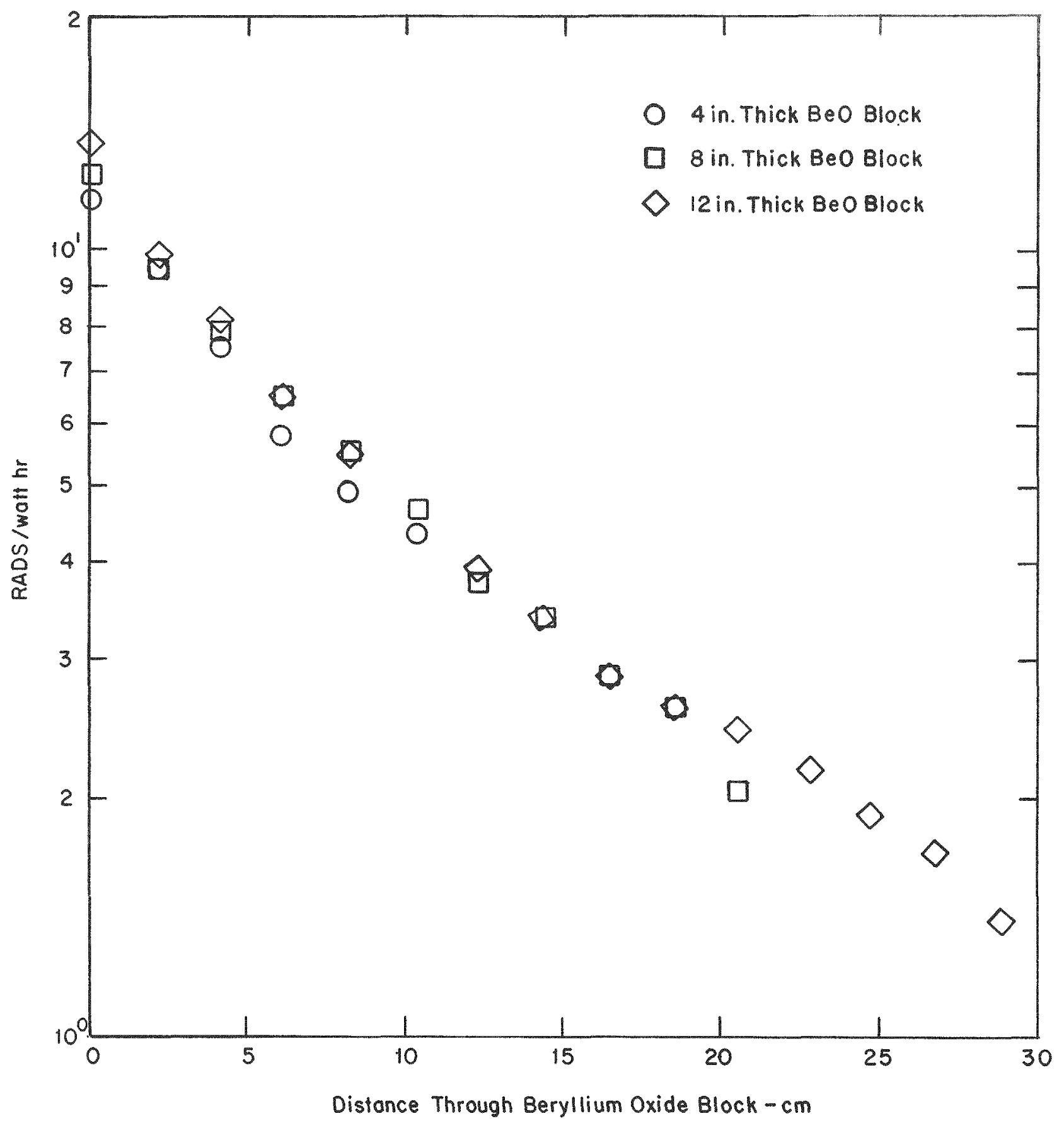

$A N C-A-313$

Figure 2. Gamma dose rate in beryllium oxide reflector. 
MEASUREMENTS OF CONTROL METHODS CONCEPTS FOR THE GAS CORE REACTOR

J. F. Kunze, C. G. Cooper, J. H. Iofthouse

The gas core (cavity) reactor concept which is being developed as a future space flight engine[1] has received extensive critical experiment testing and evaluation over the last several years at the National Reactor Testing Station $[2,3,4,5]$. These measurements have principally been performed as benchmark reactor physics experiments for the purpose of evaluating the adequacy of current computer codes in calculating the reactor physics characteristics of these reactors. However, engineering design details have also been measured, particularly where the engineering configuration is not tractable to a simple geometrical configuration that can be adequately described in a code format. An example of such measurements are the worths of control methods that might be employed in the moderator-reflector of the gas core reactor. Examples of proposed control methods are shown in Figure 1. Poison rods, drums with poison sectors, poison "caps," and poison sleeves were measured in an otherwise clean, spherically symmetric critical experiment $[6,7]$. This experiment was $12 \mathrm{ft}$ in outer reflector diameter, with a $6 \mathrm{ft}$ diameter cavity and a 4 ft diameter UF 6 gaseous core, operating at about $200^{\circ} \mathrm{F}$. The reflector-moderator was heavy water $\left(D_{2} O\right)$ into which mockups of these various control methods configurations were installed. Since a typical control system must be worth more than $5 \% \Delta k$, pulsed neutron decay techniques were adopted as the most feasible method of measuring their worths. Decay rates (for factor of $e$ ) of the neutron pulses were in the range of 10 to 200 milliseconds for the various measurements made. The various configurations were found to all be very effective in controlling and positively shutting down a gas core reactor. The results were as follows:

$\begin{array}{lr}23 \text { Control rods }(3 / 4 \text {-inch diameter }) & 3.8 \% \Delta \mathrm{k} \\ 1 \text { control drum } & 2.0 \% \Delta \mathrm{k} \\ 1 \text { cap } & 3.9 \% \Delta \mathrm{k} \\ 1 \text { sleeve } & 17 \% \Delta \mathrm{k}\end{array}$

These results coupled with the very slow prompt neutron generation time (about 3 milliseconds) in these reactors leave no doubt that they can be safely controlled.

[1] Flowing Gas Tests for the Gas Core Reactor Concept, this report.

[2] J. F. Kunze, et aI., "Cavity Reactor Critical Experiments", Nuclear Applications, 6, 104 (February 1969).

[3] G. D. Pincock and J. F. Kunze, "Cavity Reactor Critical Experiments, Vol. IV", Idaho Nuclear Corporation (October 1969) (NASA-CR-72550).

[4] G. D. Pincock and J. F. Kunze, "Cavity Reactor Critical Experiments, Vol. V", Idaho Nucleax Corporation (November 1969) (NASA-CR-72577). 
[5] J. F. Kunze and P. L. Chase, "Critical Experiments on a Modular Cavity Reactor." Idaho Nuclear Corp. (May 1970) (IN-1376, NASA$\mathrm{CR}-72681$ ).

[6] J. H. Lofthouse and J. F. Kunze, "Spherical Gas Core Critical Experiment," Idaho Nuclear Corp. (February 1971) (IN-1443, NASACR-72781).

[ ] J. F. Kunze, J. H. Lofthouse, C. G. Cooper, and R. E. Hyland, "Benchmark Gas Core Critical Experiment," Irucl. Sci. and Engineering, (to be published). 

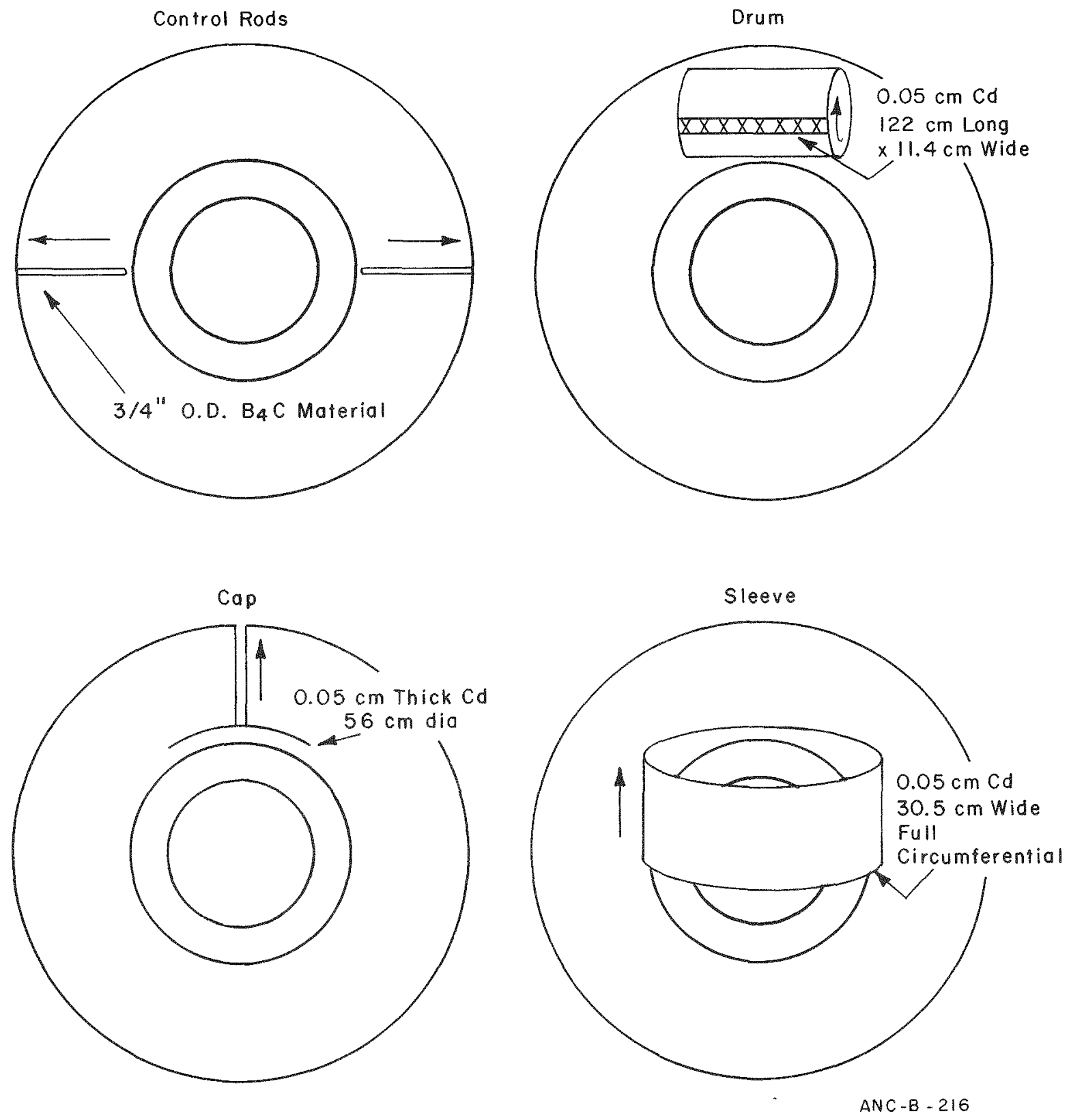

Figure 1. Measurements of Control Concepts for the Gas Core. 


\section{A. Pearson}

Measurements of the neutron spectrum produced by the CFRMF have been conducted using various techniques[1]. A current best composite spectrum gives good results when a comparison is made of measured and calculated fission rate ratios of ${ }^{235} \mathrm{U}$ and ${ }^{238} \mathrm{U}[2]$. The original method of measuring the fission rate ratios employed gamma spectrometric analysis of the uranium. A different method of measuring these ratios is available through the use of small, absolute fission chambers.

A program is currently underway to make these uranium fission ratio measurements (as well as other fissionable isotopes) using parallel plate fission chambers. The chambers being used to examine the CFRMF flux are $233,235,238 \mathrm{U}, 232 \mathrm{Th}, 239,240,241 \mathrm{Pu}$, and ${ }^{237} \mathrm{~Np}$.

The measured fission rate ratios using the fission chambers are presented in Table 1 . Included in Table 1 is the experimental fission rate ratios obtained from the ZPR-3-48[3]. The ZPR-3-48 data are included in this table for comparison purposes in view of the fact that both ZPR-3-48 and the CFRMF facilities have a typical IMFBR type spectrum.

A continuing effort is being made to complete the study of these measurements using all of the fission chambers available. Such measurements have been a standard spectral index in most fast reactor critical experiments [4]. This is an important area of spectrum evaluation effort in that, even though the technique does not measure differential neutron spectra, it does provide a verification of previously measured flux as well as sheds light on the effect of uncertainties in fission cross sections.

\section{TABLE I}

\begin{tabular}{|c|c|c|}
\hline$\sigma x$ & $\begin{array}{c}\text { CFRMF } \\
\sigma x / \sigma f(235 U) \\
\end{array}$ & $\begin{array}{r}\mathrm{ZPR}-3-48 \\
\sigma \times / \sigma f(235 \mathrm{U}) \\
\end{array}$ \\
\hline$\left.f^{(238} U\right)$ & $0.0404 \pm 0.002$ & $0.0307 \pm 0.0004$ \\
\hline$\left.f^{(239} \mathrm{Pu}\right)$ & $0.871 \pm 0.043$ & $0.976 \pm 0.010$ \\
\hline$\sigma_{f}\left(233_{U}\right)$ & \pm 0.045 & -- \\
\hline$\sigma_{f}(240 \mathrm{Pu})$ & \pm 0.015 & $0.243 \pm 0.002$ \\
\hline$\sigma_{f}(241 \mathrm{Pu})$ & $0.705 \pm 0.035$ & -- \\
\hline$\sigma_{f}(232 T h)$ & $0.00917 \pm 0.00046$ & - \\
\hline$\sigma_{f}(237 \mathrm{~Np})$ & \pm 0.012 & -- \\
\hline
\end{tabular}


[1] D. A. Pearson, "CFRMF Spectrum Determination", this report.

[2] J. J. Scoville, "The Effect of Cross Section Variation on Integrals Measured in the CFRMF", Third Conference on Neutron Cross Sections and Technology (March 1971) p. 9.

[3] H. Alter, R. S. Hubner, Status of Fast Neutron Crossmection Data Testing Using ENDF/ $\bar{B}$ Version 2 Data Files, AI-AEC-12999, p. 9.

[4] W. G. Davey, "Selected Fission Cross Sections for ${ }^{232} \mathrm{Th},{ }^{23}{ }^{3} \mathrm{U}$, ${ }^{23}{ }^{4} \mathrm{U}, 235 \mathrm{U}, 236 \mathrm{U},{ }^{237} \mathrm{~Np}, 238 \mathrm{U},{ }^{239} \mathrm{Pu}, 240 \mathrm{Pu}, 241 \mathrm{Pu}$, and $242 \mathrm{Pu}$.", Nuclear Science and Engineering, 32, pp. 35-45 (1968). 
D. A. Pearson, D. A. Millsap, J.W. Rogers

It is important to know the neutron distribution as a function of energy within a reactor. The current emphasis on the LMMBR's adds justification to our continuing efforts to improve the capability of spectrum measurements associated with fast reactors in general and the CFRMF I I in particular. It is with this goal in mind that a continual study of the spectrum of the CFRMF is being carried on utilizing several techniques. The three methods currently receiving primary attention are calculational methods, proton-recoil, and foil activation. The calculation of the neutron spectrum employs two-dimensional standard diffusion and transport calculations using the latest version of ENDF/B cross sections[2]. Measurements have been made with a calibrated protonrecoil spectrometer that employs the two detector method using hyarogen and methane gases[3]. Foil activation analys is of the neutron environment utilizing an on-line graphical analysis technique is a third method used in the continued study of the spectrum[4].

The analysis of the most recent efforts of the three independent methods of neutron spectrum determination has revealed a revised CFRMF spectrum[5]. The most accurate knowledge of the spectrum currently available is shown in Figure 1 . Efforts will continue to be made to insure that the CFRMF neutron spectrum will be among the most intensely studied and accurately determined reactor neutron environments.

[1] E. Fast, et aI., Use of the ARMF for a Fast Reactor Support Program, Idaho Nuclear Corporation report IN-1143 (November 1970).

[2] Nuclear Technology Branch Annual Progress Report for Period Ending June 30, 1969, Idaho Nuclear Corporation report IN-1317, pp. 277-282 (1970).

[3] Ibid., pp. 283-288.

[4] D. A. Pearson, Improvements in Foil Activation Data Analysis, this report.

[5] Nuclear Technology Branch Annual Progress Report for Period Ending June 30, 1970, Idaho Nuclear Corporation report IN-1407, p. 291 (1971). 


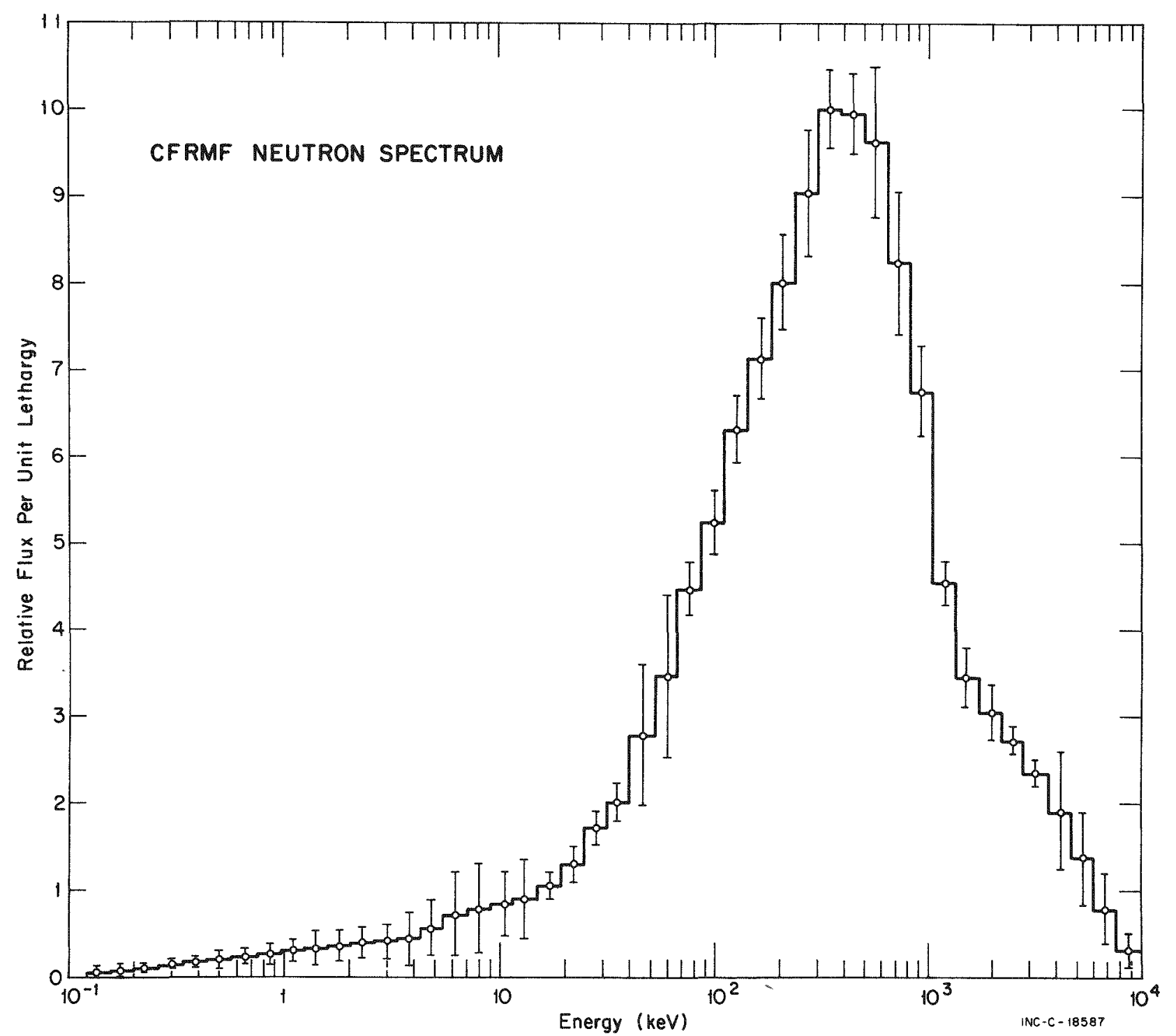




\title{
CONSISTENCY CHECKS ON EVALUATED CFRMF SPECTRA
}

\author{
Y. D. Harker
}

The credibility of using integral cross section measurements made in the CFRMF as a check on the reliability of differential cross section data places severe demands on the knowledge of the spectrum in the facility. In particular, the evaluated spectrum when integrated over a "well known" cross section should accurately predict the measured integral. Ideally, several cross sections, accenting different energy regions of the spectrum could be used to demonstrate the accuracy of the spectrum knowledge over its entire energy range. A check of this kind is undertaken here to demonstrate the consistency of the newly evaluated spectrum[1]. The method is based upon activation techniques where in this case three reactions are selected to give responses to different regions of the CFRMF spectrum.

The three reactions chosen are: ${ }^{197} \mathrm{Au}(n, \gamma)$ which is sensitive to the lower energy region, $235 \mathrm{U}(\mathrm{n}, \mathrm{f})$ which is sensitive to the mid-energy region, and $238 \mathrm{U}(\mathrm{n}, \mathrm{f})$ which is a threshold reaction sensitive to the higher energy region. The technique consists of irradiating the samples of gold and uranium in the CFRMF and determining by gamma ray spectroscopy, the saturated activities of the three reactions. Then by folding the differential cross section data from the ENDF/B-II file into the test spectrum and comparing the calculated saturated activity with the measured, the absolute normalization based upon that particular reaction is determined. If the spectrum and the cross section data are correct, then the three reactions will give to within experimental error the same absolute normalization. The degree that they are the same is a measure of the consistency of the spectrum and/or cross sections sets. In this case it is assumed that the cross section sets are reasonably correct and thus the deviations are a result of the primary errors in the test spectrum. This analysis was done on three test spectra using the same measured activities and cross section sets in each analysis, thus the only change was the test spectrum. The spectra tested are: the "best" spectrum as described in the preceding section, an earlier evaluated spectrum based upon early spectrum measured data and calculations and an early calculated spectrum using a one-dimensional diffusion code, the results of these tests are tabulated in Table 1. 
TABLE I

Spectrum Consistency Comparisons

Composite Spectrum Composite Spectrum An early one dimension Completed Feb.,1971 Completed July,1970 diffusion calculation

\begin{tabular}{|c|c|c|c|}
\hline Reaction & $\begin{array}{l}\text { Absolute flux } \\
\left(\mathrm{n} / \mathrm{cm}^{2}-\text { sec }\right.\end{array}$ & $\begin{array}{l}\text { Absolute flux } \\
\left(\mathrm{n} / \mathrm{cm}^{2}-\mathrm{sec}\right)\end{array}$ & $\begin{array}{l}\text { Absolute flux } \\
\left(\mathrm{n} / \mathrm{cm}^{2}-\mathrm{sec}\right)\end{array}$ \\
\hline${ }^{197} \mathrm{Au}(n, \gamma)$ & $6.60 \times 10^{9}$ & $7.41 \times 10^{9}$ & $1.11 \times 10^{10}$ \\
\hline${ }^{235} U(n, f)$ & $6.37 \times 10^{9}$ & $6.50 \times 10^{9}$ & $7.49 \times 10^{9}$ \\
\hline${ }^{238} \mathrm{U}(\mathrm{n}, f)$ & $6.64 \times 10^{9}$ & $6.74 \times 10^{9}$ & $6.98 \times 10^{9}$ \\
\hline Average & $6.54 \times 10^{9}$ & $6.88 \times 10^{9}$ & $8.52 \times 10^{9}$ \\
\hline$\%$ deviation & $1.8 \%$ & $5.6 \%$ & $22 \%$ \\
\hline
\end{tabular}

From this table it can be seen that the more recent composite is the most consistent of the three and is within the experimental error of the measurements.

The large deviation observed in the diffusion calculation led to an examination of where these differences could originate. It was found that the basic difference is in the region below $10 \mathrm{keV}$ where the calculated spectrum was much lower than the composites. As a result further investigation showed that this difference could arise from the treatment of the elastic scattering cross section in ${ }^{238} \mathrm{U}$ in the processing code PHROG where this cross section was treated as unshielded. In the CFRMF such a simplification (normally made in most fast reactor calculations), results in significant error because the spectrum is so heavily dependent on the ${ }^{238} \mathrm{U}$ cross sections. Modifications are currently in progress which will allow shielded elastic cross sections in the code PHROG to be calculated correctly and once the calculations are completed and if they agree with experiments over regions where they are reliable, then the spectrum predicted by the calculation will be used to extend our knowledge to lower energies than are included in the present "best" spectrum.

[1] Nuclear Technology Branch Annual Progress Report for Period Ending June 30, 1971 (This report, "Comparison of Spectrum Determinations in the CFRMF", D. A. Pearson, D. A. Millsap, J. W. Rogers.) 
EVALUATION OF THE $239 \mathrm{PU}$ CROSS SECTIONS IN THE RESONANCE REGION BELOW $45 \mathrm{eV}$ FOR THE ENDF/B VERSION III DATA FILE

O. D. Simpson, F. B. Simpson

An extensive evaluation of ${ }^{239} \mathrm{Pu}$ in the resolved resonance region has been started and will be completed by September 1 for Version III of the ENDF/B data file. The evaluation will cover the energy region of 1-300 eV. This evaluation is important to reactor calculations and constitutes a considerable improvement compared to the Version II Data File for the resolved resonance region.

The evaluation has been completed up to $45 \mathrm{eV}$ and produced the single-level resonance parameters $E_{O}$ (the neutron resonance energy), $\Gamma_{n}{ }^{\circ}$ (the reduced neutron scattering width), $\Gamma_{\gamma}$ (the capture width) and $\Gamma_{f}$ (the fission width). Three different sets of experimental data were selected for the evaluation below $45 \mathrm{eV}$ : Derrien's totals $1 \mathrm{l}]$ measured at Saclay and Gwin's fission and capture data[2] measured at Rensselaer Polytechnic Institute (RPI). The data were evaluated using the Automated Cross Section Analysis Program (ACSAP)[3].

The evaluation was done as follows: (1) The total data energy scale was normalized to that of Gwin's data. (2) Derrien's total widths were used as starting values. (3) The alpha values as determined from Gwin's fission and capture data were preserved. (4) ACSAP selected a value of $\Gamma_{n}{ }^{\circ}$ which adequately described the total fission and capture data. Table I lists the information that was used in the Doppler and resolution broadening of the theoretical cross section data as predicted by the resonance parameters of Table II. Examples of the theoretical fits to the experimental data are shown in Figures $1-3$ and includes the smooth files listed in Table III. A topical report is being prepared giving a complete analysis of the evaluation.

[1] H. Derrien, J.Blon, C. Eggermann, A. Michaudon, D. Paya, P. Ribon, "Sections Efficaces Totale et de Fission du Pu-239", Proc. of IAEA Symposium on Nuclear Data Microscopic Cross Sections and Other Data Basic for Reactors, Paris, France, October 17-2I, 1966, Vol. II, IAEA, Vienna (1967) p. 195.

[2] R. Gwin, et al., Measurements of the Neutron Fission and Absorption Cross Sections of $239 \mathrm{Pu}$ Over the Energy Region $0.02 \mathrm{eV}$ to $30 \mathrm{keV}$, Part II, ORNL-4707 (July 1971).

[3] N. H. Marshall, et al., "An Automated Cross Section Analyses Program (ACSAP)", Third Neutron Cross Section Technology Conference, Knoxville, Tennessee (March 1971). 
TABLE I

EXPERIMENTAL OPERATING CONDITIONS

\begin{tabular}{llll}
\hline & Derrien's Totals & & Gwin's Fission \& Capture \\
\cline { 2 - 3 } Machine & IINAC (Saclay) & & LINAC (RPI) \\
Burst & $100 \mathrm{nsec}$ & $100 \mathrm{nsec}$ \\
Channel Width & $100-800 \mathrm{nsec}$ & $320-640 \mathrm{nsec}$ \\
Detector Thickness & $3.6 \mathrm{~cm}$ & $4.6 \mathrm{~cm}$ \\
Flight Path & $53.7 \mathrm{~cm}$ & $25.45 \mathrm{~cm}$ \\
Sample Thickness $(I / \mathrm{n})$ & $3698 \mathrm{~b} / \mathrm{a}$ & -- \\
Resolution Shape & Gaussian & Gaussian \\
Effective Sample & $96^{\circ} \mathrm{K}$ & $298^{\circ} \mathrm{K}$ \\
Temperature & 0 & $1.5 \mu \mathrm{sec}$ \\
Moderator Effect* & & \\
*An additional term $\Delta t=1.5 \mu \mathrm{sec} / \sqrt{\mathrm{E}}$ was combined with the normal \\
resolution width to produce a wider resolution function. \\
\hline
\end{tabular}


TABLE II

${ }^{239} \mathrm{PU}$ RESONANCE PARAMETERS

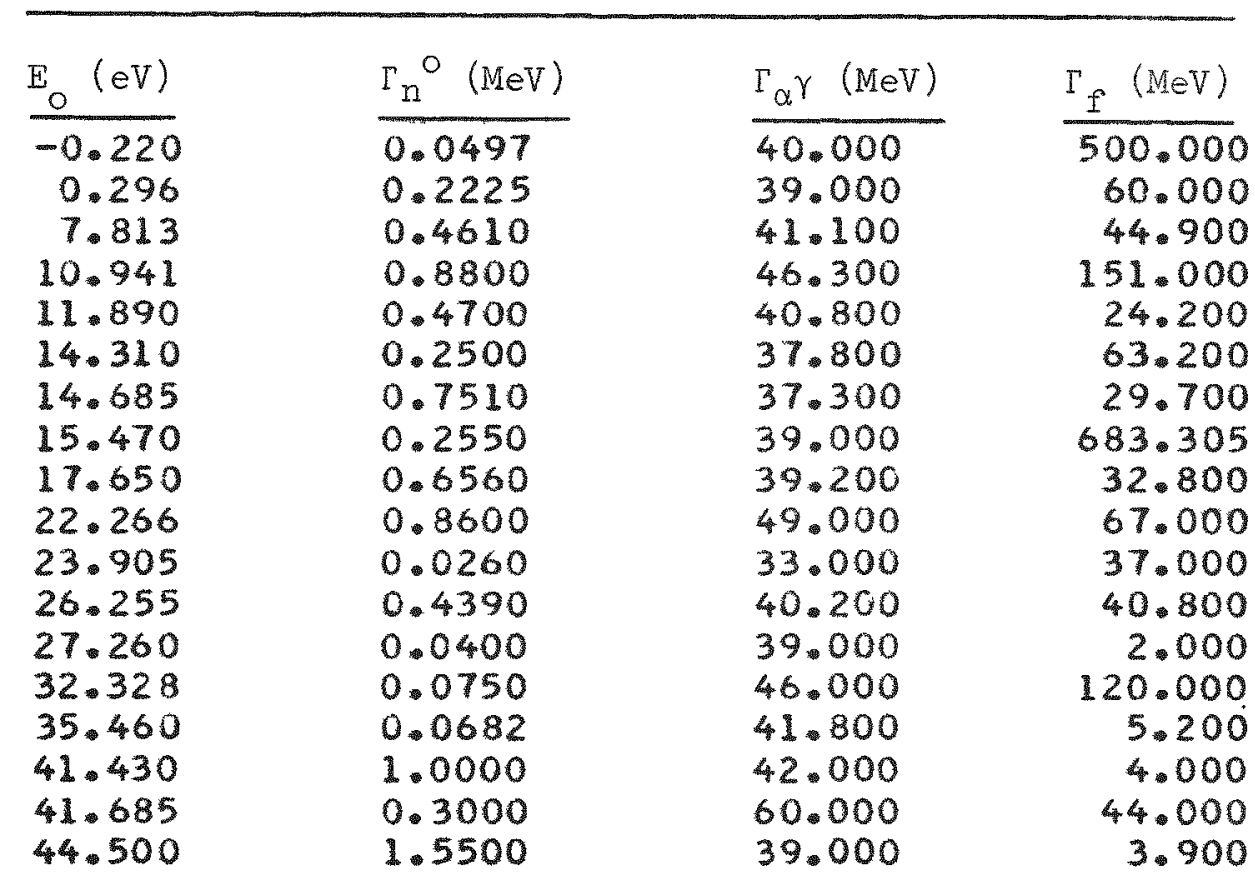


TABLE III

TOTAL SMOOTH FILE

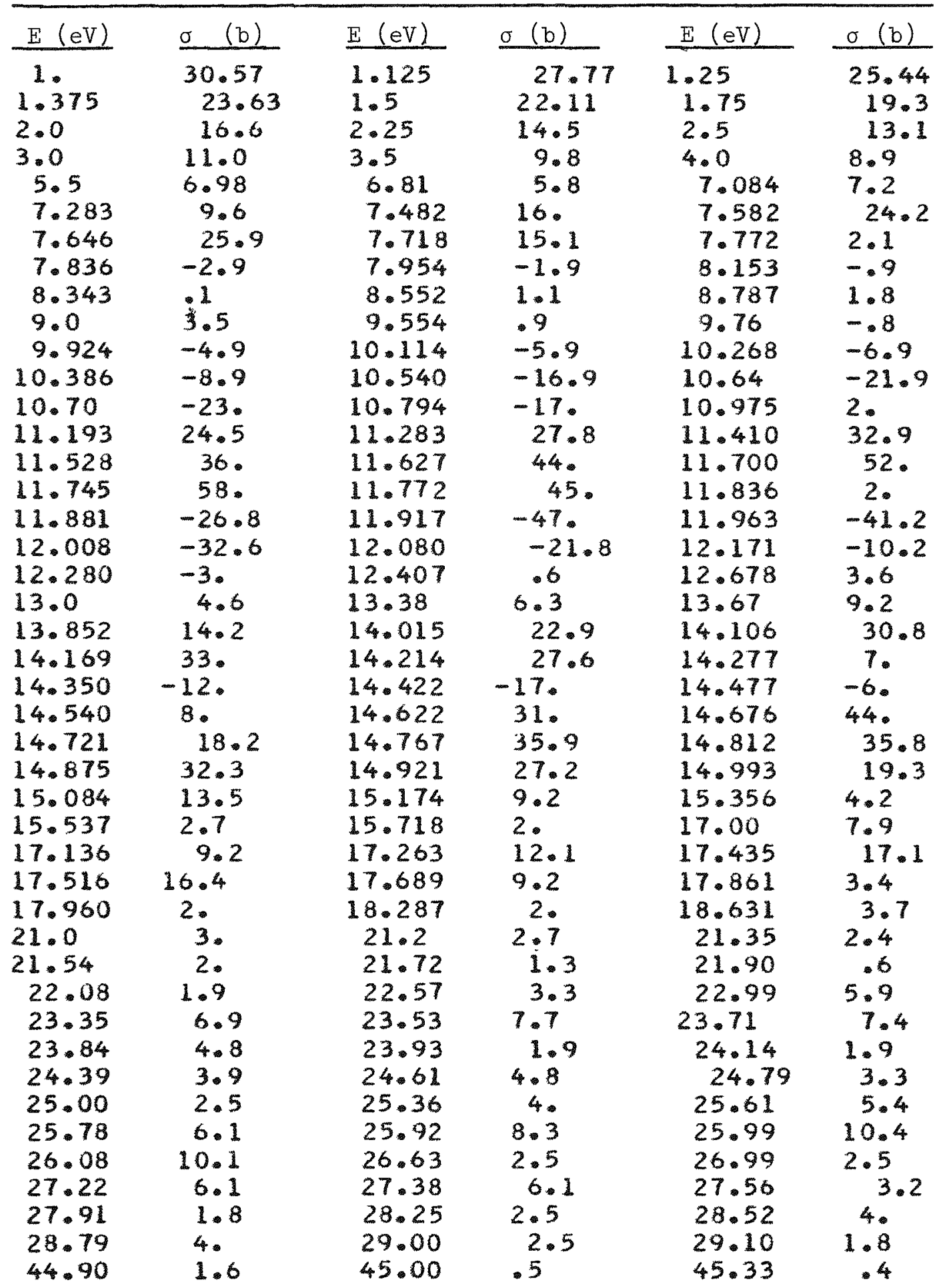


TABLE III (Contd.)

FISSION SMOOTH FILE

\begin{tabular}{|c|c|c|c|c|c|}
\hline$E(\mathrm{eV})$ & $\sigma(b)$ & $E(\mathrm{eV})$ & $\sigma \quad(b)$ & $E(\mathrm{eV})$ & $\sigma(b)$ \\
\hline 1. & 19.802 & 1.125 & 18.3 & 1.25 & 17 \\
\hline 1.375 & 16. & 1.5 & 15.1 & 1.75 & 13.5 \\
\hline 2.0 & 12.0 & 2.25 & 10.8 & 2.5 & 9.9 \\
\hline 3.0 & 8.3 & 3.5 & $7 \cdot 2$ & 4.0 & 6.3 \\
\hline $5: 5$ & $4 \cdot 3$ & 6.81 & 3.0 & 7.084 & 3.6 \\
\hline $7 \cdot 283$ & 4.5 & $7 \cdot 482$ & 6.1 & 7.582 & $7 \cdot 3$ \\
\hline 7.646 & 6.5 & 7.718 & 0 & 7.772 & -10 \\
\hline 7.836 & -13 & 7.954 & -10 & 8.153 & -8 \\
\hline 8.343 & -4 & 8.552 & -2 & 8.787 & -0.3 \\
\hline & -0.7 & 9.554 & $-3 \cdot 3$ & 9.76 & -5. \\
\hline $\begin{array}{r}9.924 \\
10.386\end{array}$ & -7 & $\begin{array}{l}10.114 \\
10.540\end{array}$ & $\begin{array}{l}-8 . \\
-19 .\end{array}$ & $\begin{array}{l}10.268 \\
10.64\end{array}$ & $\begin{array}{l}-9 \\
-24\end{array}$ \\
\hline 10.70 & -25 & 10.794 & -19. & 10.975 & 0 \\
\hline 11.193 & 22.5 & 11.283 & 25.8 & $11 \cdot 410$ & 30.9 \\
\hline 11.528 & 34. & 11.627 & 42. & 11.700 & 50. \\
\hline 11.745 & 56. & 11.772 & 43. & 11.836 & 0. \\
\hline 11.881 & $-28 \cdot 8$ & 11.917 & -49.0 & 11.963 & $-43 \cdot 2$ \\
\hline 12.008 & -34.6 & 12.080 & $-23 \cdot 8$ & 12.171 & $-12 \cdot 2$ \\
\hline $12 \cdot 280$ & -5.0 & 12.407 & $-1 \cdot 4$ & 12.678 & 1.6 \\
\hline 13.0 & 2.6 & 13.38 & 6.3 & 13.67 & 7.2 \\
\hline 13.852 & $12 \cdot 2$ & 14.015 & 20.9 & 14.106 & 27.4 \\
\hline 14.169 & 28.8 & 14.214 & $22 \cdot 3$ & 14.277 & 0. \\
\hline 14.350 & -23 & 14.422 & -39 & 14.477 & -42 \\
\hline 14.540 & -35. & 14.622 & -14 & 14.676 & -1 \\
\hline 14.721 & 1.2 & 14.767 & 25.9 & 14.812 & 28.8 \\
\hline 14.875 & 28.1 & 14.921 & 25.2 & 14.993 & 17.3 \\
\hline 15.084 & $11 \cdot 5$ & 15.174 & 7.2 & 15.356 & 2.2 \\
\hline 15.537 & 0.7 & 15.718 & 0. & 17.00 & 5.9 \\
\hline 17.136 & $7 \cdot 2$ & $17 \cdot 263$ & 10.1 & 17.435 & 15.1 \\
\hline 17.516 & 14.4 & 17.689 & 7.2 & 17.861 & 1.4 \\
\hline 17.960 & 0 . & 18.287 & 0. & 18.631 & 1.7 \\
\hline 21.0 & 1.0 & 21.2 & .7 & $21 \cdot 35$ & .4 \\
\hline $21 \cdot 54$ & 0. & $21 \cdot 72$ & -7 & 21.90 & -1.4 \\
\hline 22.08 & 0. & 22.57 & 1.4 & 22.99 & 4. \\
\hline 23.35 & 5. & 23.53 & 5.8 & 23.71 & 5.5 \\
\hline 23.84 & 2.9 & 23.93 & 0. & $24 \cdot 14$ & 0. \\
\hline 24.39 & 2. & 24.61 & 2.9 & 24.79 & 1.4 \\
\hline 25.00 & .7 & 25.36 & 2.2 & 25.61 & 3.6 \\
\hline 25.78 & $4 \cdot 3$ & 25.92 & 6.5 & 25.99 & 8.6 \\
\hline 26.08 & 8.3 & 26.63 & 0.7 & 26.99 & .7 \\
\hline 27.22 & 4 & $27 \cdot 38$ & $4 \cdot 3$ & 27.56 & 1.4 \\
\hline 27.91 & 0 & 28.25 & 0.7 & 28.52 & 2.2 \\
\hline 28.79 & $2 \cdot 2$ & 29.00 & 0.7 & 29.10 & 0. \\
\hline 44.90 & 0. & 45.00 & $-1 \cdot 1$ & 45.33 & -1.2 \\
\hline
\end{tabular}


TABLE III (Cohta.)

CAPTURE SMOOTH FILE

\begin{tabular}{|c|c|c|c|c|c|}
\hline $\mathrm{E}(\mathrm{eV})$ & $\sigma \quad(b)$ & $\mathrm{E}(\mathrm{eV})$ & $\sigma(b)$ & $E(\mathrm{eV})$ & $\sigma(b)$ \\
\hline $\begin{array}{l}1.0 \\
1.375 \\
2.0 \\
3.0 \\
5.5 \\
7.283 \\
7.646 \\
7.836 \\
8.343 \\
13.852 \\
14.169 \\
14.350 \\
14.540 \\
14.721 \\
14.875 \\
101.0 \\
131.5 \\
159.2 \\
187.2\end{array}$ & $\begin{array}{l}7.774 \\
4.7 \\
1.8 \\
0.8 \\
0.38 \\
3.0 \\
17.3 \\
8.0 \\
2.0 \\
0.0 \\
2.2 \\
9.0 \\
41.0 \\
15.0 \\
2.2 \\
0.0 \\
0.2 \\
1.3 \\
1.5\end{array}$ & $\begin{array}{l}1.125 \\
1.5 \\
2.25 \\
3.5 \\
6.81 \\
7.482 \\
7.718 \\
7.954 \\
8.552 \\
14.015 \\
14.214 \\
14.422 \\
14.622 \\
14.767 \\
14.921 \\
126.1 \\
149.0 \\
170.5 \\
197.0\end{array}$ & $\begin{array}{l}6.5 \\
4.1 \\
.9 \\
0 . \\
0.7 \\
7.8 \\
13.0 \\
6.0 \\
1.0 \\
0.0 \\
3.3 \\
19.0 \\
43.0 \\
8.0 \\
0.0 \\
0.0 \\
0.8 \\
1.4 \\
0.0\end{array}$ & $\begin{array}{l}1.25 \\
1.75 \\
2.5 \\
4.0 \\
7.084 \\
7.582 \\
7.772 \\
8.153 \\
8.787 \\
14.106 \\
14.277 \\
14.477 \\
14.676 \\
14.812 \\
14.993 \\
126.8 \\
151.2 \\
182.5 \\
301 .\end{array}$ & $\begin{array}{c}5.5 \\
2.9 \\
.4 \\
0 . \\
1.5 \\
14.8 \\
10.0 \\
5.0 \\
0.0 \\
1.4 \\
5.0 \\
34.0 \\
43.0 \\
5.0 \\
0.0 \\
0.05 \\
1.1 \\
1.5 \\
0.5\end{array}$ \\
\hline
\end{tabular}

SCATTER SMOOTH FILE

$\begin{array}{lllllll}\frac{E(e V)}{1.0} & \frac{\sigma(b)}{2.994} & \frac{E(e V)}{1.12} & \frac{\sigma(b)}{2.97} & \frac{E(e V)}{1.25} & \frac{\sigma(b)}{2.94} \\ 1.375 & 2.93 & 1.5 & 2.91 & 1.75 & 2.9 \\ 2.0 & 2.8 & 2.25 & 2.8 & 2.5 & 2.8 \\ 3.0 & 2.7 & 3.5 & 2.6 & 4.0 & 2.6 \\ 5.5 & 2.3 & 6.81 & 2.1 & 7.084 & 2.1 \\ 301.0 & -1.9 & & & & & \end{array}$




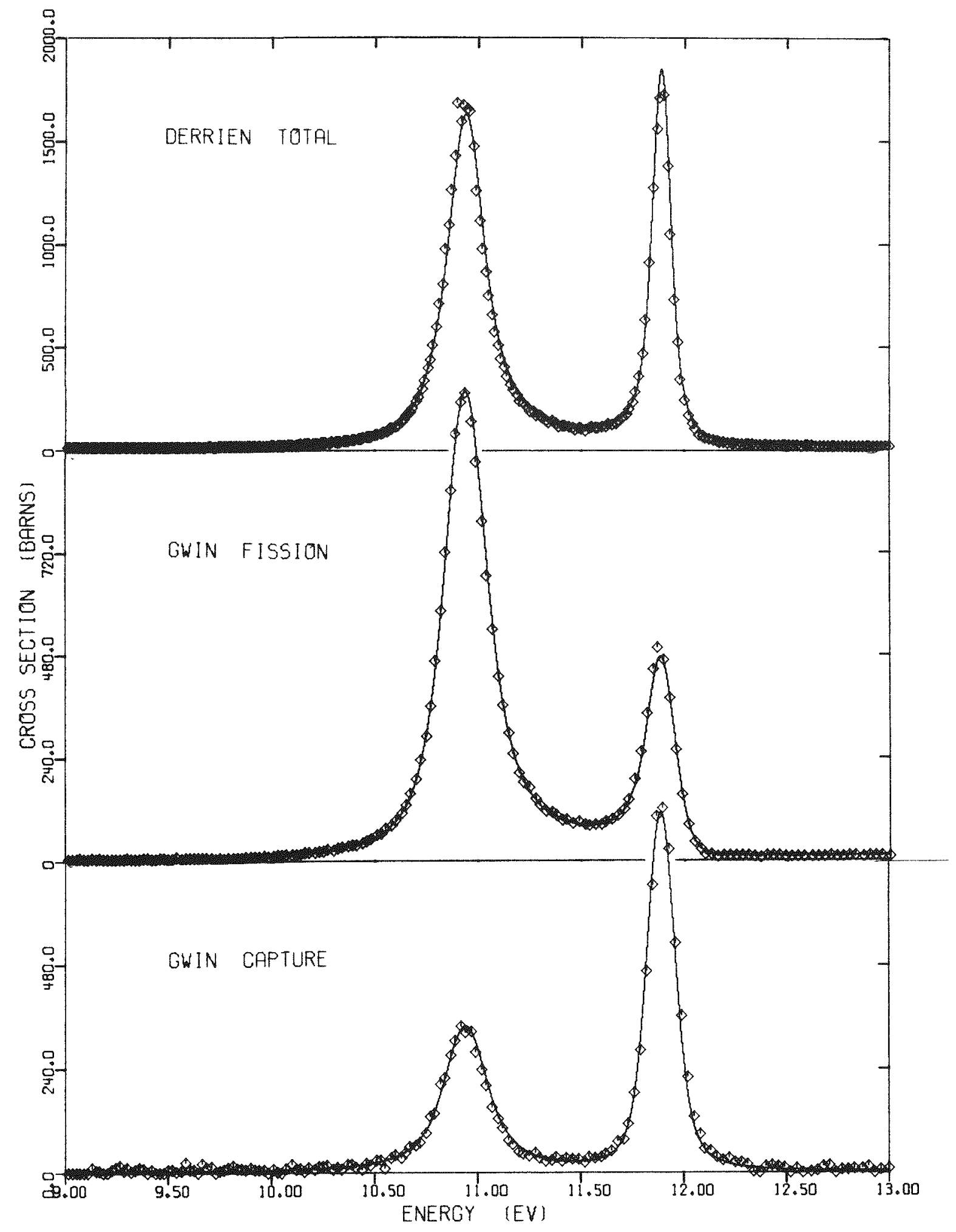

Fig. I The total, fission and capture cross sections of $239 \mathrm{Pu}$. The solid line is a theoretical fit to the data and was obtained by Doppler and resolution broadening the cross sections as predicted by the resonance parameters and smooth files of Tables II and III. 


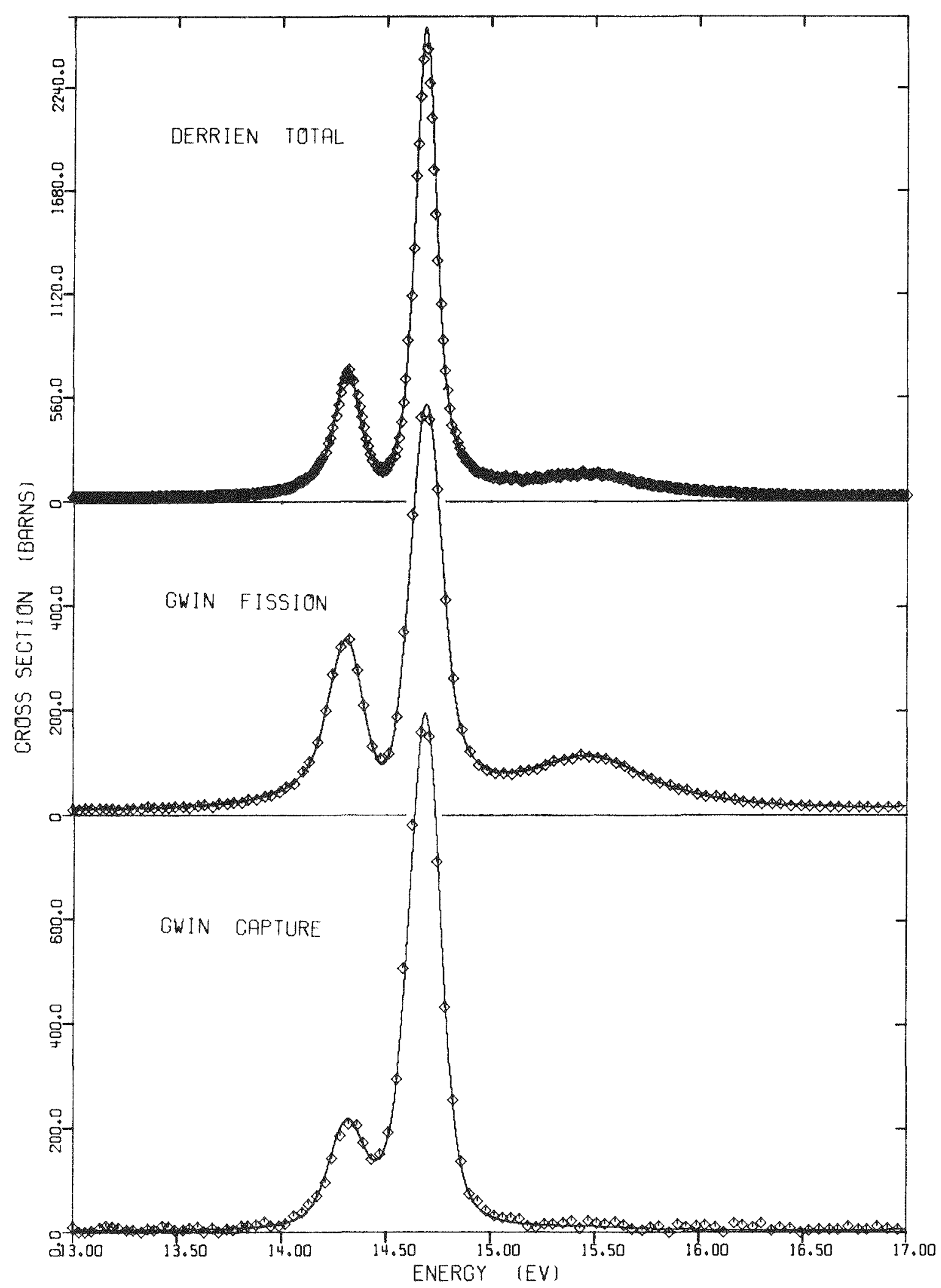

Fig. 2 The total, fission and capture cross sections of $239 \mathrm{Pu}$. The solid line is a theoretical fit to the data and was obtained by Doppler and resolution broadening the cross sections as predicted by the resonance parameters and smooth files of Tables II and III. 


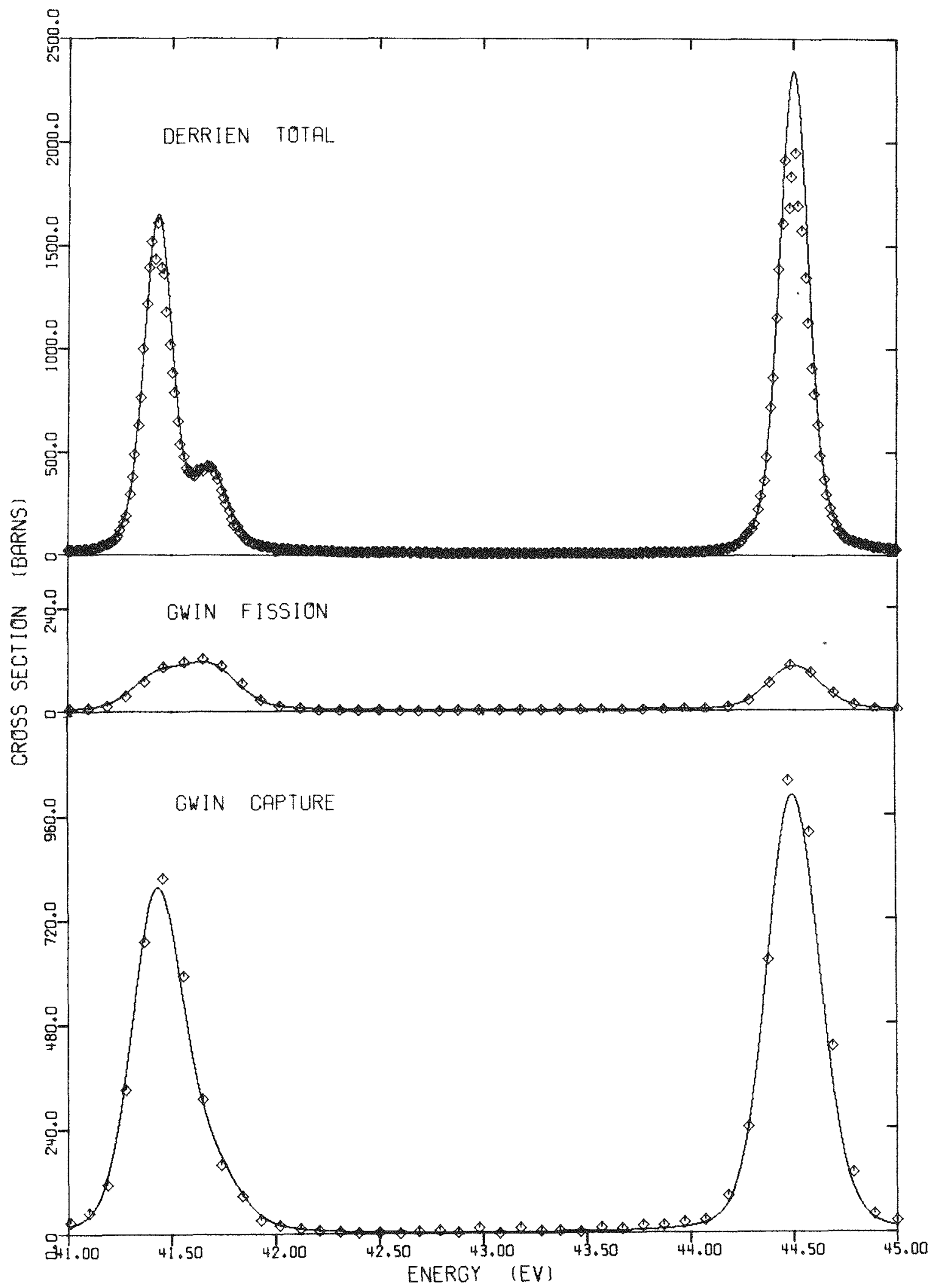

Fig. 3 The total, fission and capture cross sections of ${ }^{239} \mathrm{Pu}$. The solid line is a theoretical fit to the data and was obtained by Doppler and resolution broadening the cross sections as predicted by the resonance parameters and smooth files of Tables II and III. 


\section{U-235 RESONAINCE PARAMETERS FOR ENDF/B-III}

$$
\text { J. R. Smith, R. C. Young }
$$

Idaho Nuclear Corporation received the assignment from the Cross Sections Evaluation Working Group to revise the resolved resonance files for U-235 and Pu-239 for ENDF/B, Version III. In this section we describe the U-235 analysis.

U-235 represents probably the most challenging task in all neutron resonance analysis. This is due not only to the close spacing and assymmetry of the resonances, but also to the fact that U-235 is the most used nuclear fuel, and there are vast quantities of data from many experiments to be examined. There are also many sets of resonance parameters that have been accumulated over the past 20 years. The first task was to select the best data sets and compare them with some of the existing parameter sets to determine whether any of the latter might be adequate.

The data sets selected for detailed use in the analysis are as follows:

1. Simultaneous capture and fission measurements by DeSaussure et al. (1). The strength of this experiment is that it measured the two most important partial cross sections of U-235 simultaneously, under the same conditions of resolution and background. Moreover, care was taken to correct for such effects as backgrounds, resonance self-shielding, and scattering in the fission chamber. These data were used principally to indicate the ratio of capture to fission for the resonances. They are, however, of much lower resolution than the other data used. The most severe problem with these data is that the resolution was substantially different from that to be deduced from the experimental parameters given by the authors in their reports. Moderation time in the moderator is probably responsible for most of the resolution problem. An ad hoc solution was used in most of the analysis. An asymmetric resolution function was used, and the flight path uncertainty was increased at low energies to give a resolution that would. allow the DeSaussure data to be fit by the parameters that fit the higher resolution data sets.

2. Total cross section measurements of Michaudon(2). These data were obtained at liquid nitrogen temperatures and fairly high resolution. They turned out to give in most cases the best indication of the total widths of the resonances. The data were available only as cross section vs. energy, with results from several samples mixed together. Total cross sections are measured from transmission of samples, and the analysis should really be performed on the transmission data for each sample.

In many resonances the partial cross sections did not add to give the total cross section properly. It is very difficult, nearly impossible, to be sure whether this error of closure is due to errors in normalization of the partial cross sections, problems of normalizing the various 
sample results in the total cross sections, or in errors in handing backgrounds across resonances.

3. Fission cross sections measured by Blons et al. (3) on the Saclay linear accelerator. These data were obtained at liquid nitrogen temperature, with resolution similar to that of Michaudon's total cross section measurement. The BIons data are the best resolution fission data, but below about $35 \mathrm{eV}$ the normalization gets progressively more erratic because of difficulty in interpreting the backgrounds in the presence of a B-10 filter used to eliminate low energy overlap neutrons. Normalization by a fourth order polynominal in energy adjusted these data to match the DeSaussure normalization.

4. Fission cross sections measured by Cao et al, (4) on the Iineax accelerator at C.B.N.M. (Geel). These data are the highest resolution room temperature measurements of $\sigma_{f}$ for U-235. They are useful for comparing with the Blons data to confirm the effectiveness of the Doppler corrections in the analysis code. They go to a lower energy than the Blons data, $6 \mathrm{eV}$ vs. $17 \mathrm{eV}$. However, the Cao data are troubled. by exratic background corrections in the vicinity of resonances in filters used to determine backgrounds.

These data sets were all used in the evaluation, using the automated cross sections analysis program (ACSAP). With this versatile program it is simple to compare several sets of data against a single set of parameters. Parameters were fit to one set of data and compared to the other sets. The most effective procedure, as it turned out, was to shape-fit the Michaudon total cross sections, then adjust the capture and fission widths to match the capture and fission data of Desaussure.

Parameters below $30 \mathrm{eV}$ are listed in Table $I$. It is expected that the ENDF/B Version III file will contain parameters to approximately $80 \mathrm{eV}$.

The fits to the various data sets are shown for the region below $29 \mathrm{eV}$ in Figures 1 to 4. The dashed curves illustrate the contributions of the resonances alone. The solid lines show the complete cross sections, parameters plus smooth file. A simultaneous display of this type shows the peculiarities of the various data sets, such as the superior resolution of the low temperature measurements and the distortion in the Cao data around. $27 \mathrm{eV}$. The problems with normalization amongest the total and partial cross sections can be seen to be the most severe in the region between 8 and 16 $\mathrm{eV}$. Overall, the fit describes these sets of data very well, and should rival a good multilevel fit in accuracy of description of the cross sections of U-235. 
[I] G. DeSaussure et a., "Simultaneous Measurements of the Neutron Fission and Capture Cross Sections for U-235 for Incident Neutron Energies from $0.4 \mathrm{eV}$ to $3 \mathrm{keV}$," AEC Report ORIL-TM-1804 (1967).

[2] A. Michaudon, "Contribution a I'Etude par des Methodes du Temps de Vol de I'Interaction des Neutrons Lents Ave. I'U-235," Report CEAR2552 (1964)

[3] J. Blons, H. Derrien, and A. Michaudon, "Measurements and Analysis of the Fission Cross Sections of U-233 and U-235 for Neutron Energies Below $30 \mathrm{keV}, "$ CONF-710301, Vol. 2, p. 829 (1971).

[4] M. G. Cao et al., "Fission Cross-Section Measurement on U-235," J. Nucl. Energy 22, 211 (1968).

[5] J. J. Shore and V. L. Sailor, "Slow Neutron Resonances in U-235," Phys. Rev. 112, 191 (1958). 
TABLE I

${ }^{235} \mathrm{U}$ Resonance Parameters

RESONANCE
ENERGY

- 1.490

0.290

1. 140

2. 035

2. 920

3.147

3.609

4.848

5.449

3.600

6.210

6. 382

7.077

8.781

9.286

9.730

10.180

10.800

11.066

12.396

12.861

1.3.275

13.700

13.996

14.544

15.406

16.088

16.067

18.052

18.960

19.297

20.130

20.200

20.610

21.072

22.939

23.412

$23 \cdot t 29$

24.245

24.370

25.200

25.590

26.480

26.740

27.149

27.796

28.040

28.351

28.710

29.044
GAMMA N-

NOUGHT (MV)

3.0164

0.0056

0.0142

0.0054

0.0028

0.0126

0.0240

0.0274

0.0014

0.0141

0.0256

0.1062

0.0476

0.3791

0.0537

0.0170

0.0194

0.0284

0.1837

0.3585

0.0148

0.0109

0.0100

0.1436

0.0302

0.0604

0.0900

0.0659

0.0905

0.0266

0.7270

0.0195

0.0029

$0.0+21$

0.3274

0.0910

0.1454

0.1760

0.0545

0.0303

0.1347

0.1116

0.0925

0.0166

0.0163

0.1279

0.0059

0.0354

0.0084

0.0326
GAMMA-GAMMA

(MV)

27.000

36.000

34.500

34.874

20.000

33.210

33.696

35.945

60.000

20.003

43.469

34.972

$35.57 \%$

31.170

35.600

32.000

38.000

67.000

40.400

34.500

33.500

28.500

30.400

26.000

35.200

35.300

31.383

32.105

35.000

50.000

34.821

13. 910

49.280

40.485

40.342

32.670

28.500

43.000

31.000

35.000

25.000

25.020

32.000

30.000

42.00 ?

32.000

40.000

31.700

50.000

37.000
GAMMA $F$ (MV)

207.000

99.000

116.200

9.814

200.000

106.374

50.637

3. 587

30.117

621.891

187.363

9.548

28.233

91.000

75.000

237.020

62.500

868.000

6.250

27.500

86.030

122.800

93.500

470.000

20.900

43.300

18.617

100.855

125.000

55.000

60.179

226.091

0.720

43.515

31.658

42.330

5.000

182.000

27.000

65.030

825.000

360.000

160.000

220.000

73.500

88.000

25.000

117.330

80.000

24.000 


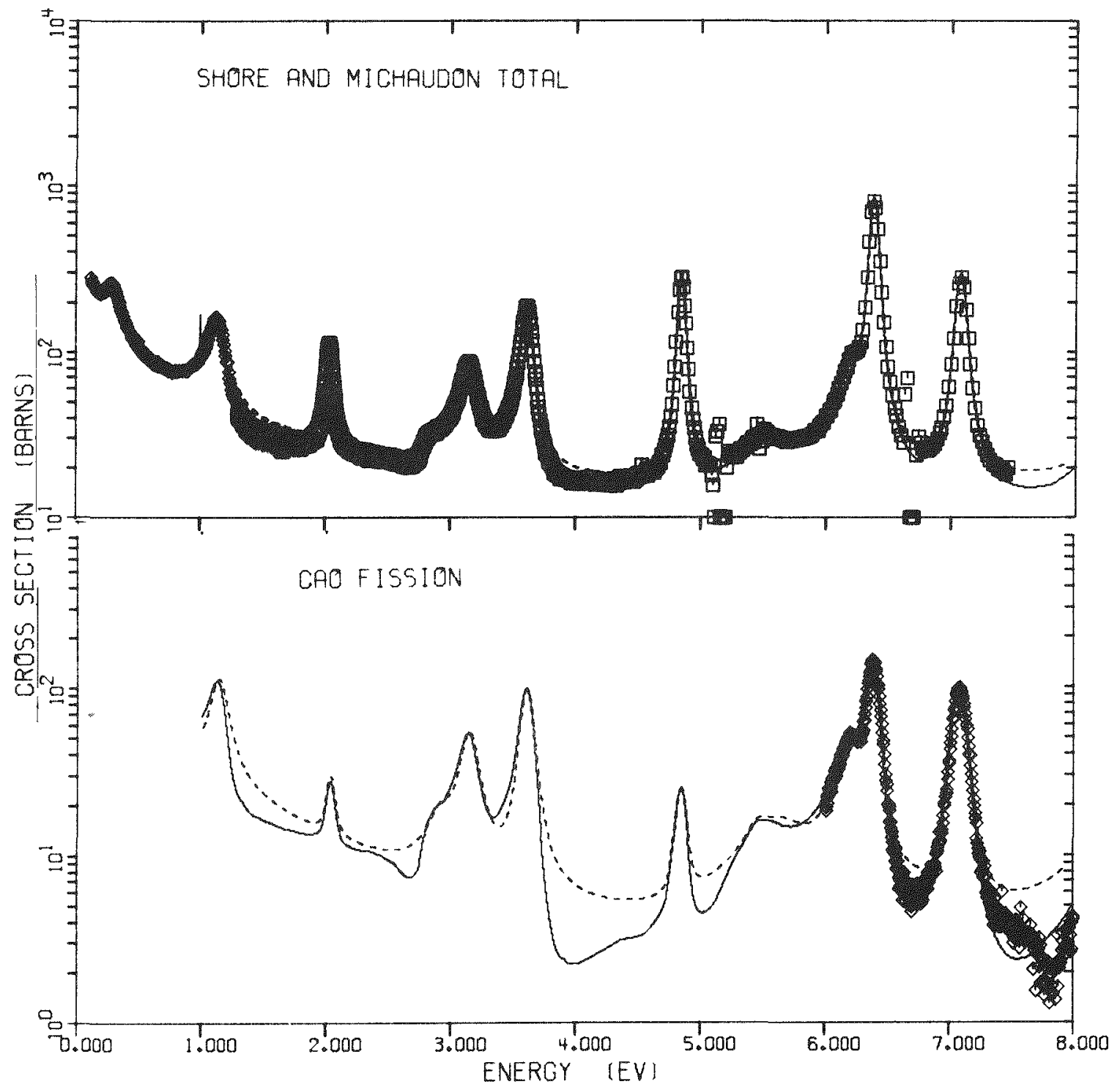

Fig. 1(A) Total, fission, and capture cross sections for U-235. The dashed lines represent the contributions of the single level resonance parameters submitted for ENDF/B, Version III. The solid lines represent the complete ENDF/B-III cross sections; including both resonance parameters and smooth file. The fit begins at $1 \mathrm{eV}$. The curves have been resolution- and Dopplerbroadened to match the conditions under which the various sets of data were collected. Shown are the total cross section measurements of Michaudon (2), fission cross section data of Cao (4), and DeSaussure (II, and capture data of DeSaussure (I). In addition the total cross section data of Shore and Sailor (5) are show below $1.8 \mathrm{eV}$. 


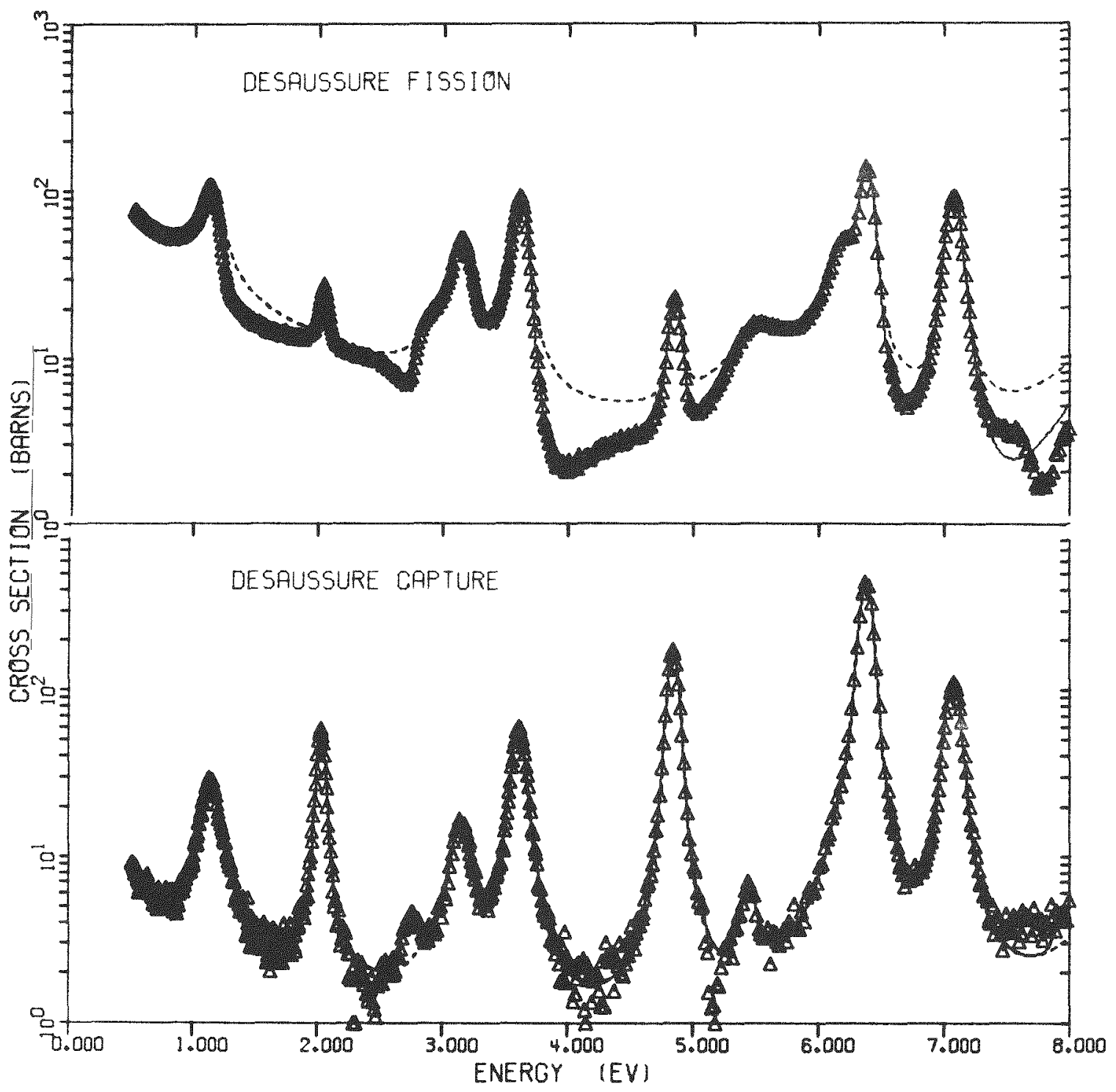

Fig. 1 (B) 


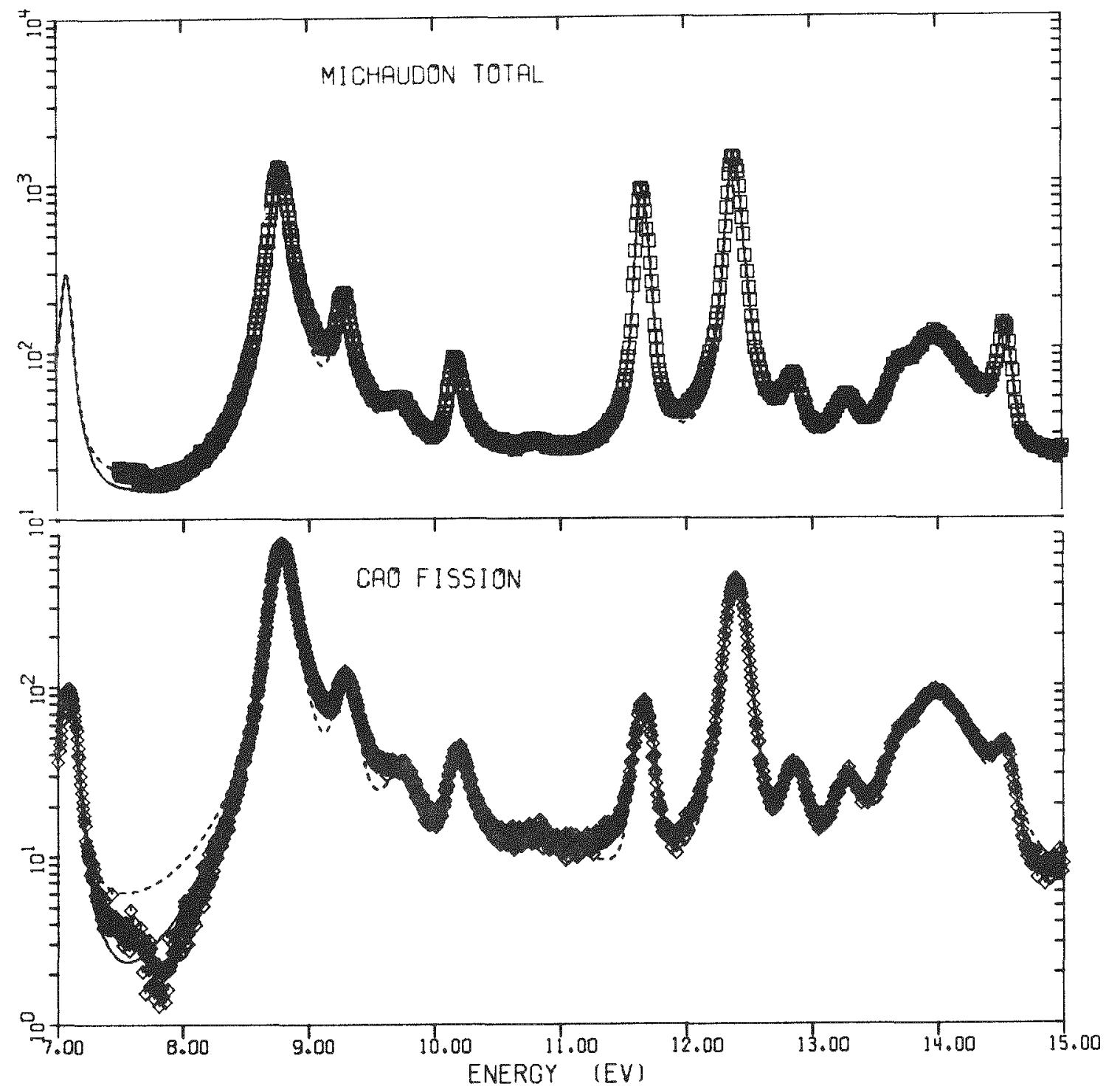

Fig. 2(A) Cross Sections of U-235 from 7 to $15 \mathrm{eV}$. In Figure 2(A) are shown the total cross section data of Michaudon (2) and the fission data. of Cao (4). In Figure $2(B)$ are the fission and capture data of DeSaussure et al., (I). In all cases the dashed curves represent calcul ttions using ENDF/B, Version-III parameters, with the solid line showing the cross sections including both resonances and smooth file (File 3). 


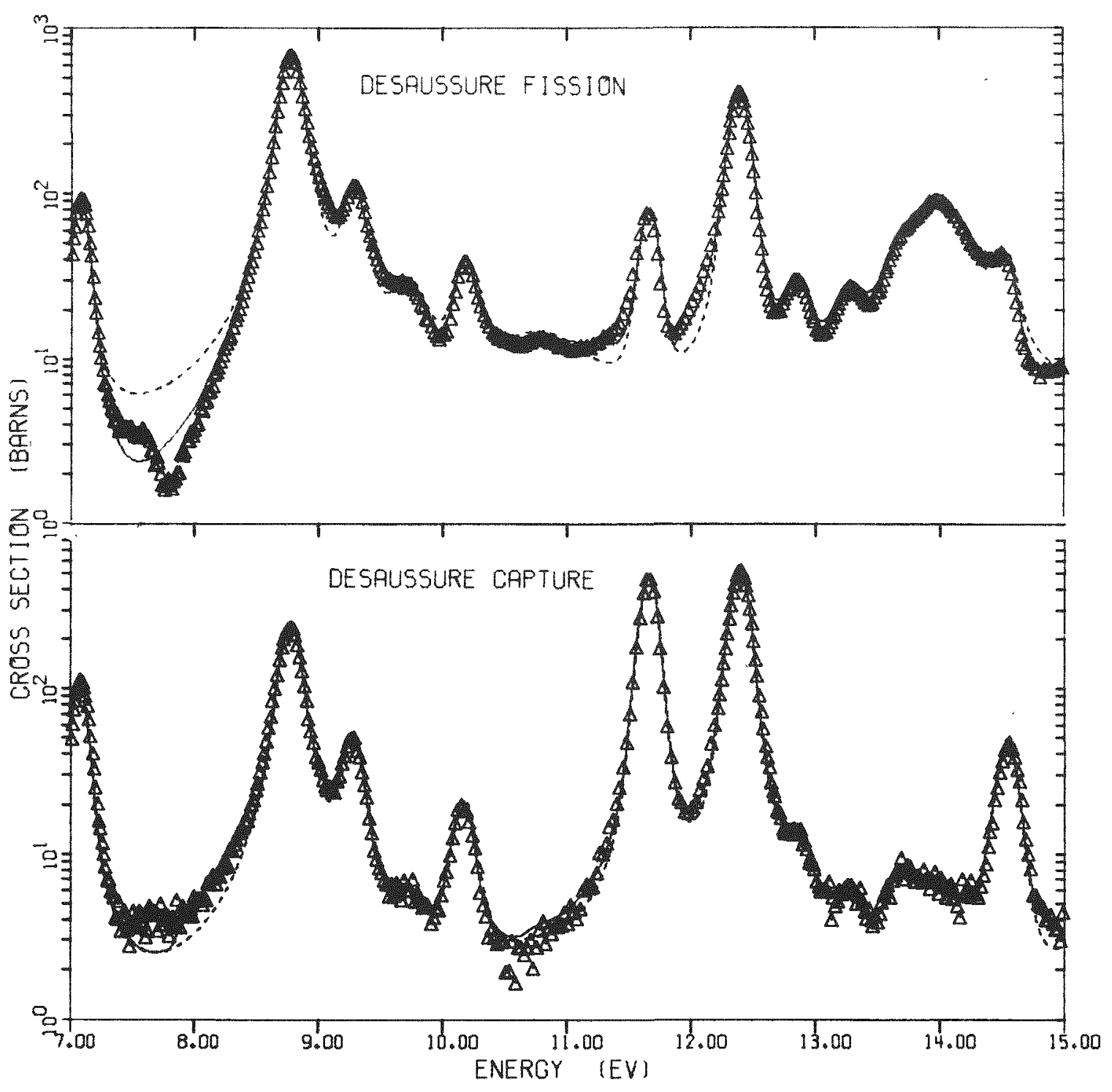

Fig. 2(B) 


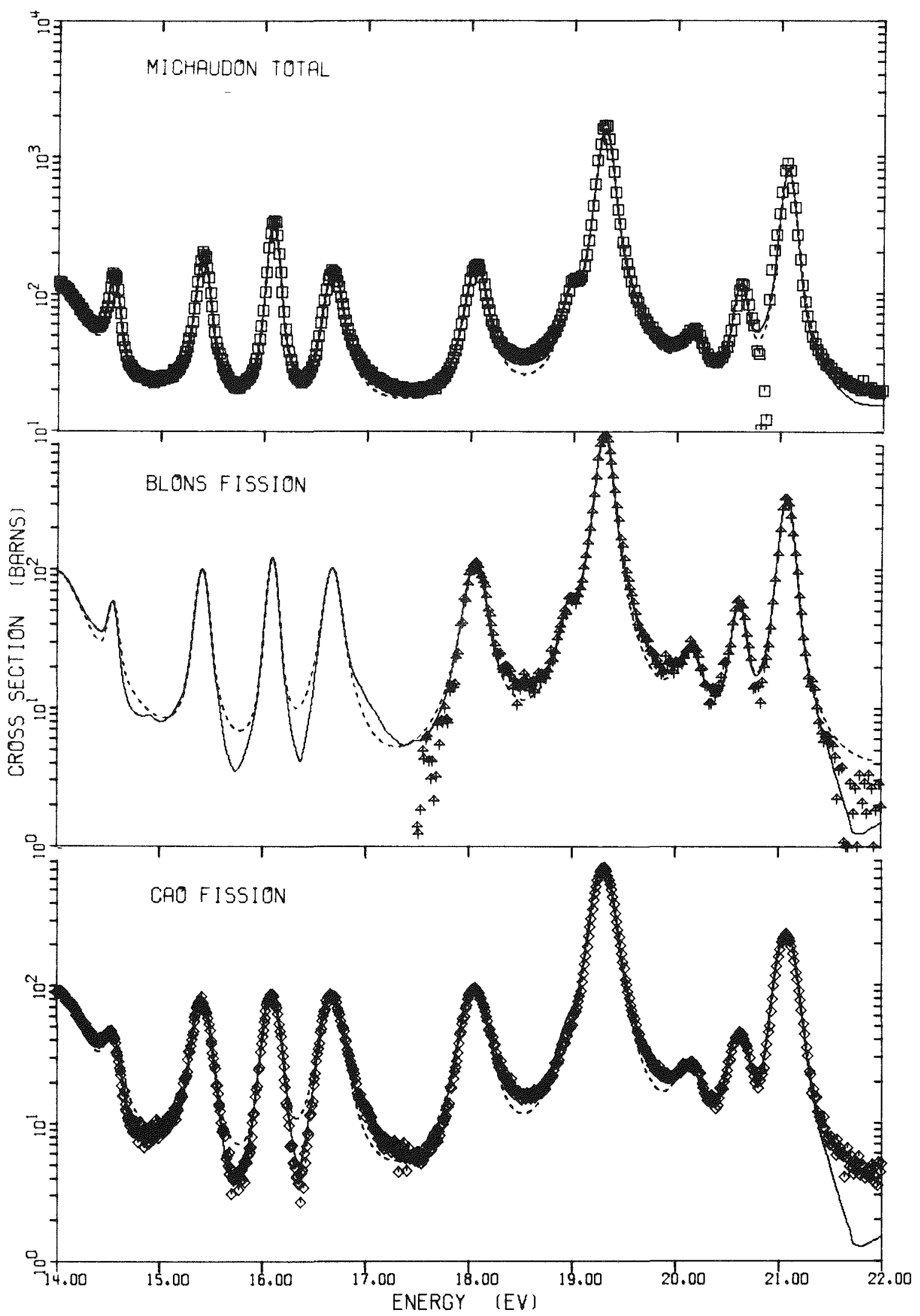

Fig. 3(A) 


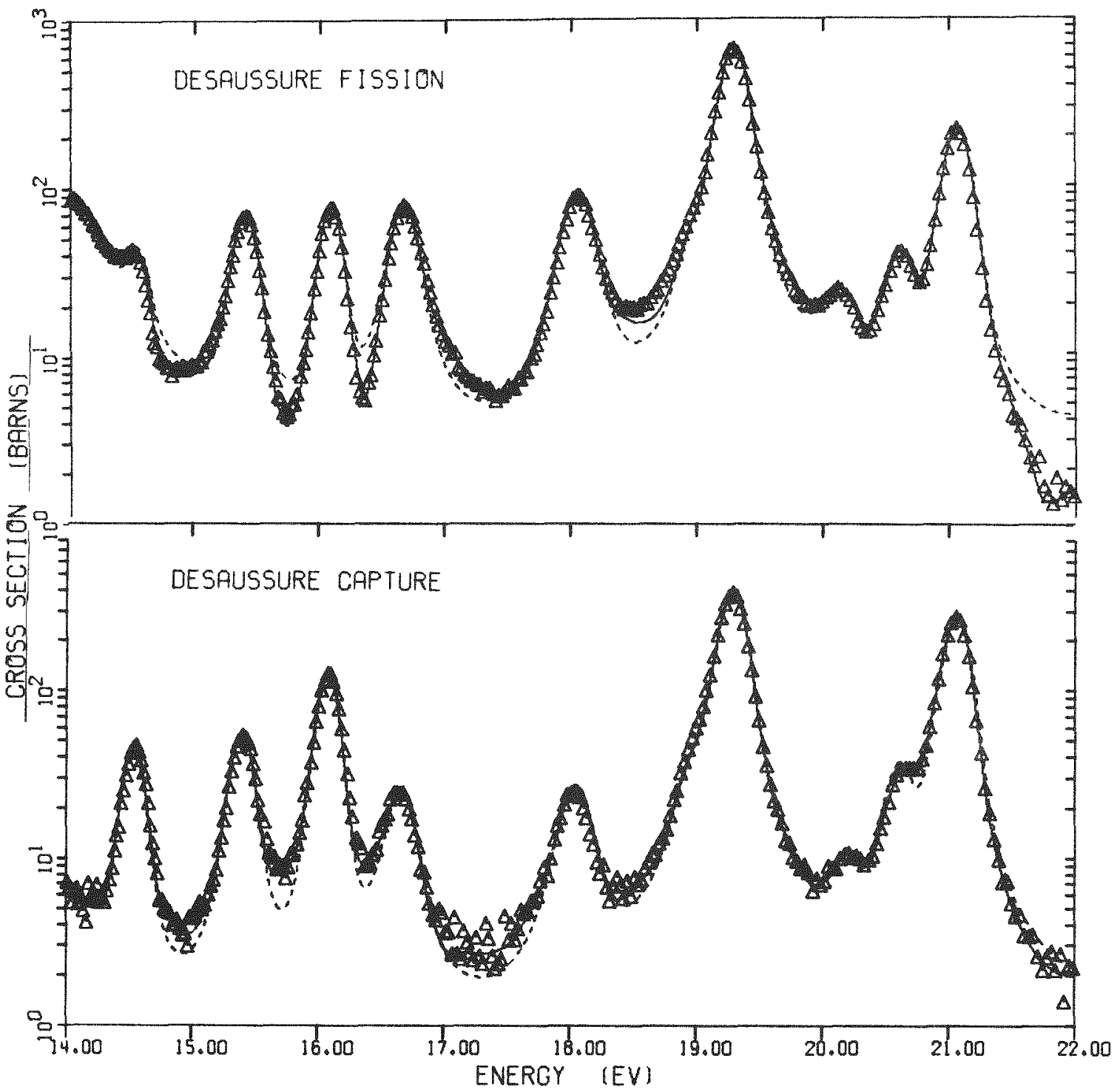

Fig. 3(B) Cross sections of U-235 from 14 to $22 \mathrm{eV}$. In Figure 3(A) are shown the total cross section data of Michaudon (2) and the fission data of Blons (3) and Cao (4). In Figure $3(B)$ are the fission and capture data of DeSaussure et al., (1). In all cases the dashed curves represent calculations using ENDF/B, Version-III parameters, with the solid line showing the cross sections including both resonances and smooth file (File 3 ). 


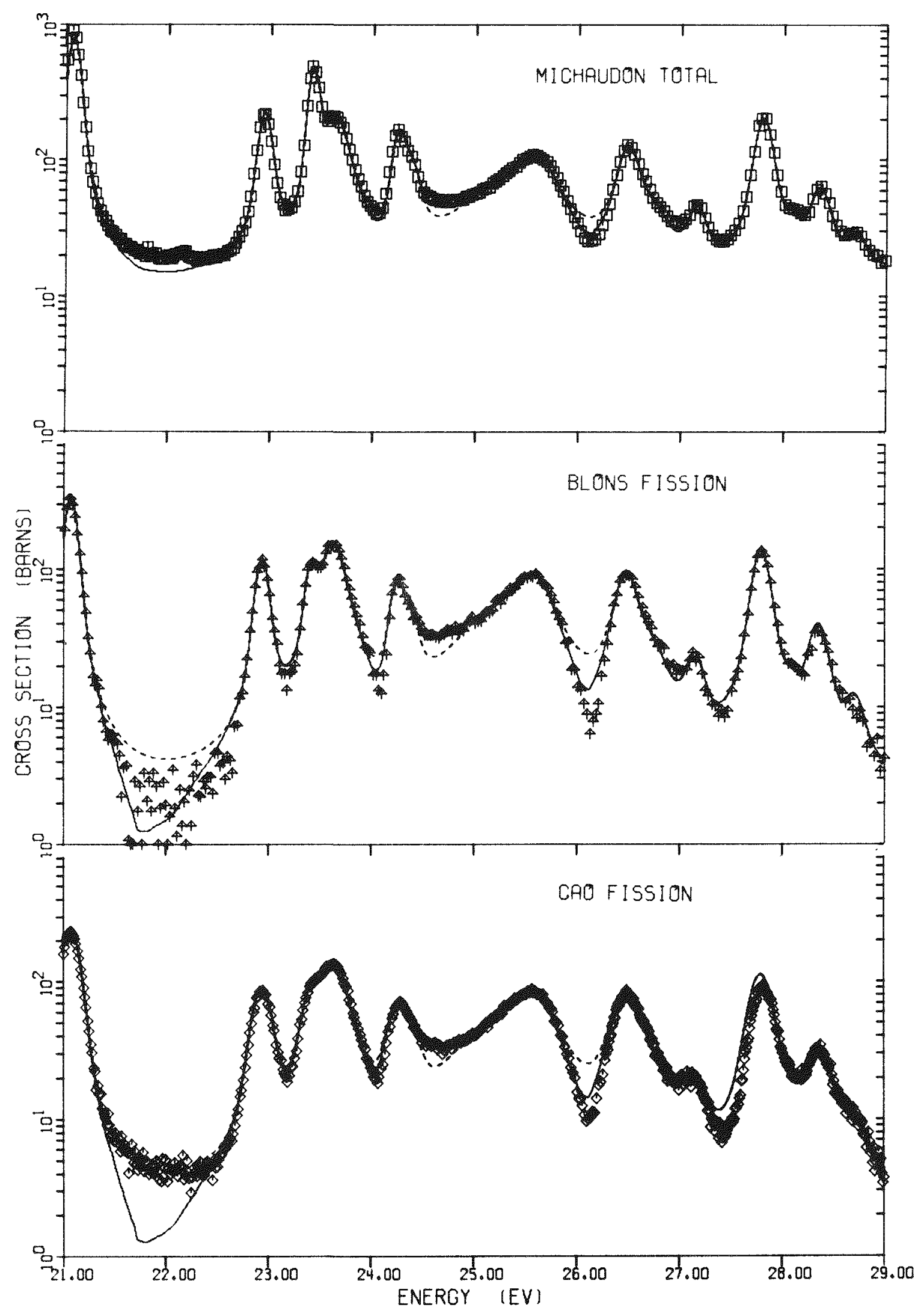

Fig. 4(A) 


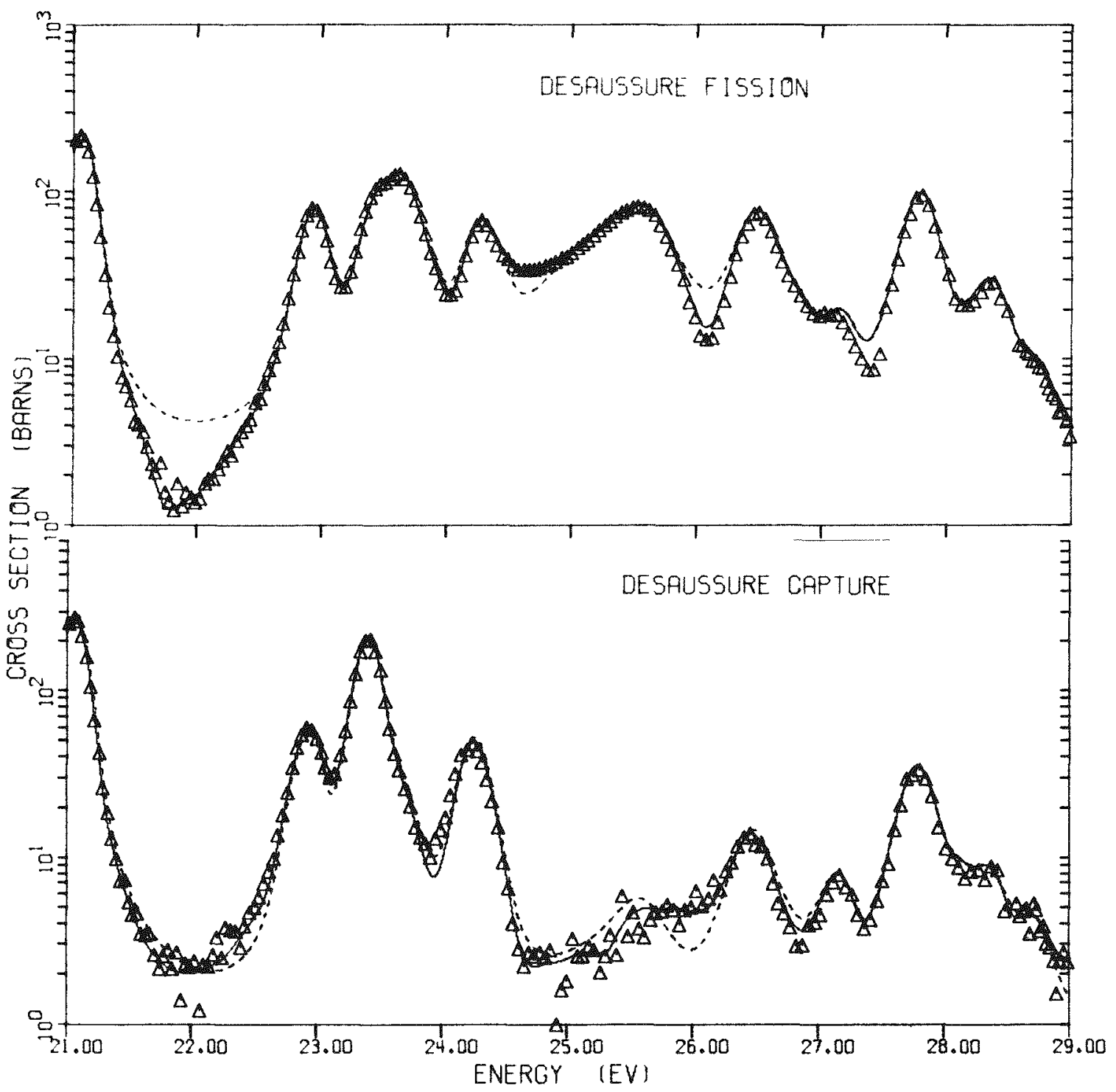

Fig. 4(B) Cross sections of U-235 from 21 to $29 \mathrm{eV}$. In Figure 4(A) are shown the total cross section data of Michaudon (2) and the fission data of Blons (3) and Cao (4). In Figure 4(B) are the fission and capture data of DeSaussure et al., (I). In all cases the dashed curves represent calculations using ENDF/B, Version-III parameters, with the solid line showing the cross section including both resonance and smooth file (File 3 ). 


\section{REVISION OF THE LOW ENERGY ENDF/B FIIES FOR ${ }^{244} \mathrm{Cm}$}

J. R. Berreth, R. A. Grimesey

Detailed, energy-dependent cross sections of the transplutonium nuclides are needed for accurate prediction of reaction rates in AEC production reactors. Precise information on $244 \mathrm{~cm}$ neutron cross sections is important because this isotope is a major link in the production chain leading to heavier nucljides having practical or scientific usefulness. New measurements [1,2,3] on ${ }^{244} \mathrm{Cm}$, both at low energies and in the resonance region, have expanded the available neutron cross section data. Using this data, revisions to the ENDF/B (Evaluated Nuclear Data File-B) have been prepared.

These measurements bring up to date the low energy evaluation of ${ }^{244} \mathrm{~cm}$ based on direct low energy measurements and new and additional determinations of resonance parameters. New resonance parameters are presented up to $500 \mathrm{eV}$ (see Table I). Low energy resonances up to and including $85.8 \mathrm{eV}$ are a composite of three sets of data, Cote
et al.LI], M. S. Moore et al.[2], and J. R. Berreth and F. B. Simpson $[3]$. The data of Berreth and Coté are total neutron cross sections. The Moore data are capture and fission cross section measurements. The fission component for the $16.8 \mathrm{eV}$ resonance is an indirect determination by Moore based on comparing their capture and fission measurements. The energy of this resonance is at the lower energy range of their measurements and, therefore, subject to some exror in the fission parameters. Since no fission measurement was available, a fission component for the $7.66 \mathrm{eV}$ resonance was used based on the average fission parameters of several large low energy resonances. Because the resonance parameters do not account for all of the low energy cross section as measured, a negative energy resonance is postulated to make up the difference between the theoretical curve as determined from the resonance parameters and the measured data below $I \mathrm{eV}$. Conditions for postulating the negative energy resonance were as follows: all of the measured resonances to $500 \mathrm{eV}$ were reflected as bound states around the $7.66 \mathrm{eV}$ resonance in order to arrive at a scattering cross section. Parameters for an assumed negative energy resonance at $-1.48 \mathrm{eV}$ were then adjusted until a fit to the total cross section data between 0.01 and $1.0 \mathrm{eV}$ was achieved. A scattering cross section bias was also determined and necessary up to $150 \mathrm{eV}$ to make the parameters, as presented, compatible with the data. The revised cross sections determined from the above information at $0.0253 \mathrm{eV}$ are $\sigma_{\text {tot }}=23.56$ barns, $\sigma_{\mathrm{sc}}=8.40$ barns, $\sigma_{\mathrm{n \gamma}}=14.28$ barns, and $\sigma_{\text {fiss }}=0.88$ barns. These total neutron cross sections data have been determined using the single-level Breit-Wigner formula. This revision should be a marked improvement because of the much more detailed data available and should enhance the accuracy of calculations involving production and use of transplutonium isotopes.

[1] R. E. Coté, R. F. Barnes and H. Diamond, Total Neutron Cross Section of ${ }^{244} \mathrm{Cm}$ ", Phys. Rev. Vol. 134, No. 6B, pp. 1281-1284 (June 22, 1964). 
[2] M. S. Moore and G. A. Keyworth, "Analysis of the Fission and Capture Cross Sections of the Curium Isotopes", Phys. Rev. C, Vol. 3, No. 4, pp. 1656-1667 (April 1971).

[3] J. R. Berreth and F. B. Simpson, Total Neutron Cross Sections of the $\mathrm{Cm}$ Isotopes from 0.01 to $30 \mathrm{eV}$ (to be published).

TABLE I

${ }^{244} \mathrm{Cm}$ RESONANCE PARAMETERS USED FOR ENDF/B REVISION

\begin{tabular}{|c|c|c|c|}
\hline $\begin{array}{l}\text { Energy } \\
\mathrm{eV}\end{array}$ & $\begin{array}{c}2 \mathrm{gI}_{\mathrm{n}} \\
\mathrm{mV} \\
\end{array}$ & $\begin{array}{r}\Gamma_{Y} \\
m V \\
\end{array}$ & $\begin{array}{l}\Gamma_{f} \\
m V \\
\end{array}$ \\
\hline-1.480 & 0.0685 & 37.000 & 2.100 \\
\hline 7.660 & 3.5700 & 37.000 & 2.100 \\
\hline 16.800 & 0.4000 & 37.000 & 1.400 \\
\hline 22.864 & 0.1840 & 37.000 & 3.700 \\
\hline 34.900 & 0.5900 & 37.000 & 2.500 \\
\hline 52.670 & 0.0780 & 37.000 & 1.700 \\
\hline 69.900 & 0.0720 & 37.000 & 3.000 \\
\hline 85.800 & 0.3900 & 37.000 & 0.650 \\
\hline 96.120 & 0.7450 & 37.000 & 1.540 \\
\hline 132.800 & 1.3500 & 37.000 & 1.170 \\
\hline 139.100 & 0.2120 & 37.000 & 2.800 \\
\hline 171.200 & 0.2360 & 37.000 & 1.300 \\
\hline 181.600 & 0.7240 & 37.000 & 2.100 \\
\hline 197.000 & 3.0600 & 37.000 & 1.000 \\
\hline 209.800 & 2.9000 & 37.000 & 0.520 \\
\hline 220.100 & 3.6400 & 37.000 & 1.250 \\
\hline 230.500 & 1.9800 & 37.000 & 0.400 \\
\hline 234.900 & 0.2500 & 37.000 & 0.900 \\
\hline 242.700 & 0.0830 & 37.000 & 2.200 \\
\hline 264.900 & 0.6150 & 37.000 & 0.900 \\
\hline 274.100 & 0.9670 & 37.000 & 0.800 \\
\hline 316.800 & 0.3090 & 37.000 & 0.300 \\
\hline 329.500 & 0.3630 & 37.000 & 0.290 \\
\hline 343.600 & 1.4000 & 37.000 & 1.160 \\
\hline 353.100 & 5.3800 & 37.000 & 1.280 \\
\hline 361.700 & 1.7900 & 37.000 & 1.030 \\
\hline 364.400 & 0.5240 & 37.000 & 2.100 \\
\hline 386.200 & 1. 3200 & 37.000 & 1.110 \\
\hline 397.600 & 1.1500 & 37.000 & 0.660 \\
\hline 415.000 & 0.9300 & 37.000 & 0.270 \\
\hline 420.600 & 4.5400 & 37.000 & 0.890 \\
\hline 426.900 & 0.6200 & 37.000 & 0.350 \\
\hline 443.400 & 4.8000 & 37.000 & 0.820 \\
\hline 470.900 & 7.6900 & 37.000 & 1.840 \\
\hline 488.900 & 0.6780 & 37.000 & 0.500 \\
\hline 491.900 & 2.4300 & 37.000 & 0.470 \\
\hline
\end{tabular}




\section{PU-242 RESONANCE PARAMETERS FOR ENDF/B-III}

$$
\text { T. E. Young, R. A. Grimesey }
$$

A new evaluation for $\mathrm{Pu}-242$ in the resolved resonance range and thermal energy region has been computed and submitted to BNI for Version III of ENDF/B. The ENDF/B is a file of the most current evaluated cross section data, a file which has become the standard for reactor calculations throughout the nation. Resolved resonance parameters for resonances below $390 \mathrm{eV}$ and all thermal energy cross sections were revised. No changes were made in the cross section values above $390 \mathrm{eV}$.

The total neutron cross section below $1 \mathrm{eV}$ was taken from References I and 2. The elastic scattering cross section at $0.0253 \mathrm{eV}$ was calculated to be $8.4 \mathrm{~b}$ by using a potential scattering cross section of $10.7 \mathrm{~b}$ and the parameters of the $2.68 \mathrm{eV}$ resonance only. A single bound level was selected which, when used in combination with the resolved resonances below $390 \mathrm{eV}$, also predicted a thermal scattering cross section of $8.4 \mathrm{~b}$.

The total, absorption, and elastic scattering cross section values in Table I were calculated from the resonance parameters in Table II using the single-level formula and $10.7 \mathrm{~b}$ potential scattering. Parameters for resonances above $20 \mathrm{eV}$ were obtained by averaging values from References 1-6, and those of the $2.68 \mathrm{eV}$ resonance were taken from Reference 1 .

Cross section values obtained at $0.0253 \mathrm{eV}$ were $26.9 \pm 1 \mathrm{~b}$ for the total, $18.5 \pm 1 \mathrm{~b}$ for the absorption, and $8.4 \pm 1 \mathrm{~b}$ for the scattering.

[1] T. E. Young, and S. D. Reeder, Nucl. Sci. Engr. 40, 389 (1970)

[2] T. E. Young, et. al., Mucl. Sci. Engr., 43, 341 (1971)

[3] R. E. Cote, et. al., Phys. Rev. 114, 505 (1959)

[4] P. A. Egelstaff, et. al., J. Nuclear Energy, 6, 303 (1958)

[5] N. Pattenden, EANDC-50-S (1965)

[6] G. Anchampangh, et. al., Phys. Rev. 146, 840 (I966). 
Table I

Thermal Energy Region Cross Sections of Pu-242

\begin{tabular}{lccc}
\hline $\begin{array}{l}\text { Energy } \\
(\mathrm{eV})\end{array}$ & $\begin{array}{c}\sigma_{\mathrm{T}} \\
(\mathrm{b})\end{array}$ & $\begin{array}{c}\sigma_{\mathrm{abs}} \\
(\mathrm{b})\end{array}$ & $\begin{array}{c}\sigma_{\mathrm{el}} \\
(\mathrm{b})\end{array}$ \\
\cline { 2 - 4 } 0.00001 & 960 & 951.6 & 8.412 \\
0.0001 & 297. & 288.8 & 8.404 \\
0.0005 & 139. & 130.6 & 8.403 \\
0.001 & 99.77 & 91.37 & 8.403 \\
0.002 & 73.06 & 64.66 & 8.402 \\
0.004 & 54.18 & 45.78 & 8.401 \\
0.007 & 43.08 & 34.68 & 8.398 \\
0.010 & 37.48 & 29.88 & 8.396 \\
0.017 & 30.81 & 22.42 & 8.390 \\
0.0253 & 26.87 & 18.48 & 8.383 \\
0.040 & 23.23 & 14.86 & 8.370 \\
0.060 & 20.66 & 12.31 & 8.353 \\
0.080 & 19.15 & 10.82 & 8.335 \\
0.10 & 18.14 & 9.821 & 8.318 \\
0.15 & 16.60 & 8.326 & 8.272 \\
0.20 & 15.72 & 7.493 & 8.225 \\
0.30 & 14.75 & 6.624 & 8.125 \\
0.45 & 14.10 & 6.135 & 7.961 \\
0.60 & 13.86 & 6.083 & 7.776 \\
0.80 & 13.91 & 6.418 & 7.489 \\
0.90 & 14.06 & 6.735 & 7.324 \\
1.00 & 14.30 & 7.157 & 7.143 \\
& & & \\
\hline
\end{tabular}


Table II

Resonance Parameters of $\mathrm{Pu}-242$

\begin{tabular}{rrr}
$\begin{array}{c}E_{\mathrm{O}} \\
(\mathrm{eV})\end{array}$ & $\begin{array}{c}\Gamma_{\mathrm{n}}{ }^{\mathrm{O}} \\
(\mathrm{MeV})\end{array}$ & $\begin{array}{c}\Gamma_{\gamma} \\
(\mathrm{MeV})\end{array}$ \\
\cline { 2 - 3 }-70.000 & $\begin{array}{r}19.0000 \\
1.2200\end{array}$ & 25.600 \\
2.680 & 0.0632 & 25.000 \\
22.540 & 0.0703 & 25.600 \\
40.930 & 6.9320 & 25.600 \\
53.700 & 0.5090 & 25.600 \\
68.000 & 1.9180 & 25.600 \\
89.100 & 0.0580 & 25.600 \\
106.000 & 1.7260 & 25.600 \\
107.500 & 0.5490 & 25.600 \\
131.800 & 1.3880 & 25.600 \\
150.000 & 0.0776 & 25.600 \\
166.000 & 4.5750 & 25.600 \\
205.000 & 0.3290 & 25.600 \\
217.000 & 0.5150 & 25.600 \\
235.000 & 0.8760 & 25.600 \\
276.000 & 0.8750 & 25.600 \\
306.000 & 0.6240 & 25.600 \\
311.000 & 12.7420 & 25.600 \\
323.000 & 5.1630 & 25.600 \\
335.000 & 2.4070 & 25.600 \\
386.000 & & 25.600 \\
& & \\
\hline
\end{tabular}


REACTOR PHYSICS CODE MODIFICATIONS FOR ENDF/B CROSS SECTION DATA

R. A. Grimesey, G. L. Singer $[1]$

This report describes improvements in the codes used by Aerojet Nuclear Company to process ENDF/B data. The purpose is to produce from the ENDF/B data multigroup cross sections for use in reactor analys is codes.

The processing code which forms the link between the ENDF/B fast data files and the reactor physics analysis codes currently in use at Aerojet Nuclear Company is ETOP[2]. ETOP is a modification of the ETOG[3] code developed by Westinghouse Nuclear Energy Systems to fit the GAM code theory and formats. Extensive modifications of ETOP have occurred during this fiscal year to meet the changing requirements of the ENDF/B data and formats. A completely new unresolved resonance subroutine was written for ETOP and PHROG[4] so that PHROG is now totally compatible for data from single isotopes in the ENDF/B format. The only case where ETOP preprocesses ENDF/B capture and fission data in the unresolved resonance range is for the case where several resonant isotopes are present in a single material.

A new subroutine was written to process multigroup elastic scattering matrices in the range of isotropic scattering in the center of mass system. This subroutine is based on the fact that a completely analytical result exists for the column sum of the elastic scattering matrix for any order of the Legendre moment in the isotropic scattering range. For the fissile and fertile elements, the structure in the resolved resonance range made the calculation of the within-group scattering term very laborious by straightforward numerical techniques. This new subroutine alleviated this difficulty and substantially improved the accuracy of the matrix calculation.

The complex structure of the ENDF/B II chromium, iron and nickel files in the resolved resonance range required extensive modification of the mesh allocation for accurate numerical integration. Each of these isotopes possess resolved resonance structure to approximately $1 \mathrm{MeV}$ and 250 or more resolved $\mathrm{s}$ and $\mathrm{p}$ wave resonances. These conditions exceeded the limits for numerical accuracy for which the original version of the code was designed. The present version of ETOP is being extended to treat up to $250 \mathrm{~s}$ wave and $500 \mathrm{p}$ wave resonances with accuracy for a single material. The $p$ wave resonances of a given material will be integrated by means of an analytical prescription to speed processing time.

Several approximations to the single-level Breit Wigner resonance formulas used in ETOG and subsequently in ETOP for $s$ wave resonances which were valid at low energy generated excessive errors in the keV region resonances of the stainless steel materials. In particular, the small angle approximation for $\sin ^{2} \phi_{\ell}$ is invalid above $100 \mathrm{keV}$ and caused excessive errors in the elastic scattering matrices for these isotopes. Even more serious errors occurred due to the assumption that $\cos 2 \phi_{\ell}$ and $\sin 2 \phi_{\ell}$ were approximately $I$ and 0 , respectively, and could be 
omitted from the calculations. These approximation have been eliminated and a more accurate form of the single-level resolved resonance equations substituted.

Excessive computation time resulted in the original version of ETOG due to resonance wing calculations being performed over the same mesh generated for the principal contribution from adjacent resonances. The program was modified so that all wing calculations, other than where the I/V capture tail is significant, are performed over a separate mesh. This change alone will reduce the computation time in the resonance subroutine by a factor of about 20 .

The inability to shield elastic scattering matrices in PHROG[4] in the resolved and unresolved resonance range is a serious limitation to PHROG for fast reactor calculations. A new elastic scattering subroutine has been outlined for PHROG where shielded scattering matrices will be calculated directly in the resonance ranges. The corresponding subroutines are being written for ETOP so PHROG will be compatible with ENDF/B elastic scattering data.

A program CSURE[5] was written to do shielded unresolved resonance calculations over an arbitrary energy mesh for ENDF/B materials. The program will read input from an INDF/B tape or from card input. This program will permit fitting unresolved range resonance parameters to experimental data when performing new evaluations. The program calculates capture, fission, total and elastic scattering pointwise cross sections and adds in any smooth background contribution. The Program SIGMA-2 was obtained from Brookhaven and converted to the IBM-350 for our usage. SIGMA-2 consisted essentially of resolved resonance equations only with practically no input or output capabilities. An extensive input-output capability was written around the SIGMA-2 code, and the new program was called SIGMA-R. SIGMA-R will produce Doppler and resolution broadened resolved resonance cross sections from single-level or multilevel Breit Wigner resonance parameters in the resolved resonance range. The program will produce pointwise cross sections for a selected mesh and will read data directly from an ENDF/B tape or accept parameters from card input.

[1] Member, Computer Science Branch, Technical Services Division, Aerojet Nuclear Company .

[2] G. L. Singer and R. A. Grimesey, ETOP, A FORTRAN-IV Program to Process Data from the ENDF/B File to the PHROG Library, (unpublished).

[3] D. E. Kusner, R. A. Dannels and S. Kellman, ETOG-I, A FORTRAN-IV Program to Process Data from the ENDF/B File to MUFT, GAM and ANISN Formats, WCAP-3845-1, ENDF 144 (December 1969).

[4] R. L. Curtis, G. I. Singer, F. J. Wheeler and R. A. Grimesey, PHROG A FORTRAN-IV Program to Generate Fast Neutron Spectra and Average MuItigroup Constants, IN-1435 (Apri1 1971).

[5] G. L. Singer and R. A. Grimesey, CSURE Cross Sections for Unresolved Resonances from ENDF/B, (to be published). 
FAST CRITICAL BENCHMARK CALCULATIONS USING ENDF/B VERSION II DATA

A. W. Brown, R. A. Grimesey

One important aspect of the evaluation and compilation of neutron cross section data for the ENDF/B data files is testing the data against fast integral experiments once it is compiled and ready for general usage. Data testing against integral experiments can point up any or all of three basic inconsistencies.

1. Errors of judgment in the evaluation of the basic cross section sets.

2. Analytic and numerical errors generated by cross section processing codes to prepare the data for multigroup reactor codes.

3. Discrepancies in integral parameters calculated due to transport theory approximations and alterations of the basic data in reactor analysis codes.

Each of these three aspects of data testing must be considered when setting up benchmark tests for nuclear data.

The Data Testing Subcommittee of the Cross Section Evaluation Working Group (CSEWG) specified 10 fast reactor critical experiments to be calculated by participating members of CSEWG for Version II of the ENDF/B data. The 10 critical experiments ranged from very soft to very hard reactor spectrums. Each member lab of CSEWG was assigned 5 benchmarks with sufficient overlap so each experiment would be calculated by at least 3 different labs and sufficient intercomparisons could be made. Idaho Nuclear Corporation calculated the 5 assemblies, Vera IB, ZPR III Assy 48, APR III Assy 1I, Zebra Core 2 and Zebra Core 3.

The parameters calculated for comparison with experiment and intercomparison between various labs were: Microscopic multigroup constants for selected isotopes in the core and blanket, eigenvalues, fission and capture ratios relative to U-235, Central reactivity worths and 26 group flux and adjoint spectra at the core center.

After all results were reported, a task force met at Brookhaven to assess the results and make recommendations for specific data changes which would yield substantial improvements for the calculation of fast reactor parameters. Their recommendations led to substantial data modifications of the Version II ENDF/B isotopes U-235, U-238, and Pu-239. These data changes resulted in significant improvement for eigenvalues and fission and capture ratios among the ten CSEWG benchmarks especially for the soft spectrum low enriched assemblies.

In adition to the data changes resulting from these integral tests of the ENDF/B data, a number of modifications have been indicated necessary for the processing codes. The PHROG(I) fast spectrum code developed by Aerojet Nuclear Company suffers from the inability to self-shield the multigroup elastic scattering matrices in the resolved and unresolved resonance 
ranges. This deficiency was apparent after intercomparison of the CSEWG benchmarks involving the low enriched assemblies. In adition, it will be necessary to make the elastic scattering contribution of diluent moderators energy dependent in PHROG to account adequately for mutual shielding in future fast reactor computations.

The maintenance and updating of the ENDF/B nuclear data files is a continuing cooperative task for the various laboratories participating in this national effort. Data testing will continue with selected benchmarks aimed at specific isotopes over specified energy ranges. Version III of ENDF/B will become available in the fall of 1971. Aerojet Nuclear Company is presently participating at all levels in this national program. 


\section{MONTE CARLO DEVELOPMENT}

F. J. Wheeler, W. E. Vesely[I], R. S. Marsden $[I]$

The RAFFLE Monte Carlo code ${ }^{[2]}$ was developed here to provide for making accurate reactor physics calculations under conditions where diffusion and transport methods cannot be used or require approximations of uncertain validity. The program solves iteration type problems to obtain the multiplication factors, normal mode fluxes and reaction rates, or it can be used to solve external source problems. The external sources may be distributed volume sources of any shape or may be incoming current sources specified on particular surfaces; the current sources may have any energy and angular distributions. For external source problems, subsequent generated fission daughters can be followed.

The report[2] concerns further developments in RAFFLE capability. Correlation techniques have been applied to compute cell cross sections directly with Monte Carlo. In addition to the scalar absorption, nufission and scatter cross sections, the entire Po and $P_{I}$ transfer matrix can be obtained. This technique is advantageous since no simplifying assumptions are needed to compute the few group or multigroup constants. Also, for many classes of problems the required computer time is comparable to that required by other, less exact, methods. A paper[3] on this technique was presented at the ANS topical meeting held in Idaho Falls in March 1971.

Another addition to the code was the inclusion of upscatter in the scattering routines, allowing a multigroup treatment in the thermal range. Scattering in the thermal groups is described by a full Po and $P_{1}$ matrix in the lab system. Use of the multithermal option in RAFFLE is very simple since all cross sections and transfer matrices can be obtained as punched card output from INCITE[4]. By taking advantage of the properties of thermal scatter the programming was done so that a multithermal treatment requires less machine time than the old one-thermal-group treatment.

[I] Member, Computer Science Branch, Technical Services Division, Aerojet Nuclear Company.

[2] W. E. Vesely, F. J. Wheeler and R. S. Marsden, The RAFFLE Monte Carlo Code and Its Use to Obtain Correlated Estimates of Collapsed Cross Sections, (paper presented at MA \& MC Topical ANS Spring 1971 Meeting, Idaho Falis, Idaho).

[4] R. I. Curtis and R. A. Grimesey, INCITE, A FORTRAN-IV Program to Generate Thermal Neutron Spectra and Multigroup Constants Using Arbitrary Scattering Kernels, IN-106? (November 1967). 
MONTE CARLO CALCULATIONS FOR A FUEL PIN CELL

A. J. Scott, E. Fast, F. J. Wheeler

The RAFFLE code has been developed as a fast, less costly method of performing Monte Carlo reactor physics calculations. It is an improvement over existing codes in that it contains various scoring techniques to calculate reactor physics parameters. The code is especially useful for Monte Carlo calculations in small regions, such as for a LOFT-I fuel pin cell. Such calculations are not merely of engineering value but also are a direct check of the accuracy of the basic reactor physics methods and cross section data that were used in the preliminary design of LOFT-I[1]. (The preliminary design methods are described in Reference 1.) The Monte Carlo calculations done with the RAFFLE code [2] solved for the neutron flux over the full energy range $(O \rightarrow I 0 \mathrm{MeV})$ using ENDF/B (Version II) data. However, the neutron absorption predicted by RAFFLE in the epithermal resonance region was not in good agreement with the preliminary design results. To verify the accuracy of the 99-group RAFFLE cross section library, a simple homogeneous, infinite medium calculation was made. When compared to the results of a PHRoG[3] calculation using the same ENDF/B data base, RAFFLE was found to predict the epithermal absorption in the resonance range about $20 \%$ higher than PHROG. Since it is known that the PHROG solution methods would not lead to errors this large, a "bug" was implied in the RAFFLE program. After some debugging runs, the error in RAFHLt was found to be due to the resonance scattering calculation. Subsequent correction of the program and recalculation of the LOFT fuel pin cell problem will be used to establish the base from which pertinent data, such as thermocouple and other instrumentation effects can be calculated for the LOFT core.

[1] E. C. Anderson, et al., Reactor Physics Analysis for the Preliminary Design of the 5-1/2 foot LOFT Core I, ANCR-I02I (to be published).

[2] W. E. Vesely, F. J. Wheelex, R. S. Marsden, The RAFFLE Monte Carlo Code, ANCR-1022 (to be published).

[3] R. I. Curtis, et aI., PHROG - A FORTRAI Program to Generate Fast Neutron Spectra and Average Multigroup Constants, IN-1435

(ApriI 1971). 
METHODS USED FOR PHYSICS ANALYSIS OF THE POWER BURST FACILITY AND COMPUTED RESULTS FOR THE DESIGN CORE

B. I. Rushton, W. R. Carpenter

The Power Burst Facility (PBF) was designed to allow testing nuclear fuels to melting and vaporization without endangering the driver core. The Reactor Physics Section, initially under Phillips Petroleum Company and finally under Idaho Nuclear Corporation, did all. of the physics analysis required from the initial feasibility studies to the finalized core design. A report has been published describing in detail the reactor physics techniques used and the computed results for the finalized core design $[1]$.

The physics techniques are interesting because of the nontypical features of the PBF which add to the complexity of the physics calculations. The nontypical features can be summarized as follows: the core is light-water moderated with a high (2.46) nonmoderator-to-moderator ratio. In addition to usual cladding material, there are calcia-stabilized zirconia insulators that further separate the fuel pellet from the surrounding moderator. There is a large central experiment hole and there are large cylindrical $\mathrm{B}_{4} \mathrm{C}$ rods that leave air ducts when withdrawn from the core. The fuel pellets are a terinary fuel composed of uranium-oxide and a zirconia-calcia diluent.

The calculational methods were tested by application to critical experiments and to operating reactors. Doppler coefficients measured under controlled conditions were calculated to evaluate ability to calculate Doppler feedback. In some cases methods based on diffusion theory were tested by comparison with calculations using more rigorous transport methods.

[1] B. L. Rushton and W. R. Carpenter, Methods Used for Physics Analysis of the Power Burst Facility and Computed Results for the Design Core, ANCR-1008 (JuIy 1971). 


\title{
LOFT CORE I PHYSICS METHODS VERIFICATION
}

\author{
B. L. Rushton
}

The Loss of Fluid Test (IOFT) which is one of the major reactor safety tests being conducted is in late design stage. As part of the design, accurate predictions of criticality and rod worth for clean oxide cores have been obtained with the calculational scheme used in PBF design(1). However, the model used in these calculations had not been tested for borated systems such as LOFT and had not been tested extensively for computing measured power distributions. Also, the rod worth verification reported in Reference 1 was for rods of different design than the cluster control assemblies used in LOFT. Therefore, it was necessary to test the model against experiments more closely resembling LOFT and to test the accuracy for predicting power distributions.

Data from a set of critical experiments performed by the Babcock and Wilcox Research and Development Division were used to test the physics techniques. The results obtained from the study were considered to be in good agreement with experiment. For the critical configurations having M/W ratios close to that of LOFT Core I, the calculated eigenvalues were 21.0\% high. The two cases studied indicated that the model used for representing the $\mathrm{B}_{4} \mathrm{C}$ poison rods overestimates their reactivity worth by about 10\%. Pyrex poison rods were accurately computed using the same method; however, the pyrex absorption cross section was much lower than that in the $\mathrm{B}_{4} \mathrm{C}$. The agreement between calculated and measured power distributions was quite good. The agreement was within 3\% for configurations having water holes and within 5\% for configurations that included pyrex rods.

The generally good agreement between experimental results and computed results show that the physics methods are adequate for preliminary design purposes. For purposes of final design work, there are areas where the calculational model should be improved to remove some of the approximations that are present in the preliminary design calculations. Complete details of the work that was done and of how it was done will be published in a forthcoming ANC report $(2)$.

[1] B. I. Rushton, "Physics Methods Used to Compute Reactivity, $\Lambda / \bar{\beta}$ eff" and Rod Worth for the PBF Critical Experiments," IDO-17276, (October 1968).

[2] E. C. Anderson, et. al., "Reactor Physics Analysis for the Preliminary Design of the 5.5 ft LOFT Core I," ANCR-I02I (to be published). 


\section{METALLOGRAPHY FOR LOFT}

B. G. Carlson

Assistance was given to the Nuclear Engineering and Analysis Branch in perfecting instrumentation fastening techniques for the LOFT program. Titanium sheathed thermocouples had to be fastened to $\mathrm{z} x-4$ tubing in a way that would minimize any change in mechanical properties of the tubing, yet be well bonded. Penetration and heat effect from the joining were measured metallographically and compared with the mechanical strength of the joint. Both spot and laser welds were used, the spot welding for straps and the laser for the junctions. These are shown in Figures 1 and 2.

Typical cross sections are shown in Figures 3 and 4 which show the shallow depth of the heat-affected zone in the $\mathrm{Zr}-4$.

Optimum joint strength consistent with minimum weld effect was attainable with laser welding but spot welding proved unuseable due to lack of strength at these minimum heat requirements. 


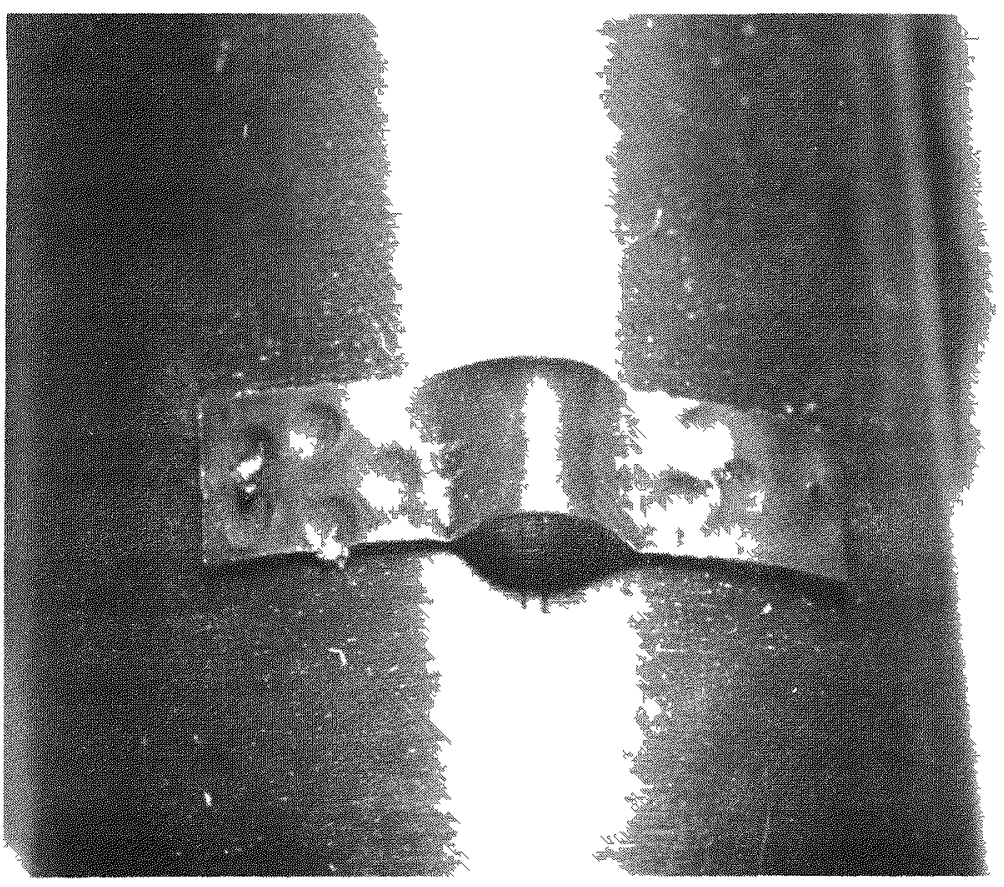

Fig. 1 Spot weld of $\mathrm{Ti}$ to $\mathrm{Zr}-4$ tube.

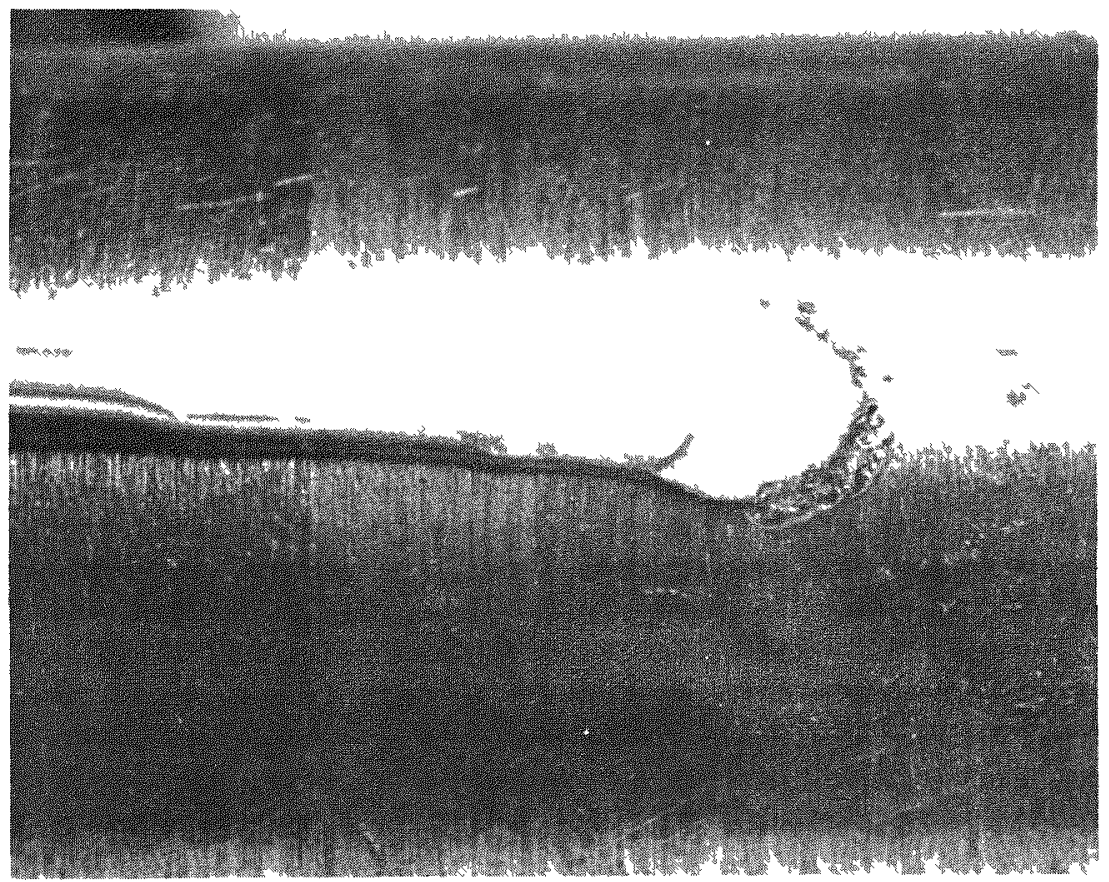

Fig. 2 Junction laser welded to $\mathrm{Zr}-4$ tube. 


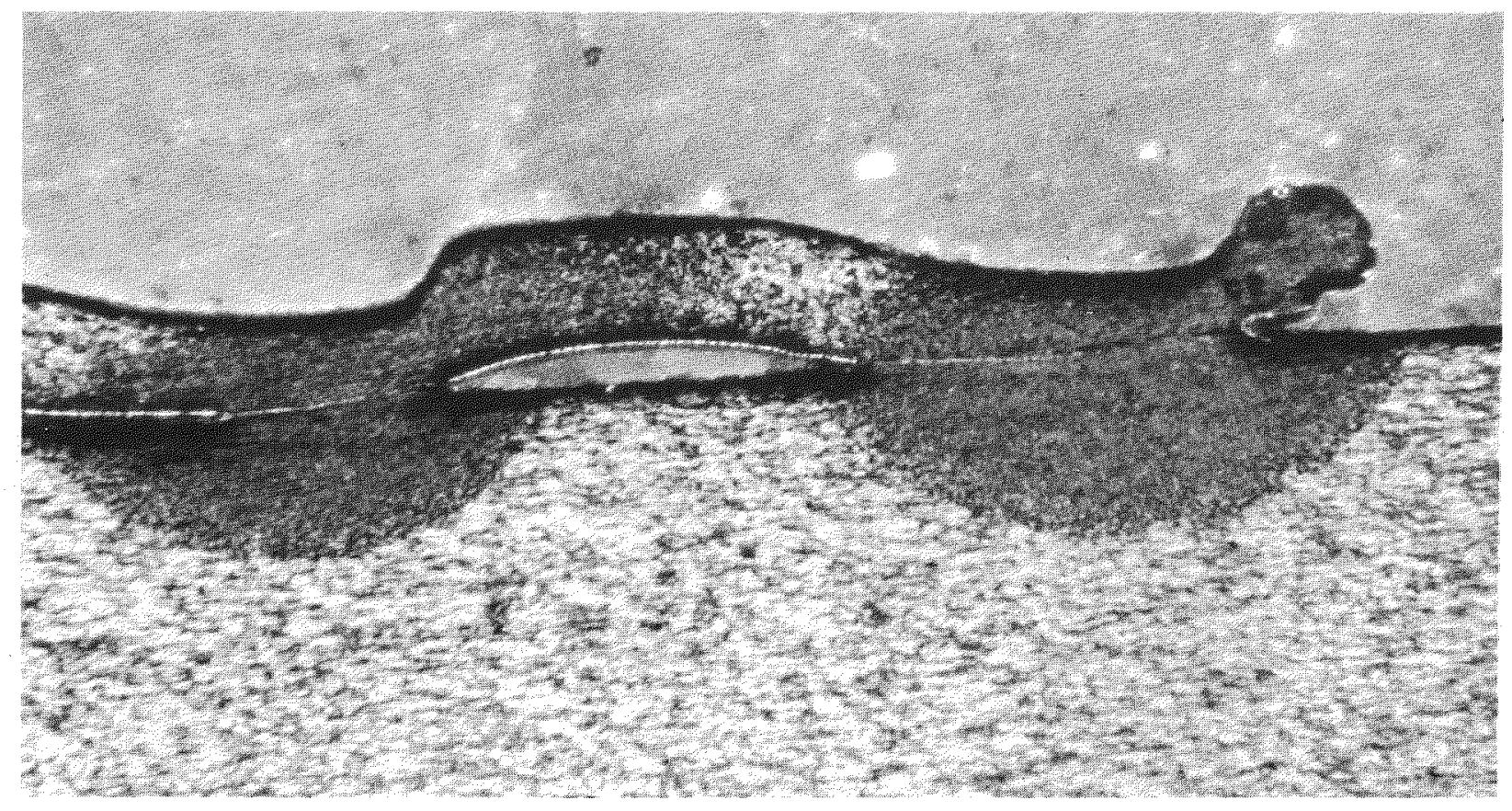

$\mathrm{HNO}_{3}+\mathrm{HF}$ Etch

$100 \mathrm{x}$

Fig. 3 Cross section through two spot welds.

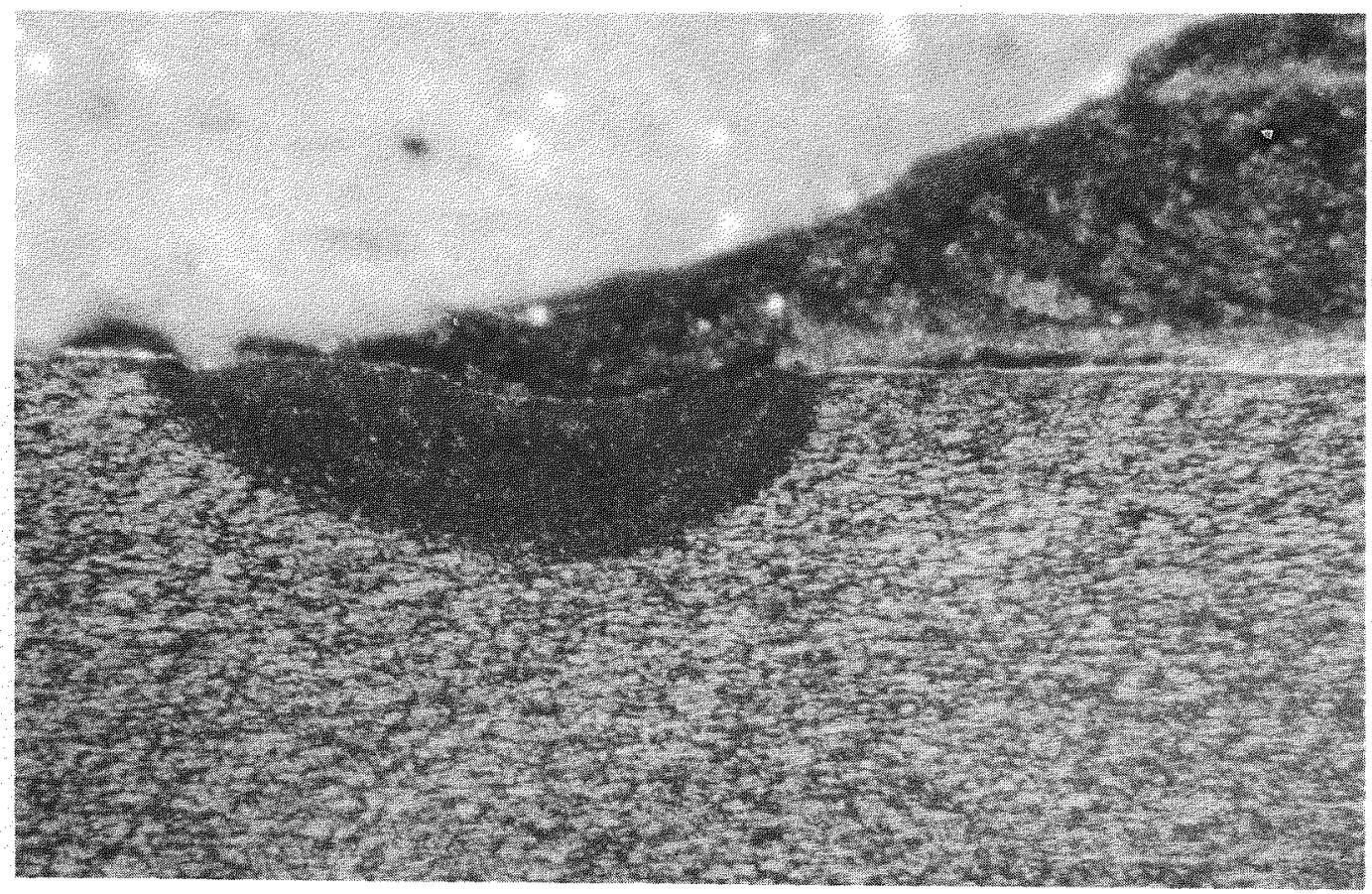

$\mathrm{HNO}_{3}+\mathrm{Hf}$ Etch

$100 \mathrm{X}$

Fig. 4 Titanium sheath laser welded to $\mathrm{Zr}-4$ tube. 
PLUTONIUM DETECTION IN BONE SECTIONS

$$
\text { L. G. Miller, W. S. S. Jee }{ }^{(1)}
$$

Prior to 1967, conventional nuclear emulsion photographic autoradiography was used to determine the distribution of Pu-239 in bone (2-4). More recently, the technique of neutron-induced fission track autoradiography has been used to characterize the temporal and quantitative distribution of plutonium in bone (5-8). Thin bone sections are attached to thin polycarbonate films and irradiated in a high neutron flux. The neutronirradiation of the very small quantities of plutonium produce fission tracks in the polycarbonate film. Using track etching techniques, the fission tracks become visible in the film. This neutron-induced autoradiography has advantages over the conventional nuclear emulsion because it eliminates long exposure times for bone containing only a small quantity of plutonium (in the order of pico pico curies per $\mathrm{cm}^{2}$ ). It also eliminates the fading of latent image (loss of the photographic image) which plagues nuclear emulsion autoradiography.

In the studies of Bleaney (5), bone sections were sandwiched between polycarbonate films and exposed to thermal neutrons. The films were detached following irradiation and then etched to reveal the fission fragment tracks. The fission fragment tracks recorded by the "sandwich" technique are technically contact autoradiographs. This technique made it difficult to know, by superposition of foil and section, whether fission fragment tracks arose from the bone surface, bone or marrow cells.

This work demonstrates the feasibility of producing high resolution detailed neutron-induced autoradiographs that give superb resolution, equal to that of Iiquid emulsion coating of thin bone sections $(2)$. The detailed neutron-induced autoradiographic process produces autoradiographs with both fission fragment tracks and a corresponding bone image.

After the bone sections were prepared, they were exposed to neutrons in the thermal column of the Materials Testing Reactor (MTR). Neutron fluence in the range of $10^{13}$ to $10^{14} \mathrm{n} / \mathrm{cm}^{2}$ was used. During the irradiation the temperature must be kept below $32^{\circ} \mathrm{C}$. Higher temperatures will anneal the tracks. Neutron irradiations were made in the MTR thermal column because of its high thermal neutron flux and low gamma heating.

Subsequent to neutron irradiation, the specimens are allowed to age for a period greater than several weeks before immersing in $28 \% \mathrm{KOH}$ at $60^{\circ} \mathrm{C}$ for one hour to etch pinholes several microns in diameter at the location of each fission track. The bone section separates from the film during the etching process. The etched film is rinsed in tap water, air dried and inspected microscopically. The film exhibits both the fission fragment tracks and a corresponding bone image.

Figure 1 is a contact neutron-induced autoradiograph of a bone section with plutonium localized in both the bone and bone marrow. In Figure 1 the short black lines are fission fragment tracks. Position and number of these tracks determines the position and concentration of plutonium in the dog bone and bone marrow. But the exact position of the fission tracks with respect to 
bone surface or bone marrow is uncertain because a bone image is not present to give the investigator the necessary orientation. If the polycarbonate film is etched immediately after thermal neutron irradiation, we obtain the same result as with a contact neutron-induced autoradiograph. If the bone sections are allowed to remain on the polycarbonate film for several weeks after irradiation, the decay radiation from the bone causes a pitting of the film surface with results shown in Figure 2. In this figure, the bone was left on the polycarbonate film for 4 weeks after irradiation. As is quite evident, the radiation from the bone produced small pitting in the surface, which show as grey areas. The etched film exhibiting both fission fragment and bone images permits a more accurate characterization of the plutonium distribution.

Since the film pitting occurs after irradiation, reactions such as $(n, \alpha),(n, p),(n, d)$ or $(n, n)$ by fast neutrons are ruled out. Radiation must be from the activation of bone constituents, i.e., calcium, phosphorus, etc. with a short half-life. The composition of bone indicates no alpha emitter would be produced and no alpha activity was found on control (plutoniumfree) $d o g$ bone sections after irradiation.

Only beta activity was found in the sample. Absorption curves with aluminum absorbers indicated that most of the activity was from phosphorus-32, a $1.71 \mathrm{MeV}$ beta emitter with a 14-day half-life. Some calcium activation should have occurred, but little activity other than phosphorus-32 was observed.

Human bones produce greater amounts of film pitting than dog bones. This clue so far has not helped to identify the decay radiation causing this pitting.

Betas from phosphorus-32 seem to be the only charged particles available to produce the pitting. Pitting by betas has never been reported in the literature. More work is needed before the source of pitting is known.

[2] J. S. Arnold, "An Improved Technique for Liquid Emulsion Autoradiography," Proc. Soc. Expt1. Biol. and Med. 85, 113 (1954).

[3] J.S. Arnold and W. S. S. Jee, "Bone Growth and Osteoclastic Activity as Indicated by Radioautographic Distribution of Pu-239." Am. J. Anat. 101, 367 (1957).

[4] J.S. Arnold and W. S. S. Jee, "The Toxicity of Plutonium Deposited in Skeletal Tissues of Beagles. I. The Relation of Distribution of Plutonium to the Sequence of Histopathologic Bone Changes, "Lab. Invest. I, 797 (1961).

[5] B. Bleaney, "Radiation Dose Rates Near Bone Surfaces in Rabbits After an Injection of Plutonium," Phys. Med. Biol. 12, 145 (1967). 
[5] B. Bleaney, "The Radiation Dose-rates Near Bone Surface in Rabbits after I.V. or I.M. Injection of Plutonium-239." Brit. J. Radiol. 42, 51 (1969).

[7] B. Bleaney, "Plutonium Deposition on Bone Surface and in Bone Marrow Following Intravenous and Intramuscular Injections," In Delayed Effects of Bone-Seeking Radionuclides, Ed. by C. W. Mays, R. D. Lloyd and W. S. S. Jee, p. 125, U. of Utah Press, Salt Lake City (1969).

[8] K. Becker and D. R. Johnson, "Non-photographic Alpha Autoradiography and Neutron-Induced Autoradiography," Science, 167, 1370 (1970). 


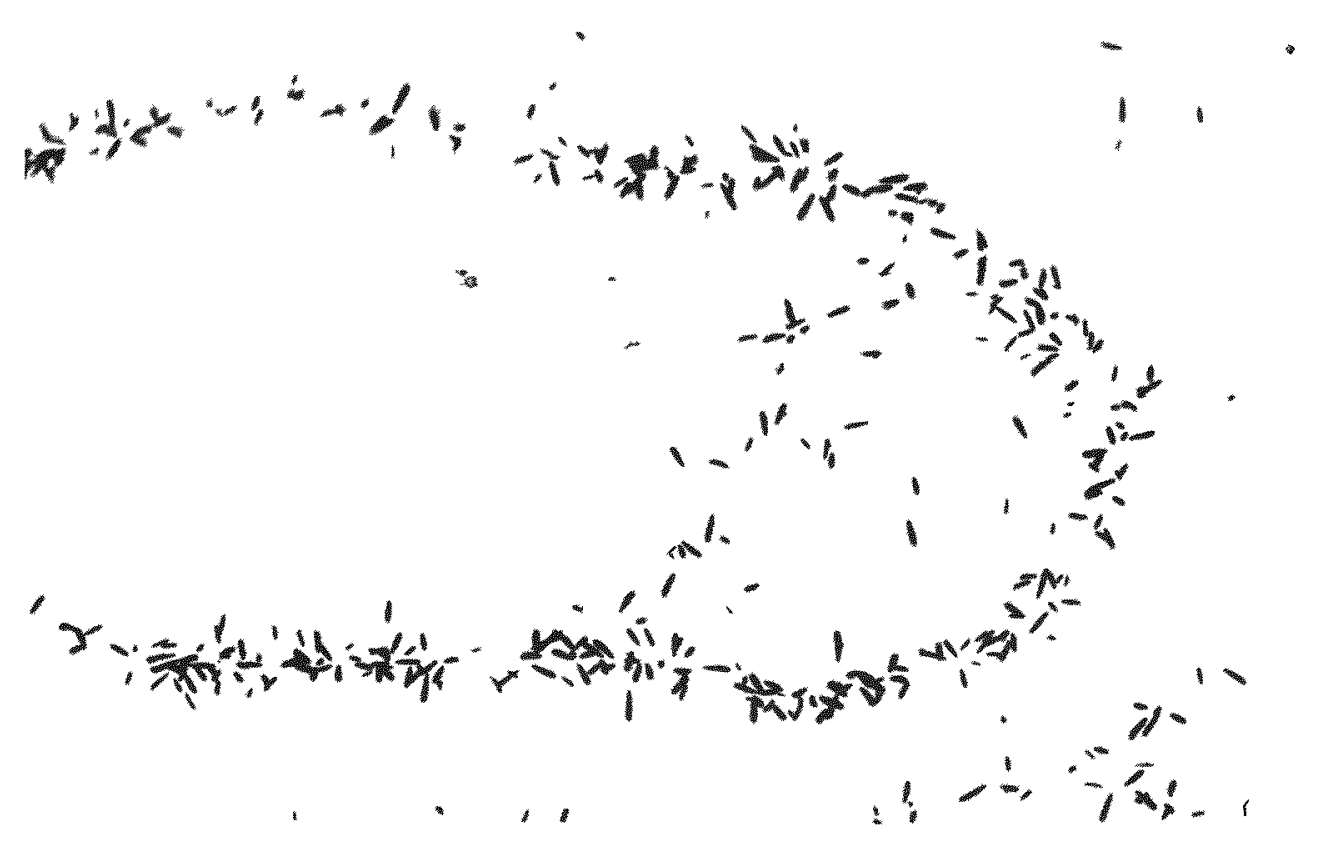

Figure 1. Contact neutron-induced autoradiograph showing bands, starproducing aggregates and diffusely distributed fission fragment tracks in the lumbar vertebral body from a dog given $0.93 \mu \mathrm{Ci}$ of $239 \mathrm{Pu} / \mathrm{kg}$ and killed 1288 days later. The fission fragment tracks are about 15p long. Note the absence of the bone image making it impossible to characterize the location of the plutonium (X 210).

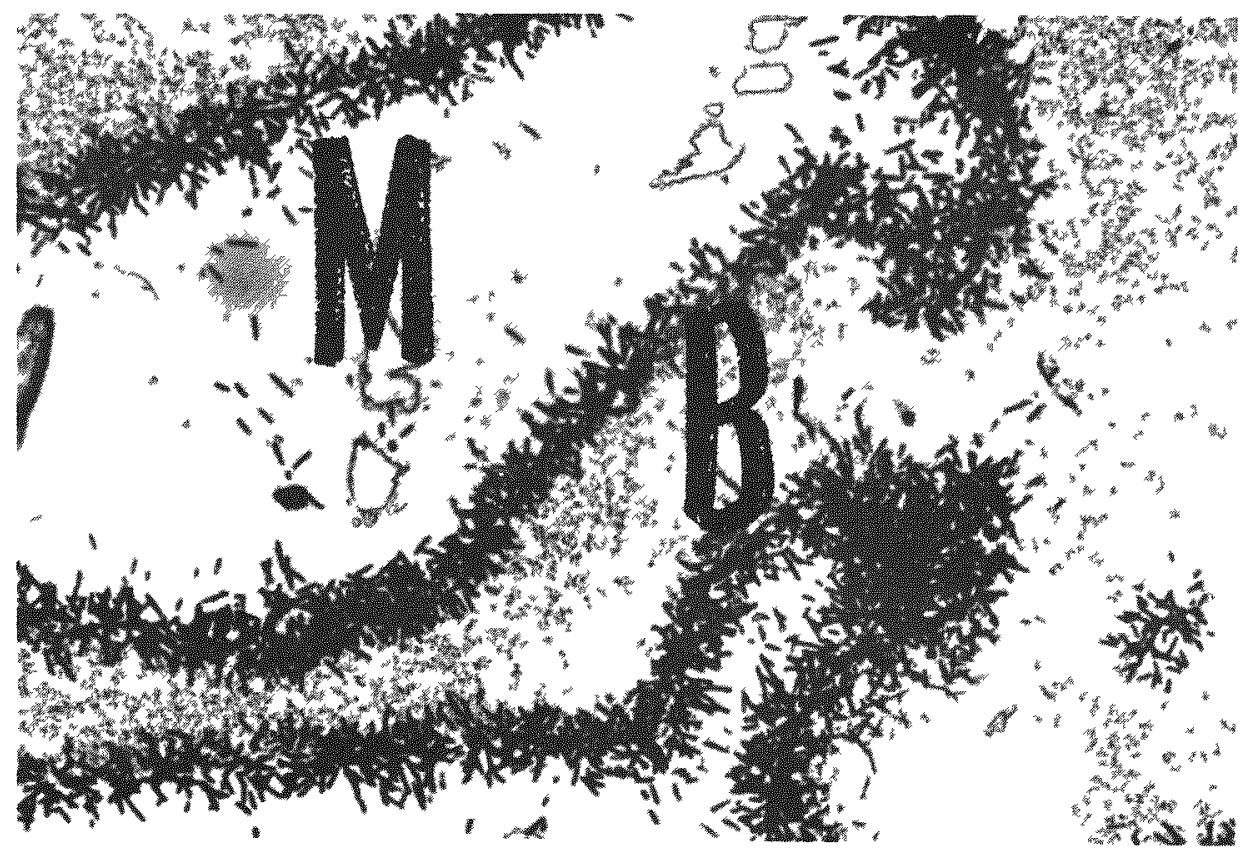

Figure 2. Detalled neutron-induced autoradiograph of portions of two bone spicules, showing fission fragment tracks distributed mostly upon bone surfaces in the lumbar vertebral body from a dog given $2.6 \mu \mathrm{Ci}$ of $23{ }^{9} \mathrm{Pu} / \mathrm{kg}$ and killed 28 days later. Few isolated tracks are found in the bond marrow. $B$ denotes bones, $M$ denotes marrow (X 210). 
DESIGN AND PERFORMANCE ANALYSIS OF THE PBF EXPERIMENT LOOP FOR THFRMAL VOLUME SWELI

J. W. Sielinsky

Introduction

The Power Burst Facility (PBF) Experiment Loop System has two primary functions. The first is to provide the required steady-state environment for a test sample and the second is to contain and control effects of reactor power burst tests. The purpose of this study was to analyze the design and performance of the loop for the thermal volume swell. This is a summary of the detailed analysis reported in the "Power Burst Facility In-Pile Tube System Design Basis Report".[I] Following a reactor power burst, the vaporization and expansion of water within the in-pile tube cause rapid water flow into the loop system. The purpose of the thermal swell accumulator system (TSA) in the loop is to relieve the closed high pressure loop of this expansion such that loop pressure design limits are not exceeded.

The TSA System operates on the principle of providing a chamber of compressible gas (nitrogen) into which the water can expand. To prevent mixing, a movable barrier is situated at the interface between the water and nitrogen. The TSA system consists of two thermal swell accumulators connected to nitrogen reservoirs which in turn are connected to a nitrogen supply system. To provide the necessary volumetric compliance, the nitrogen reservoirs will either (a) have sufficient volume to absorb the thermal swell without exceeding pressure limits, or (b) be equipped with relief valves to control overpressure.

The experiment loop system including the thermal swell accumulators (TSA) was simulated on the ANC HYDAC Computer System to evaluate the performance of the accumulators and to investigate the pressure transients throughout the loop. Two TSA systems were analyzed, a small-volume (10 $\mathrm{ft}^{3}$ ) reservoir operating in conjunction with a relief valve, and a large volume reservoir $\left(184 \mathrm{ft}^{3}\right)$ operating without a relief valve.

Method of Analysis

The experiment loop system as shown in Figure I was simulated on the HYDAC Hybrid Computer System using simulation models to describe the dynamics of each of the system components.

The equations describing the fluid dynamic effects in the loop, which were derived from the lumped parameter approximations of a distributed parameter system[2] resulted in a system described by lumped nodes. The fluid resistance and inertia effects were lumped between nodes and the fluid compressibility effects were lumped at the nodes. The equations describing the dynamic pressure drop between nodes, the pressure at a node, the pump head as a function of flow, the motion of the piston, the nitrogen volume, the motion of the relief valve, and the flow through the valves are given in Appendix $D$ of

Reference 1 . 
Because the pressurizer is connected to the loop by a small diameter pipe, it is essentially isolated from the loop. Consequenily, its pressure was assumed to remain constant during the thermal swell. The constants and parameters used for the model were based on the experiment loop system described in Reference 1.

The HYDAC analog computer was used to solve the differential equations of the simulation model, and the HYDAC digital logic unit was used to solve the logic switching involved in the piston and relief valve models.

Additional calculations were performed in the analysis to determine volume requirements for the large nitrogen volume reservoirs.

Conditions Examined

The transients caused by the thermal swell were computed for various initial conditions in the 100p. These were loop flows at 100 and $400 \mathrm{gpm}$ for test section outlet temperatures of 200 and $630^{\circ} \mathrm{F}$. Two thermal swell flow functions were considered. These were the maximum thermal swell function as shown in Figure 2 and a hypothetical step function of $5.4 \mathrm{ft}^{3} / \mathrm{sec}$ for 1.1 seconds. Other system configurations and operating information are summarized in Table $I$. In addition to the cases at 100 and $400 \mathrm{gpm}$ with the maximum and step thermal sweII functions, two cases were run with a thermal swell function which followed the maximum swell for 1.2 seconds, then decreased linearly to zero at 2.8 seconds. The purpose of these runs was to examine the reestablishment of flow after the swell has subsided.

Analytical Results

The detailed results of the analysis in the form of chart recordings of system transients is assembled in Appendix $E$ of Reference 1. Significant results are summarized and discussed below.

The primary purpose of the TSA system is to provide a compliant volume such that the loop pressure does not exceed specified limits during the thermal volume swell. On this basis, the most significant analytical results are the pressures at critical loop locations for the anticipated transient conditions. The highest pressure in the loop during normal operations occurs at the pump discharge: therefore, this location is critical from the stand point of pressure increases during the volume swell. The results showing this pressure and other information are summarized in Table II. As shown in this table, the maximum pressure at the pump discharge under the worst conditions is 2910 psig. This is below the maximum permissible pressure of 3000 psig (for short duration pressure occurrences). Other information shown indicates a maximum pressure of $2650 \mathrm{psig}$ in the outlet TSA and a maximum nitrogen flow through the relief valve of $3.6 \mathrm{ft}^{3} / \mathrm{sec}$ (the maximum permissible pressure in the outlet line is 2700 psig). Two additional cases were run to examine flow through the in-pile tube as the thermal swell flow (volumetric rate of steam formation) decreases to zero. The input of in these cases followed the maximum 
TABLE I

CONFIGURATION AND OPERATING CONDITIONS FOR LOOP MODEL

A. ermal Swell Accumulator

Diameter

15.9 inches

Stroke

88 inches

Nitrogen volume per accumulator

system

$20 \mathrm{ft}^{3}$

Piston weight

$200 \mathrm{lbs}$

Nitrogen temperature

$50^{\circ} \mathrm{F}$

Nitrogen bulk modulus

7320 psi

B. Relief Valve

Cracking pressure

Fully open pressure

Fully open flow rate

Opening time for step pressure
2400 psig

2640 psig

$4.25 \mathrm{ft}^{3} / \mathrm{sec}$ (nitrogen at $50^{\circ} \mathrm{F}$ and 2440 psig)

$10 \mathrm{msec}$ 
TABLE II

THERMAI SWELI ACCUMULATOR PERFORMANCE

\begin{tabular}{|c|c|c|c|c|c|c|}
\hline Case & $\frac{\text { Flow }}{(\mathrm{gpm})}$ & $\begin{array}{l}\text { Temper- } \\
\frac{\text { ature }}{\left({ }^{\circ}\right)}\end{array}$ & $\begin{array}{l}\text { Type of } \\
\text { Thermal } \\
\text { Swell } \\
\text { Modeled } \\
\end{array}$ & $\begin{array}{l}\text { Max Pump } \\
\text { Outlet } \\
\frac{\text { Pressure }}{\text { (psig) }}\end{array}$ & $\begin{array}{l}\text { Max } \\
\text { Downstream } \\
\text { TSA } \\
\frac{\text { Pressure }}{\text { (psig) }}\end{array}$ & $\begin{array}{l}\text { Relief } \\
\text { Valve } \\
\text { Flow } \\
\left(\mathrm{ft}^{3} / \mathrm{sec}\right)\end{array}$ \\
\hline 1 & 100 & 200 & Maximum* & 2910 & 2650 & 3.4 \\
\hline 2 & 400 & 200 & Maximum & 2910 & 2615 & 3.6 \\
\hline 5 & 100 & 200 & Step** & 2880 & 2590 & 3.0 \\
\hline 8 & 100 & 630 & Maximum & 2685 & 2591 & 2.7 \\
\hline 9 & 100 & 630 & Step & 2702 & 2600 & 2.7 \\
\hline 10 & 400 & 630 & Maximum & 2723 & 2578 & 2.7 \\
\hline 11 & 400 & 630 & Step & 2723 & 2583 & 2.7 \\
\hline \multicolumn{7}{|c|}{$\begin{array}{l}\text { *20 } \mathrm{ft}^{3} \text { volumetric input is shown on Figure } 2 \text {. } \\
\text { * Step volumetric input } 5.4 \mathrm{ft}^{3} / \mathrm{sec} \text { for } 1.1 \text { seconds, } \\
\text { total volume }-6.0 \mathrm{ft}^{3} \text {. }\end{array}$} \\
\hline
\end{tabular}


thermal swell function out to 1.2 seconds, then decreased linearly to zero at 2.8 seconds. The case at $100 \mathrm{gpm}, 200^{\circ} \mathrm{F}$ indicated that in-pile tube flow is reestablished when the thermal swell flow has decreased to $0.5 \mathrm{ft}^{3} / \mathrm{sec}$. Complete reestablishment of coolant flow does not occur until the thermal swell flow has decreased to zero. The case at $400 \mathrm{gpm}, 200^{\circ} \mathrm{F}$ indicated reestablishment of coolant flow when the thermal swell flow has decreased to $1.8 \mathrm{ft}^{3} / \mathrm{sec}$ and complete reestablishment at zero thermal swell flow.

Calculations were performed to determine the nitrogen reservoir volume required to accommodate the total volume swell without venting. The requirements are to limit the pressure between the pump and the inlet TSA to less than 2700 psig.

The computer studies described above indicated that as the thermal swell volume increases, the pressure in the loop increases until it equals the nitrogen pressure. The loop pressure then follows the subsequent increase in the nitrogen pressure as the nitrogen is compressed by piston motion. The maximum operating pressure at the pump discharge is $2535 \mathrm{psig}$ for loop conditions at $200^{\circ} \mathrm{F}$ and $100 \mathrm{gpm}$. This pressure increases to 2585 psig (assuming a 50 psi nitrogen overpressure) and piston motion is initiated. Since the pump outlet pressure is limited to $3000 \mathrm{psig}$, the maximum allowable pressure increase is therefore 3000 psig less 2585 psig or 415 psi.

Calculations were made to determine the volume required to limit the pressure rise to $415 \mathrm{psi}$ for the maximum ( $10 \mathrm{ft}^{3}$ per TSA) and reduced (5.0 $\mathrm{ft}^{3}$ per TSA) volumetric inputs. Employing the ideal gas laws with an effective gas constant of 3.0 , these volumes were found to be $184 \mathrm{ft}^{3}$ and $92 \mathrm{ft}^{3}$, respectively, for the maximum and reduced cases.

Criteria

The Thermal Swell Accumulator design criteria for both the relief valve and the large nitrogen volume concepts developed from this study are summarized in Table III.

\section{REFERENCES}

[1] L. S. Masson, Idaho Nuclear Corporation, Private Communication, (Dec. 1970).

[2] V. L. Streeter, Handbook of Fluid Dynamics, New York: McGraw-Hill Book Co., 1961, Sections 20 and 21. 
TABLE III

THERMAL SWELL DESIGN CRITERIA

Water volume capacity requirement for each TSA

Nitrogen volume vented $\left(50^{\circ} \mathrm{F}\right.$, 2640 psig)

Relief valve opening time

Cracking pressure

Fully open pressure

Reseating pressure

Fully open flow rate

Reservoir volume assuming no venting of nitrogen
$10 \mathrm{ft}^{3}$ (maximum swell)

$10 \mathrm{ft}^{3}$ (maximum swell)

$5 \mathrm{ft}^{3}$ (reduced swell)

$10 \mathrm{msec}$ (based on valve having an undamped natural frequency of $32 \mathrm{~Hz}$ and a damping factor of $0.25)$

2400 psig

2640 psig

2280 psig

$3.6 \mathrm{ft}^{3} / \mathrm{sec}$ (nitrogen at $50^{\circ} \mathrm{F}$ and 2640 psig)

$184 \mathrm{ft}^{3}$ (maximum swell)

$92 \mathrm{ft}^{3}$ (reduced swell) 


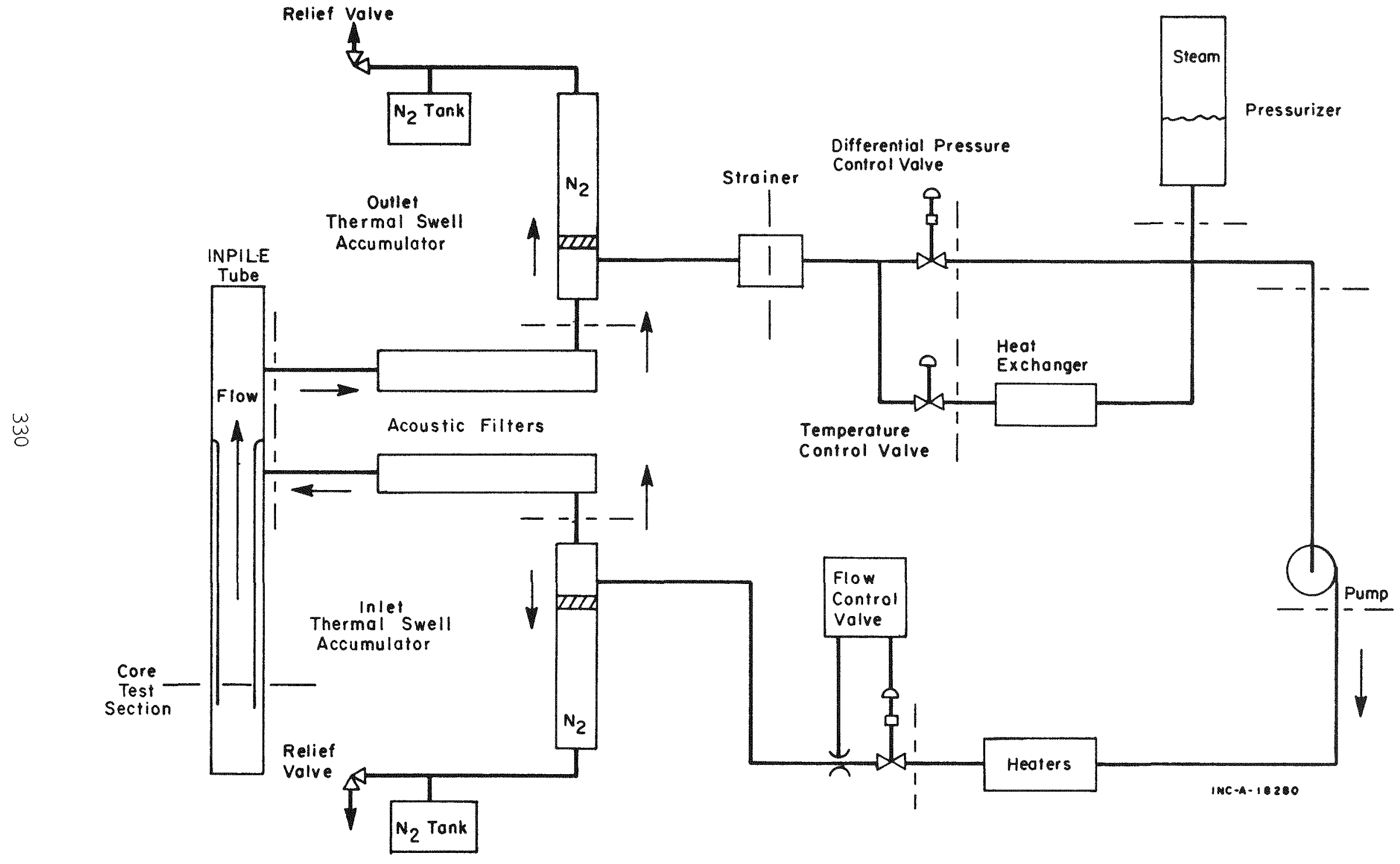

Fig. I Simplified experiment loop system. 
$10 \mathrm{~F}$

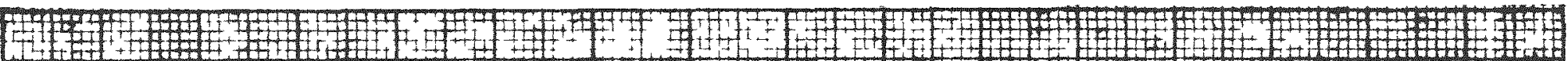

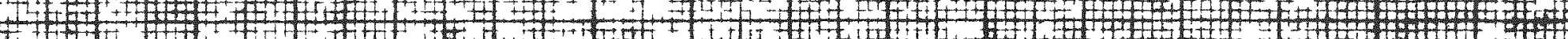
5. $1+17+1$

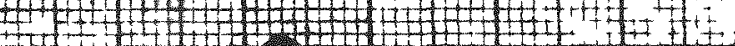

8

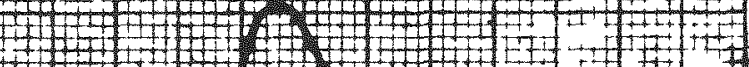

- Colculated Thermal swell volume

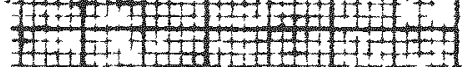
$+2+1+7+1+7$

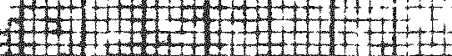

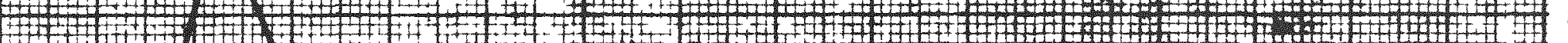

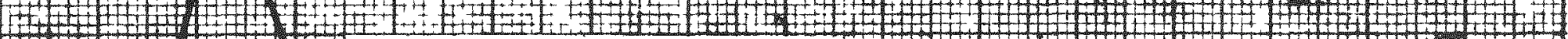

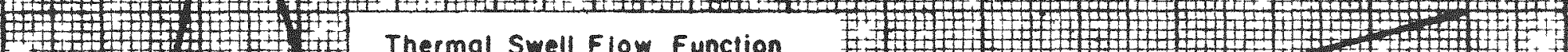
+11+10 Maximum Conditions

6 $7+12$. 7 I

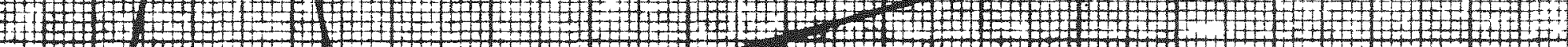

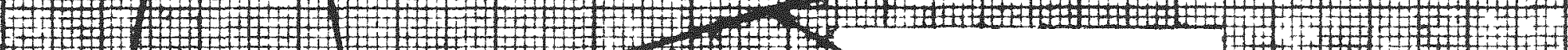
7. 21 Thermal Swell Volume

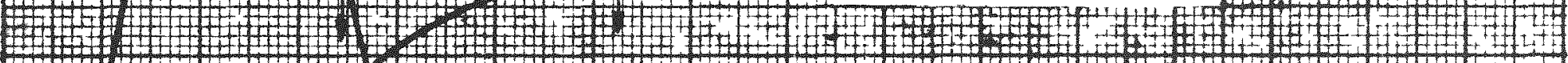
1 ( I

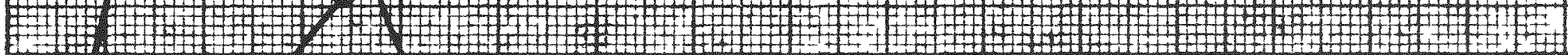

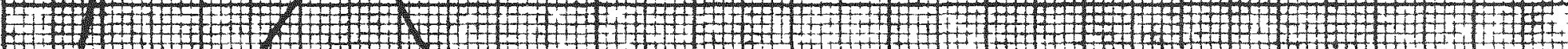
L

2 L $17+2+1$

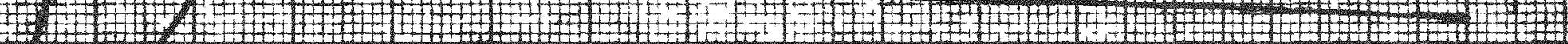
6. T2

0

0

\begin{abstract}
0.4
\end{abstract}
0.8

1.2

1.6

2.0

2.4

2.8

INC-A-1820:

Time $(\mathrm{sec})$

Fig. 2 Thermal swell volume and flow for maximum conditions. 
The purpose of this study was to determine the flow and pressure controllability of the Power Burst Facility (PBF) Experiment Loop for various induced disturbances and control failures under steady state operating conditions. This study deals with relatively slow disturbances caused by the experiments.

The Experiment Loop consists of six systems which are the Loop Coolant System; the In-Pile Tube System; the Vent, Drain and PressureSuppression System, the Loop Cleanup and Decontamination System; the High Pressure Demineralized Water System; and the Loop-Process Instrumentation System. This study being concerned with the controllability of the loop flows and pressures, is focused on the Loop Coolant System as described in "SDD 3.1 - Loop Coolant System" and the Loop-Process Instrumentation System as described in "SDD 7.2 Loop Process Instrumentation System". The other systems are interfaced with these where they affect the flows and pressures of the loop Coolant System.

The system was simulated on the ANC Hybrid Computer using the simulation models developed for it. Figure 1 shows a sketch of the particular components simulated for this study. The simulation included the pressurizer and the loop hydraulics with their associated control systems. The model for the pressurizer included the spray control, the level control, and the pressure control. The model for the loop included the pump, the flow control, the heat exchangers, the differential pressure control, and the heat exchanger manual temperature control valves.

The flow and pressure controller simulation models were adjusted for reasonably good control for the ranges of interest. For a particular set of operating conditions each controller could have been optimized, but due to the system nonlinearities they would not be optimum at other operating conditions. Therefore, each was adjusted for relatively good control over the range of operating conditions expected.

\section{Simulation Models}

In order to simulate the system on the Hybrid Computer, simulation models were developed to describe the components of the system. The models were based on actual component performance data where it was available. On some of the components, in particular the flow control valve and the differential pressure control valve, the dynamic responses were not available. The vendor was contacted, but he was not able to supply the required information. Where data were not available, models were based on best engineering judgment.

The description of the models was divided into four basic categories, the models of the loop hydraulics, the loop flow and pressure controls, the pressurizer, and the pressurizer controls. 
The model for the loop hydraulics is used to determine the various flows and pressures in the loop due to disturbances caused by valve position changes, makeup pump operation, level control valve operation, and pressurizer disturbances. Figure 2 shows the basic loop and defines the flows and pressures. The basic assumptions made for this model were:

(1) The compressibility and inertia effects of the water are fast compared to the disturbances.

(2) The pressure drop between nodes varies as a function of flow times the absolute value of flow.

(3) The flow resistance is directly proportional to the average loop water density.

(4) The change in water density around the loop is small compared to the average density.

The equations for the summation of the flows at each node, with exception of the pressurizer node which will be discussed later, were based on the relationship that for incompressible fluids the summation of flows into the node is equal to the summation of flows out of the node. The equations for the pressure drop between nodes, based on the above assumption that the inertia effects are fast compared to the disturbances, result in the pressure drop being proportional to the flow times the absolute value of flow $(\triangle \mathrm{P}=R Q|Q|$, where $R$ is the flow resistance between the two nodes being considered). For the section that has the pump in it (between nodes $P_{3}$ and $P_{1}$ in Figure 2), the pump was considered a pressure source of its no flow pressure head with its internal flow resistance lumped with the section piping flow resistance. The simultaneous flow and pressure equations were solved on the Hybrid Computer with the inputs to them from the simulation of the control systems and the pressurizer.

The models for the loop flow and pressure controls include the loop flow controls, the differential pressure controls, the makeup pump controls, the level control valve controls, the heat exchanger temperature controls, and spray flow controls.

The loop flow controls consist of the flow element, flow transmitter, compensating relay, flow recorder/controller, I/P transducer, valve positioner, and valve actuator (Figure 1). The information available on the flow element, flow transducer, compensating relay, and $I / P$ transducer indicated that their responses were fast compared to the response of the valve positioner and actuator; therefore, their responses were neglected. The compensating relay converts the output of the flow transducer to a signal proportional to mass flow rate to be used as the input to the recorder/controller. The flow element, flow transducer, and compensating relay were modeled by multiplying the loop flow by density to obtain mass flow rate for the input to the recorder/controller. The controller is three-mode, which includes proportional, reset, and rate action. The controller was modeled 
using only proportional and reset modes with the idea of adding the rate mode if reasonable control could not be obtained. The flow control valve positioner and actuator was modeled as a second order system.

The only information on the response of the valve was that for a step setpoint change, it takes approximately 10 seconds to reach the new setpoint and the output is well damped. Using a damping factor of 0.7 and a natural frequency of 0.35 radian results in the output reaching the new setpoint in 10 seconds with a 5\% overshoot with no ringing. The input for the flow controller is loop flow obtained from the hydraulic model. The output used to calculate the valve flow resistance for the hydraulic model is valve position.

The differential pressure controls regulate the pressure drop across the heat exchangers by controlling a valve in the bypass line. The controls consist of a differential pressure transmitter, an indicator/controller, and a valve positioner and actuator (Figure I). The differential pressure transmitter measures the pressure drop from the inlet to the outlet junctions of the heat exchanger and it was modeled as a first order system with a time constant of one second. The differential pressure controller is a two-mode controller with proportional band and reset action, and was modeled as such. The model for the valve positioner and actuator was the same as the flow positioner and actuator. The same type positioner and actuator is used on the two systems. The input to the model is differential pressure obtained from the hydraulic model. The output is the valve position. It is used with the valve characteristic to determine the flow resistance of the differential pressure control valve for the hydraulic model.

The model for the two parallel makeup pumps is based on the assumption that the electrical power switching is fast compared to the flow response and that flow is directly proportional to pump speed. A first order time constant of two seconds and a full flow of 1 gpm were assumed for the flow response.

The on and off switching of the makeup pumps was modeled both for manual and automatic control in the pressurizer level controls. The pressurizer controls are discussed later.

The model for the level control valves is based on the assumption that the actuators open on a two second time constant. The control to the valves is modeled for manual and for automatic control in the pressurizer level control model. The output of this model is the valve position. It is used to determine the flow resistance between the loop and the knockout drum for the hydraulic model.

The temperature and spray control valves were modeled as manual controls of the flow resistance in their respective sections for the hydraulic model. 
The loop flow and pressure control models were connected to the hydraulic model to simulate the loop system under automatic flow and pressure control. The pressurizer pressure is still required as an input to the hydraulic model.

The pressurizer is a cylindrical vessel of $2 \mathrm{ft}$. inside diameter and $10 \mathrm{ft}$. high with an inside volume of 30 cubic ft. The bottom of the pressurizer is connected to the $100 \mathrm{p}$ upstream of the $100 \mathrm{p}$ pump through approximately $50 \mathrm{ft}$. of one-inch pipe. The top of the pressurizer is connected to the loop downstream of the loop pump through a spray nozzle, a spray valve, and approximately $50 \mathrm{ft}$. of one-inch pipe. Immersion heaters are located in the lower portion of the vessel and the power to these is controlled by the pressurizer pressure controls. The level of the water is controlled by the level controls. Under steady state conditions the pressurizer is filled with steam and water with the nominal water level at $63 \mathrm{in}$.

The model for the pressurizer is basically the same as the one developed for simulating the LOFT[I] pressurizer. The inputs to the pressurizer model from the hydraulic model are the spray flow rate, the flow rate from the pressurizer to the loop, and the densities and enthalpies of these streams. Out of the pressurizer model is the pressure for the hydraulic model, the pressure for the pressurizer pressure controls, and the pressurizer level for the level controls. The input to the model from the pressure controls is the heater power. The pressurizer pressure is controlled by a system which measures pressure and controls the power to the heaters. The level is controlled by a logic system which controls the operation of the makeup pumps and the level control valves.

The pressure control system consists of a pressure transmitter, a pressure recorder/controller, a saturable core reactor controller, a saturable core reactor, and the immersion heaters. The pressure transmitter was modeled with a first order time constant of $1.0 \mathrm{sec}$. The controller was modeled as a three-mode controller with proportional band, reset, and rate action. The saturable core reactor controller and the saturable core reactor are relatively fast (time constants less than $0.1 \mathrm{sec}$ ); therefore, their responses were neglected, and the heater response was neglected compared to the slow response of heating the large water volume.

The level controls consist of a level transmitter, the makeup pump power control logic and the solenoid control logic for the level control valves. The makeup pump power control logic was modeled so that power was applied to the pumps at a low level of $60 \mathrm{in}$ and remained on until the level reached $64.5 \mathrm{in}$. The makeup pumps were modeled having a $2.0 \mathrm{sec}$. time constant as described in the hydraulic models above. The level control logic was modeled so the solenoids opened at a high level of $66 \mathrm{in}$. and remained open until the level dropped to $61.5 \mathrm{in}$. The level control actuators were modeled with $2.0 \mathrm{sec}$ time constants as described in the hydraulic models above. 
This concludes the brief descriptions of the models for the loop hydraulics, the loop flow and pressure controls, and the pressurizer and its controls. The models were simulated on the Hybrid Computer System and numerous transients were run to determine the controllability of the system for various induced disturbances and control failures at steady state loop operating conditions.

Results

The flow and pressure controllability of the PBF Experiment Loop was studied by introducing various disturbances and simulated control failures with the loop at steady state operating conditions and in automatic control. Since the control is directly dependent on the flow and pressure controller settings, each controller was adjusted such that reasonably good control was obtained for the range of flows and pressures expected. The idea was to set the controllers to cover the full range of operating conditions without having to change them. Because of the nonlinearities in the loop flow control and the differential pressure control, the control response varies with changes in operating conditions. Since this loop will be operated over a wide range of conditions, it is more meaningful to adjust the controllers to cover the expected ranges and not to optimize them for each particular operating condition.

The pressurizer pressure controller was adjusted to minimize pressure changes due to makeup pump and level control valve operations. These settings also resulted in good control for pressure setpoint changes.

The differential pressure controls were set up to cover a range of loop flows from 200 to $800 \mathrm{gpm}$. The response of this control system was very sensitive to the loop flow. At low flows (200 gpm) the control was only slightly damped and at high flows $(800 \mathrm{gpm})$ it was over damped with very slow response. This effect is caused by the nonlinearities due to flow and the valve characteristic combined with the associated piping and components. It would be very desirable to linearize the system such that its response would be essentially the same for the full range of flows.

The loop flow controls were set up to cover the range of flows from 200 to $800 \mathrm{gpm}$. The nonlinear effects of flow were not as strong for this as for the differential pressure controls. The control is well behaved at both low and high flows, although the nonlinear effects are present with faster response and some ringing at $200 \mathrm{gpm}$ and slower response and more damping at $800 \mathrm{gpm}$.

In order to have a common base to compare the results of the various disturbances, the simulation was set up to represent typical loop operating conditions such that both increases and decreases in parameters could be studied. 
The study of the disturbances was made in two series, the first series consisted of disturbances which mainly affect the pressurizer and the second series consisted of disturbances which mainly affect the loop flows and pressures. The types of disturbances that were introduced into the pressurizer were:

(I) Pressure control setpoint changes

(2) Pressurizer spray turned on and off

(3) Pressurizer heater control failures of turning fully on and off

(4) Makeup pumps manually turned on until the level control valves opened

(5) Level control valves manually jogged until makeup pumps turned on

(6) Makeup pumps manually turned on with the spray and heaters off

(7) Failure of level control valves open

(8) Makeup pumps stay on with level control valyes operational

(9) Makeup pumps stay on and level control valyes do not open

(10) Surge flow into loop to simulate water in loop expanding due to fast reactor power increase

(11) Surge flow out of the loop to simulate water in loop contracting due to fast reactor power decrease.

The types of disturbances that were induced into the system that mainly affected the loop flows and pressures were loop flow control setpoint increases and decreases, differential pressure control setpoint increases and decreases, and temperature control valve position increases and decreases. The transients that were caused by these disturbances were recorded and are included in Reference 1.

$\underline{\text { Recommendations }}$

As a result of this study several changes in the PBF Experiment Loop System, as well as changes in its operation, were recommended. It was also recommended that additional studies be made to determine the temperature controllability of the loop.

Remote Spray Flow Control. Presently the spray flow valve is operated by a turn wheel in the basement of the reactor building. The results of the pressurizer disturbances show that it is desirable to operate the pressurizer heater at about half power to decrease the pressure after a transient has caused pressure to increase above the setpoint. In order to remove the energy added to the pressurizer from the heaters the spray has to be on. 
The spray flow rate is determined by the enthalpy of the spray stream as it enters the pressurizer. The enthalpy of the spray can change significantly from low experiment power to full

experiment power. Therefore, it is recommended that the spray valve be remotely controlled at the control room and be adjusted to obtain about half heater power as the reactor is started up.

Pressurizer Heater Power Monitor. The power to the pressurizer heater should be monitored at the control room so the spray flow can be set and the pressurizer controls optimized.

A Differential Pressure Transducer Should Be Used for the Pressurizer Control System. The pressurizer controller was set at its minimum proportional band for the best pressure control. By replacing the pressure transducer now used (2500 psi range) with a differential pressure transducer with a range from 1850 to 2350 psi, the proportional band can be increased which should result in less noise problems with the controller and also provide more range for the controller settings. If automatic pressure control is required out of this 1850 to 2350 psi range, some means of switching to other ranges will be required.

Differential Pressure Control. At lower loop flow the differential pressure control is quite underdamped. A higher proportional band in the controller would damp this control, but the controller is set at its maximum proportional band. This proportional band could effectively be increased by a factor of five by replacing the differential pressure transducer now used (100 psi range) with one having a 500 psi range. But at the higher loop flows the differential pressure control response would be slower than it was for this study. It is then recommended that this control be linearized to obtain about the same controllability for the full range of loop flows. The control can be significantly linearized by replacing the "Iinear" differential pressure control valve with an "equal percent" type valve.

Limit Pressurizer Level Control Flow. The level control flow causes a pressure drop of about 55 psi in the loop pressures. This is assuming the loop is at high pressure and the water flashes to steam in the valves and restricts the flow to $I I \mathrm{gpm}$. If the water is at high pressure and below $200^{\circ} \mathrm{F}$ it will not flash to steam and will reach a flow of about $40 \mathrm{gpm}$ thus dropping the loop pressure about $500 \mathrm{psi}$. A restriction should be placed in the piping to the level control valves or the valve positions limited so the flow is restricted to less than $10 \mathrm{gpm}$ under all conditions,

Pump Cavitation Detector. Presently only low pump suction pressure is used to shut the loop pump off to protect it from being damaged. by cavitation. Pressure alone does not determine the water saturation conditions. The temperature of the water must be used along with the pressure to predict pump cavitation. A method of using both the temperature and pressure or a method using noise detection techniques should be used for pump cavitation detection. 
Operation of Loop to Check Out Actual Controls. It is recommended the loop be connected through a restriction, to simulate the InPile Tube System, at the crossover couplings and operated before the In-Pile Tube System is completed. This will check the 10op controls and provide operating experience on the actual loop operation.

Additional studies. It is recommended that the thermal and heat balance models of the system be simulated to determine the loop temperature controllability and the effects of changes in reactor power. Reviewing previous studies of the loop temperature control[2] shows that the control system is unstable for higher loop flows and ramp reactor power increases. These additional studies would determine the controller settings and modifications required to stabilize the system. The loop contraction rate estimated in this study caused severe loop pressure drops. The additional studies would better estimate these effects by calculating the surge flow rates. The effects of the heat stored in the piping walls were not included in the surge flow estimated in the study, but a thermal model would include these effects.

REFERENCES

[I]F. K. Hyer, Idaho Nuclear Corporation, Private Communication (Nov. 20, 1970).

[2]T. B. Klingler, Power Burst Facility High Pressure Water Loop System Response, GEMP-665, PBF-GE-6. 


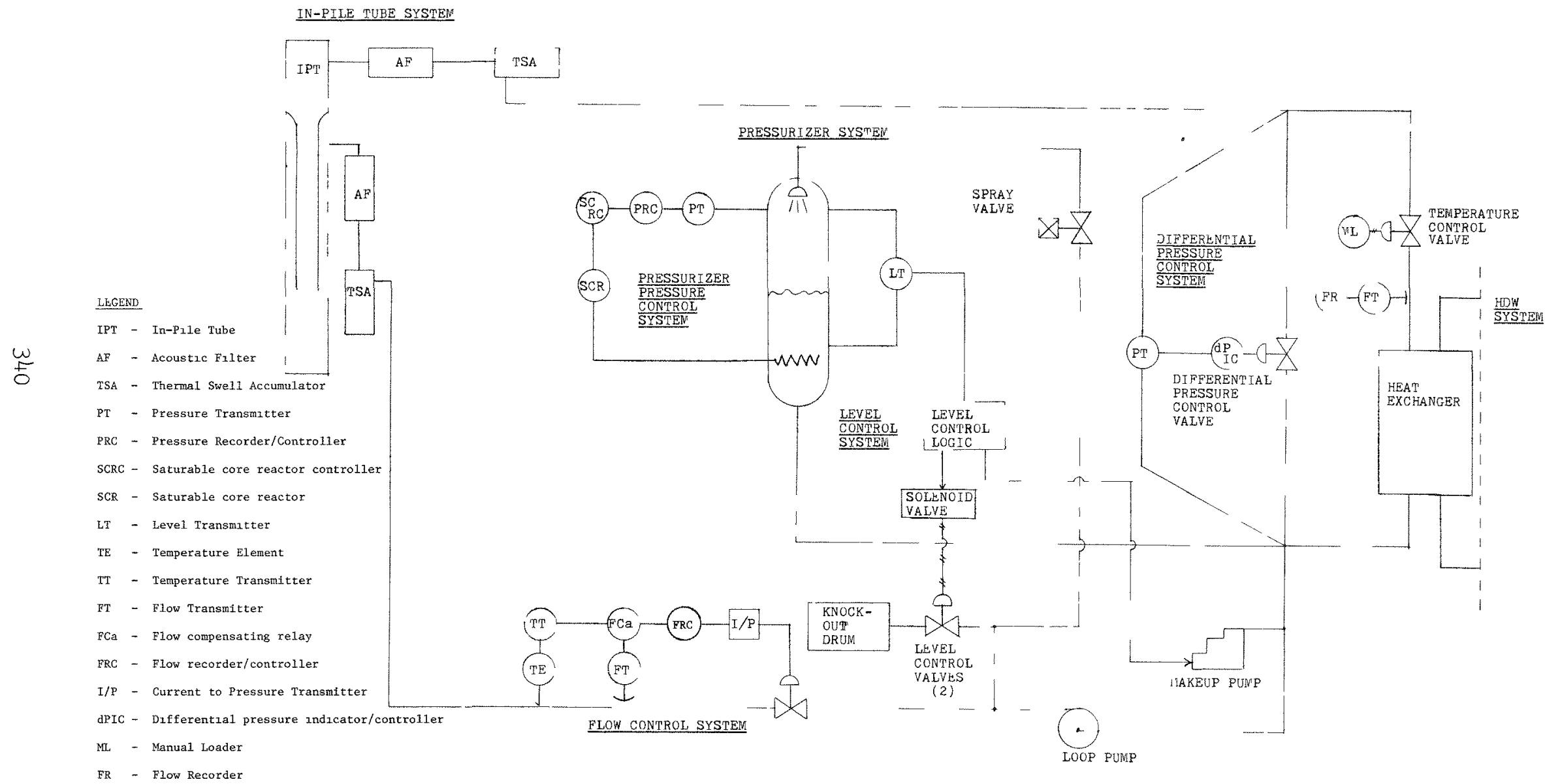

Fig. 1. Sketch of System Simulated on Hybria Computer. 


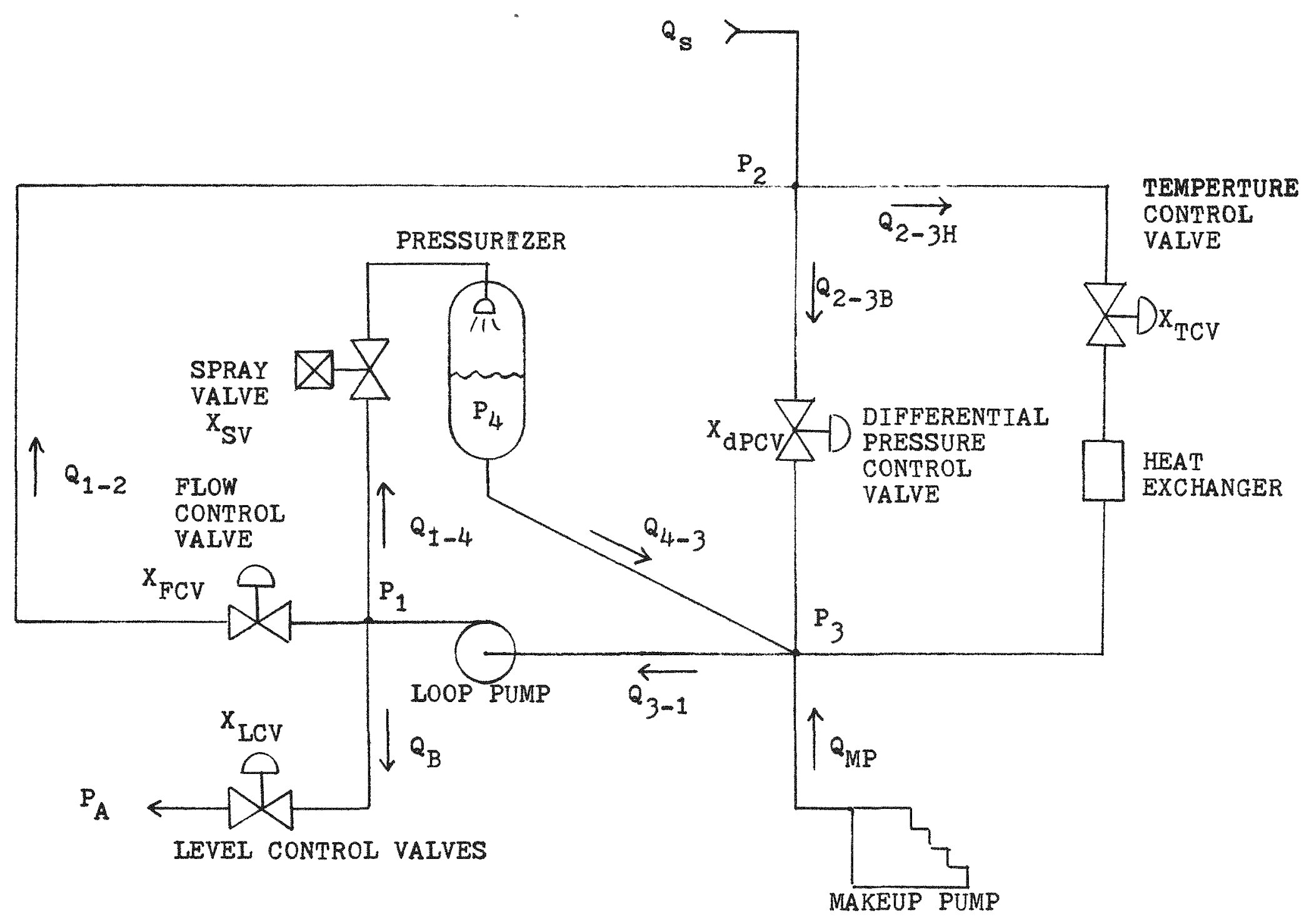

Fig. 2. Sketch Defining Flows and Pressures 


\title{
DESIGN AND RELIABILITY ANALYSIS OF LOFT PPS LOGIC CIRCUITRY
}

\author{
R. W. Keller and E. V. Waite[I]
}

As a subassembly of the LOFT Plant Protection System (PPS), the Plant Protection System Logic circuitry operates in unison with the total system, in accordance with RDT standard C 16-IT[2], to prevent unacceptable release or spread of radioactive materials, and to prevent unacceptable plant damage. The design basis for the PPS circuitry is found in FDD 1.6[3]. Since the PPS is composed of the reactor shutdown subsystem, the emergency core cooling subsystem, and the containment isolation subsystem, the PPS logic circuitry appears as a subassembly in all the related Plant Protection Systems.

Various designs were proposed for the PPS circuitry, most of which were rejected on the basis of intuitive and inductive analyses. To provide the basis for informed management decision, reliability calculations were made using probabilistic analysis techniques for three proposed circuit models (Figures 1, 2, and 3) which could not be rejected on the basis of other analyses.

\section{Circuit Models}

To facilitate the analysis each system model is divided into five main system building blocks (Figures 1, 2, and 3). These system blocks are:

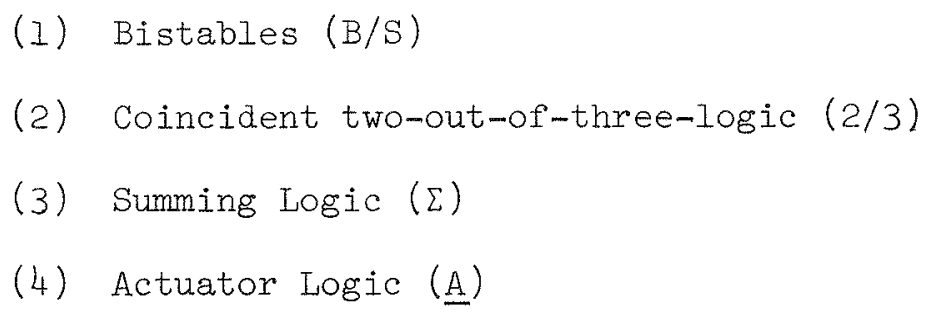

(a) Systems 1 and 3 use a 2/3 relay matrix.

(b) System 2 uses a $1 / 2$ relay matrix, ie, relay contacts connected in series.

\section{(5) Actuator (ACT)}

Not all of the system blocks need be considered in any given system model. The blocks are listed for completeness rather than as system requirements.

Each system model is driven by three independent instrument channels per plant variable (flow, temperature, pressure, etc.) as determined by the technical specifications, and each system provides redundant actuator response, eg, rod release, initiate emergency core cooling, or initiate containment isolation through two independent actuator ariving mechanisms . 
Assumptions

Components are considered as being of two kinds: (a) dynamic--those that switch or modify energy, and (b) quasi-static--those that support or contain something, eg, wires connecting dynamic components.

Only identifiable dynamic components, as shown in Figures 1, 2, and 3, are being considered and they are restricted to the following component fault categories:

(1) Primary Failure - Component fails when subjected to operational environmental parameters for which it is qualified, ie, internal random failures.

(2) Secondary Failure- Caused or forced failures, eg, operation under conditions for which it is not qualified.

(3) Command Failure - A state or system failure controlling the dynamic state of a component. Not an actual component failure, but the component is in the wrong state at the wrong time due to actuation or nonactuation by some other component. Allows failures to propagate up the tree.

Primary and secondary failure of any component is assumed to be mutually independent of failures in other components, within or without a particular model.

Causes of secondary failures are not developed and failure rates of one-half the assumed primary failure rate are used.

The considered safe (fail tripped) and unsafe (fail untripped) failures for each dynamic component were assumed equal in terms of the failure rates used for a component failing to either state. Although that is not necessarily the case in actual practice, it is sufficient for a quantitative comparison between systems. Furthermore, the failure rate data (Table I) used for this study may not be equivalent to that which will exist for the LOFT PPS circuitry, but the use of identical data in the three circuit models does validate relative comparisons between systems.

All components are assumed to have survived burn-in and are assumed to be in a period of life wherein the hazard rate is relatively constant. The basic parameter characterizing the failures is the constant component failure rate $\lambda$ which is characteristic of the exponential distribution.

In all cases no repair is assumed during the period of operation. 


\section{$\underline{\text { TABLE I }}$}

FAILURE RATE INFORMATION[4]

\begin{tabular}{lcc}
\hline & \multicolumn{2}{c}{ Failures $\lambda / 10^{6}$ Hours } \\
\cline { 2 - 3 } Failure & Primary Failure & Secondary Failure \\
Relay Failure & 1.0 & 0.5 \\
\hline Tripped (Open) & 1.0 & 0.5 \\
Untripped (Closed) & & \\
Summer Failure & 0.269 & 0.134 \\
\hline Tripped & 0.269 & 0.134 \\
Untripped & & 0.54 \\
2/3 Logic Failure & 1.076 & 0.54 \\
\hline Tripped & 1.076 & 0.769 \\
Untripped & & 0.769 \\
Bistable Failure & 1.538 & \\
\hline Tripped & 1.538 & \\
Untripped & & \\
Instrument Channel Flow, & & \\
Press., Temp. & 1.538 & \\
Fails causing trip & 1.538 & \\
Fails without tripping & & \\
Nuclear Power Range & 100.0 & \\
\hline Fails causing trip & & \\
Fails without tripping & & \\
\hline
\end{tabular}

Analysis

For the three proposed PPS circuit models, graphic models (Fault Trees[5]) were constructed of the various (considered) parallel and sequential combinations of components which could result in the following undesired events:

(1) Failure of the system to respond when required - System Reliability. (Components fail untripped--Unsafe failures)

(2) System responds when not required - Plant Availability. (Components fail tripped--Safe failures)

Several methods are available which will yield the quantitative information necessary for system comparison. [6] In this case Kinetic Tree Theory[7] developed at the NRTS was employed. The fault trees as drawn provide simple inputs to computer programs PREP and KITT[8]. The only other data required as inputs are the failure rates (Table I) assumed for the primary and secondary failures leading to $I$ and 2 above. 
System Reliability (Safety)

When considering system safety, to simply state that the system failed to respond is not sufficient. The event must be described as a failure of the system to initiate rod release, ECC, or containment isolation when the appropriate plant variable exceeds a predetermined limit. To establish a reliability envelope each circuit model was studied with a single variable input (three instrument channels). Two runs were made. The first run utilized an instrument failure rate of $1.538 \times 10^{-6}$ failures/hr (representing flow, temperature, and pressure) and the second run utilized an instrument failure rate of $100 \times 10^{-6}$ failures/hr (representing nuclear power range). Thus bounds were established that encompassed a range of expected probabilities of safety failures.

Figures 4, 5, and 6 are plots of the probability of system failure versus continuous operating time for systems I, II, and III, respectively. The plot indicates that when the same assumptions are applied to components and the individual systems (refer to assumptions), the physical arrangement of those components into functionally different systems does not significantly affect the postulated undesired event in terms of the probability of occurrence of that event. System selection could not be based on this information.

Although the intent of these analyses was not to exactly determine the reliability of any system, it should be noted that the probability of system failure could be theoretically maintained at any desired level by requalifying (complete test and repair) the system at that point in time where the probability of failure becomes unacceptable.

Plant Availability

Spurious reactor shutdowns are as unacceptable as safety failures in achieving mission success, ie, safe operation at a predetermined power level under specified conditions for a set period of time. Thus with equally safe systems those with the greatest probability for spurious reactor shutdown become unacceptable. Availability studies postulating system response when no plant variable exceeds its preset limit were performed for three cases. These cases are: (a) singlevariable (three channel) input, (b) five-variable input not using nuclear power range instrumentation, and (c) five-variable input including nuclear power range instrumentation.

In these studies the first case was established to obtain a check on the computer input data and to indicate the performance of a singlevariable system. The second case was established to compare system operation without nuclear instrument inputs to the third case which included the nuclear instrumentation.

The plots of systems I, II, and III representing the probability of system response when protective action is not necessary versus time are shown in Figures 7,8 , and 9. 
From the plots System I is the least likely to fail resulting in spurious actuator operation. System II has the greatest probability for spuriously tripping the actuators. This high probability of failure is due to the single failure vulnerability of any $2 / 3$ logic block, either summing logic or relay matrix. Single failure of the svstem building blocks in Systems I or III will not cause actuator trips. The increase in plant availability of System I over System III is due to the added 2/3 coincidence logic at the Bistable level. This early coincidence, as opposed to the single final coincidence in System III, allows system I to be free from spurious actuator trips caused by spurious action on unrelated instrument channels.

The relative comparisons between Systems I and III indicate that (considering the five-variable case including nuclear power range instrumentation) after 1900 hours of continuous operation System III is more than twice as likely to result in a spurious plant shutdown as System I.

\section{Maintenance and Testing}

The block diagrams of Figures 1, 2, and 3 were used to study the flexibility of each system model to maintenance and testing procedures.

System I (Figure 1) has provision for complete testing from instrument channel to actuator logic through a two-step operation. This is accomplished through the tripping of an instrument channel and its related $2 / 3$ logic. Although this is a more complex testing scheme than required for Systems I or II, the System will not fail because of single unrelated instrument and channel failures in other PPS sets. This additional flexibility enhances the testing and repair features in this System.

System II (Figure 2) allows little flexibility for on-line testing and repair. In fact, only the instrument channels can be tested on-line without causing protective response. This means that after the system is operational the 2/3 logic, the summer logic, and the actuator logic cannot be tested or repaired. This is obviously an undesirable feature.

System III (Figure 3) allows for complete testing and repair from instrument channel through actuator driver on an operational system. The testing can be accomplished through the tripping of a single instrument channel. This is the simplest testing scheme available. However, this simplicity also causes the system to revert to a possible single failure mode during the testing and repair of a single instrument channel.

\section{Conclusions}

From the safety analysis all three models appear to be relatively equal. For this reason, no decision can be made on the basis of safety. Further analysis of this type should be performed to insure that the system built does not violate the more important basic assumptions stated or that the graphic model be adjusted to accommodate the differences in what was assumed to what is actually installed. 
Plant availability considerations indicate that System $I$ is the least susceptible to failures resulting in spurious actuator trips. System III is quite reliable, but it does lack the test and repair capabilities of System I. System III can be completely tested from instrument channel to actuator driver in one operation, but due to its two-out-of-N coincidence scheme it is more susceptible to spurious actuator trips during maintenance operation. Because of the simple redundant logic after the bistable of System II it is the most susceptible to spurious trips of the three systems proposed. Likewise it cannot be completely tested or repaired while the reactor is operational.

System I is recommended on the basis of its availability and the improved characteristics during operational maintenance periods.

\section{REFERENCES}

[1] Computer Science Branch, Technical Services Division.

[2] RDT Standard C 16-1T, Supplementary Criteria and Requirements for RDT Reactor Plant Protection Systems (December 1969).

[3] FDD 1.6, "Plant Protection Systems", Idaho Nuclear Corp., LOFT Project Division.

[4] M. E. Stewart, J. F. White, T. O. Zalie, Idaho Nuclear Corp., Private Communication (December 1969).

[5] D. F. Haasl, "Advanced Concepts in Fault Tree Analysis", System Safety Symposium, June 8-9, 1965, Seattle: The Boeing Company, 1965.

[6] P. Crosetti, "Fault Tree Analysis with Probability Evaluation", IEEE Transactions on Nuclear Science, Vol. NS-18, (1), Feb. 1971.

[7] Dr. W. E. Vesely, Analysis of Fault Trees by Kinetic Tree Theory, In-1330, Oct. 1969.

[8] W. E. Vesely and R. E. Narum, PREP and KITT: Computer Codes for the Automatic Evaluation of a Fault Tree, IN-1349, August 1970. 


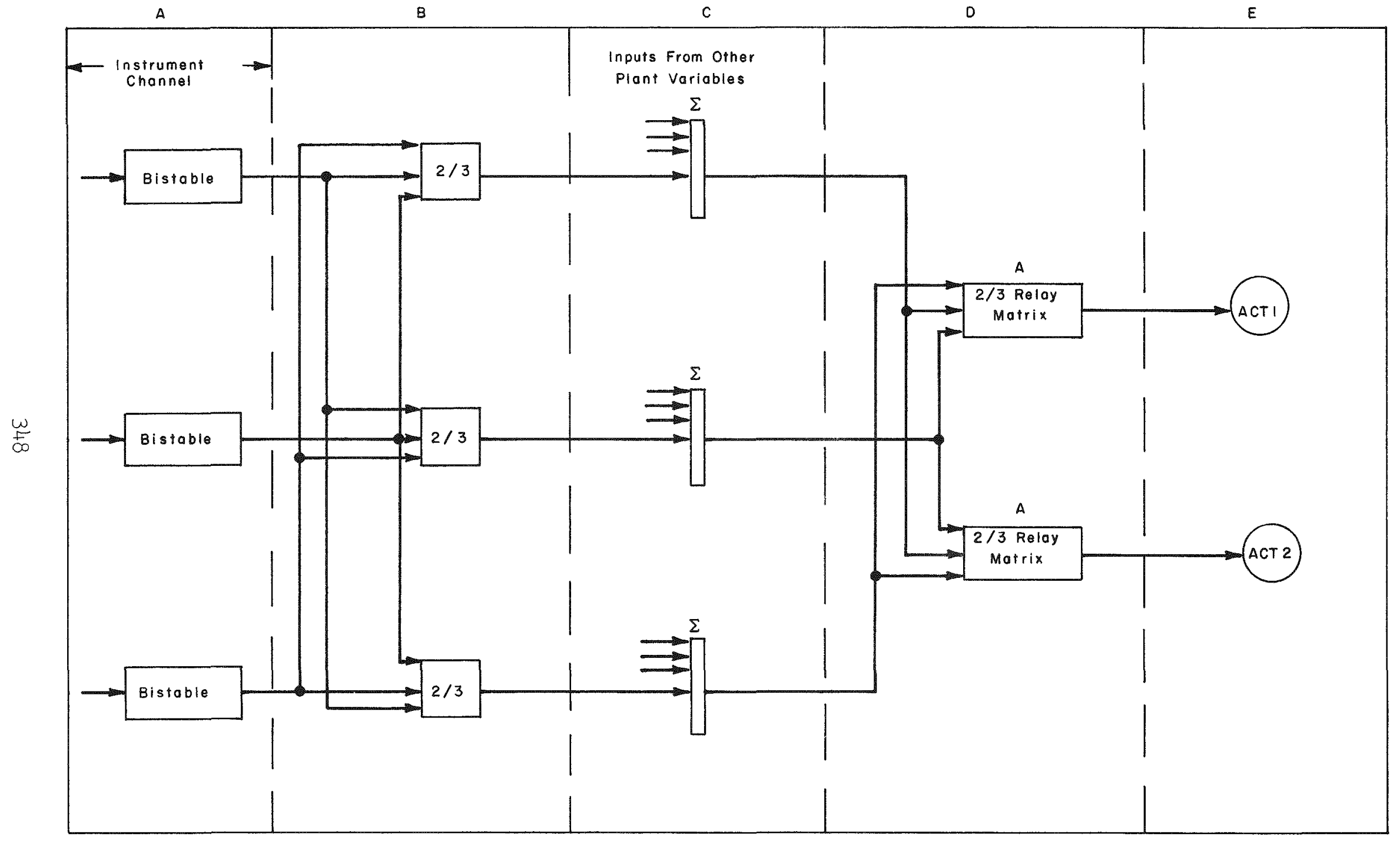

Fig. I System I block diagram.

ANC-B-364 
D

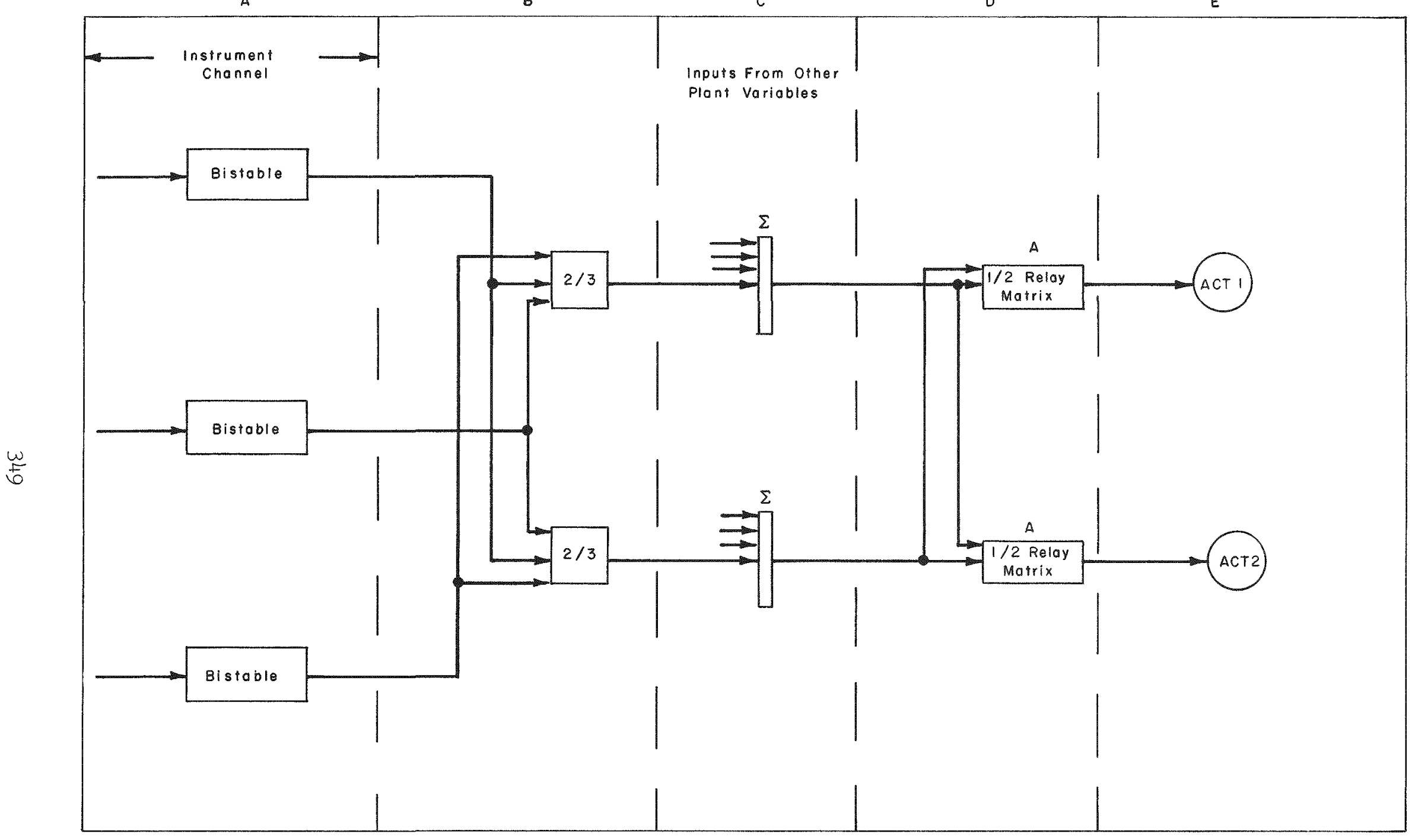

Fig. 2 System II block diagram. 
A

B

C

D

E

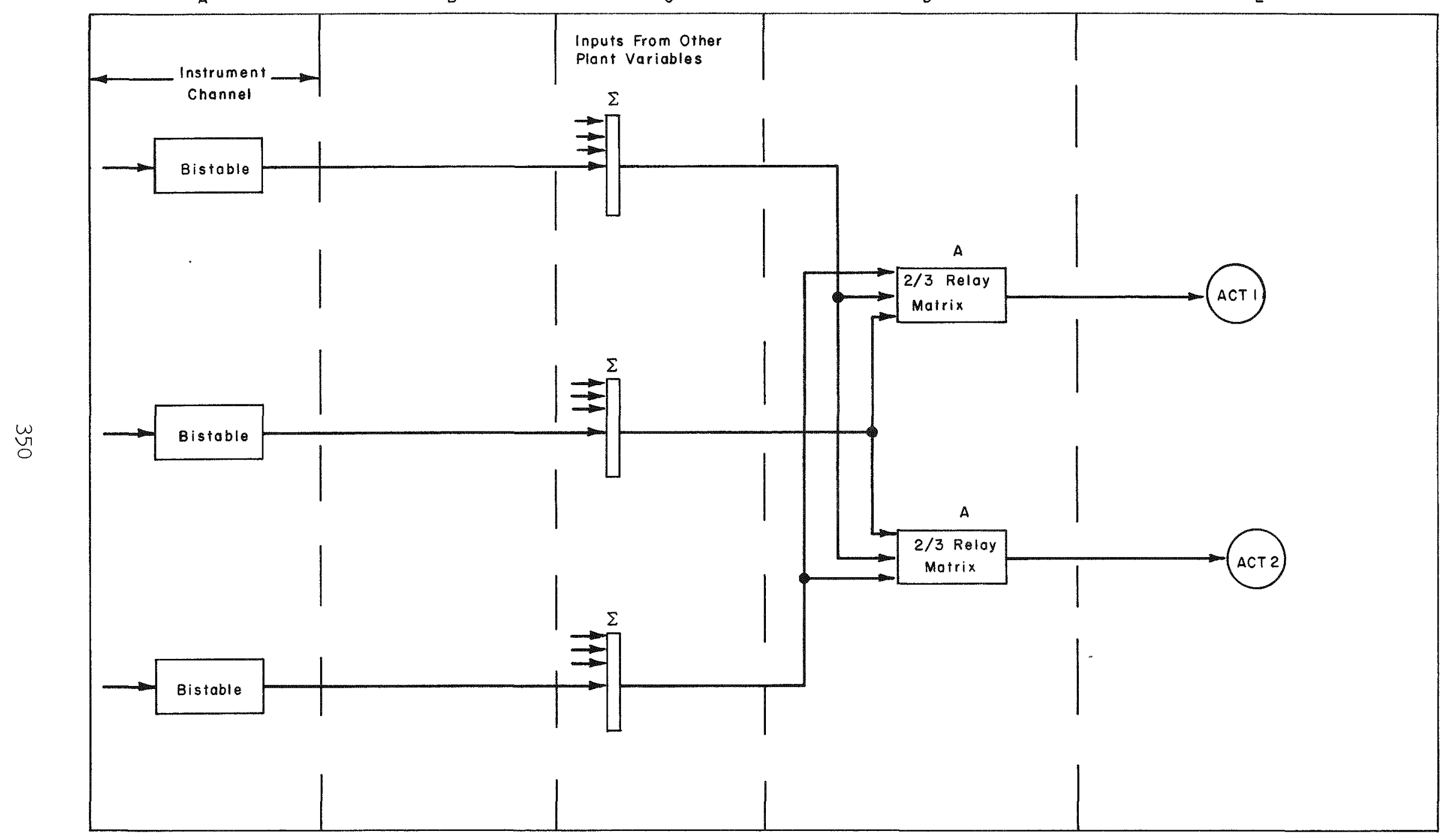

Fig. 3 System III block diagram.

ANC-B-363 


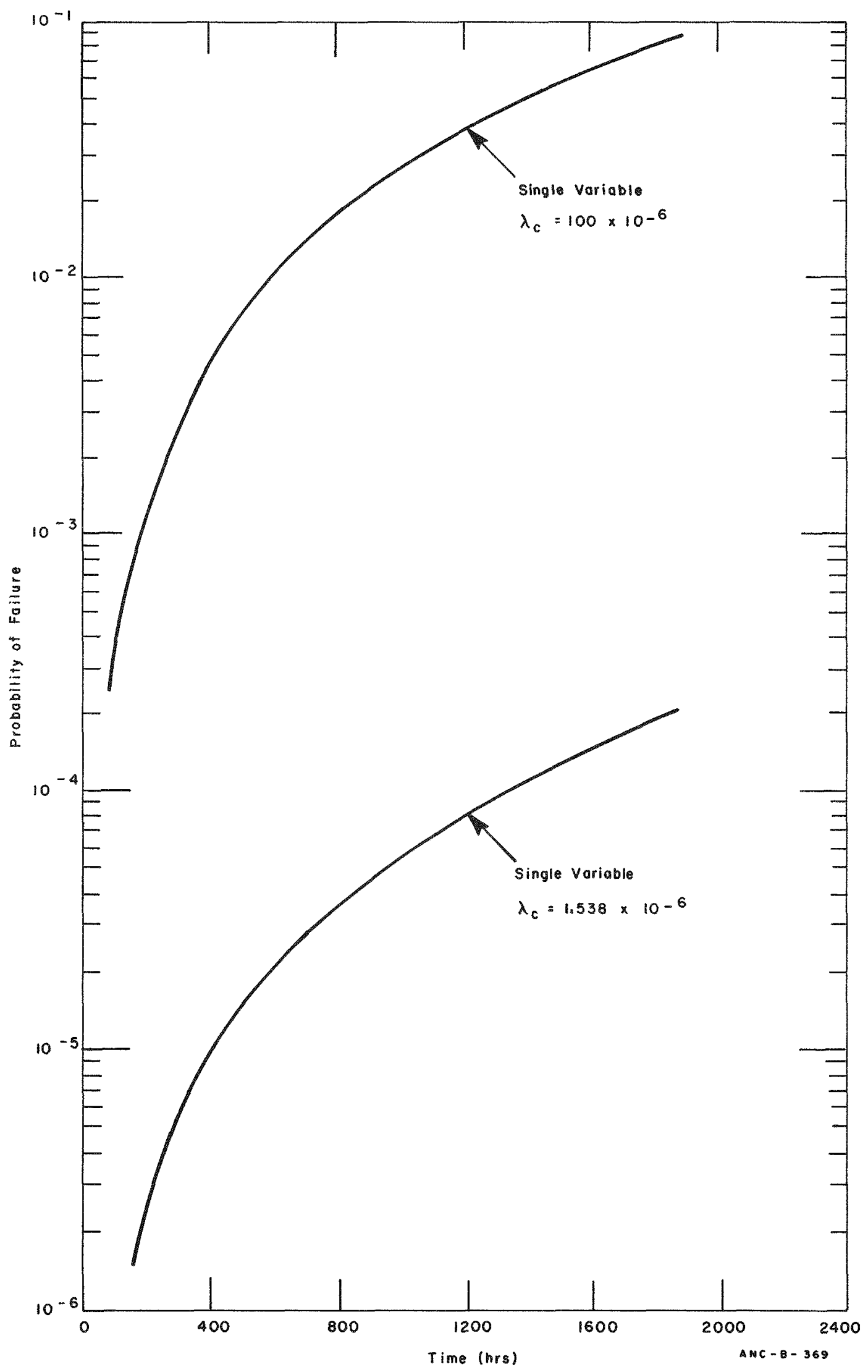

Fig. 4 Reliability -- System I. 


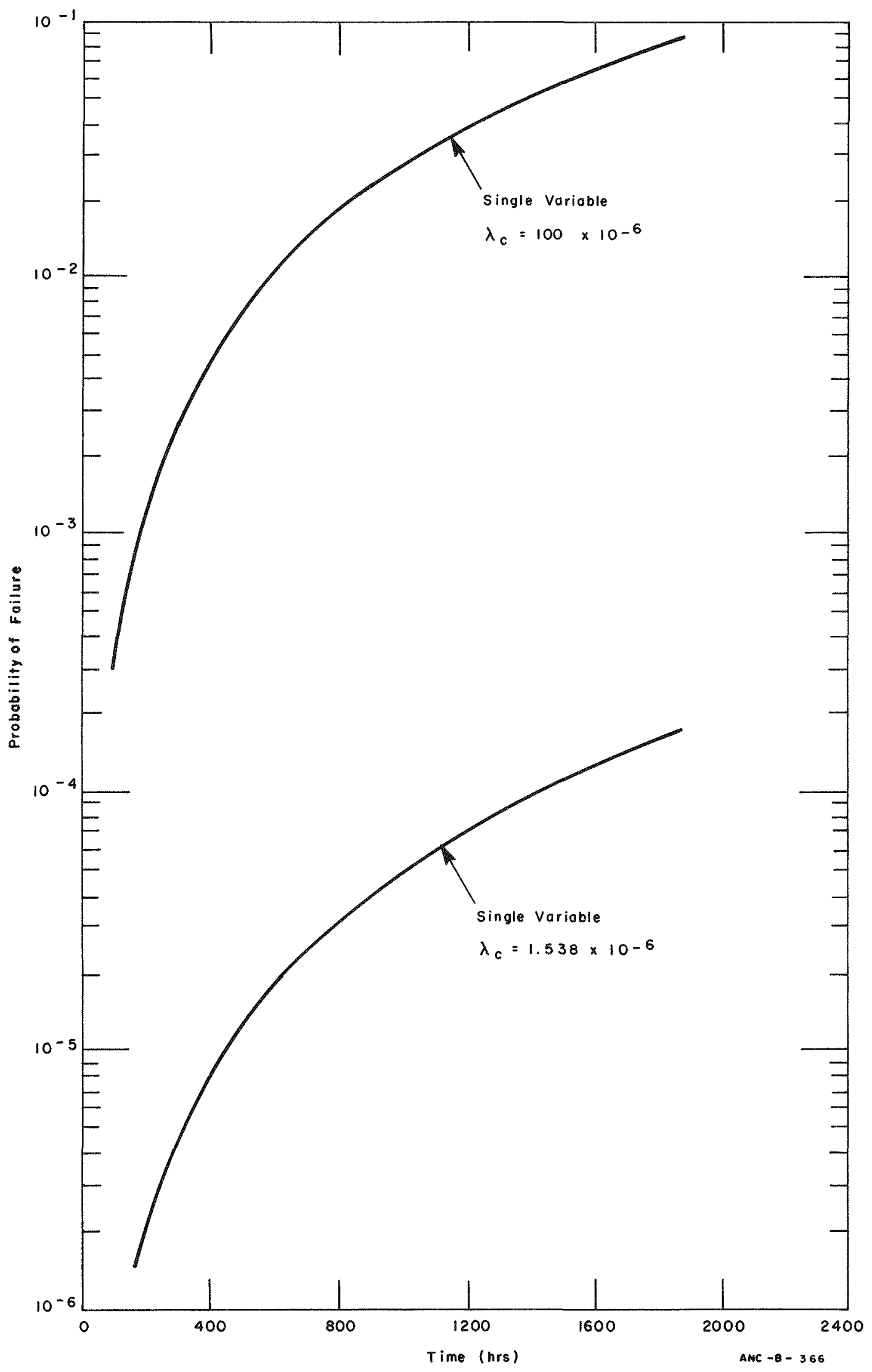

Fig. 5 Reliability -- System II. 


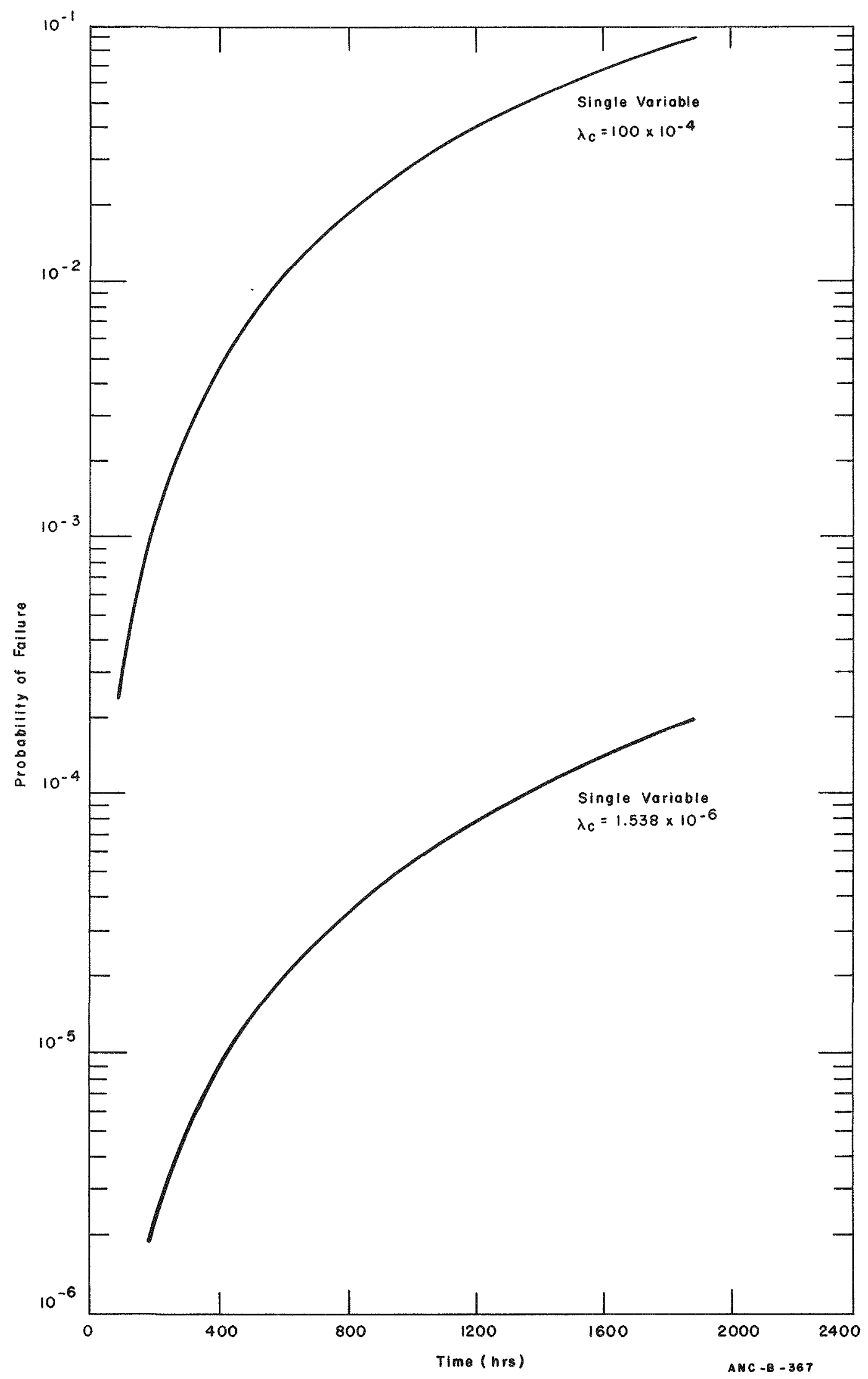

Fig. 6 Reliability -- System III. 


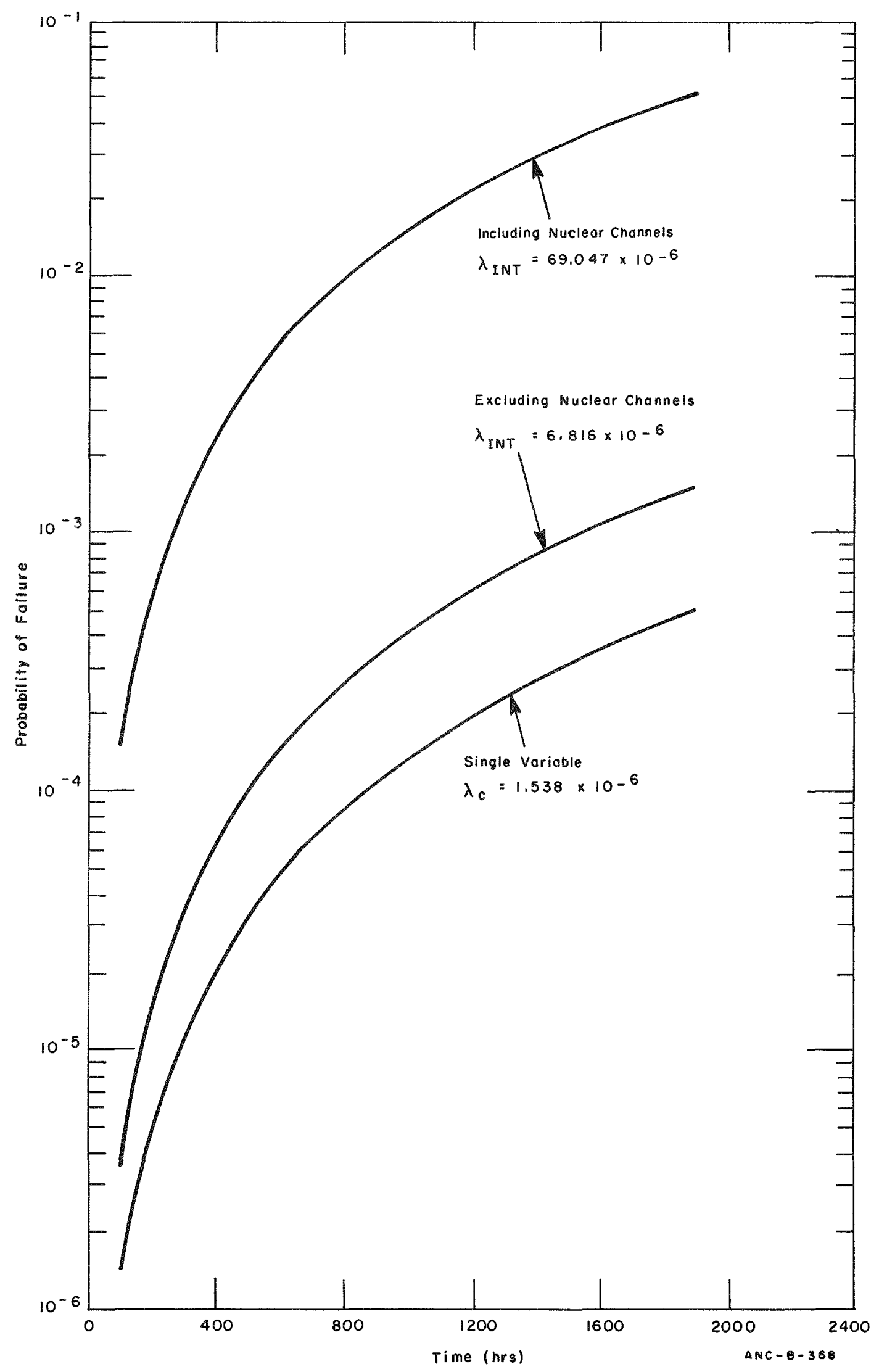

Fig. 7 Plant availability -- System I. 


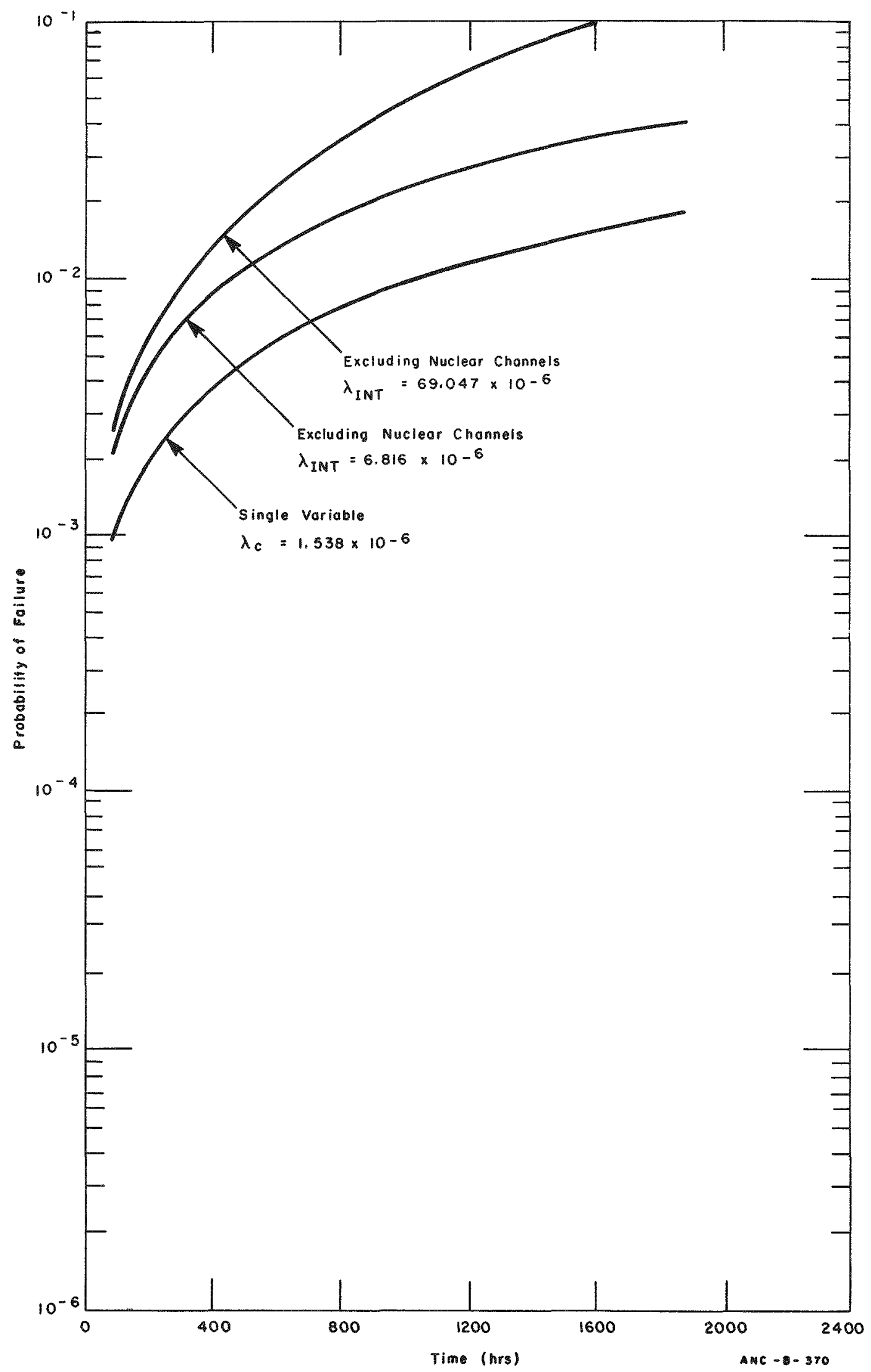

Fig. 8 Plant availability -- System II. 


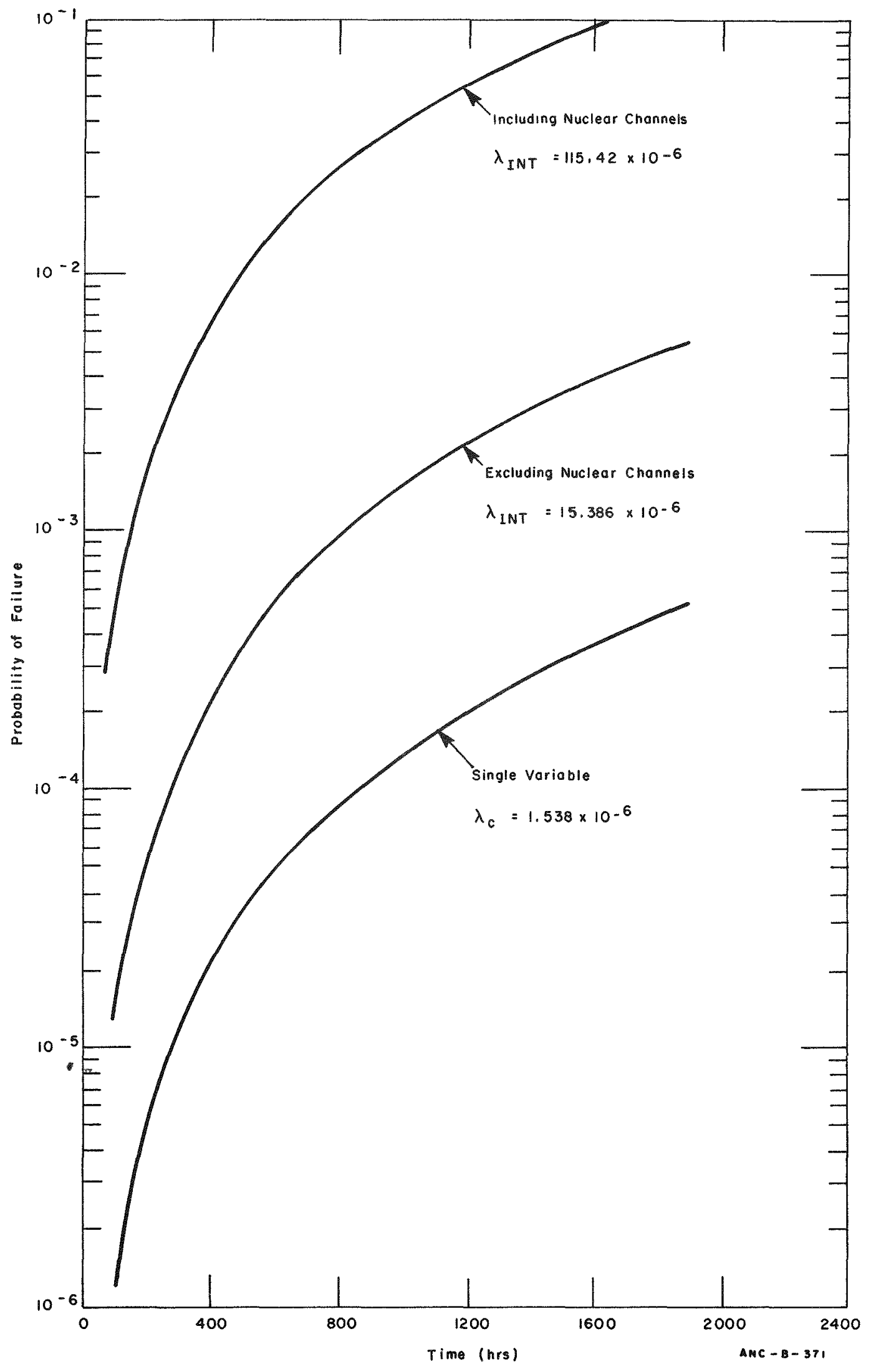

Fig. 9 Plant availability -- System III. 
F. RESEARCH AND DEVELOPMENT FOR NONREACTOR RELATED DISCIPLINES 
○

- 


\title{
IARGE SCALE IRRADIATION OF BIOLOGICAL SAMPIES FOR MERCURY ANALYSIS
}

\author{
J. R. Berreth, D. A. George
}

The increasing concern on possible contamination of game birds, fish and other food stuffs for human consumption with dangerous levels of mercury prompted the State of Idaho to approach the $A B C$ and Idaho Nuclear Corporation in June of 1970 , to perform neutron activation analysis for $\mathrm{Hg}$ on 1000 samples. The only facility available to perform the large scale irradiation was the Materials Testing Reactor (MTR), which had been put on a standby basis in April of 1970. At the State's request, the AEC granted permission to operate the MTR for 48 hours in order to perform the 1000-sample irradiation. The types of samples were quite varied but the immediate concern was possible dangerous levels of Hg in pheasants.

Because of the large number of samples to be irradiated in a high flux at one time, the core region of the reactor was the only practical location. Quartz was chosen for encapsulating the samples because it would not activate and would be able to withstand the gamma heat gertaticr and internal pressure buildup. The samples would be counted without removal from the quartz vials.

The quartz vials were prepared from $7 \mathrm{~mm}$ I.D. by $8 \mathrm{~mm} \mathrm{O.D.} \mathrm{tubing}$ and the samples were lyophilized directly in the tubes. Prior to sealing, the lower end of the tube and its contents were frozen in a liquid nitrogen bath then the vial was flamed shut with a hydrogen torch. The average length of the sealed vials was $1-1 / 2 \mathrm{in}$. with the $60 \mathrm{mg}$ of dry sample occupying $1 / 3 \mathrm{cr}$ less of the volume. The vials were placed in arrays of six in a circle plus one in the center. These arrays of 7 vials were then stacked 6 layers high, with spacers between each layer, into an $1-1 / 8$ in. $0 . D$. by $10-1 / 2$ in. long aluminum capsule. Thus, each capsule contained a total of 42 vials. The aluminum capsules purposely had holes at top and bottom for water to circulate around the vials to keep them cool while in the reactor.

Each capsule had two $\mathrm{Hg}$ standards and one empty vial which were used for calibration of the Hg peaks and subtraction of any activity due to the quartz. The Hg standards were prepared by freeze drying some pheasant meat previously determired to be free of $\mathrm{Hg}$ and then spiking a piece similar to the sample size with a $100 \lambda$ solution containing $1.0 \mu \mathrm{g}$ of $\mathrm{Hg}$ as $\mathrm{Hg}\left(\mathrm{NO}_{3}\right)_{2}$. The sriked sample was then freeze dried and sealed. In this manner the spike was as nearly as possible the same as the sample in order to account for any differences in self-absorption of the low energy gamma rays of the ${ }^{197} \mathrm{Hg}$ formed.

Nine aluminum capsules containing mostly pheasant samples were placed in the "A" positions of the reflector area of the core in the MIR. Sirce the neutron flux intensity decreases as you approach the ends of the core, only two 10-1/2 in. capsules were placed in each "A" position, one on each side of the peak flux which is near the core center line, thus minimizing the variation in the flux that each capsule received. The neutron intensity varied from approximately $2.5 \times 4 \times 10^{13} \mathrm{n} / \mathrm{cm}^{2} \mathrm{sec}$. 
Shown in Figure $I$ are the locations of the capsules in the grid work of the reactor. The capsules in the "L" positions were irradiated at a higher flux $\left(2-4 \times 10^{14} \mathrm{n} / \mathrm{cm}^{2} \mathrm{sec}\right)$ and consisted of the samples to be counted at a later date. Although not all of the grid is available for irradiations (some space is needed for fuel and beryllium reflector) one can readily see there is a large volume available for capsule activations.

The reactor was operated for 48 hours. The advantage of this long irradiation was that the 15 hour sodium activity in the samples would approach saturation, whereas the longer lived $\mathrm{Hg}$ activity $(197,203 \mathrm{Hg})$ would continue to increase with time. After irradiation, the samples were allowed to stand for six days, then opened under water (to provide radiation shielding mostly from the $A I$ capsules) and moved to the laboratory. Each quartz vial was treated with $8 \mathrm{~N} \mathrm{HNO}_{3}$ to remove ary surface activity, and mounted on individual 2 in. $x 3$ in. counting cards. The radiation present due to each sample was approximately $30 \mathrm{mR}$ beta and 3 to $5 \mathrm{mR}$ gamma at the time of mourting. Actual gamma counting on the large $\mathrm{Ge}(\mathrm{L} i)$ detectors was started approximately 10 days after removal from the reactor.

Some loss of quartz encapsulated samples occurred, particularly with the samples that received high irradiation doses. Only four samples were lost in the over 300 samples that were irradiated in the $3 \times 10$ neutrcn flux region. Somewhat cver ino samples were lost in the 2 to $4 \times 10^{14}$ neutron flux region. Several factors brought about this loss. The higher gamma intensity caused increased degeneration of the tissue material, resulting in higher gas pressures and increased stored energy in the quartz. Furthermore, no annealing of the stored energy could occur because the quartz temperature was kept low to minimize gas pressure buildup. Most of the breakage occurred after the irradiation. Loss of the sensitive quartz vials occurred during opening of the aluminum capsules, during the mounting procedure, or they broke just on sitting before they had a chance to be counted.

A few experiments were conducted with pheasant muscle tissue to check the possible loss of $\mathrm{Hg}$ during lyophilization. Muscle tissue taken from a pheasant known to have $\mathrm{Hg}$ was irradiated in sealed vials, both as fresh and as freeze dried tissue. The few experiments performed were not conclusive, but variations in $\mathrm{Hg}$ activity indicated $15 \%$ to $50 \%$ less activity in the dried samples. It is possible loss may vary. with freeze drying time, vacuum pumping rate, precooling conditions or some other phenomenon, none of which we were able to check. It may be there is a variation of $\mathrm{Hg}$ content in the muscle tissue themselves. 


\section{PLAN OF MTR REACTOR LATTICE AND BERYLLIUM REFLECTOR}

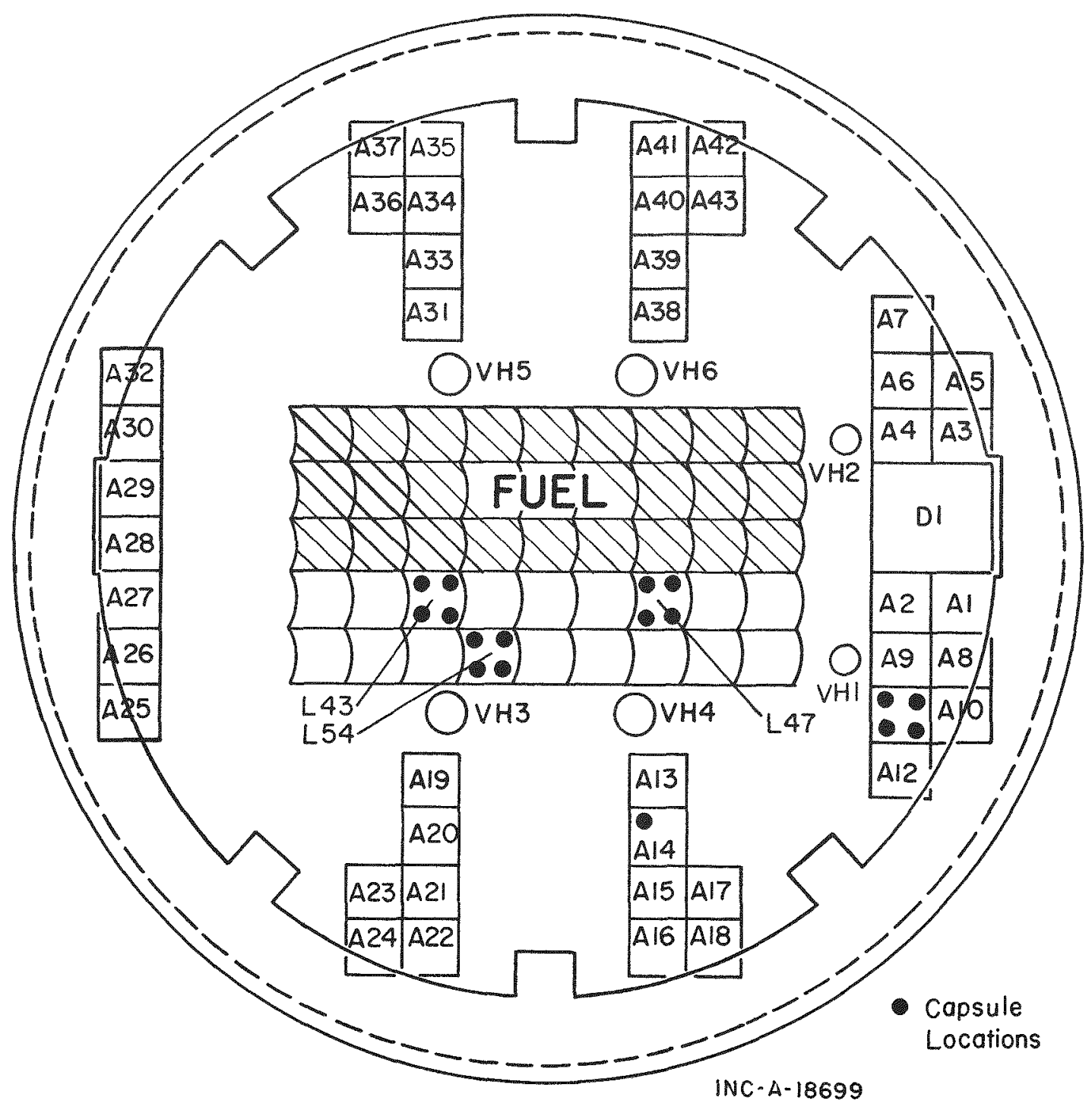

Figure 1. Materials Testing Reactor lattice plan. The small circles show the capsule locations in relation to the fuel. 


\section{AEROSPACE MEDICAL ACTIVATION ANALYSIS PROGRAM}

J. R. Berreth, J. E. Cline, L. D. MeIsaac, P. D. Randolph

E. H. Turk, D. A. George, M. J. Laird

Neutron activation analysis is being recognized as a sensitive and precise method of detecting trace elements[1]. Realizing the method's utility, the Air Force Aerospace Medical Group (AAMG) requested that Idaho Nuclear Corporation start a program of neutron activation analysis on samples of blood and serum. Chemical elements from atomic No. Il through 82 which are activated by thermal neutrons are being analyzed by use of the radioactivities which they produce. The samples were irradiated in the ETR pneumatic rabbit facility using newly developed plastic rabbits $[2,3]$. Because of the wide range of half lives encountered and the large amounts of sodium present, each sample was divided into two parts for separate treatments. One part was irradiated for 2 minutes and gamma counted immediately for determination of short lived materials. This same sample was re-irradiated at a later date for 3 hours and analyzed with no chemical treatment. The second sample was irradiated for 3 hours with immediate chemical treatment to remove the large amounts of sodium activity and gamma counted for determining intermediate half lived materials. Chemical treatment consisted of dissolving the sample in hot concentrated. $\mathrm{HNO}_{3}$ and heating to dryness. After this, the sample was dissolved in conc $\mathrm{HNO}_{3}$ and $\mathrm{HClO}_{4}$ was added and again evaporated to near aryness. The residue was then taken up in conc $\mathrm{HCl}$ and passed through a $10 \mathrm{~cm} \times 8 \mathrm{~mm}$ diameter column of hydrated antimony pentoxide (HAP) column [4]. The column retains the radioactive ${ }^{24} \mathrm{Na}$ but allows all other chemical elements to pass through. With this procedure some volatile elements, such as $\mathrm{Br}$, are partially lost during digestion. Therefore, one reason for the 3-hour irradiation with no chemistry was to determine the concentration of the elements such as $\mathrm{Br}$, which are partially or completely lost in the digestion step.

In order to calibrate the technique, series of standard samples with elements of known composition were prepared and irradiated under the same conditions as the unknowns. Cobalt monitors were irradiated with both the samples and the standards to provide an accurate external flux measurement for comparison purposes.

Samples were gamma counted using a $35 \mathrm{cc}$ Ge(Li) detector with a 4096 channel analyzer and magnetic tape readout. Analysis of the data was done by means of an automatic spectrum analysis program known as GAUSS [5]. Detailed and careful comparisons of the sample spectra with the standard element spectra were made, thereby providing quantitative determinations of the elements present in the sample. Typical elements found in the samples were $\mathrm{Na}, \mathrm{Cl}, \mathrm{K}, \mathrm{Br}, \mathrm{Fe}, \mathrm{Cu}, \mathrm{Se}, \mathrm{Hg}, \mathrm{Au}$ and $\mathrm{Zn}$. Limits of detection were in the submicrogram range.

[1] Dick Tracy, Salt Lake Tribune (August 23, 1971).

[2] J. R. Berreth and F. O. Cartan, "ETR Pneumatic Rabbit Irradiation Facility", this report.

[3] D. A. George, "Plastic Irradiation Capsules", this report. 
[4] F. Girardi, E. Sabbioni, "Selective Removal of Radio-Sodium from Neutron Activated Materials by Retention on Hydrated Antimony Pentoxide", Journal Radioanal. Chem., Vol. I, (1968) pp. 169-178.

[5] R. G. Helmer, et al., "Photopeak Analysis Program for Photon Energy and Intensity Determinations", Nuc. Instr. and Meth. 57, (1967) pp. 46-57. 
ELEMENTAL DETERMINATIONS IN IDAHO WILDLIFE SAMPLES

AND IN AIR FILTER SAMPLES

J. E. Cline, P. D. Randolph, E. L. Murri, [I]

L. D. McIsaac, M. J. Laira

Introduction The technique of neutron activation analysis offers many advantages over other analysis techniques in that it represents a survey type of measurement where many elements present in trace quantities can be simultaneously and non-destructively analyzed with relatively high sensitivity and precision. It has particularly useful application in the measurement of trace quantities of the heavier elements in biological tissues where the bulk of the sample consists of hydro-carbons, nitrogen and water.

Wildlife Samples One thousand samples were analyzed by the techniques of neutron activation, primarily for mercury and selenium, expected to be present in quantities ranging up to several parts per million. Also present in the samples were traces of fifteen other elements. Since the experiment was specifically designed to detect mercury and selenium, several other elements that activate with significantly longer or shorter lived activities were not observed.

A list of the wildlife samples supplied by the State of Idaho and irradiated in the MTR is given in Table I. In addition to these 1000 samples, 60 mercury standards and 32 empty quartz ampules were irradiated. Approximately $10 \%$ of these samples were ruptured, either during the irradiation itself or during the preparation of the samples for analysis. The primary cause of the capsule failures is the large latent energy buildup in the quartz ampules during irradiation.

The samples were divided into two groups, one containing the priority samples and the other containing the remainder. The priority group consisted of approximately 300 samples of pheasant, chinook salmon, mourning doves, partridges, ducks and human blood and their associated standards and empty capsules. The results from these samples were desired within two weeks following the beginning of the irradiation so that adequate information would be available prior to the hunting season. These samples were counted using five high-volume, high-resolution $\mathrm{Ge}(\mathrm{Li})$ spectrometers. The samples, in the quartz ampules, were counted at a fixed distance of approximately $4 \mathrm{~cm}$ from the center of the ampule to the nearest face of the detector. The counting time for each sample was $1000 \mathrm{sec}$, live time. All 21 samples from a given half capsule (containing the standard and empty capsule) were counted using the same spectrometer so that the quantities of mercury observed could be directly and easily compared to the $1 \mu \mathrm{g}$ of mercury in the standard. Considerations of absolute neutron flux and detection efficiencies need not be made in such measurements. The 330 samples were counted in two 16-hr days. 
$\underline{\text { TABLE I }}$

LIST OF IDAHO WILDLIFE SAMPLES

$\begin{array}{lrlr}\text { Pheasant breast samples } & 123 & \text { Human blood samples } & 46 \\ \text { Other pheasant samples, i.e., } & & \text { Hungarian partridges } & 4 \\ \text { kidney, brain, thigh, liver } & 20 & \text { Quail } & 17 \\ \text { Pheasant feathers } & 5 & \text { Ducks } & 43 \\ \text { Mourning dove breast samples } & 83 & \text { Geese } & 2 \\ \text { Red meat and food } & 288 & \text { Bobcats } & 13 \\ \text { Grain } & 23 & \text { Fungicide } & 1 \\ \text { Human tissue } & 47 & \text { Vegetation } & 38 \\ \text { Chinook salmon } & 6 & \text { Eagles } & 23 \\ \text { Other types of fish } & 172 & \text { Human urine } & 36 \\ \text { Water samples } & 10 & & \text { TOTAL }\end{array}$

From two of the spectrometers, data were fed into a small digital processor (PDP-9) for near-instantaneous analysis, supplying a continued monitoring of the quality of the data being recorded. The other three spectrometers recorded the data onto IBM compatible magnetic tape for batch analysis using the IBM 360/75 computer.

The remaining 700 samples and standards were collected on a much more leisurely basis. Student help from Idaho State University, paid by a grant to the University, was recruited to count the samples. These samples were counted, in a manner similar to that employed above, using one $\mathrm{Ge}(\mathrm{Li})$ spectrometer. The samples were counted at a rate of approximately 100 samples per week. Data were collected on magnetic tape, inspected, and batched for processing following the completion of the counting.

The 300 high-priority samples were analyzed for mercury through an examination of the quantity of both the $65-\mathrm{hr} 197 \mathrm{Hg}$ and the 46-day ${ }^{203} \mathrm{Hg}$ activities. Although the 8-day cooling period between the end of the irradiation and the initiation of the counting reduced the $197 \mathrm{Hg}$ activity by nearly a factor of 10, it reduced the dominant ${ }^{24} \mathrm{Na}$ (15 hrs) by about a factor of 10,000. This resulted in a relative enhancement of the ${ }^{197} \mathrm{Hg}$ activity to the point where it could be observed without the need for costly chemical separations to remove the ${ }^{24} \mathrm{Na}$ activity. A typical 4096-channel pulse-height spectrum of the gamma rays from one of the mercury-containing pheasant samples is given in Figure I. These data were taken approximately 200 hours following the termination of the irradiation. The peaks at 77 and $279 \mathrm{keV}$ are due to the isotopes $197 \mathrm{Hg}$ and $203 \mathrm{Hg}$, respectively. Also prominent in the spectra is the presence of $122 \mathrm{Sb}, 124 \mathrm{Sb}$ and ${ }^{24} \mathrm{Na}$. 


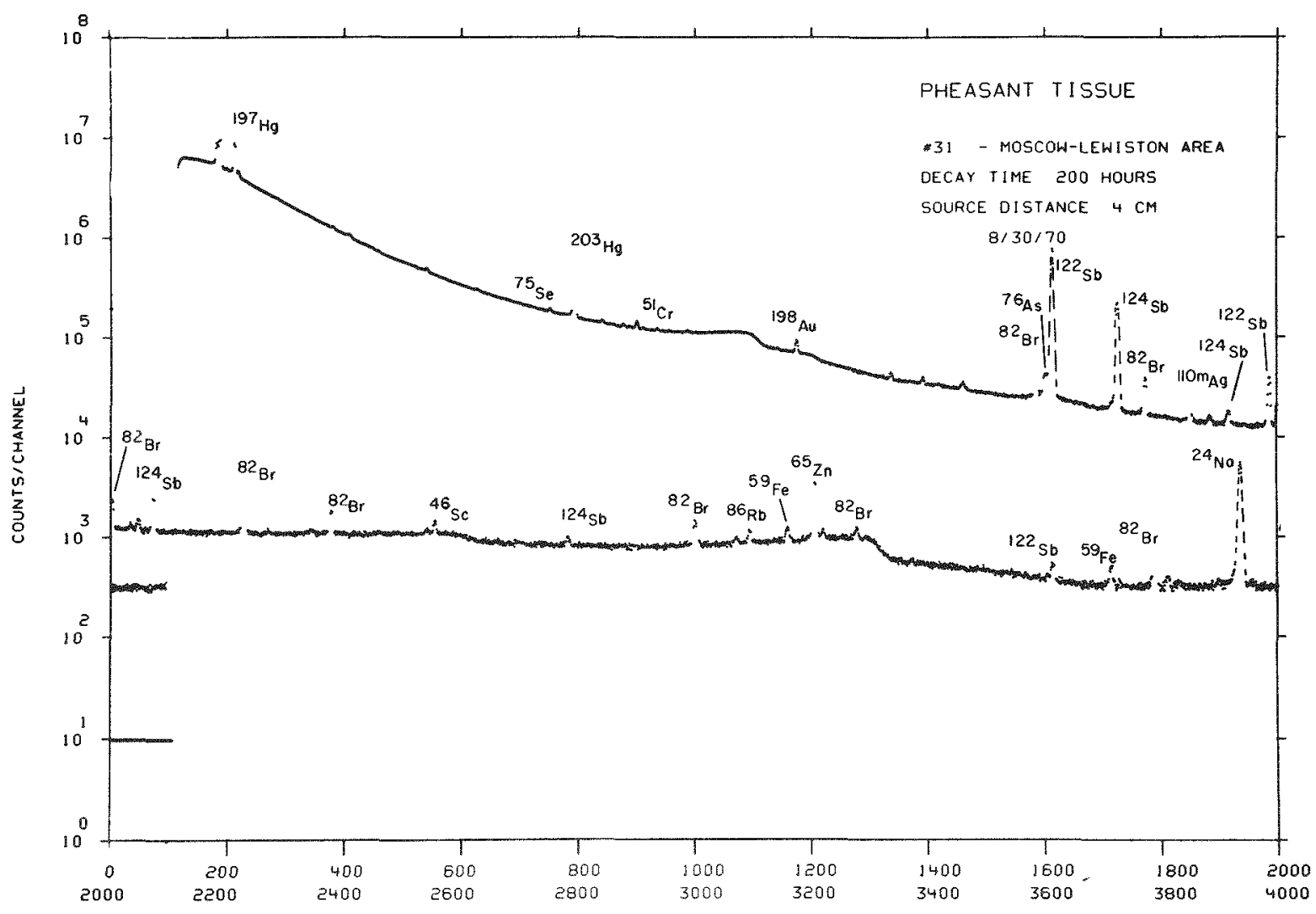

CHANNEL NUMBER

Figure 1

To analyze these and similar data to determine the mercury content of the samples, Gaussian functions together with straight-line backgrounds were least-squares fit in each spectrum to the peaks at 77 and $279 \mathrm{keV}$ to obtain the areas (in counts) of the peaks. These areas were corrected for decay of the two isotopes and compared to similarly obtained peak areas in the standard for that particular sample. Since the standard contained $1 \mu \mathrm{g}$ of mercury $( \pm \sim 2 \%)$, the ratio yielded the $\mu g$ content in the sample itself.

The neutron flux profile across the lo-inch long sample was such that approximately a $10 \%$ correction should be made to the mercury content of the capsules in the two layers adjacent to that containing the standard. This correction was considered negligible when compared to the uncertainties in the loss of mercury in the lyophilization process [2], and hence, the correction was not made.

The remaining samples were counted over a period of two months, starting about 45 days after the irradiation. A typical gamma-ray pulse-height spectrum taken during this period is given in Figure 2. These data are of the sample as in Figure 1, but were taken at 80 days. The ${ }^{197} \mathrm{Hg}$ isotope has totally decayed leaving only the ${ }^{203} \mathrm{Hg}$ ( 46 days). 


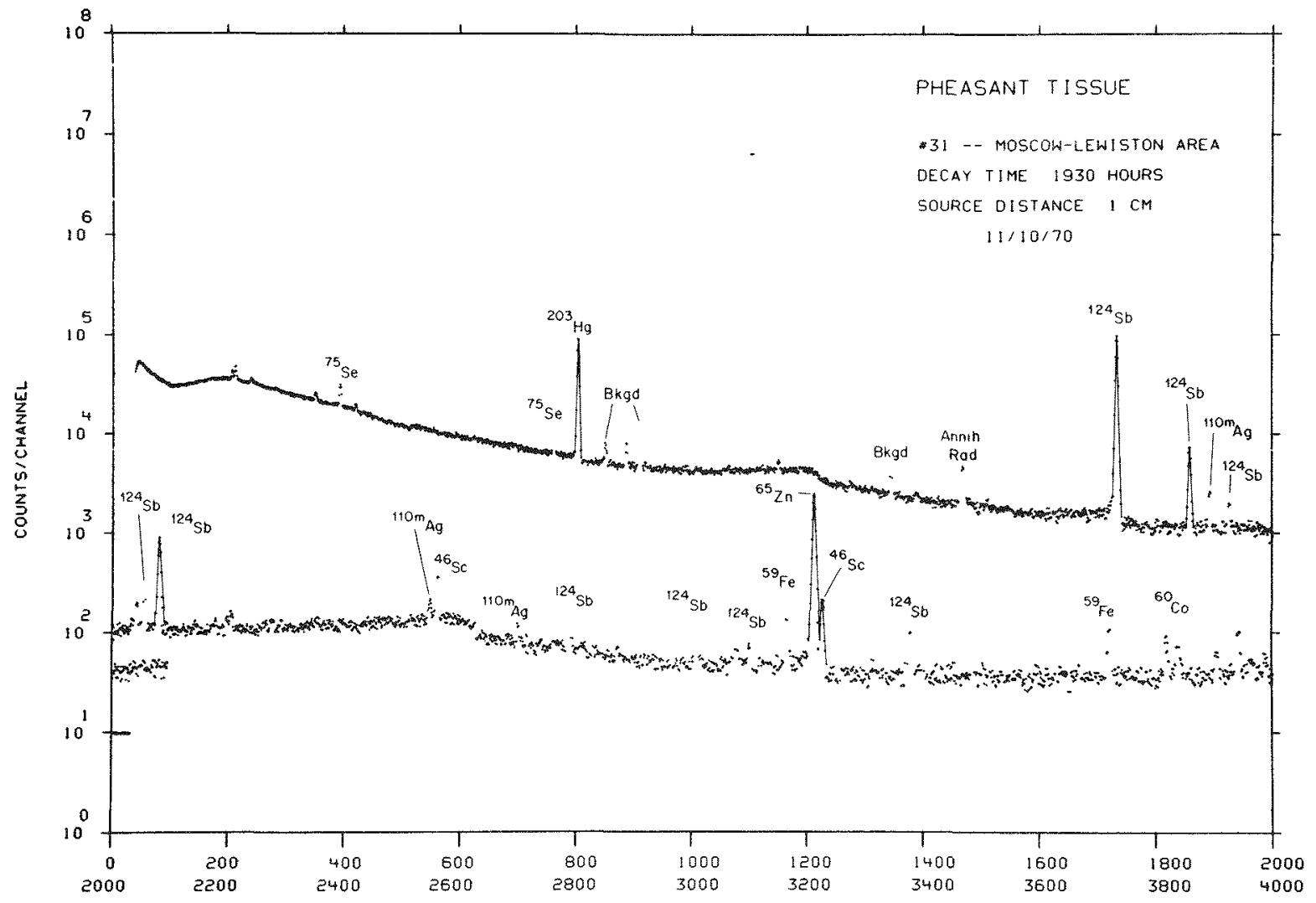

Figure 2

Present also in the sample is ${ }^{75} \mathrm{Se}$. This radioisotope emits gamma rays of 264 - and $279-\mathrm{keV}$ energy, the latter being the same energy within $0.5 \mathrm{keV}$ as the gamma ray emitted by $203 \mathrm{Hg}$. Corrections, therefore, had to be made to the intensity of the $279-\mathrm{keV}$ peak to account for the contribution from ${ }^{75} \mathrm{Se}$. This correction amounted to approximately $40 \%$ of the size of the peak from ${ }^{75} \mathrm{Se}$ at $264 \mathrm{keV}$. For samples with mercury content about $0.5 \mu \mathrm{g}$, such as that of Figure 2, the correction was of small significance, but highly influenced the lower limit of sensitivity.

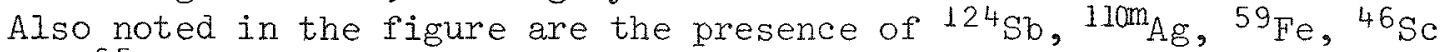
and ${ }^{65} \mathrm{Zn}$. These represent other trace elements in the sample.

The data from the samples were collected on magnetic tape and batched together for processing on the IBM $360 / 75$ computer. Figure 3 shows a block diagram of the steps in the analysis program used on the large machine. This program contains an automatic peak-search routine which uses the techniques of auto-correlation. This portion supplies the input to the peak fit portion which fits a Gaussian function and a straight-line background to each peak. The nuclide identification procedure consists basically of comparing the gamma-ray energies from the analysis with a list of energies and nuclide assignments sorted from a master nuclide data library. This sort is accomplished on the 


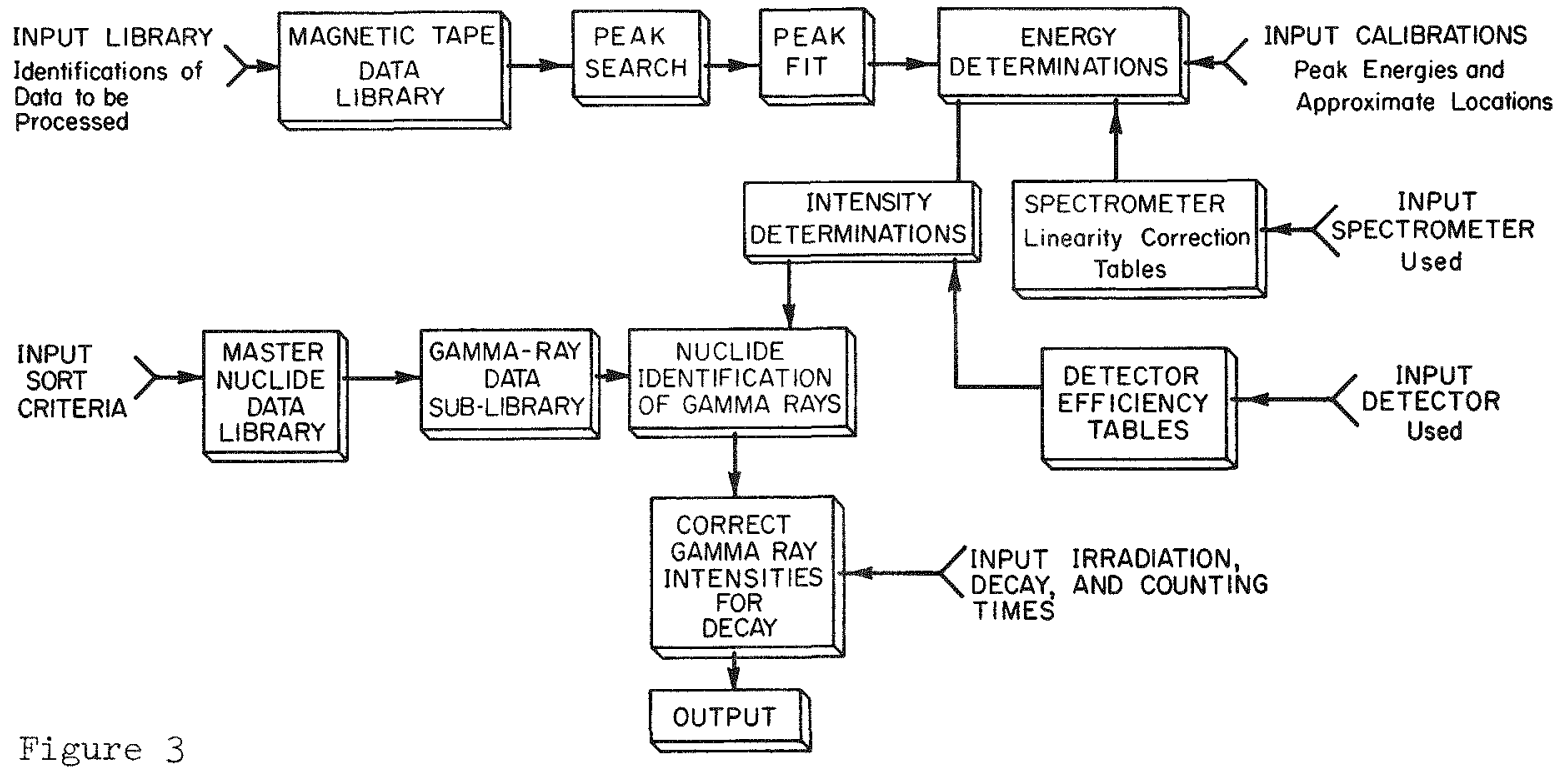

basis of atomic number, half-life, gamma-ray energy, intensity and nuclide production mode restrictions. After nuclidic assignment to a gamma-ray, the intensity of that gamma ray is corrected for decay during the decay time and during the finite irradiation and counting times.

Computer time required per spectrum for complete analysis in batch analyses of 550 spectra was about 20 seconds on the IBM $360 / 75$. This resulted in a computer cost per sample of $\$ 0.50$ when lowest priority computer time was used.

The results of the mercury determinations have been published ${ }^{[3]}$. In addition to the mercury, approximately 16 elements were identified in these measurements. One of the more important of these was selenium, an element required for the existence of healthy tissue. Table II shows the elements found in pheasant sample \#3l, the isotope of that element observed in the data, the half-life and the parts-per-million quantities. At the bottom of the table are the elements that would undoubtedly have been observed with shorter irradiation and decay times than those used to optimize the experiment for mercury. 
TABLE II

ANALYSIS OF PHEASANT SAMPLE \#3I

\begin{tabular}{|c|c|c|c|}
\hline Element & Isotope & & \\
\hline$\frac{\text { Detected }}{N a}$ & $\frac{\text { Detected }}{24 \pi}$ & $\frac{\text { Half-Life }}{150 h}$ & $\frac{\text { Parts/Million }}{383}$ \\
\hline $\mathrm{Sc}$ & $46 \mathrm{Sc}$ & $83.8 \mathrm{a}$ & 0.004 \\
\hline $\mathrm{Cr}$ & ${ }^{51} \mathrm{Cr}$ & $27.8 \mathrm{~d}$ & 0.9 \\
\hline $\mathrm{Fe}$ & ${ }^{59} \mathrm{Fe}$ & $45.1 \mathrm{a}$ & 70 \\
\hline Co & ${ }^{60} \mathrm{Co}$ & $5.3 \mathrm{y}$ & 0.04 \\
\hline $\mathrm{Zn}$ & $65 \mathrm{Zn}$ & $243.6 \mathrm{~d}$ & 20 \\
\hline As & ${ }^{76} \mathrm{As}$ & $26.5 \mathrm{~h}$ & 1.5 \\
\hline $\mathrm{Se}$ & ${ }^{75} \mathrm{Se}$ & $120.4 \mathrm{~d}$ & 0.7 \\
\hline $\mathrm{Br}$ & ${ }^{82} \mathrm{Br}$ & $35.3 \mathrm{~h}$ & 1.0 \\
\hline $\mathrm{Rb}$ & ${ }^{86} \mathrm{Rb}$ & $18.7 \mathrm{a}$ & 1.5 \\
\hline $\mathrm{Zr}$ & $95 \mathrm{Zr}$ & $65.0 \mathrm{~d}$ & 4 \\
\hline $\mathrm{Ag}$ & $110 \mathrm{~m}_{\mathrm{Ag}}$ & $253.0 \mathrm{~d}$ & 0.2 \\
\hline $\mathrm{Sb}$ & $122 \mathrm{Sb}$ & $2.8 d$ & 2.4 \\
\hline & ${ }^{124} \mathrm{Sb}$ & 60.3 a $\}$ & \\
\hline La & $14^{0} \mathrm{La}$ & $40.2 \mathrm{~h}$ & 0.01 \\
\hline Ir & ${ }^{192} \mathrm{Ir}$ & $74.2 \mathrm{~d}$ & 0.0005 \\
\hline $\mathrm{Au}$ & $198 \mathrm{Au}$ & $2.7 a$ & 0.001 \\
\hline $\mathrm{Hg}$ & $197 \mathrm{Hg}$ & $65.0 \mathrm{hl}$ & \\
\hline & $20{ }^{3} \mathrm{Hg}$ & $46.6 \mathrm{a}\}$ & 1.7 \\
\hline
\end{tabular}

Elements which might have been detected with shorter irradiation and decay times: $\mathrm{Mg}, \mathrm{AI}, \mathrm{Cl}, \mathrm{K}, \mathrm{Ca}, \mathrm{V}, \mathrm{Mn}$, $\mathrm{Ca}, \mathrm{Cd}$, In, Sm, Eu, W, Pb.

For the present measurements, the sensitivity for mercury detection was about $0.002 \mu \mathrm{g}$, corresponding to $20.008 \mathrm{ppm}$. This is the smallest quantity of mercury which would yield an analyzable peak either at $77 \mathrm{keV}$ (at earlier times) or at $279 \mathrm{keV}$ in the spectra. From the data in Figures $l$ and 2 , it is quite apparent that this sensitivity is very subject to the type and quantities of the other activities in the spectra. In these samples, and for all the samples in this experiment, the sensitivity is limited primarily by the presence of antimony which is responsible for the major peaks in each spectrum. The accuracy, however, of the mercury determinations was limited almost solely by the uncertainties in the loss of the mercury in the sample preparation. In some of the test, the losses were as high as 50\%. Such losses would undoubtedly also be experienced for some of the other volatile elements, particularly bromine. 
Air Filters An air sampling station was located in the downtown area of Cleveland, Ohio. Two thousand cubic meters of air were drawn through a filter $25 \times 20 \mathrm{~cm}$ in area. Small portions of this filter paper, about $10 \mathrm{~cm}^{2}$, were irradiated simultaneously with a known amount of gold in a neutron flux of about $2 \times 10^{13} \mathrm{n} / \mathrm{cm}^{2} \mathrm{sec}$. Two irradiations were made, one of 5-minute duration and the other of 5 hours. The gold in a neutron flux of about $2 \times 10^{13} \mathrm{n} / \mathrm{cm}^{2} \mathrm{sec}$. Two irradiations were made, one of 5-minute duration and the other of 5 hours. The irradiated samples were counted with a large volume, high-resolution $\mathrm{Ge}(\mathrm{Li})$ spectrometer at decay times of 4 minutes, 15 minutes, 1 hour, 20 hours, 45 hours, 139 hours, and 314 hours. Some of the gamma-ray pulse-height spectra from these measurements are presented in Figures 4-8. No chemical separations were made on either of these samples. Twenty-eight elements were identified in the measurements. Table III gives a list of these elements, the isotope used for the determination, the half-life, the gamma-ray energy, the amount of that element that was determined to be in the sample, and the minimum amount of that element needed to be present for the element to have been detected. The values in the last column are based on the assumption that all elements other than that particular element were present in the measured abundances. Thus the elemental sensitivities in a practical experiment are subject to the quantities and types of the other elements present in the sample.

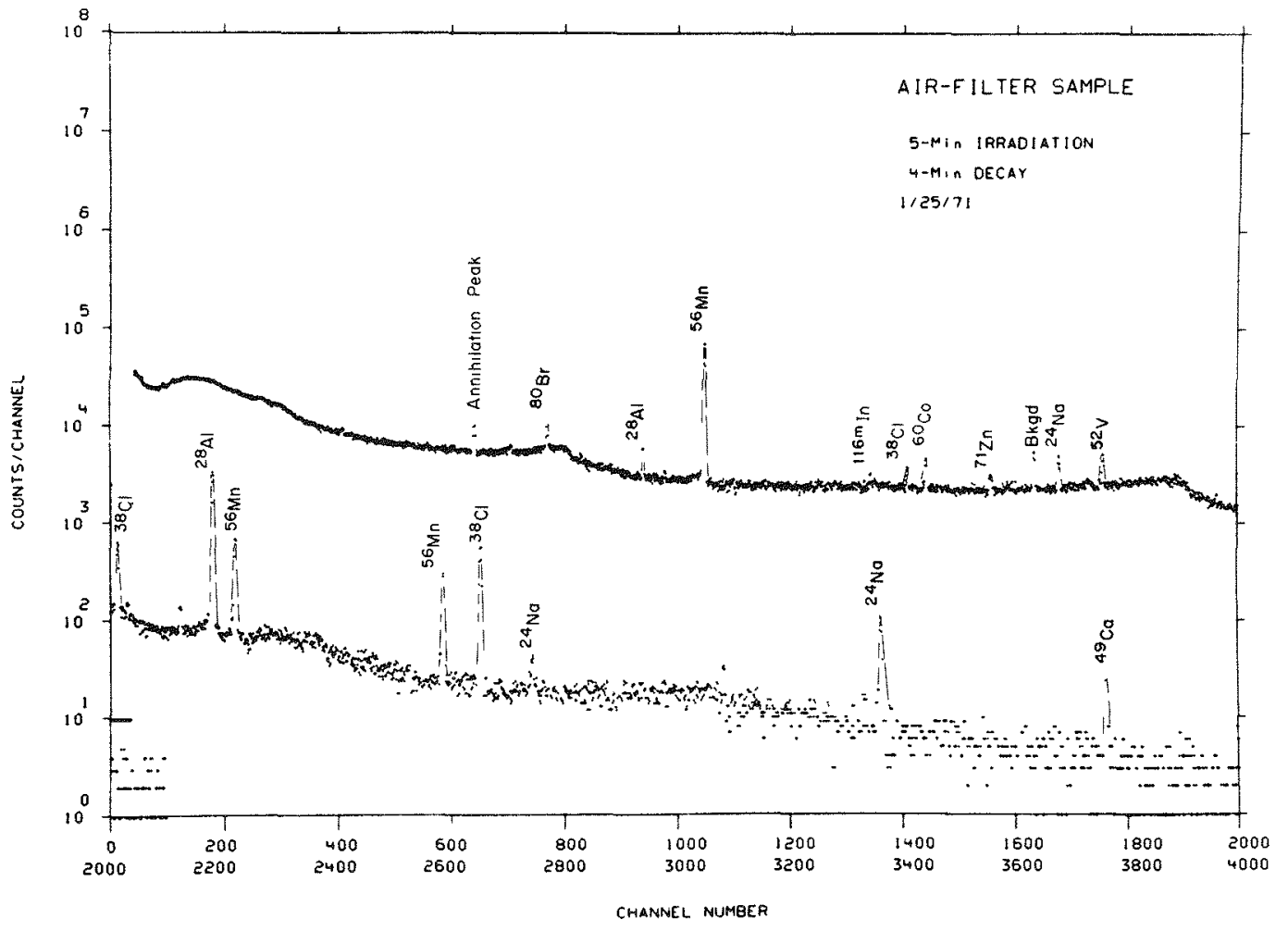




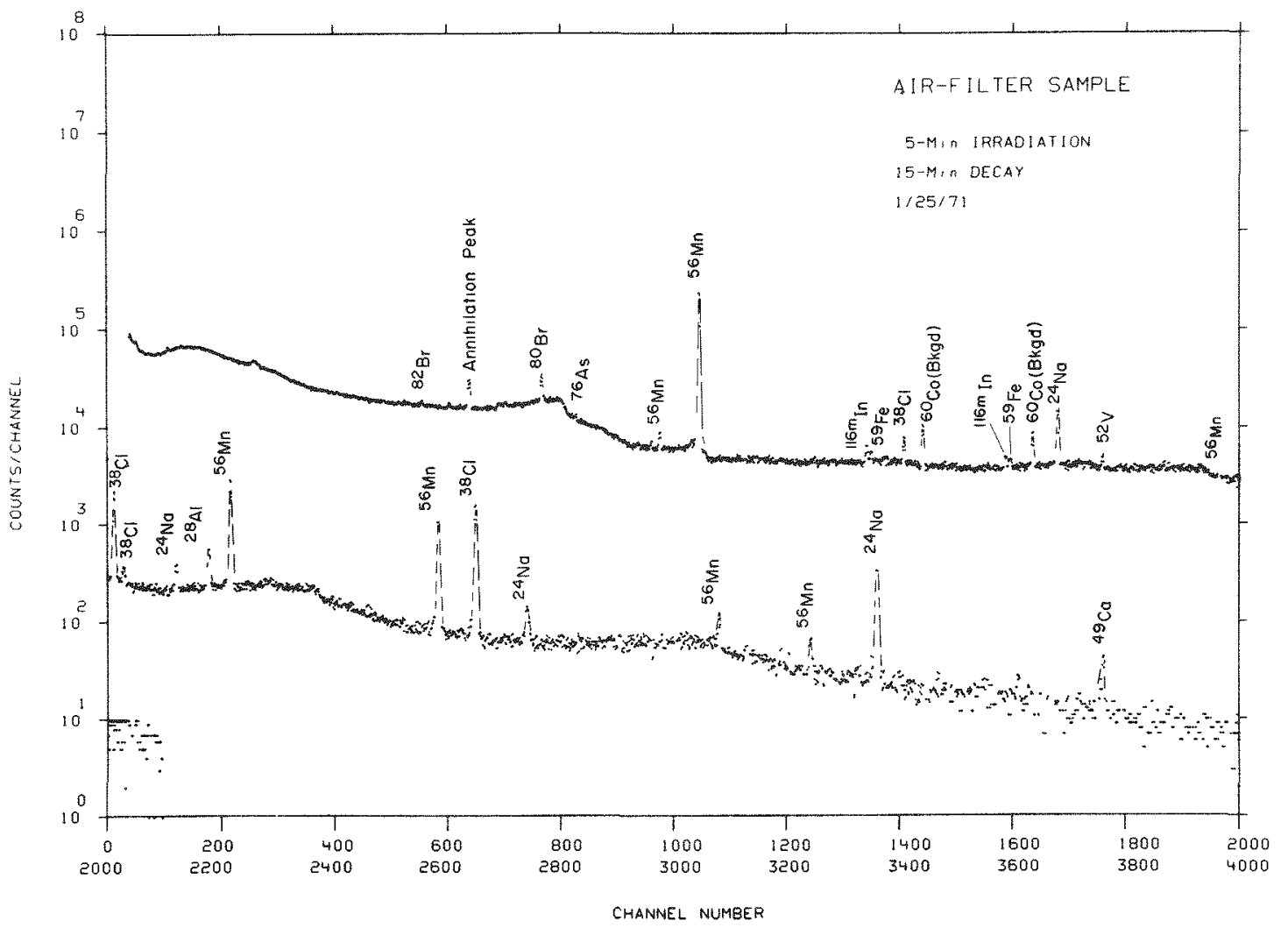

Figure 5

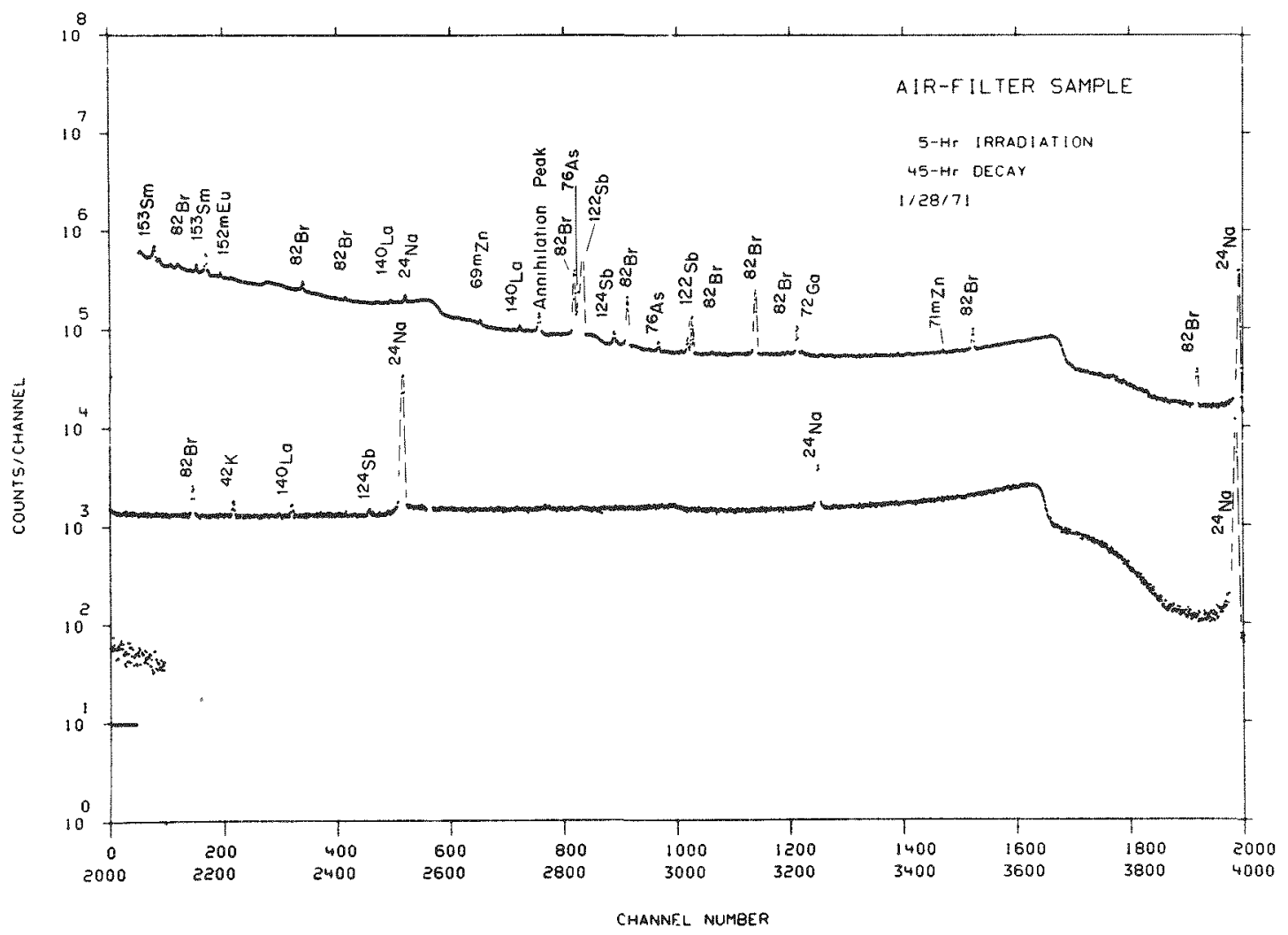

Figure 6 


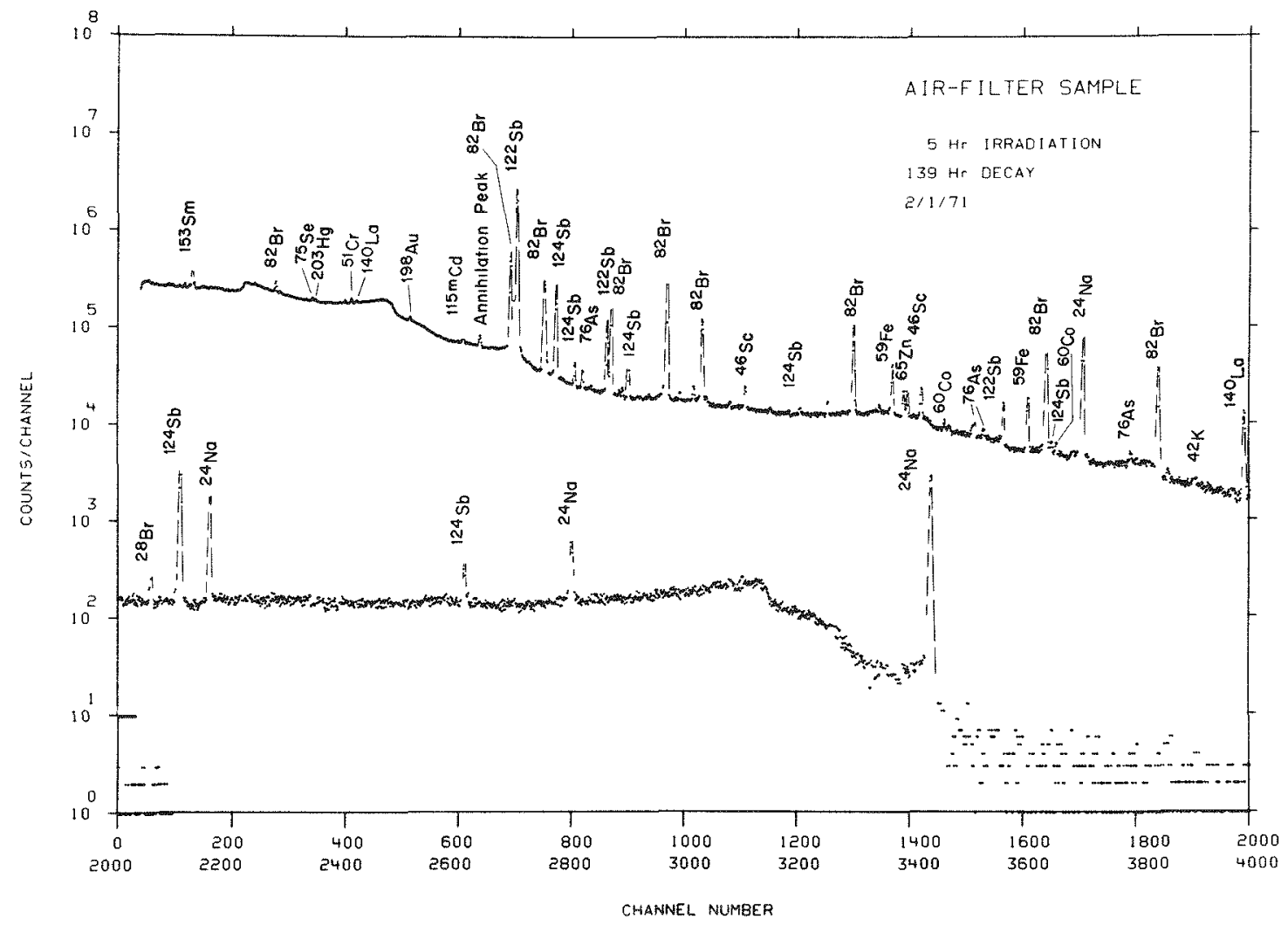

Figure 7

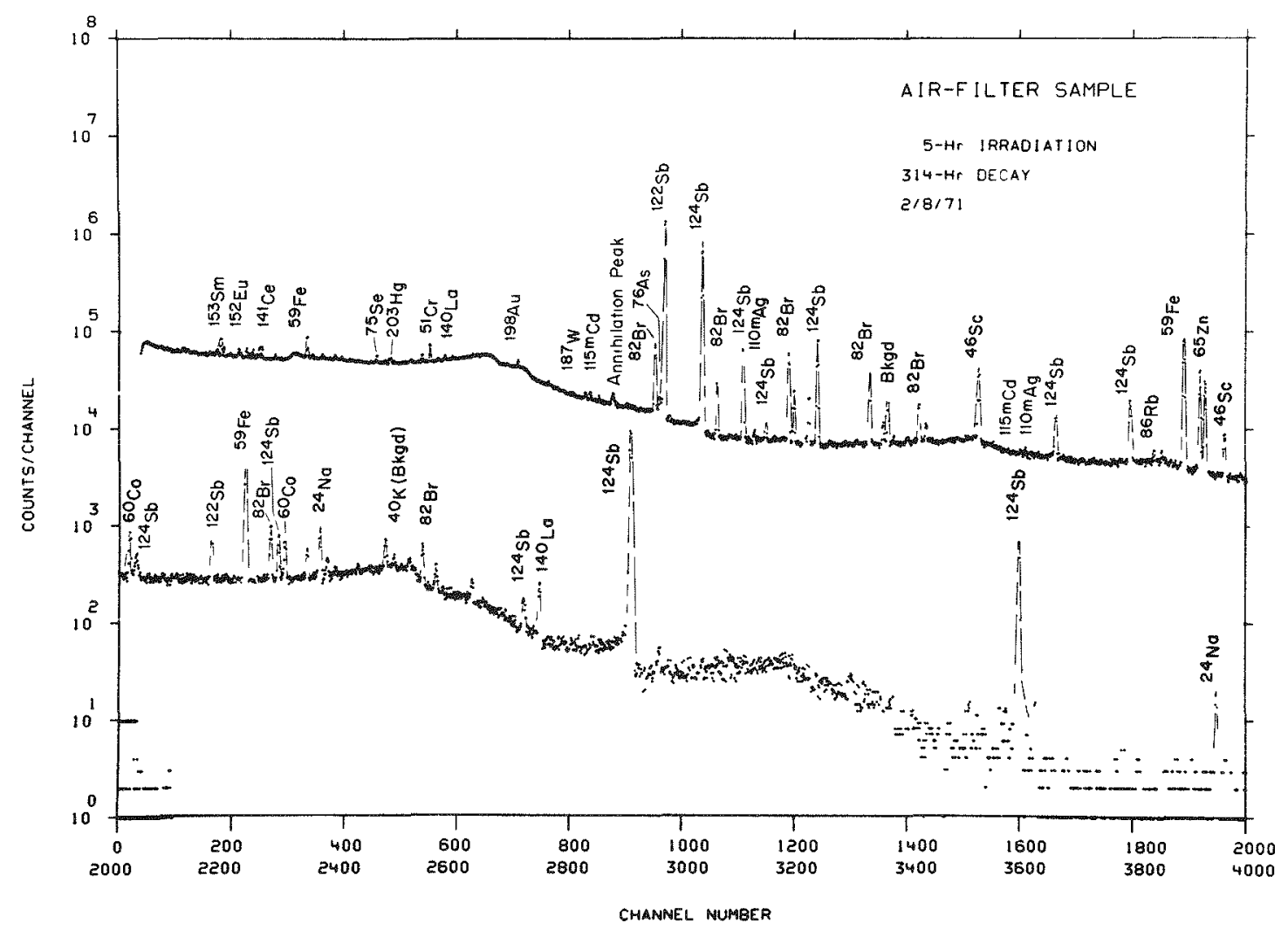

Figure 8 
TABLE III

ELEMENTS DETECTED IN AIR FILTER SAMPLE

Element

Detected

Na

$\mathrm{Mg}$

AI

$\mathrm{Cl}$

$\mathrm{K}$

$\mathrm{Ca}$

$\mathrm{Sc}$

$\mathrm{V}$

$\mathrm{Cr}$

$\mathrm{Mn}$

$\mathrm{Fe}$

Co

$\mathrm{Zn}$

$\mathrm{Ga}$

As

$\mathrm{Se}$

$\mathrm{Br}$

$\mathrm{Rb}$

Ag

$\mathrm{Ca}$

In

$\mathrm{Sb}$

La

$\mathrm{Ce}$

$\mathrm{Sm}$

$\mathrm{Eu}$

$\mathrm{Au}$

$\mathrm{Hg}$
E $(\mathrm{keV})$ $\frac{T_{1 / 2}}{15} \frac{\gamma}{\text { used }}$

$24 \mathrm{Na}$

$2 / \mathrm{Mg}$

${ }^{28} \mathrm{AI}$

${ }^{38} \mathrm{Cl}$

$42 \mathrm{~K}$

${ }^{49} \mathrm{Ca}$

${ }^{46} \mathrm{Se}$

$52 \mathrm{~V}$

${ }^{51} \mathrm{Cr}$

$56 \mathrm{Mn}$

$59 \mathrm{Fe}$

${ }^{60} \mathrm{Co}$

$65 \mathrm{zn}$

${ }^{72} \mathrm{Ga}$

$76 \mathrm{As}$

7 be

$82 \mathrm{Br}$

$86 \mathrm{Rb}$

$11 \cup \mathrm{m}_{\mathrm{Ag}}$

$115 \mathrm{Cd}$

$116^{\mathrm{m}} \mathrm{In}$

$122 \mathrm{Sb}$

$124 \mathrm{Sb}$

$140 \mathrm{La}$

${ }^{141} \mathrm{Ce}$

$153 \mathrm{Sm}$

15. ${ }^{\mathrm{m}} \mathrm{Eu}$

$198 \mathrm{Au}$

$20{ }^{3} \mathrm{Hg}$
$9.5 \mathrm{~m} \quad 1014.4$

$2.3 \mathrm{~m} \quad 1778.9$

$37.3 \mathrm{~m} 2167.3$

$12.4 \mathrm{~h} 1524.7$

$8.8 \mathrm{~m} 3084.4$

83.8 a 889.3

$3.8 \mathrm{~m} 1434.0$

27.8 a 320.1

2.6 h $\quad 1810.7$

45.1 d 1099.2

$5.3 \mathrm{y} \quad 1173.2$

243.6 a $\quad 1115.5$

$14.1 \mathrm{~h} 833.9$

26.5 h 559.1

120.4 d 264.7

35.3 h 776.5

18.7 a 1076.8

253 d 657.7

53.5 h 527.8

$54.0 \mathrm{~m} \quad 1293.6$

2.8

60.3 a 602.7$\}$

40.2 h 1596.2

33 a 145.7

$46.8 \mathrm{~h} \quad 103.2$

$9.2 \mathrm{~h} \quad 121.9$

2.7 d 411.8

46.6 d 279.1

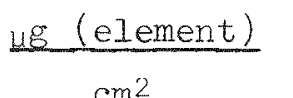

$\mathrm{cm}^{2}$

7.7

0.12

10.0

6.5

1.1

18.0

0.0026

0.042

0.105

1.24

107.0

0.026

2.45

1.0

0.26

0.05

1.0

0.13

0.011

0.03

0.00023

1.7

0.020

0.035

0.00045

0.06077

0.00059

0.018
Minimum amount present that would have been detected

$\sim 0.007$

20.10

20.002

20.007

20.1

22.0

20.0003

20.008

$\sim 0.03$

20.0005

21.0

20.005

20.5

20.3

20.02

20.04

20.005

20.05

20.001

20.03

20.00007

20.002

20.002

$\sim 0.005$

20.00005

20.00005

$\sim 0.0002$

20.005 
[1] Present Address: Consumer Power Co., Jackson, Michigan.

[2] J. R. Berreth and D. A. George, "Sample Preparation and Irradiation", Journ. Idaho Acad. of Science, Special Research Issue \#2 (April 1971).

[3] D. W. Brock, "Results and Interpretation", Journ. Idaho Acad. of Science, Special Research Issue \#2, (April 1971). 
TELEMETERED PROFILING ISOTOPIC SNOW GAUGE

R. A. Coates, I. O. Johnson, E. W. Killian, R. E. Brown, G. O. English, W. R. Beck, P. D. Randolph

The western region of the United States is generally semi-arid, and much of the water available comes in the form of snow stored in the mountains during the winter. For example, some 85 percent of the stream flow in Idaho comes from snow runoff water. The economic value of the water use is great, approximately $\$ 1.4$ billion per year in the Columbia. River Basin alone. More accurate methods than heretofore available for measuring snow packs during the winter and during melt are needed in order to make better forecasts for power dam regulation, flood control and irrigation.

To improve this situation a laboratory prototype of a snow gauge that profiled snow density has been developed by the U. S. Forest Service at the Central Sierra Snow Laboratory in California. Under contract to the Division of Isotopes Development, Idaho Nuclear Corp. has built an engineered, telemetered version of this gauge.

A prototype telemetered isotopic snow gauge that measures the density of a snow pack as a function of depth was installed and demonstrated in California with the field unit at Donner Pass and the base station at Berkeley. Telephone telemetry is used between the two locations. The system is designed for eventual low-power untended operation in remote locations and in severe winter environments. The gauge consists of a field unit, a field logic unit, a telemetry link, and a base station. It operates on the principle of measuring the attenuation of monoenergetic gamma rays that pass a fixed distance through successive horizontal layers of snow. Operation of the system is digital throughout with a base station processor exercising complete control. The field unit consists of two parallel access tubes $7.5 \mathrm{M}$ high spaced $66 \mathrm{~cm}$ apart and mounted vertically with their bottom ends in the ground. At the top of this assembly is a lift unit containing a stepping motor that simultaneously lifts a CsI scintillation detector located in one tube and a 10-millicurie $137 \mathrm{Cs}$ radioactive source in the other. The main engineering requirements in the design of this gauge are that it have low power drain and that the detector and control logic have good gain and operational stability in an environment where the temperature can range from $-20^{\circ} \mathrm{F}$ to $70^{\circ} \mathrm{F}$ and with gross count rates of 1000 to 20,000 per sec. The detector system uses a fixed-gain, charge sensitive preamplifier and a window discriminator whose window width and base line is fixed. System gain is controlled from the base station by digitally stepping the detector high voltage. The field logic unit uses low power IC logic throughout, with discrete components being avoided where possible. The logic section contains a serial to parallel converter, an 8-bit command register, a 48-bit output multiplexer and a parallel to serial converter. It also contains data handling logic, which is set up by base station command, a constantcurrent source high-voltage supply, the window discriminator, a digital high voltage control, motor control circuits, and voltage regulators. 
Telemetry is by telephone at 110 bits per second, with an interrogation rate of once every two seconds, in a bit serial, half duplex mode.

The gauge operates by lifting the source and detector a fixed-step distance while counting. Either a preset-time or a preset-count mode can be used under base station command, and lift steps of $1 / 2,1,2$ or 4 inches are available.

The base station consists of a PDP-8/e processor and a teletype together with a small plotter. The processor controls the snow gauge operation and performs the data analysis. The teletype is used to enter commands, and to list the calculated results in the form of snow density versus position. Data taking can be done manually or automatically. A sample of the plotter output is shown in Figure $I$.

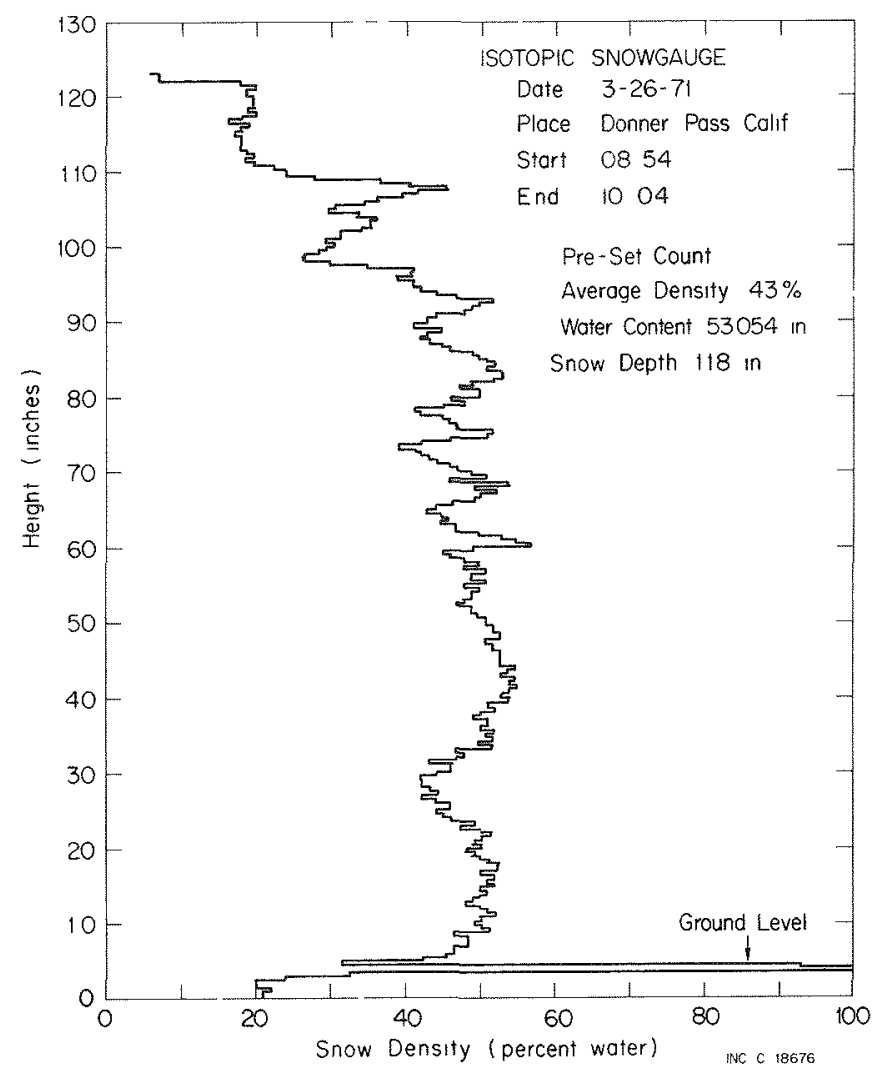

Fig. 1 Sample of plotter output from telemetered profiling isotopic snow gauge.

The operation of this engineered prototype was quite successful. In order to further demonstrate this gauge and to get it in the hands of a wider class of users, 3 more gauges will be built to be used by the Soil Conservation Service, the Bureau of Reclamation and the National Weather Service. 
PPM MEASUREMENTS OF IRON-IN-OIL BY RADIOACTIVE X-RAY FLUORESCENCE ANALYSIS [1]

R. J. Gehrke, I. L. Packer ${ }^{2]}$, B. A. Woody [2], W. A. Bruton $\left.{ }^{2}\right]$

Engine wear metal analysis, obtained by laboratory measurement of iron-in-oil samples, is performed routinely on military aircraft and is now being performed by certain commercial operators. This analysis, when correlated to wear measurements obtained during engine overhaul, can serve as an indicator of possible incipient failure. The applicability of nondispersive X-ray fluorescence spectroscopy to measure trace quantities (<50 ppm) of iron in oil was investigated.

These low level concentrations of iron in oil have been detected by using high resolution $\mathrm{Si}(\mathrm{Li})$ spectrometer systems (<400 eV FWHM) and by optimization of excitation sources. The potential of several primary radioactive excitation sources, including ${ }^{71} \mathrm{Ge},{ }^{73} \mathrm{As},{ }^{244} \mathrm{Cm},{ }^{238} \mathrm{Pu}$ and secondary excitation sources, including ${ }^{147} \mathrm{Pm} / \mathrm{Cu},{ }_{147} \mathrm{Pm} / \mathrm{Zn},{ }_{147} \mathrm{Pm} / \mathrm{Ni}$, and $238 \mathrm{Pu} / \mathrm{Cu}$ has been investigated. Problems associated with sourcetarget detector geometry have also been examined in order to achieve maximum sensitivity for each source. Using these techniques it is presently possible to detect concentration levels of $3 \mathrm{ppm} F e$ in oil in acquisition times of 20 minutes.

[I] R. J. Gehrke, I. L. Packer, B. A. Woody and W. A. Bruton, Applications of Low Energy X-and Gamma Rays, (Gordon and Breach, New York, in press).

[2] United Aircraft Research Laboratories, East Hartford, Connecticut. 
-

a 


\section{G. EXPERIMENTAL FACILITIES}

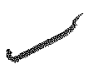


-

a 


\section{UPGRADE OF THE EIR PNEUMATIC RABBIT IRRADIATION FACILITY}

$$
\text { J.R. Bexreth, F. O. Cartan [I] }
$$

Placing the Materials Testing Reactor (MTR) on a standby basis created an acute need for a high neutron flux pneumatic rabbit irradiation facility. The only operating facility for short-term neutron irradiations of any type was an ETR pneumatic rabbit facility with a thermal flux of $6 \times 10^{11} \mathrm{n} / \mathrm{cm}^{2} / \mathrm{sec}$ which was too low for most requirements. The old facility extended into the reactor tank just to the top of the core region at the periphery of the tank. A considerable increase in thermal flux, to the order of $3 \times 10^{13} \mathrm{n} / \mathrm{cm}^{2} / \mathrm{sec}$, would better serve the needs of the current research programs. The least expensive way to obtain a facility of more usable neutron flux appeared to be to relocate the pneumatic rabbit into a nearby beryllium reflector piece. In order to keep radiation levels to a minimum, plastic rabbits were still the most desirable means of sample containment during irradiation. But plastic will only withstand limited amounts of gamma radiation and the gamma fluxes in the reflector area of the ETR are considerable, therefore, some means of suppressing the gamma flux was likely necessary.

The nearest area available with the desired flux and requiring the least modification was the $Q-5$ position. In order to obtain a reasonable flux the rabbit facility would have to extend into the reflector piece about 10 inches. At this distance the ganma heating was about 5 watts/ gram - considerably more than a plastic rabbit could withstand for any length of time. Calculations showed that one could place sufficient lead into the 3 in. $x 3$ in. position to decrease the gamma heating to approximately 0.3 watts per gram.

Relocating the facility in the Q-5 position only required a new shuttle tube section inside the tank and the short lead shielded section in the reflector piece. Figure 1 shows a schematic of the shielded facility. The lead shielded section is a separate insert in the Q-5 position. It consists of a lead annulus approximately $5 / 8 \mathrm{in}$. thick and $12 \mathrm{in.}$ long encased in aluminum. Water flows between the shuttle tube and the lead annulus to provide the necessary cooling.

The new ETR pneumatic rabbit irradiation facility provides a thermal neutron flux of $1.9 \times 1013 \mathrm{n} / \mathrm{cm}^{2} / \mathrm{sec}$. The gamma heating, as measured by iron dosimetry, is 0.3 watts/gram. Standard high density polyethylene rabbits with air cooling may be irradiated up to 10 hours in the facility, thereby, providing a much more useful short-term irradiation tool than previously available.

[1] Currently with Allied Chemical Corporation, Idaho Chemical Programs, NRTS 


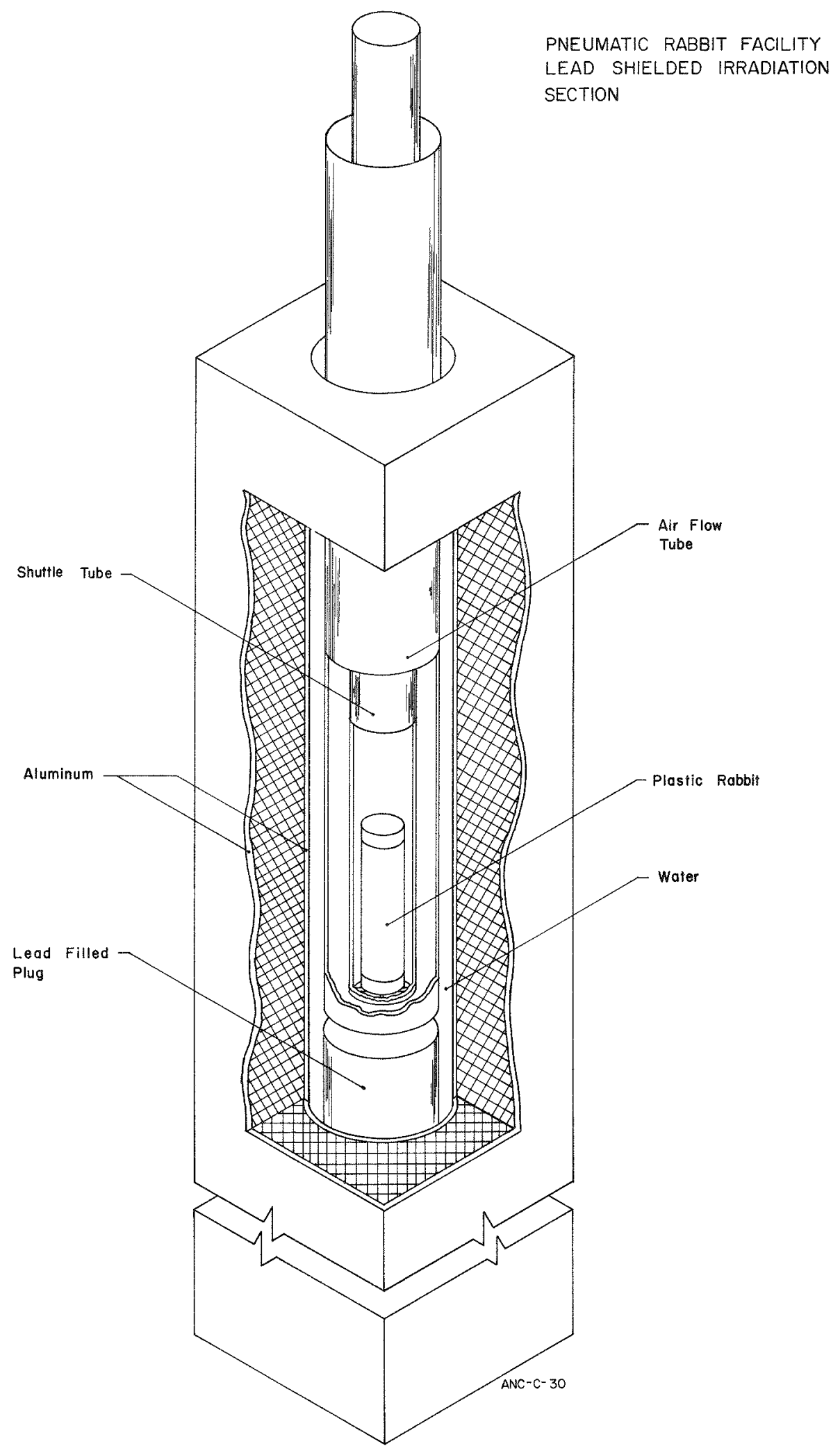

Figure 1. Exploded view of the ETR pneumatic rabbit shielded irradiation section. 


\section{COMPARISON OF SPECTRUM DETERMINATIONS IN THE CFRMF}

D. A. Pearson, D. A. Millsap, J.W. Rogers

It is important to know the neutron distribution as a function of energy within a reactor. The current emphasis on the IMFBR's adds justification to our continuing efforts to improve the capability of spectrum measurements associated with fast reactors in general and the CFRMF[I] in particular. It is with this goal in mind that a continual study of the spectrum of the CFRMF is being carried on utilizing several techniques. The three methods currently receiving primary attention are calculational methods, proton-recoil, and foil activation. The calculation of the neutron spectrum employs two-dimensional standard diffusion and transport calculations using the latest version of ENDF/B cross sections[2]. Measurements have been made with a calibrated protonrecoil spectrometer that employs the two detector method using hydrogen and methane gases[3]. Foil activation analysis of the neutron environment utilizing an on-line graphical analysis technique is a third method used in the continued study of the spectrum[4].

The analysis of the most recent efforts of the three independent methods of neutron spectrum determination has revealed a revised CFRMF spectrum[5]. The most accurate knowledge of the spectrum currently available is shown in Figure 1 . Efforts will continue to be made to insure that the CFRMF neutron spectrum will be among the most intensely studied and accurately determined reactor neutron environments.

[1]. E. Fast et al., "Use of the ARMF for a Fast Reactor Support Program", IN-1143, (November 1970).

[2]. Nuclear Technology Branch Annual Progress Report for Period Ending June 30, 1969, IN-1317 pp. 277-282.

[3]. Nuclear Technology Branch Annual Progress Report for Period Ending June 30,1969 , IN-1317 pp. 283-288.

[4]. Nuclear Technology Branch Annual Progress Report for Period Ending June 30, 1971, ANCR-1016, this report, "Improvements in Foil Activation Data Analysis", D. A. Pearson.

[5]. Nuclear Technology Branch Annual Progress Report for Period Ending June 30, 1970, IN-I407 p. 291. 


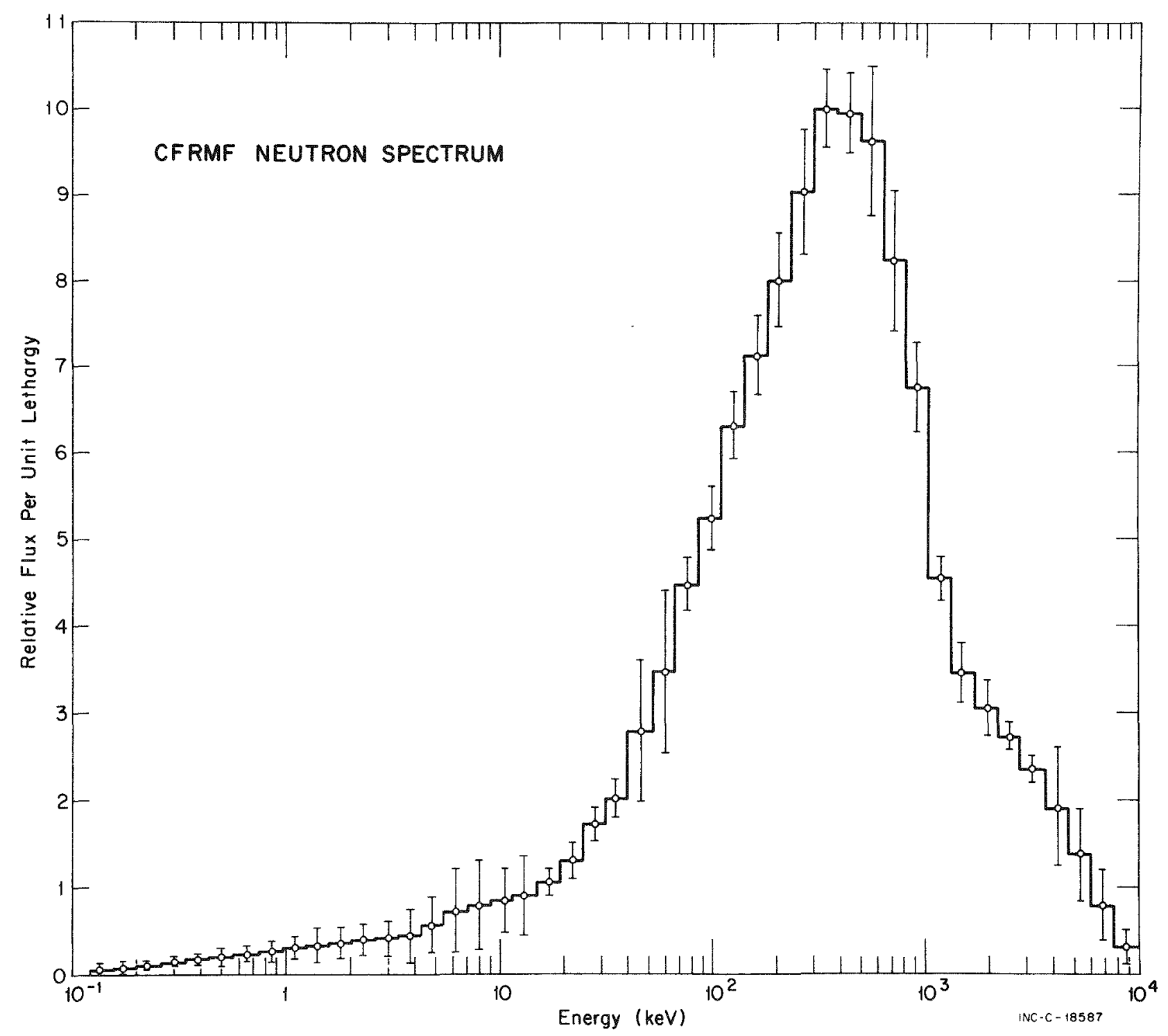

Fig. I CFRMF neutron spectrum. 


\section{SUBCRITICAL MULTIPLICATION OF 252 CA NEUTRONS}

T. Watanabe, I. G. Miller, J. F. Kunze

Most neutron radiography with californium-252 requires moderation of the fast neutrons from the source followed by collination of these thermalized neutrons into a beam. To compensate for neutrons lost during moderation and collimation, tests have been made on a subcritical assembly, the Nuclear Test Gauge (NTG) from a l-milligram californium-252 source $(1)$.

The source, in a Plexiglas holder, was inserted near the center of the NTG (Figure I) either on or off the axis. The neutrons were moderated. by beryllium and were extracted from the core with a divergent collimator having a length/inlet-aperture-diameter ratio of 50. Thermal neutron fluxes at an image plane 40 inches from the source were measured by indium foil activation.

Image-plane fluxes from the source in the NTG (operating at $k_{\text {eff }}=$ 0.941 to 0.988 , corresponding to source multiplications** of 17 to $8 \overline{9}$ ) were 5 to 25 times higher than those from the nonamplified source moderated by about 11 inches of $\mathrm{H}_{2} \mathrm{O}$ (Figure 2). As a result, the exposure time for comparable radiographs was reduced from 18 hours with neutrons from the water-moderated source to only 30 minutes with neutrons from the source in the NTG at $k_{\text {eff }}=0.998$.

Studies are continuing to determine the best source, collimator, and external shielding configurations to decrease the unwanted gamma background at the imaging plane. Various types of films and converters are being tested. The multiplication method appears to offer a very safe and practical means of inexpensively boosting the output of a californium source. For even at this high multiplication of 0.998 , the booster was still safe from a flooding accident, i.e., would remain subcritical if flooded. Furthermore, for neutron activation analysis, the booster gives large volumes having highly boosted flux compared to that obtainable from an unboosted source.

* Californium-252 Progress, No. 3, 26 (April 1970) (USAEC)

* Source neutron multiplication $=1 /(1-k$ eff $)$

[1] To be published in Materials Evaluation. 


\section{NUCLEAR TEST GAGE (NTG)}

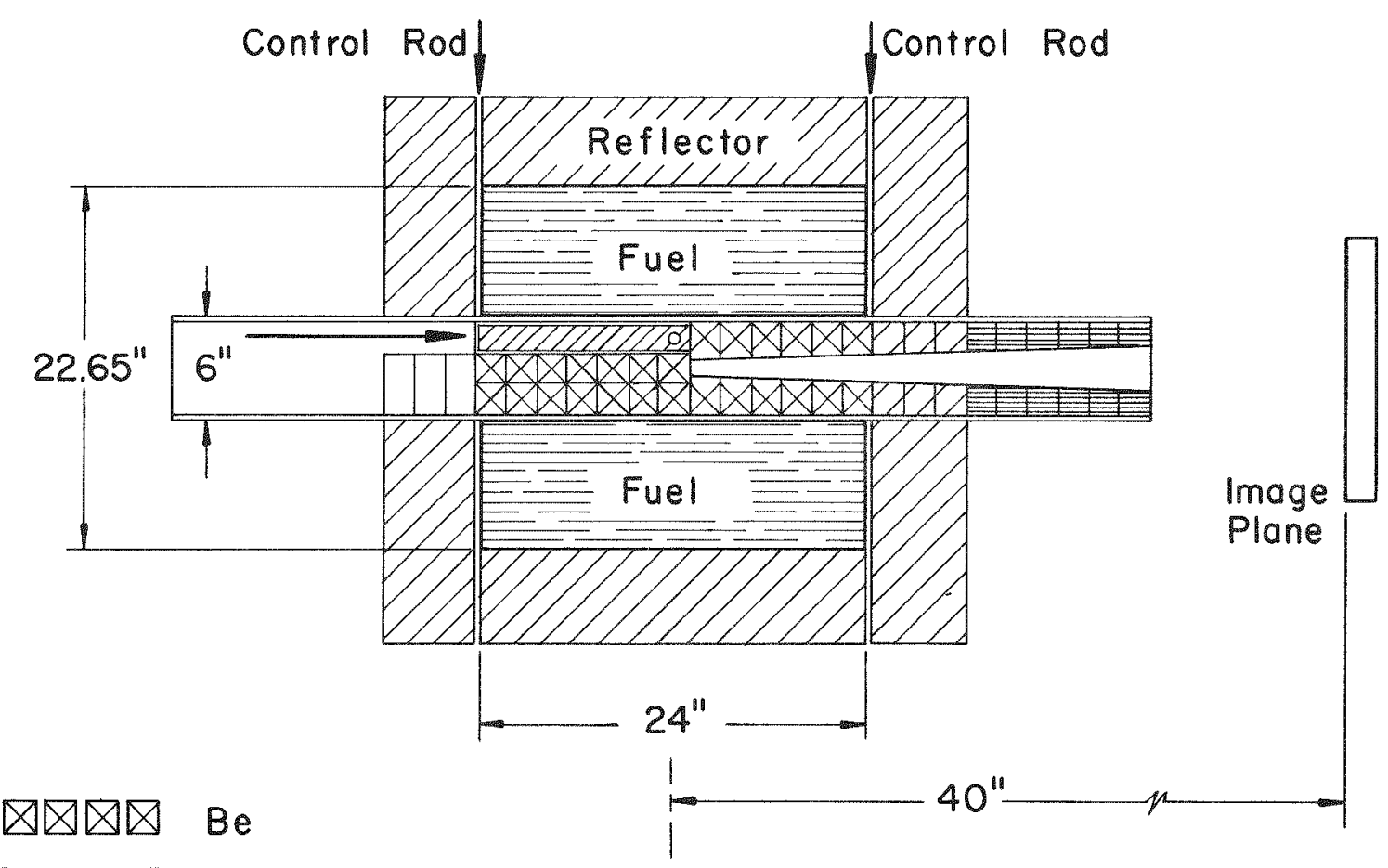

Bin Borated Polyethylene

ZZZZA Polyethylene

$\mathrm{Pb}$

INC-A-16625

Figure 1. Diagram of the Nuclear Test Gage showing the neutron radiography collimator diverging from the right side and the californium neutron source positioned in the central tube near the center. 


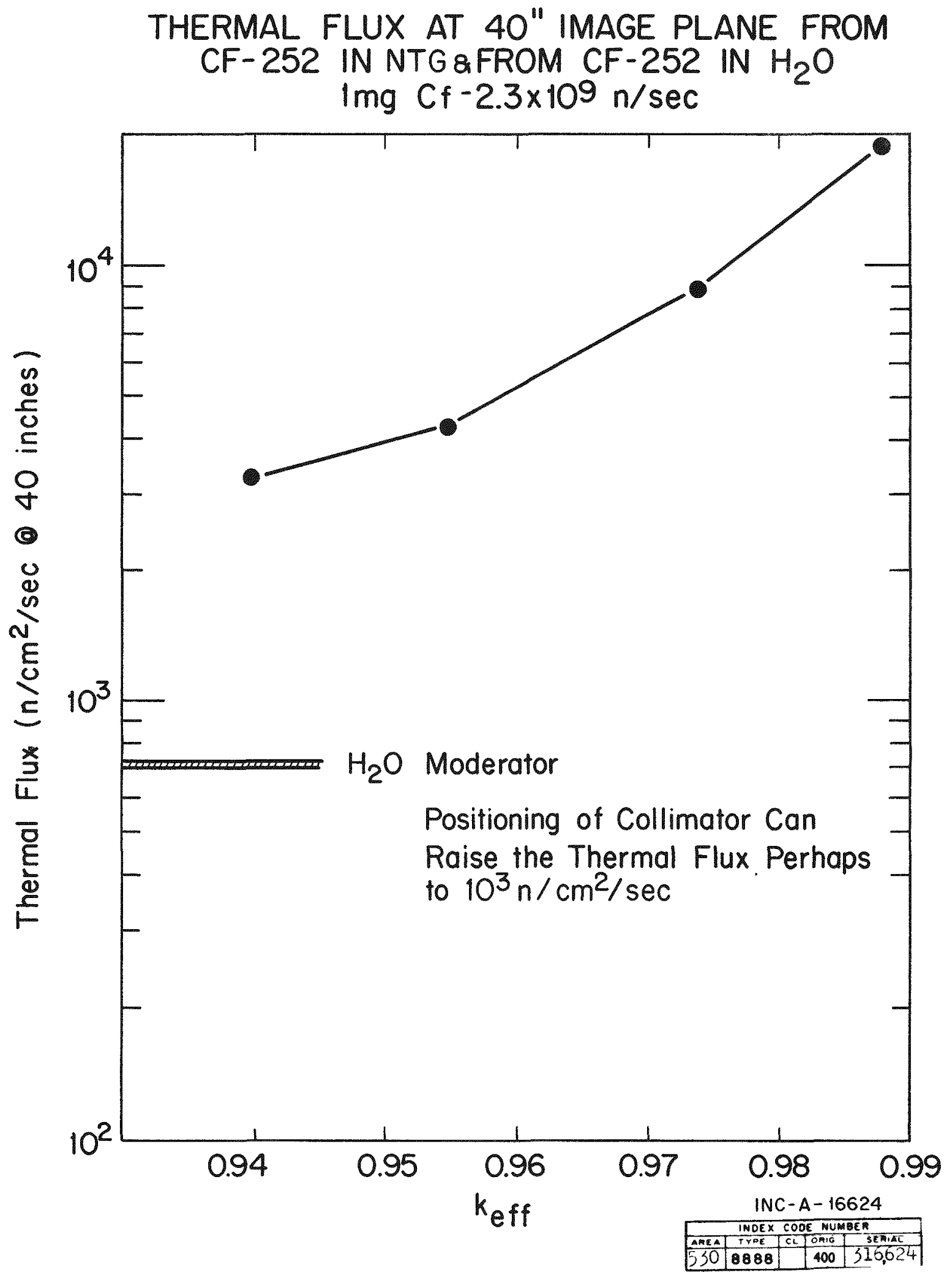

Figure 2. Thermal flux at 40" image plane from $\mathrm{Cf}-252$ in NTG and from $\mathrm{CF}-252$ in $\mathrm{H}_{2} \mathrm{O} I \mathrm{mg} \mathrm{CF}-2.3 \times 10^{9} \mathrm{n} / \mathrm{sec}$. 


\section{SPLIT TABLE REACTOR STARTUP AND AUTOROD TESTING}

D. H. Suckling, J. H. Lofthouse, J. F. Kunze

The Split Table Reactor at the Low Power Test Facility is a versatile critical facility for quickly and easily mocking up a variety of reactors and performing actual measurements to supplement or check reactor physics design calculation. This reactor facility was re-commissioned for operation beginning in June 1971. The reactor had previously been operated on a number of programs through the Oak Ridge Operations Office, principally on critical experiments related to fast compact reactor space power plants.

The Safety Analysis Report (1) and operating procedure documentation $(2,3)$ has been completely rewritten to permit operation of this critical experiment table for mocking up a variety of reactor configuration-from highly thermal to fast reactors, with U-235 fissile loadings as large as $200 \mathrm{~kg}$.

Figure 1 is an overall view of the hydraulically driven table and matrix. Table separation adds an extra margin of shutdown safety beyond that possible with the regular complement of 8 scram (safety) rods and 4 shim rods. These rods can be mounted for insertion at any point in the aluminum matrix. The safety rods are reflector material. The aluminum matrix is of hexagonal shape, 1.75 inches across flats. It accommodates a variety of hexagonal shaped materials in stock, including such items as $\mathrm{Be}, \mathrm{BeO}$, stainless steel, tungsten, molybdenum, and Plexiglas to accommodate the sheet fuel.

The initial operation of the STR in June involved testing of a newly fabricated "auto-rod" device. This instrument is a precision servo control mechanism used to drive a fine (dynamic) control rod, principally to adjust for changes in reactivity of the order of $10^{-7} \Delta \mathrm{k}$ or less. Tests were made to examine a low-enriched fuel rod (such as is used in most light water electric power producing reactors). The rod was slowly traversed through the reactor, while the "auto-rod" maintained steady reactor power. These initial tests indicated that variations of $1 \mathrm{gm}$ of fissile material along the rod length could easily be detected. Future plans are to install a spatial thermal flux filter so that only a small portion of the rod is being examined for reactivity variations. Ultimately, this type of device could serve as a reactivity quality-control device for a fuel production facility.

A mockup of the portable neutron radiography reactor was installed in the STR in late June. A cross sectional view of it is shown in Figure 2. Testing of the reactor will begin in the summer of 1971, and will be directed toward optimizing a neutron radiography beam for a given power level.

[1] C. G. Cooper, "Safety Analysis Report for the Split Table Reactor (STR) at the Low Power Test Facility," internal report.

[2] J. F. Kunze, et. al., "Technical Operating Limits for the Split Table Reactor (STR) Critical Experiment Assembly at the Low Power Test Facility," internal report. 
[3] J. H. Lofthouse, "Standard Reactor Operating and Maintenance Procedures for the Split Table Reactor at the Low Power Test Facility," internal report. 


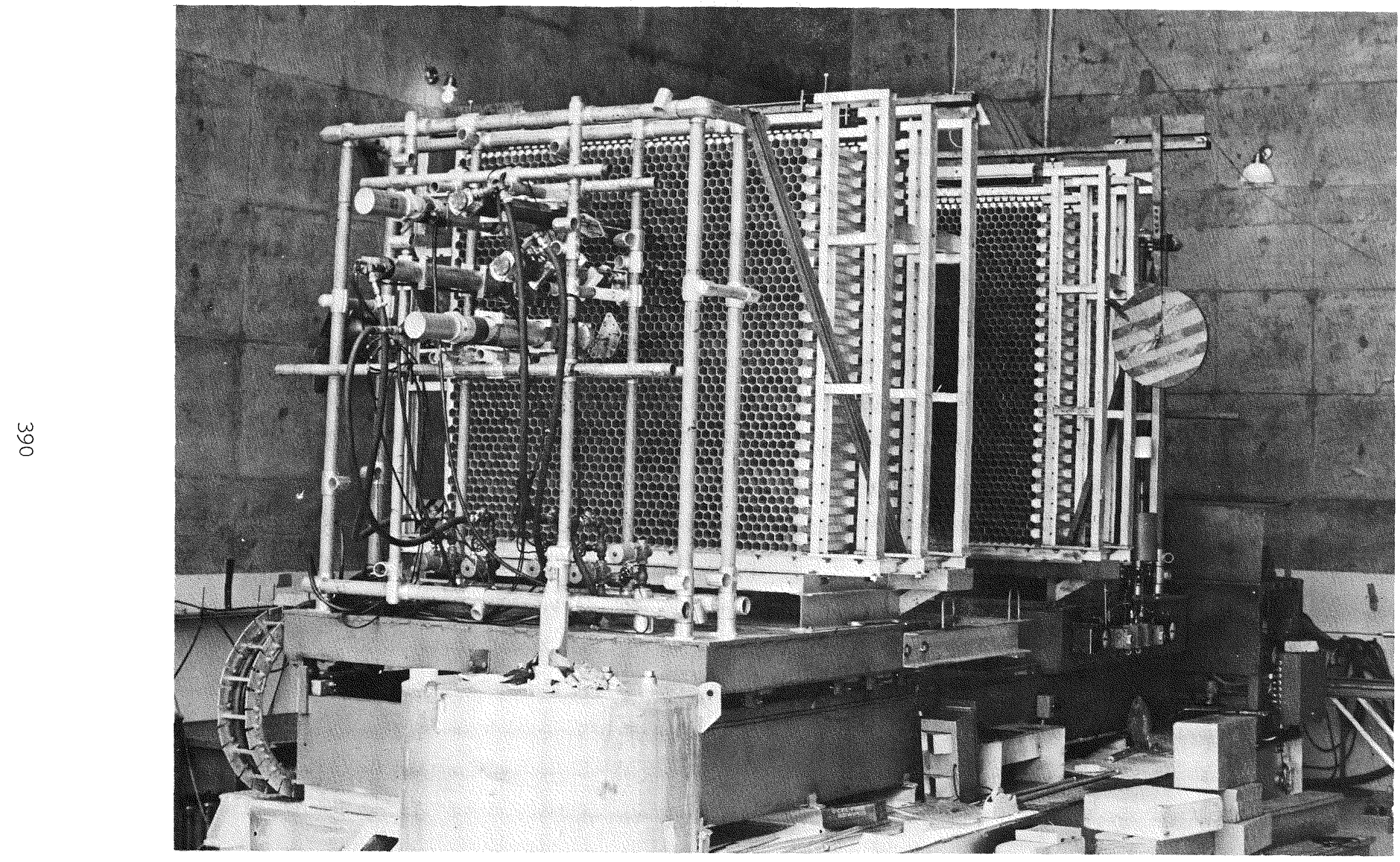

Figure 1. Split Table Reactor 


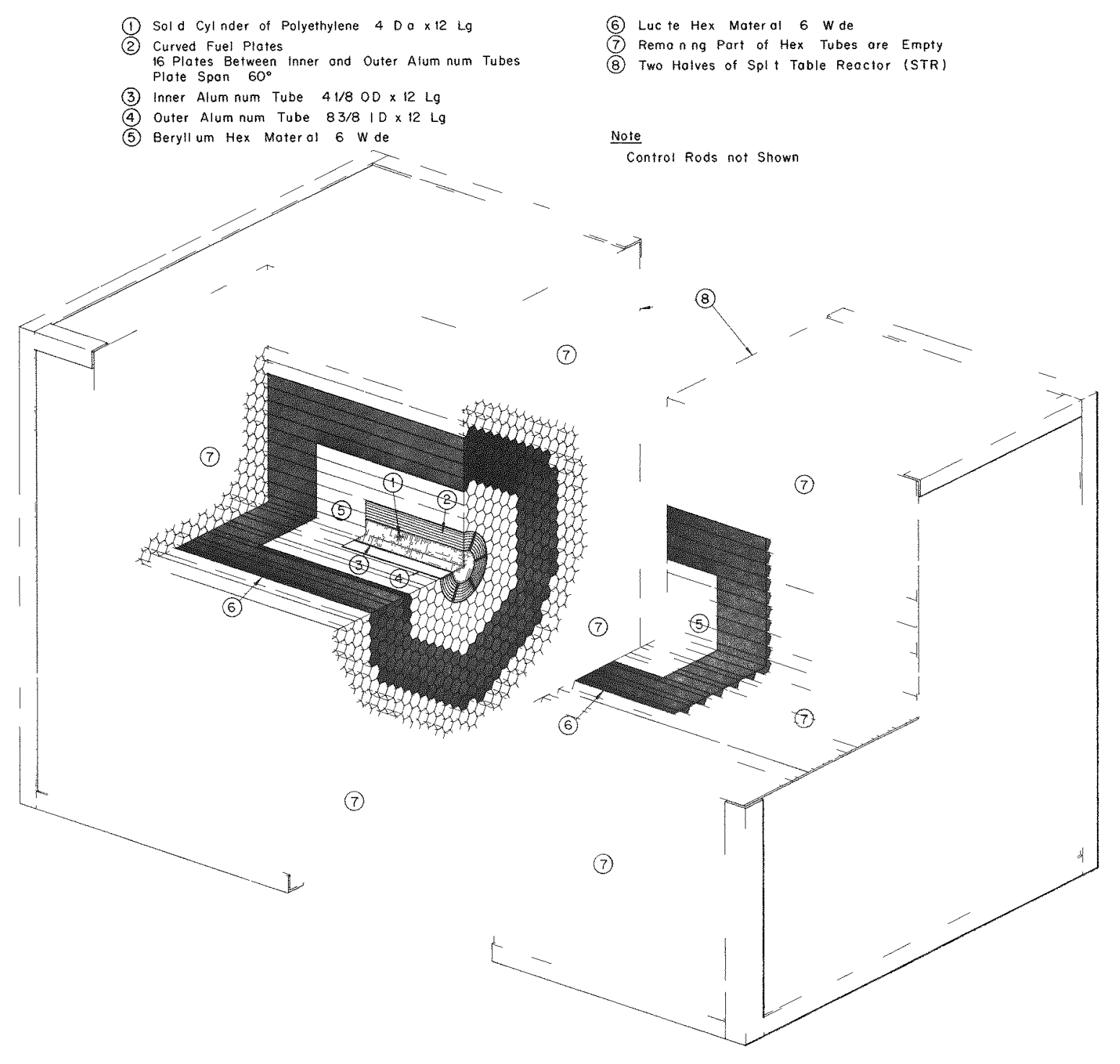

Flgure 2. Neutron radiography reactor mockup in the Split Table Reactor. 
An Electromagnetic Isotope Separator is needed to supply both stable and radioactive samples for integral cross section measurements of fission products in support of the IMFBR program. The contract for purchase of a separator was awarded to Nucletec, S.A. of Geneve, Switzerland, with delivery and installation due in september, 1971. This machine has a $1500 \mathrm{~mm}$ radius sector magnet, $80 \mathrm{kV}$ acceleration potential, the most advanced set of electromagnetic lenses available with aberrations removed to the 5th order, and the latest designed ion source which is constructed to have greater stability and virtually eliminate the need to continually adjust the alignment. The machine is to meet the performance requirements of having a resolution of not less than 2000, a dispersion at mass 250 of $0.6 \mathrm{~cm}$ between adjacent masses and a ion beam current capability of 1 ma.

The pre-delivery preparation of Room 120 of the MTR "alpha" wing is proceeding. Necessary electrical power, cooling water, and vent systems have been designed and are now being installed. The normal power for the separator will be from the commercial power buss; however, the mechanical vacuum pumps will be operated off the emergency power system to insure no loss in vacuum from a power loss. The vacuum pumps will be vented into the hood vent system already installed in the wing and cooling water for the magnet and diffusion pumps will be from the demineralized water supply also present in the wing. This machine will provide the capability of obtaining milligram quantity samples of various isotopes for analysis in reactor spectra, measurement of differential cross sections integral cross sections, and in general, enhance the overall laboratory capability. 


\section{NEW METALLURGY FACILITY}

W. C. Francis, G. E. Korth

Early in 1971, a new laboratory and office building, designed for housing much of the Metallurgy and Materials Science Branch equipment and personnel, was completed at ARA-I. This site is adjacent to Aerojet Nuclear's only alpha Hot Cell and is conveniently located between EBR-II and Central Facilities Area and close to the SPERT-PBF complex. The new laboratories now contain all of the Branch's tensile, creep, and hardness testing equipment as well as metallography, microscope and probe facilities. Scheduled to be moved to this area sometime in FY-72 are the fatigue laboratory and the fabrication equipment, still in the Central Facilities Area and in the Test Reactor Area. With the completion of this threephase move, the Branch's entire operations will be centralized at ARA with the expectation of providing more efficient service to NRTS customers and the AEC.

The laboratory at ARA includes a new Hitachi HU-200F transmission electron microscope which was installed in February 1971. This new instrument replaces the old RCA microscope used at TRA and with it, the electron microscopy capability is now considerably updated compared to the past. The higher accelerating voltage of up to $200 \mathrm{KV}$ permits thicker metal foils to be examined with less electron interaction due to the shorter wave-length. Capabilities include direct magnification to $250,000 \mathrm{X}$, resolutions to $3.4 \mathrm{~A}$, specimen tilting device capable of $\pm 30^{\circ}$, hot stage to $1000^{\circ} \mathrm{C}$ and cold stage to $-160^{\circ} \mathrm{C}$. 
THE OAK RIDGE ELECTRON LINEAR ACCELERATOR RADIOACTIVE SAMPLE CHANGER

H. G. Miller, D. R. Staples, F. B. Simpson

With the phaseout of the Materials Testing Reactor (MrR), including the loss of the MTR fast chopper, the U.S. capability of measuring cross sections of highly radioactive small samples was lost. To re-establish these capabilities a radioactive sample changer has been designed, constructed and installed on one of the experimental beam ports of the Oak Ridge Electron Linear Accelerator (ORELA).

The sample changer is very versatile and some of its unique features are listed in Table I. The changer has been interfaced to the SEL-8IOB data acquisition computer and can be automatically cycled in any desired sequence. The cycling time is independent for each of the four sample positions. Liquid nitrogen and helium cryostats have been designed and adapted to the sample changer for transmission measurements at these temperatures.

A photograph of the unshielded changer is shown in Figure 1. The radioactive cast that is used for transporting the samples from Idaho to Oak Ridge can be seen on the left side of the photograph.

Figure 2 is an assembly drawing showing different views of the support table, transport cask and lead shielding. A block diagram of the control system is shown in Figure 3. The system is capable of making cross section measurements on very small samples. It has been used successfully in the measuring of the total neutron cross section of $\mathrm{Cm}-244$ using a circular collimation system. One of the samples used had a total weight of only $6 \mathrm{mg}$ of $\mathrm{Cm}-244$. This was accomplished by pressing the material into an aluminum matrix. The total neutron cross section of Pu-242 has been measured at liquid nitrogen temperatures using the liquid nitrogen cryostat in conjunction with a rectangular collimation system.

As yet the cross section of a highly penetrating radioactive sample has not been measured but the system has been checked out and is ready for these type of measurements. 
Table I

Features of the ORELA Radioactive Sample Changer

$\begin{array}{ll}\text { Collimation } & \text { Circular or Rectangular } \\ \text { Beam Size } & 0.025 \text { to } 3.0 \text { in. } \\ \text { Sample Size } & \text { Having above areas and up to } 4 \text { in. Iong } \\ \text { Shielding } & \begin{array}{c}\text { Adequate for } 20,000 \text { curies (could be } \\ \text { increased if required) }\end{array} \\ \text { Sample positions } & \text { Four } \\ \text { Sample Temperatures } & \text { Room, liquid nitrogen or liquid helium } \\ \text { Positioning accuracy } & \pm 0.0002 \text { in. which is accomplished with } \\ & \text { a precision ball screw } \\ \text { Position indication } & \text { Glow transfer tubes } \\ \text { Digital Logic } & \text { Texas Instrument } 7400 \text { series and } \\ & \text { Fairchild } 900 \text { series } \\ \text { Control System } & \text { Manual or computer } \\ \text { Cycling Sequence } & \text { Programmable } \\ \text { Cycling Time } & \text { Independent for each sample }\end{array}$




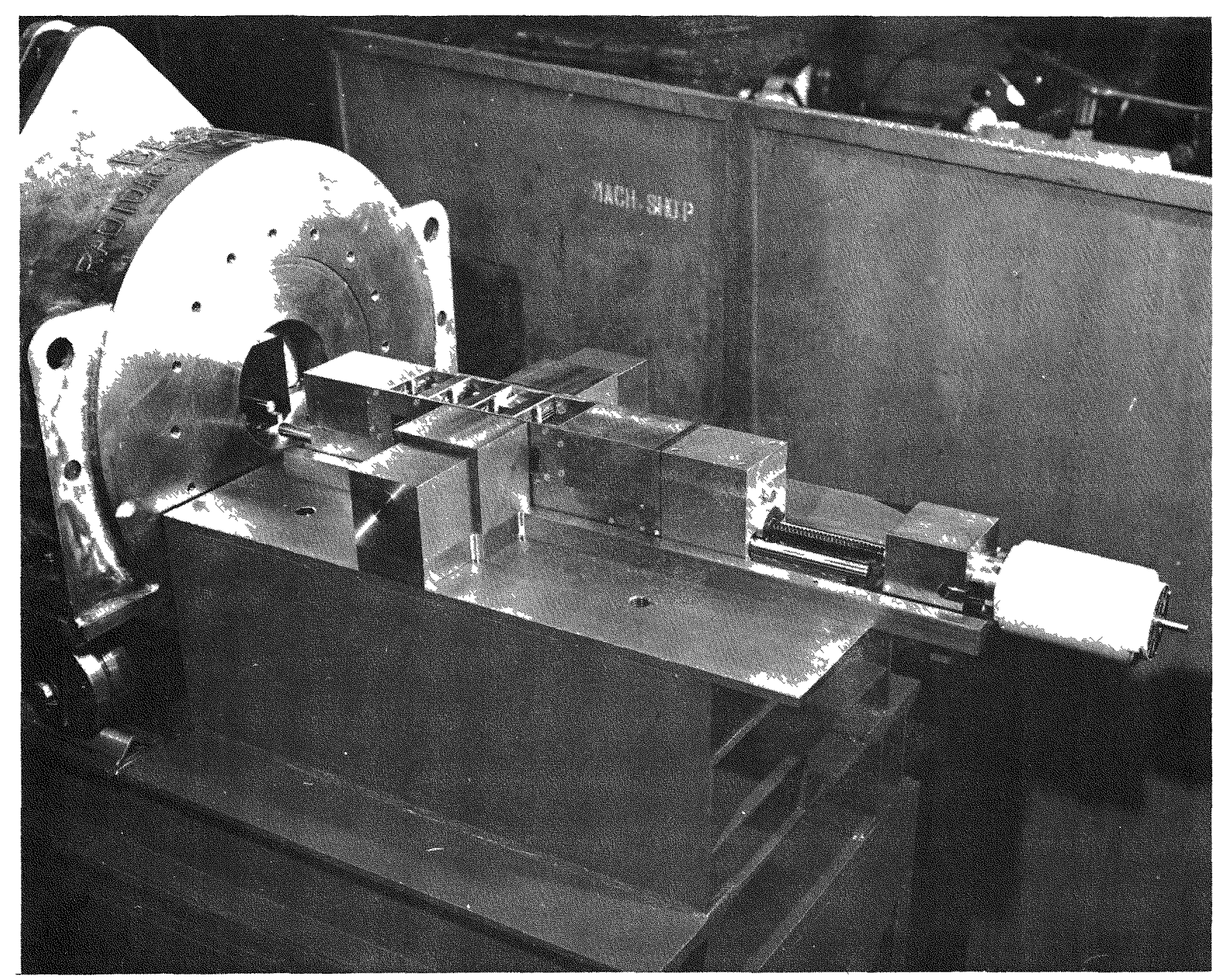

Fig. I A photograph of the unshielded sample changer. The large cask on the left side is used for transporting the radioactive samples from Idaho to Dak Ridge. The carrier which holds the four sample blocks is transferred from the cask onto the sample changer by means of a metal rod inserted through the end cover plate of the cask. 


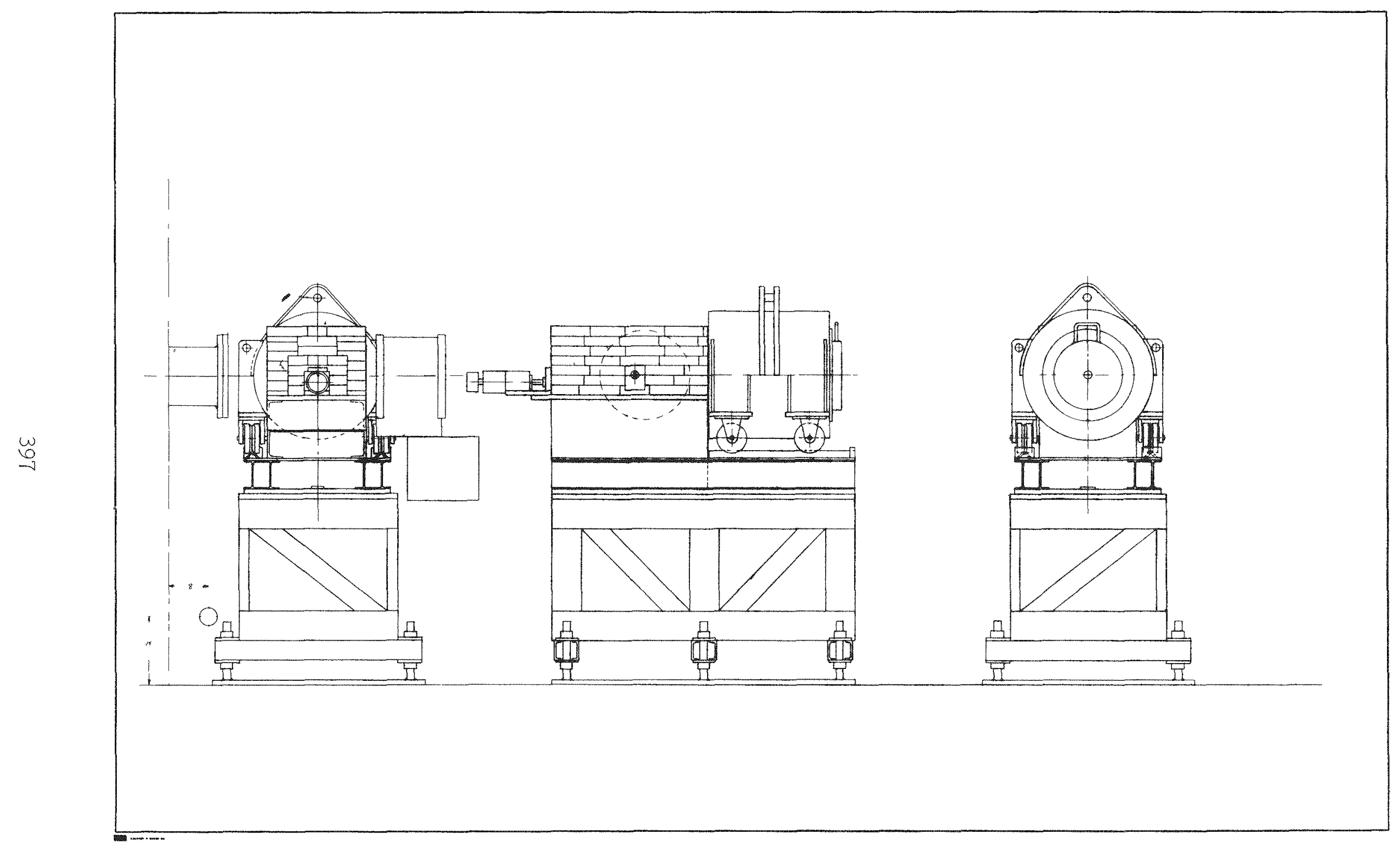

Fig. 2 An assembly of the ORELA sample changer showing the support table, transport cask, and lead shielding. 


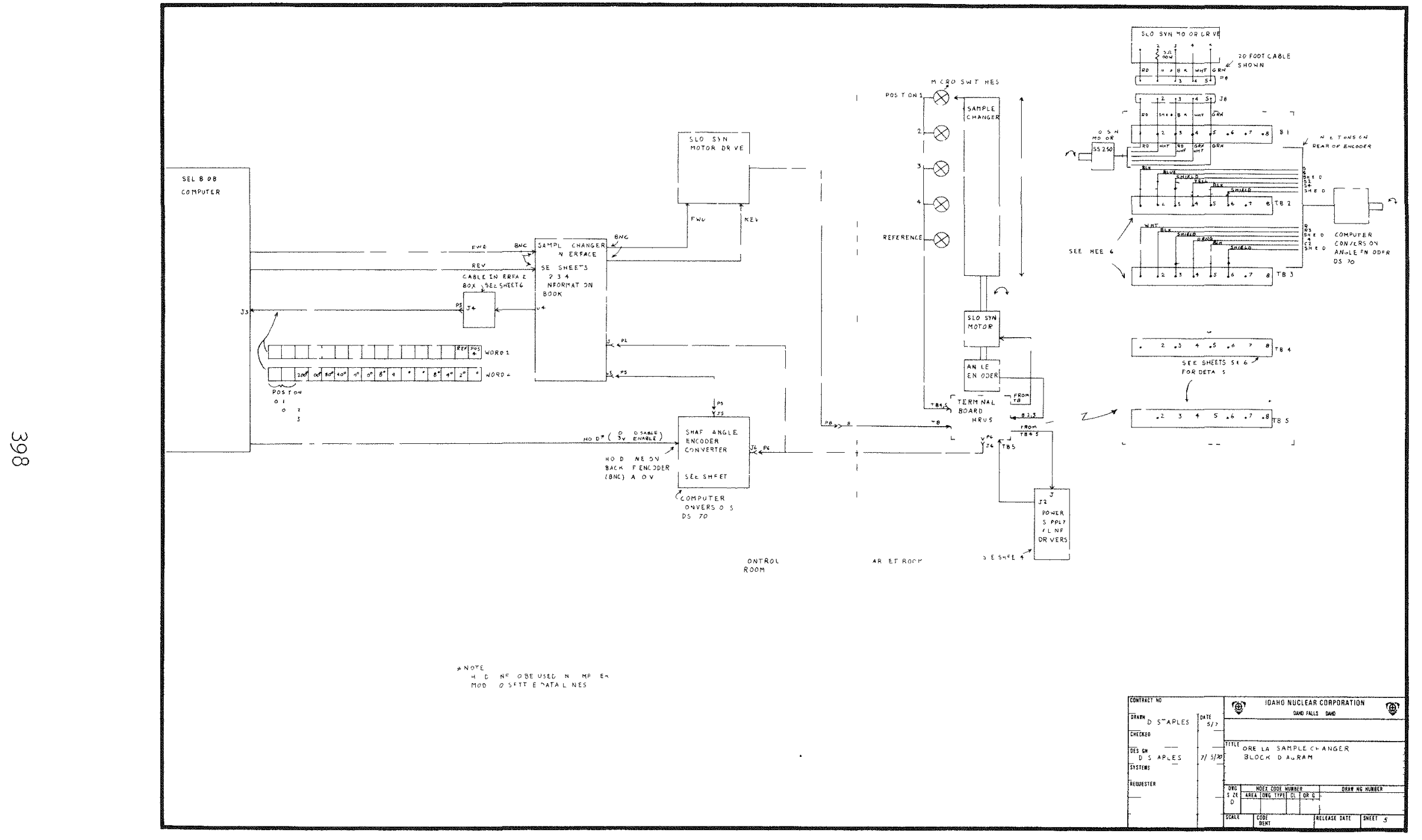


THE NEED FOR HIGH FLUX NEUTRON BEAMS

R. M. Brugger

During the year the University of Utah conducted a study to determine the feasibility of reactivating the MTR and to determine the need for additional facilities in the U.S. Members of the Nuclear Technology Division acted as consultants to the University of Utah and provided information for this study. The study[1] was completed and the report presented to the NSF in June 1971. The major findings of the study are: [2]

"1. High flux neutron beams are becoming an essential scientific investigative tool in a variety of scientific investigations.

There is a significant scientific need for additional high-flux neutron beam facilities in the U.S., especially in the areas of chemical and biological structure, solid state physics, chemistry, molecular dynamics, study of liquids, medical radiography, nuclear physics and magnetic structure. Furthermore, in-core radiations for neutron activation analysis in environmental monitoring of trace elements requires some work in high-flux reactors.

"2. Only two major centers exist at present for high-flux studies.

The only major U.S. reactors now in operation with neutron fluxes at the beam ports clearly in excess of $1 \times 10^{14}$ neutrons $/ \mathrm{cm}^{2} \mathrm{sec}$ are at Brookhaven and at Oak Ridge. These two reactors with a combined total of $13 \mathrm{high-flux}$ ports are nearing saturation, however. The MTR, presently not operating, has 15 beams with fluxes in excess of $1 \times 10^{14}$ neutrons $/ \mathrm{cm}^{2} \mathrm{sec}$ and an additional 10 beams of lower flux. Many very fine intermediate-flux (defined herein as $5 \times 10^{13}-1 \times 10^{14}$ neutrons/ $\mathrm{cm}^{2} \mathrm{sec}$ ) reactors operating throughout the U.S. are providing excellent data relevant to some of the areas listed in Item 1, above. They cannot contribute significantly to solution of aifficult problems in biological structures and certain inelastic neutron scattering studies on solids, liquids and molecular dynamics where flux levels become critical.

"3. The MTR is a suitable reactor for meeting the present and future scientific needs of the U.S. for additional high-flux neutron beams.

While the MTR does not quite match the high fluxes found at Brookhaven and at Oak Ridge and is admittedly inferior on this point to these two reactors, it is sufficiently comparable to justify its operation, considering the saturation of the two above reactors. The larger number of high flux holes in and luxurious amounts of floor space about the MTR compensate in part for the reduced fluxes. This study furthermore revealed that the 40 megawatt MTR is, in fact, a research tool for high flux work superior to the many excellent reactors presently operating below the 20 megawatts power level. 
"4. The operational costs of the MTR including adequate scientific support will be approximately $\$ 3.8-4.1$ million per year.

This amount is comparable with that found at Brookhaven and Oak Ridge once all hidden costs are included.

"5. The U.S. expertise in neutron beam research is recognized throughout the world.

This study indicated, however, that this prominence is concentrated primarily at the AEC national laboratories. This results logically from the general lack of neutron beam support by agencies other than the AEC.

"6. There is not presently an adequate system operating in the U.S. which makes possible extensive academic use of high-flux neutron beam facilities.

This is primarily tied to the manner in which neutron beam research is funded and not the intrinsic nature of the science. Academicians in Europe share more directly in neutron beam research. As several of the excellent intermediate flux reactors are located at universities, MIT, Iowa State, and Missouri, these are of course used by academic personnel.

"7. The MTR should be owned and operated by the AEC.

Safety and licensing requirements for reactor operation require extensive experience in the management of reactors. Therefore, it was concluded that the MIR should remain the property of the AEC and be operated by one of their contractors for any other governmental agency or group of investigators desiring the use of the facility. It is realized of course that the $\mathrm{AEC}$ must be recompensed for this service. Evidence indicates however that contractors of the $A E C$, under the $A E C$ 'S direction, are in a position to provide this function for less cost with more efficient operation than any alternative operational structure."

[1] The Feasibility of Reactivating the Materials Testing Reactor and Report on the Needs for Additional High-Flux Neutron Beam Research Facilities in the United States, A Report to the National Science. Foundation, D. M. Grant and R. J. Pugmire, Dept. of Chemistry, University of Utah, Salt Lake City, Utah (1971).

[2] Ibid., pages 4-6. 
H. TECHNIQUES AND INSTRUCTIONS

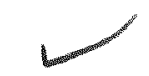


-

$a$ 


\section{GAMMA-RAY AND NEUTRON SPECTROSCOPY}

\section{PRECISE COMPARISON AND MEASUREMENT OF GAMMA-RAY ENERGIES}

WITH A Ge(Li) DETECTOR III: $1300-3600 \mathrm{keV}$

R. G. Helmer, R. C. Greenwood, R. J. Gehrke

In previous papers $[1,2]$, the authors have reported on energy measurements for gamma rays from 50 to 420 and 400 to $1300 \mathrm{keV}$ with $\mathrm{Ge}(\mathrm{Li})$ spectrometers. This project is now being extended into the region from 1300 to $3600 \mathrm{keV}$. As in the previous papers, the energies of some crossover transitions will be obtained by summing the measured energies of the cascade transitions. As an example of this procedure, the energies of several gamma rays from the decay of $11 \mathrm{~m}_{\mathrm{Ag}}$ are given in Table $I$.

For gamma rays above about $1300 \mathrm{keV}$, the following three peaks are observed:

1. The full-energy (FE) peak corresponding to absorption in the detector of the total gamma-ray energy,

2. the single-escape (SE) peak from the escape of one annihilation photon of $\mathrm{m}_{\mathrm{O}} \mathrm{c}^{2}$ (or $511 \mathrm{keV}$ ) following absorption of the gamma ray by pair production, and

3. the double-escape (DE) peak resulting from the escape of both annihilation photons from the pair production process.

In principle, it would be very desirable to determine the energies of these gamma rays by measuring their $D E$ peak energies. (Also it would be useful to be able to use $D E$ and $F E$ peaks in the spectrum for energy calibration and measurement.) This would allow one to "bootstrap" up from lower energies in चl MeV steps. For example, well-known calibration lines at $\sim 300 \mathrm{keV}$ could be used to determine the energies of $\approx I .3 \mathrm{MeV}$ transitions, which in turn could be used to calibrate the $D E$ peak energies of transitions at $\approx 2.3 \mathrm{MeV}$. In principle, this technique could be very accurate since, from the latest adjustment of the fundamental constants, [3] the value of $2 \mathrm{~m}_{\mathrm{O}} \mathrm{c}$ (the nominal FE-DE energy difference) is $1022.008 \pm 0.003 \mathrm{keV}$. This error is only $3 \mathrm{ppm}$.

However, such a technique involves two complications. The first is the "field effect", which is the acceleration (by the electric field gradient across the sensitive volume of the detector) of the primary electrons produced by the interaction of the gamma ray. The apparent energy for any event is shifted by an amount equal to the voltage drop along the path of the electron. The positron, created in a pair production interaction, will contribute to the energy shift in the opposite direction as the electrons. Since in pair production an electron and a positron are created, the $\mathrm{DE}$ peak is expected to have a small field effect shift, as compared to the FE peaks which are primarily from photoelectric and compton scattering events. The magnitude of these field effect shifts has been measured for three detectors with different configurations; namely, planar, coaxial, and "five-sided" (or closed end) coaxial. These results are given in Table II. It is assumed (as a 
first-order approximation) that for a planar detector, there is no field effect when the gamma rays enter the detector perpendicular to the electric field. It is to be noted that the field effect can be as large as about five times the typical uncertainties in the energies, which is 222 ppm.

The second complication in the use of $\mathrm{DE}$ and $\mathrm{FE}$ peaks in the same energy measurement is more basic. We have no assurance that the energy difference between the $F E$ and $P E$ peaks for a given transition is exactly $2 \mathrm{~m}_{0} \mathrm{c}^{2}$. In fact, it is known [4] that, at least in a nonmetal, the positron will annihilate with an electron which is slightly bound. This means that some energy is transferred to the atom and each annihilation photon carries off an energy less than $m_{O} c^{2}$. If the energy transferred to the atom is able to produce electron-hole pairs, the corresponding energy will appear in the DE peak. The FE-DE peak energy difference will then be less than $2 \mathrm{~m}_{\mathrm{O}} \mathrm{c}^{2}$. We have used the transitions illustrated in Figure 2 to obtain a measurement of this energy difference. As given in Table III, we have measured the energy of the $1045 \mathrm{keV}$ line and the energy difference between the 645-keV FE peak and the 669-keV DE peak. The latter measurement was made with the gamma rays entering a planar detector perpendicular to the electric field. Two values of the uncertainty in this FE-DE peak energy difference are given in the table. The first (and smaller) value is based on an operational definition of the energy scale where the ${ }^{198} \mathrm{Au}$ energy [1,2] is taken to exactly 411.794 $\mathrm{keV}$. The second exror includes the error in the 4ll-keV value of $8 \mathrm{eV}$ (i.e., it is based on the $m_{0} c^{2}$ value of $511.004 \pm 0.0016 \mathrm{keV}$ ).

The data in Table II imply that on the operational scale the FE-DE peak energy difference is less than $2 \mathrm{~m}_{\mathrm{O}} \mathrm{c}^{2}$. The larger uncertainty is such that the discrepancy may not be significant on the $m_{0} c^{2}$ scale. Here we have the possibility that either the FE-DE peak energy difference is less then $2 \mathrm{~m}_{\mathrm{O}} \mathrm{c}^{2}$ or the accumulated errors in the energy scale are such as to cause the discrepancy with $2 \mathrm{~m}_{\mathrm{O}} \mathrm{c}^{2}$.

Additional study of this problem of the FE-DE peak energy difference will be made (currently in progress) before we attempt to utilize measured DE peak energies (relative to calibration lines which are FE peaks) to determine gamma-ray transition energies.

[I] R. C. Greenwood, R. G. Helmer and R. J. Gehrke, Nucl. Instr. and Methods 77 (1970) 141.

[2] R. G. Helmer, R. C. Greenwood and R. J. Gehrke, publ. in Nucl. Instr. and Methods.

[3] B. N. Taylor, W. H. Parker and D. N. Langenberg, Rev. Mod. Phys. 4 I (1969) 375.

[4] P. G. Varlashkin and E. F. Zganjar, Nucl. Phys. Al30 (1969) 182. 


\section{TABLE I}

GAMMA-RAY ENERGIES FROM DECAY OF IIUmAg

\begin{tabular}{|c|c|c|}
\hline $\begin{array}{c}\text { Energy Value } \\
(\mathrm{keV})\end{array}$ & $\begin{array}{c}\text { Average Value } \\
(\mathrm{keV})\end{array}$ & Method \\
\hline $446.791 \pm 0.010$ & -- & measured \\
\hline $620.342 \pm 0.016$ & $-\infty$ & measured \\
\hline $657.744 \pm 0.017$ & -- & measured \\
\hline $677.601 \pm 0.018$ & -- & measured \\
\hline $686.998 \pm 0.019$ & --- & measured \\
\hline $706.669 \pm 0.020$ & - & measured \\
\hline $744.254 \pm 0.020$ & $-\infty$ & measured \\
\hline $763.928 \pm 0.019$ & -- & measured \\
\hline $818.018 \pm 0.022$ & -.- & measured. \\
\hline $884.667 \pm 0.018$ & -.- & measured \\
\hline $937.483 \pm 0.020$ & $\overline{--}$ & measured \\
\hline $\begin{array}{l}1384.265 \\
1384.265 \\
1384.270\end{array}$ & & $\begin{array}{ll}706+677 & \text { sum } \\
763+620 & \text { sum } \\
937+446 & \text { sum }\end{array}$ \\
\hline 1475.757 & $\begin{array}{l}1384.267 \pm 0.029 \\
1475.757 \pm 0.034\end{array}$ & $818+657$ \\
\hline $\begin{array}{l}1505.010 \\
1505.004\end{array}$ & & $\begin{array}{ll}818+686 & \text { sum } \\
884 & +620 \text { sum }\end{array}$ \\
\hline $\begin{array}{l}1562.266 \\
1562.262\end{array}$ & $1562.264 \pm 0.033$ & $\begin{array}{ll}818+744 & \text { sum } \\
884+677 & \text { sum }\end{array}$ \\
\hline
\end{tabular}

TABIE II

FIELD EFFECT FOR Ge(Li) DETECTORS (PRELIMINARY RESULTS)

\begin{tabular}{|c|c|c|c|c|c|c|}
\hline \multicolumn{3}{|c|}{ Detector } & \multirow[b]{2}{*}{$\begin{array}{l}\text { Gamma-Ray } \\
\text { Energy } \\
\text { (keV) } \\
\end{array}$} & \multicolumn{3}{|c|}{ Field Effect (eV) } \\
\hline Geometry & $\begin{array}{l}\text { Voltage } \\
\text { (volts) } \\
\end{array}$ & $\begin{array}{c}\text { Electric } \\
\text { Field } \\
\text { (volts } / \mathrm{cm} \text { ) } \\
\end{array}$ & & $\begin{array}{l}\text { FE Peak } \\
\text { Shift } \\
\end{array}$ & $\begin{array}{c}\text { DE Peak } \\
\text { Shift } \\
\end{array}$ & $\begin{array}{l}\text { FE Peak Shift } \\
\text { minut } \\
\text { DE Peak Shift }\end{array}$ \\
\hline planar ${ }^{a}$ & 2000 & 250 & 2600 & $256+20$ & $-24+10$ & $280 \pm 22$ \\
\hline planar ${ }^{a}$ & 2000 & 250 & 2180 & -- & - & $254 \pm 29$ \\
\hline $\operatorname{coaxial}^{b}$ & 2500 & 150 & 2180 & -- & -- & $25 \pm 30$ \\
\hline $\begin{array}{l}\text { five sided } \\
\text { coaxialc }\end{array}$ & 2000 & 125 & 2180 & -- & -- & $301 \pm 42$ \\
\hline
\end{tabular}

a) Gamma rays entering parallel to electric field.

b) Gamma rays entering along axis of coaxial cylinder (i.e., perpendicular to electric field).

c) Gamma rays entering parallel to electric field of closed end of the coaxial cylinder. 


\section{TABLE III}

MEASUREMENT OF FE-DE PEAK ENERGY DIFFERENCE

PRELIMINARY RESULTS FOR ${ }^{124} \mathrm{Sb}$ GAMMA RAYS

\begin{tabular}{|c|c|c|c|}
\hline Item & $\begin{array}{l}\text { Energy } \\
(\mathrm{keV})\end{array}$ & $\begin{array}{l}\frac{\text { Uncex }}{4 I I} \\
\text { Scale }\end{array}$ & $\begin{array}{r}\frac{\mathrm{y}(\mathrm{eV})}{\mathrm{M}_{\mathrm{O}} \mathrm{C}^{2}} \\
\text { Scale } \\
\end{array}$ \\
\hline Energy of $1045-\mathrm{keV}$ gamma ray & 1045.114 & 12 & 24 \\
\hline Energy difference $(669-\mathrm{DE})-(645 \mathrm{FE})$ & 23.121 & 4 & 4 \\
\hline FE-DE energy difference & 1021.993 & 13 & 24 \\
\hline $2 \mathrm{M}_{0} \mathrm{C}^{2}$ value & 1022.008 & 3 & 3 \\
\hline Discrepancy & 0.015 & 13 & 24 \\
\hline
\end{tabular}



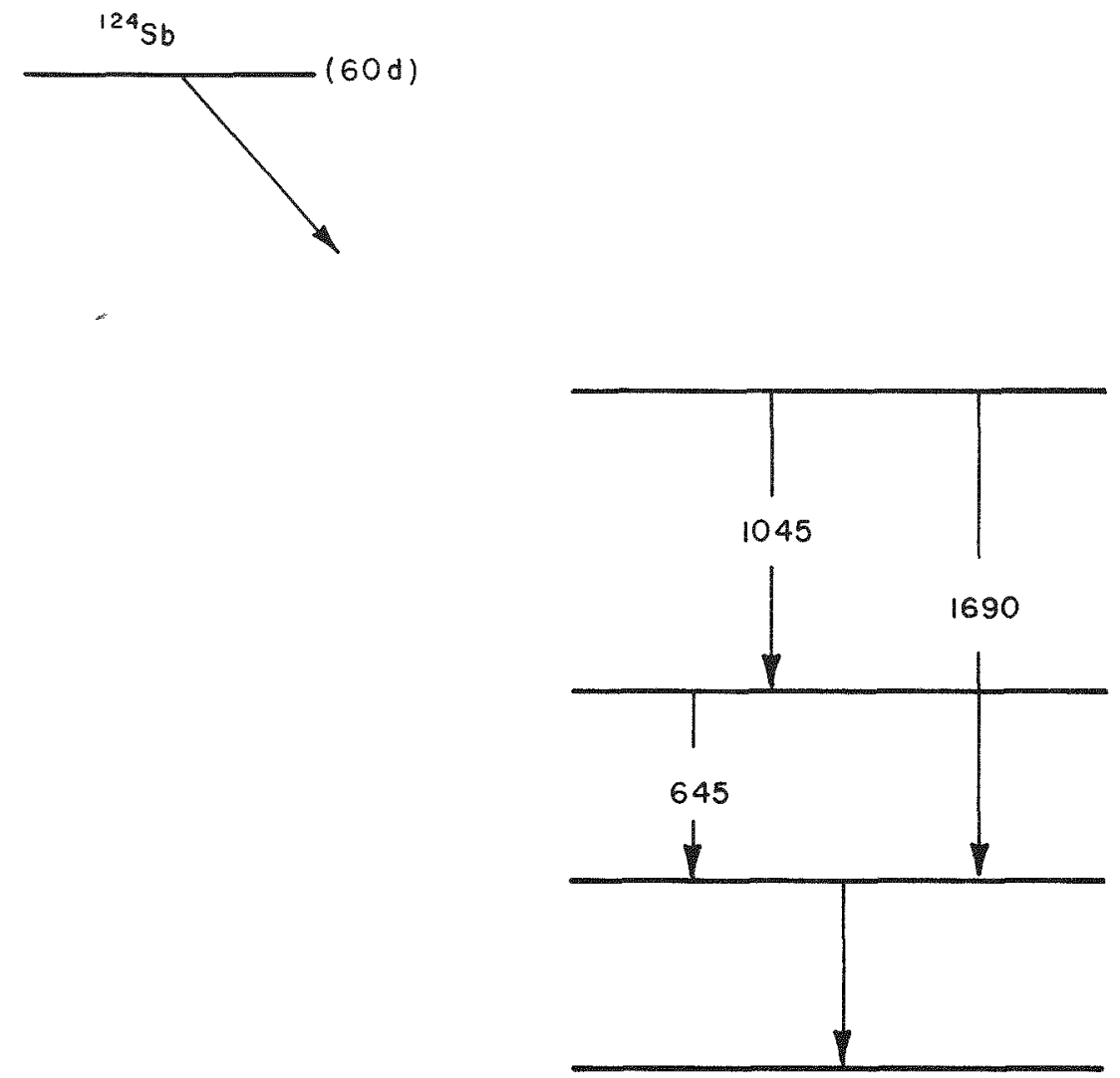

Measurements

$E=1045$

Gamma Energy

$D=668(1690$ DEP $)-645$

Energy Difference

\section{Calculation}

$\Delta_{\text {FEP }}-D E P=1690-668(\mathrm{DEP})$

$=1045+645-668$

$=E-D$

A N C-A-165

Figure 1 Partial level scheme of ${ }^{124} \mathrm{Sb}$ showing transitions used to determine FE-DE peak energy differences. 
PRECISE COMPARISON AND MEASUREMENT OF GAMMA-RAY ENERGIES WITH A Ge(Li) DETECTOR II: 400 to $1300 \mathrm{keV}$

R. G. Helmer, R. C. Greenwood, R. J. Gehrke

In a journal publication[I], the authors reported the results of a study of the energies of many gamma rays that are useful as energycalibration standards below $420 \mathrm{keV}$. The work reported herein, which has been submitted for publication, is an extension of that study to the energy range 400 to $1300 \mathrm{keV}$. In this range, the set of gamma-ray energies that had previously been determined with sufficient accuracy to provide good energy-calibration standards is not extensive enough for precise calibration of $\mathrm{Ge}(\mathrm{Li})$ spectrometers. The availability of such calibration standards is a necessary prerequisite if the full potential of $\mathrm{Ge}(\mathrm{Li})$ spectrometers for precise gamma-ray energy measurements is to be realized. In addition to being highly valuable in decay-scheme studies, precise gamma-ray energy values are frequently invaluable in making unique isotopic identifications in samples containing unknown radioactive constituents.

In order to extend the list of gamma-ray calibration standards available from 400 to $1300 \mathrm{keV}$, we have chosen to make use of crossover gamma-ray transitions for which the energies of the cascade transitions can be determined accurately. For cascade transitions below $400 \mathrm{keV}$, their energies can be measured quite precisely with the techniques and calibration lines in ref. [1]. In the cases where a member of the cascade has an energy greater than $400 \mathrm{keV}$, it has been possible to determine its energy by measuring the energy difference between it and a close-lying transition whose energy had already been determined.

In the process of generating additional calibration energies, two "sets" of gamma-ray energies have been of special value. These two particular "sets" involve (I) the transitions from the decay of $198 \mathrm{Au}$ along with those from ${ }^{182} \mathrm{Ta}$ and ${ }^{59} \mathrm{Fe}$ and (2) the transitions from the decay of ${ }^{192} \mathrm{Ir}$ and $160 \mathrm{~Tb}$. The manner in which these sets have allowed us to obtain precise gamma-ray energy standards, up to $1300 \mathrm{keV}$, from cascade energy sums and from small measured energy differences, is illustrated in Figure 1. In addition to providing a mechanism to obtain calibration energies, it has proven quite valuable to have two such independent sets of gamma-ray energies, since they provide a method of checking for errors that accumulate as the sets are constructed. After all the energies in the two sets have been determined, values of the energy differences for the $1177-(160 \mathrm{~Tb})$ and $1189-\mathrm{keV}$ (182 $\mathrm{Ta})$ lines as well as the $1271-(160 \mathrm{~Tb})$ and $1291-\mathrm{keV}\left({ }^{59} \mathrm{Fe}\right)$ lines can be computed. Comparisons of the measured differences with these computed values give a very sensitive test of the accumulated errors in the energy suming process.

In addition to the isotopes involved in these two sets, energy values have been determined for the gamma rays emitted by 19 other iso,topes. The energy values measured for these other isotopes should be especially valuable as calibration energies since the key transitions in the two primary sets, at 884 and $1087 \mathrm{keV}$, are weak ( $<1 \%$ of the 
strongest transition) and difficult to use routinely. The isotopes $95 \mathrm{~m}_{\mathrm{Tc}}$ and ${ }^{185} \mathrm{Os}$ provide a convenient group of calibration lines in the region 700 to $900 \mathrm{keV}$. However, since these two isotopes are difficult to produce, they have been used, in this work, principally to aid in the calibration of gamma rays emitted by isotopes that are more easily produced and more convenient to use.

The basic group of our measurements in the region from 400 to 1300 $\mathrm{keV}$ consists of a series of energy differences for closely spaced lines. A typical spectrum is shown in Figure 2 and portions of others showing a few pairs of lines of special interest are given in Figures 3-4. For each energy difference, an average value was computed for each detector system used (up to four detector systems were available for use). Comparison of the results from the diffexent detectors provide a test for systematic errors in the data, especially for those in the linearity corrections.

During the course of this work two tests were made specifically to verify our ability to make accurate energy-difference measurements in the region above $I \mathrm{MeV}$. The first of these involved making a two-step measurement of the 1177 (160 Tb) - 1189 (182 Ta) energy difference with $1173\left({ }^{60} \mathrm{Co}\right)$ as an intermediate line. A sample of the spectra obtained for the 1173-1177 difference is shown in Figure 3. Each of the three differences shown below were obtained from independent sets of spectra. The resulting $\mathrm{Tb}-\mathrm{Co}$ and Co-Ta differences are:

and

$$
\mathrm{Tb}-\mathrm{CO} \quad 4.725 \pm 0.002 \mathrm{keV}
$$

$$
\text { Co-Ta } 15.814 \pm 0.002 \mathrm{keV} \text {. }
$$

These values imply a Tb-Ta difference of

$$
11.089 \pm 0.003 \mathrm{keV}
$$

in good agreement with the directly measured value of

$$
11.085 \pm 0.002 \mathrm{keV} \text {. }
$$

The discrepancy between these two differences is then

$$
4 \pm 4 \mathrm{eV} \text {. }
$$

The second test of our ability to measure energy differences is based on measurements for the gamma rays at 1221 and $1231 \mathrm{keV}$ from 182 Ta. From the energies of the low-energy transitions from $182 \mathrm{Ta}$ it is possible to compute this difference; the value is

$$
9.612 \pm 0.003 \mathrm{keV} \text {. }
$$

The corresponding measured difference is

$$
9.606 \pm 0.002 \mathrm{keV} \text {. }
$$


The discrepancy between these values is

$$
6 \pm 3 \mathrm{eV} \text {. }
$$

The results of both of these tests support the proposition that we are able to measure energy differences in this energy region with an accuracy of a few $\mathrm{eV}$, and that we are able to obtain reasonable estimates of the uncertainties in the differences.

As an illustration of the cascade-crossover and energy difference methods used here, the determination of the energies in the $192 \mathrm{Ir}-160 \mathrm{~Tb}$ set will be described (see Figure 1). In ref. [I] average energies were reported for the gamma rays of 295,308 and $316 \mathrm{keV}$. Also available for use, after adjustment to the current reference energy scales, are an energy value for the $136-\mathrm{keV}$ gamma ray measured by Muller et al.[2], a value for the $468-\mathrm{keV}$ gamma ray measured by Reidy [3], and the energies of the 468-, 588-, 604- and $612-\mathrm{keV}$ gamma rays determined from the internal-conversion measurements of Murray et al. 44. Also, energies for the gamma rays at $136,468,604$ and $612 \mathrm{keV}$ were obtained from the relative $\mathrm{K}$ internal-conversion electron momenta reported by Daniel et al.[5]. These relative momenta were converted to absolute momenta in a manner such that the average energy of the three lines near $300 \mathrm{keV}$ was the same as the average of the three energies given in ref. [1].

In adition to these measured values, energies of the lines at 604 and $612 \mathrm{keV}$ were computed from the appropriate sums (see Figure 1).[9] The energy of the $588-\mathrm{keV}$ gamma ray was then computed from the measured value as well as the values of the 604-588 and 612-588 energy differences. Finally, the $884-\mathrm{keV}$ energy was computed from the appropriate energy sum.

The energies of the gamma rays from $160 \mathrm{~Tb}$ are based on the measurement of the energy difference between the $879-\mathrm{keV}$ line from $160 \mathrm{~Tb}$ and the $884-\mathrm{keV}$ gamma ray from ${ }^{192} \mathrm{Ir}$. The quality of the data and the separation of the peaks for these two transitions are shown in Figure 4. From the resulting energy of the 879-keV line and the previously reported energies of several low-energy lines, the energies of the lines at 962 , 966, 1177 and $1271 \mathrm{keV}$ have been computed.

Energy values measured for transitions below $400 \mathrm{keV}$ (many of which were needed in the cascade-crossover combinations) are given in Table I. All of the gamma-ray energies between 400 and $1300 \mathrm{keV}$ from this study, along with those from previous papers, are listed in Table II.

As noted in earlier tests of the consistency of the two basic gamma-ray energy sets (see Figure l) can be made easily. These checks are of particular importance because each energy set is susceptible to significant systematic errors. The energy set involving the gamma rays from ${ }^{198} \mathrm{Au}$ depends on a single measurement of the energy of the $675-\mathrm{keV}$ gamma ray. And, in the energy set based on ${ }^{192} \mathrm{Ir}$, the $884-\mathrm{keV}$ line is essentially a sum of the energies of three transitions of about $300 \mathrm{keV}$. Thus, a small systematic error at $300 \mathrm{keV}$ is magnified by a factor of 3 at $884 \mathrm{keV}$. 
The first two of these tests involve the energy differences $1177(160 \mathrm{~Tb})-1189(182 \mathrm{Ta})$ and $1271 \cdot(160 \mathrm{~Tb})-1291\left({ }^{59} \mathrm{Fe}\right)$. The results of these tests are given in Table III. In computing the uncertainties in the energy differences in column 2, we have not included any errors for the $884-\left({ }^{192} \mathrm{Ir}\right)$ and $1087-\mathrm{keV}\left({ }^{198} \mathrm{Au}\right)$ gamma rays, since these are common to both comparisons. Thus, the errors in the computed differences in column 2 include only the contributions from the energy differences and the measurement errors for the low-energy transitions involved in the cascade sums. As noted in Table III, the average discrepancy between the energy sets is

$$
17 \pm 4 \mathrm{eV}
$$

A third consistency check of the two basic energy sets can be made from a comparison of the energy values determined for the $646-\mathrm{keV}$ gamma ray from $1850 \mathrm{~s}$ as obtained relative to the $675-\left({ }^{198} \mathrm{Au}\right)$ and $884-\mathrm{keV}$ (192 Ir) Iines. Although this comparison results in rather large errors, it has the advantage of providing a Au-Ir comparison without involving the transitions from ${ }^{59} \mathrm{Fe}, 160 \mathrm{~Tb}$ and $182 \mathrm{Ta}$. The result of this comparison is given in Table IV. Again, the energies of the 675- and 884-keV Iines are assumed to be exact in the computation of the uncertainty in the discrepancy.

The average of all three independent measurements of the discrepancy between the two basic energy sets is

$$
17 \pm 4 \mathrm{eV}
$$

Since tests of our ability to measure energy differences indicate that they are accurate to a few $\mathrm{eV}$, we consider this discrepancy to be significant.

On the basis of this result, we conclude that there is a real discrepancy in the energies of the two sets above $0.8 \mathrm{MeV}$. One can also ask whether the magnitude of this discrepancy, $17 \mathrm{eV}$, is reasonable; or whether it indicates some errors in the energies outside the uncertainties that have been assigned. If we recompute the error in the above discrepancy including all of the measurement errors (i.e., including measurement uncertainties in the 675-, 884- and 1087-keV gamma rays), but not the reference scale error we obtain

$$
17 \pm 14 \mathrm{eV}
$$

This indicates that the discrepancy that we observe is quite reasonable.

The gamma-ray energies given in Table II represent the values which have been obtained in the direct manner described above. However, the energy difference measurements in Tables III and IV give additional information that should be considered in the determination of the best gamma-ray energy values. Therefore, we have prepared a list of adjusted energies for which the average discrepancy between the two basic sets is reduced from $I 7 \pm 4 \mathrm{eV}$ to $I \pm 4 \mathrm{eV}$. 
Since we have no basis for knowing if one energy set is more accurate than the other, we have adjusted each of the lines which is basic to the discrepancy measurements in Tables III and IV [i.e., 884 $(192 \mathrm{Ir})$ and 675 and $\left.1087\left({ }^{198} \mathrm{Au}\right)\right]$ by the same amount. Specifically, those energies measured with respect to the 675- and $1087-\mathrm{keV}$ lines from $198^{\circ} \mathrm{Au}$ have been lowered by $8 \mathrm{eV}$, and those determined relative to the $884-\mathrm{keV}$ gamma ray of ${ }^{192}$ Ir have been increased by $8 \mathrm{eV}$. The energies measured with respect to the $\approx 600-\mathrm{keV}$ lines from ${ }^{192}$ Ir have been increased by $5 \mathrm{eV}$. (This value is based on the assumption that any systematic error in the energies of this energy set probably results from the energy values used at $\approx 300 \mathrm{keV}$. Since the $\approx 600-\mathrm{keV}$ energies are essentially sums of two of these values, one might expect the error to be two-thirds as large at $\approx 600 \mathrm{keV}$ as at $884 \mathrm{keV}$.) In those cases where a gamma-ray energy is based on more than one of these calibration lines, or is not traceable to any of these calibration lines, an average adjustment is made. The final energy values are given in Table $V$. The errors quoted are the same as those in Table II. Below $550 \mathrm{keV}$, no adjustments have been made.

In summary, we have been able to provide a set of gamma-ray energies that, together with those of ref. [1], should be sufficient for the calibration of any $\mathrm{Ge}(\mathrm{Li})$ gamma-ray spectrum up to an energy of 1300 $\mathrm{keV}$. The uncertainties in the reported energies increase from $\approx 15 \mathrm{eV}$ at $600 \mathrm{keV}$ (or $25 \mathrm{ppm}$ ) to $\approx 24 \mathrm{eV}$ at $110 \mathrm{keV}$ (or $22 \mathrm{ppm}$ ). However, there is a discrepancy between the two basic energy sets of $17 \mathrm{eV}$ at $1100 \mathrm{keV}$. Since this discrepancy is real, but reasonable, adjusted energies which removed this discrepancy are given in Table $\mathrm{V}$.

It is of interest to note that at $1100 \mathrm{keV}$ the measurement errors are $\approx 13 \mathrm{eV}$ while the contribution from the reference energy is $\approx 22 \mathrm{eV}$. This suggests that a significant improvement in these energies could be obtained from a more accurate measurement of the 4lI-keV gamma ray from ${ }^{198} \mathrm{Au}$. Significant improvements could also occur from more accurate values for the energies of the three gamma rays near $300 \mathrm{keV}$ from ${ }^{192} \mathrm{Ir}$, or the $675-\mathrm{keV}$ line from ${ }^{198} \mathrm{Au}$.

Since our energy differences at $1100 \mathrm{keV}$ have errors of $<5 \mathrm{eV}$, the accuracy of the gamma-ray energies in this region depends almost completely on the accuracy of the best calibration energy in this region.

[1] R. C. Greenwood, R. G. Helmer and R. J. Gehrke, Nucl. Instr. and Meth. 77 (1970) 141 .

[2] D. E. Muller, H. C. Hoyt, D. J. Klein and J.W. M. DuMond, Phys. Rev. 88 (1952) 775 .

[3] J. J. Reidy, private communication.

[4] G. Murray, R. I. Graham and J. S. Geiger, Nucl. Phys. 63 (1965) 353.

[5] H. Daniel, P. Jahn and W. Todt, Bull. Acad. Sci. USSR, phys. ser. 30 (1966) 2107; and P. Jahn, W. Todt and H. Daniel, Phys. Lett. 20 (I966) 395. 
[6] G. Murray, R. L. Graham and J. S. Geiger, Nucl. Phys. 45 (1963) 177.

[7] G. Kaye and R. I. Graham, Can. J. Phys. 42 (1964) 1865.

[8] J. S. Geiger, Atomic Energy of Canada Limited report AECL-3166 and private communication.

[9] Since the energy values for the $\approx 300-\mathrm{keV}$ transitions are an average of the results from several experiments, the degree of independence of their measurement errors is difficult to estimate. Therefore, in the calculation of the measurement errors associated with the energy sums, the measurement errors for the three $\approx 300-\mathrm{keV}$ Iines were assumed to be independent. The same assumption is made in the calculation of all other energy sums in this paper. In some cases, as here, this may result in errors which are underestimated since some degree of non-independence must be expected. 

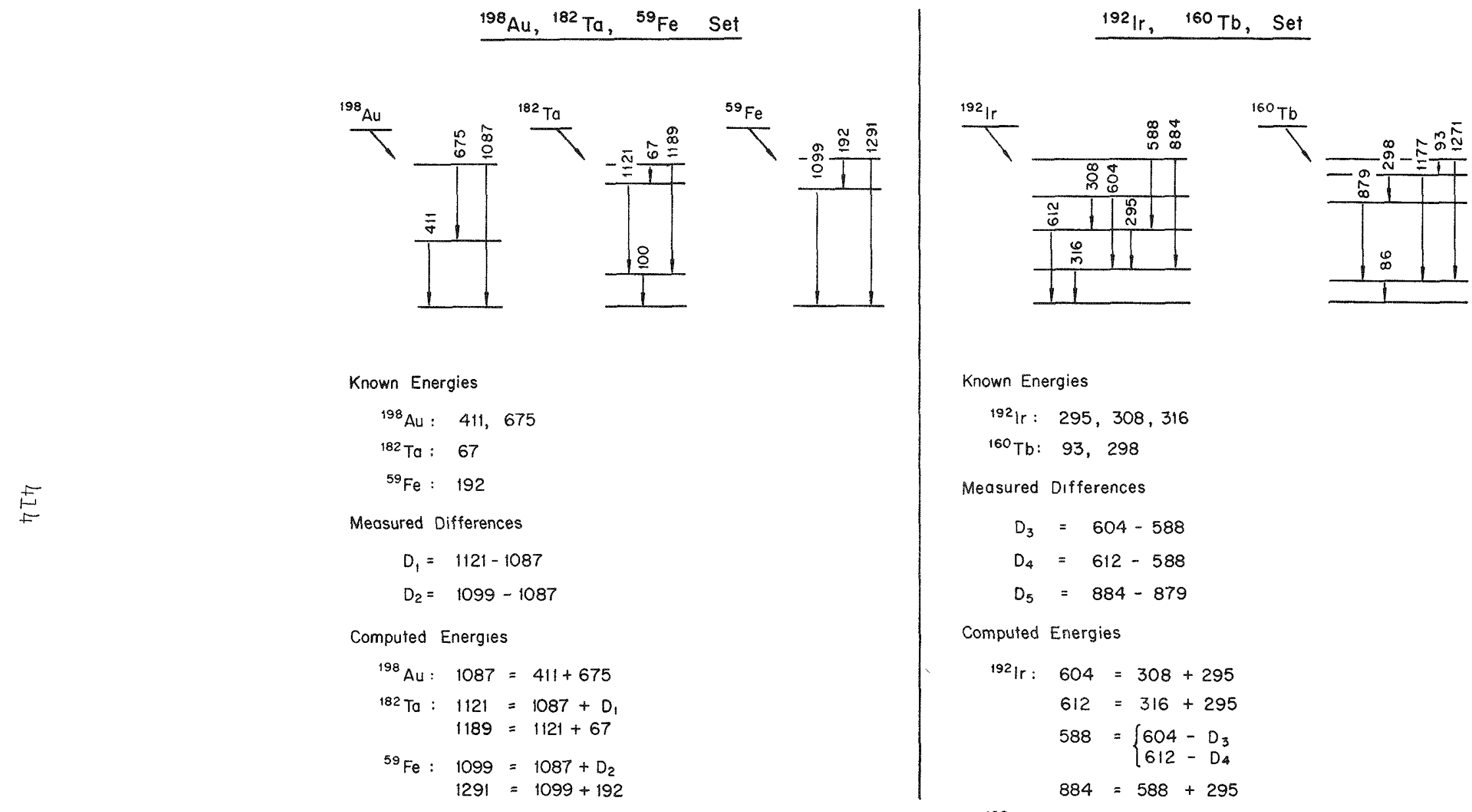

Known Energies

${ }^{192} \mathrm{Ir}: 295,308,316$

${ }^{160} \mathrm{~Tb}: 93,298$

Measured Differences

$$
\begin{aligned}
& D_{3}=604-588 \\
& D_{4}=612-588 \\
& D_{5}=884-879
\end{aligned}
$$

Computed Energies

$$
\begin{aligned}
& { }^{192} \mathrm{Ir}: \quad 604=308+295 \\
& 612=316+295 \\
& 588=\left\{\begin{array}{l}
604-D_{3} \\
612-D_{4}
\end{array}\right. \\
& 884=588+295 \\
& { }^{160} \mathrm{~Tb}: 879=884-\mathrm{D}_{5} \\
& 1177=879+298 \\
& 1271=879+298+93
\end{aligned}
$$




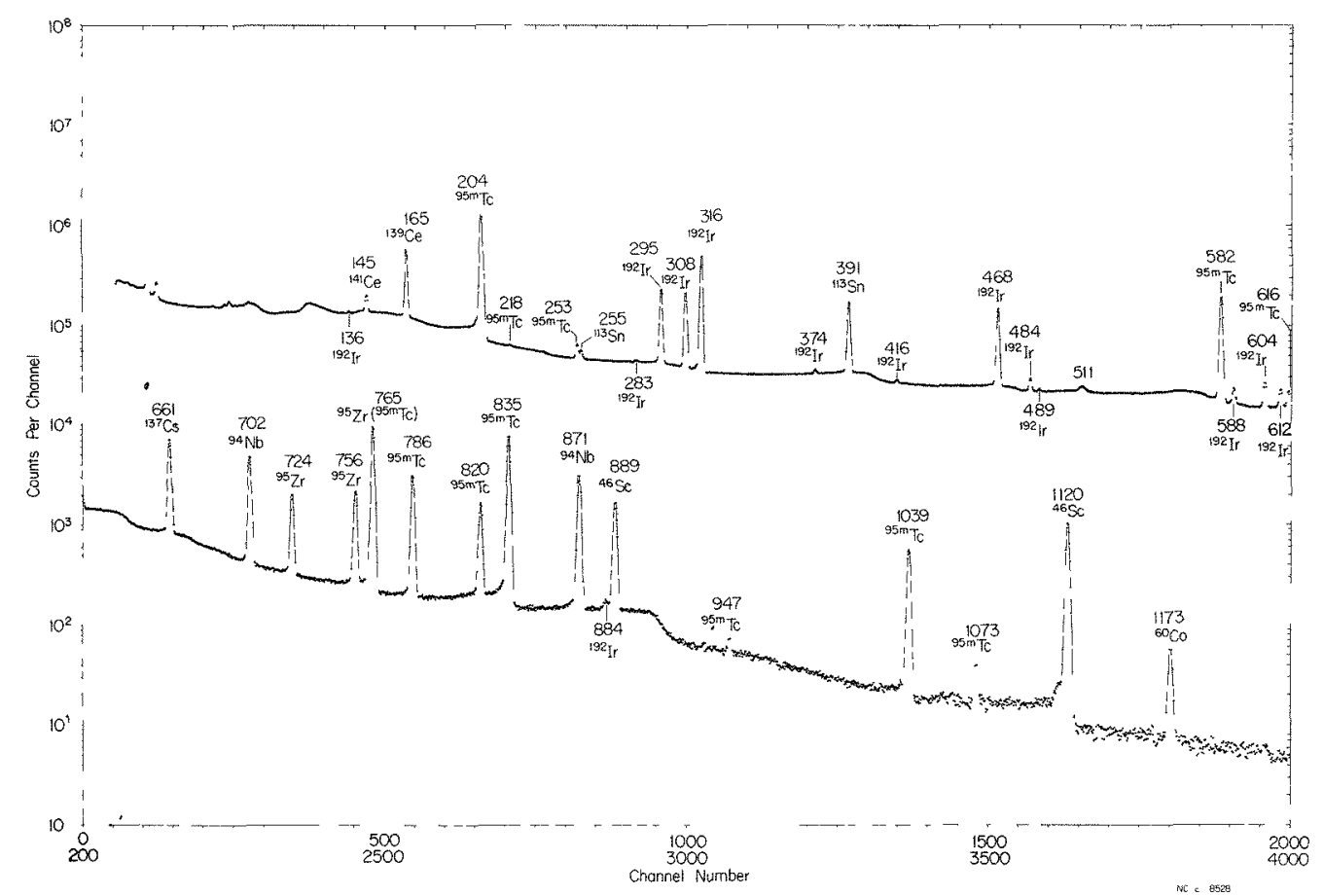

Figure 2 Sample spectrum for the measurement of gamma-ray energies. In this case, the energies of the lines from ${ }^{94} \mathrm{Nb}$ and $95 \mathrm{Zr}-{ }^{9} \mathrm{Nb}$ were determined with respect to the other gamma-ray energies.

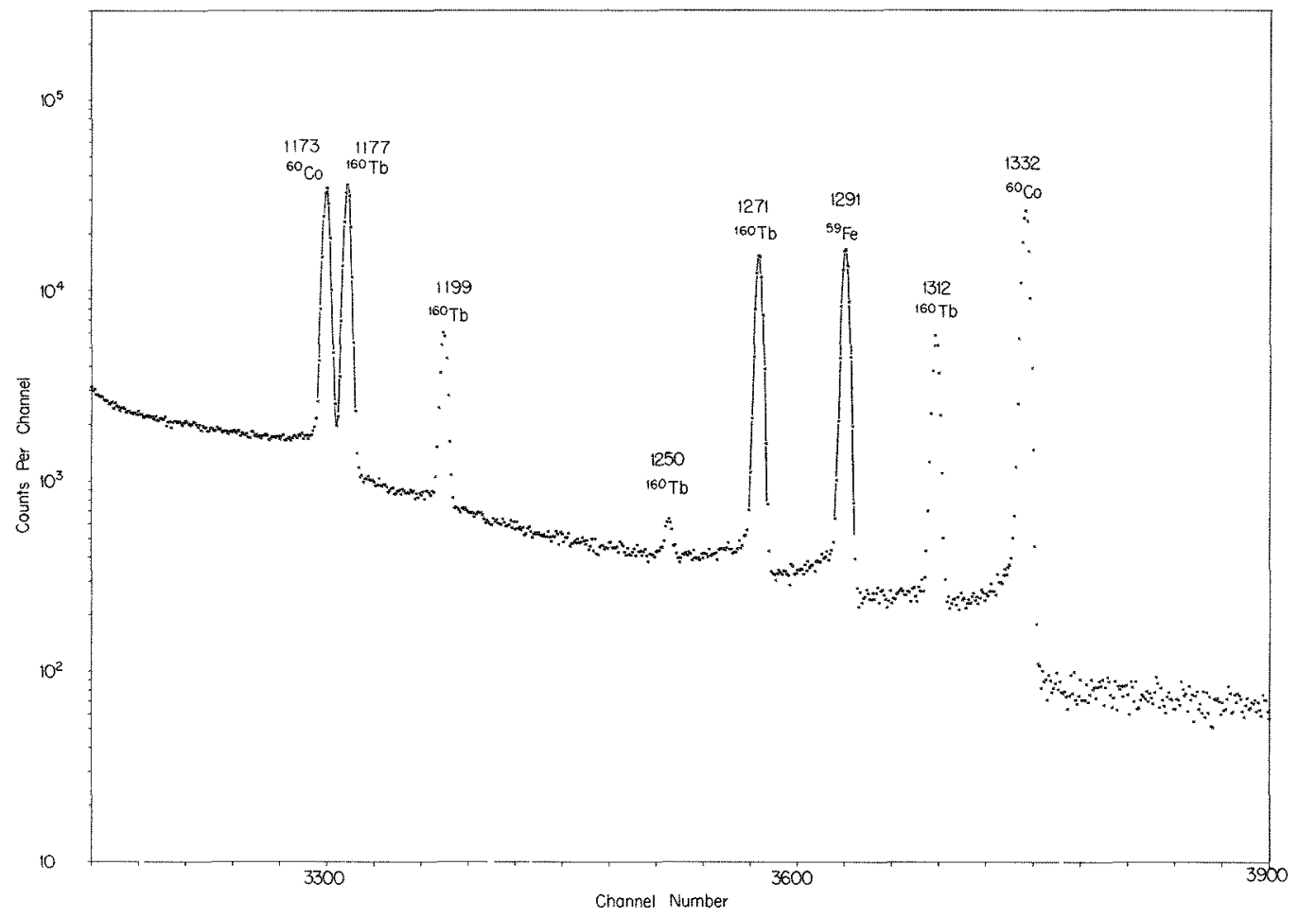

Figure 3 Portion of a gamma-ray spectrum illustrating the resolution of the peaks for the measurement of the $1173\left({ }^{60} \mathrm{Co}\right)-1177\left({ }^{160} \mathrm{~Tb}\right)$ and the $1271(160 \mathrm{~Tb})-129 I\left({ }^{59} \mathrm{Fe}\right)$ energy differences. 


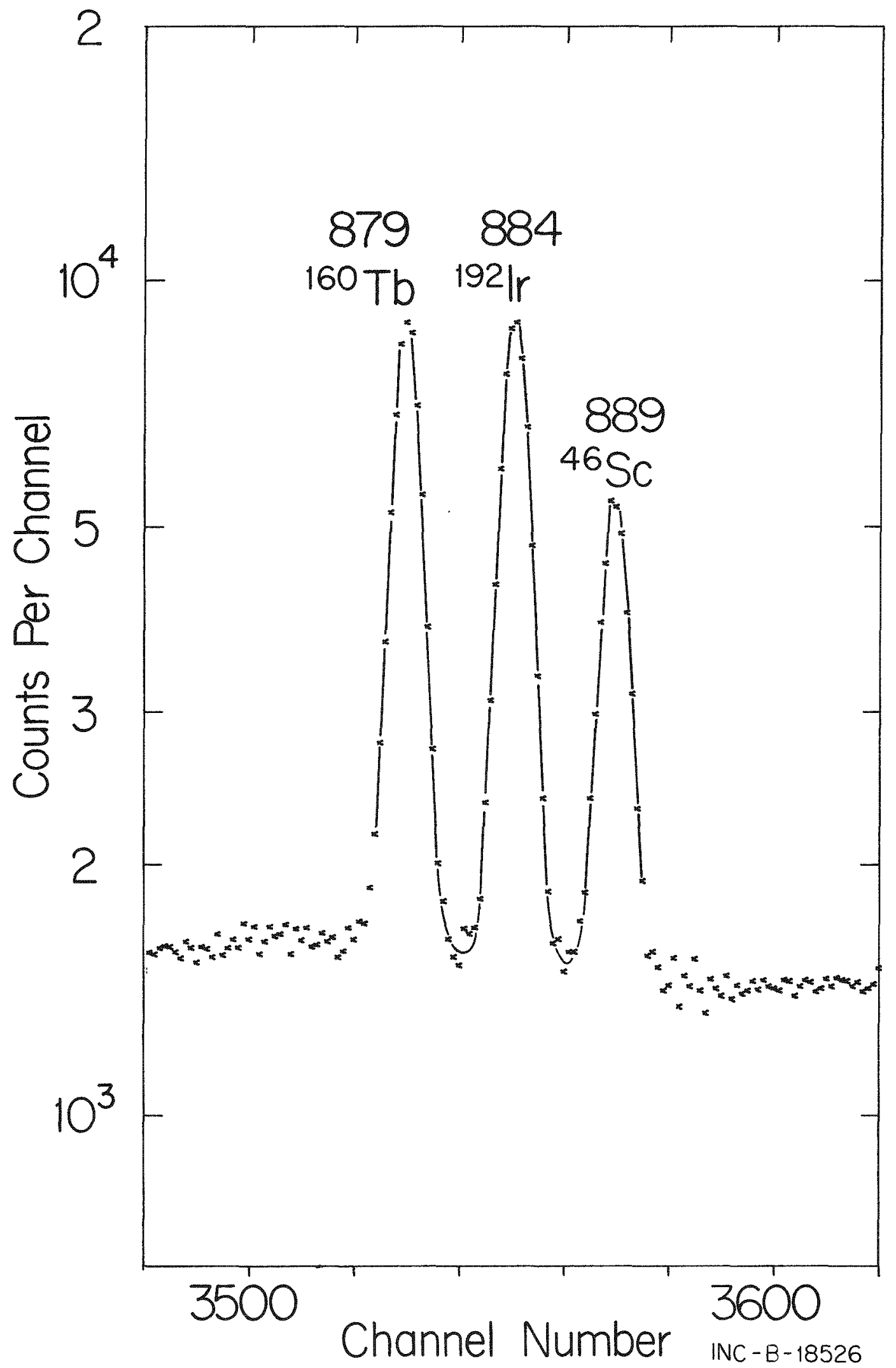

Figure 4 Portion of a spectrum showing the resolution of the peaks for the measurement of the $879\left({ }^{160} \mathrm{~Tb}\right)-884(1.92 \mathrm{Ir})-889\left({ }^{46} \mathrm{Sc}\right)$ energy differences. 
TABLE I

GAMMA-RAY ENERGIES DETERMINED BELOW $400 \mathrm{keV}$

\begin{tabular}{|c|c|c|c|c|}
\hline \multirow[b]{2}{*}{$\begin{array}{l}\text { Parent } \\
\text { Isotope }\end{array}$} & \multirow[b]{2}{*}{$\begin{array}{l}\begin{array}{l}\text { Energy Value } \\
(\mathrm{keV})\end{array} \\
\end{array}$} & \multicolumn{3}{|c|}{ Error Components $(\mathrm{eV})$} \\
\hline & & $\begin{array}{l}\text { Measurement } \\
\text { Error } \\
\end{array}$ & $\frac{\text { Refer }}{\text { WK }_{\alpha 1}}$ & $\frac{\text { ence Error }}{198 \mathrm{Au}-411}$ \\
\hline${ }^{75} \mathrm{Se}$ & $\begin{aligned} & 66.055 \pm 0.009[\mathrm{a}] \\
& 96.733 \pm 0.002 \\
& 121.115 \pm 0.003 \\
& 136.000 \pm 0.005 \\
& 198.596 \pm 0.007 \\
& 264.651 \pm 0.008 \\
& 279.528 \pm 0.008 \\
& 303.913 \pm 0.007[\mathrm{a}] \\
& 400.646 \pm 0.009\end{aligned}$ & $\begin{array}{l}9 \\
2 \\
3 \\
4 \\
7 \\
6 \\
5 \\
4 \\
4\end{array}$ & $\begin{array}{l}1 \\
1\end{array}$ & $\begin{array}{l}5 \\
5 \\
6 \\
8\end{array}$ \\
\hline${ }^{109} \mathrm{Cd}$ & $88.037 \pm 0.005$ & 5 & 1 & \\
\hline $153 \mathrm{Gd}$ & $97.432 \pm 0.003$ & 3 & 1 & \\
\hline${ }^{57} \mathrm{Co}$ & $\begin{array}{l}122.063 \pm 0.004 \\
136.473 \pm 0.004\end{array}$ & $\begin{array}{l}3 \\
3\end{array}$ & & $\begin{array}{l}2 \\
3\end{array}$ \\
\hline $1850 \mathrm{~s}$ & $\begin{array}{l}125.358 \pm 0.004 \\
162.854 \pm 0.008 \\
234.158 \pm 0.010\end{array}$ & $\begin{array}{l}3 \\
7 \\
9\end{array}$ & & $\begin{array}{l}2 \\
3 \\
5\end{array}$ \\
\hline${ }^{59} \mathrm{Fe}$ & $\begin{array}{l}142.648 \pm 0.004 \\
192.344 \pm 0.006\end{array}$ & $\begin{array}{l}3 \\
4\end{array}$ & & $\begin{array}{l}3 \\
4\end{array}$ \\
\hline $95 \mathrm{mTc}$ & $\begin{array}{l}204.117 \pm 0.005 \\
253.066 \pm 0.006\end{array}$ & $\begin{array}{l}4 \\
5\end{array}$ & $\begin{array}{l}2 \\
3\end{array}$ & \\
\hline
\end{tabular}

[a] These values were computed of the energy differences 264-198 and $400-96 \mathrm{keV}$. 
TABLE II

SUMMARY OF GAMMA-RAY ENERGIES (UNADJUSTED) BETWEEN 400 AND $1300 \mathrm{keV}$

\begin{tabular}{|c|c|c|c|}
\hline $\begin{array}{l}\text { Parent } \\
\text { Isotope }\end{array}$ & $\begin{array}{c}\text { Gamma-ray Energy } \\
(\mathrm{keV})\end{array}$ & $\begin{array}{l}\text { Parent } \\
\text { Isotope } \\
\end{array}$ & $\begin{array}{c}\text { Gamma-ray Energy } \\
(\mathrm{keV})\end{array}$ \\
\hline${ }^{20} 3_{\mathrm{Pb}}$ & $\begin{array}{l}401.315 \pm 0.013 \\
680.503 \pm 0.017\end{array}$ & ${ }^{137} \mathrm{Cs}$ & $661.646 \pm 0.019^{d}$ \\
\hline${ }^{198} \mathrm{Au}$ & $\begin{array}{r}411.794 \pm 0.008^{\mathrm{a}} \\
675.879 \pm 0.018^{\mathrm{b}}\end{array}$ & ${ }^{94} \mathrm{Nb}$ & $\begin{array}{l}702.622 \pm 0.019 \\
871.091 \pm 0.018\end{array}$ \\
\hline & $1087.671 \pm 0.024$ & ${ }^{95} \mathrm{Zr}-{ }^{95} \mathrm{Nb}$ & $\begin{array}{l}724.179 \pm 0.018 \\
756.710 \pm 0.019\end{array}$ \\
\hline${ }^{192} \operatorname{Ir}$ & $\begin{array}{l}468.062 \pm 0.010^{c} \\
484.570 \pm 0.011 \\
588.567 \pm 0.012^{\mathrm{e}} \\
604.396 \pm 0.012^{f} \\
612.445 \pm 0.013^{f} \\
884.515 \pm 0.018\end{array}$ & $\begin{array}{l}{ }^{58} \mathrm{Co} \\
{ }^{54} \mathrm{Mn} \\
56\end{array}$ & $\begin{array}{l}765.781 \pm 0.019 \\
810.752 \pm 0.021 \\
834.822 \pm 0.021 \\
846.745 \pm 0.019\end{array}$ \\
\hline${ }^{7} \mathrm{Be}$ & $477.593 \pm 0.012$ & $160_{\mathrm{Tb}}$ & $879.356 \pm 0.018$ \\
\hline${ }^{85} \mathrm{Sr}$ & $513.996 \pm 0.016$ & & $\begin{array}{l}962.287 \pm 0.020 \\
966.143 \pm 0.020\end{array}$ \\
\hline${ }^{207} \mathrm{Bi}$ & $\begin{array}{r}569.684 \pm 0.013 \\
1063.643 \pm 0.024\end{array}$ & & $\begin{array}{l}1177.926 \pm 0.024 \\
1271.842 \pm 0.026\end{array}$ \\
\hline $95 \mathrm{~m}_{\mathrm{TC}}$ & $\begin{array}{r}582.063 \pm 0.013 \\
786.179 \pm 0.017 \\
820.603 \pm 0.019 \\
835.127 \pm 0.018 \\
1039.242 \pm 0.022\end{array}$ & $\begin{array}{l}{ }^{88} \mathrm{Y} \\
59_{\mathrm{Fe}}\end{array}$ & $\begin{aligned} 889.250 & \pm 0.018 \\
1120.524 & \pm 0.025 \\
898.013 & \pm 0.019 \\
1099.232 & \pm 0.025\end{aligned}$ \\
\hline${ }^{228} \mathrm{Th}$ & $583.169 \pm 0.013^{e}$ & & $1291.572 \pm 0.028$ \\
\hline $1850 \mathrm{~s}$ & $\begin{array}{l}592.061 \pm 0.014 \\
646.111 \pm 0.017 \\
717.419 \pm 0.018 \\
874.806 \pm 0.019 \\
880.264 \pm 0.019\end{array}$ & $\begin{array}{l}65_{\mathrm{Zn}} \\
182 \mathrm{Ta}\end{array}$ & $\begin{array}{l}1115.526 \pm 0.025 \\
1121.280 \pm 0.026 \\
1189.030 \pm 0.027 \\
1221.384 \pm 0.027 \\
1230.997 \pm 0.028\end{array}$ \\
\hline $110 \mathrm{~m}_{\mathrm{Ag}}$ & $\begin{array}{l}657.748 \pm 0.017 \\
706.664 \pm 0.020 \\
744.249 \pm 0.020 \\
763.923 \pm 0.019 \\
818.013 \pm 0.022 \\
884.659 \pm 0.018 \\
937.475 \pm 0.020\end{array}$ & $\begin{array}{l}{ }^{60} \mathrm{Co} \\
{ }^{22} \mathrm{Na}\end{array}$ & $\begin{array}{l}1257.398 \pm 0.028 \\
1273.711 \pm 0.028 \\
1289.134 \pm 0.029 \\
1173.208 \pm 0.025 \\
1274.519 \pm 0.028\end{array}$ \\
\hline
\end{tabular}

a) From ref. [4] and [6].

b) From ref. [7].

c) Evaluated from data in ref. $[3],[4]$ and $[5]$.
d.) From ref. [8].
e) Evaluated from our data and that of ref. [4].
f) Evaluated from sums and data of ref. [4] and [5].


TABLE $\cdot$ II

COMPARISONS OF GAMMA-RAY ENERGY SETS ABOVE $1 \mathrm{MeV}$

\begin{tabular}{|c|c|c|c|}
\hline \multirow[b]{2}{*}{ Isotopes and Transitions } & \multicolumn{2}{|c|}{ Energy Difference (keV) } & \multirow{2}{*}{$\begin{array}{c}\text { Discrepancy } \\
(\mathrm{eV})\end{array}$} \\
\hline & Computed & Measured & \\
\hline $1177(160 \mathrm{~Tb})-1189(182 \mathrm{Ta})$ & $11.104 \pm 0.004$ & $11.085 \pm 0.002$ & $19 \pm 5$ \\
\hline $1271\left(160_{\mathrm{Tb}}\right)-1291\left({ }^{59} \mathrm{Fe}\right)$ & $19.730 \pm 0.008$ & $\begin{array}{l}19.716 \pm 0.003 \\
\text { Average }\end{array}$ & $\frac{14 \pm 9}{17 \pm 4}$ \\
\hline
\end{tabular}

\section{TABLE IV}

COMPARISON OF GAMMA-RAY ENERGY SETS BELOW I MeV BY MEANS OF THE 646-keV GAMMA RAY FROM 1850 s

\begin{tabular}{|c|c|c|c|c|}
\hline & 198 & Energy Set & 1921 & Energy Set \\
\hline & Isotope & $\begin{array}{l}\text { Energy Value } \\
\quad(\mathrm{keV}) \\
\end{array}$ & Isotope & $\begin{array}{l}\text { Energy Value } \\
\quad(\mathrm{keV})\end{array}$ \\
\hline Basic energy & $198 \mathrm{Aul}$ & 675.879 & $192 \mathrm{Ir}$ & 884.515 \\
\hline Energy difference & & $14.229 \pm 0.003$ & & $4.254 \pm 0.007$ \\
\hline Intermediate energy [b] & $137 \mathrm{Cs}$ & $661.650 \pm 0.003$ & $1850 \mathrm{~s}$ & $880.261 \pm 0.007$ \\
\hline Energy difference & & $15.529 \pm 0.002$ & & $234.158 \pm 0.009$ \\
\hline Final energy & $185 \mathrm{Os}$ & $646.121 \pm 0.004$ & $1850 \mathrm{~s}$ & $646.103 \pm 0.012$ \\
\hline & & . & & \\
\hline
\end{tabular}

[a] These errors include only the measurement contributions and assume the basic energies are exact.

[b] The intermediate energies are computed only from the differences given and do not correspond to the values elsewhere in the paper. 
TABIE V

FINAL ADJUSTED GAMMA-RAY ENERGIES BETWEEN 400 AND $1300 \mathrm{keV}$. SEE TEXT FOR DESCRIPTION OF THE ADJUSTMENTS MADE.

\begin{tabular}{|c|c|c|c|}
\hline $\begin{array}{l}\text { Parent } \\
\text { Isotope }\end{array}$ & $\begin{array}{c}\text { Gamma-ray Energy } \\
(\mathrm{keV})\end{array}$ & $\begin{array}{l}\text { Parent } \\
\text { Isotope }\end{array}$ & $\begin{array}{c}\text { Gamma-ray Energy } \\
(\mathrm{keV})\end{array}$ \\
\hline${ }^{203} \mathrm{~Pb}$ & $\begin{array}{l}401.315 \pm 0.013 \\
680.495 \pm 0.017\end{array}$ & ${ }^{137} \mathrm{Cs}$ & $661.638 \pm 0.019$ \\
\hline \multirow[t]{2}{*}{${ }^{198} \mathrm{Au}$} & $\begin{array}{l}411.794 \pm 0.008 \\
675.871 \pm 0.018\end{array}$ & $94 \mathrm{Nb}$ & $\begin{array}{l}702.627 \pm 0.019 \\
871.099 \pm 0.018\end{array}$ \\
\hline & $1087.663 \pm 0.024$ & $95 \mathrm{Zr}$ & $\begin{array}{l}724.184 \pm 0.018 \\
756.715 \pm 0.019\end{array}$ \\
\hline \multirow[t]{4}{*}{${ }^{192} \operatorname{Ir}$} & $\begin{array}{l}468.062 \pm 0.010 \\
484.570 \pm 0.011\end{array}$ & & $765.786 \pm 0.019$ \\
\hline & $\begin{array}{l}588.572 \pm 0.012 \\
604.401 \pm 0.012\end{array}$ & ${ }^{58} \mathrm{Co}$ & $810.757 \pm 0.021$ \\
\hline & $\begin{array}{l}612.450 \pm 0.013 \\
884.523+0.018\end{array}$ & ${ }^{54} \mathrm{Mn}$ & $834.827 \pm 0.021$ \\
\hline & & ${ }^{56} \mathrm{Co}$ & $846.751 \pm 0.019$ \\
\hline${ }^{7} \mathrm{Be}$ & $477.593 \pm 0.012$ & $160 \mathrm{~Tb}$ & $879.364 \pm 0.018$ \\
\hline${ }^{85} \mathrm{Sr}$ & $513.996 \pm 0.016$ & & $\begin{array}{l}962.295 \pm 0.020 \\
966.151 \pm 0.020\end{array}$ \\
\hline${ }^{207} \mathrm{Bi}$ & $\begin{array}{r}569.689 \pm 0.013 \\
1063.635 \pm 0.024\end{array}$ & & $\begin{array}{l}1177.934 \pm 0.024 \\
1271.850 \pm 0.026\end{array}$ \\
\hline \multirow[t]{2}{*}{$95 \mathrm{mrc}$} & $\begin{array}{l}582.068 \pm 0.013 \\
786.184 \pm 0.017 \\
820.608 \pm 0.019\end{array}$ & $46_{S C}$ & $\begin{array}{r}889.258 \pm 0.018 \\
1120.516 \pm 0.025\end{array}$ \\
\hline & $\begin{array}{r}835.132 \pm 0.018 \\
1039.247 \pm 0.022\end{array}$ & ${ }^{88} Y$ & $898.021 \pm 0.019$ \\
\hline $228 \mathrm{Th}$ & $583.174 \pm 0.013$ & ${ }^{59} \mathrm{Fe}$ & $\begin{array}{l}1099.224 \pm 0.025 \\
1291.564 \pm 0.028\end{array}$ \\
\hline \multirow[t]{4}{*}{$185 \mathrm{Os}$} & $\begin{array}{l}592.066 \pm 0.014 \\
646.111 \pm 0.017\end{array}$ & $65 \mathrm{zn}$ & $1115.518 \pm 0.025$ \\
\hline & $717.424 \pm 0.018$ & $182 \mathrm{Ta}$ & $1121.272 \pm 0.026$ \\
\hline & $874.814 \pm 0.019$ & & $\begin{array}{l}1189.022 \pm 0.027 \\
1221.376+0.027\end{array}$ \\
\hline & & & $1230.989 \pm 0.028$ \\
\hline \multirow[t]{5}{*}{$110 \mathrm{~m}_{\mathrm{Ag}}$} & $657.744 \pm 0.017$ & & $1257.390 \pm 0.028$ \\
\hline & $706.669 \pm 0.020$ & & $1273.703 \pm 0.028$ \\
\hline & $\begin{array}{l}744.254 \pm 0.020 \\
763.028 \pm 0.019\end{array}$ & & $1289.126 \pm 0.029$ \\
\hline & $\begin{array}{l}103.928 \pm 0.019 \\
818.018 \pm 0.022 \\
884.667 \pm 0.018\end{array}$ & $60 \mathrm{Co}$ & $1173.208 \pm 0.025$ \\
\hline & $937.483 \pm 0.020$ & ${ }^{22} \mathrm{Na}$ & $1274.511 \pm 0.028$ \\
\hline
\end{tabular}


DEPERMINATION OF RELATIVE PHOTOPEAK EFICIENCY AND SYSTEM LINEARITY FOR Ge(Li) GAMMA-RAY SPECTROMETERS II]

R. J. Gehrke, J. E. Cline, R. L. Heath

In the paper, Reference I, a simple precise method is described for determining both the relative detection efficiency and the linearity of a $\mathrm{Ge}(\mathrm{Li})$ spectrometer. The method employs three readily available radioactive sources, ${ }^{75} \mathrm{Se}(120 \mathrm{~d}),{ }^{82} \mathrm{Br}(35 \mathrm{~h})$, and ${ }^{56} \mathrm{Co}(77 \mathrm{~d})$, that emit gamma rays covering the energy range $90-3500 \mathrm{keV}$. The gamma-ray energies and relative intensities for these isotopes have been precisely measured. A method is suggested to reproducibly set the "effective zero" of the analyzer using the above gamma-ray sources.

[1] Nucl. Instr. and Methods 91 (1971) 349. 
CALIBRATION OF THE EFFICIENCY OF A Si(Li) PHOTON SPECIROMETER IN THE ENERGY REGION 5 TO $125 \mathrm{keV}[\mathrm{I}]$

R. J. Gehrke, R. A. Lokken[2]

A technique for measuring the absolute photopeak efficiency of a $30-\mathrm{mm}^{2} \times 3-\mathrm{mm} \mathrm{Si}(\mathrm{Li})$ spectrometer in the energy region from 5 to 125 $\mathrm{keV}$ was developed. These measurements are made by comparing the photopeak counting rate obtained from the Si(Li) spectrometer with the respective photon emission rate using a series of absolutely calibrated $\gamma$-ray sources. A simple procedure to obtain an accurate relative efficiency curve is also given. This alternate procedure measures the relative efficiency by using three readily available radionuclides (i.e., $145 \mathrm{Pm}, 155 \mathrm{Eu}$ and ${ }^{241} \mathrm{Am}$ ) whose relative photon intensities have been measured with the Si(Li) spectrometer calibrated for absolute photopeak efficiency. In addition, the K-conversion coefficients of the $\gamma$-ray transitions from $60 \mathrm{~m}_{\mathrm{Co}}(58.6 \mathrm{keV}), 94 \mathrm{~m}_{\mathrm{Nb}}(40.7 \mathrm{keV})$, and $176 \mathrm{~m}_{\mathrm{Lu}}(88.4 \mathrm{keV})$ and the L-conversion coefficients for those of $192 \mathrm{~m}_{\mathrm{Ir}}$ $(58.0 \mathrm{keV})$ and $210 \mathrm{~Pb}(46.5 \mathrm{keV})$ were determined from their $\mathrm{x}-\mathrm{ray} / \mathrm{\gamma}-\mathrm{ray}$ intensity ratios and their respective fluorescence yields. The values obtained for these conversion coefficients are $42.1 \pm 4.5,995 \pm 150$, $1.18 \pm 0.12,2040 \pm 430$ and $14.3 \pm 1.5$, respectively.

[1] R. J. Gehrke and R. A. Lokken, Nucl. Instr. and Methods (in press).

[2] Associated Western University Graduate Fellow from Utah State University, Logan, Utah. 


\title{
CALIBRATION OF Ge(Li) SPECTROMETER SYSTEMS
}

\author{
R. J. Gehrke, I. D. McIsaac
}

Progress continues on measuring the photopeak efficiency of specialized Ge(Li) spectrometer systems. One Ge(Li) spectrometer used in activation analysis experiments has been calibrated for photopeak efficiency for samples consisting of known volumes of radioactive solutions. This is the type of sample normally encountered in activation analysis when radiochemical separations are necessary. Using techniques previously developed at our laboratory, [1] the relative efficiency was measured as a function of source-detector distance and volume of solution in the sample. In this series of experiments, the relative shapes of the curves did not change appreciably $(<8 \%)$ for different source-detector distances or different volumes. The container cross section was always the same with the height of the solution varying proportionately with the volume. The reason for this effort is to better define the relative efficiency for samples differing from point source geometry and to eliminate the need to make separate calculations for sample selfabsorption of lower energy $\gamma$-rays. Below $600 \mathrm{keV}$ the relative shape of this efficiency curve differs significanty from the efficiency curve obtained with point sources.

Another Ge(Li) spectrometer which will be used for scanning fuel pins is being calibrated for absolute photopeak efficiency and system Iinearity. This Ge(Li) detector is a closed-end circular coaxial and is located at the end of a 7-ft. Iong collimator. The slit formed by the collimatox has a height of $1 / 2$ in. and a width which can be varied from 0 to $50 \times 10^{-3}$ in. The collimator slit is centered on the detector container face.

The relative photpeak efficiency was obtained from the relatiye intensities of the gamma rays emitted from ${ }^{75} \mathrm{Se}, 82 \mathrm{Br}$ and ${ }^{56} \mathrm{Co} .[1]$ reduce the intensity required of the ${ }^{75} \mathrm{Se},{ }^{82} \mathrm{Br}$ and ${ }^{56} \mathrm{Co}$ sources but to approximate the effect of a $7-f t$. Iong collimator, these sources were counted separately through an iron collimator 15-in. long with a $3 / 4 \mathrm{in.} \times 45 \times 10^{-3}$-in. wide slit. It is assumed that the shape of the relative photopeak efficiency through the 7 -ft. long collimator will not differ appreciably above $100 \mathrm{keV}$ from the relative photopeak efficiency obtained with the 15-in. long collimator. A I/4-in. horizontal displacement of the vertically positioned slit resulted in essentially the same relative photopeak efficiency.

Two irradiated cobalt wires $1 / 2$ in. $\times 40 \times 10^{-3}$-in. diameter and calibrated for absolute gamma-ray emission rate will be used to obtain the absolute photopeak efficiency at $1173 \mathrm{keV}$ and $1332 \mathrm{keV}$ through the 7-ft. collimator. These absolute photopeak efficiencies will then be used to normalize the relative photopeak efficiency curve to provide an absolute curve for energies ranging from $100 \mathrm{keV}$ to $3000 \mathrm{keV}$.

[1] R. J. Gehrke, J. E. Cline and R. I. Heath, Nucl. Instr. and Methods 11 (1971) 349 . 
A 2-mg source of ${ }^{252} \mathrm{Cf}$, with a neutron output $25 \times 10^{9}$ neutrons/sec has recently been acquired in order to evaluate the utility of such small ${ }^{252} \mathrm{Cf}$ neutron sources for prompt neutron activation (neutron capture gamma-ray) analysis. Experiments are being conducted with this source using two basically different ${ }^{252} \mathrm{Cf}$ source-sample-detector geometries. These are: (I) an internal target geometry, with the sample located close to the ${ }^{252} \mathrm{Cf}$ source in a tangential beam tube; and (2) an external beam geometry, in which the sample is irradiated in a beam of neutrons external to the biological shield.

For our initial experiments, the ${ }^{252} \mathrm{Cf}$ source has been located within a biological shield consisting of a minimum of 22 in. of water and borated paraffin plus 1 to 1.5 in. of lead. This biological shield is penetrated by a 2-in. diameter tangential "beam channel" which passes close to the ${ }^{252} \mathrm{Cf}$ source. With this facility we have been able to conduct experiments using both the internal and external sample arrangements. A schematic drawing of the facility, as arranged for the internal target geometry, is shown in Figure 1 .

Experiments to date have been concerned primarily with optimizing the design geometry of the internal sample arrangement to achieve maximum sensitivity (for detection of prompt gamma rays) with minimal contribution to the sample prompt gamma-ray spectrum from background gamma radiation. We can see from Figure 1 that, in order for the detector to "see" only the sample material in the region of highest neutron flux, a suitable gamma-ray collimator must be located in the "beam channel" between the sample and the detector. It is obvious then that, since the sample end of this collimator will itself be in a fairly high neutron flux region, this portion, at least, of the collimator must be constructed of a low neutron capture cross section material. Initially, this portion of the collimator was constructed of lead, but the resultant $207 \mathrm{~Pb}(n, y)$ spectrum was found to contribute excessively to the background spectrum. Subsequent experiments have demonstrated that bismuth is a suitable material for use in the high-flux end of the collimator. Our most recent gamma-ray collimator design is shown in detail in Figure 2. The alternate layers of borated polyethylene were inserted to eliminate neutron streaming along the "beam channel". Neutrons will, however, be scattered off the samples into the Ge(Li) detector. To minimize this problem, without unduly attenuating the beam of highenergy prompt gamma rays, a 4-in. plug of borated polyethylene has generally been inserted in the collimator, at the detector end, as shown in Figure 2.

Examples of prompt gamma-ray spectra measured with this internal sample arrangement (using the gamma-ray collimator shown in Figure 2) are illustrated in Figures 3 and 4 , the spectra being taken using samples of $100 \mathrm{gm}$ tungsten and $42.2 \mathrm{gm}$ of basalt, respectively. The $W(n, y)$ spectrum can be compared with the corresponding spectrum obtained using the thermal-neutron capture gamma-ray facility on the Materials 
Testing Reactor, shown in Figure $I$ on page 41 of this report. (This latter spectrum was obtained using a $12.5-\mathrm{gm}$ sample of tungsten irradiated in a thermal-neutron flux of $26 \times 10^{6} \mathrm{n} / \mathrm{cm}^{2} \mathrm{sec}$ for a period of 16 hours with the prompt gamma rays being detected in a $6 \mathrm{~cm}^{3} \mathrm{Ge}$ (Li)

detector.) Examination of the prompt gamma-ray spectrum obtained from the basalt sample, which is shown in Figure 4, reveals that well resolved peaks are observed for all the principal (> few tenths percent) constituent elements, except oxygen and magnesium, of basalt. Elements identified in the spectrum include aluminum, silicon, sodium, potassium, calcium, titanium and iron. 


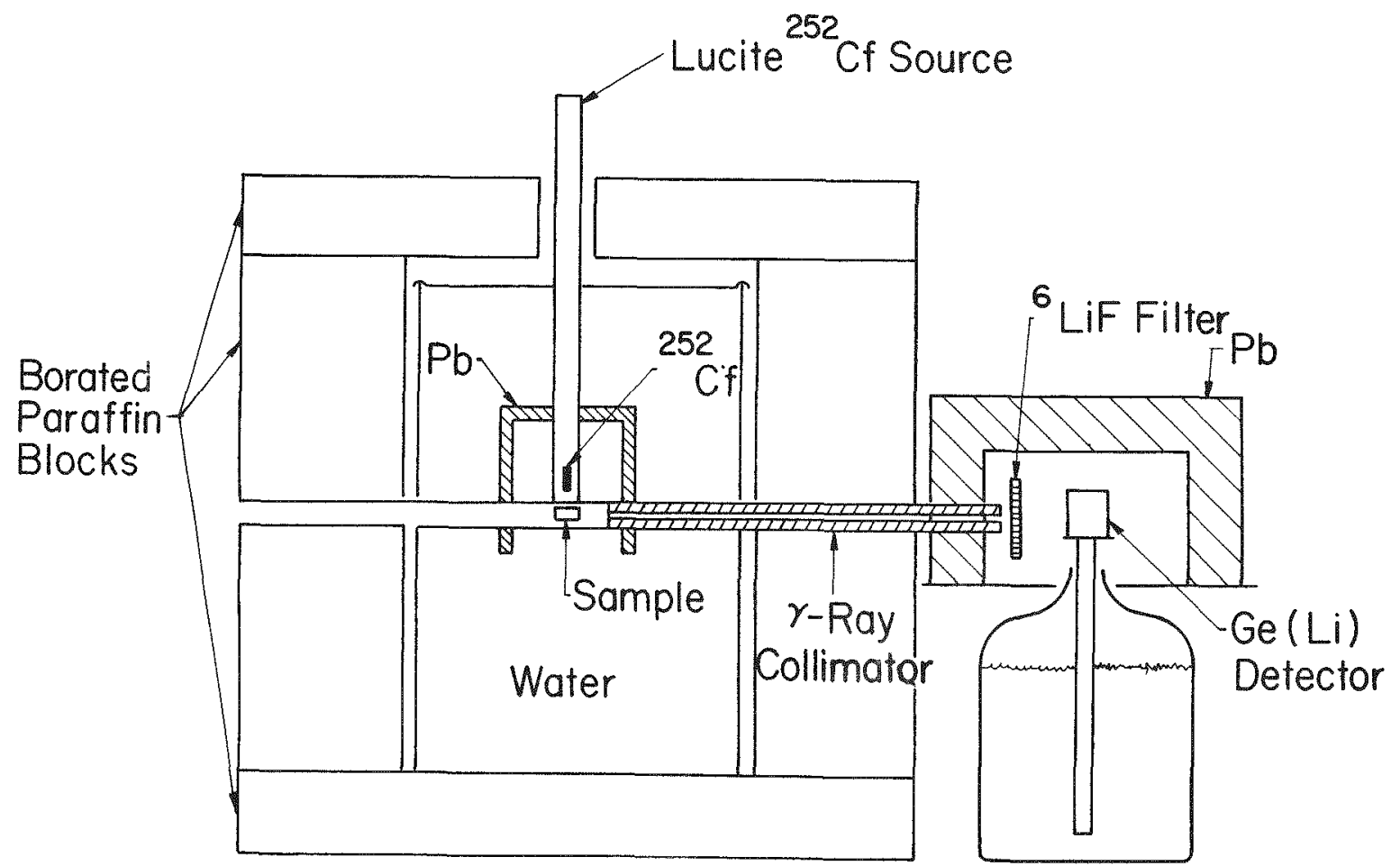

Figure 1 A schematic drawing of the experimental arrangement utilized for prompt neutron capture gamma-ray assay with the $2-m g{ }^{252} \mathrm{Cf}$ source using the internal target geometry.
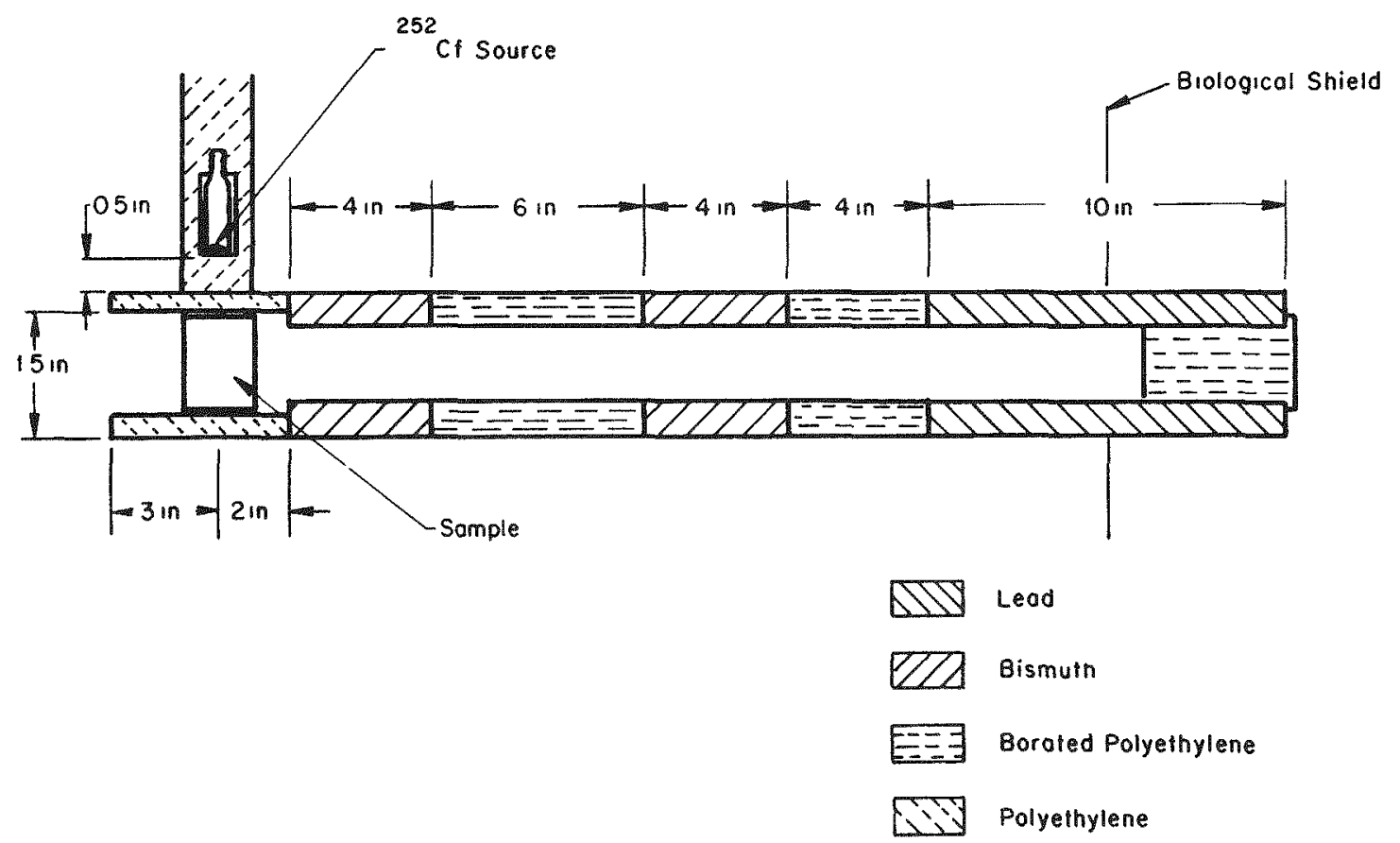

Figure 2 Design of a gamma-ray collimator for use with the internal target geometry. 


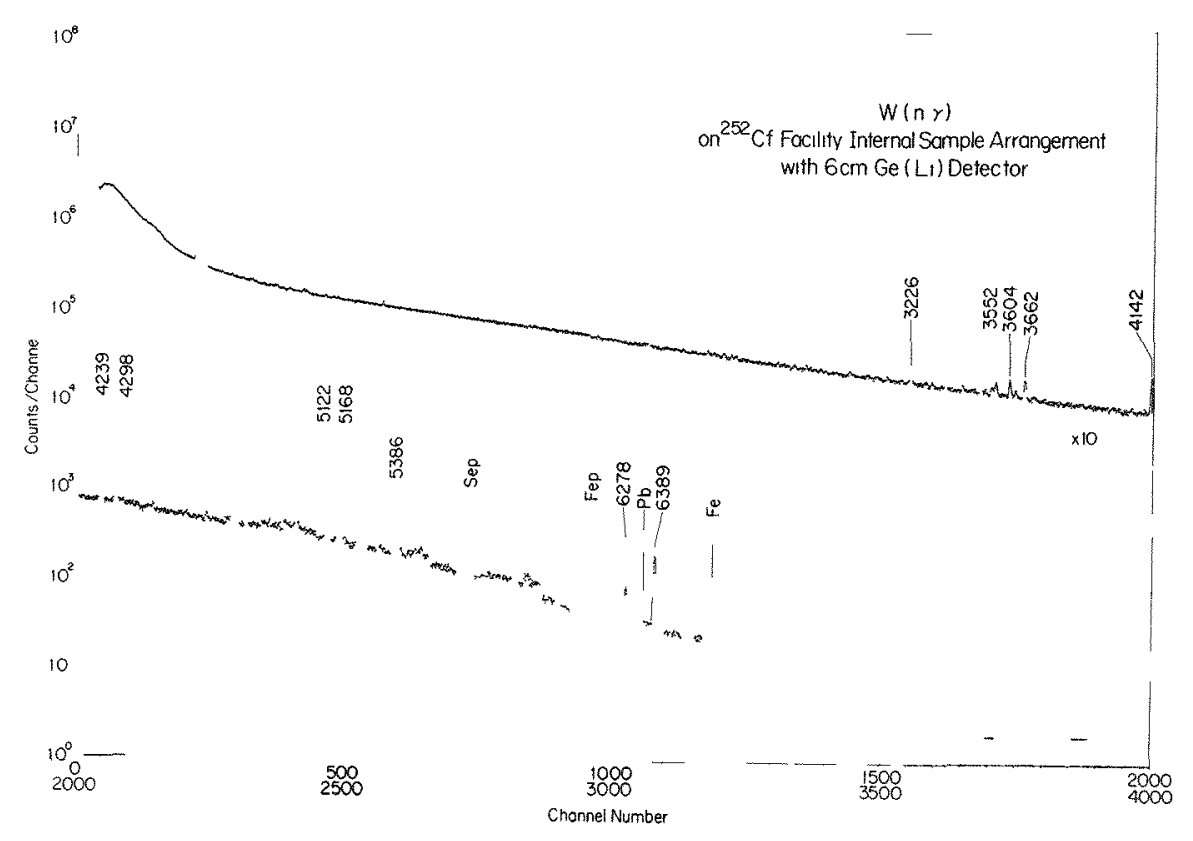

Figure 3 Prompt gamma-ray spectrum obtained with a sample consisting of $100 \mathrm{gm}$ of tungsten using the internal target geometry arrangement. A $6 \mathrm{~cm}^{3} \mathrm{Ge}(\mathrm{Li})$ detector was used to obtain this spectrum in a counting time of 7 hours. (The energies associated with lines in this spectrum are double-escape peak energies.)

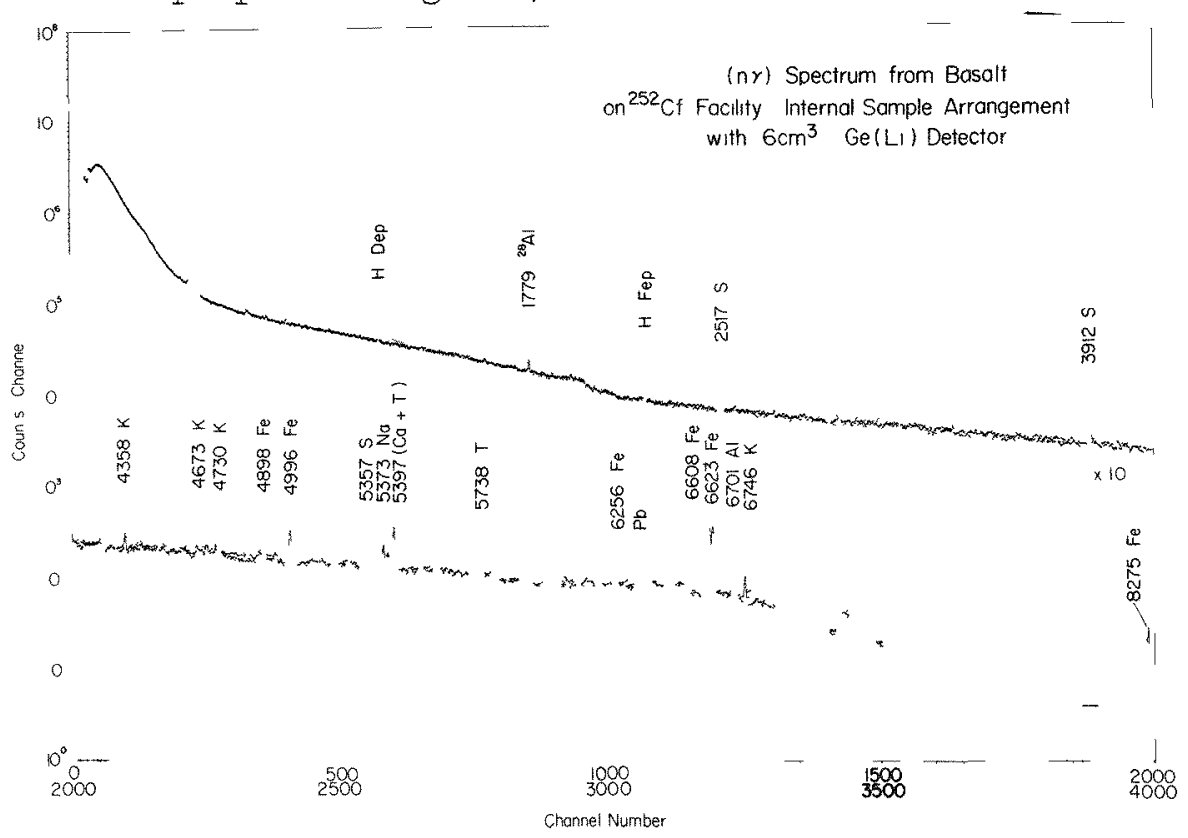

Figure 4 Prompt gamma-ray spectrum obtained with a sample consisting of $42.2 \mathrm{gm}$ of basalt using the internal target geometry arrangement. A $6 \mathrm{~cm}^{3} \mathrm{Ge}(\mathrm{Li})$ detector was used to obtain this spectrum in a counting time of 7 hours. (Unless otherwise noted, the lines observed in this spectrum are double-escape peaks and hence the prompt gamma-ray energies are 1.022-MeV higher.) The small ${ }^{207} \mathrm{~Pb}(n, y)$ line which is seen in this spectrum is from background. 
A TECHNIQUE FOR ASSAY OF L-10 BOTTLES OF PLUTONIUM NITRATE[1]

J. E. Cline, E. B. Nieschmidt, A. L. Connelly, E. L. Murri [2]

A technique has been developed for the assay of I-10 "Ioad-out" bottles containing plutonium nitrate for application to the nuclear fuel safeguards program. The method, utilizing non-destructive Ge(Li) spectrometry, is capable of measuring total plutonium content as well as relative isotope abundances of ${ }^{238} \mathrm{Pu},{ }^{239} \mathrm{Pu}, 240 \mathrm{Pu}, 24{ }^{24} \mathrm{Pu}$ and ${ }^{241} \mathrm{Am}$ isotopes. Accuracies of about $5 \%$ may be obtained for plutonium concentration and for relative abundances of $239 \mathrm{Pu}$ and ${ }^{241} \mathrm{Pu}$, about $10 \%$ for ${ }^{238} \mathrm{Pu}$ and ${ }^{241} \mathrm{Am}$, and about $15 \%$ for ${ }^{240} \mathrm{Pu}$. The assay may be performed quickly and simply.

[1] Idaho Nucleax Corporation report IN-1433 (October 1970).

[2] Present Address: Consumer Power Co., Jackson, Michigan. 
EVALUATION OF A GAS SCINTILLATION COUNTER FOR MEASURING FISSION CROSS SECTIONS IN HIGH ALPHA ENVIRONMENT

\author{
F. B. Simpson, L. G. Miller
}

The fission cross sections of the curium and californium even-odd isotope are important in the $\mathrm{Cf}-252$ production chain. Because of this interest, tests were made using a fast gas scintillation counter to see if it is feasible to measure the fission cross section of Cm-245 in the presence of a high alpha background resulting from $\mathrm{Cm}-244$ impurities in the samples. The objective of this experiment was to find a technique of discrimination between the fission fragments and alpha pile up. The approach that was taken was the use of a fast scintillation gas to help eliminate alpha pile-up, an impurity in the gas to reduce the decay time, and, the requirement of coincidences between the two fission fragments.

To test the system, Cf-252 was electroplated on $6 \times 10^{-5}$ inch thick nickel foil and used in conjunction with a $\mathrm{Cm}-244$ foil $\left(10^{9} \mathrm{\alpha} / \mathrm{min}\right)$. In order not to perturb the fission fragment distribution too much a very thin backing is required so that one can look at the fission fragments on both sides of the foil. Pulse-height distributions were taken on both sides of the foil with $\mathrm{Cf}-252$ in the chamber at different gas pressuresto get the best distribution. The distribution through the thin foil is somewhat perturbed compared to the bare surface.

A $98 \%$ He and a $2 \% \mathrm{~N}_{2}$ gas mixture was used at $780 \mathrm{~mm}$ of $\mathrm{H}_{2}$ pressure. After the best fission fragment distribution was obtained, the $\mathrm{Cm}-244$ source was inserted into the chamber and the pulse distribution repeated. With the addition of $\mathrm{Cm}-244$ in the chamber, pulse distributions were taken in the coincidence and chance-coincidence modes as well as with each separate phototube. The coincidence count rate represented the fission count rate and the chance-coincidence, the background or noise level. Figure 1 is a bias curve as a function of the count rate for phototube $A$ and $B$ along with the coincidence and chance-coincidence counting rates. These data were taken with only the Cf-252 foils in the chamber. In Figure 2 are distributions taken under the same conditions but with the $\mathrm{Cm}-244$ foil added to the chambers. Also included for comparison are the distributions of the alpha pulses resulting from having only the $\mathrm{Cm}-244$ foil in the chamber. From these data one can see that excellent discrimination can be obtained between the fission fragments and alpha $\left(10^{9} \alpha / \mathrm{min}\right)$ pulses.

In order to make final tests on the system, fission cross section measurements were made on U-235 using the Lawrence Radiation Laboratory (LRL) Electron Linear Accelerator with and without the $\mathrm{Cm}-244$ foil in the chamber. The $\mathrm{Cm}-244$ foil represented and alpha background of $10^{9} \mathrm{\alpha} / \mathrm{min}$. These results showed that with the use of this fast scintillation fission chamber in the coincidence mode that the large alpha pulse rate could be rejected in the presence of a few fission pulses. It demonstrated that this type of system is capable of producing high resolution time-of-flight data up to $14 \mathrm{MeV}$ without any interference from the accelerator-gamma-flash. 


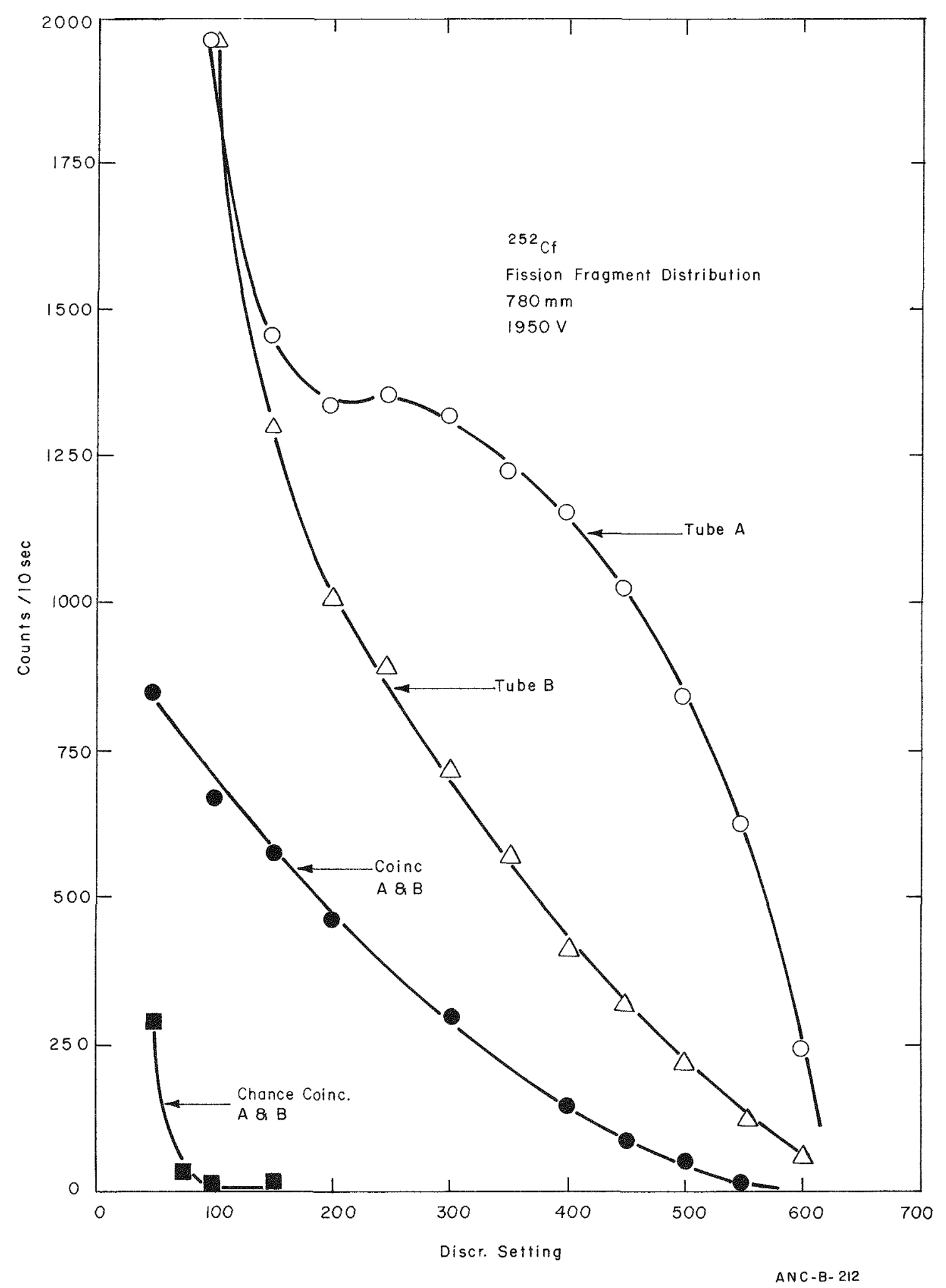

Figure 1. Bias curves as a function of count rate for single time coincidences and change-coincidence with ${ }^{252} \mathrm{Cf}$ in the chamber. 


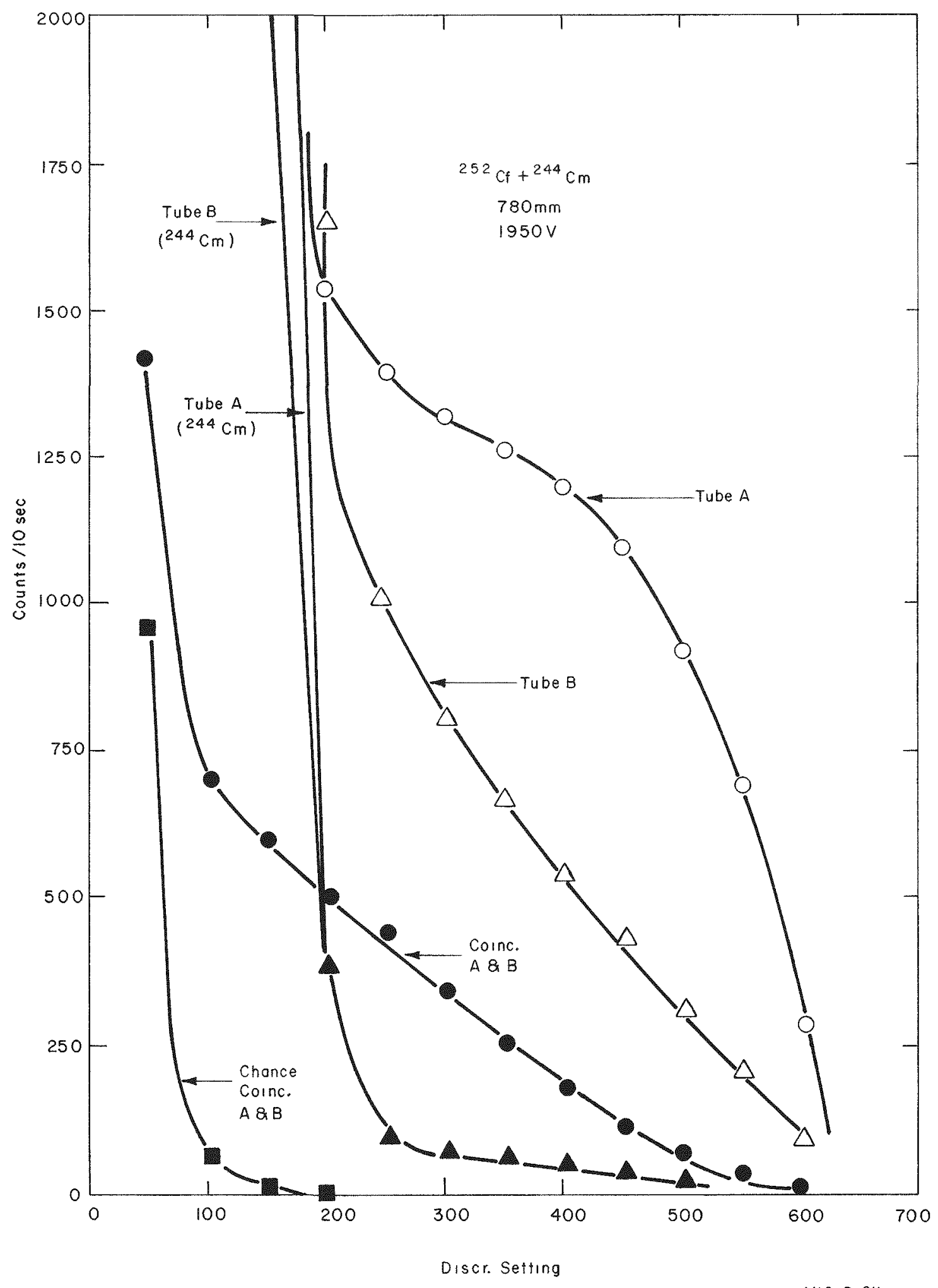

$A N C-B-211$

Figure 2. Bias curves as a function of count rate for single, true coincidence and chance-coincidences with $252 \mathrm{Cf}$ and $244 \mathrm{~cm}$ in the chamber. Also curves for singles with only $244 \mathrm{~cm}$ in the chamber. 
FAST NEUTRON MEASUREMENT METHODS

J. W. Rogers

In the effort to measure the neutron energy spectrum of the CFRMF, the development of spectrometer systems along with their evaluation and improvement continues. Two types of spectrometers; gas-filled protonrecoil counters and ${ }^{6} \mathrm{Li}$ semiconductor-sandwiches, are currently being used and investigated. This effort is to obtain the best possible measurement of the CFRMF spectrum by up-to-date methods so that the integral cross sections of materials may be tested in terms of activation and reactivity analyses.

Proton Recoil Spectrometer: Measurements of the neutron energy spectrum in the CFRMF with a proton-recoil spectrometer have met with a severe limitation due to the gamma-ray level increase. This increase is due to the higher power level operation of the reactor for the activation of low cross section materials. Consequently, attempts have been made to enhance the gamma-ray discrimination in proton recoil measurements by detector selection and pulse shape analysis. Some improvements in gamma-ray discrimination have been realized from this effort especially in the energy independence of pulse rate-of-rise measurements. However, the gamma-ray level is so intense that measurements below $\sim 20 \mathrm{keV}$ in neutron energy appear to be futile.

The primary source of this gamma activity is thought to be from fission products built into the ${ }^{235} \mathrm{U}$ annulus surrounding the measuring position. If this annulus can be replaced, it is expected that conditions favorable for proton-recoil measurements will be obtained. However, the removal without replacement of the $235 \mathrm{U}$ would probably be satisfactory since it does not change the real flux spectrum substantially but is used primarily to tailor the adjoint flux.

More spectrum measurements will be obtained using the improved data accumulation and handling capabilities now available, both with and without the $235_{\mathrm{U}}$ annulus present.

${ }^{6} \mathrm{Li}$ Sandwich Spectrometer: An additional effort to measure the neutron energy spectrum of the CFRMF has been through the use of the ${ }^{6}$ Li-semiconductor sandwich spectrometer technique. This technique has been used by many experimenters $[1,2,3,4,5]$ who have tried several variations in spectrometer design. Our interest lies in its use at neutron energies between $\sim 0.3 \mathrm{MeV}$ and $\sim 6 \mathrm{MeV}$ to overlap the proton recoil measurement and to extend the experimental determinations of the neutron spectrum measurements to an energy region beyond which there is no significant neutron population.

An additional reason for using this technique arises from the gammaray background level that has arisen in the CFRMF due to its use in activation of low cross-section material. This gamma-ray background level makes the proton-recoil method very difficult but should not affect the ${ }^{6} \mathrm{Li}$ semiconductor method. 
The in-house availability of surplus detector heads and electronics has made it possible to gain experience with this technique. The unavailability of additional detector heads has, so far, prevented the actual measurement of the neutron spectrum in the CFRMF.

The two detector heads that are available are described to the extent known in Table 1 . Detector \#1 contains the ${ }^{6} \mathrm{Li}$; detector $\# 2$ has none. These detectors are assumed to be junction type semiconductors rather than surface barrier.

TABLE 1

Detector Heads

\#1

Detector Case

Semiconductors

Semiconductor Spacing

Resistivity

Wafer Thickness

Sensitive Area.

Depletion Depth

Sensitive Material

Sensitive Material Thickness

Alpha Resolution

Rated Bias
A1

Si p type

$1 \mathrm{~mm}$

3,000 ohms

400 microns

$150 \mathrm{~mm}^{2}$

100 microns

${ }^{6} \mathrm{LiF}$

$12 \mu \mathrm{g} / \mathrm{cm}^{2}$

$42 \mathrm{keV}$

50 volts

\section{非}

A1

Si p type

?

12,000 ohms

500 microns

$150 \mathrm{~mm}^{2}$

?

None

None

$59 \mathrm{keV}$

140 volts

It is necessary to operate the detectors in an evacuated environment to prevent alpha particle energy straggling. This poses a problem since the detectors must be 25 feet from the preamplifier and are not constructed to hold adequate vacuum without continuous pumping. The remoteness of the detectors also cause electronic noise problems due to the long electrical cables required.

The ${ }^{6} \mathrm{LiF}$ is deposited directly on the surface of one silicon wafer in detector 1 which makes it impossible to make a background measurement to correct for the neutron reactions in the silicon alone. Detector \#2 is sufficiently different that a background taken with it cannot be assumed to be entirely valid. Detector 1 also has one wafer that has untolerable electronic noise rendering it useless unless it can be repaired.

Detector $\#_{1}$ is quite limited in that it has only a 100 micron depletion depth limiting it to the measurement of neutron energies of about $2 \mathrm{MeV}$ or greater. The very thin ${ }^{6} \mathrm{LiF}$ deposit greatly limits the efficiency of the detector. The close spacing of the wafers broadens the resolution although it slightly enhances the relative efficiency. 
The proposed solutions to these problems are to get new detectors designed for the current application if they become available or to attempt repairing detector 非 2 and using a removable ${ }^{6} \mathrm{LiF}$ foil in it. No commercially fabricated detectors of the type required are currently available but it is hoped that they may be in the near future.

\section{References}

1. H. B1uhm and D. Stegemann, "Theoretical and Experimental Investigations for Improved Application of the "Li Sandwich Spectrometer", Nucl. Instr. and Meth. 70, 2 (1969).

2. M. G. S. Silk and S. B. Wright, "Neutron Spectrum Measurements Over A Wide Range Using "Li Semiconductor Spectrometer", AERE-R-6060 (1969).

3. V. V. Verbinski and N. S. Bokhari, "A Fast Neutron Spectrometer for Reactor Environments", Nuc1. Instr. and Meth. 46, 309 (1967).

4. R. J. Huber, "In-Core Experiments with ${ }^{6} \mathrm{Li}$ Sandwich Fast Neutron Spectrometer", ANS-SD-1 (1964).

5. T. A. Love and R. B. Murray, "The Use of Surface-Barrier Diodes for Fast Neutron Spectroscopy", IRE Trans. Nucl. Sci. NS-8 (1961). 


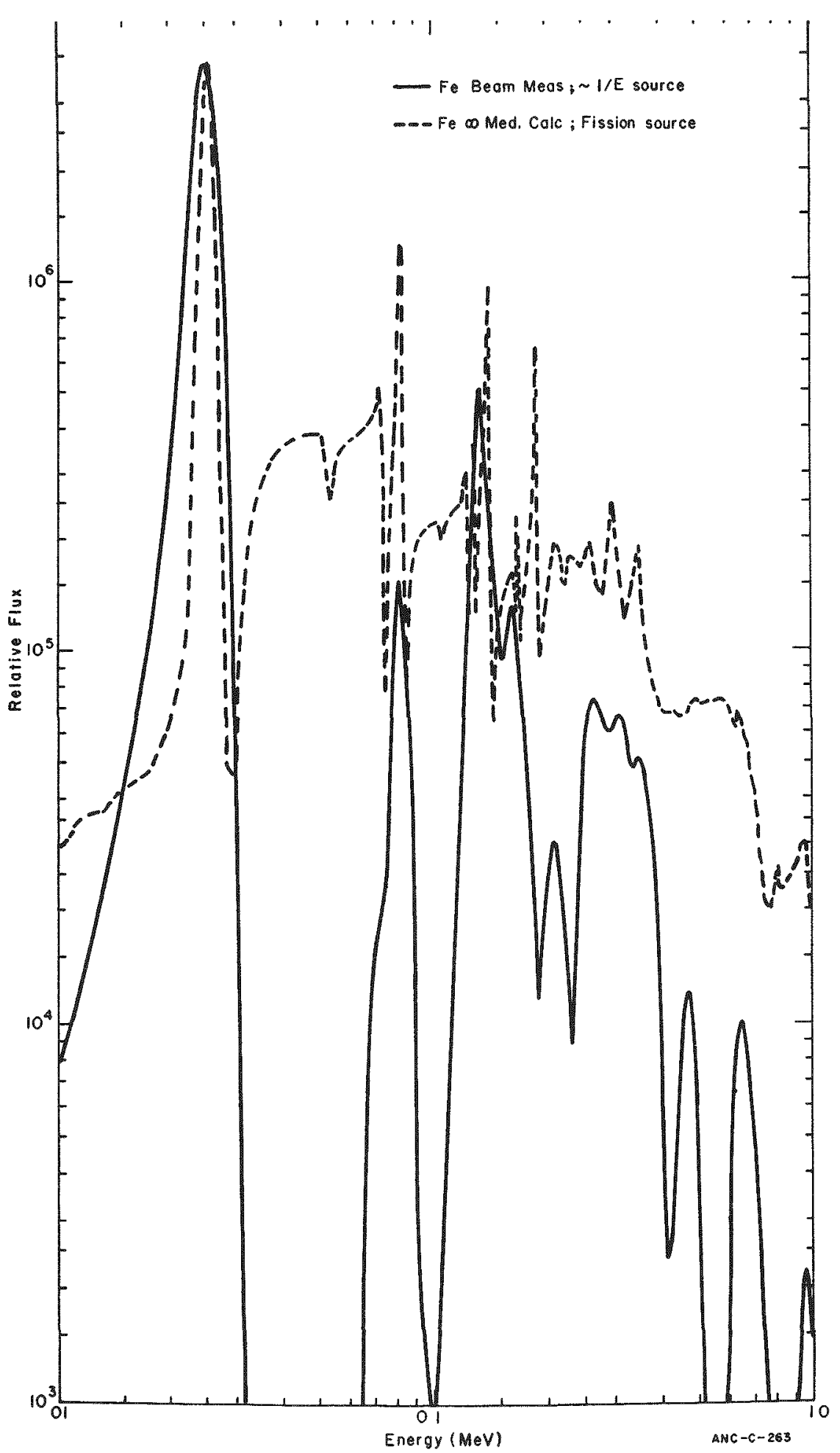

Figure 1. A comparison of a proton-recoil measurement in a beam of neutrons filtered by iron and an infinite media calculation by slowing down theory. 


\section{ANALYSIS OF DOW CHEMICAL COMPANY, ROCKY FLATS DIVISION PLUTONIUM WASTE BARRELS \\ J. E. Cline}

As part of the waste management program, the AEC desires to measure and audit the contents of all packages of waste sent to burial grounds. As part of this effort, Idaho Nuclear Corporation was asked to use their recently developed methods to assay, nondestructively, barrels sent by Rocky Flats to Idaho for storage.

Barrels of Rocky Flats plutonium waste number $771-7753,771-7873$, 771-7799, 771-7959 and 771-7961 were analyzed for $\mathrm{Pu}$ content. The first three of these barrels, together with one that was assembled from known plutonium waste packages, were used as standards to establish the calibration scale. Analysis was done using Ge(Li) spectrometric techniques.

Analysis Technique - Absorption Determination Although 239Pu emits gamma rays of reasonably high energy ( $413 \mathrm{keV})$, absorption of the gamma rays by the material in the barrel, the matrix, and by the plutonium itself is appreciable. Therefore, the absorption must be determined and appropriate corrections must be made. The method used is that of differential gamma-ray absorption using the radiation from the source itself.

Assumptions :

1. That an average path length through the matrix can be defined for the gamma rays emitted from the plutonium. This assumption is not valid if

a. the plutonium exists in large chunks which are opaque to its own radiation;

b. a sizable fraction of the plutonium exists inside a heavily shielded container such that the radiations are heavily absorbed.

2. That an average gamma-ray attenuation coefficient can be defined for the matrix. This is valid if

a. the matrix in the barrel is all light element material (i.e., atomic number less than that for calcium, 20) or

b. the bulk of the plutonium is being contained by a known element, such as a lead sheet or thin lead bottle.

If assumptions 1 and 2 are valid, then using the 129-keV gamma ray from ${ }^{239} \mathrm{Pu}$ as \#I and the $413-\mathrm{keV}$ gamma ray as $\# 2$, the following expression can be generated: 


$$
\operatorname{gms}\left(23{ }^{3} \mathrm{Pu}\right)=K\left(I_{2}\right)_{\text {obs }} e^{\frac{\mu_{2}}{\mu_{1} \mu_{2}} * \frac{4.36}{\frac{I_{1}}{I_{2}} \text { obs }}}
$$

where

$$
\begin{aligned}
K= & \text { constant of proportionality, } \\
\left(I_{2}\right)_{\text {obs }}= & \text { observed intensity of gamma ray \#2 }(4 I 3 \mathrm{keV}), \\
\left(I_{I}\right)_{\text {obs }}= & \text { observed intensity of gamma ray \#I (I29 keV), and } \\
\mu_{I} \text { and } \mu_{2}= & \text { gamma-ray absorption coefficients in the matrix material } \\
& \text { for gamma rays \#I and \#2, respectively. }
\end{aligned}
$$

Measurements A barrel was loaded with 12 pasteboard containers containing known mixed plutonium waste, $16.6 \mathrm{gms} 23{ }^{2} \mathrm{Pu}$, and $30 \mathrm{lbs}$. of rags. This barrel was placed on a turntable, rotating at $0.5 \mathrm{rpm}$. The center axis of the barrel $(57 \mathrm{~cm}$ in diameter) was located 1.25 meters from a 4.5-cm diameter $x 4.5-\mathrm{cm}$ long $\mathrm{Ge}(\mathrm{Li})$ gamma-ray detector. A 1.0-mm thick cadmium shield was placed over the detector to absorb the intense $60-\mathrm{keV}$ radiation from ${ }^{241} \mathrm{Am}$. The barrel was loaded so that the vertical center of the Pu distribution was in the center of the barrel. The position of the detector was adjusted to this center. In such a fashion, a 1000-sec live-time pulse-height accumulation was made of the gamma rays emitted from the rotating barrel.

Transmission measurements were made through the center of the barrel for radiations from ${ }^{137} \mathrm{Cs}, 662 \mathrm{keV}$, and ${ }^{60} \mathrm{Co}, 1117$ and 1332 $\mathrm{keV}$. These were used to determine the "average" atomic number of the matrix material and to measure the average density of the matrix.

From these two sets of measurements, the values of $K, \mu_{2}$ and $\mu_{1}$ were obtained in Equation (1), above.

A set of coordinates was drawn on the barrel as shown in Figure 1. A G-M counter, fitted with a $1 / 4^{\prime \prime} \times 3^{\prime \prime}$ slit was placed at each of the 64 positions on the coordinate grid and the radiation reading recorded. These data helped to determine the distribution of the plutonium in the barrel and to locate the center of this distribution.

Rocky Flats "Known" Barrels Barrels 771-7753, 771-7799, and $771-7 \overline{873}$ were reported by Dow to contain 4, 173, and 180 gms of plutonium, respectively. All three types of measurements were made on these barrels. The data from the first two barrels, when inserted into Equation (1), yielded ${ }^{239} \mathrm{Pu}$ contents of 3.7 and $196 \mathrm{gms}$, in quite good agreement with the Dow values. Barrel \#7873 showed anomalous absorption of the gamma rays, indicating the presence of heavy material in the environment of the plutonium. This is consistent with the 
contents label on the barrel, "heels", which are waste products from plutonium extraction procedures.

Rocky Flats "Unknown" Barrels Similarly, all the measurements were made on barrels 771-7959 and 771-7961, the Rocky Flats "baddies". In both of these barrels the plutonium was quite localized and severe gamma-ray absorption was indicated, similar to that in the barrel containing "heels".

\section{Analysis of Data and Results}

Grid Coordinate Readings Tables I-VI show the relative readings obtained from the G-M counter at the grid positions shown in Figure I for the five Rocky Flats barrels and for the standard. It is noticed that the distribution of plutonium in 771-7753 is quite uniform. For all other barrels, including the standard, the plutonium is quite localized - as shown by the "contour lines" on the Tables.

Gamma-ray counts Table VII lists the results of the gamma-ray counting. As noticed, the gram ${ }^{239} \mathrm{Pu}$ determinations are shown with maximum and minimum limits. For the standard, these limits represent the uncertainties in the gram contents of the individual containers. For barrels 7753 and 7799 , the maximum value represents that calculated from Equation (1), assuming a light-element matrix (verified by the transmission measurements) and no self-absorption in plutonium. These assumptions can be justified on the basis of the high value for the intensity ratio.

This intensity ratio, column 4, for the remaining three barrels is anomalously low, indicating severe absorption of the lower-energy gamma ray by some dense material. In these two cases, the lower limit is based upon the rather ideal case of evenly distributed plutonium, i.e., no self-absorption of the gamma rays, encased in a sufficient thickness of a heavy element, such as $\mathrm{Pb}$, to produce the observed intensity ratio. In the case of barrel 771-7959, this amounted to $0.5 \mathrm{~g} / \mathrm{cm}^{2}$ of lead (about $0.44 \mathrm{~mm}$ ). The upper limit is based upon an assumption that the plutonium is mixed in with a rather heavy absorbing material and that some self-attenuation exists.

If it can be assumed that barrel $771-7873$ contains 180 grams, as reported on the barrel label, then we can make the following comparison. The intensity ratios for this barrel and for the two "baddies" are quite comparable and it may be reasonable to assume that the total absorption is also comparable. In such a case, a direct ratio of the intensities of the 413-keV radiation from the barrels yields 1565 grams for 771-7959 and 1005 grams for 771-7961. Therefore, it is very possible that the plutonium content of these barrels is near that listed under the "maximum" column.

Since the grid scans indicated a fairly localized plutonium concentration for both of the unknown barrels, an $x$-ray photo was taken of that region in which the plutonium was located. The photo revealed three rather large ( $6^{\prime \prime}$ square) ill-defined objects which show no well-defined 
boundaries. Therefore, it is still very difficult to guess as to what is causing the gamma-ray shielding and the limits on the plutonium are those given in Table VII, with the true value very likely closer to the upper limit.

This method of assaying bartels, while still not as accurate as desired, has demonstrated its value as a nondestructive auditing instrument. 


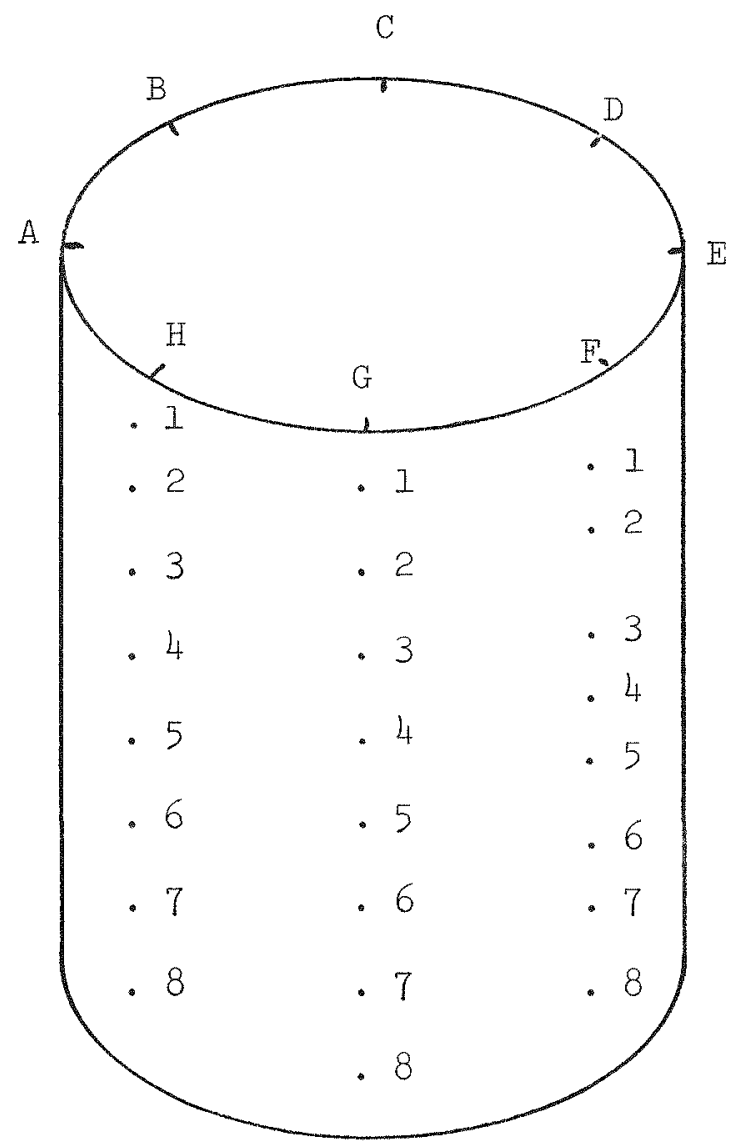

Figure 1 Grid positions for the five Rocky Flats barrels, and for the standard. 
Barrel weight:

Barrel \#:

$\gamma-\operatorname{scan}$ set up at height:

32 Ibs. rag

Barrel tare:
135 Ibs.

standard

52 1bs.
PIutonium Content:

$16.64 \mathrm{gms}^{239} \mathrm{Pu}$ $0.21 \mathrm{gms}^{241} \mathrm{Pu}$

51 lbs. matrix

\begin{tabular}{|c|c|c|c|c|c|c|c|c|}
\hline & A & $B$ & $\mathrm{C}$ & D & $E$ & $F$ & G & $\mathrm{H}$ \\
\hline 1 & 0.3 & 0.35 & 0.40 & 0.50 & 0.40 & 0.30 & 0.20 & 0.40 \\
\hline 2 & 0.6 & 0.80 & 0.80 & 1.00 & 0.55 & 0.60 & 0.50 & 0.70 \\
\hline 3 & 1.0 & 1.40 & 1.10 & 0.95 & 0.70 & 0.95 & 0.90 & 1.05 \\
\hline 4 & 1.0 & 1.10 & 1.40 & $\overline{1} . \overline{10}$ & 2.00 & 2.50 & -1.25 & 2.00 \\
\hline 5 & 1.1 & 1.30 & 1.55 & 1.60 & 8.00 & 4.00 & 1.25 & 0.90 \\
\hline 6 & 0.9 & 1.10 & 1.35 & 1.20 & 7.00 & 4.00 & 1.10 & 10.70 \\
\hline 7 & 0.8 & 1.55 & 1.85 & 0.80 & 1.00 & 1.20 & 1.15 & 0.90 \\
\hline 8 & 0.7 & 1.40 & 2.00 & 0.75 & $0 . \overline{90}$ & 1.20 & 1.40 & 1.00 \\
\hline
\end{tabular}

TABLE II

Barrel weight:

Barrel \#:

$\gamma$-scan set up at height: $66 \mathrm{~cm}$
Plutonium Content:

$4 \mathrm{gms}$

\begin{tabular}{lcccccccccc}
\hline & A & B & C & C & D & E & F & G & H \\
2 & 0.3 & 0.3 & 0.4 & 0.4 & 0.5 & 0.4 & 0.5 & 0.5 \\
3 & 0.4 & 0.4 & 0.4 & 0.4 & 0.5 & 0.5 & 0.6 & 0.4 \\
4 & 0.4 & 0.4 & 0.4 & 0.4 & 0.5 & 0.6 & 0.5 & 0.5 \\
5 & 0.4 & 0.5 & 0.4 & 0.5 & 0.5 & 0.6 & 0.5 & 0.5 \\
6 & 0.5 & 0.5 & 0.4 & 0.6 & 0.6 & 0.5 & 0.5 & 0.5 \\
7 & 0.4 & 0.4 & 0.4 & 0.6 & 0.4 & 0.5 & 0.5 & 0.5 \\
8 & 0.4 & 0.4 & 0.4 & 0.5 & 0.3 & 0.5 & 0.5 & 0.5 \\
\hline
\end{tabular}


$\begin{array}{llc}\text { Barrel weight: } & 158 \text { lbs. } & \text { Plutonium content: } \\ \text { Barrel \#: } & 771-7799 & 173 \mathrm{gms} \\ \text { r-scan set up at height: } & 66 \mathrm{~cm} & \end{array}$

\begin{tabular}{|c|c|c|c|c|c|c|c|c|}
\hline & A & B & C & $D$ & $E$ & $F$ & G & $\mathrm{H}$ \\
\hline 1 & 0.5 & 0.5 & 0.5 & 0.5 & 0.5 & 1.0 & 0.5 & 1.0 \\
\hline 2 & 1.0 & 1.3 & 1.0 & 1.0 & 0.5 & 1.0 & 0.5 & 1.0 \\
\hline 3 & 2.5 & 2.0 & 1.5 & 2.0 & 1.5 & 1.5 & 1.5 & 2.0 \\
\hline 4 & 3.0 & 2.5 & 3.0 & 3.0 & 5.0 & 0.0 & 4.0 & 4.0 \\
\hline 5 & 4.5 & 3.5 & 4.5 & 6.0 & 11.0 & 15.0 & 9.0 & $\overline{6} . \overline{0}$ \\
\hline 6 & 5.5 & 3.0 & 4.0 & 7.0 & 11.0 & 14.0 & 7.0 & 7.0 \\
\hline 7 & 11.0 & 3.5 & 4.0 & 7.0 & 14.0 & 10.0 & 8.0 & 10.0 \\
\hline 8 & 7.0 & 3.5 & 4.0 & 6.0 & 8.0 & 9.0 & 7.0 & 8.0 \\
\hline
\end{tabular}

TABLE IV

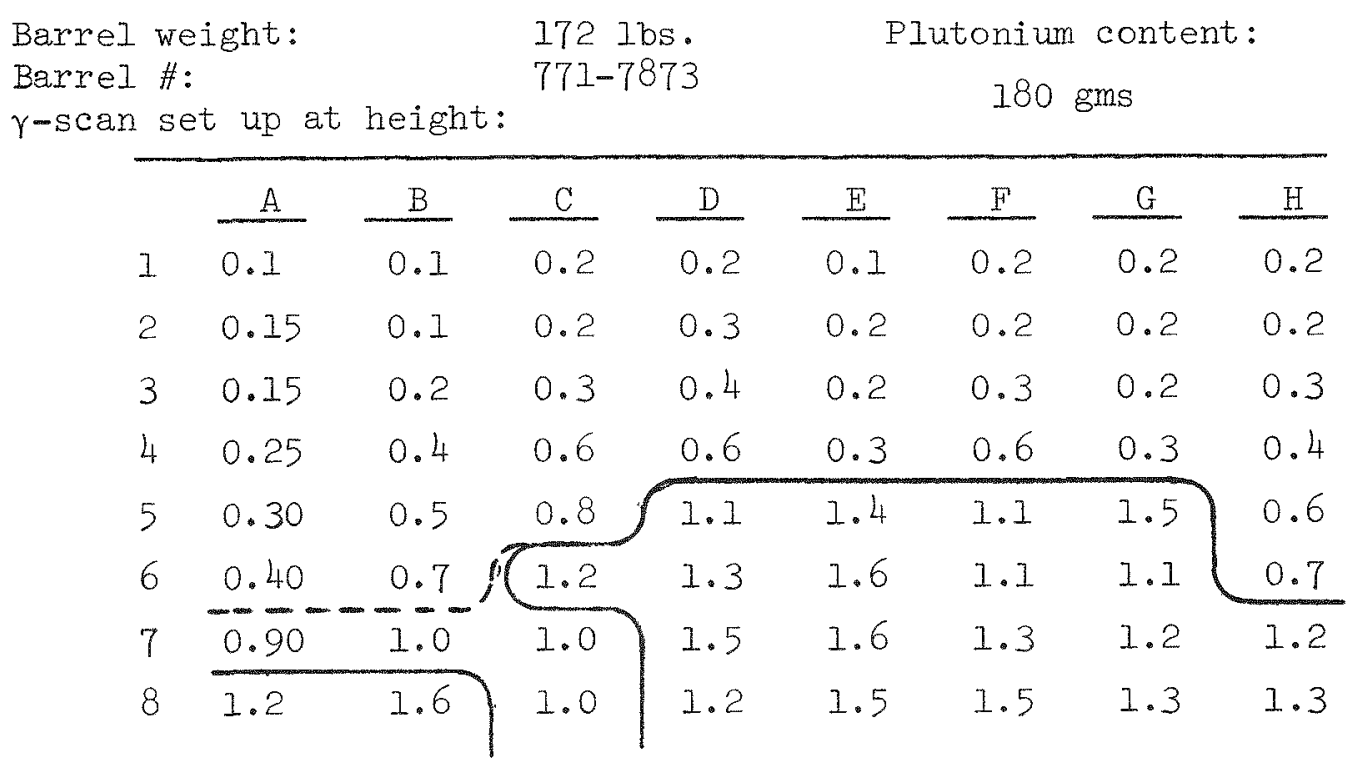




\section{TABLE V}

Barrel weight:

152 Ibs. Plutonium content:

Barrel \#:

$771-7959$

$\gamma$-scan set up at height: $76 \mathrm{~cm}$

Unknown

\begin{tabular}{|c|c|c|c|c|c|c|c|c|}
\hline & A & $B$ & $\mathrm{C}$ & D & $E$ & $F$ & G & $\mathrm{H}$ \\
\hline 1 & 3.0 & 6.0 & 3.0 & 2.0 & 2.0 & 3.0 & 2.5 & 2.0 \\
\hline 2 & 5.0 & 9.0 & 6.0 & 6. & 4.0 & 6.0 & 5.0 & $7 . \overline{0}$ \\
\hline 3 & 10.0 & 16.0 & 11.0 & 8.0 & 11.0 & 14.0 & 8.0 & 8.0 \\
\hline 4 & 10.0 & 10.0 & 8.0 & 8.0 & 9.0 & 7.0 & 7.0 & 8.0 \\
\hline 5 & 5.0 & 5.0 & 4.5 & 3. & & .0 & .0 & 5.0 \\
\hline 6 & 3.0 & 2.0 & 3.0 & 2.0 & 2.0 & 2.5 & 3.0 & 2.5 \\
\hline 7 & 2.0 & 2.0 & 2.0 & 1.5 & 2.0 & 2.0 & 2.5 & 2.5 \\
\hline 8 & 2.0 & 2.0 & 1.5 & 1.5 & 1.5 & 1.5 & 1.5 & 1.5 \\
\hline
\end{tabular}

TABLE VI

Barrel weight: 139 lbs.

Barrel \#:

$\gamma$-scan set up at height: $66 \mathrm{~cm}$
Plutonium content:

Unknown

\begin{tabular}{|c|c|c|c|c|c|c|c|c|}
\hline & A & $B$ & C & D & $\mathrm{E}$ & $F$ & $G$ & $\mathrm{H}$ \\
\hline$I$ & 1.5 & 1.5 & 0.5 & 0.5 & 1.0 & 1.0 & 1.0 & 1.0 \\
\hline 2 & 1.5 & 1.5 & 1.0 & 1.0 & 1.0 & 1.0 & 1.0 & 1.0 \\
\hline 3 & 2.0 & 1.5 & 1.5 & 2.5 & 1.0 & 1.5 & 1.1 & 1.5 \\
\hline 4 & $\overline{6} . \overline{0}$ & $4 . \overline{0}$ & 3.0 & 2.5 & 2.5 & 2.5 & 2.5 & 10.0 \\
\hline 5 & 11.0 & 5.0 & 9.0 & 5.0 & 3.5 & 3.0 & 3.5 & 16.0 \\
\hline 6 & 9.0 & 6.0 & 11.0 & 6.0 & 3.5 & 3.0 & 3.5 & 7.0 \\
\hline 7 & 4.0 & 5.0 & 9.0 & 6.0 & 3.0 & 3.0 & 3.0 & 3.0 \\
\hline 8 & 3.0 & 4.0 & 3.0 & 4.0 & 3.0 & 3.0 & 3.0 & 2.0 \\
\hline
\end{tabular}


TABLE VII

RESULTS OF GAMMA RAY COUNTIIG OF ROCKY FLATS BARRELS

\begin{tabular}{|c|c|c|c|c|c|}
\hline \multirow[b]{2}{*}{ Barrel \# } & \multirow{2}{*}{$\begin{array}{c}\text { Area of } \\
129-\mathrm{keV} \\
\text { Peak } \\
\end{array}$} & \multirow{2}{*}{$\begin{array}{c}\text { Area of } \\
413-\mathrm{keV} \\
\text { Peak } \\
\end{array}$} & \multirow{2}{*}{$\begin{array}{l}\text { Ratio of areas } \\
I_{129} \\
I_{413}\end{array}$} & \multicolumn{2}{|c|}{ grams $239 \mathrm{Pu}$} \\
\hline & & & & $\underline{\text { minimum* }}$ & maximum* \\
\hline Standard & 20953 & 5275 & 3.97 & 16.5 & 16.8 \\
\hline $771-7753$ & 5254 & 1272 & 4.13 & 3.7 & 4.0 \\
\hline $771-7799$ & 147512 & 44225 & 3.33 & 160.0 & 195.0 \\
\hline $771-7873$ & 36032 & 19595 & 1.83 & 100.0 & 200.0 \\
\hline $771-7959$ & 179041 & 170399 & 1.05 & 600.0 & 1700.0 \\
\hline $771-7961$ & 199007 & 109421 & 1.82 & 370.0 & 1100.0 \\
\hline
\end{tabular}

*See section "Gamma-ray Counts". 


\section{PLASTIC IRRADIATION CAPSULES}

D. A. George

Plastic irradiation capsules have been used exclusively as an outer container for pneumatic rabbit neutron irradiations. Most all materials must, in addition, be sealed in an inner container before irradiation. There have been a variety of inner containers used with varying degrees of success. Recent irradiation requirements have created the need for a well constructed inner plastic rabbit which is inexpensive, has a rigid form and can be quickly and tightly sealed to prevent spread of contamination and to withstand some degree of pressure buildup. These inner containers should also be chemically inert and not become significantly activated during irradiation. Two standard inner containers as well as a modified outer rabbit have been designed and tested. These newly developed plastic containers have met the above requirements very well.

Figure 1 shows the appearance of these rabbits. They are constructed of Marlex $5003^{[1]}$ high density polyethylene by inexpensively machining them from bar stock. The lid is simply a $0.100^{\prime \prime}$ thick plug which forms an

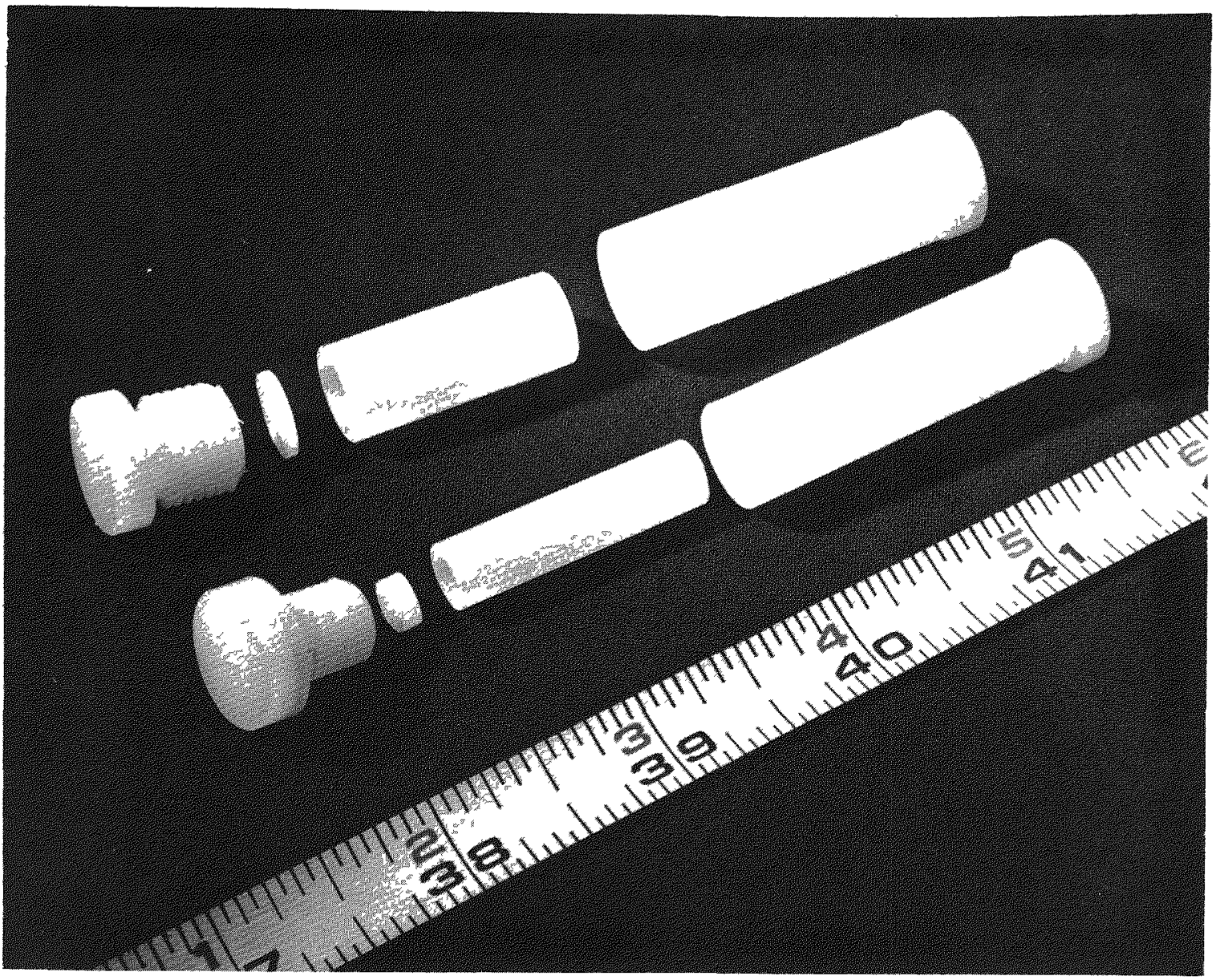

Figure 1 The two types of inner and outer plastic irradiation capsules. 
interference fit and is pressed flush with the body of the capsule. This end is then heat sealed. Since these inner capsules utilize every available bit of space in the larger outer rabbits, tolerances were quite close. A means of heat sealing the lid without distorting the body and maintaining tolerance has been developed. This consists of a Teflon[2] form (see Figure 2) enclosing the capsule and leaving the end to be sealed protruding about 0.020". To seal, simply place the form on a hot plate covered with a thin sheet of Teflon for a few seconds to allow the heat to fuse the body of the capsule with the lia. The temperature required to fuse Marlex 5003 can be attained on most any hot plate. Eiquids may also be encapsulated by keeping the capsule in an upright position until the sealing process is complete.

Using this technique and keeping the irradiation time within prescribed limits assures against loss of samples during irradiation due to packaging techniques.



Figure 2 Teflon form used during heat sealing of inner capsule. An inner capsule is protruding from the end of the form. 
Two capsule sizes are used to meet the broad irradiation requirements. The smaller capsule ( $0.75 \mathrm{cc}$ ) is particularly suited for inert and dry biological materials whereas the larger capsule ( $1.8 \mathrm{cc}$ ) will nicely hold the same amounts of wet biological material and provide somewhat more volume to compensate for pressure buildup due to dissociation or decomposition during irradiation. The new outer rabbit is the same in overall size as the old one except that the usable volume has been increased by a modification of the body design.

With these capsules we can irradiate up to $0.5 \mathrm{~g}$ of wet biological materials for periods up to five hours in the new ETR rabbit facility at an irradjating temperature of $180^{\circ} \mathrm{F}[3]$. Non-pressure producing materials may be irradiated up to 10 hours. Since the activity of the capsule is very low, samples may be gamma counted without opening the capsules, thus providing an additional benefit in that the radioactive material remains contained, thereby minimizing possible radioactive contamination to the plant or personnel.

[I] Trademark of Phillips Petroleum Company.

[2] Trademark of E. I. duPont delNemours \& Company.

[3] J. R. Berreth and F. O. Cartan, "ETR Pneumatic Rabbit Irradiation Facility", this report. 


\section{EXPERIMENTAL REACTOR TECHNOLOGY}

FUEL CLADDING THICKNESS MEASUREMENTS USING X-RAY FLUORESCENCE SPECTROSCOPY

R. J. Gehrke, J. E. Cline, I. G. Miller

Introduction The application of $x$-ray fluorescence as a gauge for measuring the thickness of aluminum clad reactor fuel plates has been investigated. Preliminary results indicate that this technique could be a quick and simple method of scanning fuel plates for thin spots in the cladding. Because of the simplicity of this technique, the thickness measuring equipment could be installed in a fuel fabricating plant and controlled automatically by a small digital processor. This digital processor would also analyze the data and record at which locations thin spots occur.

Experimental I $x$-rays of uranium are produced by fluorescence when a sample of uranium is bombarded by $x$-rays whose energy exceeds $17 \mathrm{keV}$ ( $>I_{\text {III }}$ absorption edge). The pulse-height spectrum of these I $x$-rays is composed of four resolved components, $L_{\ell}, I_{\alpha}, L_{\beta}$, and $L_{\gamma}$. The $I_{\alpha}$ $(13.6 \mathrm{keV})$ and $L_{\beta}(16.9 \mathrm{keV})$ components are approximately of equal intensity and are the more intense $L x$-rays. Because the mass absorption coefficients in aluminum for the $U I_{\alpha}\left(\mu_{A I}=12.7\right)$ and for the $U I_{\beta}$ $\left(\mu_{A I}=6.5\right) x$-rays are quite different, the cladding thickness is determined by measuring the $I_{\alpha} / L_{\beta} x$-ray intensity ratio of the $U L$ $x$-rays transmitted through the aluminum cladding. This ratio is a logarithmic function of the cladding thickness as illustrated by Figure 1. Because the absorption coefficients of uranium at $13.6 \mathrm{keV}$

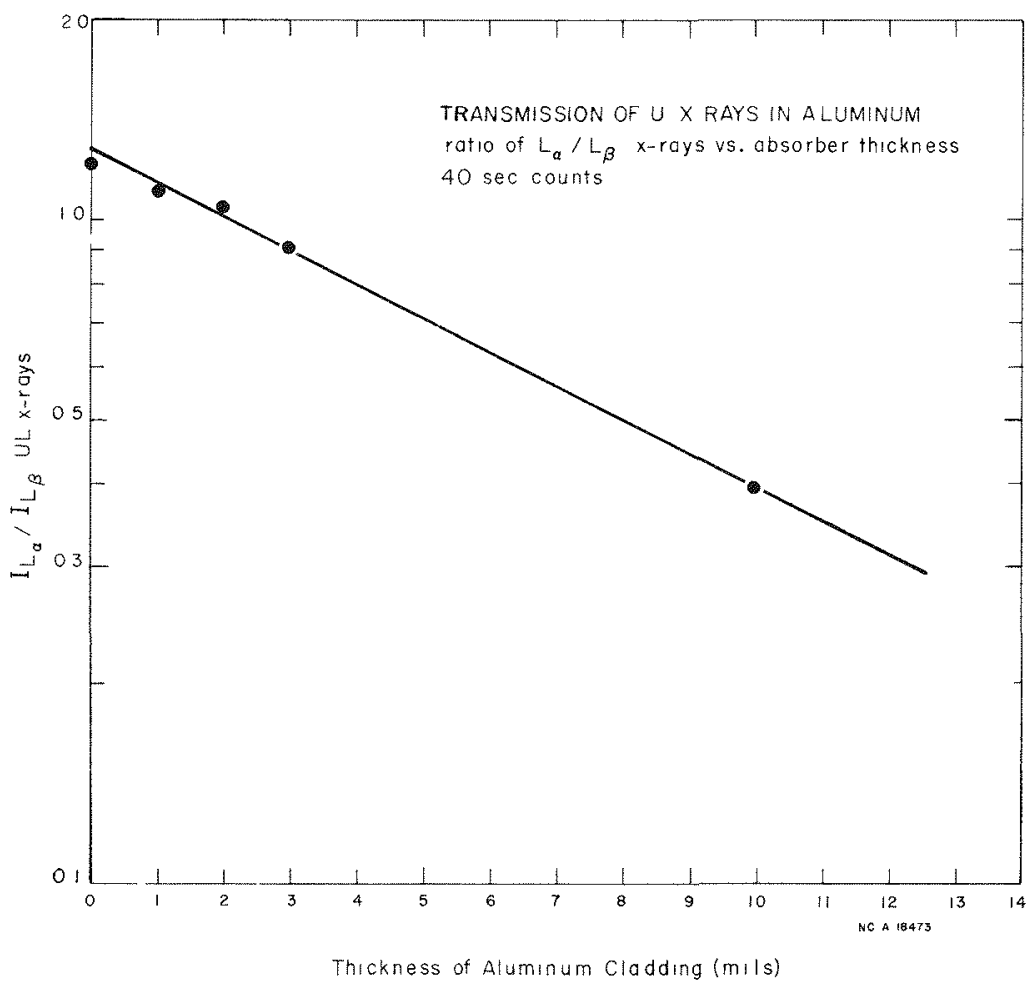

Figure 1 
and at $16.9 \mathrm{keV}$ are about a factor of ten greater than those of aluminum, and the density of uranium is a factor of seven greater than aluminum, only those U L x-rays formed by $x$-ray fluorescence at the cladding-core interface pass through the clading before they are totally absorbed. Measurements of the $L_{\alpha} / L_{\beta}$ intensity ratio yield a cladding thickness resolution of $0.0005^{\prime \prime}$.

The instrument consists of a two-dimensional scanning mechanism, a $\mathrm{Si}(\mathrm{Li})$ spectrometer and collimated Mo $\mathrm{K} \mathrm{x}$-ray beam ( $\mathrm{Ag} \mathrm{K} \mathrm{x}$-rays are a preferred radiation source). Because the $U \mathrm{I} x$-rays can be partially resolved by commercially available proportional counters, it may be possible to substitute a proportional counter for the $\mathrm{Si}$ (Li) detector. The calibration of the $L_{\alpha} / L_{\beta} x$-ray intensity ratio for cladding thickness is easy to obtain using fuel plates with known cladding thickness.

To illustrate the feasibility of this technique for measuring thin spots (dog bones) in aluminum clad fuel plates, a scan was made of an enriched ${ }^{235} \mathrm{U}$ fuel plate (\#ZA-18-4A) with a $25 \times 10^{-3}$ in. diameter Mo $\mathrm{K} x$-ray beam. The overall thickness of this plate is $50 \times 10^{-3}$ in. and the cladding is $15 \times 10^{-3} \mathrm{in}$. thick. No attempt was made to make a complete scan of the ends of the plate or to determine how quickly a complete scan could be made. The data were analyzed by a small PDP-8 computer coupled to the $x$-ray fluorescence spectrometer and a plot of cladding thickness vs. scanning location on the fuel plate is shown in Figure 2. It is estimated that a scanning time of 35-50 minutes per fuel plate (both sides and both ends) could be attained using this technique and fuel plates could be automatically scanned 24 hours a day. Unlike the ultrasonic probe technique of thickness measurement, this $x$-ray fluorescence technique can make aluminum cladding thickness measurements even when only one or two mils of clading cover the uranium core.

Figure 2

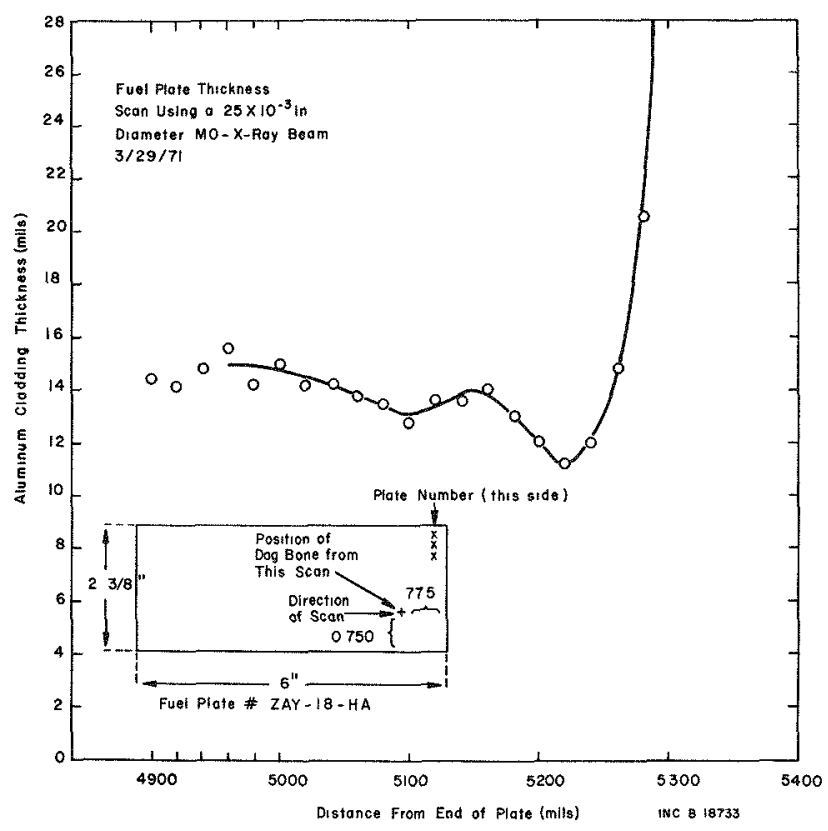


IRON INFLUENCE ON FAST NEUTRON FLUX DISTRIBUTIONS

J.W. Rogers

The neutron spectrum resulting from fission neutrons in an iron environment contains significant structure to the iron cross section that may be useful for the calibration of proton-recoil detectors. A comparison of an infinite medium calculation[I] and a beam transmission measurement[2] has been made to demonstrate the degree of predictability of the structure. The calculation was made using continuous slowing down theory in an infinite medium of iron with a fission source. The measurement was made with a proton-recoil spectrometer (hydrogen gas counter) in an iron filtered beam whose source was the leakage neutrons from a reactor (MTR). This comparison was made to investigate independently determined peaks and valleys that exist in fast neutron fluxes transmitted through iron. The peaks and valleys observed in the resulting spectrum constitute the points in energy which may be useful for calibration of a proton-recoil spectrometer.

In the comparison of time-of-flight measurements of transmission through thin samples of iron and proton-recoil measurements of transmission through thick filters of iron reasonable agreement in energy locations has been observed at the transmission peaks $[2,3]$. However, attempts to observe flux depressions in smooth flux distributions due to iron resonance perturbations have not been successful in that the depressions do not appear at the energy locations expected $[4,5]$. In the comparison of measurements in bulk media of iron and infinite or semi-infinite media calculations of the flux perturbations (cross section maxima and minima) disagreement in energy location has been greater than expected[6].

A comparison of the proton-recoil measurement from the thick iron filter and the infinite media calculation by continuous slowing down theory is shown graphically in Figure 1 . The differences in the overall shapes of these spectra are due to differences in resolutions, efficiencies, geometries, neutron sources, absorption and angular scattering affects. The spectra were scaled such that they have equal magnitudes at $25 \mathrm{keV}$. It is clearly seen that there are only two distinct flux variations that agree in energy location well enough to be used as calibration points. These are the peaks at $25 \mathrm{keV}$ and $82 \mathrm{keV}$ corresponding to two of the extreme cross section minima. This suggests that in using iron resonance perturbations in neutron spectra for energy calibration purposes that the use of peaks in the flux is more reliable than the use of depressions. However, in general, measurements in bulk media[6,7] have not yielded the same structure seen in the calculation, and this is especially true of the fine structure at $82 \mathrm{keV}$. All measurements and calculations show the $25 \mathrm{keV}$ peak although in some cases there appears to be energy calibration discrepancies. Consequently, the use of bulk media iron spectra for calibration purposes is not recommended untiI these discrepancies are resolved. 
[1] E. T. Burns and M. Becker, "Fast Neutron Spectrum Models and Their Application to Specific Materials", Trans. Am. Nucl. Soc. 13, 688 (1970).

[2] J.W. Rogers, "A Method for Calibrating A Proton-Recoil Spectrometer", Nuc1. Instr. and Meth. 80, 313 (1970).

[3] J.E. Powell and J.W. Rogers, "Fast Neutron Spectrum Measurements in the MPR Scandium and Iron Filtered Beams", Nucl. Instr. and Meth. 87, 29 (1970).

[4] A. M. Broomfield and N. D. Carter, "Proton-Recoil Counter Techniques in ZEBRA," AEEW-M-905 (1969).

[5] G. I. Morgan et al., "Energy Calibration of a Proton-Recoil Proportional Counter", ORNL-TM-3347 (1971).

[6] B. K. Malaviya, et al., "A. Study of Neutron Transport in Iron with an Assessment of Data Files", Trans. Am. Nucl. Soc. 13, 757 (1970).

[7] R. J. Cerbone, "Neutron and Gamma-Ray Spectra in a Spherical Iron Assembly", Trans. Am. Nucl. Soc. 12, 399 (1969). 


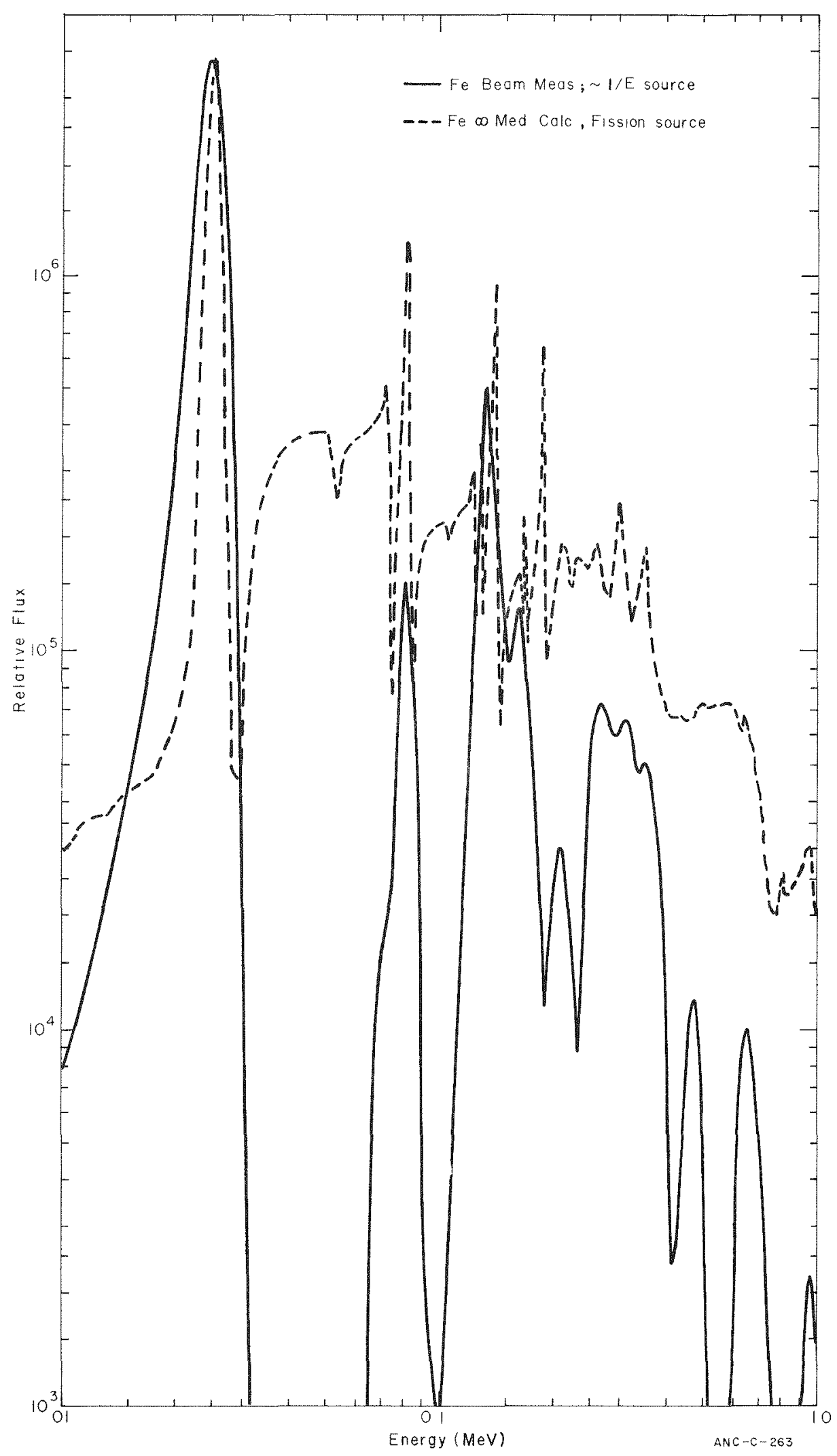

Fig. I A comparison of a proton-recoil measurement in a beam of neutrons filtered by iron and an infinite media calculation by slowing down theory. 
IN-PLANT FUEL CIAD THICKNVESS MEASUREMENT FACILITY USING THE

BETA AUTORADIOGRAPHY TECHNIQUEE

L. G. Miller

Until recently no satisfactory method has been available for fuel plate clad thickness inspection during fabxication. The cladding failure of several ATR fuel plates has made it necessary to inspect clad thickness of fuel plates prior to their assembly into elements.

The availability of milligram quantities of californium-252 makes it a desirable neutron source for the irradiation of fuel plates in a clad thickness measurement facility. The method of measuring cladaing thickness is to irradiate with neutrons the surface layer of fuel meat uniformly. Then the fission product $\beta$-activity is measured through the clad and this transmission can be converted to the clad thickness. This facility utilizing the beta autoradiography technique can be built in-plant for the needs of a fuel fabricator (see preceding article for details of the technique).

The facility would be divided into two parts, irradiation and inspection. The safety and constant intensity of californium-252 make it ideal for use as a neutron source to irradiate the fuel plates. Two different methods could be used to irradiate the fuel plates. The scintillation scanning method would be a completely atomated inspection facility and would require development of the scanning detector system. The photographic plate method would be one-man operation requiring small investment and no additional development.

For the scintillation scanning method, the irradiation facility would consist of a conveyor passing through a sphere containing a moderated californium source. The fuel plates would be automatically fed through the sphere on the conveyor and restacked at the opposite side similar to that shown in Figure 1. The plates would be automatically delayed the correct decay time then fed through the beta scanning equipment. The beta scanner, controlled by a simple inexpensive computer such as a PDP-8/S, would scan both sides of the dog bone regions on each plate. Plates having sufficient cladding, i.e., a predetermined minimum thickness, would be stamped "approved" and sent through and stacked. Those having insufficient cladding would be stamped "reject" and transferred to a reject bin. The cladding inspection could be made on the plates after they have been rough sheared to size. A batch of plates would be stacked at the input to the irradiation facility. Sixteen hours later the approved plates could be removed from the scanner output.

The photographic plate method is shown in Figure 2. After the plates are exposed to neutrons, photographic plates are clamped onto the ends of the fuel plates. Later the film is developed, and qualitatively inspected. Plates that indicate thin cladding would then be scanned with a densitometer. 
A californium-252 source was chosen for the neutron source because of its high neutron yield, long life-time and low gamma production. Neutron flux measurements taken from our moderated I mg Cf-252 source indicates that a 1 to $2 \mathrm{mg}$ source will produce the required activation of the fuel plates if the plates are passed through the moderator at about 4 inches per minute. Only $1 \%$ per month corrections need be made in the scanner calibration for the decay of the californium. Since there is no danger from a criticality standpoint and the neutron field will be contained within the moderator sphere, there should be no trouble in licensing such a device at a fabricator's plant. The system can be disassembled, shipped and reassembled at another plant with little effort.

To determine the amount of fuel in each plate, the induced activity is measured immediately after exposure to the neutron source. Since this activity from fission will be much greater than that due to natural decay of U-235, U-238, etc., this scan can be on gross gammas and will be directly proportional to the amount of fissionable isotopes in the plate.

With a $2 \mathrm{mg}$ californium-252 source the fuel fabricator could examine his total output of fuel plates each day assuming he produces two fuel elements per day. This could be increased to 6 fuel elements per day if facility use was increased to 3 shifts per day.

It would require one full-time person to load and unload fuel plates, and load and unload film each morning. This person would then read the film each afternoon. Upkeep (parts and labor) for this system should be less than $\$ 1,000$ per year. Clad thickness for accuracy for either system would be \pm 0.001 inch in a routine operation although accuracies using this technique are possible to \pm 0.0005 inch. 


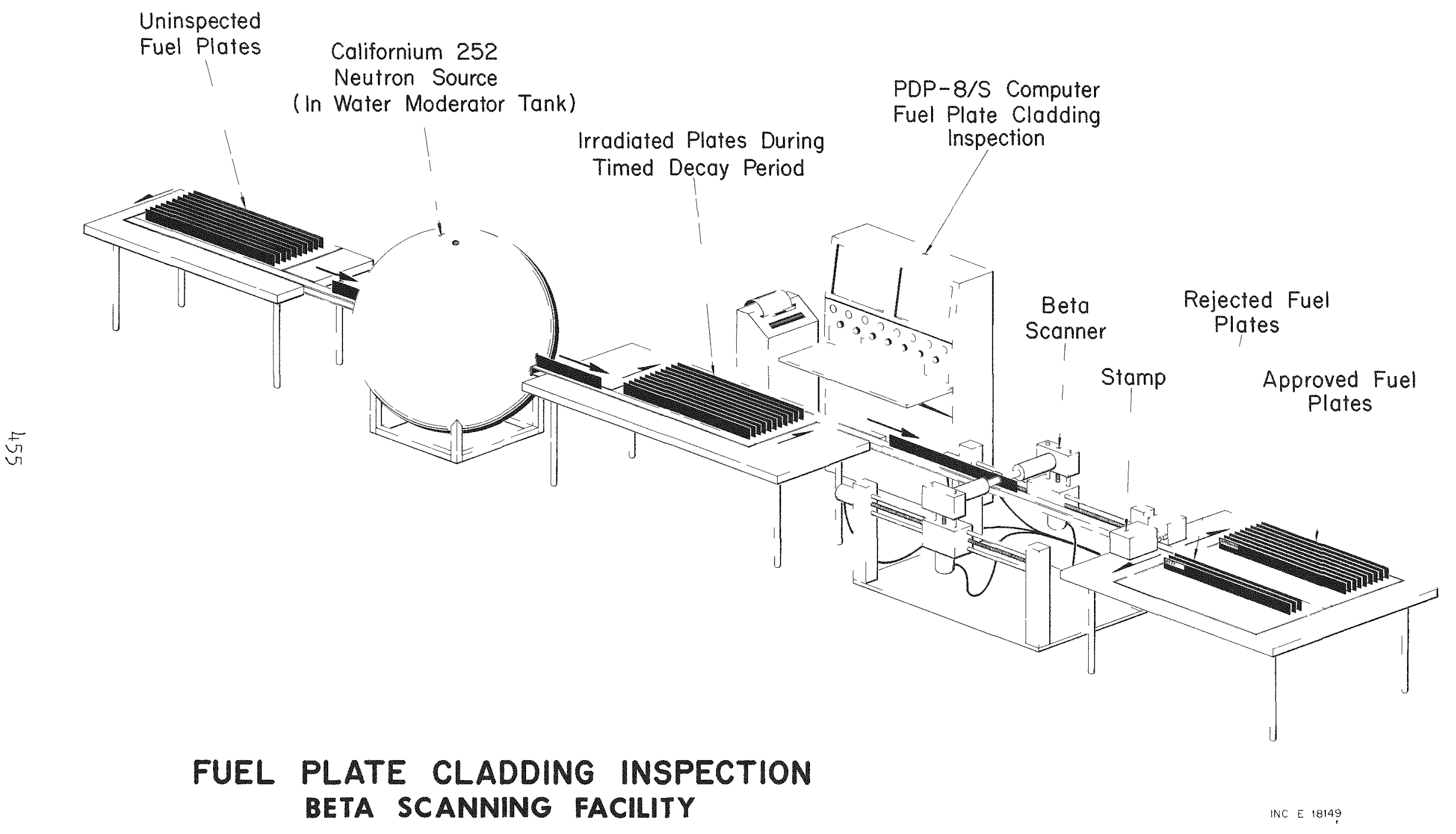

Figure 1. This scintillation scanning facility is completely automated with complete information on each plate read-out on a teletype. 


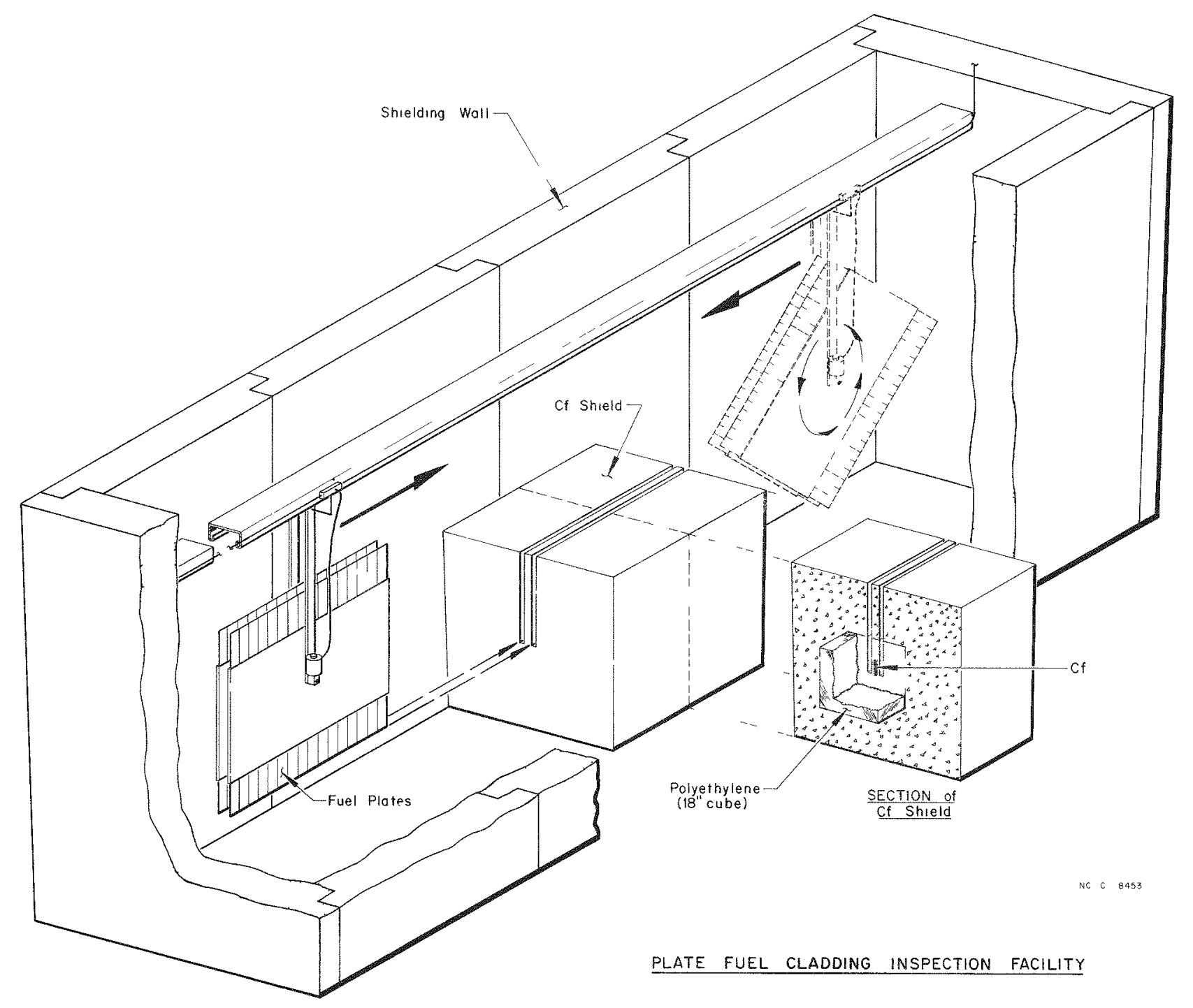

Figure 2. Photographic plate method. 
USE OF METAL ALLOYS FOR NETURON SPECTRUM MEASUREMENTS

\author{
D. A. Pearson
}

Measurements of the flux spectrum test is of vital importance to the IMFBR program. Irradiation experiments conducted in the test reactor (primarily EBR-II) that require spectrum measurement or monitoring have generally relied on the multiple foil activation technique for this information. This procedure, however, is cumbersome in that it involves encapsulation and subsequent removal of a number (perhaps a dozen) of small foils of various materials. These foils may be damaged or lost during the hot-cell operations required for their removal from some types of capsule. A continuing effort toward the fabrication of a metal alloy which would contain the necessary materials to determine the neutron spectrum within a specified energy region is being made[1].

Two separate foils have been made by the Metallurgical and Materials Science Branch. One foil is suited to the "resonance" region and contains the elements to produce the reactions of ${ }^{197} \mathrm{Au}(\mathrm{n}, \gamma){ }^{198} \mathrm{Au},{ }^{63} \mathrm{Cu}(\mathrm{n}, \gamma)^{64} \mathrm{Cu}$, ${ }^{55} \mathrm{Mn}(\mathrm{n}, \gamma){ }^{56} \mathrm{Mn},{ }^{59} \mathrm{Co}(\mathrm{n}, \gamma)^{60} \mathrm{Co}$, and ${ }^{50} \mathrm{Cr}(\mathrm{n}, \gamma)^{51} \mathrm{Cr}$. The second alloy which is a "fast" neutron flux monitor has the following elements to produce the reactions such as ${ }^{54} \mathrm{Fe}(\mathrm{n}, \mathrm{p}){ }^{54} \mathrm{Mn},{ }^{27} \mathrm{AI}(\mathrm{n}, y)^{24} \mathrm{Na},{ }^{64} \mathrm{Zn}(\mathrm{n}, \mathrm{p})^{64} \mathrm{Cu}$, ${ }^{115} \mathrm{In}\left(\mathrm{n}, \mathrm{n}^{1}\right)^{115 \mathrm{~m}} \mathrm{In},{ }^{46} \mathrm{Ti}(\mathrm{n}, \mathrm{p})^{46} \mathrm{Sc},{ }^{58} \mathrm{Ni}(\mathrm{n}, \mathrm{p})^{58} \mathrm{Co}$.

As in all foil activation analyses, the induced activity must be determined. The most common method of doing this is with the use of a $\mathrm{Ge}(\mathrm{Li})$ crystal and a multi-channel analyzer.

A typical gamma-ray spectrum obtained from the "resonance" and "fast" alloys is shown in Figures 1 and 2 respectively. From these figures, it is at least qualitatively apparent the detector resolution is sufficient for determining the magnitude of each of the induced activities. The obvious advantage of the alloy is that only one or two foils need to be handled and counted although repeated counting at various decay times may be desirable. Furthermore, the ratio of various gamma-rays from a number of foils made from the same alloy irradiated. in a reference spectrum may be used as a check on the homogeneity of the alloy.

A continuing effort is being made to standardize the alloy sothat a single foil may be included with and ultimately replace multiple foil arrays that are currently being used.

[1] Nuclear Technology Annual Report Ending June 30, 1970, IN-1407 p. 303. 


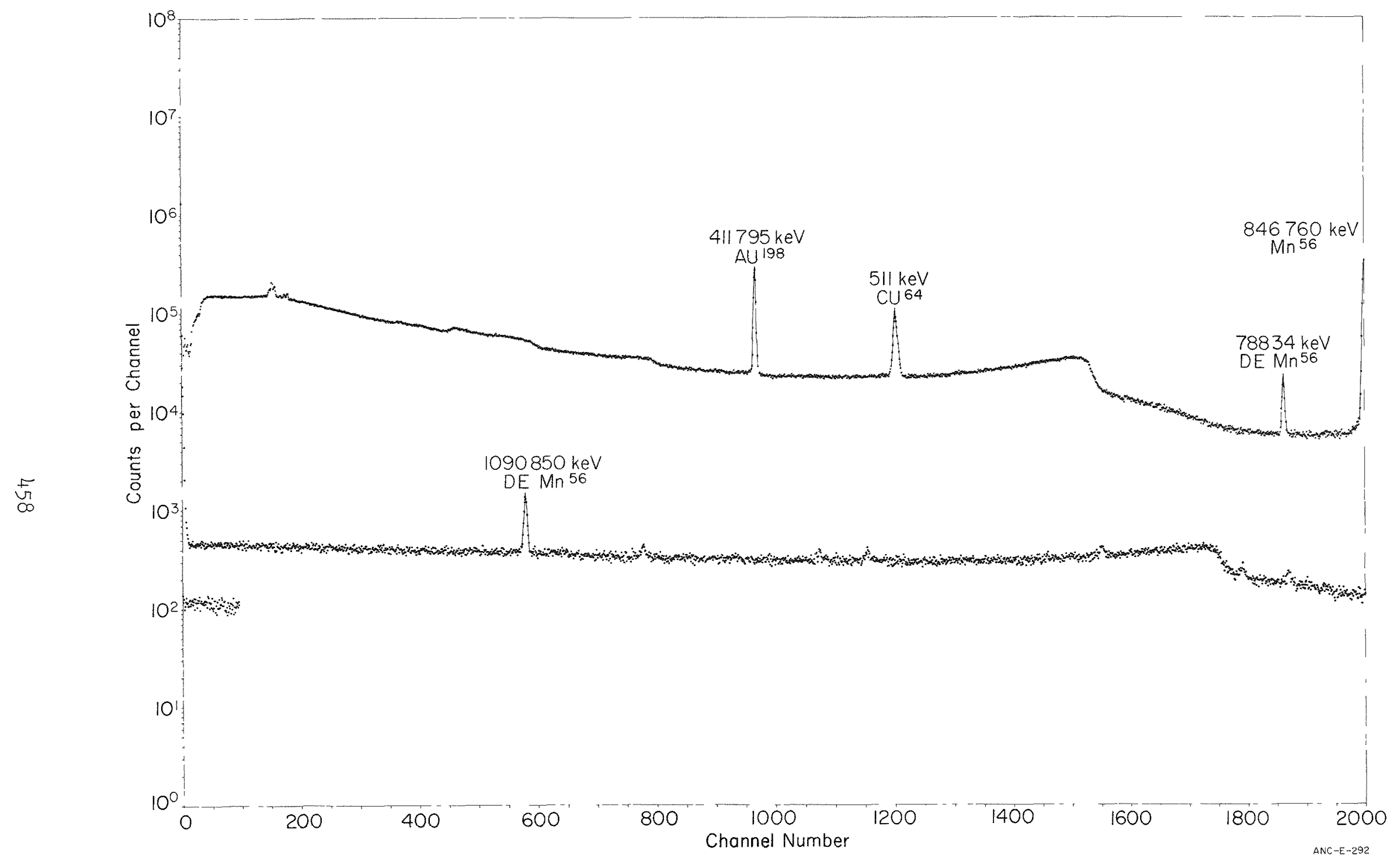

Fig. I Resonance neutron flux monitoring alloy spectrum. 


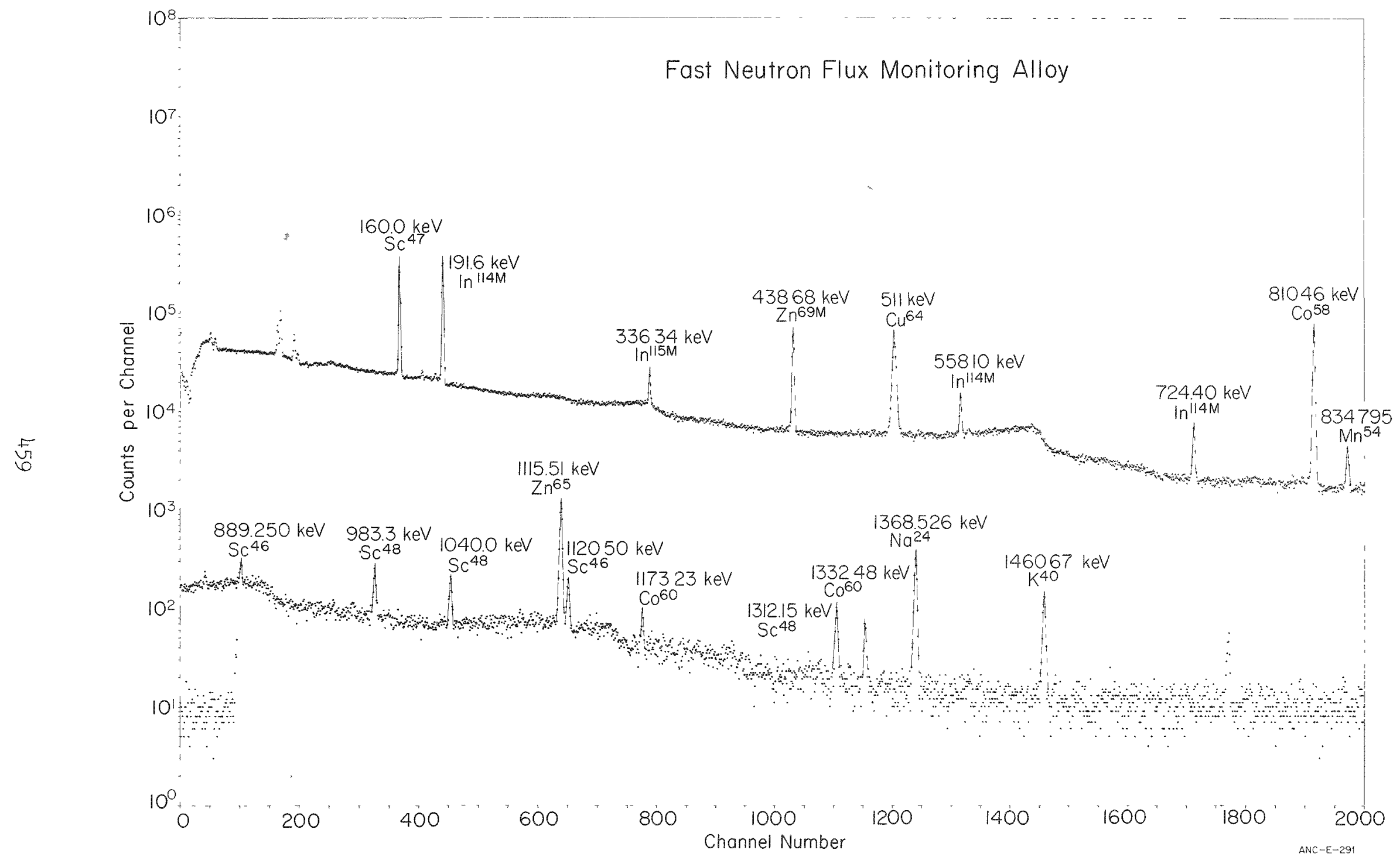

Fig. 2 Fast neutron monitoring alloy spectrum. 
IMPROVEMENTS IN FOIL ACTIVATION DATA ANALYSIS

D. A. Pearson

A major problem to overcome in the determination of a neutron spectrum through the use of foil activation analysis is with the data analysis procedure which involves the use of an "unfolding" code[1]. In order to alleviate the time consuming and expensive procedure of submitting repeated computer problems with manual corrections and manipulations of each run, an on-line graphics approach to the problem has been developed.

The code (SPECTRA) used to determined the neutron flux from the foil activation data utilizes a trial spectrum supplied as tabular input, and by requiring that the squared difference between the measured and calculated detector activities be a minimum, it is possible to obtain a convergence fit to the new spectral shape. The SPECTRA code is very input sensitive as certain combinations of detectors and errors can make the matrix equations unstable. If this occurs, the code will calculate unrealistic values. When unrealistic values are obtained, the user must re-submit the problem after making the necessary adjustments to the trial spectrum. Repeated runs are necessary to arrive at a final physically acceptable solution. This process is very time consuming and expensive. The code was revised so that an on-Iine graphics approach was possible. The basic mathematical techniques used and the method of solution was not changed. However, since the code operation was altered, the name was changed to INSPECT.

INSPECT enables the user to submit experimental data to the computer, and through the interactive graphics capability, guide the code in iterating toward a realistic solution thus, eliminating the need for repeated problem submittals. This is a time saving aid as well as a necessary function in view of the complexity of the mathematical model used to unfold the neutron spectrum. Figure 1 shows the flow of data and the important manipulations available to the operator.

INSPECT has proven to be a very valuable aid in our efforts to determine the neutron spectrum of a reactor. This code has played an important role in the investigation of the CFRMF spectrum[2]. A neutron cross-section comparison and evaluation program is now being added as a sub-routine to INSPECT using the on-line graphics approach. Interactive graphics and the INSPECT code form a very efficient means of processing foil activation data.

[1] C. R. Greer, J. A. Holbleib, J. V. Walker, A Technique for Unfolding Neutron Spectra from Activation Measurements, SC-RR-67-746

(December 1967).

[2] D. A. Pearson, D. A. Milisap, J. W. Rogers, Comparison of Spectrum Determinations in the CFRMF, this report. 


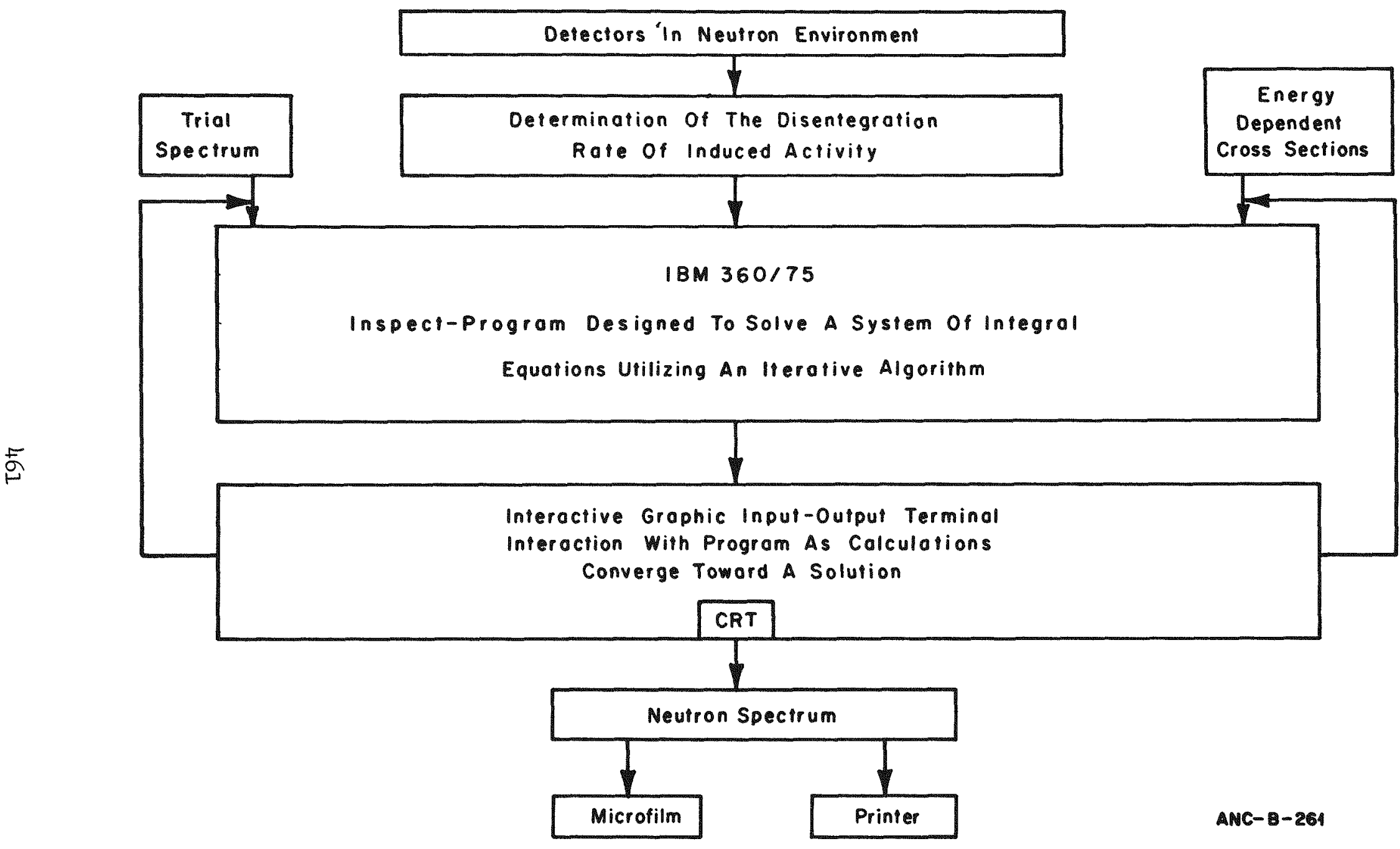

Figure I. Flow Data 
FABRICATION AND EVALUATION OF SAMPLES FOR THE IRRADIATION PORTION OF THE FBR PHYSICS CONSTAIIS PROGRAM

R. G. Nisle

The irradiation portion of the FBR Physics Constants Program at the National Reactor Testing Station is being undertaken for the purpose of evaluating the reactivity response of mixed-oxide fuels as a function of fast reactor irradiation. The gross reactivity response is a complicated function of burn-up, fuel production, fission-product build-up and decay, neutron spectrum and sample density. It is not enough, therefore, to simply measure the reactivity response before and after an irradiation. One would like to know the flux spectrum, as well as intensity, during irradiation and certainly the flux spectrum in which the reactivity measurements are made. A non-destructive method of analysis for fuel and absorber content is also needed. Spectrum measuring methods are described in this report. The non-destructive analysis by reactivity measurement methods have been described previously[I].

The sample capsule is shown pictorially in Figure 1. Mixed oxide pellets, $20 \mathrm{wt} \% \mathrm{PuO}_{2}$ in $\mathrm{UO}_{2}$, were fabricated by a commercial supplier and were loaded, four-to-a-capsule, at this laboratory. A series of tests were then made on the assembled capsules to evaluate their suitability for the experiment in question. A complete report of these tests will soon be issued[2].

After sealing by TIG welding in a helium atmosphere, the capsules were radiographed and dye-penetrant tested for leaks. A pressure test showed that the weld would hold to 7000 psig. The content was then evaluated by the following series of tests: (1) gamma spectroscopy, (2) neutron radiography, and (3) reactivity measurements. The first method yields a value of absolute content which can be compared with the manufacturer's quoted value, and the latter two provide a means for testing the uniformity of content.

The results of these tests indicate that the contents of these capsules are as quoted by the manufacturer of the pellets and as uniform as quoted. The reactivity and neutron radiography methods were found to be very sensitive to sample content. The accuracy of the gamma spectroscopic method is hampered by an uncertainty in the value of $\beta$, the branching ratio of the $413.7 \mathrm{keV}$ gamma ray arising from the natural decay of ${ }^{239} \mathrm{Pu}$.

[I] R. G. Nisle, I. E. Stepan, D. A. Millsap, E. Fast, "Fission Product Transient Measurements in the Advanced Reactivity Measurement Facilities", IN-1096, Idaho Nuclear Corporation (1967).

[2] R. G. Nisle, "The Fabrication and Evaluation of Samples for the Irradiation Portion of the FBR Physics Constants Program, ANC-1001, (1971) 


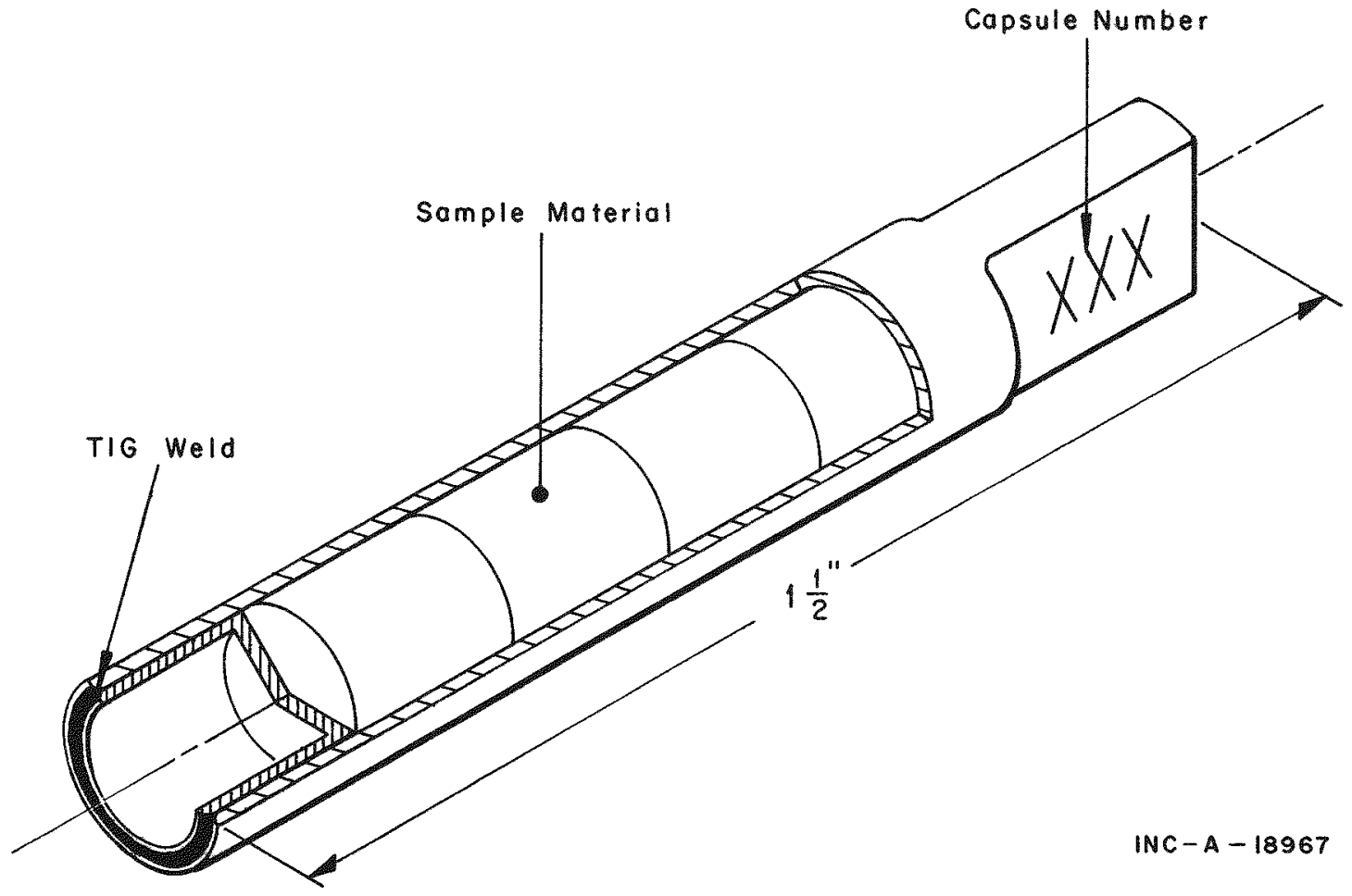

Figure 1. Pictorial view of sample capsule. 
NON-DESTRUCTIVE ANALYSIS OF FUEL PINS IN THE ARMF

I. E. Stepan, N. F. Allard, C. R. Suter

Studies are being made in the ARMF to determine the applicability of such a facility for the rapid non-destructive measurement of fertile and fissile material content in reactor fuel pins. If feasible these measurements could be used for quality assurance of fuel pins that are to be used in power reactors.

Samples used in these preliminary studies were 3 inch fuel pins clustered together in bundles of four. The samples contained either enriched, natural or depleted uranium. A sample that was similar in geometry but contained no fuel was also measured. The samples were positioned for a traverse through the center of the ARMF-I core in a 5 foot long flared top square tube having internal dimensions of 1 inch by 1 inch.

A Slo-Syn motor, which is a pulse type motor, was used as the drive mechanism. A Slo-Syn translator was used to control the motor. Figures 1 and 2 illustrate the experimental apparatus. The translator produces a uniform pulse-rate output for uniform speed. Variable control is obtained with an adjustable potentiometer mounted on the unit. The onoff time of the control unit derives its signal from the ARMF read-out system. Thus when the data read-out unit interrogates the regulating rod encoder, the translator receives a signal and drives the motor which in turn passes the sample through the core.

The samples were withdrawn a distance of 53 inches in 18 minutes, although the active section of the core is only 25 inches in length. The ARMF read-out system was set to interrogate 16 times per second, and to take an average of every 256 interrogations. Other interrogation and average rates are available but the best results are obtained from these settings. These averages are printed out on IBM cards and can be reduced to net reactivities with the computer code DUZIT[I]. Figure 3 illustrates the results of a typical measurement.

The net reactivity of a sample was measured with an accuracy of $0.5 \%$. The total worth of a natural uranium sample was measured to be worth $3721.5 \pm 16.3 \mu \mathrm{k}$. This sample was measured 53 times. Other results are given in Table 1 .

\section{TABLE I}

\begin{tabular}{|c|c|c|c|c|}
\hline Sample & Type & $\begin{array}{l}\text { Sample } \\
\text { Mass g } \\
\end{array}$ & Net Worth $(\mu \mathrm{k})$ & Specific Reactivity $(\mu k)$ \\
\hline 1 & Enriched & 170.06 & 5156.1 & 30.3 \\
\hline 2 & Natural & 171.54 & 3721.5 & 21.7 \\
\hline 3 & Depleted & 172.36 & 721.8 & 4.2 \\
\hline
\end{tabular}


The considerably different sensitivities and their magnitudes noted above, provide optimism for continued effort in this study. Other positions (these data are from the center of the ARMF-I water hole) avajlable in the reactor will be considered. Also, currently under construction, is a cadmium lined dry tube facility in which further traverses will be made. (The cadmium tube will allow the study of long rods which, without it, would result in too much reactivity addition to the reactor.) A "window" in the cadmium at the reactor center line provides a high sensitivity thermal reactivity signal and at the same time should give the capability of determining where a content heterogeneity occurs in a long pin.

[I] D. H. Gipson and R. A. Peereboom, ARMF Data Analysis Program, IDO-17021. 


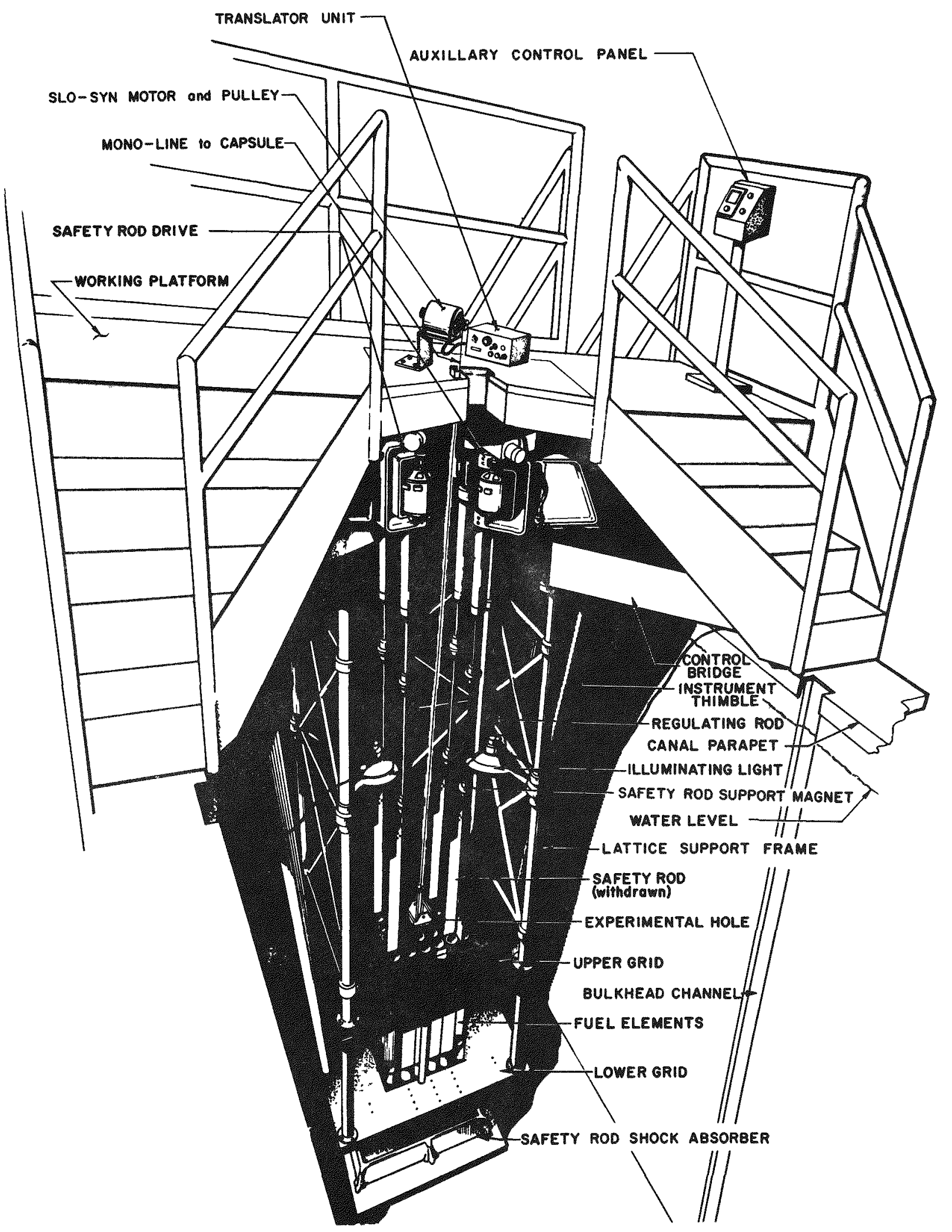

Figure 1. ARMF with experimental apparatus. 


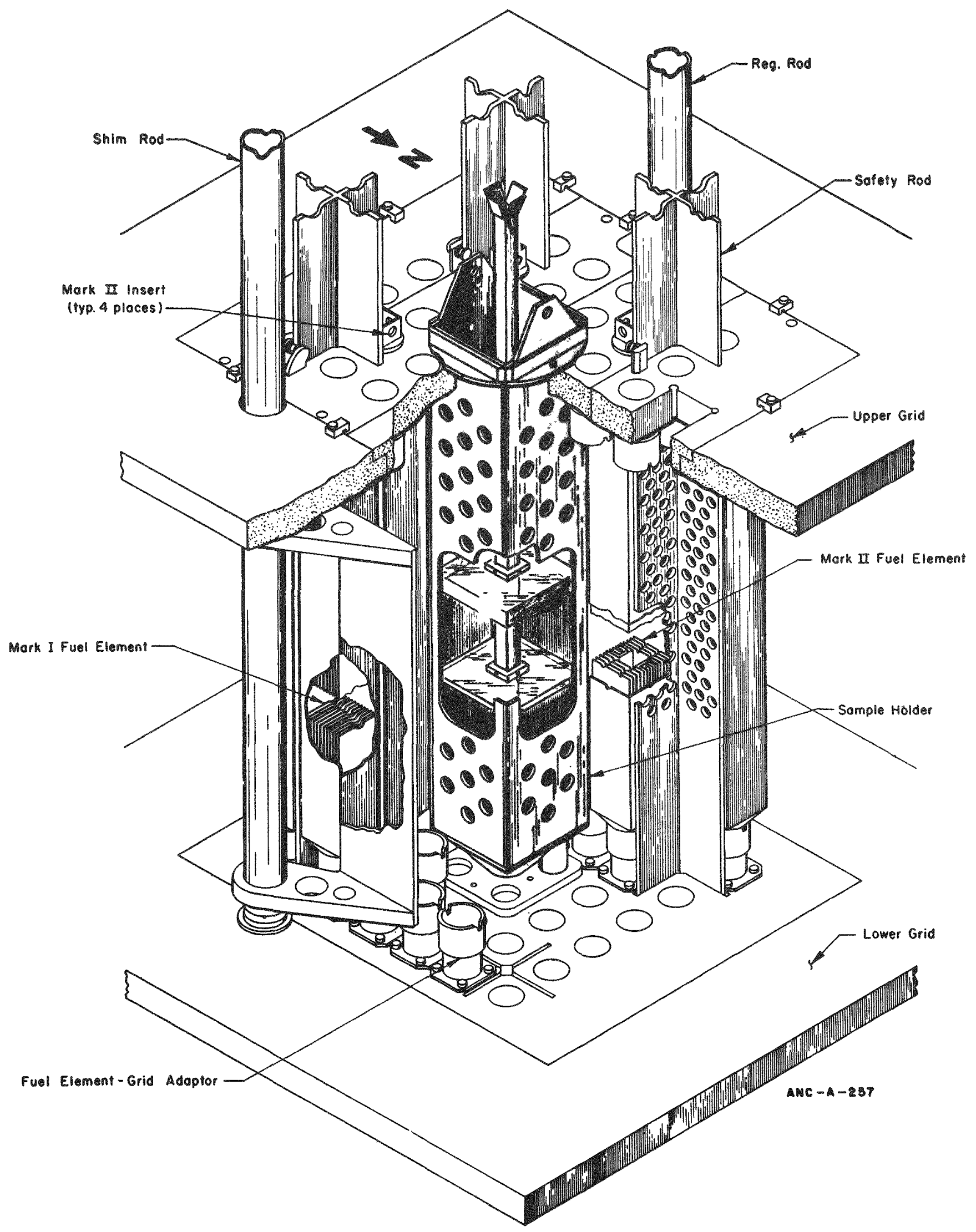

Figure 2. In-core measuring equipment. 


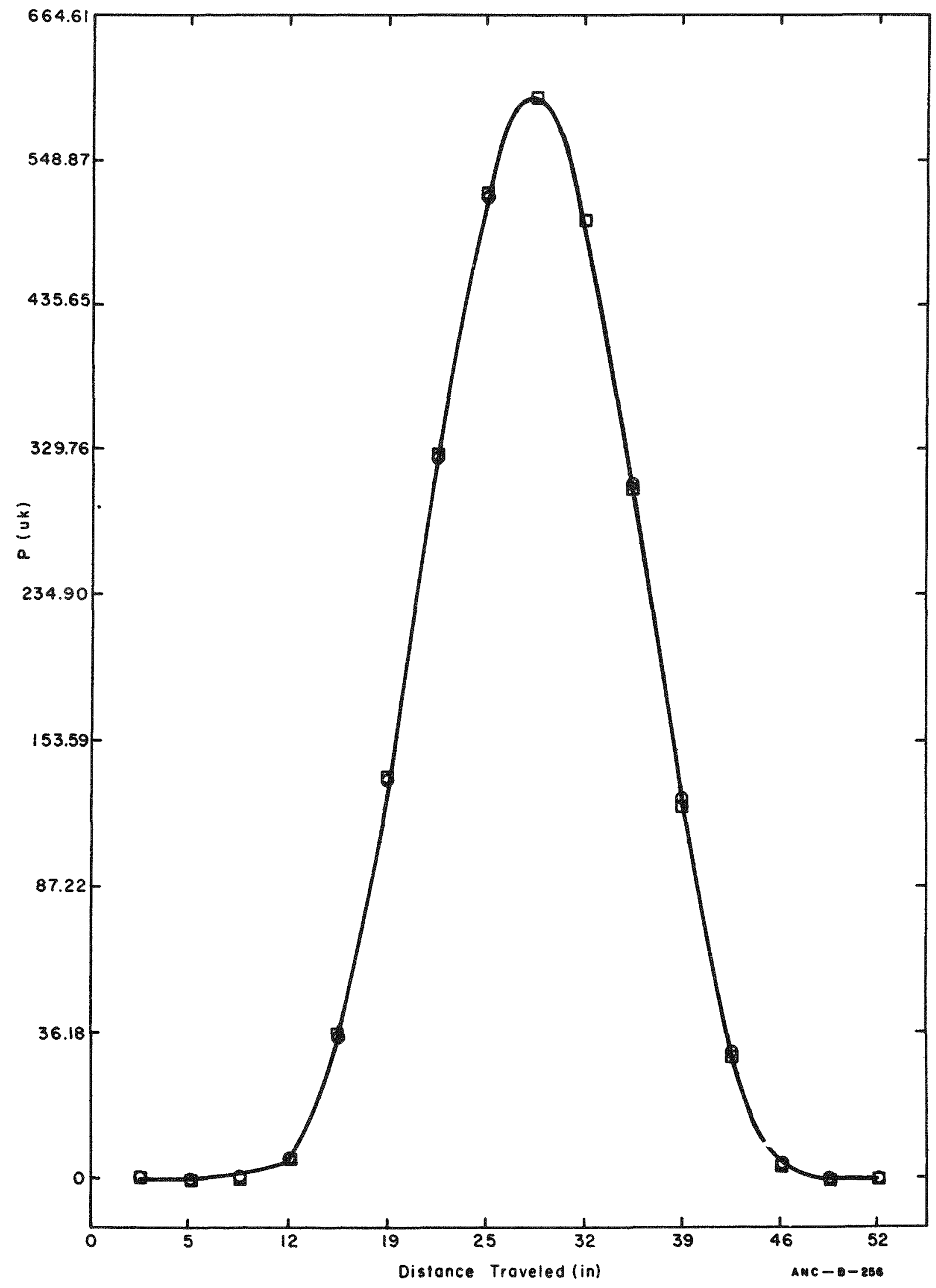

Figure 3. Sample traverse measurement. 


\title{
PREPARATION OF STANDARDS FOR BETA AUTORADIOGRAPHY
}

J. K. Cranda11

During the year a major problem arose at the ATR because of failure of the fuel elements caused by thin cladding. A method of autoradiography was developed to identify thin cladding before the elements were used. Standards were needed in order to assign accurate values of cladding thickness of test reactor fuel plates as determined by this $\beta$-autoradiography. These standards were fabricated in the Metallurgy Laboratory according to procedures established for the fabrication of sample fuel plates for the ETR INC-16 irradiations. The standards consisted of unclad fuel core material in each of the three fuel loadings of the ATR 7F fuel element. The fuel core, consisting of UA1 powder, was prepared by powder metallurgy blending and compacting techniques. The green compacts were enclosed in aluminum picture frames and hot and cold rolled to the desired thicknesses of .020" and .030". The frames were then stripped off and the standards placed in plastic bags until needed for the $\beta$-autoradiography. These standards established the cladding thickness scale to $\pm 0.00005^{\prime \prime}$.

\section{PREPARATION OF TEST CAPSULES FOR NASA}

\author{
J. K. Cranda11
}

Metallurgical support was provided to a study of containment vessels for the NASA program. Mock-up capsules were assembled from pre-machined parts in preparation for assembly of test capsules for melt-down studies. Six molybdenum capsules $1 / 2$ in. in diameter by $11 / 8$ in. long were loaded with fully enriched $\mathrm{UO}_{2}$ and were sealed by electron-beam welding of the end caps. Two similar capsules containing depleted $\mathrm{UO}_{2}$ and one empty capsule were closed by epoxy-cementing the end caps. The nine capsules were then loaded symmetrically in two 6 in. hemispheres which were welded to form a sphere. Depleted $\mathrm{UO}_{2}$ was poured through an opening in one of the hemispherical sections to fill the void space surrounding the capsules. This opening was then welded shut to complete the spherical mock-up assembly. Final test assemblies fabricated using the same procedure are planned for $F Y-72$. 


\section{FORMING AU-NI ALLOY BELLOWS}

\section{G. O. Hayner}

Work is being carried out to fabricate the bellows of a packless valve from 10 mil thick 75 per cent gold - 25 per cent nickel alloy foil. This highly corrosion resistant alloy is required because of the short life of stainless steel bellows in an environment of nitric and hydroflouric acids at approximately $160^{\circ} \mathrm{F}$. It is estimated that the Au-Ni alloy will give many times the service life in this application.

The Au-Ni alloy foil, however, has been determined to have a breaking strength of nearly $2 \times 10^{5}$ psi at room temperature in conjunction with less than one per cent ductility. This low formability level necessarily ruled out fabrication of the bellows by hydroforming techniques (the method currently employed to form the bellows from stainless steel sheet). Elevated tensile tests were made on this material and it was determined from these results that the per cent elongation increased to approximately 7 per cent at $950^{\circ} \mathrm{F}$. Therefore, it was decided to individually form each convolution (of the bellows) at elevated temperature. Then these in turn could be electron beam welded together. Therefore, punches and dies were ordered to punch out the flat washer-1ike blanks shown in the lower left corner of Figure 1 and forming dies were fabricated to form the inside and outside diameters of the blanks to the required radii (shown in the upper portion of Figure 1). A blank, after forming, is shown in the lower right corner of Figure 1. Heat is provided during the forming operation by means of cartridge heaters enclosed in a separate assembly. Two formed blanks are then held in a special fixture and an electron beam lap weld is made on the inside radius of each pair while it is turned by a rotary fixture. These welded pairs are then fitted on a solid spindal, and the outside radii welded in a similar fashion.

Work on this project is continuing and no conclusions have been drawn for the overall feasibility of this fabrication method. 

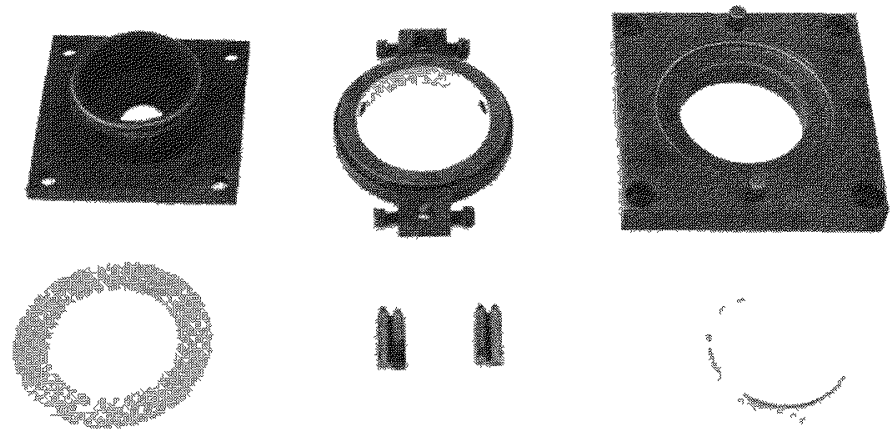

Fig. 1 Forming dies and washer before (left) and after (right) forming. 


\section{PREPARATION OF TRANSDUCER HOUSINGS BY EB WELDING}

\section{G. 0. Hayner}

Electron beam welding has been used to fabricate transducer housings which are used for eddy current oxide thickness measurements in the ETR and ATR canals. This work involved joining preformed fully annealed one mil thick 304 stainless steel faces to 304 stainless steel cylinders with ten mil thick walls. The welding was performed by using a rotary fixture mounted on an $\mathrm{x}-\mathrm{y}$ platform within a vacuum chamber. A photograph of this facility is given in Figure 1 .

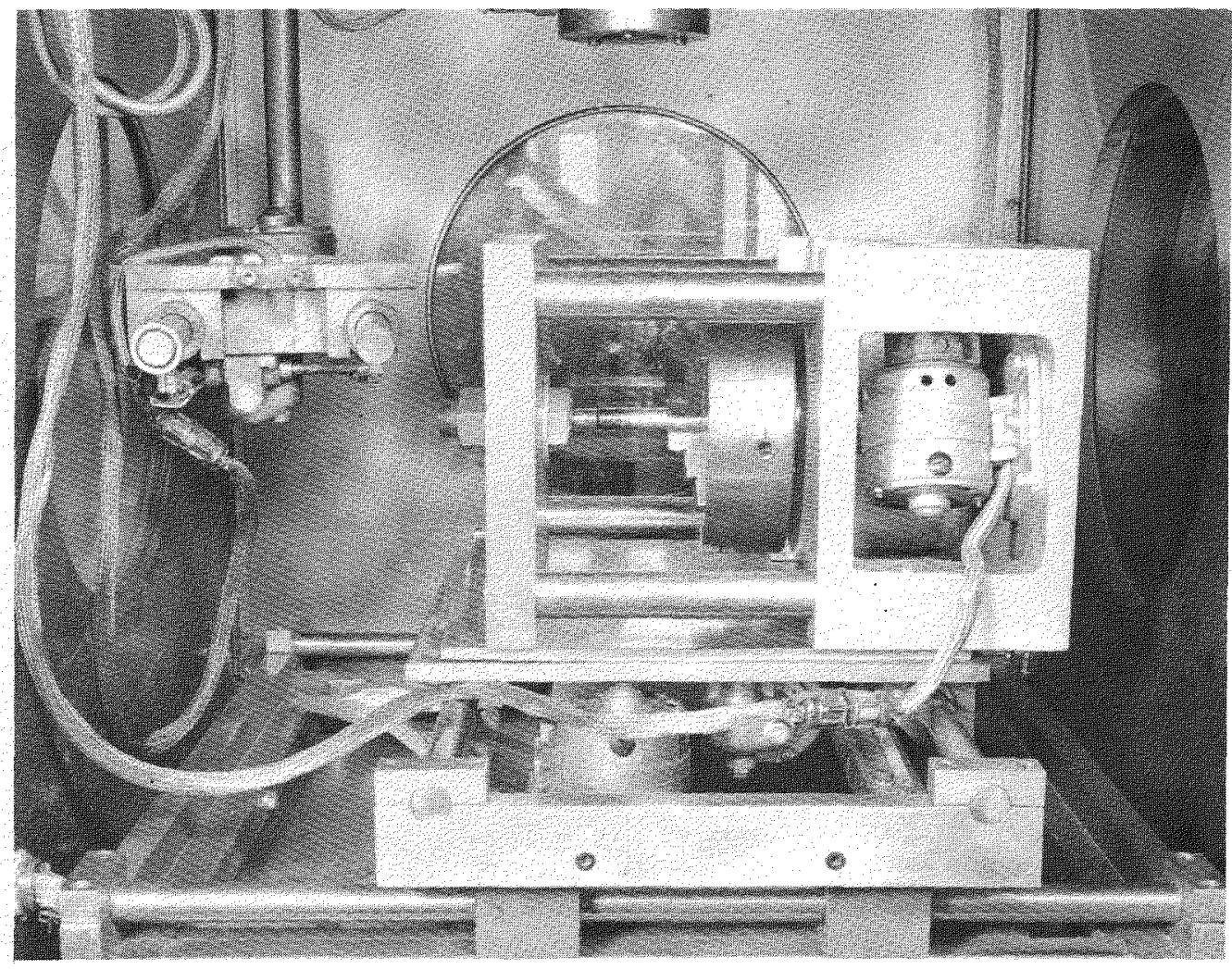

Fig. 1 Photo of the inside of the electron beam vacuum chamber. The $x-y$ platform is shown at the bottom of the picture. The rotary fixture is seen in the center sitting on the $x-y$ platform.

A disassembled transducer housing, prior to welding is shown in Figure 2. The assembly after welding has been completed, is shown in Figure 3. This particular electron beam welding application permitted welding to be completed in approximately 15 minutes for 
each with zero per cent rejection rate on the first seven completed, after a 20 man-hour development effort. Before this technique was developed, each unit required approximately five man-hours of machining time with somewhat over 50 per cent rejection rate. It has been our experience after welding many of these assemblies that proper time must be devoted to set up of the electron beam gun prior to the time that welding is initiated. If this step is not carried out properly, and if the gun is not realigned periodically during the welding operation, a high rejection rate will result. However, with proper controls and a certified technician to perform the welding, this can be avoided. 

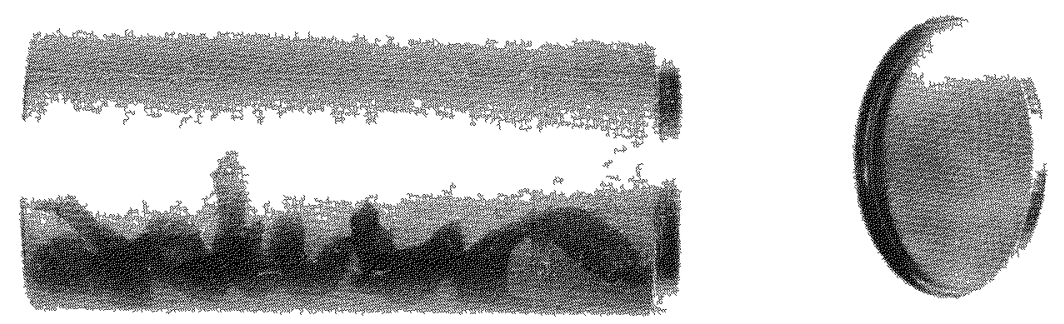

Fig. 2 Transducer assembly before welding. This type of housing is approximately $0.5 \mathrm{in}$. in diameter.

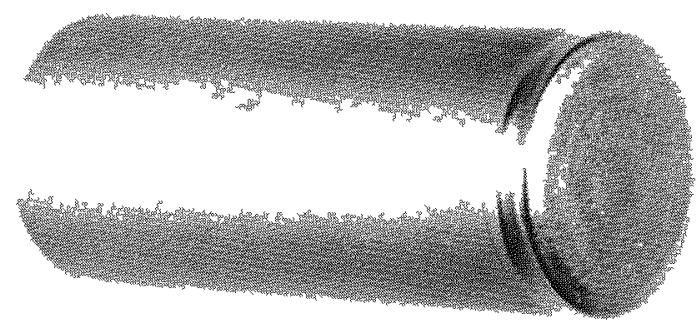

Fig. 3 Welded transducer assembly. Notice the thin and uniform weld area. Also, note that no bulging of the face has occurred. 


\title{
FABRICATION OF FLUX MONITOR FOILS
}

\author{
G. 0. Hayner
}

Two flux monitor foils have been fabricated by powder metallurgy techniques. The use of these foils is discussed elsewhere in this report.

The weights of the powders used to fabricate foil 非 1 and \#2 are given in Table I. All of the powders were screened to -325 mesh particle size and the powders comprising each alloy were then blended in a mechanical rotary blender for four hours. The blended powders for alloys $\#_{1} 1$ and $\left.\right|_{2}$ were charged in a $0.500 \mathrm{in}$. $\mathrm{x} 1.875 \mathrm{in}$. die and a 2.000 in. $x 2.250 \mathrm{in}$. die, respectively. Green compacts were made using approximately 30 tons/in. 2 of ram pressure. The thicknesses of these compacts were 0.20 in. and 0.22 in. for alloys $\# 1$ and $\# 2$, respectively. These compacts were then fitted in picture frames of copper and aluminum for alloys 1 and 2, respectively. Cladding of the same material was electron beam welded to the outside perimeter of each picture frame. A total of four rolling assemblies of alloy 1 composition and one rolling assembly of alloy 2 composition were made with the material listed in Table I. Alloys 1 and 2 were then hot rolled at $800^{\circ} \mathrm{C}$ and $500^{\circ} \mathrm{C}$, respectively, to a final core thickness of approximately 0.035 in. After rolling, the cladding on both assemblies was either pulled or machined off.

After fabrication, both alloys appeared to be silvery in appearance, however, alloy \#2 appeared to have poor mechanical properties, was fairly porous, and could not be punched. Alloy 非 could be punched, was not porous, and appeared to be quite brittle. No specific property data was obtained on either alloy.

Samples of both of the above alloy foils were sent to the Analytical Chemistry Branch for analysis for the weight per cent of the elements in each foil and the variance in the weight per cent of the elements in samples taken from the same foils. Table I lists the data which has been obtained to date. The more precise analyses given below would indicate that good homogeneity was obtained within a given foil and the powder metallurgy technique used for fabricating the foils produces foils that meet the uniformity and stability requirements for use as flux monitor foils. 


\section{TABLE I}

WEIGHT PER CENT AND HOMOGENEITY OF THE ELEMENTS WHICH FORM ALLOY \#I AND 2

\begin{tabular}{|c|c|c|c|c|}
\hline Alloy 非 & Element & Sample & $\begin{array}{l}\text { Weight Per Cent } \\
\text { Desired } \\
\end{array}$ & $\begin{array}{l}\text { Weight Per Cent } \\
\text { Obtained }\end{array}$ \\
\hline & $\mathrm{Au}$ & $\begin{array}{l}1 \\
2\end{array}$ & $\begin{array}{l}4.8 \\
4.8\end{array}$ & [a] \\
\hline & $\mathrm{Cu}$ & $\begin{array}{l}1 \\
2\end{array}$ & $\begin{array}{l}40.6 \\
40.6\end{array}$ & $\begin{array}{l}41.0^{[c]} \\
40.4[c]\end{array}$ \\
\hline & $\mathrm{Mn}$ & $\begin{array}{l}1 \\
2\end{array}$ & $\begin{array}{l}27.1 \\
27.1\end{array}$ & $\begin{array}{l}26.4^{[b]}[b] \\
26.4^{[b]}\end{array}$ \\
\hline . & Co & $\begin{array}{l}1 \\
2\end{array}$ & $\begin{array}{l}0.3 \\
0.3\end{array}$ & [a] \\
\hline & $\mathrm{Cr}$ & $\begin{array}{l}1 \\
2\end{array}$ & $\begin{array}{l}27.1 \\
27.1\end{array}$ & $\begin{array}{l}27.6^{[c]} \\
27.9^{[c]}\end{array}$ \\
\hline \multirow[t]{6}{*}{ Alloy 非 } & Al & $\begin{array}{l}1 \\
2 \\
3\end{array}$ & $\begin{array}{l}27 \cdot 3 \\
27 \cdot 3 \\
27 \cdot 3\end{array}$ & $\begin{array}{l}26 \cdot 5^{[c]} \\
25 \cdot 5^{[d]} \\
26.5^{[c]}\end{array}$ \\
\hline & $\mathrm{Fe}$ & $\begin{array}{l}1 \\
2 \\
3\end{array}$ & $\begin{array}{l}18.2 \\
18.2 \\
18.2\end{array}$ & $\begin{array}{l}18.1^{[\mathrm{b}]} \\
17.3^{[\mathrm{b}]} \\
17.9^{[\mathrm{b}]}\end{array}$ \\
\hline & $\mathrm{Ni}$ & $\begin{array}{l}1 \\
2 \\
3\end{array}$ & $\begin{array}{l}9.1 \\
9.1 \\
9.1\end{array}$ & $\begin{array}{l}9.0[c] \\
7.8[d] \\
9.2^{[c]}\end{array}$ \\
\hline & $\mathrm{Ti}$ & $\begin{array}{l}1 \\
2 \\
3\end{array}$ & $\begin{array}{l}18.2 \\
18.2 \\
18.2\end{array}$ & $\begin{array}{l}17.5[\mathrm{c}] \\
17.5[\mathrm{c}] \\
17.6^{[\mathrm{c}]}\end{array}$ \\
\hline & In & $\begin{array}{l}1 \\
2 \\
3\end{array}$ & $\begin{array}{l}9.1 \\
9.1 \\
9.1\end{array}$ & $\begin{array}{l}9.1[\mathrm{c}] \\
8.0[\mathrm{~d}] \\
9.1[\mathrm{c}]\end{array}$ \\
\hline & $\mathrm{Zn}$ & $\begin{array}{l}1 \\
2 \\
3\end{array}$ & $\begin{array}{l}18.2 \\
18.2 \\
18.2\end{array}$ & $\begin{array}{ll}17.9 & {[\mathrm{c}]} \\
18.3 & {[\mathrm{~d}]} \\
17.8 & {[\mathrm{c}]}\end{array}$ \\
\hline
\end{tabular}

[a] Analysis values have not been obtained for these elements as yet. 


\section{TABLE I (Contd.)}

[b] The expected error which can be associated with these values is $0.50 \%$ relative standard deviation.

[c] The expected error which can be associated with these values is approximately $1.0 \%$ relative standard deviation.

[d] The approximate range which can be associated with these values is approximately $\pm 5 \%$ to $\pm 10 \%$ of each value given. 
CHOPPER TEST INSTRUMENTATION

FOR USE IN QUALITY ASSURANCE AND MAINTENANCE

R. L. Sumstine, B. G. Nelson

Failures of choppers used in operational amplifiers at ATR and ETR brought about a study for an instrument that would check the choppers for proper operation. The instrument will be used to check newly purchased choppers and keep check on choppers used in the amplifiers on a routine maintenance program.

A chopper test unit was designed to test the choppers used in the amplifiers at ATR and ETR. The unit can check photo choppers, mechanical choppers, and solid state choppers. The following tests are performed by the chopper tester:

$$
\begin{aligned}
& \% \text { dwell time of both contacts } \\
& \% \text { off time } \\
& \text { noise and contact bounce } \\
& \text { photo efficiency }
\end{aligned}
$$

A dynamic chopper tester was also designed to test the chopper under actual operation in a typical amplifier. This unit will test for offset produced by the chopper and long term drift in the output of the amplifier.

The chopper tester block diagram is shown in Figure 1. An excitation voltage is applied to the chopper. The reference voltage is applied to the chopper contacts by selector switch $\mathrm{S1}$. The dwell time is a measurement of the time that the chopper contacts are closed. The measurement is made with a keyed integrator that converts time to voltage. This voltage is displayed on the meter in percentage of dwell time. The off time is a measurement of the percent of time that the wiper is not on either contact, and is measured and displayed the same as dwell time. The noise measurement is a measure of the noise and contact bounce that is generated by the contacts and wiper, and is displayed on the meter as a figure of merit. The photo efficiency is a measurement of the ratio of the "on" to "off" resistance of the photo ce11, and is displayed on the meter as a percentage of photo efficiency. The test circuit is an internal calibration source used to calibrate and check the chopper tester.

The dynamic chopper tester block diagram is shown in Figure 2. This test unit uses a Philbrick USA-4-JX operational amplifier. The circuit has a gain of 10 with zero input to check for offset and drift of the chopper under test. The chopper to be tested is installed in the operational amplifier and is monitored on the meter and recorder output. The meter range is plus and minus 5 volts.

The chopper tester shown in Figure 3 and the dynamic chopper tester shown in Figure 4 were constructed and designed by the Physical Instrumentation Section. The test instrumentation is now being used by the reactor instrument groups at ATR and ETR. 


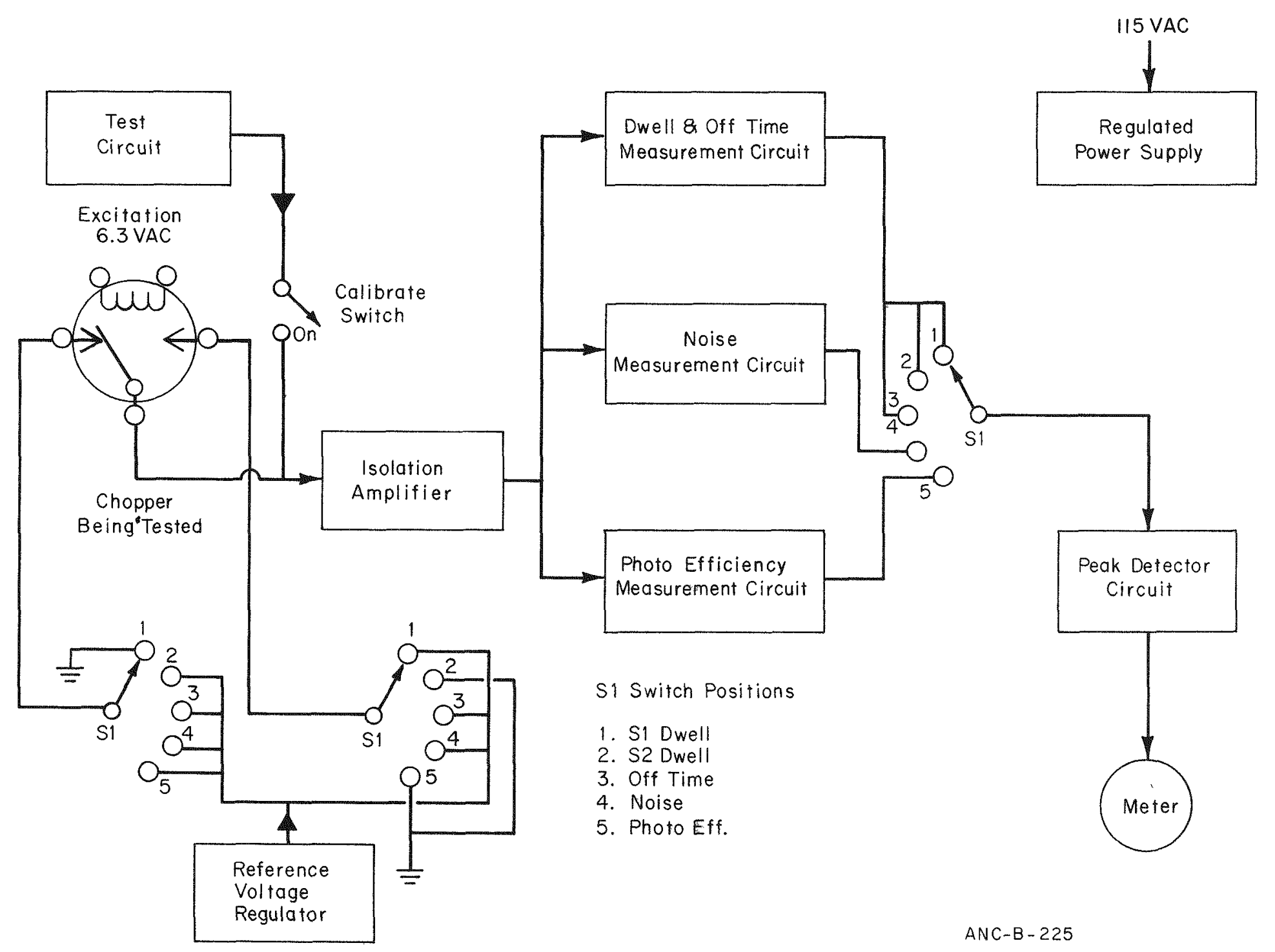

Fig. I Chopper tester model 0571 block diagram. 


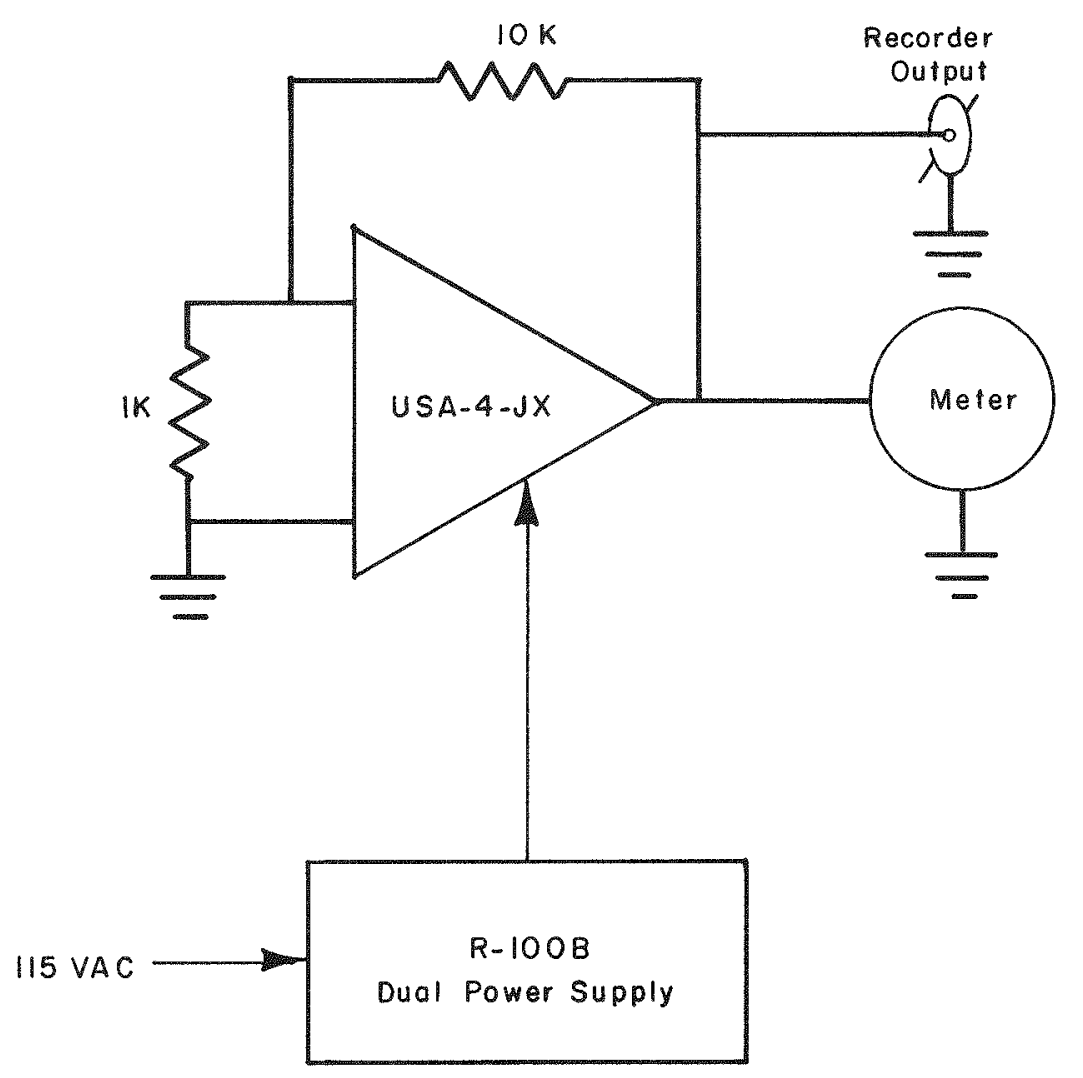

ANC-A-219

Fig. 2 Dynamic chopper tester model 0671 block diagram. 


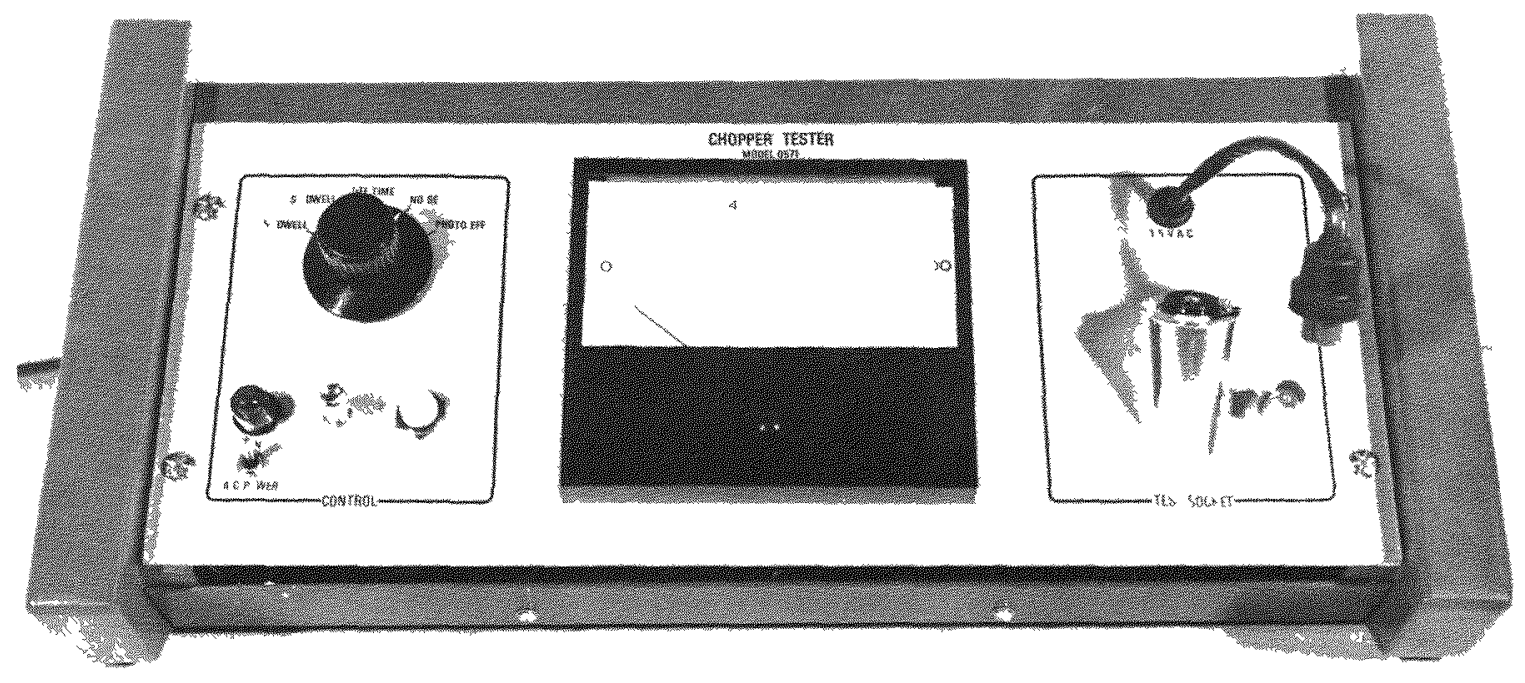

Figure 3. Chopper Tester Mode1 0571. 


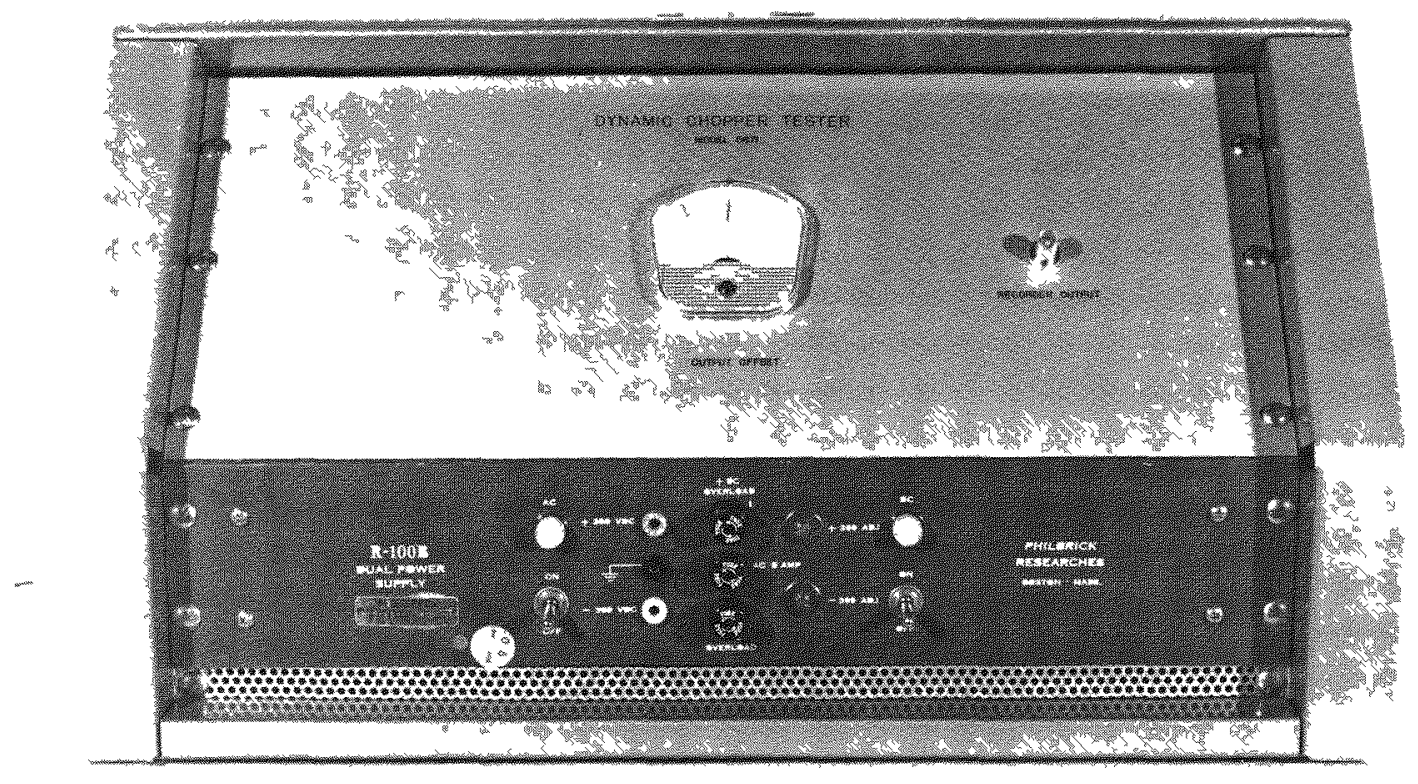

Figure 4. Dynamic Chopper Tester Mode1 0671. 


\section{AUTOMATIC UNDERWATER RADIQACTIVE WIRE SCANNER}

R. L. Sumstine, B. G. Nelson, F. A. Meichle

The Engineering Test Reactor (ETR) radioactive wire scanner system provides automatic underwater scanning of radioactive wires in the ETR canal. Advanced Test Reactor (ATR) wires are also scanned with this system. High maintenance and inadequate data handling facilities justified the upgrade of the system electronics and motor drive, but constant operation prevented any system upgrade. Increasing difficulties in transfering the ATR radioactive wires to the ETR provided the impetus to move the ETR scanner to the ATR canal. This move provided an opportune time to upgrade the scanner system.

To achieve minimum down time, the electronics and motor control units were designed and fabricated before the move was to be made. The present scanning mechanism, excluding the motor, limit switches and position indicator was retained.

The radioactive wire scanner block diagram is shown in Figure 1 and the control and data console is shown in Figure 2. The radioactive wire is drawn across the face of a scintillation counter. The counts are amplified and fed to an eight decade counter through a single channel analyzer. The counter is contained in the scanner controller. The wire can be scanned in preset 0.1 inch increments from 0.1 to 47.0 inches. Preset counting time can be selected in 1 second increments from 1 to 999 seconds. By selecting a slower clock rate the preset time can be increased to .I to 99.9 minutes in .1 minute steps if required.

Controls are provided on the scanner control unit to allow the following items to be programmed and recorded on the teletypewriter: sample number, date, and background count.

The readout format is shown in Figure 3. 


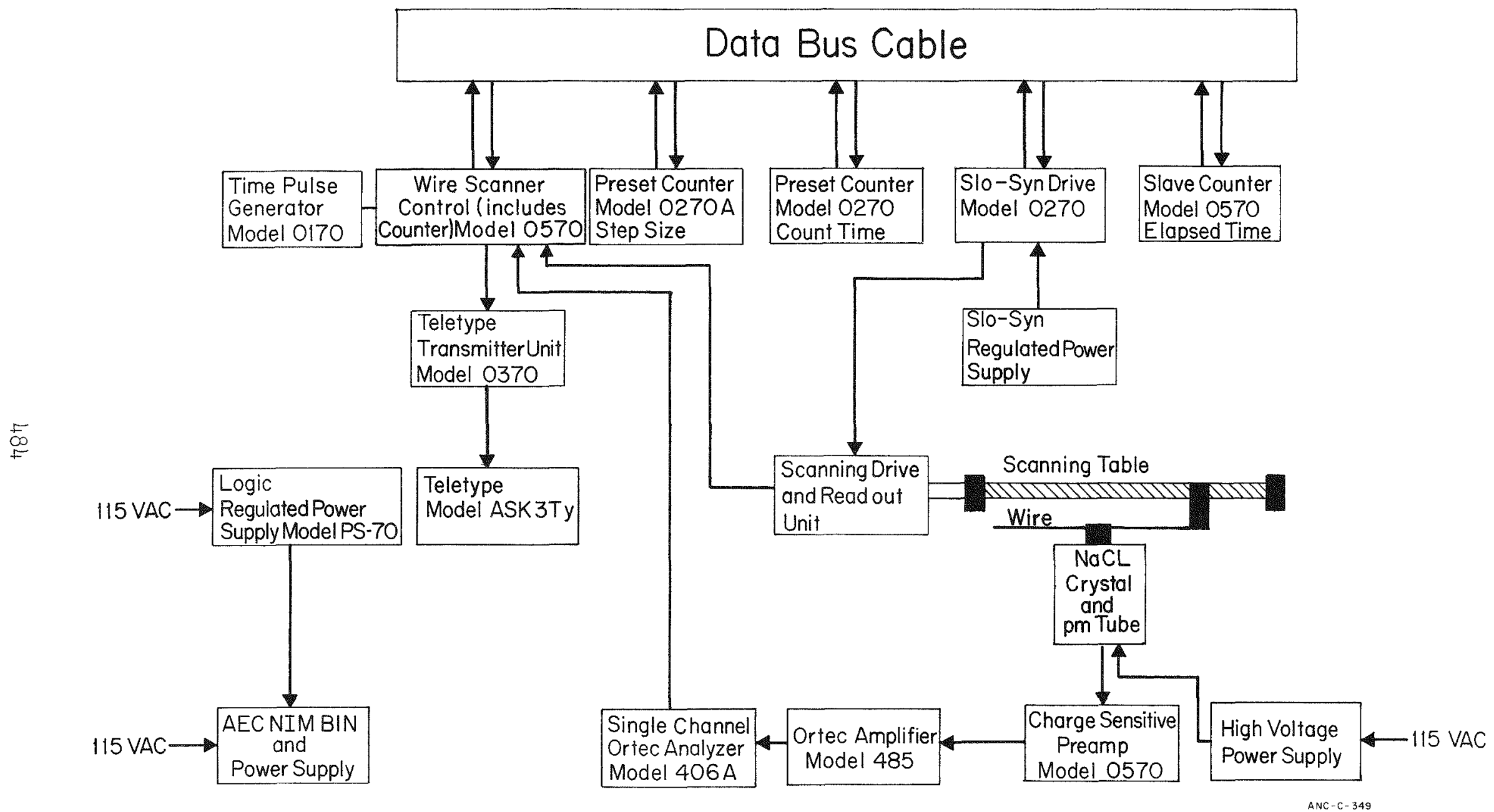

Figure 1. Automatic Underwater Radioactive Wire Scanner Block Diagram. 


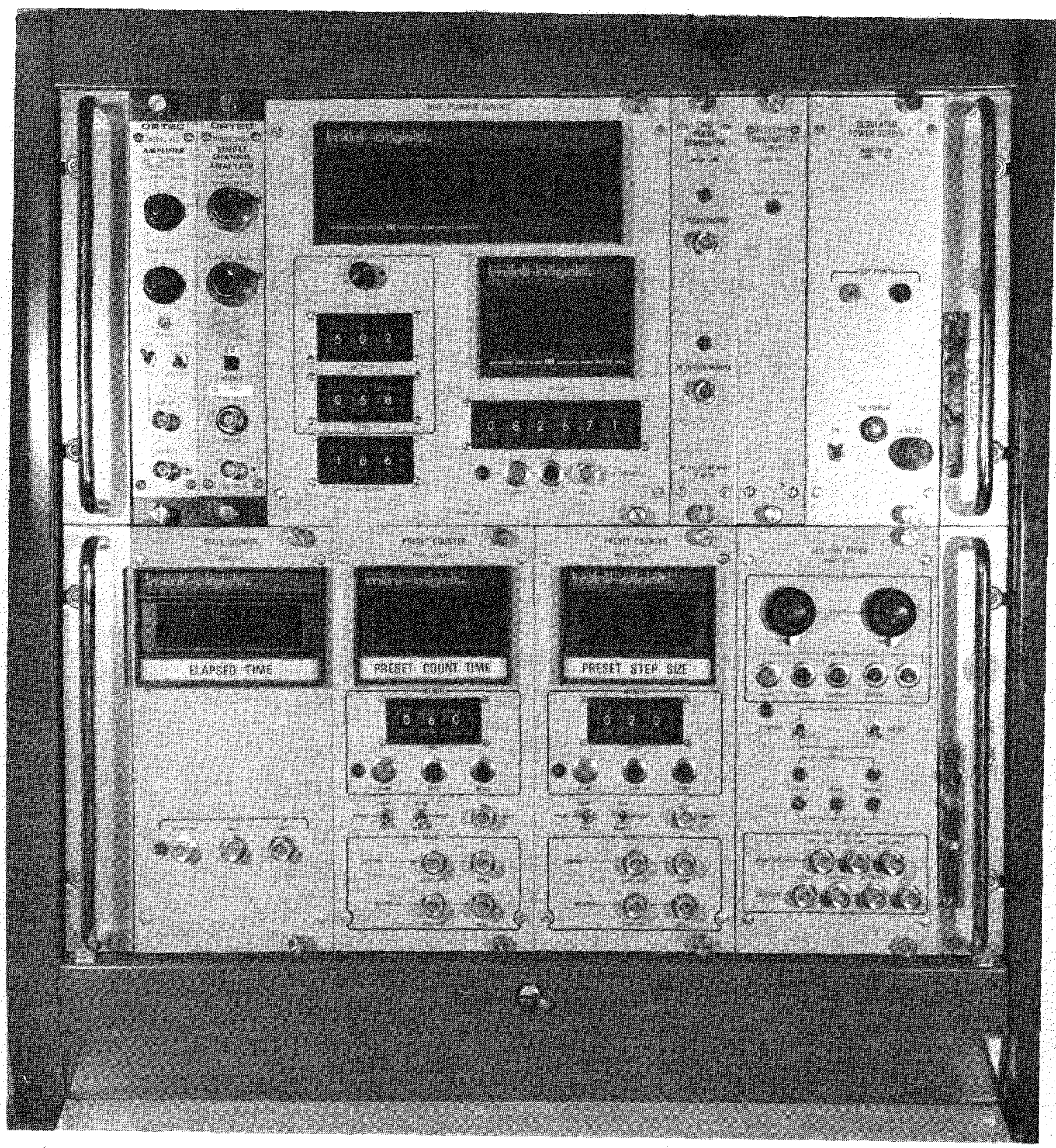

Figure 2. Automatic Underwater Radioactive Wire Scanner Control and Data Sys tem Modules. 


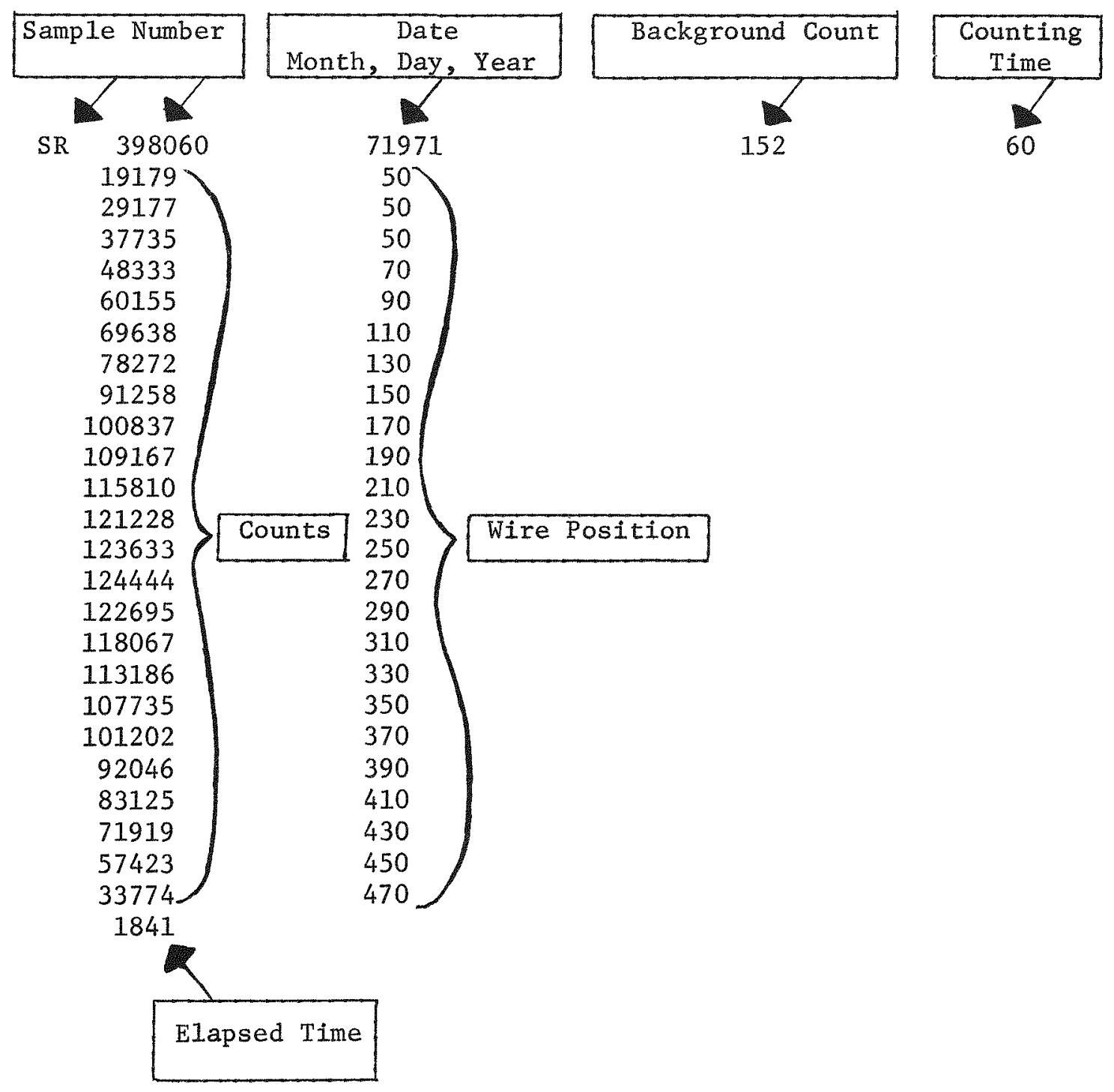

Figure 3. Wire Scanner Teletypewriter Format. 


\title{
III. APPLIED MATHEMATICS AND COMPUTER SOFTWARE
}

NEW SOFTWARE FOR GAMMA-RAY ANALYSIS

\author{
A. I. Connelly, R. C. Davies $[1]$
}

Two pieces of computer software have been completed which are to be used in the analysis of gamma-ray spectra. The first is based on an algorithm by Rutishauser[2]. It is used to invert a symmetric matrix in place with only the upper triangular part in core and that stored in compressed form using only $1 / 2 N(N+1)$ core storage locations, approximately one half that of the full matrix. The algorithm has the advantage of requiring only $1 / 2 \mathbb{N}^{2}(\mathbb{N}+3)$ multiplication and division operations whereas Gauss-Jordon techniques require $\mathrm{N}^{3}$ such operations, i.e. the Rutishauser algorithm requires approximately half as many. This approximately halves the time required for the matrix inversion.

The second piece of software is a non-linear least squares Gauss fitting program for use on PDP-9's and PDP-15's based on that presently being used in the GAUSS V Program 3$]$ in which up to five gaussians on a straight line background may be fit to a section of a spectrum. This program uses the matrix inversion routine mentioned above. The PDP-9 version requires $1027_{10}$ core locations or slightly more than IK of core. The PDP-15 version requires $1023_{10}$ core locations or slightly less than $1 \mathrm{~K}$ of core.

The Gauss fitting routine is to be integrated into the Gamma-Ray Analysis Package 4 presently being used on the graphics equipped PDP-9 in the Physics Branch. This will allow a user to operate on his data with algorithms of the same power as used in the GAUSS V Program on the IBM 360 with the added advantage that he will be able to do this interactively. Both the matrix inversion routine and the Gauss fitting routine use the floating point package developed by $W$. R. Myers[5].

An isotope library has also been added to the Gamma-Ray Analysis Package. Each time the spectrum rotation is stopped the library is searched for an identifying isotope corresponding to the energy of the displayed bright dot. The bright dot is an intensified point of the spectrum used for location purposes.

The library consists of an isotope name and its corresponding energy. Each time rotation is stopped, the program searches the library for an energy which most approximates the energy of the bright dot. The isotope name for the found energy is then displayed.

[1] Member, Computer Science Branch, Technical Services Division, Aerojet Nuclear Company.

[2] H. Rutishauser, "Algorithm 150" Comm. ACM, V. 6, No. 2, p. 67 (1963).

[3] R. G. Helmer and M. H. Putnam, unpublished report on GAUSS V. 
[4] R. C. Davies and E. W. Killian, "Gamma-Ray Spectrum Analysis for a PDP-9", Nuclear Technology Branch Annual Progress Report for Period Ending June 30, 1970, IN-1407 (Dec. 1970) p. 364.

[5] W. R. Myers, PD-9, A Floating Point Arithmetic Package for a PDP-9 Computer, Idaho Nuclear Corporation internal report (1969). 
AUTOMATIC PEAK LOCATION AND LIBRARY LOOK-UP

M. S. Cole $\mathrm{e}^{[1]}$

Software has been written for the PDP-8 which utilizes the Nuclear Data 50/50 system to collect and store spectral data. The analysis uses a correlation technique[2] for locating peaks in the data, and the results of the search may be printed on the teletype or stored for later use. If the results are stored, they may be compared to a standard library. Correlations between spectral events and library entries are printed on the teletype, providing an automatic qualitative analysis of the spectrum.

[I] Member of Computer Science Branch, Technical Services Division, Aerojet Nuclear Company.

[2] See "Application of Correlation Techniques to Isolate Structures in Experimental Data", W. W. Black and "Automatic Location and Area Determination of Photopeaks", A. I. Connelly and W. W. Black. 
SPECTRUM STRIPPING USIIG A PDP-9

R. C. Davies ${ }^{[I]}$

Typical gamma-ray spectra contain components from several elements. In order to analyze the spectra, the contribution of each recognized element is removed by subtraction of a "standard" spectrum. This "stripping" procedure is continued until all of the components have been identified and removed.

The following is a description of a stripping overlay for the program COINC[2]. The overlay is designed to do floating point stripping of coincidence and singles data.

Spectrum stripping can be accomplished with the COINC program using the following forms of the GET and/or RDSLOT commands:

\begin{tabular}{|c|c|c|}
\hline $\mathrm{GET} ; \mathrm{X}$ & or & RDSLOT; $\mathrm{X}$ \\
\hline $\mathrm{GET} ; \pm \mathrm{Y}^{*} \mathrm{X}$ & or & RDSLOT; $\pm Y * X$ \\
\hline $\mathrm{GET} ;+\mathrm{Y} / \mathrm{Z}^{* \mathrm{X}}$ & or & RDSLOT: $\pm Y / Z * X$ \\
\hline
\end{tabular}

The commands GET3 and RDSLO3 are the same as the GET and RDSLOT commands given above except they read data from unit \#3 of the DECtape.

The results are stored in memory as 17-bit signed integers and on a scratch tape as floating point numbers. The scratch tape values are used in the stripping computations to preserve the accuracy through several stripping steps. The memory values are for display only.

The overlay also adds the form GET; ${ }^{*} X$ and/Or RDSLOT; $+* X$ to the command structure. This form $\left( \pm^{*}\right)$ indicates that the experiment $X$ is to be multiplied by the quotient of the floating point registers $R I$ and R2. These registers can be loaded with various quantities which allow the same sequence commands to strip different sets of data.

As an example of the usage of the strip routine, assume that there are two spectra with experiment identifications 2 and 3 . Assume also that there is a peak between channels 23 and 27 in the $F$ spectrum of each experiment and that the ratio of the areas of the peak in the two spectra is to be multiplied by each channel in experiment 3 and subtracted channel by channel from experiment 2. The following commands could be used:

INIT; Zero out the scratch tape.

GET3;3 Experiment 3 is loaded into memory from unit 3.

F; $\quad$ Display the $F$ spectrum.

W;23,27 Display the peak only. 


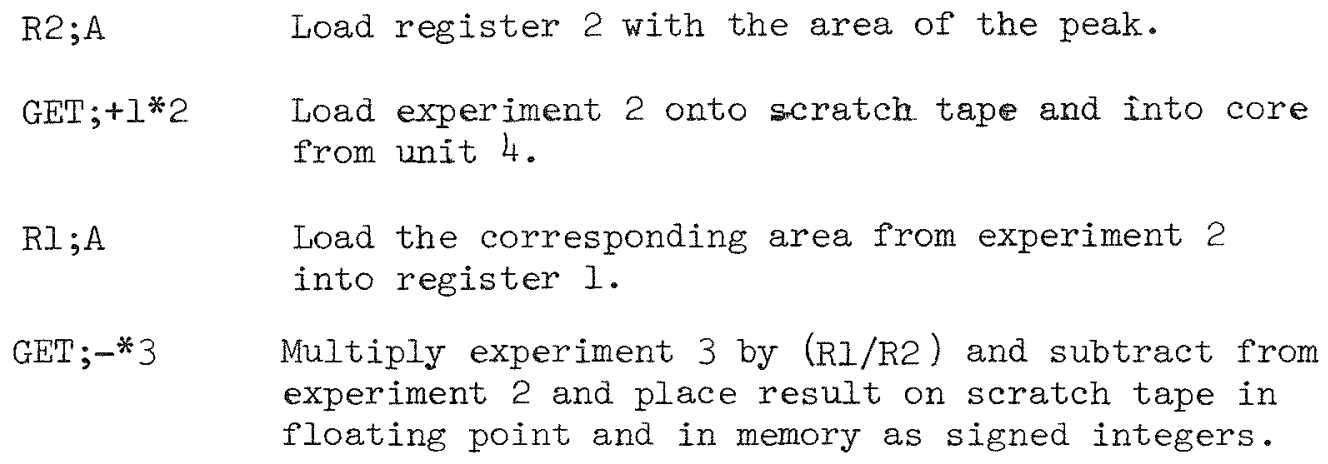

Many times when stripping coincidence data it is desirous to add areas together to obtain a better ratio of a spectrum to be stripped. To do this, the commands $R I+V$ and $R 2+V$ have been added. $V$ can be in area, integer, or other values as indicated in reference [2].

This stripping procedure has been used at the NRTS, and at the NASA Manned Spacecraft Center, for the analysis of gamma coincidence spectra of lunar material.

[1] Member, Computer Science Branch, Technical Services Division, Aerojet Nuclear Company.

[2] W. W. Black and C.W. Richardson, User's Manual for the Multiparameter Data Acquisition Program COINC Version 5B with Teletype and Panel Controls, Idaho Nuclear Corporation Manual. 


\section{COMPUTER PROGRAM FOR THE ANALYSIS OF} CONTINUOUS ELECTRON SPECTRA

R. G. Helmer, R. G. Hughes ${ }^{[I]}$

Unstable nuclei containing an excess of neutrons generally decay by the emission of an electron from the nucleus. The energy spectrum of these electrons is continuous, from o to the total decay energy, in contrast to the monoenergetic lines resulting from emission of $\alpha$ particles, $\gamma$ rays and internal-conversion electrons. In the study of beta spectra, the shape of the spectrum (i.e., $N(E)$ Vs. E) is the quantity of interest, since information concerning the nuclear matrix elements involved in the transition can frequently be obtained from analysis of the shape. In the first approximation for a single transition, the theoretical shape of the spectrum is given by the statistical division of the energy available (a constant $=E_{0}$ ) between the electron and the associated neutrino. Refinements to these calculated spectral shapes take into account the finite nuclear size and the screening of the atomic electrons. These effects modify the potential that the outgoing electron sees and thereby the electron wave function. The final step in any complete theoretical treatment would be to consider the influence of the specific nuclear states involved on the relative contribution of the various beta-decay matrix elements.

The relative importance of the beta-decay matrix elements is usually determined from experimental results rather than the theory. By combining the results of several types of experiments this is generally possible. One such class of experiments is the spectral shapes discussed here. For a single transition the spectrum $\mathbb{N}(E)$ is fitted with a function:

$$
\mathbb{N}(E)=F(Z, E) S(E)\left(E_{O}-E\right)^{2}
$$

where

$$
F(Z, E)=\text { Fermi function }
$$

and the shape factor

$$
S(E)=a+b E+\frac{C}{E}+d E^{2}
$$

In an analysis, the parameters to be determined are $E_{0}, a, b, c$ and $a$. (Usually, one or more of the parameters $b, c$, and d are zero.) $F(Z, E)$ is a known function.

If a measured spectrum consists of more than one component, the problem becomes very complex. The first step in such an analysis is to fit the data with several components to determine the values a and $\mathrm{E}_{0}$ for each component under the assumption that all the shape factors are constant $[i . e ., S(E)=a]$. Any further steps in the analysis for several components depends on the existence of other information (e.g., shapes of single components from beta-gamma coincidence measurements) that is available. 
In order to be able to carry out the first step in this type of analysis, a computer program has been obtained from Argonne National Laboratory. (This program was originally written at Argonne National Laboratory under the supervision of one of the authors, R. G. Helmer.) The program has been modified to run on the IBM $360 / 75$ computer. Also the method of computing the Fermi function $F(Z, E)$ has been improved. This program is constructed to use the energy level information that is available to constrain the computed $E_{0}$ values. This greatly improves the quality of the analysis when several components are present. 
ANALYSIS OF CONVERSION-ELECTRON SPECTRA

R. G. Helmer, R. A. Lokken[I], D. J. McMillan [2]

In the decay of most radioactive isotopes, a number of excited nuclear states are populated. In the transition from one of these excited states to a lower one, the excess energy is usually released in the form of a gamma ray. As an alternative mode of de-excitation this energy can be transferred to an atomic electron which is then ejected from the atom. As shown in the spectrum in Figure 1, monoenergetic electron lines result from each of the several atomic shells and subshells.

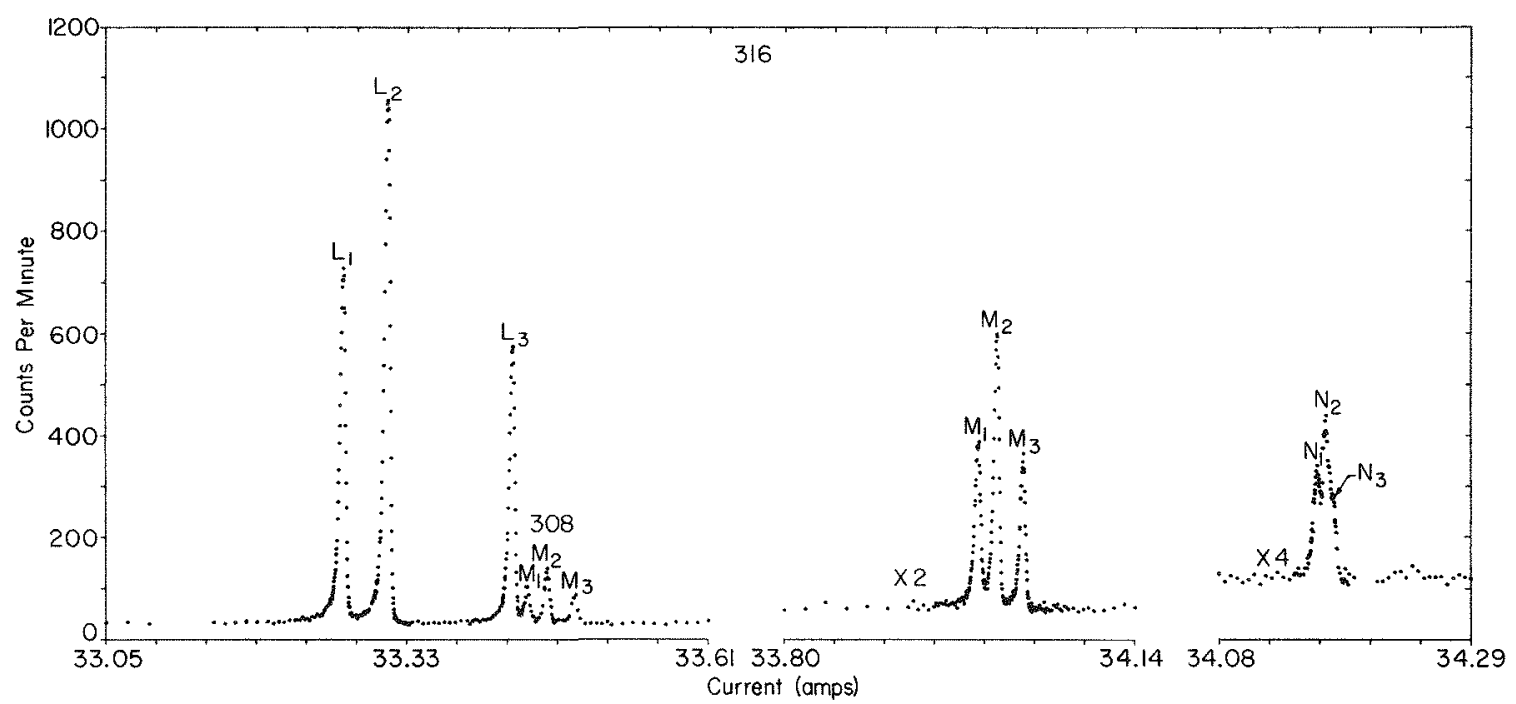

Figure 1

A (singles) gamma-ray spectrum contains information primarily about the energy spacing of nuclear levels and only very indirectly about the angular momentum characteristics (or multipolarity) of the gamma-ray transition. In contrast, the intensities of the electron lines (as in Figure 1) give direct information about the multipolarity of the transition. This information can be obtained in either of two ways. The multipolarity can be deduced from the ratio of the intensities of the conversion line and the corresponding gamma ray. The second method, which is generally more sensitive at lower energies, is to use the ratio of the intensities of the conversion lines within one shell (e.g. $I_{1}, I_{2}$ and $\left.I_{3}\right)$. This technique avoids the difficulty of normalizing the intensity of the gamma rays. Therefore, in analyzing conversion-electron spectra, such as Figure 1 , the line intensities are of primary interest. 
One complication in the analysis of high-resolution conversionelectron spectra is the natural width of the atomic shells. In any process of absorption or emission involving one or two atomic electrons, it is found that the atomic levels exhibit a spread in energies. Theoretical consideration[3] of these processes (especially $x$-ray emission) indicate that the energy spread should have a Lorentzian distribution. That is

$$
f(E) \propto \frac{1}{\left(E-E_{0}\right)^{2}+\left(\frac{\Gamma}{2}\right)^{2}}
$$

where $E$ is the most probable energy and $\Gamma$ is the width of the function at half ${ }^{\circ}$ the maximum height. It can be shown that the convolution of two Lorentzian functions of widths $\Gamma_{1}$ and $\Gamma_{2}$ gives a Lorentzian function of width $\Gamma=\Gamma_{1}+\Gamma_{2}$ (i.e., $50+5=55$ ). (This is in contrast to the convolution of two Gaussian functions where the widths add in quadrature, $\mathrm{W}=\mathrm{W}_{1}^{2}+\mathrm{w}_{2}^{2}$ or $\left." 50+5^{\prime \prime}=50.25.\right)$

If the widths of the atomic shells were known accurately, there would be no complication. But the widths have not been accurately determined experimentally. Therefore, in the analysis of spectra, such as Figure l, the level widths may become added variables. Most of the data on widths comes from the widths of $x$-ray lines [4]. Since these transitions involve two levels, they do not relate directly to the case of interest here. Another possible source of information on atomic level widths is the theoretical calculation of $x$-ray transition probabilities $[5]$. Since the probability per unit time that a hole in an atomic level is filled is proportional to the width of that level, these calculated probabilities should, to the extent that they are correct, give the level widths. If the proportionality factor is exactly the fluorescence yield, $\left(\omega_{i}\right)$ one has $\Gamma_{i}+T_{i} / \omega_{i}$ for the $i$ th subshell.

There are two basic methods of analyzing conversion-electron spectra. First, one can simply subtract a background and integrate the area under the peaks. This method has the advantage of not requiring any assumptions about the differences in natural widths. A disadvantage is that the lines involved must be completely resolved. Another disadvantage is that of taking into account the long, low-energy tail of the line, and especially the differences in these tails due to differences in the natural width.

The second method of analysis involves the least-squares fit of a "standard" line shape to each peak. In this case, the natural widths can be taken into account by convoluting a Lorentzian function of the appropriate width with the standard line shape. This method has the advantages of allowing the line shape to vary correctly (if Equation (I) is correct) and of fitting unresolved peaks. The disadvantages of this method is the increase in the number of variables to be determined when the natural widths are not known. 
We have developed techniques to carry out the analysis of conversionelectron spectra by means of the least-squares fitting method with a standard line shape. These techniques are combined in a computer program which carries out the following operations:

1. Reads spectral data, the line shape and control information.

2. Corrects data for source decay and computes counting rates at each point.

3. Shifts line shape to approximate location of line(s).

4. Convolutes Lorentzian with line shape, if desired.

5. Carries out least-squares fit to determine approximate line intensities.

6. Makes iterative adjustment of the line positions to determine best position; after each adjustment the intensity is recomputed.

7. Prints results indicating the final intensities, their uncertainties, the quality of the fit, and the contribution of each component.

8. A plot of the resulting fit can be made.

A plot of the results of a fit to the three $L$ subshell lines for the $295-\mathrm{keV}$ gamma ray from the decay of ${ }^{192} \mathrm{Ir}$ is shown in Figure 2.
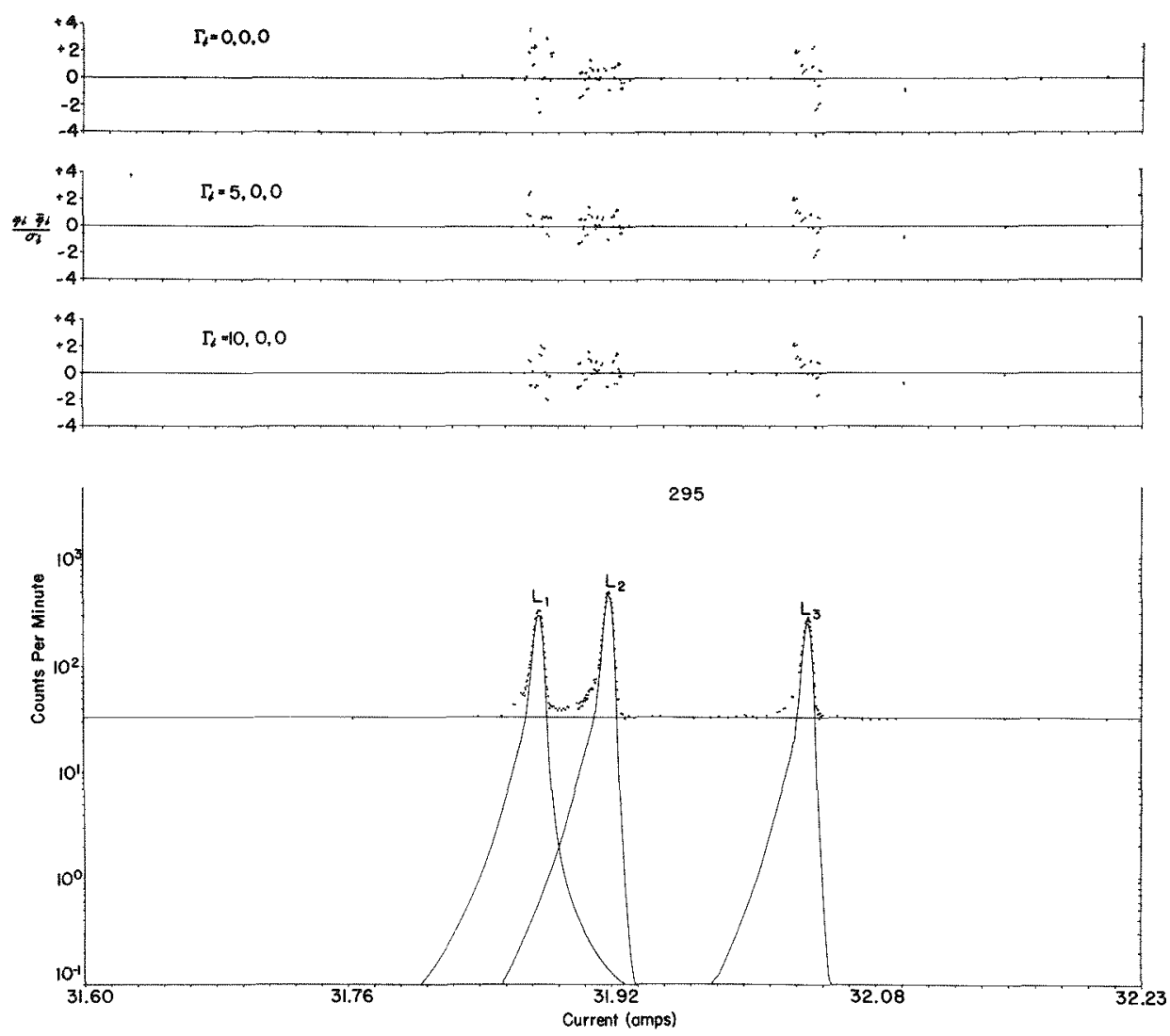

Figure 2 
[1] Graduate student, Utah State University, Logan, Utah.

[2] Present address: Department of Physics, Wisconsin State University, Stevens Point, Wisconsin.

[3] See for example, E. V. Condon and G. H. Shortley, The Theory of Atomic Spectra, p. 82.

[4] G. E. Nelson, W. John and B. G. Saunders, Phys. Rev. A2, 542 (1970).

[5] J.H. Scofield, Phys. Rev. 179, 9 (1969). 


\section{PLOTTING ROUTINE FOR GAMMA-RAY SPECTRA}

\section{S. D. McClure}

The plotting routine was written to handle the large volume of spectra that is processed on the $\mathrm{Ge}(\mathrm{Li}$ ) spectrometer systems. The logarithm of the counts in a channel is plotted versus the channel number (i.e., the points are equally spaced). A spectrum usually contains 4096 data points. The Calcomp Digital Plotter Package is utilized with output to the FR-80 microfilm system as well as the Calcomp Plotter.

The program has a variable plotting format which includes the following features: (1) a plot of a pulse-height spectrum can be split into as many as five sections, each displaced vertically from one another; (2) a spectrum can be split into equal length sections with each section displayed on a separate plot; (3) a specific portion from a spectrum can be extracted and plotted; (4) specific sections from different spectra can be extracted and displayed as one plot; (5) a spectrum can be corrected for channel capacity overflows prior to being plotted; (6) a spectrum or a section of a spectrum can be shifted vertically; (7) each axis can be independently scaled.

The gamma-ray spectra can be read from:

1. cards

2. analyzer magnetic tape - 18 binary bits per channel

3. analyzer magnetic tape - 24 binary bits per channel

4. analyzer magnetic tape - 36-bit binary coded decimal

5. library tape form.

Axes are drawn with 9 tick marks per decade on the log scale and every inch along the x-axis. One line of alphanumeric information can be printed above the $x$-axis for identification.

This program was used as a basis to set up a plot on the FR-80 microfilm system using the integrated graphics system. In this case, the output has three fixed format plots, 4096 channels plotted in one section, 4096 channels plotted 2000 channels/section across axis, and 1024 channels plotted in one section.

The program plots the spectrum on a semi-log scale, drawing a box around it to form the ordinate and abscissa. These are then labelled with the channel numbers and decade notations. A title block is printed in the upper right hand corner of the box which includes: (1) isotope name, (2) half life, (3) detector used, (4) absorber used, (5) source distance, (6) date. There are also grid options available: (1) no grid drawn, (2) 10 grid lines per decade, (3) 5 grid lines per decade.

Examples of the plotting routine are shown in Figures 1 and 2; the energy tagging was done by hand. 
This program provides a fast and convenient method of plotting for routine processing, plus flexibility to provide plots for any type of reports.

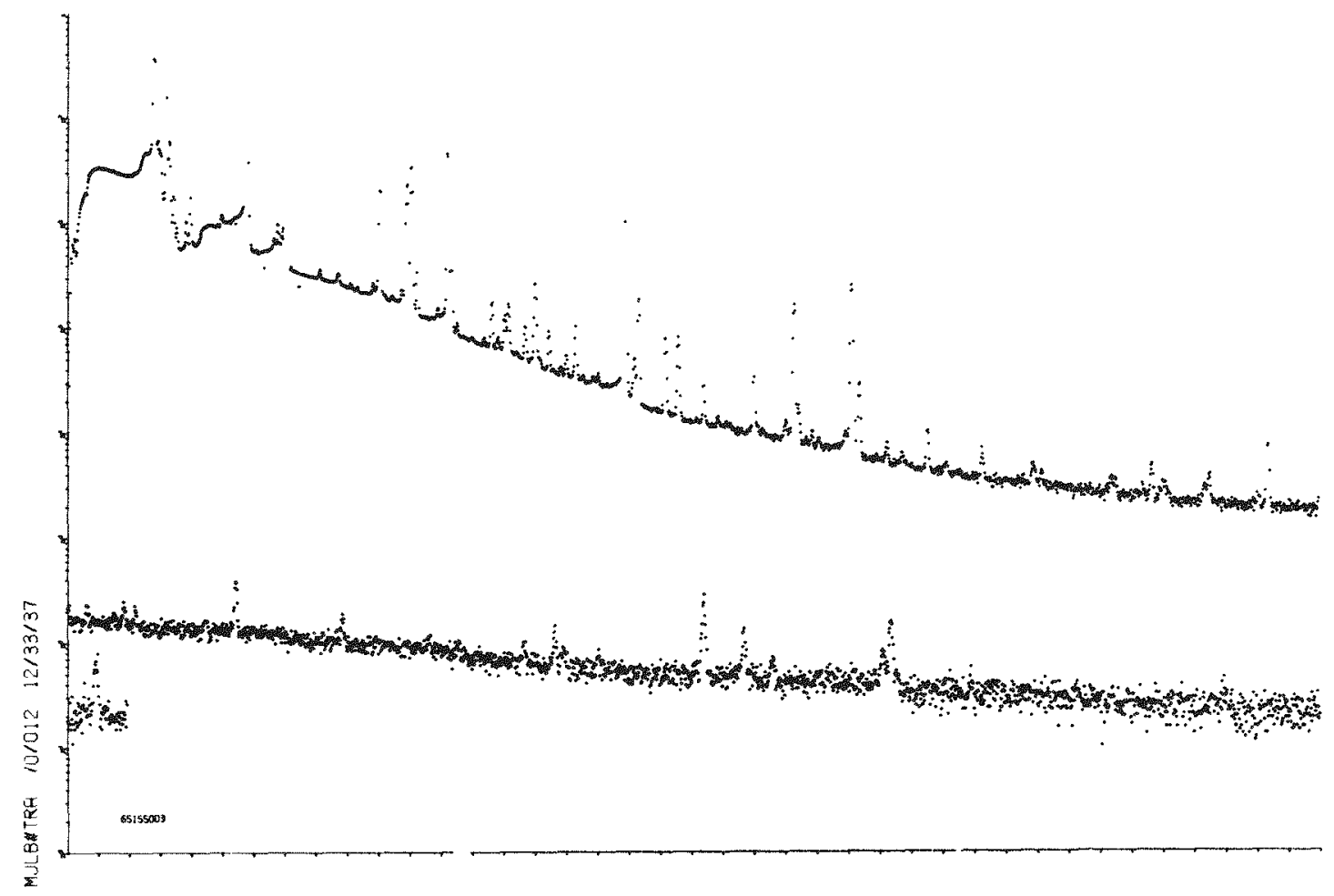

Fig. I Plotting routine for gamma-ray spectra.

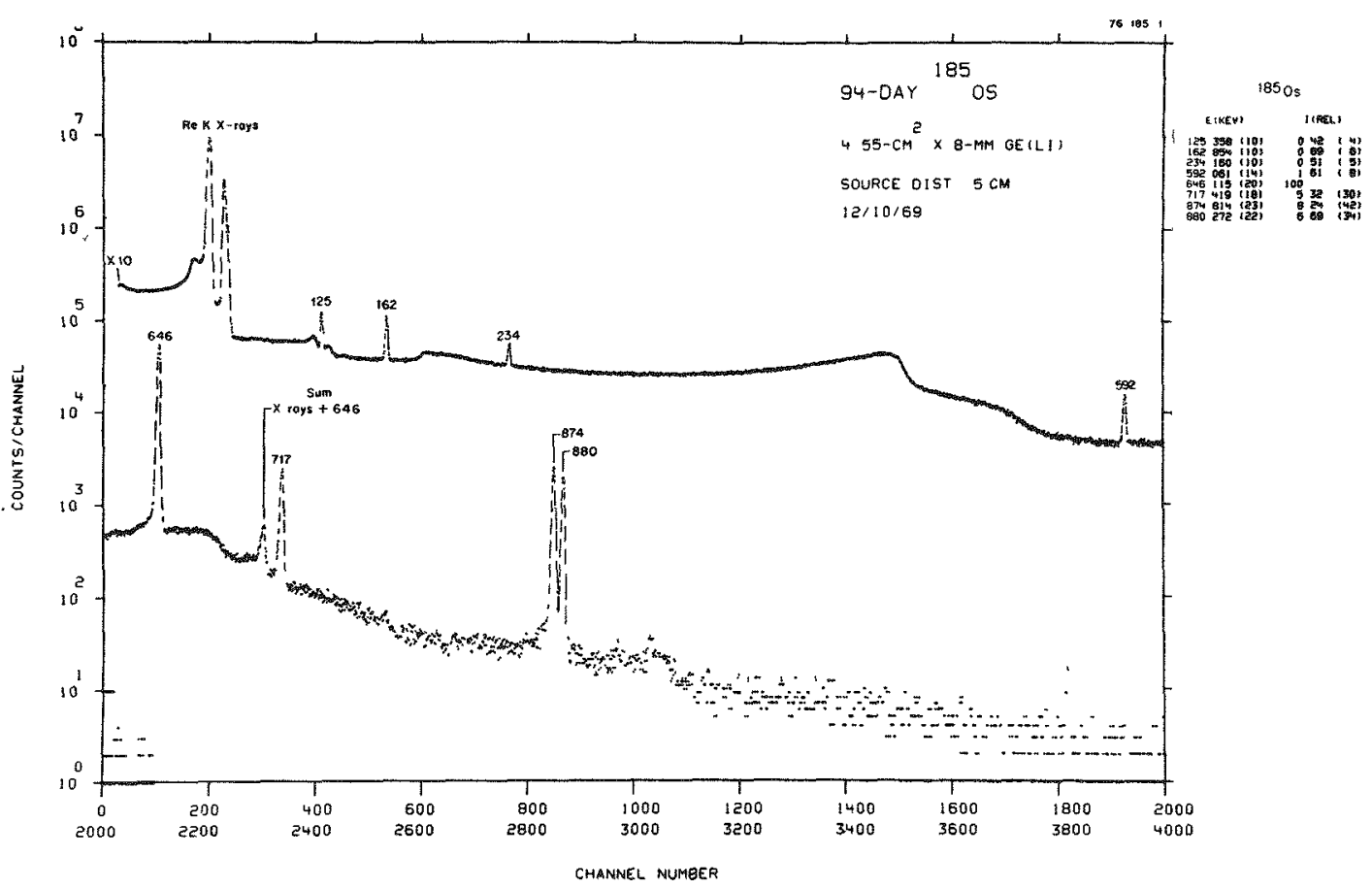

Fig. 2 Example of a gamma-ray spectrum processed using improved technique. 
R. G. Helmer, R. C. Greenwood, S. D. McClure

In the interpretation of nuclear decay-scheme data, it is frequently important to not only have the best possible values (consistent with the gamma-ray datal for the energies of the observed levels but also have realistic estimates for the uncertainties of these values. A computer program has been written to compute these "best" nuclear level energies and their associated uncertainties from a set of energies and uncertainties for gamma-ray transitions whose positions in a level scheme are known. This calculation is carried by means of a linear least-squares fit in the following manner:

Let the energy of level \#I be 0 . If $I_{j}$, are the level energies to be computed (with $L_{\mathcal{I}}=0$ ) and $E_{i j}$ is the energy of transition between the $i^{\text {th }}$ and $j^{\text {th }}$ levels, one minimizes the function:

$$
R^{2}=\sum_{\text {all } \gamma \text { rays }}\left(\frac{L_{j}-L_{j}+E_{i j}}{\sigma_{i j}}\right) .
$$

At the minimum, we set $\frac{\partial R^{2}}{\partial L_{k}}=0$, which gives $n$ equations in $n$

unknowns. The solution of this set of equations gives the level energies $L$ and the associated uncertainties.

As indicated in Figure 1 , the output of the program includes a comparison of the measured and computed level energy differences (i.e., $E_{i j}$ and $L_{j}-I_{i}$ ) as well as the computed level energies. 
N15 LEVELS

NO. LEVELS $=10$ NO. GAMMA RAYS = 19 ENERGY OF LEVEL 1 0.0

\begin{tabular}{|c|c|c|c|c|c|c|c|}
\hline \multirow{3}{*}{ LEVEL 1} & \multirow{3}{*}{\multicolumn{2}{|c|}{ LEVEL 2}} & \multicolumn{2}{|c|}{ GAMMA } & CALC. & \multirow{3}{*}{$\begin{array}{c}\text { DIFF } \\
-0.0690\end{array}$} & \multirow{3}{*}{$\begin{array}{c}\text { RATIO } \\
-0.6896\end{array}$} \\
\hline & & & ENERGY & ERROR & ENERGY & & \\
\hline & & & 5298.8000 & 0.1000 & 5298.8690 & & \\
\hline 1 & 4 & & 6323.4000 & 0.1000 & 6323.4936 & -0.0936 & -0.9362 \\
\hline 1 & 5 & & 5270.2000 & 0.1000 & 5270.0908 & 1092 & 920 \\
\hline 1 & 6 & & 7300.9000 & 0.2500 & 7300.9076 & -0.0076 & 0306 \\
\hline 1 & 7 & & 8322.7000 & 0.4000 & 8312.4483 & C. 2517 & 293 \\
\hline 1 & 8 & & $9152.8 n 00$ & 0.5000 & 9151.8284 & 0.9716 & 431 \\
\hline 2 & 5 & & -1884.9300 & 0.0600 & -1884.9464 & 0.0164 & 2733 \\
\hline 2 & 9 & & 1999.7900 & 0.1000 & 1999.8417 & -0.0517 & 5171 \\
\hline 2 & 10 & & 3678.2020 & 0.2000 & 3678.1753 & 0.0247 & 1233 \\
\hline 3 & 9 & & 3855.7000 & 0.2500 & 3858.0100 & -0.3100 & 2398 \\
\hline 3 & 10 & & 5534.3000 & 0.1500 & 5534.3436 & -0.0436 & 905 \\
\hline 4 & 9 & & 2831.4000 & 0.2000 & 2831.3853 & 0.0147 & .0735 \\
\hline 4 & 10 & & 4509.5000 & 0.1500 & 7189 & -0.2189 & -1.4594 \\
\hline 5 & 9 & & 3884.2000 & $0.500 \mathrm{c}$ & 3884.7881 & -0.5881 & -1.1762 \\
\hline 5 & 10 & & 5563.3000 & 0.1000 & 5563.1217 & 0.1783 & .7827 \\
\hline 6 & 10 & & $3532 \cdot 3000$ & 0.2000 & 3532.3049 & -0.0049 & -0.0244 \\
\hline 7 & 10 & & 2520.7800 & 0.1000 & 2520.7643 & 0.0157 & 0.1573 \\
\hline 8 & 10 & & 1681.5160 & 0.1800 & 1681.3841 & 0.1259 & 0.6995 \\
\hline 9 & 10 & & 1678.2900 & 0.0600 & 1678.3336 & -0.0436 & -0.7269 \\
\hline
\end{tabular}

CHISQ $=1.6436001 \quad$ NDIF $=10 \quad$ QUAL FIT $=1.6436000$

$\begin{array}{cclc}* & \text { ENERGY } & \text { LEVEL } & \text { RELATIVE } \\ & & & \\ \text { ERROR } & \\ 1 & 0.0 & 0.0 & 0.0 \\ 2 & 7155.0372 & 0.1131 & 7155.0372 \\ 3 & 5298.8590 & 0.1080 & 5298.8690 \\ 4 & 6323.4936 & 0.1067 & 6323.4936 \\ 5 & 5270.0908 & 0.1000 & 5270.0908 \\ 6 & 7300.9076 & 0.2097 & 7300.9076 \\ 7 & 8312.4483 & 0.1573 & 8312.4483 \\ 8 & 9151.8284 & 0.2353 & 9151.8284 \\ 9 & 9154.8789 & 0.1108 & 9154.8789 \\ 10 & 10833.2125 & 0.1024 & 10833.2125\end{array}$

Figure 1 Example of the output obtained from the "Best" Level Energy Program; for an adjustment of the ${ }^{15} \mathrm{~N}$ levels. 


\section{AUTOMATED CROSS SECTION AINALYSIS PROGRAM (ACSAP)}

N. H. Marshall, J.W. Codding, O. D. Simpson, J. R. Smith, R. C. Young

In recent years advances in experimental equipment and techniques have produced a flood of neutron cross section data. This is particularly true in the resolved resonance region, which is being extended to ever higher energies by the improving resolution of neutron sources. A need to develop data analysis techniques capable of dealing with this wealth of data has resulted.

For example, the resonance analyses of U-235 and Pu-239, described elsewhere in this report $(1,2)$ utilized amounts of data that would have been impossible to handle a few years ago. The efforts of Idaho Nuclear Corp. to deal with this analysis problem have been centered in the development of the Automated Cross Sections Analysis Program (ACSAP) $(3,4)$.

A flow chart of ACSAP is shown in Figure 1. Much as in the classical shape fitting procedure, theoretical cross section data computed using initial guess parameters are first Doppler and resolution broadened, then compared with the input data. After adjusting the desired parameters, the program reiterates a specified number of times before producing an output listing and plot of the results.

During the past year the flexibility and utility of ACSAP have been vastly increased. Algorithms have been modified to speed convergence to a valid set of parameters. The program will now use data from magnetic disc storage as well as data read in from cards. The shape of the resolution function may be changed at will. A subroutine MERGE has been added, to give the capability for simultaneous fitting of different cross sections. A subroutine AREA is being written to add area analysis capability.

The key to the increased flexibility of ACSAP, both in the operational use of the program and its adaptability for adding new features, lies in its revised format. The whole program has been restructured to put it in modular form. The many operations of ACSAP, such as calculating cross sections, fitting data, and plotting both experimental and calculated data in a great variety of combinations can now be selected at will by the evaluator, and performed in the order he desires. The ACSAP functions are essentially independent of each other, the only requirement being that all essential data be entered before a selected function is requested. Each function is selected. by means of a function card which contains a keyword identifying the operation it represents. A stipulated function will be completed before the next function card is read. This allows the evaluator to designate the order and frequency with which ACSAP will perform its various operations.

An example of the arrangement of ACSAP function cards for a short run is shown in Figure 2. The TITLE card enters titling information, which will appear at the top of each output page and on the plot. The ISOTOPE card enters isotope information such as atomic weight, statistical weighting factor, etc. The PARAMETER cards enter the initial guess resonance parameters. There is one PARAMETER card for each resonance. The 
CONDITIONS card enters experimental conditions data, e.g., sample temperature, flight path, burst width. The IIST card will produce an output listing of theoretical cross sections computed using the current values of isotope and parameter information. The evaluator may specify the desired energy range and his choice of the single-Ievel Breit-wigner or the multilevel Reich-Moore formula. He may list (or plot) total, absorption, capture, fission, and/or scattering cross sections. He may also stipulate alpha or eta if he wishes. The AXIS card causes a plot axis to be produced on either a Calcomp plotter or on microfilm. The PLOT card describes the information to be plotted. Several sets of data may be drawn on the same axis by entering additional plot cards. To determine resonance parameters, ACSAP requires FIT cards and an ADJUST card. The FIT cards describe the experimental points to be fit. There is one such card per resonance. The ADJUST card stipulates the number of iterations desired, the cross section to be fit, and the formula to be used. A summary of function cards currently used appears in Table 1.

The expanded capabilities of ACSAP were utilized fully in the resonance analyses of U-235 and Pu-239 to produce new resonance parameters for inclusion in ENDF/B, Version III. These analyses are described in other sections of this report $(1,2)$. The figures displaying the data and fits in these other sections were reproduced directly from ACSAP output plots. They represent a small sample of the plotting versatility available from this program.

The flexibility of ACSAP, its ease of use, and its parameter adjustment algorithms make ACSAP a powerful tool for the neutron cross section evaluator.

[1] J. R. Smith and R. C. Young, "Evaluation of the U-235 Cross Sections in the Resonance Region for the ENDF/B, Version III," this report.

[2] O. D. Simpson and F. B. Simpson, "Evaluation of the Pu-239 Cross Sections in the Resonance Region for the ENDF/B Version III," this report.

[3] O. D. Simpson, N. H. Marshall, C. L. Dunford, "Automatic Analysis of Cross Section Data Using Interactive Graphics as an Aid." Presented at the Conference on Computer Systems in Experimental Nuclear Physics, Skytop, Pennsylvania, March 1969.

[4] N. H. Marshall, O. D. Simpson, C. L. Dunford, "An Automatic Cross Section Analysis Program for Obtaining Neutron Cross Section Resonance Parameters." Presented at the AEC Semiannual Computer Information Meeting, Idaho Falls, Idaho, October 1969. 
Table 1

Summary of ACSAP Function Cards

Keyword

ADJUST

AXIS

BIAS

CLEAR

COMMENTS

CONDITIONS

DATA

DIFFER

FIT

HOLDUP

ISOTOPE

LIST

MERGE

PARAMETER

PLOT

PUNCH

RESOIUTION

TIME

TITLE

VALLEY
Function Performed.

Causes resonance parameters to be adjusted for a designated number of iterations

Draws plot axis on Calcomp plotter or on microfilm

Enters various kinds of bias information, e.g., $\sigma / \mathrm{V}, \sigma$

Reinitializes ACSAP for a new run

Allows comments to be printed on output

Enters data on how experimental cross sections were measured

Allows experimental data to be entered

May be used to enter difference data (experimental minus theory) or to create new difference tables

Used to enter experimental data points to be fit

Allows correction to the resolution function for moderator holdup time

Used to enter isotope information (such as atomic weight)

Produces listing of theoretical cross sections

Combines parameters from fits to different partial cross sections into a consistent set.

Used to enter resonance parameter information

Produces plot of either experimental or theoretical data (or both) on Calcomp plotter or on microfilm

Used to output theoretical data on cards, tape, or disk

Permits new resolution shapes to be entered

Prints elapsed time in seconds

Titling information appears on all printed and plotted output

Used to enter points to be fit in valleys between resonances 504 


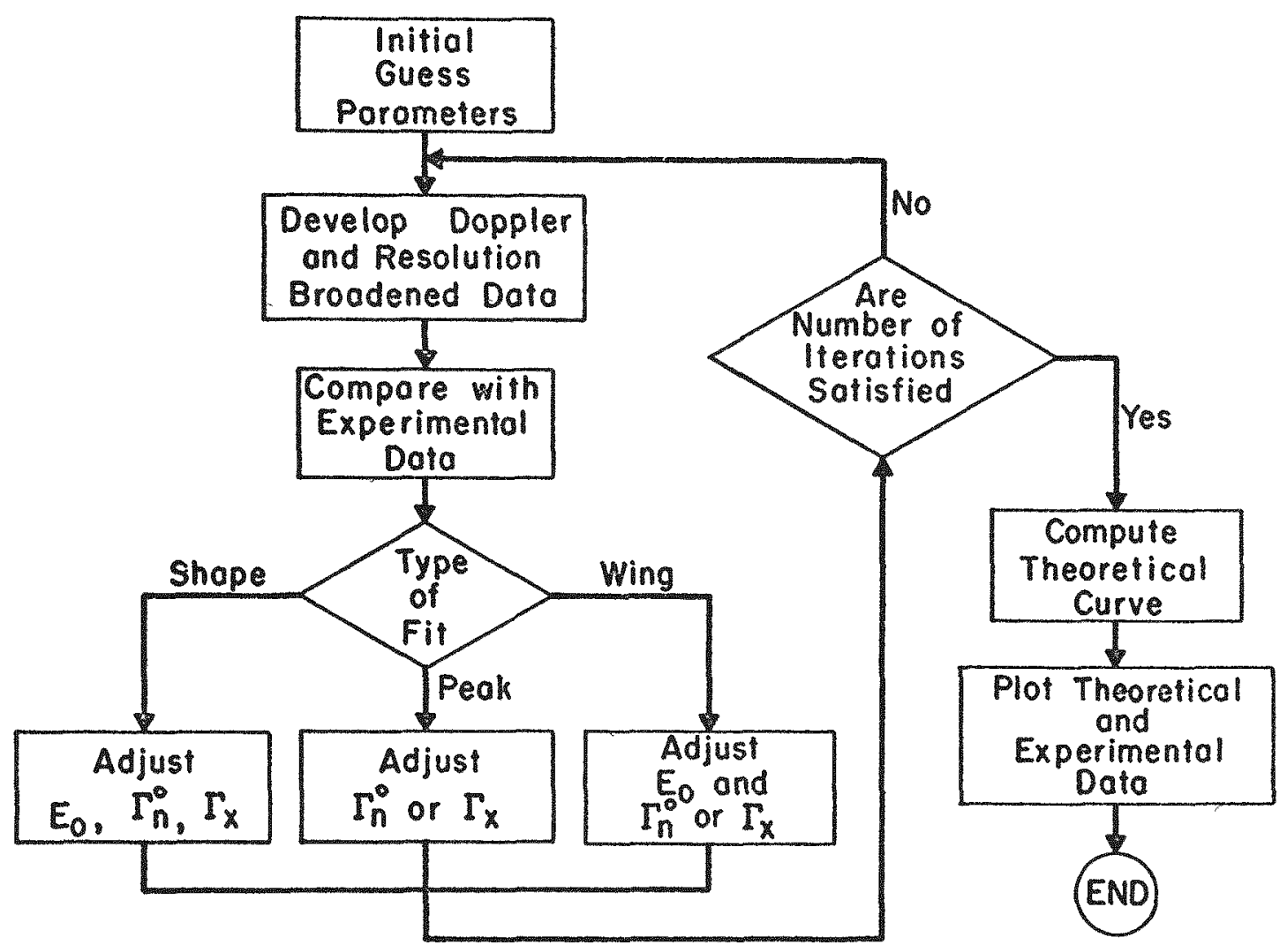

Fig. I Much like the classical shape fitting, theoretical cross section data computer using initial guess parameters are first Doppler and resolution broadened, then compared with the input data. After adjusting the desired parameters, the program reiterates a specified number of times before producing an output listing and plot of the results. 


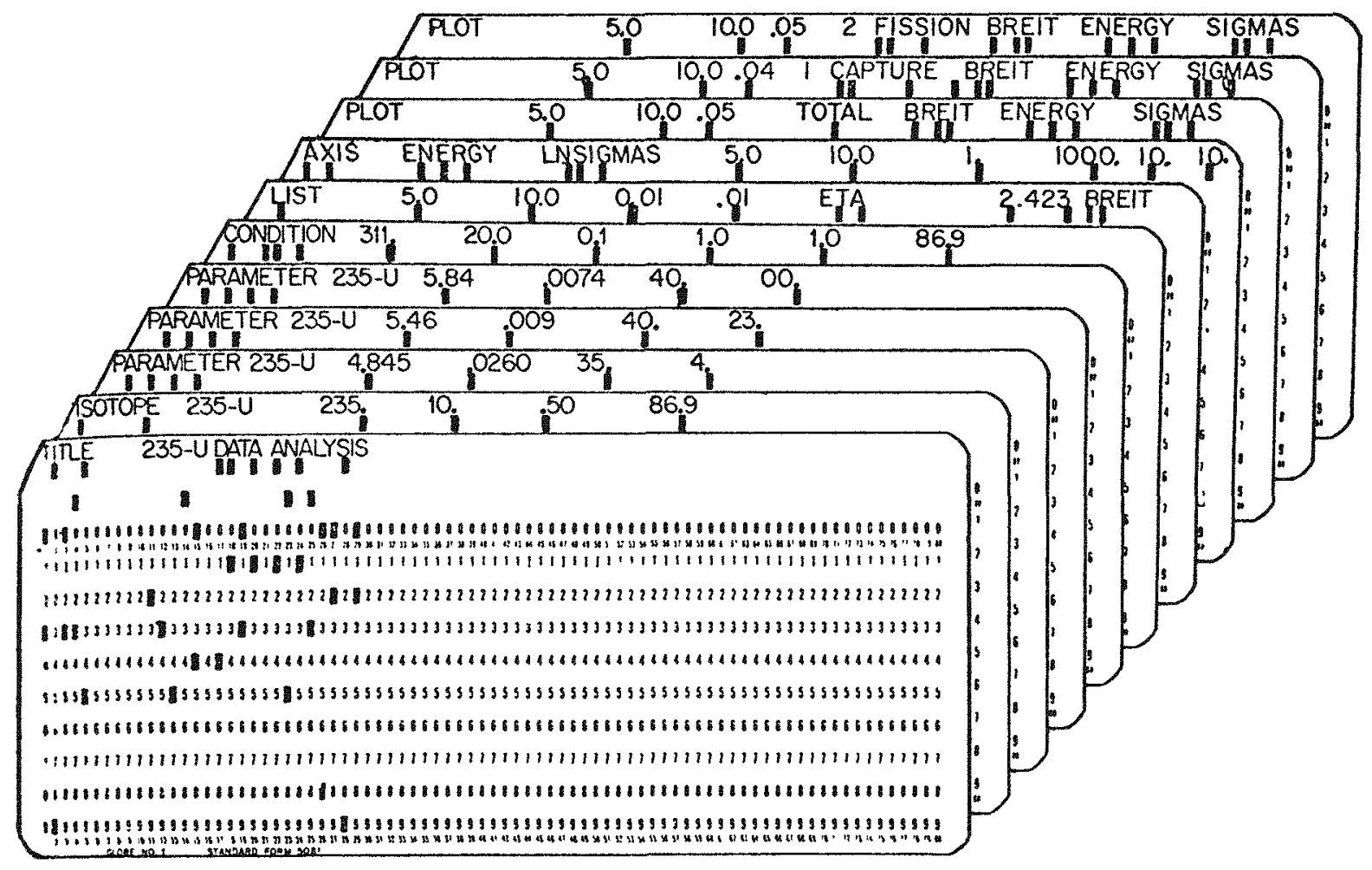

Fig. 2 Input function cards for a short ACSAP run. Key words at the first of each card provide programmability. 
SPECTRUM CALCULATIONS FOR THE CFRMF

D. A. Millsap

Computer codes, in this case ETOP* and PHROG[2], are used to calculate the flux spectra in the CFRMF. Frequent changes are made in these processing codes. ETOP processes the ENDF/B cross section information to provide a 68-group library for PHROG; and PHROG provides spectrum averaged broad group cross sections suitable for various multi-group transport or diffusion theory reactor codes. Generally, the changes that have been made in both of these codes, in the ENDF/B library, or in the calculational model, have yielded only minor changes in the calculated spectrum of the CFRMF. In the past year, however, two modifications in the overall calculational "chain" have given spectrum results worthy of comment.

Figure I gives the calculated real flux spectra referred to in the preceding paragraph. The 26-group calculation No. 8-31-70 is a typical 26-group calculated spectrum and is used here as a reference spectrum. It is essentially identical to that reported previously[3]. The 44group spectrum No. $12-9-70$ is a spectrum obtained subsequent to modifications[4] in ETOP and PHROG which changed the group averaged unresolved capture cross sections by 5 to 10 percent. There appears to be no major influence on the spectrum (this observation is somewhat subjective in view of the concurrent change in group structure); however, as a result of the change in group structure one does see a slightly different location for the flux peak. The 26-group calculation gives a peak in the group which covers the energy range $302 \mathrm{keV}$ to $498 \mathrm{keV}$. The 44-group calculation peaks in the group covering $498 \mathrm{keV}$ to $639 \mathrm{keV}$. Though of passing interest, one would expect to see little chenge in calculated activations or reactivities resulting from the change in group structure.

The 44-group spectrum No. 2-18-7I is of significantly greater interest. Major changes[4] were made in the mesh scheme in ETOP for the resolved resonance range. These changes resulted in higher diffusion constants in the resonance range $(52.5 \mathrm{keV}$ or less). The effect on the calculated spectrum is noticeable below $5.53 \mathrm{keV}$ and is increased $2-3$ times the previous level through the energy range $0.454 \mathrm{keV}$ to $3.36 \mathrm{keV}$. Such changes in calculated spectrum are sufficient to give significantly greater calculated activation integrals for certain relatively high absorbing materials such as gold and $235 \mathrm{U}$. It will also effect reactivity calculations for these materials. This is an important result since it brings the calculated spectrum more in line with the composite spectrum of Pearson[5] and would undoubtedly lead to improvement in the consistency checks performed by Harker[6].

There are additional changes in the cross section codes now in progress which could have an important effect on the flux in this same energy range, roughly $0.5 \mathrm{keV}$ and $5 \mathrm{keV}$. PHROG is being changed to

*ETOP is a modified version of ETOG-I[I]. 
allow resonance self-shielding of scattering cross sections. This will have the effect of reducing the scattering cross sections, therefore, increasing diffusion constants. This in turn would tend to increase the flux still further in the resonance range, though the magnitude of such an increase is, of course, unpredictable. Any change in flux level will. see a corresponding change in calculated activation integrals and reactivities of resonance absorbers.

[1] D. E. Kusner et al., ETOG-], A FORTRAN IV Program to Process Data From the ENDF/B File to the MUFT, GAM and ANISIN Formats, WCAP-3845-1, ENDF 144 (December I969).

[2] R. L. Curtis et al., PHROG - - A FORTRAN IV Program to Generate Fast Neutron Spectra and Average Multi Group Constants, IN-1435 (Apriı 1971).

[3] D. A. Millsap, "Reactor Analysis of the CRRMF", Nuclear Technology Branch Annual Progress Report for Period Ending June 30, 1970, IN-1407 (December 1970) pp. 286-288.

[4] G. L. Singer, "Excerpts from the ETOP Log Book", private communication, Idaho Nuclear Corporation.

[5] D. A. Pearson, "CFRMF Spectrum Determinations", this report.

[6] Y. D. Harker, "Consistency Checks on Evaluated CFRMF Spectra", this report. 


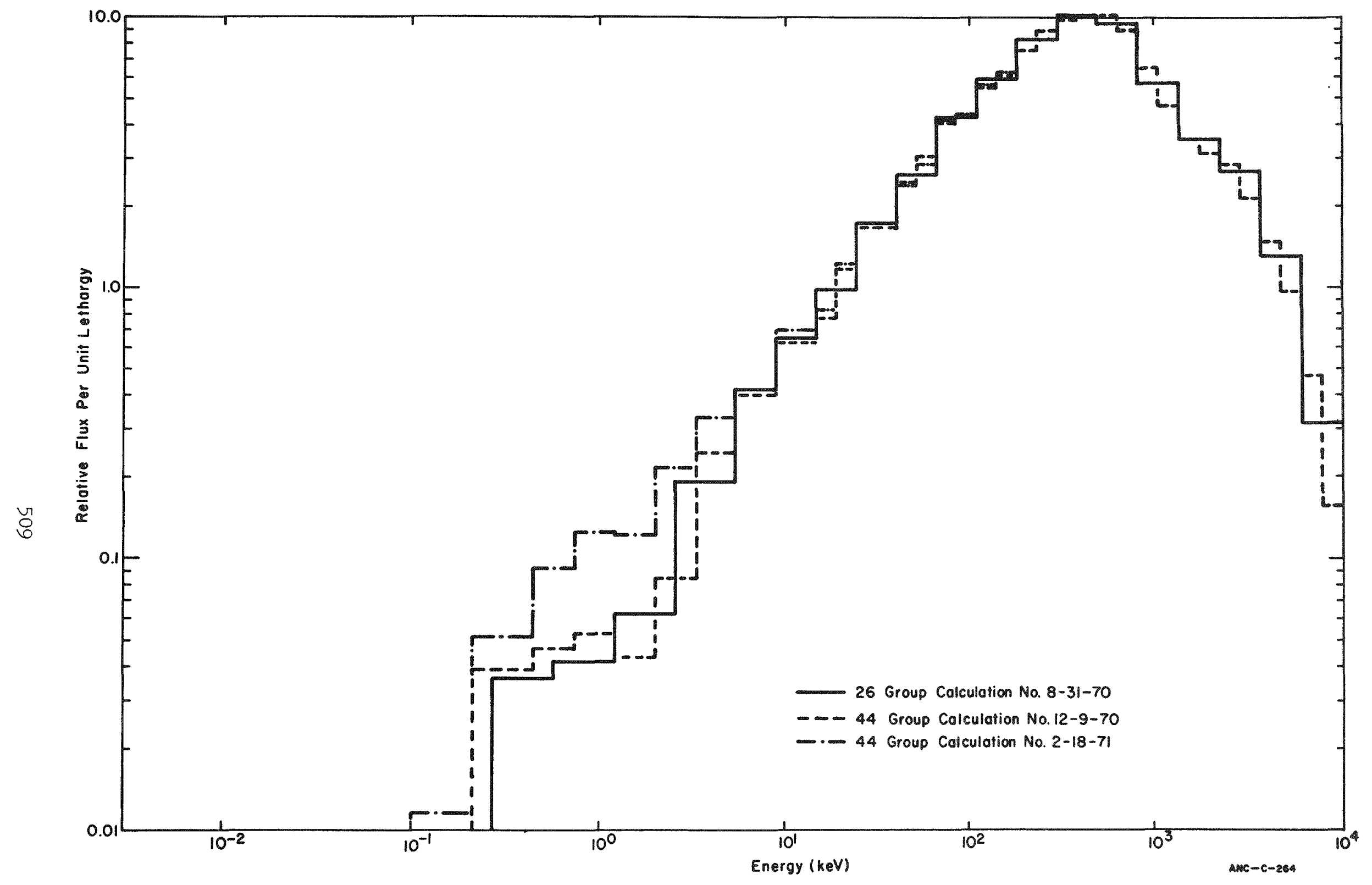

Figure 1. CFRMF - various calculations of the real flux spectrum: 
Comparisons of calculated and measured reactivities of materials in critical assemblies provide a valuable check on calculational models and in particular the cross section sets used for the materials being studied. Several such comparisons have been made using perturbation theory techniques on samples placed in the fast spectra of the CFRMF. The materials for which data are presented were selected primarily because they were existing samples, most of which have been previously measured and calculated for the spectra of the ARMF-I and ARMF-II reactors.

The samples chosen for comparison were beryllium, carbon, magnesium, aluminum, molybdenum, gold, lead and bismuth. The measured and calculated reactivities are given with the sample weights in Table I. All measurements for the samples were made at the midplane of the CFRMF centerhole position. The reactivity calculations were made using a modified version of PERT[1], with real and adjoint fluxes obtained from a 26-group diffusion theory calculation by MONA[2]. Cross sections for all calculations were obtained from PHROG[3] and INCITE[4] and are derived from Version-II ENDF/B data except for lead and bismuth. The cross sections for these two materials are from pre-ENDF/B PHROG Iibrary tapes.

The agreement seen from Table I between measurement and calculation is considered good for all materials except magnesium and gold. The current ENDF/B magnesium absorption cross section set appears to be far too high, a situation noted in previous calculations[5] for this element using a pre-ENDF/B cross section set. The discrepancy for gold presents a more serious problem, particularly because gold has often been considered one of the basic standards. However, subsequent to the results presented here, new calculations of the CFRMF spectrum[6] indicate that the calculated flux is too low in the energy range $0.454 \mathrm{keV}$ to $3.36 \mathrm{keV}$. This may well account for some of the discrepancy in gold. New reactivity calculations for gold using later determinations of the real and adjoint fluxes will have to be made. The other materials with the possible exception of molybdenum should be little effected by changes in flux at this energy range since their major response is due to higher energy reactions such as inelastic scattering, $n-2 n$, or $n-\alpha$. 
[1] H. P. Flatt, PERT - - A Perturbation Theory Code, AI-Memo-64-102 (May 1964).

[2] G. E. Putnam, MoNA, A Multi Group One-Dimensiona] Neutronics Analysis code, (to be published).

[3] R. L. Curtis, et al., PHROG - - A FORTRAN IV Program to Generate Fast Neutron Spectra and Average Multigroup Constants, IN-1435 (Apri1 1971).

[4] R. L. Curtis and R. A. Grimesey, INCITE - - A FORTRAN IV Program to Generate Thermal Neutron Spectra and Multigroup Constants Using Arbitrary Scattering Kernals, IN-1062 (November 1967).

[5] D. A. Millsap, Analysis of ARMF Measurements by One-Dimensional Multigroup Diffusion Calculations, IN-1067 (July 1968) pp. 31-32.

[6] D. A. Millsap, "Spectrum Calculations for the CFRMF", this report. 
TABLE 1

A Comparison of Calculated and Measured Reactivities

\begin{tabular}{|c|c|c|c|c|c|c|}
\hline & Weight & Measured & & Iculated Re & ity $(\mu \mathrm{k})$ & \\
\hline Sample & $(\mathrm{gm})$ & Reactivity $(\mu \mathrm{k})$ & Total & Absorption & Scatter & $n, 2 n$ \\
\hline Beryllium & 85.91 & -103.0 & -102.9 & -8.9 & -118.3 & +24.3 \\
\hline Carbon & 79.21 & -49.3 & -55.4 & -0.13 & -55.4 & -- \\
\hline Magnesium & 78.15 & -20.3 & -44.4 & -21.0 & -22.4 & -- \\
\hline Aluminum & 121.4 & -19.1 & -19.8 & -1.2 & -18.6 & -- \\
\hline Molybdenum & 228.0 & -35.3 & -33.2 & -12.2 & -21.0 & +0.03 \\
\hline Gold & 87.0 & -18.5 & -14.6 & -7.8 & -6.8 & --- \\
\hline Lead & 505.1 & -11.8 & -9.6 & -- & -9.6 & $\ldots$ \\
\hline Bismuth & 444.7 & -9.2 & -9.7 & $\ldots$ & -9.7 & +0.04 \\
\hline
\end{tabular}


The Coupled Fast Reactivity Measurement Facility (CFRMF) is a critical facility designed to duplicate the flux spectra obtained in an LMFBR. This spectra is then used to measure integral cross sections needed in the IMFBR program. In the CFRMF, special sleeves can be individually removed thus giving at least a limited possibility of flux tailoring so as to create various spectra that can be used to deduce problems in various cross section sets. In order to examine some of the applications of such flux tailoring various calculations have been made for the four possible CFRMF configurations: (1) as constructed (2) with ${ }^{10} \mathrm{~B}$ sleeve only, (3) with ${ }^{235} \mathrm{U}$ sleeve only, (4) with both sleeves removed. In addition to 26 -group real and adjoint fluxes, the reactivities of a few matexials were calculated. Cross sections were obtained using the PHROG[I] and INCITE[2] codes. The one-dimensionaI diffusion theory code MONA[3] was used to calculate the real and adjoint fluxes, and the code PERT[4] was used for the reactivity calculations. Version-II ENDF/B cross sections were used for all calculations except for the reactivity calculations for lead and bismuth. For these materials, cross sections from an earlier PHROG library were used.

Thus far the CFRMF has been operated only in the configuration of its original construction. This consists of a 6 in. $x 6$ in. $x 24$ in. block of depleted uranium $(0.193 \% 235 \mathrm{U})$ surrounded by a $1 / 4$ inch layer of boral. The central measurement hole is surrounded by a stainless steel dry tube, an inner sleeve of enriched uranium (the "235U sleeve"), and an outer sleeve of boron (the " $10_{\mathrm{B}}$ sleeve", $90.7 \% 10_{\mathrm{B}}$ ). The objective of the ${ }^{10} \mathrm{~B}$ sleeve was to remove any remaining thermal neutrons which might penetrate the uranium block and to remove or smooth out the influence of resonance energy "windows". The primary objective of the ${ }^{235} \mathrm{U}$ sleeve was to flatten the adjoint flux.

The calculated central real and adjoint fluxes for the four configurations are presented in Figures 1 - 4. Figures 1 and 2 show that the ${ }^{10_{B}}$ sleeve depresses the real flux almost over the entire energy range (relative to Figure 4) and becomes particularly effective below about $10 \mathrm{keV}$. Figures 1 and 3 show that the $235 \mathrm{U}$ sleeve enhances the adjoint flux with a general tendency to flatten that flux. In its position closest to the sample it completely nullifies the otherwise powerful. effect of the ${ }^{10_{B}}$ sleeve on the adjoint flux.

Table 1 gives the calculatedreactivities of a few samples in the four configurations. With only the $10_{\mathrm{B}}$ sleeve present the sensitivity to absorption is lowest while the sensitivity to scattering events is highest. With only the ${ }^{235} \mathrm{U}$ sleeve in place the sensitivity to absorption is at its highest while sensitivity to scattter is lowest. Unfortunately, the relatively higher sensitivity to absorption occurs primarily due to an increase in low energy $(<100 \mathrm{eV})$ absorption. In the particular case of gold $-11.2 \mu \mathrm{k}$ of the total of $-18.8 \mu \mathrm{k}$ absorption reactivity ("235 $\mathrm{U}$ sleeve only" configuration) is due to the thermal contribution. 
OveraII, the CFRMF "as constructed" is probably the best configuration for most reactivity measurements. Supplementary readings in the "10 $\mathrm{B}$ sleeve only" configuration might be useful for certain materials. Each material would have to be evaluated on an individual basis. Neither of the configurations with the $10_{B}$ sleeve removed would be of interest unless some other sleeve was inserted in place of the ${ }^{1} 0_{B}$ to remove the thermal neutrons.

TABLE 1

Calculated Reactivities of Samples in the CFRMF - Various Configurations

\begin{tabular}{|c|c|c|c|c|c|c|c|c|}
\hline \multicolumn{5}{|c|}{ CFRMF as Constructed } & \multicolumn{4}{|c|}{ With $10_{B}$ Sleeve Only } \\
\hline & Total & $\mathrm{Abs}$. & Scatter & $n, 2 n$ & Total & Abs. & Scatter & $n, 2 n$ \\
\hline $\mathrm{Be}$ & -101.0 & -9.4 & -117.6 & +26.0 & -112.3 & -8.4 & -127.0 & +23.1 \\
\hline C & -55.3 & -0.14 & -55.2 & -- & -59.2 & -0.12 & -59.1 & - \\
\hline $\mathrm{Mg}$ & -43.5 & -20.3 & -23.2 & -- & -43.4 & -18.8 & -24.6 & - \\
\hline Al & -20.3 & -1.2 & -19.1 & -- & -20.6 & -1.1 & -19.5 & -- \\
\hline Au & -14.6 & -7.8 & -6.8 & -- & -13.7 & -7.0 & -6.7 & -- \\
\hline $\mathrm{Pb}$ & -10.0 & --1 & -10.0 & $-\infty-\infty$ & -9.5 & - & -9.5 & - \\
\hline \multirow[t]{2}{*}{$\mathrm{Bi}$} & -10.1 & -- & -10.1 & +0.04 & -9.6 & -- & -9.7 & +0.04 \\
\hline & \multicolumn{4}{|c|}{ With $235 \mathrm{U}$ SIeeve OnIy } & \multicolumn{4}{|c|}{ With Both Sleeves Removed } \\
\hline $\mathrm{Be}$ & -74.5 & -10.3 & -92.8 & +28.6 & -101.7 & -8.8 & -117.3 & +24.4 \\
\hline $\mathrm{C}$ & -44.3 & -0.14 & -44.1 & & -54.3 & -0.12 & -54.2 & -- \\
\hline $\mathrm{Mg}$ & -50.2 & -30.0 & -20.2 & - & -50.6 & -27.4 & -23.2 & -- \\
\hline A] & -17.5 & -1.5 & -16.0 & - & -18.7 & -1.4 & $-17 \cdot 3$ & -- \\
\hline $\mathrm{Au}$ & -24.6 & -18.8 & -5.8 & & -18.9 & -13.2 & -5.7 & -- \\
\hline $\mathrm{Pb}$ & -9.4 & -0.01 & -9.4 & -- & -8.9 & --- & -8.9 & -- \\
\hline $\mathrm{Bi}$ & -9.2 & $-\cdots$ & $-9 \cdot 3$ & 0.05 & -8.8 & $-\cdots$ & -8.8 & +0.05 \\
\hline
\end{tabular}

[I] R. I. Curtis, et al., PHROG - - A FORTRAN IV Program to Generate Fast Neutron Spectra and Average Multigroup Constants, IN-1435 (April 1971).

[2] R. I. Curtis and R. A. Grimesey, INCITE - - A FORTRAN IV Program to Generate Thermal Neutron Spectra and Multigroup Constants Using Arbitrary Scattering Kernals, IN-1062 (November 1967).

[3] G. E. Putnam, MONA, A Multigroup One-Dimensional Neutronics Analysis Code, (To be published).

[4] H. P. Flatt, PERT - - A Perturbation Theory Code, AI-Memo-64-102 (May 1964). 


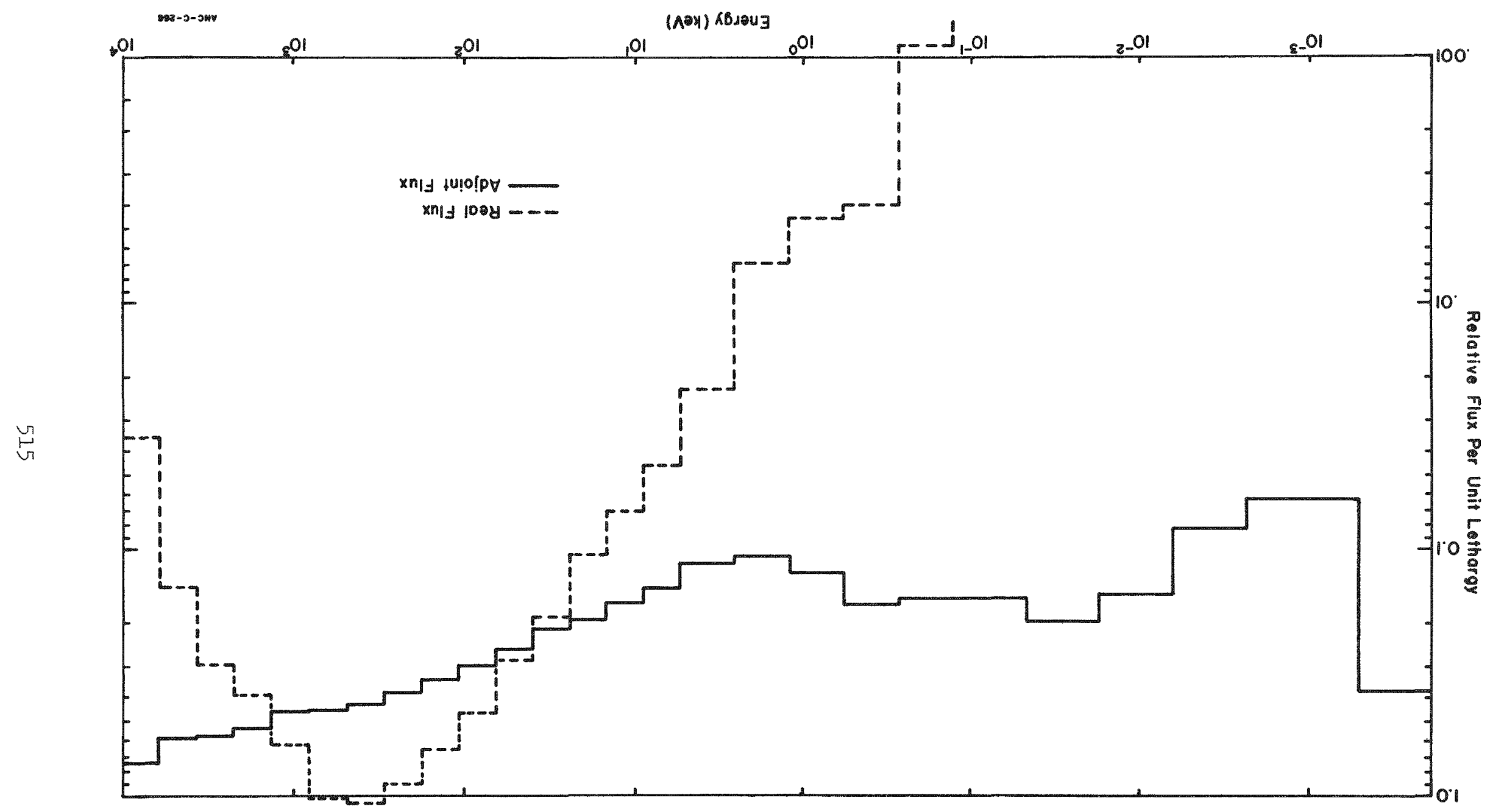

Figure 1. CFRMF as constructed. 


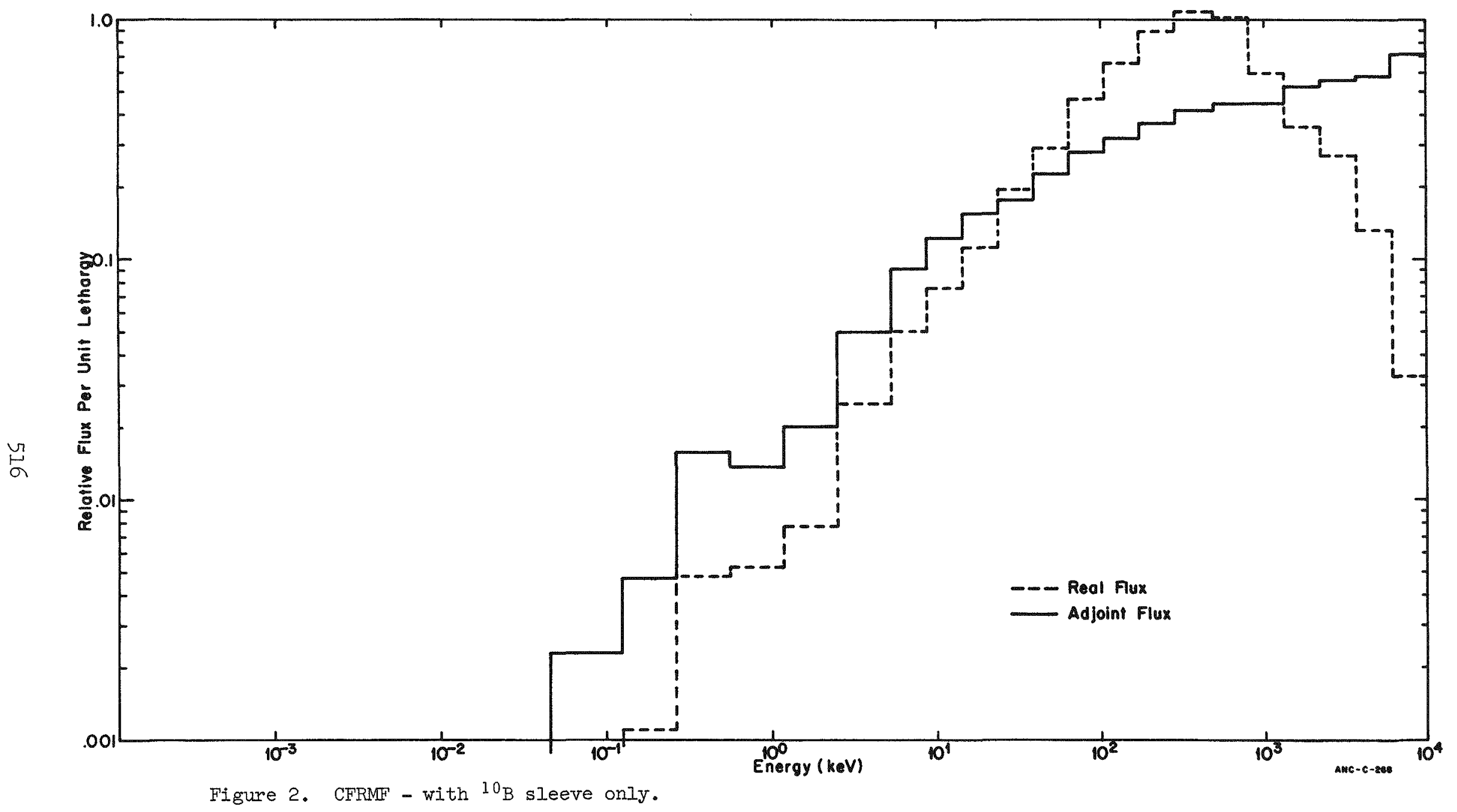




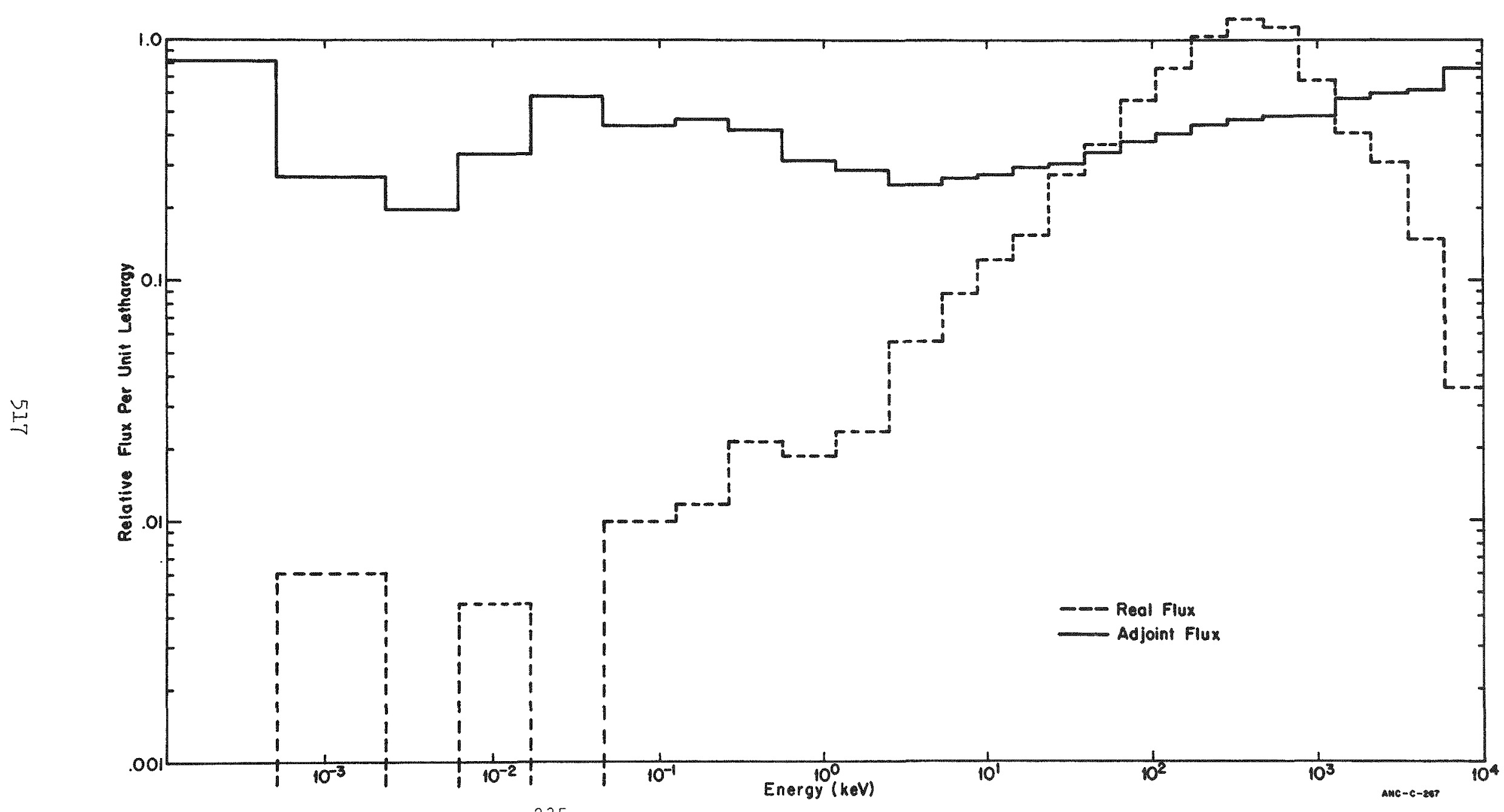

Figure 3. CFRMF - with $235 \mathrm{U}$ sleeve only. 


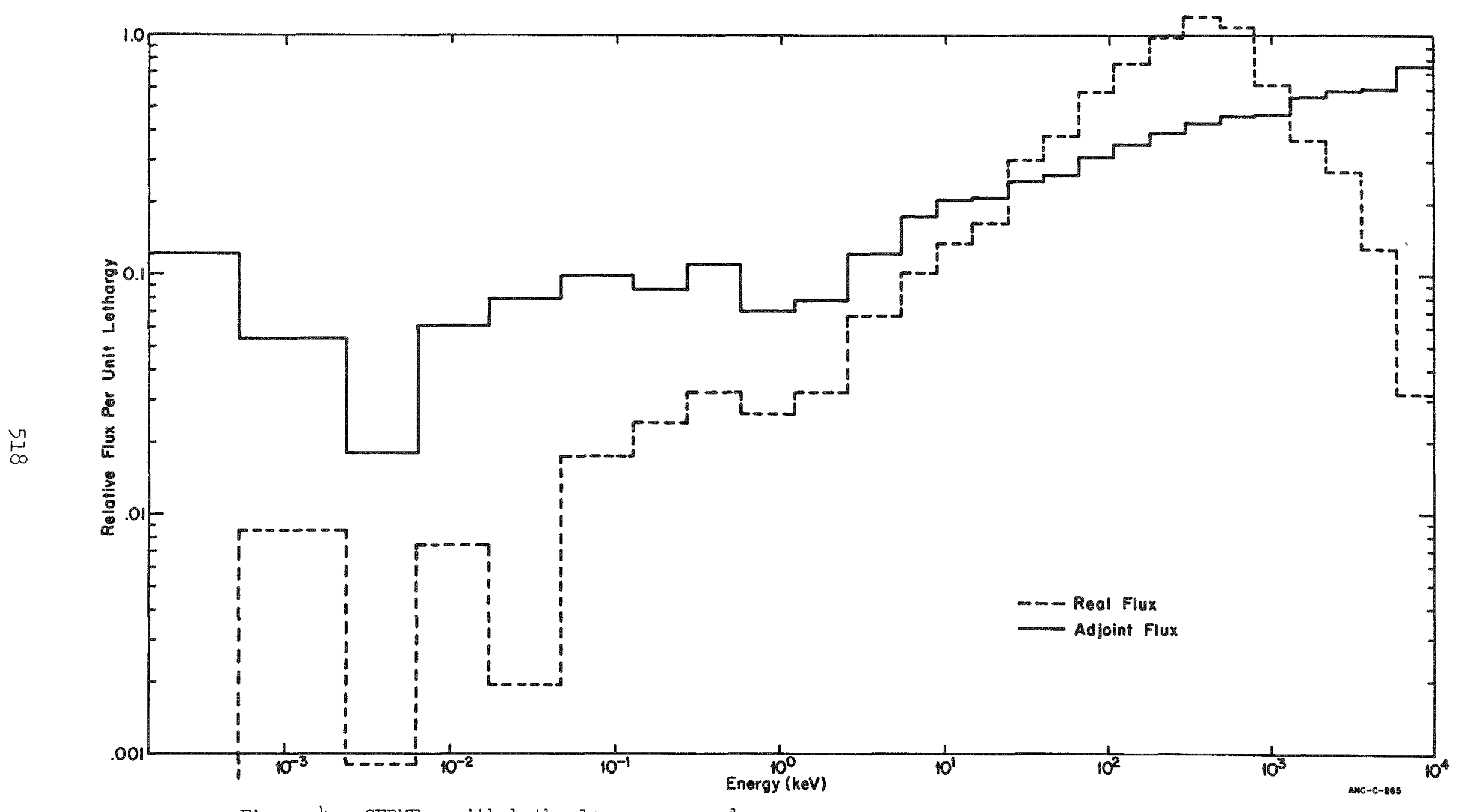

Figure 4. CFRMF - with both sleeves removed. 
General Version 1 of the snow gauge software was written for a snow gauge base station consisting of a $4 \mathrm{~K}$ PDP-8/I computer with ASR 33 teletype, internal clock, and output/input multiplexer; a Honeywell 512 plotter; and a Bell Telephone MODEM Model 103A or 103F. With this configuration the base station software will control one field station. By expanding the computer memory to $8 \mathrm{~K}$ words the base station will control up to 8 field units. A general description of the snow gauge is given in Reference [1].

The major functions now performed by the base station software include:

a. A Keyboard Monitor This monitor allows the base station operator to issue commands to the field unit via the teletype keyboard.

b. Density Calculations The program computes and profiles the density of a snow pack and computes moisture content in inches of water.

c. Plot Data The profile of a snow pack can be plotted on the Honeywell plotter as depth versus density.

d. Position Control The user has complete freedom in moving the detector and source to any position between the upper and lower limits of travel.

The current philosophy of the complete snow gauge system dictates that the base station be the master of all operations. The field unit initiates only one action without direction from the base station. This action is initiated in case communication between the two units is lost. The base station is required to issue a command (or interrogation) to the field unit at least once every 30 seconds. If the field unit does not receive a command within this time interval, it assumes communication has been lost and will perform the following operations: 1) move detector and source to its lower limit of travel, and 2) reduce power to standby.

The base station software automatically interrogates the field unit once every two seconds when the field unit has been powered up. No other base station commands are required to keep communications going between the field unit and base station. Once the field unit is powered up, all field unit components will remain on full power until the computer is stopped or until the field unit is told to power down.

Data in the field unit can be collected in two modes. In the first mode the output from a $200 \mathrm{cps}$ clock provides pulses to the lift motor and nuclear events are detected and stored in a 16-bit data register. Because data is collected for a fixed length of time (approximately 
5.1 seconds) this mode is called "fixed time mode". The interval of travel, either $1 / 2^{\prime \prime}$, $1^{\prime \prime}, 2^{\prime \prime}$ or $4^{\prime \prime}$ of the detector and source during this fixed length of time, is selected by the gating of a specific number of the total clock pulses, depending on the interval selected, to the lift motor. One eighth as many lift pulses are generated in the $1 / 2^{\prime \prime}$ interval as in the $4 "$ interval. When 1024 clock pulses have been generated the operation is terminated until a new command is given.

The other mode of data collection is the "fixed count mode". In this mode, the lift motor is fed pulses which are generated by the detection of nuclear events at the same time clock pulses are stored in the data register. After 1024 nuclear events have been detected, the operation is terminated. How far the detector and source travels while these 1024 events are being detected is selectable in the same manner as the fixed time mode.

Keyboard Monitor (KBM) The keyboard monitor is a teletype listening subroutine which allows the base station operator to control the field unit and alter the normal control sequence.

The computer signals the operator that it is in the keyboard monitor by typing the character "*". When this character is typed, the user can then type one of the 8 keyboard commands. These commands and their functions are explained under "Keyboard Commands". After the command has been typed, the program will branch out of the keyboard monitor and proceed to the subroutines which perform the required operations. At the completion of the operation, the keyboard monitor is re-entered and an "*" typed on the teletype.

A keyboard command cannot be issued by the operator unless the program is in the keyboard mode. Many of the operation subroutines require some form of input (either a unit number or a command string). The fact that a subroutine is waiting for input is indicated by the computer typing the character " $>$ ". This mode of operation is not the keyboard mode and no keyboard commands can be issued. The following example illustrates the differences between the two modes. In this example a keyboard command TIME was typed. The user then enters the correct time. In this and all following example, underlined entries were typed by the user. The "2" symbol indicates the depressing of the RETURIV key on the teletype. Comments are given after the back slash " $/$ ". These comments are for clarification of the example only and are not typed by the user.

\title{
EXAMPLE 1: KEYBOARJ COMMAID
}

\author{
* TIME L / In keyboard mode enter time subroutines \\ $>$ 0945) / Enter time \\ * / Subroutine finished-back to keyboard monitor.
}

Control can be returned from an operational subroutine to the keyboard monitor by the simultaneous operation of the CTRL and C keys on the teletype. Example 2 illustrates this function. The "^c" characters are typed by the computer to indicate a CTRI, C was typed by the operator. 


\section{EXAMPLE 2: RETURN TO KEYBOARU MONITOR}

* TIME

$>\uparrow C$

I No time was entered control passed back to KBM

* SNOW GAUGE VI

*

/ Ready for another keyboard command.

Keyboard Commands All of the keyboard commands which can be typed by the user when the program is in the keyboard mode of operation are explained in the following paragraphs:

A) $\quad \mathrm{PU}$ (Power Up) This command will cause power to be applied to all the electronics at the field station. It also initiates operation of the internal computer clock. From this point on the field unit will be interrogated at least once every 2 seconds.

When this command is typed the computer will respond by typing a ">". The routine is now waiting for the user to type a unit number. This number will be the single digit number corresponding to the identification code which is hardwired into the field unit.

Following is an example of the power up command for unit 1.

\section{EXAPLE 3: POWER UP}

$\begin{array}{ll}\text { * } \mathrm{PU}, & / \text { Power up } \\ >\text { > } 2 & / \text { Unit I } \\ * & \end{array}$

B) $\quad$ PD (Power Down) This command will move the detector and source to the bottom limit of its travel and reduce all power in the field unit electronics to standby.

The power down command operates in the same manner as power up.

\section{EXAMPLE 4: POWER DOWN}

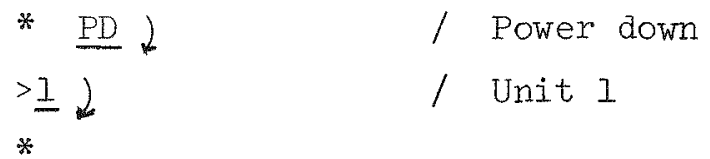

C) TIME (Set Time) This command is used to set the computer clock to the correct time of day. Example 1 illustrates how this command is used. All times are based on a 24-hour clock with 12 o'clock midnight at time 0000. Four digits must always be typed. The computer 
will then keep the correct time until it is either stopped or powered down. The clock must be reset when power is restored.

D) $B$ (Bottom Detector) This command is used to move the detector to the lower limit of its travel. The field unit is set into the continuous downward motion mode until the detector bottom flag is set. The field unit electronics is left in the "power on" state.

\section{EXANPL 5: BOTTOM LETECTOR}

$\begin{array}{ll}\text { * B 2. } & / \text { Move detector and source to lower limit } \\ & / \text { of travel } \\ & \text { * } 2\end{array}$

E) CAL (Calibrate High Voltage) This command is used to calibrate the high voltage so the window of the discriminator is directly over the photopeak of the source. Once initiated, the following operations are performed automatically by the software:

1. The high voltage is stepped down until a preset value is reached. This value is preset and can be changed at the aiscretion of the operator. This value must be small enough to insure that the photopeak is on the low side of the window discriminator.

2. Data is now taken in the fixed time mode and summed for three time intervals (approximately 15.3 seconds). The value thus obtained is compared with the previous value. The previous value will be zero for the first time through the 100p. If the new value is greater than the previous value, the high voltage is incremented by one and a new count accumulated. This process is repeated until two consecutive data points are less than its previous value. This insures that the window of the discriminator has been moved up and over the top of the photopeak. At this time, the high voltage is reduced two times which should place the window directly in the center of the photopeak. If for any reason the routines are unable to find the photopeak before the high voltage is incremented to its upper limit, the message DET UPPER LM will be typed and the calibration operation will be terminated.

3. When the high voltage has been set correctly, a calibration constant is computed. This constant is the average countrate over ten 5.1 second fixed time intervals, ie, counts are accumulated for ten fixed time intervals and the sum divided by 10 to obtain an average counting rate over one time interval. 
d. This calibration constant is then printed on the teletype together with the decimal value of the 5 most significant bits of the 7-bit voltage up-down counter.

It should be pointed out that the field unit hardware will not allow the high voltage to be changed unless the detector lower limit flag is set. For this reason the CAL routines will move the detector to the lower limit of its travel before any of the above steps are taken.

At the completion of a calibration run, that is, after the printing of the calibration constant, the operator is asked to enter a value for $\rho_{S}$ (density of standard absorber in snow equivalence). This value and the calibration constant is used to calculate snow density in the AUTO mode. Following is an example showing how the CAL conmand is used.

\section{EXAPLE 6: CALIBRATE DETECTOR}

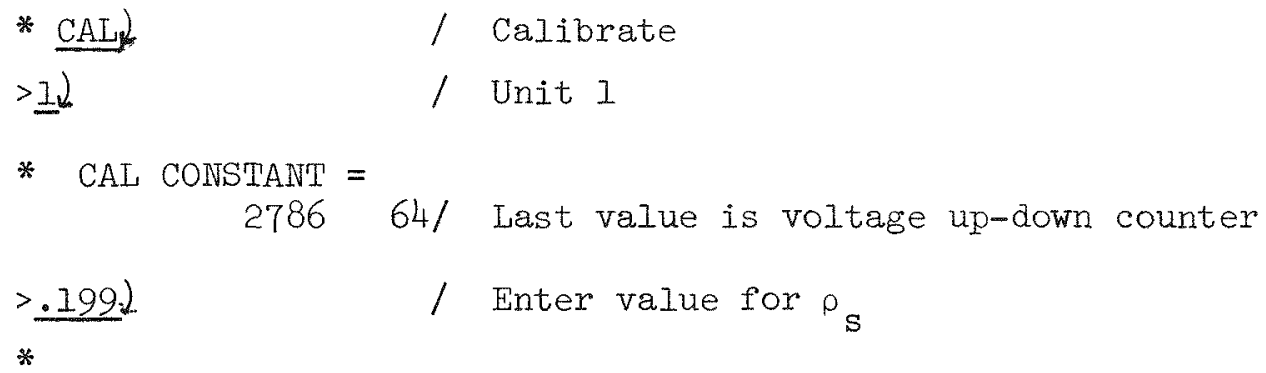

F) GT (Go To) The GT command allows the operator to move the detector and source to any height (within the upper and lower limits). The input to this command is in the form of a command string of two entries separated by a comma. The first entry is the unit number. The second is the height in inches from the lower limit of travel.

\section{EXAPPLE 7: MOVE JETECTOR}

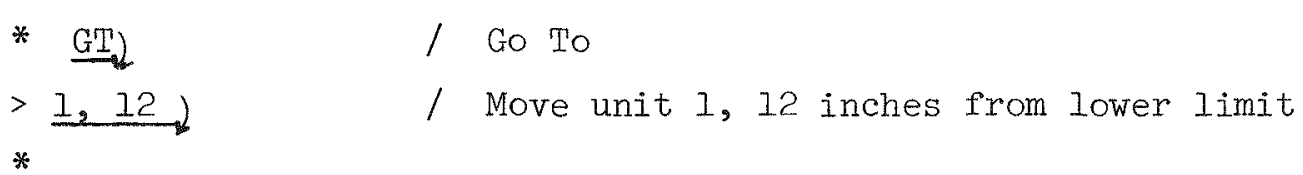

G) MANUAL (Manual) This mode of operation gives the user complete freedom in the selection of commands to be sent to the field. Input to this command is in the form of a command string of up to 7 fields, each field separated by commas. Each entry in a field determines how a command is consuructed. Once a command is constructed, it is sent to the unit. Upon completion of execution of the command the routine waits for another command string. A CTRL, C is typed to return control back to the keyboard monitor (see section on "Keyboard Monitor"). 
The following examples illustrate how the MANUAL command is used. In Example 8 (a) the MANUAI command was typed when the computer was in the keyboard monitor. Examples 8 (b) through 8 (d) indicate the construction of subsequent commands once the program is in the manual mode. The octal values of various field unit registers are printed when the field unit has completed command execution. These registers are as follows: unit register, command register, status register, position register and data register.

\section{EXAMPL 8(A): MANUAL OPERATION}

\begin{tabular}{|c|c|c|c|c|c|}
\hline * MA & & & & 1 & $\begin{array}{l}\text { Enter manual mode from keyboard } \\
\text { monitor }\end{array}$ \\
\hline$>I$ & $, T, N S$ & & & $/$ & $\begin{array}{l}\text { Take data, fixed yoltage, fixed } \\
\text { time, no motion slow }\end{array}$ \\
\hline$I$ & 200 & 0040 & 0000 & & 2632 \\
\hline
\end{tabular}

\section{EXAMPL 8(B): *}

$>I, N_{2}+, T_{2} N S_{2}$

/ No data, raise high voltage I step, fixed time, no motion

$/$ Waiting to construct another command

( $\neq$ NOTE: No data is printed, because no data was requested.)

\section{EXAMPL $8(\mathrm{c}): \neq$}

$$
>1, D, F, T, I, I, 3)
$$

$1200 \quad 0000 \quad 0001 \quad 2630$

I 200000000012700 / Data second increment
/ Take data, fixed voltage, fixed time going, from 1 inch to 3 inches in l-inch increments.

( $¥$ NOTE: In this example, data is typed at the end of each increment.)

\section{EXAMPLE 8(D)}

$>I, D, F, C, N F 2$

$1300 \quad 0000 \quad 0010 \quad 2803$

$>\uparrow C$

* SNOW GAUGE VI

*
/ Take data, fixed voltage, fixed count, no motion fast

Terminate manual operation return to KBM 
H. AUTO (Automatic Data) This mode of operation allows the user to take data in one of four incremental steps, compute density, equivalent inches of water and keep a running total of inches of

accumulated water. In addition, the density can be plotted in histogram form on the Honeywell plotter. The AUTO mode allows the user to enter up to 5 intervals. An interval is defined to be a contiguous area of the snow pack over which data is to be taken in fixed incremental step size. Data can be taken in either the fixed time mode or fixed count, but only one can be used at a time.

In the fixed time mode the following equation is solved to compute density $\rho$ :

$$
\rho=\rho_{S}-\frac{I}{K} \ln e\left(\frac{N(z)}{N_{S}}\right)
$$

where

$$
\begin{aligned}
\rho_{\mathrm{S}}= & \text { density of standard absorber in snow equivalence } \\
\mathrm{K}= & \text { absorption coefficient in } \mathrm{cm}^{3} / \mathrm{gm} \text { for pipe separation of the } \\
& \text { field unit } \\
\mathrm{N}(\mathrm{z})= & \text { counts obtained in the interval step ending at highest } \\
& \mathrm{z} \text { inches } \\
N_{S}= & \text { calibration constant (see CAL command). }
\end{aligned}
$$

For the fixed count mode the following equation is solved for $\rho$ :

$$
\rho=\rho_{S}+\frac{I}{K} \ln _{e}\left(\frac{T(z)}{T_{S}}\right)
$$

where

$$
\begin{aligned}
T(z) & =\text { number of clock pulses received in interval step } \\
T_{S} & =\frac{(1024)^{2}}{N_{S}} \text { where } N_{S} \text { is the calibration constant. }
\end{aligned}
$$

Equivalent inches of water is computed by solving:

$$
\Delta W_{i}=\Delta z_{i} \rho_{i}
$$

where

$$
\Delta W_{i}=\text { equivalent inches of water }
$$




$$
\begin{aligned}
\Delta z_{i} & =\text { step size in inches } \\
\rho_{i} & =\text { density for that step. }
\end{aligned}
$$

At the end of each interval, an average density is computed which is defined to be the total inches of water divided by total inches of movement.

Following is an example of how a run is set up in the AUTO mode:

\section{EXAPLE 9: ALTO MOLE}

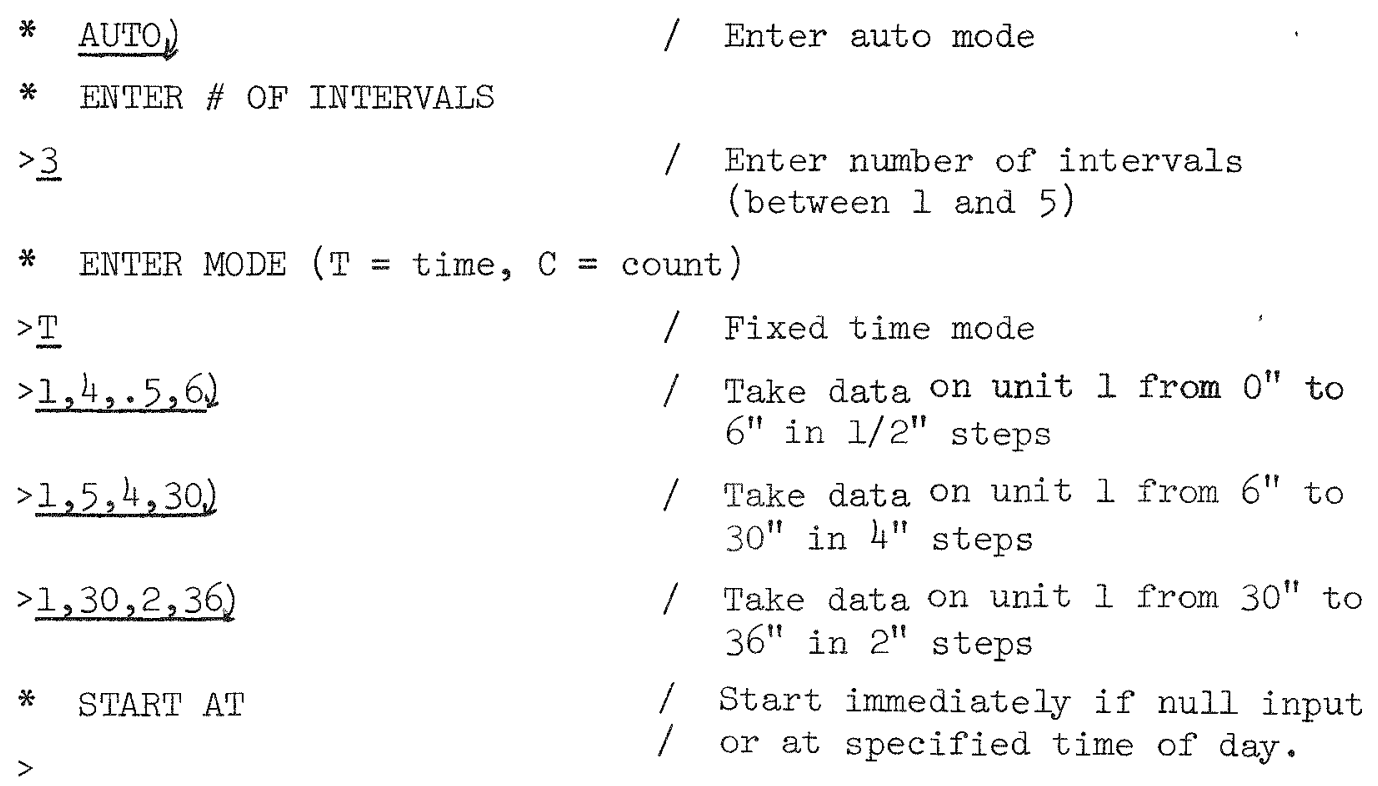

When the execution of a particular interval is begun, the source and detector is first moved to the beginning position and data is collected in the step-size increments until the ending position is reached. At the end of each step, the position, density, $\mathrm{H}_{2} \mathrm{O}$ equivalent and cumulative inches of $\mathrm{H}_{2} \mathrm{O}$ is printed on the teletype.

The power to plotter need not be on during a run in the AUTO mode. Everything will proceed normally if it is turned off except, of course, no plot will be made.

Error Messages and Command Errors Several error messages are output to the teletype when the computer detects an error either in the operation or in reception of data from the field unit.

The most common error message is the question mark "?". This message will be typed when a command is typed to the computer which it doesn't recognize. 
There are two error messages associated with an error in communication between the field unit and base station. The first being a detected error in the redundant transmission of data. This error is indicated by the message "ERROR I UNIT I" being typed by the computer. The message will be followed by 2 sets of data consisting of 3 groups of 4 octal digits. The first set of data was the data received for the first transmission; the second set is the data received for the second transmission. The data format is as follows: (1) 5 bits of voltage up-down counter plus 3 I.D. bits; (2) 8 bits of the command register; and (3) 6 bits of the status register plus 3 bits of position register.

A transmission error will terminate the current operation and return the program flow back to the keyboard monitor.

The second type of error which can be detected in communication is indicated by the computer typing the message "ERROR \#2". ERROR \#2 is considerably more serious than ERROR 1. This error probably indicates a malfunction in the field unit whereas ERROR I most generally indicates an error in movement of the data from the field unit to the base station.

ERROR \#2 is indicated when the computer tries 8 times unsuccessfully to set a command in the command register of the field unit. Every time a command is constructed in the computer and sent to the field unit, the field unit immediately sends back a redundant transmission with the new command in the command register. The computer checks the received command with the value which was sent; if there is a difference, the command is resent. This process is repeated for a total of 8 times. If they still differ at the end of the 8th try, the message ERROR \#2 is typed.

[1] R. A. Coates et al., "Telemetered Profiling Isotopic Snow Gauge", this report. 
COMPUTER RELIABILITY ASSURANCE

A. I. Connelly

The acquisition of precise data from experiments is necessary in order that meaningful information may be derived from it. The control of a set of variables in a process is necessary for the process to go to a desired end or to prevent the process from attaining undesired states with possible deleterious consequences. When digital computers are central to the data acquisition or process control functions it is desirable to have some method of assuring that the functions are properly performed without disturbing the experiment or process. With conventional systems having many indicators, charts and alarms erratic behavior can be noted with relative ease by operators familiar with the normal modes of operation and corrective action can then be taken. There is no need to wait for a final analysis of recorded data to receive indication of abnormality. In computer-based systems many important functions take place internal to the system without any indication to the external world, e.g., data may be recorded on magnetic or paper tape and thus not be readily accessible to human interpretation. Critical variables may be displayed by the system, but this does not provide the same degree of assurance of proper operation as a conventional array of indicators.

A digital computer system is a collection of independent, interacting subsystems. Even the central processing unit of most computers is such a collection. Thus in order to assure proper operation it is possible to at least partially check out one subsystem by using the others or by using the same subsystem in different ways. Indication of subsystem failure may be given to another subsystem, which may also be computer based, or to human operators for corrective action. Thus the overall system of computers, peripherals, and operators is more reliable thereby giving greater assurance that precise data will be recorded and processes properly controlled.

Algorithms for central processing unit self-checking and disk checking have been developed for the Digital Equipment Corporation (DEC) PDP-15. The algorithms operate properly on computers without malfunctions; however, the ability to detect malfunctions is open to question. The question can only be resolved by causing selective malfunctions while the programs are running. This has not been done because producing such failures is rather difficult due to the nature of the PDP-15 logic. The algorithms have been demonstrated to detect known machine malfunctions and reference manual (instruction description) errors. 


\section{NASA X-RAY FLUORESCENCE AIND DIFFRACTION SOFTWARE}

M. S. Cole $\mathrm{e}^{[1]}, \mathrm{C} \cdot$ W. Richardson [2]

Software has been developed for the NASA Manned Spacecraft Center Lunar Receiving Laboratory to control $X$-ray diffraction and X-ray fluorescence spectrometers interfaced to a PDP-8 computer. As well as controlling the spectrometers simultaneously, the data may be analyzed on-line or stored in a library for later analysis. The analysis routines compute corrected lattice spacings and peak intensities for the diffraction spectra, and provide a quantitative analysis of the fluorescence spectra for up to eight elements.

[1] Member of Computer Science Branch, Technical Services Division, Aerojet Nuclear Company.

[2] Present address: Bingham Mechanical and Metal Products Inc., Idaho Falls, Idaho. 
REAL-TIME DEBUGGING SOFTWARE FOR THE PDP-8

M. S. CoIe $[1], C \cdot W \cdot$ Richardson ${ }^{[2]}$

A program debug package has been developed for the PDP-8 which allows for insertion of break-points, restoring the break-point instruction, opening locations to change or examine them, beginning execution at a specified location, execution of a subroutine, and examining (and/or changing) a floating-point number. The debug routines may be used while the primary program is operating, allowing dynamic analysis of the system.

[1] Member of Computer Science Branch, Technical Services Division, Aerojet Nuclear Company.

[2] Present address: Bingham Mechanical and Metal Products, Inc., Idaho Falls, Idaho. 
USING FUNCTION BOX FOR A BACKUP CONTROL DEVICE

R. C. Davies [1]

A small keyboard, usually consisting of ten or twelve keys, interfaced to a computer system, and implemented through the computer software, is commonly referred to as a "function box". On a small computer system, this auxiliary keyboard is usually used for interactive control of displays (spectrum rotation, scale, etc.) or for simple interaction with a computer-controlled experiment. The application described here allows this "auxiliary" device to assume many of the functions of the primary alphanumeric keyboard, acting as a backup input medium. This procedure has been implemented on the gamma spectroscopy system in the NASA manned spacecraft center low-level counting facility, where it has been successfully used as an alternative to the teletype keyboard.

The function box consists of 12 keys which interrupt the computer processor when depressed. The numeric input capability of the function box, discussed here, together with the panel functions[2] allow experiments to be conducted without the use of the teletype. The box can also be used to control display functions[3].

The following diagram shows the key labeling on the box. The numeric keys are used to input digits, the "-" key is used to delimit numbers, and the "t" key is used to initialize the box.

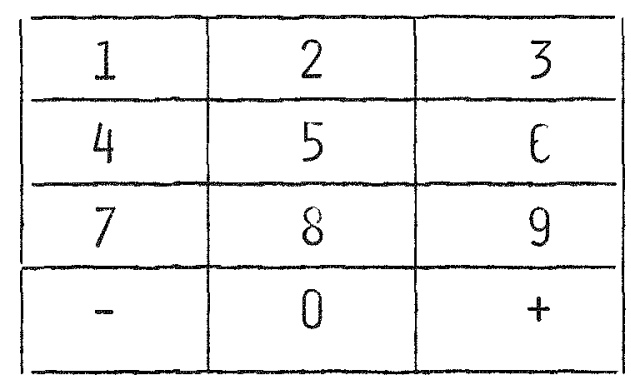

\section{FunCTION BOX}

The variables which can be viewed and/or changed are identified by a number. The variables and their corresponding number identifier are given in reference [3].

When the "-" key is pressed, the current number identifier is displayed on the scope. This number may be changed by depressing the desired numeric keys followed by another "-" key or left unchanged by pushing down only on the "-" key. In either case the second "-" key will cause the program to display the value of the indicated variable. The value may be changed by the same procedure as indicated above for changing indicator values. The thira depression of the "-"key terminates the numeric input. 
The "+" may be used to initialize the box at any point. This avoids entering in mistyped numbers.

[1] Member, Computer Science Branch, Technical Services Division, Aerojet Nuclear Company.

[2] Nuclear Technology Branch Annual Progress Report for Period Ending June 30, 1969, IN-1317, p. 357 (1970).

[3] W. W. Black and C. W. Richardson, User's Manual for the Multiparameter Data Acquisition Program COINC Version 5B with Teletype and Panel Controls, Idaho Nuclear Corporation Manual. 
DEVICE-LEVEI SOFTWARE FOR A PDP-9 OR - 15 SERIAI DATA MULTIPLEXER (LT-19) AND A COMPUTEK DISPLAY TERMINAL

R. C. Davies ${ }^{[1]}$

The LI-19 software is a Digital Equipment Corporation compatible handler. It is written for a multi-user environment from the LT-19 multiplexer. The handler is general in the sense that, with very few program changes, it will function as desired. It must be noted that although not every data structure is implemented, the hander does give the user the data in a form that he can easily manipulate.

A device handler has also been written to use the Computek terminal as an ASCII input/output terminal. The handler functions in much the same way as the teletype handler does under the KM9-I5 monitor. It is intended to add vector capabilities to the handler in the near future.

Both of these handlers operate under the KM9 or KMI 5 keyboard monitors and can be used in either a single-level or automatic priority interrupt.

[1] Member, Computer Science Branch, Technical Services Division, Aerojet Nuclear Company. 


\section{A. SMALL FLOATING POINT ARITHMETIC PACKAGE FOR THE PDP-8 FAMILY OF MINICOMPUTERS}

W. R. Myers

The typical PDP-8 computer installation has only 4096 words of core memory; the floating point arithmetic package supplied by Digital Equipment Corporation requires 1024 of these locations to implement six floating point operations, formatted output, free-format input, and the square root function. 1408 words are required if the exponent, logarithm, arctangent, sine and cosine functions are included.

A more versatile package has been written which requires only 832 locations for fourteen floating mode instructions, formatted output and free-format input. A total of 1152 locations are required if the function subroutines are included. The application program can thus utilize 6 to 10\% more of the core memory and may be written to make better use of the memory because of the more versatile floating mode instruction set. 
A COMPREHENSIVE BASIC DATA ACQUISTTION AND CONTROL MONITOR FOR THE PDP-9 AND PDP-15 COMPUTERS

W. R. Myers

Because of the many applications in which Digital Equipment Corporation PDP-9 and PDP-15 computers are used at NRTS, a comprehensive skeleton data acquisition-oriented control program was written. This program will serve as the base structure on which most applications programs will be built.

The monitor includes control routines for all of the standard peripheral devices, paper tape reader and punch, teletype keyboard and printer, drum, disc, DECtape, clock and program refreshed display. It includes a keyboard command decoding structure and special routines to allow conversational interaction from the teletype. There is a multi-level priority program queuing routine, a resident floating point arithmetic interpreter, and a resident on-line program debugging capability.

The basic program occupies 2500 memory locations, but major software modules may be deleted for special applications. All sections are commented in great detail, so that the applications programmer may easily understand and modify the coding to suit particular needs. The program has been tested in several demonstration applications. 
-

- 
I. COOPERATIVE PROGRAMS WITH EDUCATIONAL INSTITUTIONS 
○

- 
As the major operating contractor at the NRTS, Idaho Nuclear Corporation continued to stress the importance that the U. S. Atomic Energy Commission places on the stimulation and support of education. The program of the Nuclear Technology Division continued to emphasize activities to strengthen ties between the research programs at the National Reactor Testing Station and those presently being conducted by regional universities. This effort, which had enthusiastic response in past years, was limited in $\mathrm{FY}-71$ because of funding curtailments, both within the $\mathrm{AEC}$ and at the universities. However, within these limits the Nuclear Technology Division continued to encourage and implement cooperative educational programs in a variety of ways. Examples of activities in this area during the period covered in this report are briefly described below.

\section{Idaho Nuclear Corporation Educational Fund}

Idaho Nuclear Corporation contributed $\$ 125,059$ this past year to educational activities with a portion of these contributions related to programs within the Nuclear Technology Division. Such connected funding during FY-71 was a grant of $\$ 5,000$ to the University of Utah in support of their study of the MTR as a National Neutron Center for Interdisciplinary Research.

\section{Support of Secondary Schools}

The staff of the Nuclear Technology Division has been engaged in a number of cooperative programs with the local school districts. They are assisting the personnel of Idaho School District \#91 in implementing their two small computers into programs for computer assisted instruction (CAI) and computer techniques in problem solving. 


\section{Association of Western Universities Program}

Under the auspices of the Association of Western Universities (AWU) scientists are able to visit and use the facilities of the National. Reactor Testing Station to conduct research projects, participate in existing programs, or carry out independent research leading to advanced degrees. During the Fiscal Year 1971 the following participated: (1) Mr. Larry Kroger, a student from the University of Wyoming continued his $\mathrm{PhD}$ research on the nuclear level scheme studies of selected actinide nuclei; (2) Mr. J. Huckabee, of the University of Wyoming, completed his $\mathrm{PhD}$ thesis research on studies of trace elements in antelope by activation analysis; (3) Mr. R. A. Lokken of Utah State University conducted $\mathrm{PhD}$ thesis research in spallation-reaction product cross sections; (4) Dr. D. J. McMilian of Vanderbilt completed the term of his AEC Postdoctoral Fellowship, during which he did research on nuclear level structure; (6) Mr. R. G. Hughes, a student from Utah State University, carried out research on beta-ray spectrum shapes; (7) Mr. Clinton Schaeffer of New Mexico University is participating in research on the gas-core reactor program; (8) Mr. R. S. Kunter, University of Idaho, participated in microprobe evaluations of fission products in fuels; and (9) R. R. Perkes, R. L. Speth and L. O. Weber of Ricks College, Rexburg, Idaho participated in neutron activation analysis studies of soil. 


\section{Adjoint Professorships}

In several of the situations where a graduate student at a regional university has completed his course work toward an advanced degree and plans to carry out thesis research at the NRTS, arrangements are made whereby the staff member of the Nuclear Technology Division responsible for supervising the on-site research is appointed to the university staff as an Adjoint Professor. This arrangement has in the past proved to be a highly beneficial one, greatly improving the communication between the university and the NRTS. During this fiscal year, four member of the Division have served in this capacity with Utah State University. They are J. E. Cline, R. L. Heath, R. G. Helmer and C. W. Reich.

J. F. Kunze was one of the 2 principal teachers in a 5-day, 2-credit course at the University of Utah on "Nuclear Power-Benefits and Risks"; the course was designed specifically for high school science teachers. In addition, he served as a Visiting Associate Professor in Mechanical Engineering at the University of Utah, teaching about $I$ day per month and consulting on nuclear engineering problems.

\section{NRTS Eaucational Program}

Idaho Nuclear Corporation, in cooperation with the University of Idaho, administers a continuing educational program at the NRTS. This program leads to the degree of Master of Science in various technical. fields. The following members of the Nuclear Technology Division taught courses: J. J. Scoville, O. D. Simpson, C. R. Brinkman, G. E. Korth and S. R. Gossman.

\section{University Seminars and Colloquium Presented}

The staff of Nuclear Technology is frequently called upon to provide seminars and colloquia at universities. In FY-71 these were:

Staff

R. M. Brugger

J.F. Kunze

C. R. Brinkman

C. W. Reich
University

University of Missouri

University of New Mexico

University of Utah

Utah State University
Topic

Thermal Reactor Research

The NRTS Gas-Core Reactor

Techniques at INC in Fatigue Testing

Systematic Features in the Level Structure of Deformed Odd-Mass Nuclei 
-

- 
J. RELATED ACTIVITIES OF THE BRANCH PERSONNEL 
-

- 
REIATED ACTIVITIES OF DIVISION PERSONNEL

Besides their direct research accomplishments and their activities in support of education, Division personnel are often called upon to assume other professional responsibilities and duties. During the past year the following staff members have been so engaged:

J.M. Beeston: Member, ASTM Committee, E-10 Subcommittee on Radiation Induced Changes in Metallic Materials, AEC.

J.R. Berreth: Member, Research Materials Committee, AEC.

R. M. Brugger: Program Committee of Reactor Physics Division of American Nuclear Society; Nuclear Cross Sections Advisory Committee, Subcommittee on Evaluation of Thermal Cross Sections of NCSAC; Applied Physics Division, Argonne National Laboratory Review Committee, AEC; Reviewer, Nuclear Technology.

T. J. Boland: Advisory Committee, Electronics Technology, ISU.

E. E. Burdick: ROD Program Committee, Denver Meeting, ANS: General Chairman, RSTD Division Meeting, ANS, Idaho Falls, Idaho 1972 Meeting; Local Sections Committee, ANS.

C. R. Brinkman: Irradiation Effects on Reactor Structural Materials Committee, AEC; Elevated Temperature Behavior Task Force on Creep-Fatigue, ASME.

J. E. Cline: Reviewer for Phys. Rev. and J. Nucl. Applications; Alternate, Nuclear Detector Subcommittee, lvational Academy of Sciences; Thermal Cross Sections and Radioactive Samples Subcommittee, NCSAC.

W. C. Francis High Temperature Fuels Committee, AEC; Secretary, Nuclear Components Committee; American Society of Nondestructive Testing, Nuclear Metallurgy Committee, American Institute of Mining, Metallurgical and Petroleum Engineers (TMS).

R. A. Grimesey: Cross Sections Evaluation Working Group, AEC.

R. L. Heath: Member, Board of Editors, Nuclear Instruments and Methods; Contributing Editor, Handbook of Chemistry and Physics; Member, National Technical Program Committee, ANS; Member, Executive Committee, I \& R Division, ANS; Reviewer for the Physical Review, Analytical Chemistry, Nuclear Applications and Nuclear Science and Engineering; Chairman, Nuclear Detector Subcommittee, National Academy of Sciences.

R. G. Helmer: Reviewer for the Physical Review.

C. H. Hogg: Member, ASTM Committee, E-IO Subcommittee on Neutron Dosimetry, AEC; Irradiation Standards and Procedures Committee of the AEC Irradiation Effects on Reactor Structure Materials Program. 
N. C. Kaufman: Finance Chairman, RSTD Division Meeting, ANS, Idaho Falls, Idaho 1972 Meeting.

J.F. Kunze: Secretary, Reactor Physics Division of Alvs; Reviewer for Nuclear Applications; Member of Reactor Safety Committee, University of Utah; Education Committee, Idaho Society of Professional Engineers.

H. I. McMurry: Reviewer, Nuclear Science and Engineering, Air Pollution Committee (on Polycylic Organic Matter) of the National Academy of Sciences; Mathematics Committee, Idaho School District \#91.

F. A. Meichle: Vice President, Idaho Section of Instrument Society of America.

L. G. Miller: Neutron Radiography Standards Committee, American Society of Nondestructive Testing.

D.A. Pearson: Alternate, ASTM Committee, E-10, Subcommittee on Neutron Dosimetry.

C. W. Reich: Reviewer for Nuclear Physics and J. Inorganic and Nuclear Chemistry.

I. A. Rowett: General Secretary, RSTD Division Meeting, September I972, Idaho Falls, Idaho Meeting, ANS.

J. J. Scoville: Reviewer for Nuclear Science and Engineering.

F. B. Simpson: Ex officio Member of Transplutonium Committee, AEC.

O. D. Simpson: Alternate, Nuclear Cross Sections Advisory Committee, AEC.

J. R. Smith: Member, Cross Sections Evaluation Working Group, AEC.

K. A. Strong: Reviewer for J. Chem. Phys.

E. H. Turk: Awards Committee, Idaho Section of American Chemical Society.

R. E. Schmunk: Reviewer for J. Phys. Chem. Solids, and J.Appl. Phys.

G. K. Wachs: National Shielding Information Center Advisory Committee, AEC. 
K. PUBLICATIONS 
-

- 


\section{NUCLEAR TECHNOLOGY DIVISION}

Presentation of Technical Papers

\section{$\operatorname{AUTHOR}(\mathrm{S})$ \\ E. B. Nieschmidt}

J. E. Cline

R. L. Heath

P. D. Randolph
R. C. Greenwood

C.W. Reich

J. J. H. Berlijn

C. W. Reich

R. G. Helmer

R. C. Greenwood

G. E. Korth

C. R. Brinkman

G. W. Gibson

J. E. Cline

B. G. Carlson

E. I. Murri
SUBJECT

Measurement of Spallation CrossSections for $590 \mathrm{MeV}$ Protons on Thick and Thin Targets of Iron and Nickel

Application of High-Resolution Photon Spectrometry to Analytical Problems

The Engineering of the Isotopic Snow Profiling Gage

General Information Exchange Meeting Present Progress Report and Future Plans

Octupole Vibrational Bands in $174 \mathrm{Yb}$

Coriolis-Mixing Analysis of Rotational Bands in $182 \mathrm{Ta}$

The Effects of Fast Neutron

Irradiation on the Mechanical Properties of Hafnium

Assaying of Boron in Nuclear Reactor Fuel Using ${ }^{60} \mathrm{Co}$ Tagging
GROUP

IEEE Conference

National Meeting, Applied Spectroscopy

Idaho Nuclear Energy Commission and Soil

Conservation Service

PLACE, DATE

San Diego, California July 21-23, 1970

New Orleans, Louisiana October 4-11, 1970

Sun Valley, Idaho october 27-28, 1970

Richland, Washington october 13-15, 1970

Houston, Texas American Physical
Society, Nuclear Physics Division

American Physical Society, Nuclear Physics Division

Metallurgical Society

Cleveland, Ohio october 19-22, 1970

American Society of

Cleveland, Ohio Non-Destructive Testing, Fall Conference 


\section{$\operatorname{AUTHOR}(S)$}

T. Watanabe

L. G. Miller

O. D. Simpson

J. R. Smith

J. W. Rogers

J. H. Lof thouse

J. F. Kunze

J. J. Scoville

R. G. Nisle

B. W. Howes

U Y Y. D. Harker

D. A. Pearson

A. L. Conneliy

J. J. Scoville

Y. D. Harker

N. H. Marshall

O. D. Simpson

J. R. Smith

\section{SUBJECT}

Increased Neutron Radiographic

Capability of ${ }^{252} \mathrm{Cf}$ with a

Subcritical Assembly

Filtered Beam Techniques

Spherical Gas Core Reactor

Physics Measurements

Measurements of Fast Neutron Capture

Integrals in the CFRMF

Comparison of Foil Activation

Techniques and Other Methods in Fast

Neutron Spectrum Measurements

On-Line, Real-Time Power Spectrum Measurement

The Effect of Cross Section Variation on Integrals Measured in the CFRMF

Fast-Neutron Capture Integral Measurements of $99 \mathrm{Tc}, 109 \mathrm{Ag}$ and Other Fission Products

The Automated Cross Section Analys is Program (ACSAP)
GROUP

American Society of Non-Destructive Testing, Fall Conference

EANDC Symposium on Neutron Standards and FIux Normalization

American Nuclear Society, Winter Meeting

American Nuclear

Society, Winter Meeting

American Nuclear

Society, Winter Meeting

Digital Equipment

Computer User's Society (DECUS) FalI Symposium

Third Conference on

Neutron Cross Sections and Technology

Third Conference on

Neutron Cross Sections and Technology

Third Conference on Neutron Cross Sections and Technology
PLACE, DATE

Cleveland, Ohio october 19-22, 1970

Argonne, Illinois October 21-23, 1970

Washington, D.C. November 15-19, 1970

Washington, D.C. November 15-19, 1970

Washington, D.C. November 15-19, 1970

Houston, Texas November 19-21, 1970

Knoxville, Tennessee March 15-17, 1971

Knoxville, Tennessee March 15-17, 1971

Knoxville, Tennessee March 15-17, 1971 
$\operatorname{AUTHOR}(S)$

O. D. Simpson

J.R. Smith

J. W. Rogers

S. Gebhardt

J. E. Cline

F. Shields

L. Pearson

D. Brock

F. Shields

E. Norberg

J. E. Cline

R. A. Grimesey

G. L. Singer

W. E. Vesely

F. J. Wheeler

J. M. Beeston

R. I. Heath

R. S. Frankel

R. J. Gehrke

J. Barstow

R. G. Helmer

R. C. Greenwood

R. J. Gehrke

\section{SUBJECT}

Neutron Filtered Beams as Standard Sources

Mercury Residue in Idaho Fish

An Analysis of Mercury Residue

in Idaho Pheasants

Some Problems Encountered

Processing ENDF/B Data with ETOG and ETOP

The RAFFLE Monte Carlo Code and Its Use to Obtain Correlated Estimates of Collapsed Cross Sections

Effects of Radiation on Structural Metals

Energy Dispersive X-ray

Spectrometry with Computer Data

Reduction

Measurement of Precise $\gamma$-ray Energies with Detectors - 400 to $1300 \mathrm{keV}$

PIACE, DATE

Third Conference on Neutron Cross Sections and Technology

Conference on Mercury in the Western Environment

Conference on Mercury in the Western Environment

American Nuclear

Society Topical Meeting

American Nuclear

Society Topical Meeting

on Reactor Mathematics

ASTM Symposium

National Symposium of the ISA Analytical Instrument Division

American Physical Society
Knoxville, Tennessee March 15-17, 1971

Portland, Oregon

February 25-26, 1971

Portland, Oregon

February 25-26, 1971

Idaho Falls, Idaho

March 29-31, 1971

Idaho Falls, Idaho

March 29-31, 1971

Niagra Falls, New York June 29-July 1, 1970

Austin, Texas

Apri1 19-21, 1971

Washington, D. C. Apri1 26-29, 1971 
$\operatorname{AUTHOR}(S)$

R. C. Greenwood

C. W. Reich

R. C. Davies

J. E. Cline

P. D. Randolph

E. I. Murri

M. J. Laird

R. I. Heath

D. Brock

F. Shields

E. Norberg

J. E. Cline

M. J. Graber

G. W. Gibson

R. E. Schmunk

R. J. Gehrke

R. A. Lokken

J. F. Kunze

J. H. Lofthouse

D. H. Suckling

R. E. Hyland
SUBJECT

Absolute Cross Sections for $2-\mathrm{keV}$

Neutron $20{ }^{4} \mathrm{~Pb}$ and $207 \mathrm{~Pb}$

Operating the Keyboard Monitor from Disk or Drum Using DECtape or

Magnetic Tape

Elemental Determinations in Idaho Wildiffe Samples

An Analysis of Mercury Residue

in Idaho Pheasants

A Metallurgical Evaluation of

Simulated BWR Emergency Core

Cooling Tests

Efficiency Calibration of a Low

Energy Si(Ii) Photon Spectrometer

Flow-Mixing, Reactivity Effects in

the Gas Core Reactor
GROUP

American Physical

Society

Symposium of Digital

Equipment Computer User's

Society

Idaho Academy of Science and Northwest Scientific Association

Idaho Academy of Science and Northwest Scientific Association

American Nuclear Society

American Nuclear Society

American Nuclear Society
PIACE, DATE

Washington, D.C. ApriI 26-29, 1971

Atlanta, Georgia May 13-15, 1971

Moscow, Idaho April 15-17, 1971

Moscow, Idaho Apri1 15-17, 1971

Boston, Massachusetts June 13-17, 1971

Boston, Massachusetts June 13-17, 1971

Boston, Massachusetts June 13-17, 1971 
NUCLEAR TECHNOLOGY DIVISION

Papers Published in the Open Literature

AUTHOR (S)

R. A. Grimesey

W. Serrano

R. A. Dannels

T. Watanabe

S. D. Reeder

A. I. Conneliy

W. W. BIack

R. C. Greenwood

C.W. Reich

S. H. Vegors, Jr.

J. W. Codding, Jr.

R. I. Tromp

F. B. Simpson

R. M. Brugger

G. J. RusselI

B. W. Johnson

G. P. DeVault

C. W. Reich

J. E. Cline

T. E. Young

F. B. Simpson

R. E. Tate

J. M. Beeston

\section{TITLE}

Legendre Moments of the Elastic-Scattering Cross Section for $s$-Wave Neutrons

Total Neutron Cross Section of Technetium-99

from 0.01 to $1000 \mathrm{eV}$

Automatic Location and Area Determination of Photopeaks

Excited $\mathrm{K}^{\pi}=0^{+}$Bands in $172 \mathrm{Yb}$

Total Neutron Cross Section and Resonance

Parameters of Promethium-147

Very Intense Neutron Source (VINS)

Gamma-Ray Studies of the $\gamma$-Vibrational Band of 166 Er

The Low-Energy Total Neutron Cross Section of Plutonium-242

Beryllium Metal as a Neutron Moderator and Reflector Material

\section{PUBI,ICATION}

Nuclear Science and Engineering 41, 140-143 (July 1970)

Nuclear Science and Engineering 41, 188-192 (August 1970)

Nuclear Instruments and Methods 82, 141-148 (1970)

Physics Letters 33B, 213-215 (1970)

Nuclear Science and Engineering (January 1971)

Nuclear Technology, 10, 188-203 (February 1971)

Nuclear Physics A159, 181-201 (1970)

Nuclear Science and Engineering 43, 341-342 (March 1971)

Nuclear Engineering and Design 14, 445-474 (1970) 
NUCLEAR TECHNOLOGY DIVISION

Papers Published in the Open Literature

$\operatorname{AUTHOR}(\mathrm{S})$

R. J. Gehrke

J. E. CIine

R. L. Heath

C. R. Brinkman

J. M. Beeston

J. M. Beeston

C. R. Brinkman

$\underset{I}{U}$

I. D. MeIsaac

E. L. Murri

W. Benson

D. Brock

J. Gibica

J. E. Cline

R. G. Helmer

R. C. Greenwood

C. W. Reich

C.W. Reich

R. G. Helmer

R. C. Greenwood

\section{TITLE}

Determination of Relative Photopeak Efficiency and System Linearity for Ge(Li) Gamma-Ray Spectrometers

The Effect of Hydrogen on the Ductile Properties of Irradiated Pressure Vessel Steels

Axial Fatigue of Irradiated Stainless Steels Tested at Elevated Temperatures

Decay Scheme Studies of $18 \mathrm{~min}{ }^{14} \mathrm{Ba}$ and $11 \mathrm{~min}$ $142 \mathrm{Ba}$

Mercury in Idaho Pheasants and Other Gamebirds

Level Structure of ${ }^{182} \mathrm{Ta}$

Coriolis Coupling in Doubly oda Deformed Nuclei The $\frac{1}{2}-[510]$ and $\frac{3}{2}[512]$ Neutrons States in $182 \mathrm{Ta}$
PUBLICATION

Nuclear Instruments and Methods 11, 349-356 (1971)

Irradiation Effects on Structural Alloys for Nuclear Reactor Applications, ASTM STP 484, pp. 42-73 (1970)

Irradiation Effects on Structural Alloys for Nuclear Reactor Applications, ASTM STP 484 , (1970)

Nuclear Physics A156, 212-224 (November 1970)

Journal of Pesticide Research (in press)

Nuclear Physics Al68, 449-486 (June 1971)

Nuclear Physics Al68, 487-508 (June 1971) 
IN-1407 Nuclear Technology Branch Annual Progress Report for Period Ending June 30, 1970, R. M. Brugger and Staff, December 1970

IN-1409 The Use of Valence Force Potentials in Calculating Crystal Vibrations, II. 'Dipole Coordinate' Approximation for Longand Intermediate-Range Forces, A. W. Solbrig, Jr., July 1970

IN-1410 Application of Solbrig's Cell-Dipole Method to Molecular Crystals, H. L. McMurry, October 1970

IN-1411 Two Contributions to the Theory of Digital Filtering, A. I. Connelly, July 1970

IN-1416 CORA - A Few Group Diffusion Theory Code for One-Dimensional Reactor Analysis, E. C. Anderson and G. E. Putnam, August 1970

IN-1421 Molecular Potential Function in Terms of Group Valence Force Symmetry Coordinates, H. L. McMurry, April 1971

IN-1424 Valence Force Potentials for Calculating Crystal Vibrations in Silicon, A. W. Solbrig, Jr., September 1970

IN-1427 The Slow Neutron Inelastic Scattering Program at the MTR (1956-1970), R. M. Brugger and Staff, September 1970

IN-1433 A Technique for Assay of L-IO Bottles of Plutonium Nitrate, J. E. Cline, E. B. Nieschmidt, A. L. Connelly and E. L. Murri, October 1970

IN-1435 PHROG - A FORTRAN IV Program to Generate Fast Neutron Spectra and Average Multigroup Constants, R. L. Curtis, G. L. Singer, F. J. Wheeler and R. A. Grimesey, April 1971

IN-1437 Metallurgy and Materials Science Branch Annual Report Fiscal Year 1970, W. C. Francis, Editor, November 1970

IN-1440 The Effect of Fast Neutron Irradiation on the Mechanical Properties of Hafnium, G. E. Korth, C. R. Brinkman, J. M. Beeston, October 1970

IN-1443 Spherical Gas Core Reactor Critical Experiment, J. H. Lofthouse, and J. F. Kunze, February 1971 (NASA CR-7278I)

IN-1448 Gamma Rays Emitted by the Fissionable Nuclides and Associated Isotopes, J. E. Cline, December 1970

IN-I420 Electron Ground States in Molecular and Valence Bonded Crystals, H. L. McMurry, October 1970

IN-1452 Ultrasonic Level Monitor, T. J. Boland, J. B. Thompson, March 1971 
IN-I453 A Metaliurgical Evaluation of Simulated BWR Emergency Core Cooling Test, M. J. Graber, W. F. Zelezny and R. E. Schmunk, May 1971

LA-DC-12255 A Survey of Nonrotational States of Deformed Odd-A Nuclei $(150<A<190)$, M. E. Bunker, (LASL) and C. W. Reich (1971) 